RECEIVED

MAR 041996

OSTI

OAK RIDGE NATIONAL LABORATORY

MARTIN MARUETRA

\title{
Circular Arc Fuel Plate Stability Experiments and Analyses for the Advanced Neutron Source
}

\author{
W. F. Swinson \\ R. L. Battiste \\ G. T. Yahr
}

August 1995

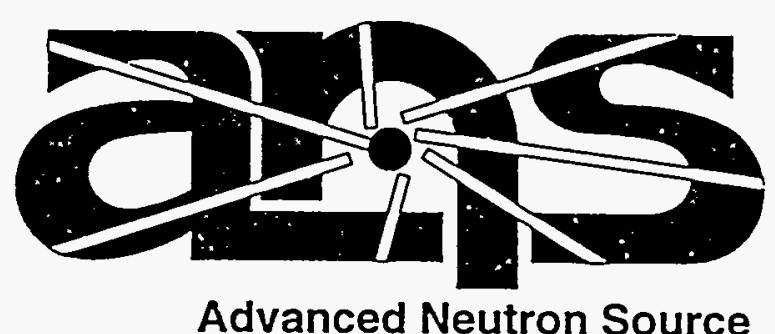

MANAGED BY

MARTIN MARIETTA ENERGY SYSTEMS, INC.

FOR THE UNITED STATES

DEPARTMENT OF ENERGY 
This report has been reproduced directly from the best available copy.

Available to DOE and DOE contractors from the Office of Scientific and Technical Information, P.O. Box 62, Oak Ridge, TN 37831; prices available from (615) 576-8401, FTS 626-8401.

Available to the public from the National Technical Information Service, U.S. Department of Commerce, 5285 Port Royal Rd., Springfield, VA 22161.

This report was prepared as an account of work sponsored by an agency of the United States Government. Neither the United States Government nor any agency thereof, nor any of their employees, makes any warranty, express or implied, or assumes any legal liability or responsibility for the accuracy, completeness, or usefuiness of any information, apparatus, product, or process disclosed, or represents that its use would not infringe privately owned rights. Reference herein to any specific commercial product, process, or service by trade name, trademark, manufacturer, or otherwise, does not necessarily constitute or imply its endorsement, recommendation, or favoring by the United States Government or any agency thereof. The views and opinions of authors expressed herein do not necessarily state or reflect those of the United States Government or any agency thereof. 
Engineering Technology Division

\title{
CIRCULAR ARC FUEL PLATE STABILITY EXPERIMENTS AND ANALYSES FOR THE ADVANCED NEUTRON SOURCE
}

\author{
W. F. Swinson \\ R. L. Battiste \\ G. T. Yahr
}

August 1995

\author{
Prepared by the \\ Oak Ridge National Laboratory \\ Oak Ridge, Tennessee 37831 \\ managed by \\ LOCKHEED MARTIN ENERGY SYSTEMS, INC. \\ for the \\ U.S. DEPARTMENT OF ENERGY \\ under contract DE-ACO5-84OR21400
}




\section{TABLE OF CONTENTS}

LIST OF FIGURES $\ldots \ldots \ldots \ldots \ldots \ldots \ldots \ldots \ldots \ldots \ldots \ldots \ldots \ldots \ldots \ldots$

ACRONYMS AND SYMBOLS $\ldots \ldots \ldots \ldots \ldots \ldots \ldots \ldots \ldots \ldots \ldots \ldots$

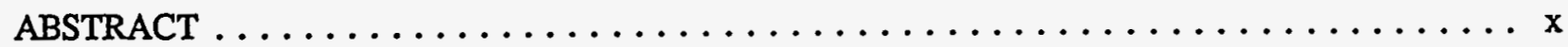

1. INTRODUCTION $\ldots \ldots \ldots \ldots \ldots \ldots \ldots \ldots \ldots \ldots \ldots \ldots \ldots \ldots \ldots \ldots$

2. ANALYTICAL ASSESSMENT OF CIRCULAR ARC PLATES $\ldots \ldots \ldots \ldots \ldots \ldots$

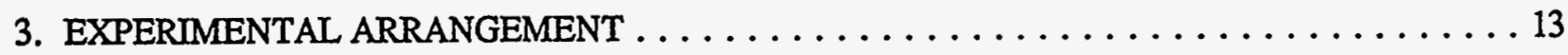

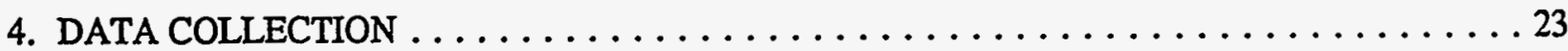

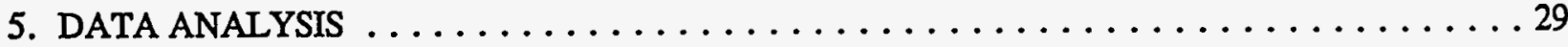

6. CONCLUSIONS ............................... 41

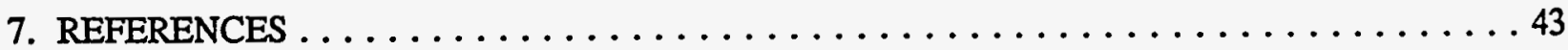

Appendix A: MODEL THEORY $\ldots \ldots \ldots \ldots \ldots \ldots \ldots \ldots \ldots \ldots \ldots \ldots \ldots \ldots \ldots \ldots$

Appendix B: ESTIMATE OF FLOW BLOCKAGE EFFECTS $\ldots \ldots \ldots \ldots \ldots \ldots \ldots \ldots \ldots$

Appendix C: THERMAL STRESSES IN THE PLANE OF THE ARC WITH ONE

BOUNDARY SUPPORT FLOATING $\ldots \ldots \ldots \ldots \ldots \ldots \ldots \ldots$.

Appendix D: DATA COLLECTED $\ldots \ldots \ldots \ldots \ldots \ldots \ldots \ldots \ldots \ldots \ldots \ldots \ldots \ldots \ldots \ldots$ 



\section{LIST OF FIGURES}

Figure

Page

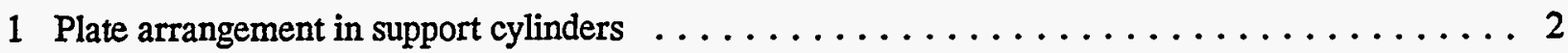

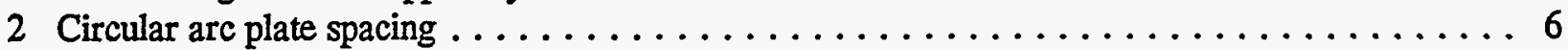

3 Free body diagram of circular arc plate with pressure load. . . . . . . . . . . . . 7

4 Free body diagram of circular arc with dummy load $\ldots \ldots \ldots \ldots \ldots \ldots \ldots$

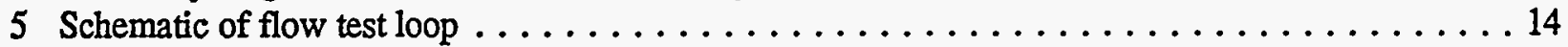

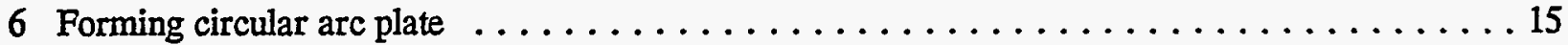

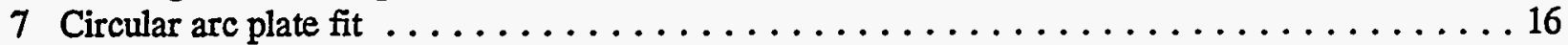

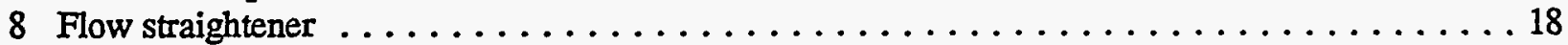

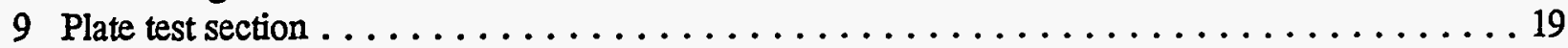

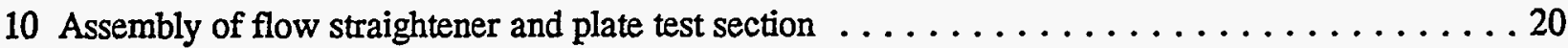

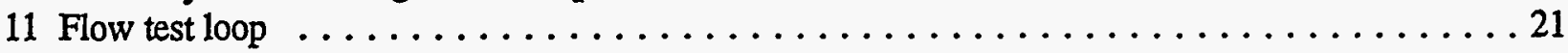

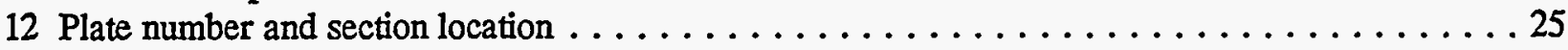

13 Failed test plates . . . . . . . . . . . . . . . . . . . . . . . 27

14 Pressure difference for plate 13 with respect to distance along plate. . . . . . . . . . . 30

15 Average pressure difference as a function of flow velocity. $\ldots \ldots \ldots \ldots \ldots \ldots \ldots \ldots$

16 One-quarter cross section pressure difference related to flow velocity $\ldots \ldots \ldots \ldots \ldots \ldots \ldots$

17 One-half cross section pressure difference related to flow velocity $\ldots \ldots \ldots \ldots \ldots \ldots \ldots$

18 Deflection of the three central plates as related to the flow velocity $\ldots \ldots \ldots \ldots \ldots \ldots$

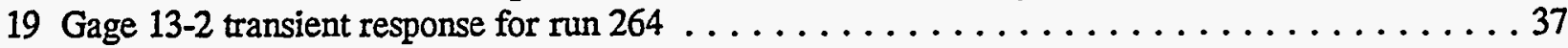

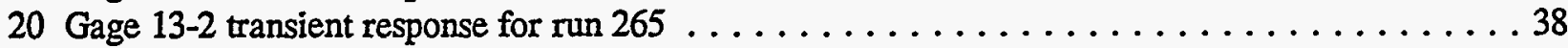

21 Pressure drop for Series $I$ and for Series $\Pi$ data . . . . . . . . . . . . . . . . . . 39

22 Pressure difference across plates $14,13,12$ at entrance, Series $\Pi \ldots \ldots \ldots \ldots \ldots \ldots \ldots$ 



\section{ACRONYMS AND SYMBOLS}

ANS Advanced Neutron Source

A longitudinal section area per unit length

$A_{b} \quad$ area of wake caused by flow blockage

E modulus of elasticity

$F \quad$ dummy load on plate and force on plate caused by blockage

$f_{\alpha} \quad$ component of the plate outer boundary force along the tangent direction of the outside support cylinder

$f_{\text {ro }} \quad$ component of the plate outer boundary force along the radial direction to the outside support cylinder

$h$ channel thickness

I area moment of inertia

$L \quad$ dimension parameter

$l_{r} \quad$ projection of plate along support cylinder radius

$M \quad$ moment in plate

$\mathrm{M}_{1} \quad$ plate outer boundary moment caused by thermal load

$\mathrm{M}_{\varphi} \quad$ plate outer boundary moment caused by pressure load

$M_{0} \quad$ plate inner boundary moment caused by thermal load

$\mathrm{M}_{\mathrm{op}} \quad$ plate inner boundary moment caused by pressure load

$N \quad$ tangent load in plate

$N_{l} \quad$ plate outer boundary tangent load caused by thermal load

$N_{\varphi} \quad$ plate outer boundary tangent load caused by pressure load

$N_{0} \quad$ plate inner boundary tangent load caused by thermal load

$N_{c p} \quad$ plate inner boundary tangent load caused by pressure load

$p \quad$ pressure

$p_{d y n} \quad$ dynamic pressure

$\Delta p \quad$ average pressure difference across a plate

$r_{c} \quad$ circle arc radius

$r_{i} \quad$ inner support cylinder radius

$r_{0} \quad$ outer support cylinder radius

$s_{c} \quad$ channel spacing at outer boundary 


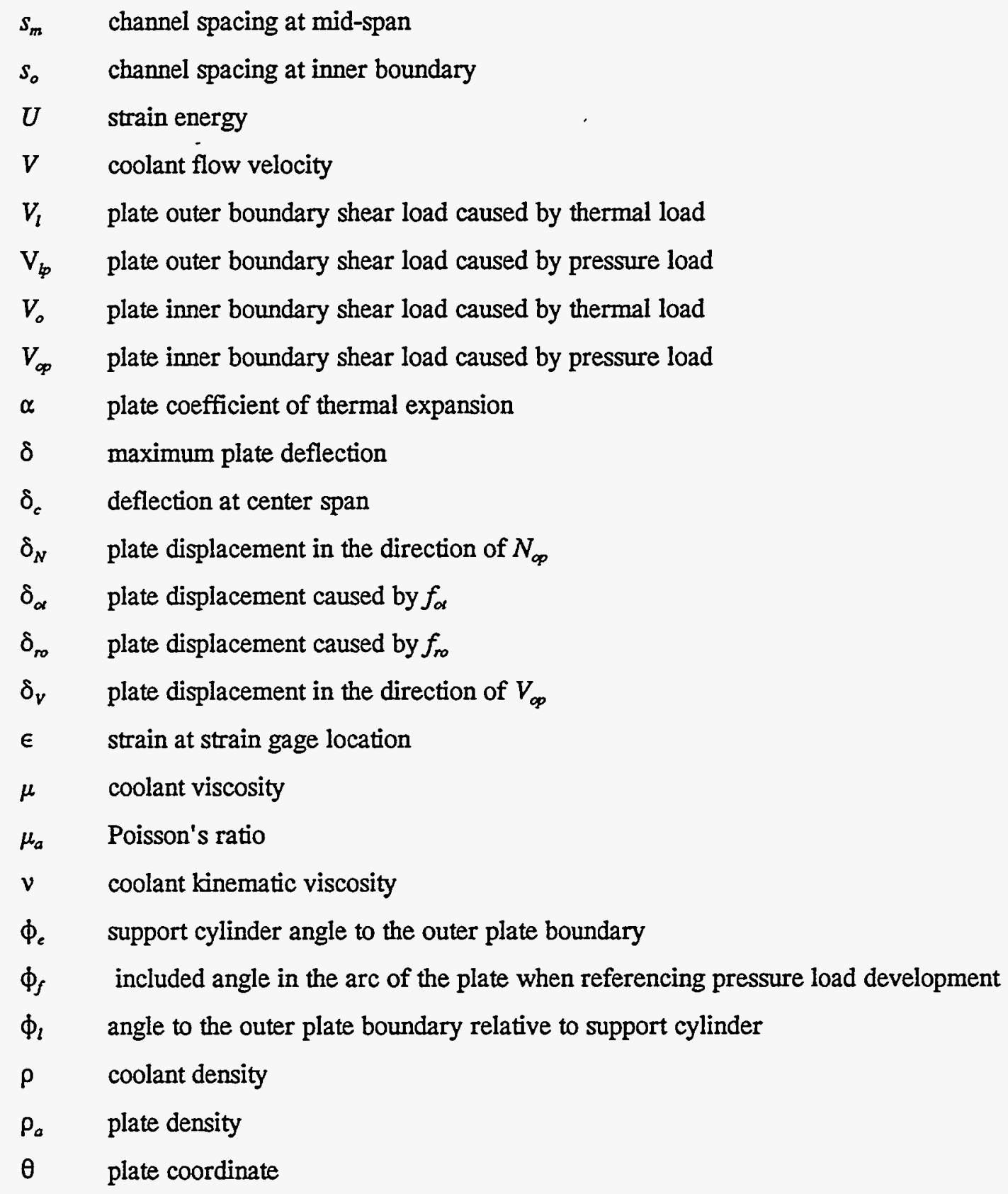


$\theta_{a} \quad$ included angle in the arc of the plate when referencing thermal load development

$\theta_{c}$ angle from origin to force, $F$, on plate

$\theta_{c} \quad$ plate tangent angle at outer support cylinder

$\theta_{\text {o }}$ plate tangent angle at inner support cylinder 


\begin{abstract}
The thin fuel plates planned for the Advanced Neutron Source are to be cooled by forcing heavy water at high velocity, $25 \mathrm{~m} / \mathrm{s}$, through thin cooling channels on each side of each plate. Because the potential for structural failure of the plates is a design concern, considerable effort has been expended in assessing this potential. As part of this effort, experimental flow tests and analyses to evaluate the structural reponse of circular arc plates have been conducted, and the results are given in this report.
\end{abstract}




\section{INTRODUCTION}

To arrive at a functional design for the Advanced Neutron Source (ANS) reactor, many topics and areas related to the design require investigation. One of these areas deals with the structural response of the fuel plates during reactor operation and is the subject of this report.

Figure 1 illustrates how thin, involute plates are arranged in the reactor. The plates consist of a uranium silicide fuel encased in aluminum. The plates are cooled by forcing coolant, heavy water, upward through narrow channels as illustrated. Principally, the loads exerted on the fuel plates come from the coolant flow and from temperature variations in the plates and their surroundings.

In assessing the structural response of the fuel plates to the coolant-flow load, four initial questions need to be answered:

1. What is the plate deformation as a function of coolant velocity?

2. What are the plate stresses as a function of coolant velocity?

3. What is the potential of a plate instability that would result in a sudden plate collapse at some specific coolant velocity?

4. What is the potential of a plate instability that would result in a resonance or flutter of the plate?

As the ANS design evolves, different plate geometries and coolant flow velocities will be required. Therefore, rather than answering the preceeding design questions for a single plate geometry and coolant velocity, techniques for calculating the answers to these design questions will be important in assessing the evolving design.

Basic to answering all of these questions is an understanding of the load imposed on the plates by a highly complicated flow field. Highly turbulent flow is not a continuum, and characterizing it as such for this application of loading the plates has not correlated with experiment. A considerable amount of the energy in the flow field is rotational and unknown. The flow in and out of a given control volume is three dimensional and unsteady. As applied to flow fields in the coolant channels, the flow in each channel is unique. The loads imposed on the plates by the coolant will be related to the pressure distribution on each side of a plate, the shear stresses on the plate surfaces, and some inertia loads. Loads that produce the most moment in the plate will induce the most deformation and highest stresses; thus, the pressure distribution on each side of a plate is the primary load variable and deserves the most attention.

Some limited answers to the design questions noted earlier have been found and reported in ORNL/TM-12353 ${ }^{1}$ and ORNL/TM-12629. ${ }^{2}$ The work centered on the fuel plates proposed for the ANS two-element core. Full-scale epoxy models of the involute fuel plates were fabricated, tested, and analyzed. The model data were related to the prototype through model theory. As related to the prototype, the answers found from these efforts included:

1. The maximum involute plate deflection is expected to be on the order of 6 to $8 \%$ of the channel height at an operating flow velocity of $25 \mathrm{~m} / \mathrm{s}$.

2. The magnitude of stresses induced in some regions of the plates were on the order of the yield stress of the aluminum material. Potential effects of the stress magnitudes on the plates and possible solutions to these potential problems are being assessed.

3. There was no evidence of a potential plate collapse in a coolant velocity range from 0 to 50 $\mathrm{m} / \mathrm{s}$. This was similar to the results found by Smissaert ${ }^{3}$ and Groninger and Kane, ${ }^{4}$ in working with flat plates. 


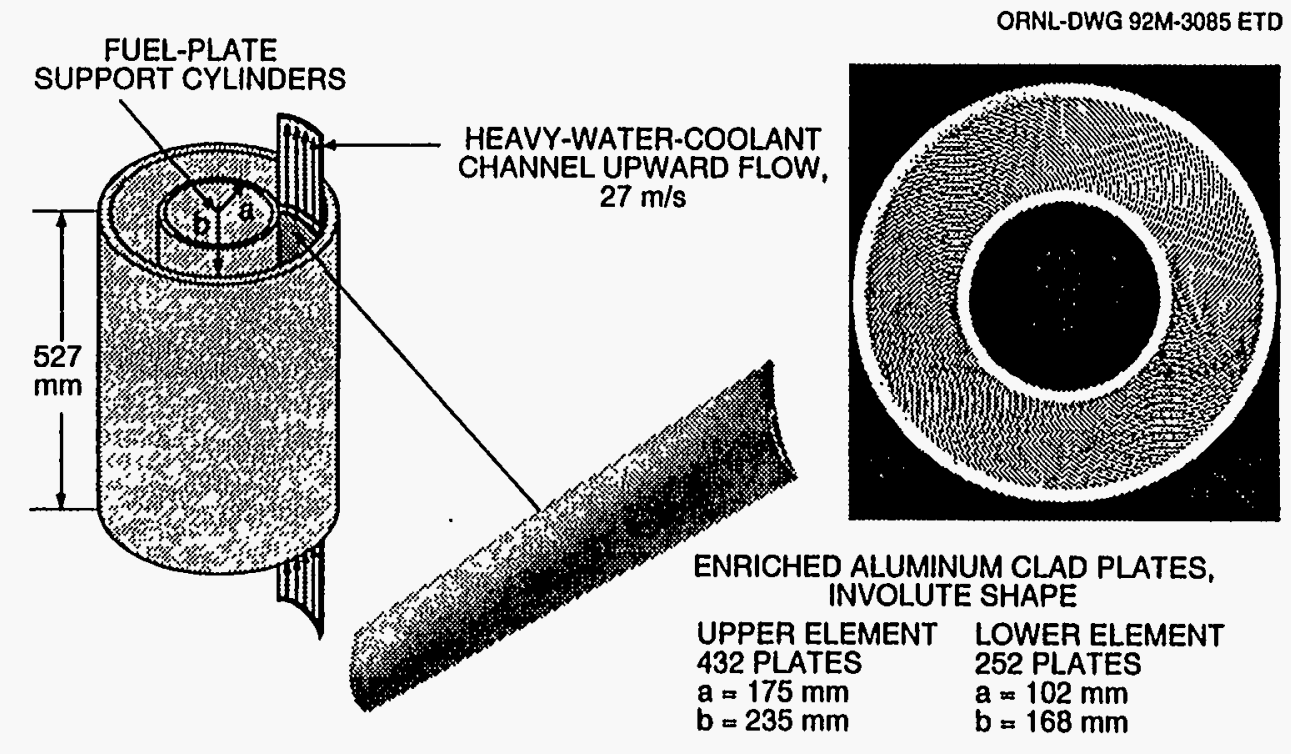

Fig. 1. Plate arrangement in support cylinders. 
4. There was no evidence of any significant resonance or flutter of the plates in the planned operating coolant velocity range. There was some evidence of a small amount of flutter in the upper plates at a prototype velocity of $50 \mathrm{~m} / \mathrm{s}^{2}$ However, using model laws intended for steady-state response to go from a model transient response to a prototype transient response is questionable. A more appropriate procedure for doing this was developed and is given in Appendix A. Results from this procedure indicate that no flutter should be anticipated in the coolant velocity range from 0 to $33 \mathrm{~m} / \mathrm{s}$.

In addition to the specific answers for the two-element core, the effort also indicated that the primary load on a plate was the difference in pressure distributions on each side of the plate.

Using the epoxy model data as a basis, some progress was made in developing techniques for calculating the deformation and stress in a plate with different geometry and at different coolant flow velocities. This part of the effort is reported in ORNL/TM-12712. ${ }^{5}$ An analytical solution for an involute plate subjected to a pressure load was developed using energy techniques. With the experimental data as a basis, an empirical relation was found that related the pressure load to the coolant flow velocity. This relation is

$$
\frac{\Delta p}{\rho V^{2}}=0.040\left(\frac{V h}{v}\right)^{0.177}
$$

where

$$
\begin{aligned}
& \Delta p=\text { average pressure difference across a plate } \\
& h=\text { channel thickness } \\
& V=\text { coolant flow velocity } \\
& \rho=\text { coolant density } \\
& v=\text { coolant kinematic viscosity. }
\end{aligned}
$$

With the pressure load of Eq. (1) and the analytical solution developed, deformation and stress in involute plates with different geometries and different coolant velocities can be calculated. The results correlated reasonably well for design purposes with the experimental results of the epoxy models.

At this point some two new questions evolved from the effort using the epoxy models and include:

1. Is the load in Eq. (1) an appropriate approximation of the load on the plates at prototypic velocities?

2. If the induced stress magnitudes in the involute plates prove to be a design problem, are there ways to alleviate the problem?

To answer these new questions, a flow experiment was designed. Question 1 could be answered by making the plates out of aluminum so that prototypic flow velocities could be realized. One answer to question 2 could be found by making the plates into circular arcs rather than involute in shape. Stress magnitudes in circular arcs from pressure loads are approximately one-fifth the stress magnitudes in the involute plates. 


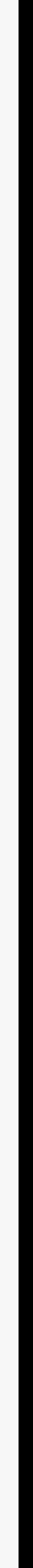




\section{ANALYTICAL ASSESSMENT OF CIRCULAR ARC PLATES}

The circular arc plates selected for the experiment were designed as an alternate for the involute plates found in the lower element of the two-element core. This involute plate had the largest deflection and stress magnitudes resulting from pressure loading and is where the biggest advantage in substituting a circular arc for the involute arc could be realized. The principal disadvantage of a circular arc instead of an involute is that the channel spacing is not uniform. An exact three-point match of the circular arc to the involute arc did not produce optimum channel spacing. Therefore, a trial and error procedure was used to find circular arc plates that had channel spacings of $1.27 \mathrm{~mm}$ at the plate support boundaries and minimized the channel spacing at mid-span. Data for the circular arc plates (Fig. 2) include:

$r_{\mathrm{i}}=102 \mathrm{~mm}$, inner support radius;

$r_{0}=168 \mathrm{~mm}$, outer support radius;

$\theta_{0}=70$ degrees, entrance angle;

$\theta_{\mathrm{e}}=13.12$ degrees, exit angle;

$\mathrm{r}_{\mathrm{c}}=86.11 \mathrm{~mm}$, circle arc radius;

$\mathrm{s}_{\mathrm{o}}=1.27 \mathrm{~mm}$, channel spacing at inside boundary;

$\mathrm{s}_{\mathrm{m}}=1.43 \mathrm{~mm}$, channel spacing at mid-span;

$\mathrm{s}_{\mathrm{s}}=1.27 \mathrm{~mm}$, channel spacing at outer boundary;

237 = number of plates that the lower element will accept;

$85.47 \mathrm{~mm}=$ the arc length of the plate; and

$0.537=$ void fraction, channel cross-sectional area divided

by total cross-sectional area of plates and channels.

An analytical solution for the circular arc plate was developed for finding the plate stresses and deformation when the load was pressure. The technique was based on energy concepts and is similar to the analytical solution for an involute plate that was reported in ORNL/TM-12712.5

A free body diagram of the circular arc plate loaded by pressure is shown in Fig. 3 . The moment equation at any section, $\theta$, is

$$
M=M_{o p}-V_{o p} r_{c} \sin (\theta)+N_{o p} r_{c}[1-\cos (\theta)]+p r_{c}^{2}[1-\cos (\theta)],
$$

where $M_{o p}=$ moment at the origin, $\theta=0$,

$V_{o p}=$ shear load at origin,

$N_{o p}=$ tangent load at origin. 


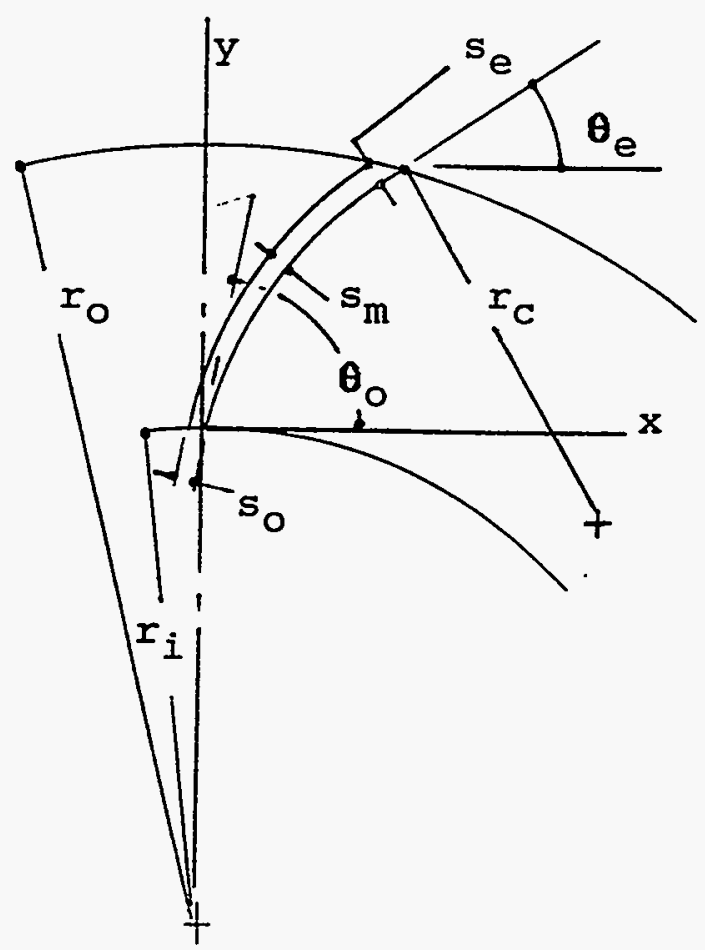

Fig. 2. Circular arc plate spacing. 


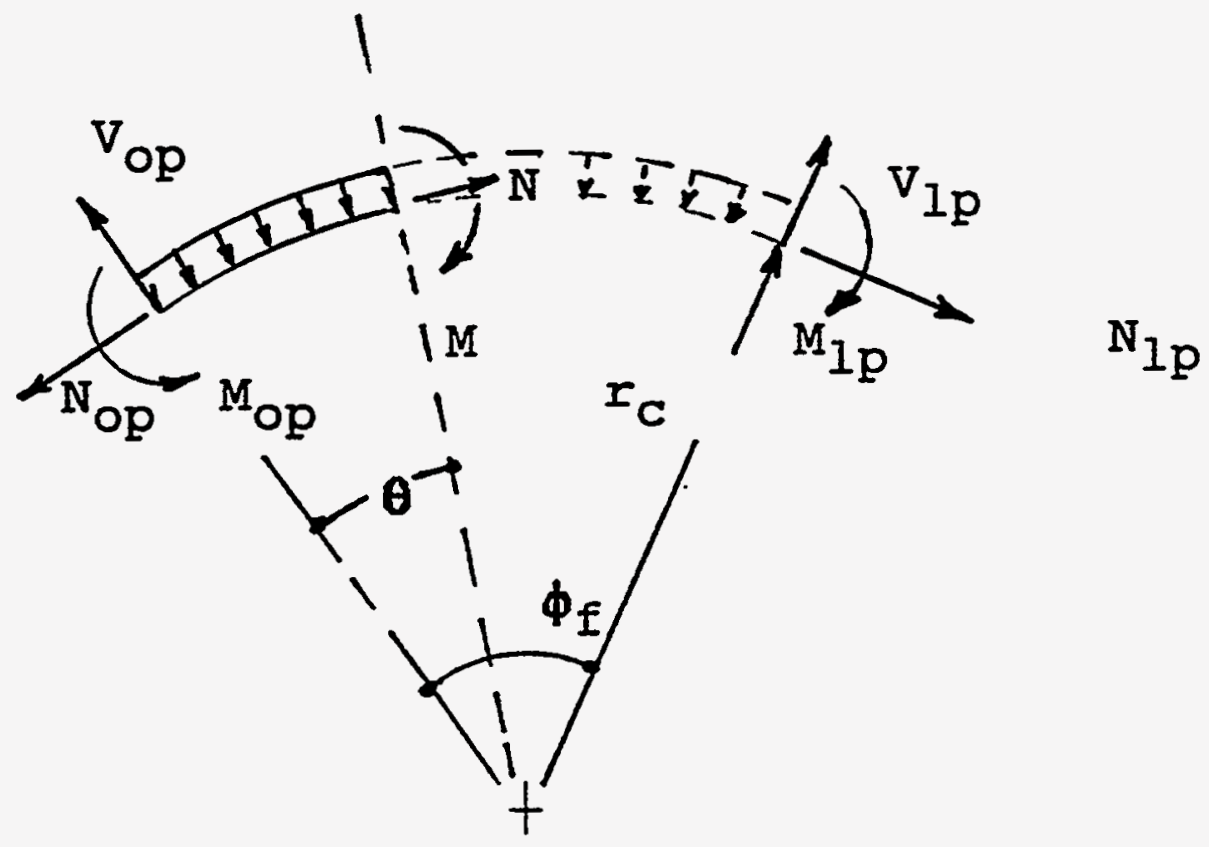

Fig. 3. Free body diagram of circular arc plate with pressure load. 
Partial derivatives of Eq. (2) are

$$
\begin{gathered}
\frac{\partial M}{\partial M_{o p}}=1, \\
\frac{\partial M}{\partial N_{o p}}=r_{c}[1-\cos (\theta)], \\
\frac{\partial M}{\partial V_{o p}}=-r_{c} \sin (\theta) .
\end{gathered}
$$

The tangent load equation at any section, $\theta$, is

$$
N=N_{o p} \cos (\theta)+V_{o p} \sin (\theta)-p r_{c}[1-\cos (\theta)]
$$

Partial derivatives of Eq. (4) are

$$
\begin{gathered}
\frac{\partial N}{\partial M_{o p}}=0, \\
\frac{\partial N}{\partial N_{o p}}=\cos (\theta), \\
\frac{\partial N}{\partial V_{o p}}=\sin (\theta) .
\end{gathered}
$$

Using strain energy, $U$, and Castigliano's theorem, the change in slope at the origin as load is applied is zero and is expressed as

$$
0=\frac{\partial U}{\partial M_{O P}}=\frac{r_{c}}{E I} \int_{0}^{\phi_{f}} M \frac{\partial M}{\partial M_{O P}} d \theta+\frac{r_{c}}{A E} \int_{0}^{\phi_{f}} N \frac{\partial N}{\partial M_{O P}} d \theta
$$


where

$$
\begin{aligned}
E & =\text { modulus of elasticity } \\
\Phi_{f} & =\text { included angle of the plate } \\
A & =\text { longitudinal section area per unit length } \\
I & =\text { area moment of inertia. }
\end{aligned}
$$

The deflection, $\delta_{v}$, of the circular arc in the direction of $V_{o p}$ is zero at the origin, or

$$
0=\delta_{V}=\frac{\partial U}{\partial V_{o p}}=\frac{r_{c}}{E I} \int_{0}^{\Phi_{f}} M \frac{\partial M}{\partial V_{o p}} d \theta+\frac{r_{c}}{A E} \int_{0}^{\Phi_{f}} N \frac{\partial N}{\partial V_{o p}} d \theta
$$

The deflection, $\delta_{N}$, of the circular arc in the direction of $N_{o p}$ is zero at the origin, or

$$
0=\delta_{N}=\frac{\partial U}{\partial N_{o p}}=\frac{r_{c}}{E I} \int_{0}^{\phi_{f}} M \frac{\partial M}{\partial N_{o p}} d \theta+\frac{r_{c}}{A E} \int_{0}^{\phi_{f}} N \frac{\partial N}{\partial N_{o p}} d \theta
$$

When Eqs. (6), (7), and (8) are expanded with Eqs. (2)-(5) the results yield three equations and three unknowns, which can be solved for the unknowns $M_{o p}, N_{o p}$, and $V_{o p}$

With values for $M_{o p}, N_{o p}$, and $V_{o p}$ parameters such as stress and deformation can be calculated. For the plate selected, the maximum moment and tangent load occur at the support boundaries; therefore, the maximum stress will also occur at the boundaries. The curvature of the plate has negligible effect on the stress magnitude, and the stress can be readily calculated; however, calculating the deformation requires some additional analysis.

The maximum deflection for this symmetric circular arc occurs at center span. The deflection can be evaluated through energy principles and the dummy load technique. For this application, the deflection at center span, $\delta_{c}$, is expressed as

$$
\delta_{c}=\int_{0}^{\theta_{c}} \frac{M}{E I} \frac{\partial M}{\partial F} r_{c} d \theta+\int_{\theta_{c}}^{\phi_{f}} \frac{M}{E I} \frac{\partial M}{\partial F} r_{c} d \theta+\int_{0}^{\theta_{c}} \frac{N}{A E} \frac{\partial N}{\partial F} r_{c} d \theta+\int_{\theta_{c}}^{\Phi_{f}} \frac{N}{A E} \frac{\partial N}{\partial F} r_{c} d \theta,
$$

where $F$ is the dummy load applied at the point where the deflection is desired (Fig. 4). To evaluate the partial derivatives, the dummy load technique requires the solution of $M_{O F}, N_{O F}$, and $V_{O F}$, as shown in Fig. 4 , and with $F$ equal to unity. First, the moment equation and the tangent load equation for the arc with a concentrated force, $F$, applied at center space are expressed as 


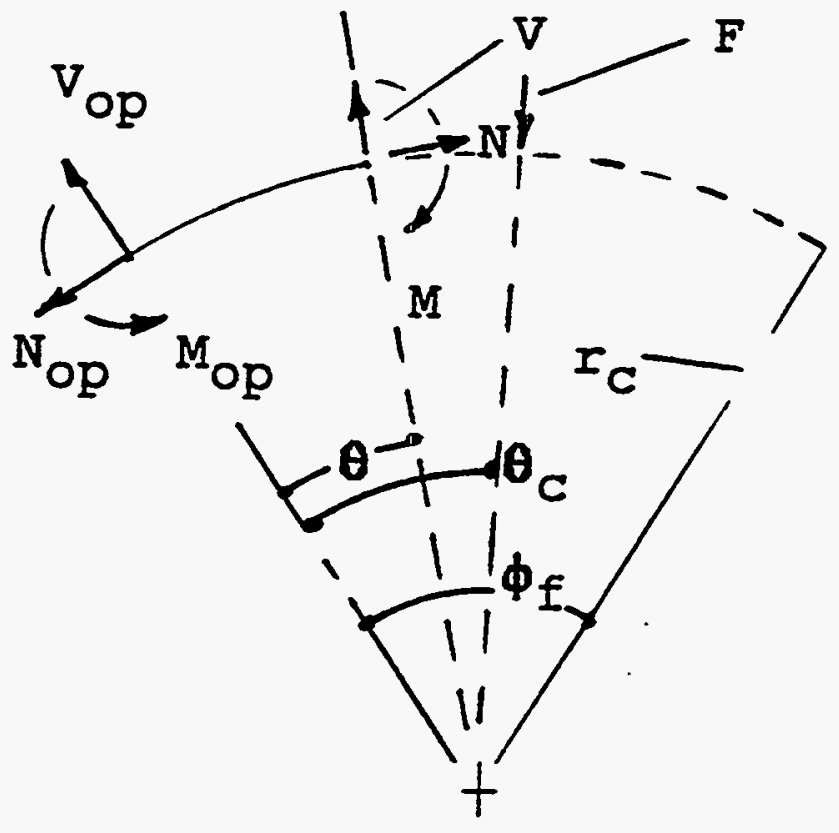

Fig. 4. Free body diagram of circular arc with dummy load. 


$$
\begin{gathered}
M_{F}=M_{O F}-V_{O F} r_{c} \sin (\theta)+N_{O F} r_{c}[1-\cos (\theta)] \\
N_{F}=N_{O F} \cos (\theta)+V_{o F} \sin (\theta) \\
\text { for } 0 \leq \theta \leq \theta c, \\
M_{F}=M_{O F}-V_{O F} r_{c} \sin (\theta)+N_{O F} r_{c}[1-\cos (\theta)]+F r_{c} \sin \left(\theta-\theta_{c}\right) \\
N_{F}=N_{O F} \cos (\theta)+V_{O F} \sin (\theta)-F \sin \left(\theta-\theta_{c}\right) \\
\text { for } \theta_{c} \leq \theta \leq \Phi_{f} .
\end{gathered}
$$

Knowing that the boundaries are fixed, three energy equations reflecting that the slope and deflection are zero at $\theta=0$ can be written to solve for $M_{O F}, N_{O F}$, and $V_{O F}$. These equations are written as

$$
\begin{gathered}
0=\int_{0}^{\theta_{c}} \frac{M_{F}}{E I} \frac{\partial M_{F}}{\partial M_{O F}} r_{c} d \theta+\int_{\theta_{c}}^{\phi_{F}} \frac{M_{F}}{E I} \frac{\partial M_{F}}{\partial M_{O F}} r_{c} d \theta, \\
0=\int_{0}^{\theta_{c}} \frac{M_{F}}{E I} \frac{\partial M_{F}}{\partial N_{O F}} r_{c} d \theta+\int_{\theta_{c}}^{\phi_{F}} \frac{M_{F}}{E I} \frac{\partial M_{F}}{\partial N_{O F}} r_{c} d \theta+\int_{0}^{\theta_{c}} \frac{N_{F}}{A E} \frac{\partial N_{F}}{\partial N_{O F}} r_{c} d \theta+\int_{\theta_{c}}^{\phi_{F}} \frac{N_{F}}{A E} \frac{\partial N_{F}}{\partial N_{O F}} r_{c} d \theta, \\
0=\int_{0}^{\theta_{c}} \frac{M_{F}}{E I} \frac{\partial M_{F}}{\partial V_{O F}} r_{c} d \theta+\int_{\theta_{c}}^{\phi_{F}} \frac{M_{F}}{E I} \frac{\partial M_{F}}{\partial V_{O F}} r_{c} d \theta+\int_{0}^{\theta_{c}} \frac{N_{F}}{A E} \frac{\partial N_{F}}{\partial V_{O F}} r_{c} d \theta+\int_{\theta_{c}}^{N_{F}} \frac{\partial N_{F}}{A E} r_{c} d \theta .
\end{gathered}
$$

Expanding Eqs. (11) with Eqs. (10) yields three equations and three unknowns that are solved for $M_{O F}, N_{O F}$, and $V_{O F}$ in terms of $F$. By setting $F$ equal to unity, $M_{O F}$ and $N_{O F}$ become the partial derivatives needed in the integrals of Eq. (9). The variables $M$ and $N$ of Eq. (9) are expressed with Eq. (2) and Eq. (4). Expanding Eq. (9) in this way allows the maximum deflection of the circular arc with a pressure load to be calculated.

Calculations for the circular arc plate loads per unit length for this test (Fig. 3) have

$$
\begin{aligned}
& M_{o p}=0.00000784 \cdot(p), \text { units of newtons, } \\
& N_{o p}=-0.0851 \cdot(p), \text { units of newtons per meter, and } \\
& V_{o p}=0.000541 \cdot(p), \text { units of newtons per meter, }
\end{aligned}
$$

where the pressure, $p$, is in pascals.

The maximum stress from the pressure load calculates to be $\sigma=96.16 \cdot(p)$, units of pascals. The maximum deflection of the circular arc loaded by pressure calculates to be $\delta=0.000000157 \cdot(p)$, units of millimeter. 
Calculations for $M_{O F}, N_{O F}$, and $V_{O F}$ resulting from a load applied to the circular arc allow for the effects of flow blockage to be estimated. This development is given in Appendix B.

A thermal analysis of the circular arc plate was done applying these same energy concepts. The results do not apply directly to the flow test of the plates; therefore, the development is located in Appendix C. 


\section{EXPERIMENTAL ARRANGEMENT}

The essential elements in the experiment are shown in the schematic of the experimental flow loop in Fig. 5. The purpose of the flow straightener was to produce a fully developed turbulent flow field. From the flow straightener, the flow entered the test section containing the dummy aluminum fuel plates. The test section contained five active plates and six flow channels. On entering the test section, the flow velocity approximately doubled. The outer flow boundary of the flow straightener and test section were the same, but about half of the interior flow area in the test section was taken up with plates and caused the flow velocity to increase. This is the same effect as in the planned reactor, where the flow area in a fully developed turbulent flow field is reduced as the fuel plates are encountered. Strain gages were located on the three central test plates at locations along the flow path so that plate deflection could be monitored during coolant flow. Pressure taps were located in the four central flow channels along the flow paths so that the different channel pressures could be monitored during flow. To prevent leaks, sealants were applied as the test components were bolted together.

The circular arc plates used in the tests were formed from flat, 1.27-mm-thick, 6061-0 aluminum plates. The plate edges that were in the coolant path and would interrupt the coolant flow were rounded to keep the flow reasonably smooth during the tests. The flat plates were formed into circular arcs by pressing them between stainless steel mandrels, which had been machined to the specified contour (Fig. 6). The whole assembly was annealed in the oven to remove stresses introduced in the forming process. To prevent the pressures on the plate from becoming excessive because of differences in the expansion coefficients of the aluminum and steel parts, spring washers were used and can be seen on the threaded cylindrical rods holding the assembly together. The annealing cycle consisted of bringing the assembly from room temperature to the annealing temperature of $413^{\circ} \mathrm{C}$, which required approximately 6 hours. The assembly was "soaked" for 2 hours at $413^{\circ} \mathrm{C}$, and then the oven was turned off and allowed to cool below $260^{\circ} \mathrm{C}$ before removing the assembly. A graphite lubricant was required to prevent the aluminum plate from sticking to the stainless steel mandrels. Fig. 7 shows how well the formed plate matched the mandrel without any "spring back."

The flow straightener and one of the flanges used in the final assembly are shown in Fig. 8. Two aluminum circular arc plates and two aluminum spacers holding the two plates apart form the boundaries for the flow cavity. The cross-sectional area of the flow straightener's flow cavity is identical to the crosssectional area of the flow cavity found in the test section. The test section's flow cavity consists of the test plates and coolant channels combined. The large stainless steel parts shown in Fig. 8 are used to contain the pressures produced by the coolant flow and to hold the boundaries of the flow cavity in place. The many bolts seen in Fig. 8 put pressure on the outer edges of the boundary plates and spacers to maintain the boundary positions and prevent leaks. In addition to the bolt pressures holding the plates and spacers in the desired position, two rows of dowel pins along the cavity length were used to pin all the parts together. Some of the small dowel pin holes that contain the dowel pins can be seen beside some of the bolts in Fig. 8.

The test section containing the plates and channels was assembled in the same manner as the flow straightener. However, instead of having only two spacers to space the two boundary plates as in the flow straightener, five plates with individual channel spacers were used to space the boundary plates and form the test flow cavity. A top view of the test section with one of the assembly flanges in place is shown in Fig. 9. As was noted earlier, the flow cavity in the test section contains two boundary plates, five active plates, and six flow channels. Considering the flow boundary conditions involved, the three central plates and the four channels that bound these plates were more representative of the plates and channels anticipated in the operating reactor and were instrumented for the tests. Each of the three central plates 


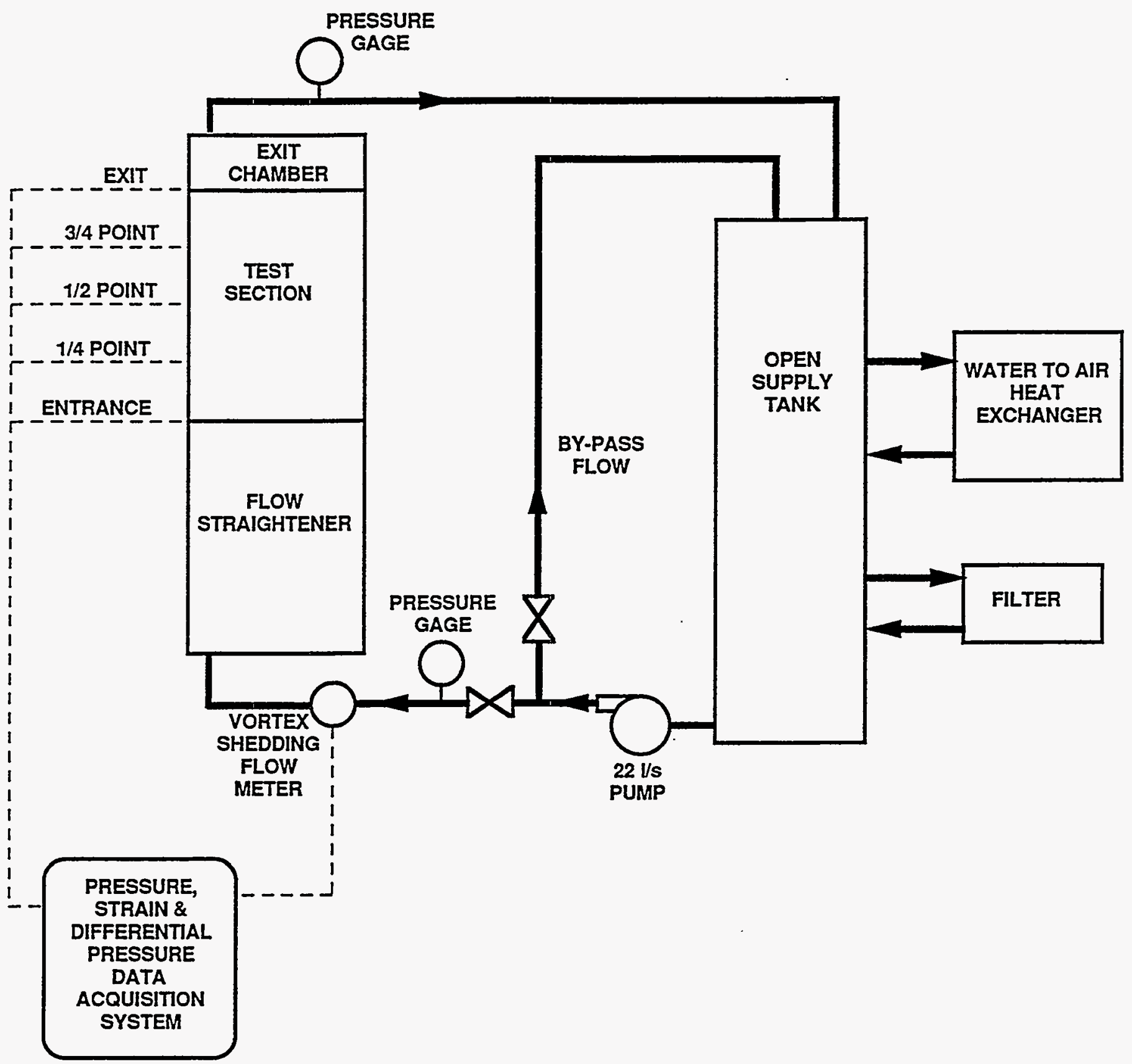

Fig. 5. Schematic of flow test loop. 


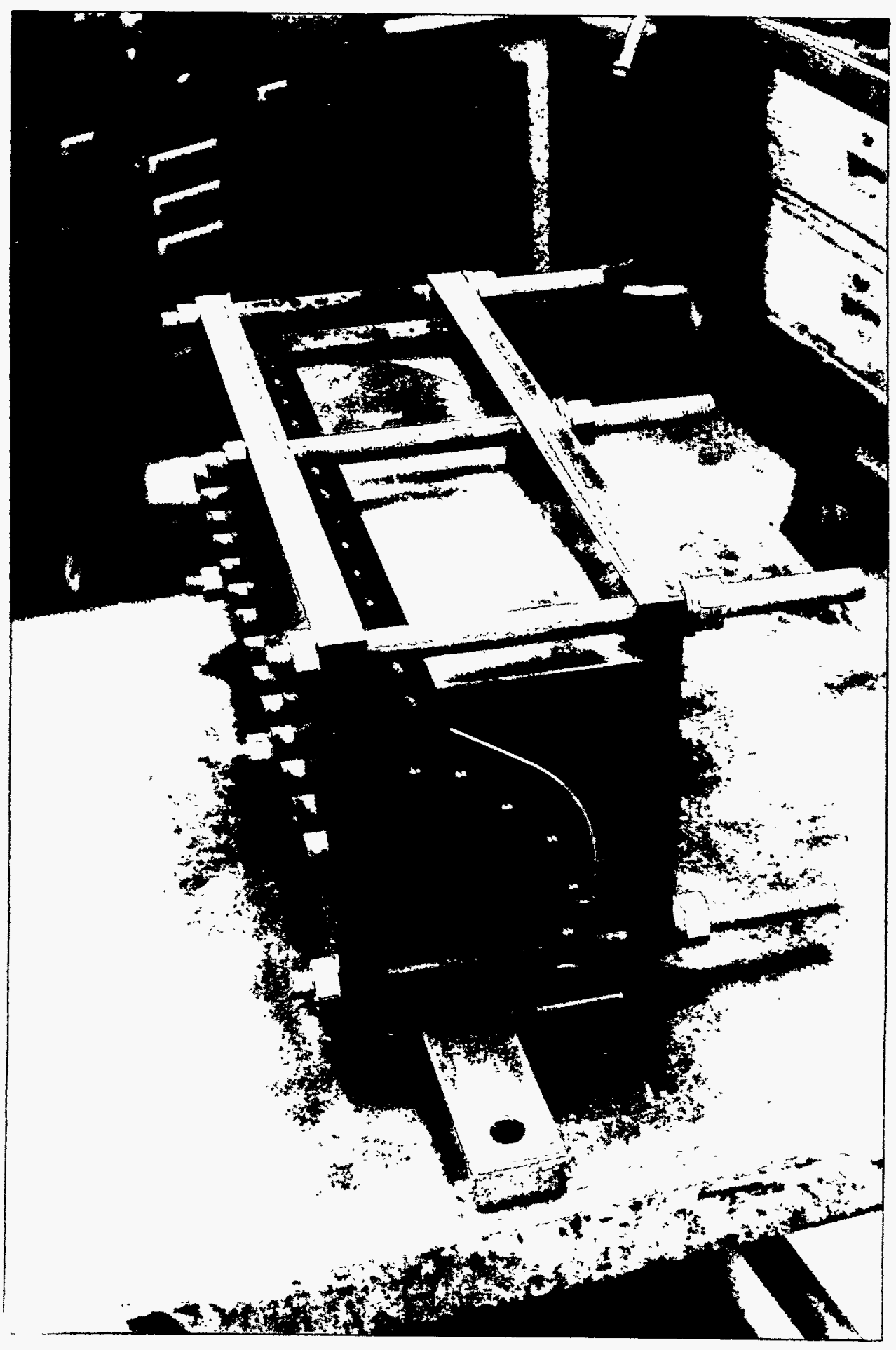

Fig. 6. Forming circular arc plate. 

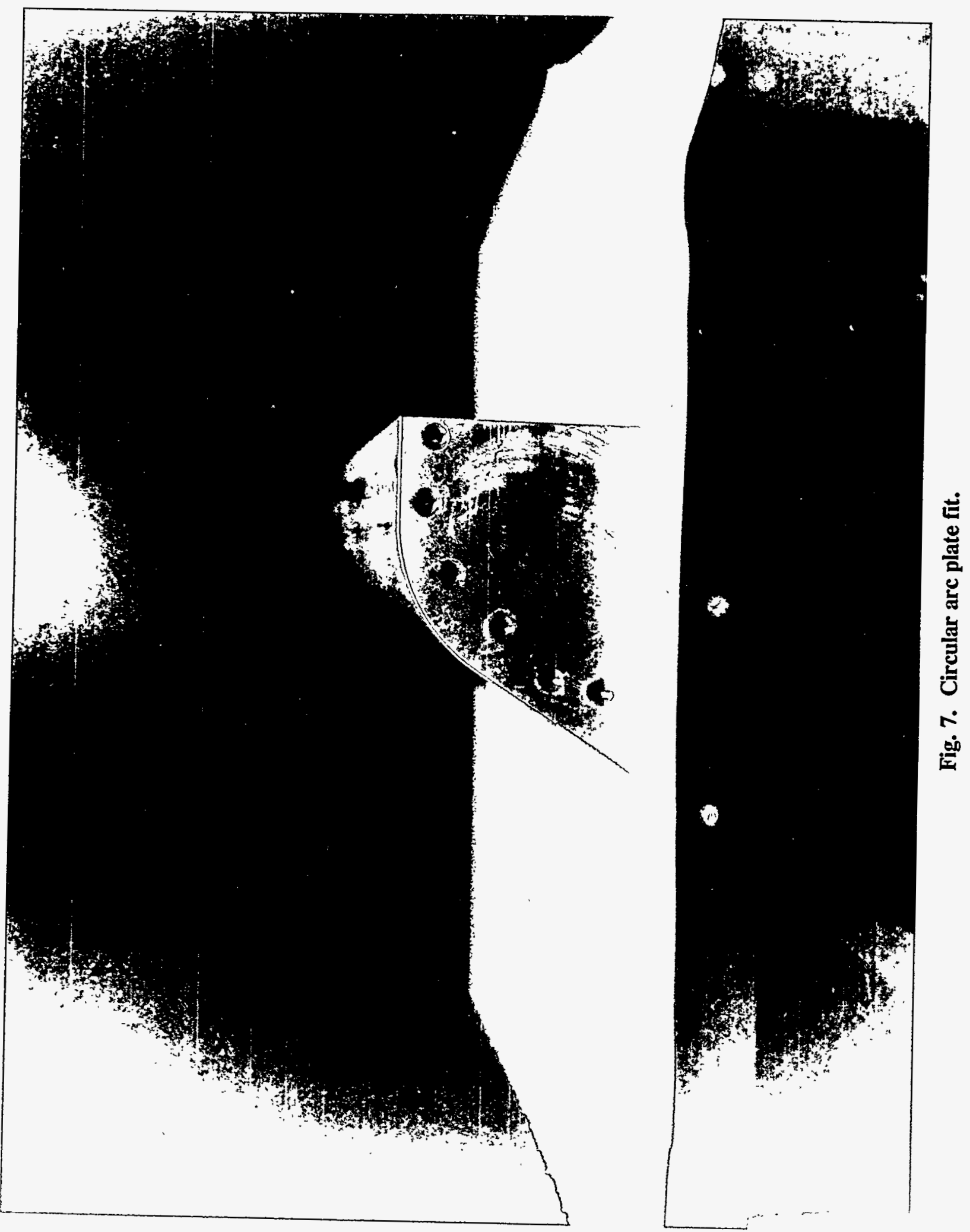
had five strain gages attached to the plate surface for indicating the deflection of the plates during coolant flow. The gages were located with respect to the test section length at the entrance, one-quarter, one-half, three-quarter, and exit cross sections. At these same cross sections, pressure tubes were located in the four channels bounding these plates to indicate the static pressure in the channels during coolant flow. An assembly of the flow straightener and the test section is pictured in Fig. 10. The pressure tubes and the strain gage lead wires can also be seen in Fig. 10.

All of the machine work was done to a tolerance of $\pm 0.05 \mathrm{~mm}$. When the plates were formed on the mandrels and annealed, no deviation of the plates from the mandrels was detectable. The overall tolerance of the four channels surrounding the gaged test plates after assembly could not be measured reasonably. However, the channel spacings near the entrance and exit regions of these four instrumented channels were measured to be $\pm 10 \%$ or $\pm 0.13 \mathrm{~mm}$.

The assembled system is shown in the flow loop from two different views in Fig. 11. The data collection was computer controlled and consisted of 15 strain gage readings, 20 channel pressure readings, pressure readings upstream and downstream of the test assembly, flow rate, and coolant temperature. Each test run consisted of scanning and recording the readings from all sensors three times with zero coolant flow (initial zero readings), adjusting the flow to the desired flow rate and, again, scanning and recording the readings from all sensors three times (loaded data), and finally stopping the flow and scanning and recording each reading three times (post zero data). Having three initial zero scans, three loaded scans, and three post-zero scans for each run was helpful in assessing the reliability of the data. If all of the zero scans (initial and post-zero scans) did not agree within a reasonable tolerance, data from that sensor was questionable and was not used in forming conclusions about the structural response of the plates to flow. For the pressure sensors, a reasonable tolerance was taken as $\pm 649 \mathrm{~Pa}$. For the strain gages, a reasonable tolerance was taken as \pm 20 microstrain. In addition to having agreement in the initial and post zeros if the loaded data had reasonable agreement within its three data scans, the plates were taken to be in a fixed deformed shape. If the three loaded data scans did not have agreement, the plate was taken to be in a transient deformed shape. For each test run, one of the strain gages was selected and connected to a strip chart to give a better indication of any transient deformation associated with that plate.

Each of the pressure tubes was connected to a separate calibrated pressure transducer. The variations in the calibrated pressures were found to be $\leq 0.5 \%$. The pressure transducer converted the pressure sensed into a voltage reading that was recorded by the computer control. A 10-volt signal corresponded to $3.447 \mathrm{MPa}$.

Each strain gage was shunt calibrated and the variations found to be $50.8 \%$. The strain gage conditioning circuit produced a voltage output that was recorded by the computer control. One microvolt corresponded to one microstrain.

The flow meter was calibrated and the variations found to be $\leq 1 \%$. The conditioning circuit for the flow meter also had a voltage output from which the flow rate could be calculated. The calculations took the form

$$
\text { flow rate }=(\text { output volts-1) } \cdot(5.126), \mathrm{L} / \mathrm{sec} \text {. }
$$

The strain gages measured strain at a point, and to convert strain data to maximum plate deflection required a further calibration. It was not realistic to measure physically maximum plate deflection as a function of strain gage reading in the assembled test section. Therefore, the approach used was to verify the analytical solution, which was outlined in the previous section, and use it to establish the relation of maximum plate deflection to strain gage reading. To verify the analytical solution, one flow channel was blocked on each end with a pliable clay, and a vacuum was produced in this channel using a vacuum pump. The plate strain gage readings were recorded for given pressure loads, and then the sequence was 


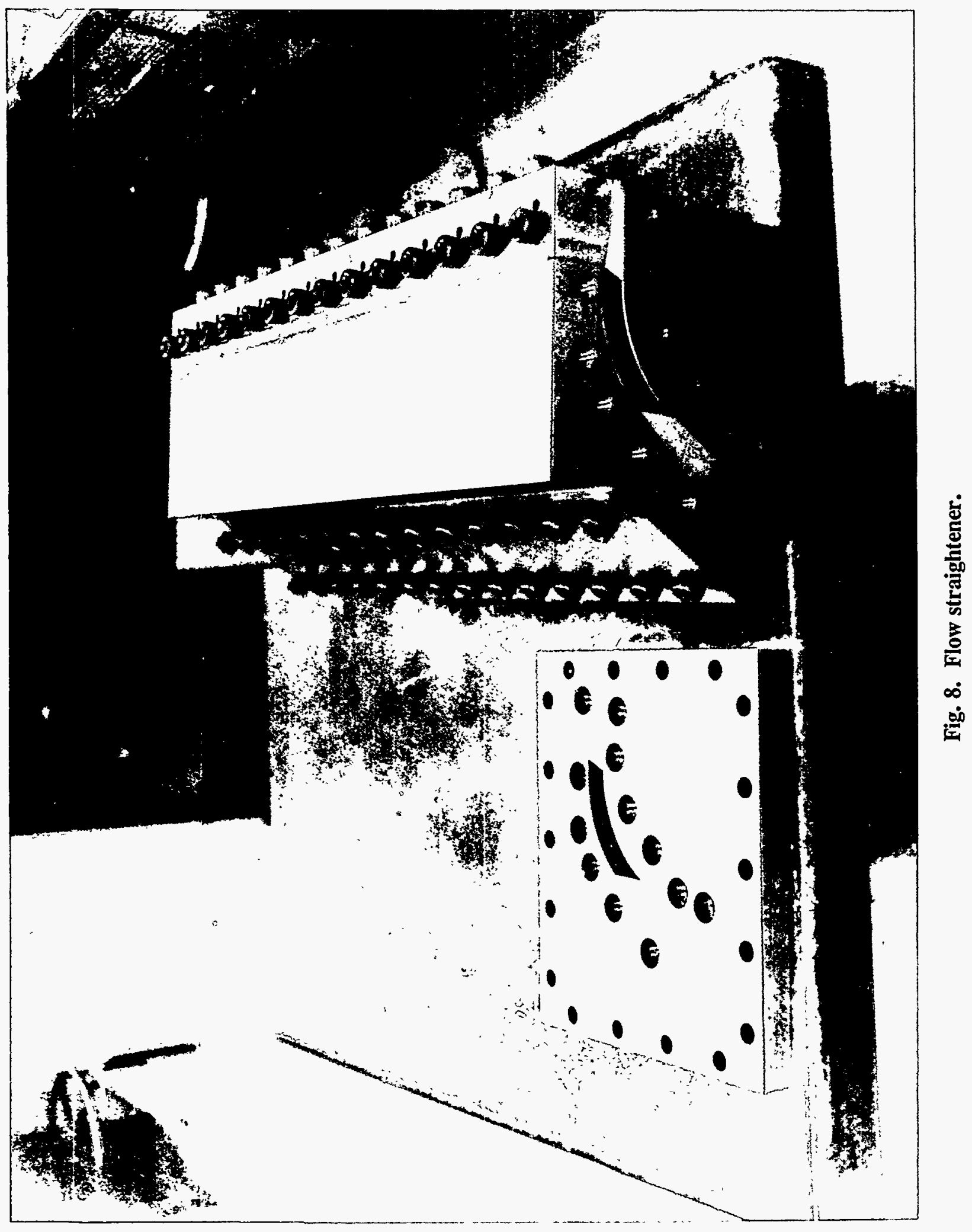




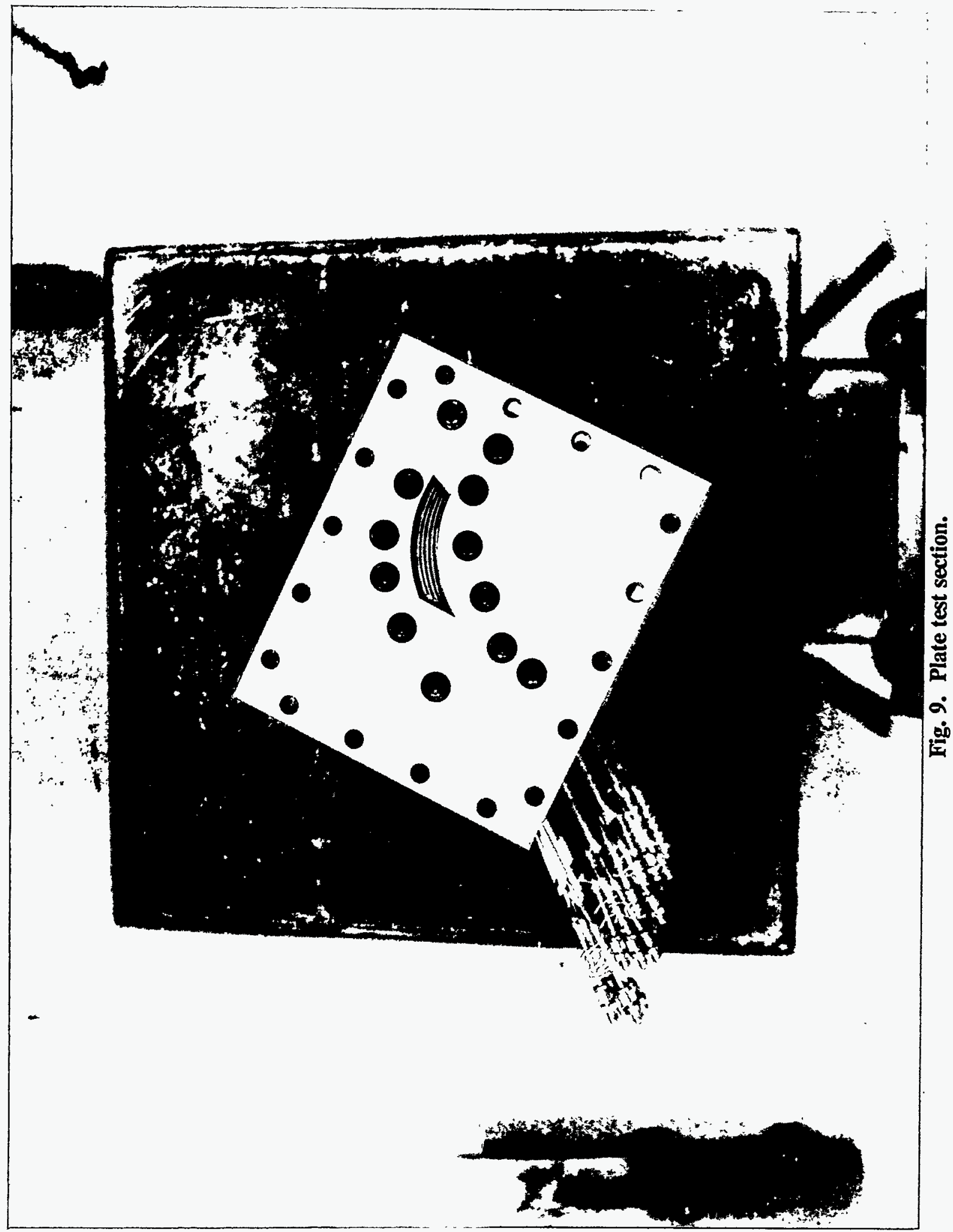




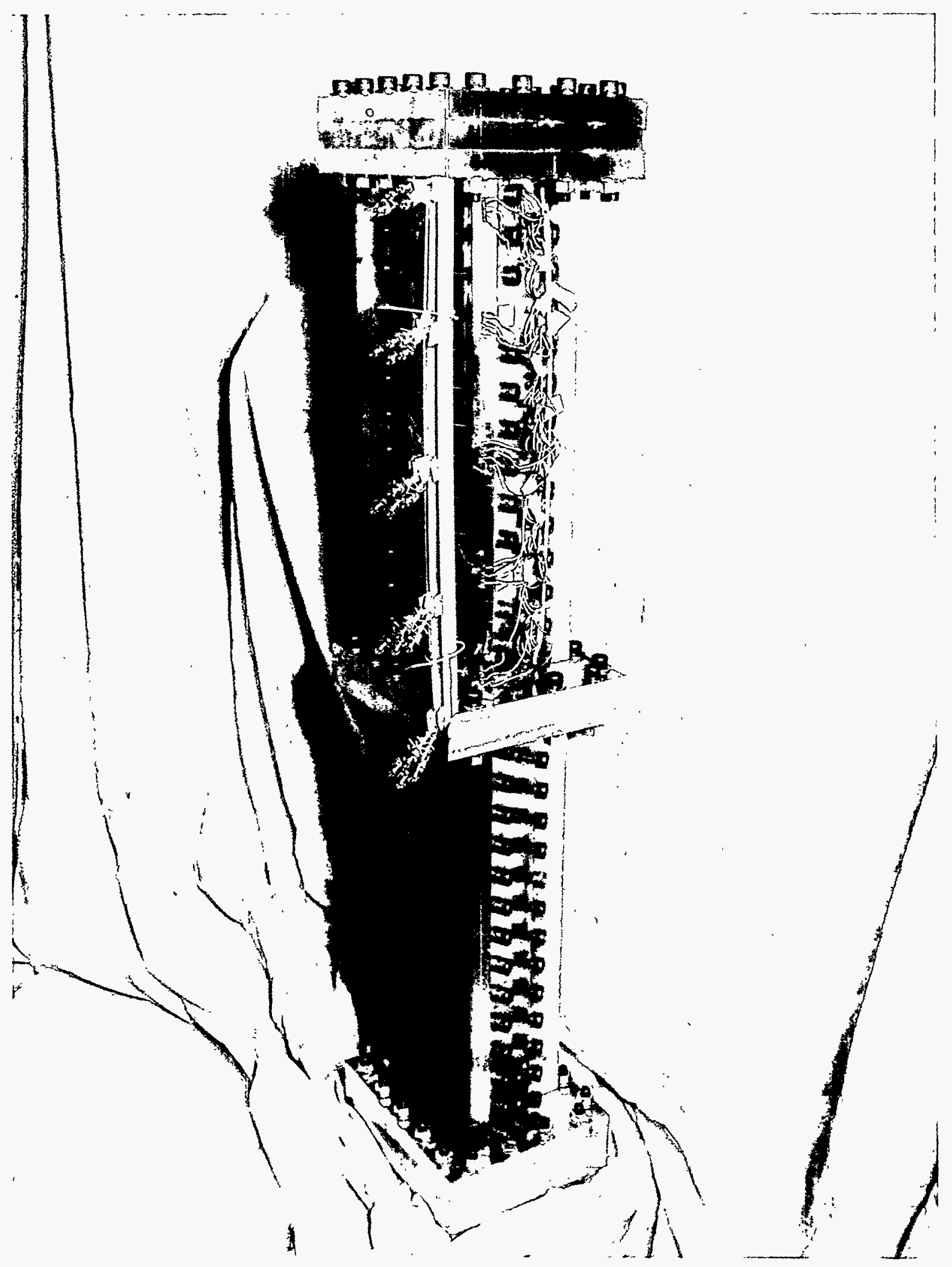

Fig. 10. Assembly of flow straightener and plate test section. 


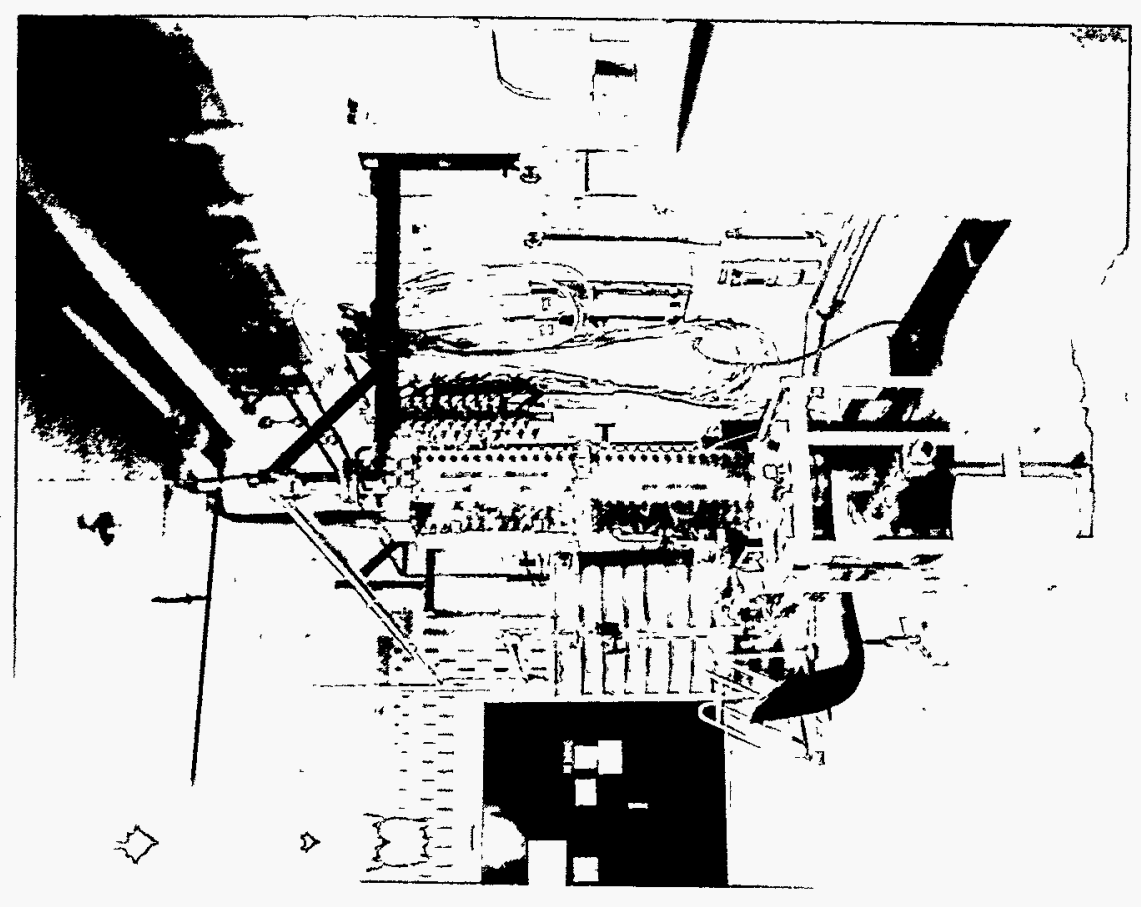

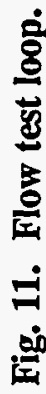


repeated using each of the four instrumented channels as the vacuum domain. The strain gage at the center cross section of each plate was used to verify the analytical solution. These gages were least affected by the end effects produced by the clay seals and would better represent the analytical solution. The average measured strain reading was $1.304 \cdot\left(10^{-9}\right)$, units of strain per $\mathrm{Pa}$. The strain value calculated using the analytical solution was $1.252 \cdot\left(10^{-9}\right)$, units of strain per $\mathrm{Pa}$. These values were in reasonable agreement $(4 \%)$, and the calculated maximum plate deflection was related to the strain gage reading as

$$
\delta=125 \cdot(\epsilon) \mathrm{mm} \text {, maximum plate deflection, }
$$

where $\epsilon$ is the strain gage reading in units of strain. 


\section{DATA COLLECTION}

A copy of the collected data is included in Appendix D. The locations of the data points are identified by the plate number and the section location (Fig. 12). The channels are identified by the two plate numbers that bound the channel. The three central plates, 12,13 , and 14, are instrumented with strain gages, and the four central channels, 1012, 1213, 1314, and 1411, contain the pressure taps. Table 1 correlates the data points with the recorded computer readings that are given in Appendix D.

An initial check of the instrumentation showed that gage 12-5 (XE-3) was an open gage, and no data were available at that point. A dummy resistor was installed to keep the readout from going off scale; thus, the reading at that point is not data.

Data runs 207 through 216 were made with gage 13-5 (XE-02) connected to a strip chart recorder; therefore, the record appearing in Appendix D for this point is not data but a dummy resistor reading.

Data runs 217 through 268 were made with gage 13-2 (XE-11) connected to the strip chart recorder; therefore, the data appearing in Appendix D for this point and these runs are a dummy resistor reading. On run 218, gage 13-2 (XE-11) showed erratic response on the strip chart. It was concluded after studying all of the gage data that almost all of the gages experienced this erratic behavior. It is thought that this was caused by breakdown of the insulation coating used on the gage tabs. The coolant would in an intermittent manner conduct stray currents between the gage tabs with the result being erratic gage response. Evidence supporting this view came when the coolant was drained from around the plates, the erratic gage response stopped, and a very stable gage response was observed. Gages 13-5 (XE-2) and 12-2 (XE-12) were the only two gages that remained stable during most of the tests. Since the erratic strain gage response was intermittent, by observing the record of the gage connected to the strip chart, the erratic response could be separated from the stable response, yielding some data. It should be emphasized that the pressure data, which was very stable, and the strain gage data are in some respects redundant. It was observed from the tests on the epoxy plates, that using the pressure difference from each side of a plate to find the steady-state plate response to coolant flow gave essentially the same result as that predicted from the strain gage response. ${ }^{1,2}$ Strain gages are more sensitive than the pressure sensors to time-varying data, and, because of this, are more helpful in assessing plate vibrations. With the limited strain gage data from these tests, the potential of plate vibrations was assessed, and with the more complete pressure data, the steady-state deformations of the plates were assessed.

On runs 219 through 222, it was observed that the connecting pipe from the pump to the flow straightener had considerable vibration, but on either side of the flow rates in this range the vibration was not so evident. An examination of the data indicated that by the time the flow entered the plate test section, this vibration has dissipated.

On run 229 the flow meter malfunctioned. Runs 230 through 236 were used to determine the flow meter problem and to evaluate repair of the flow meter. The problem was a failed solder joint in the electronics, which was probably caused by the vibration experienced in runs 219 through 222 .

Data were recorded on runs 237 through 247 with gage 13-2 (XE-11) connected to the strip chart recorder. On run 248 , another solder joint failed in the flow meter circuitry and was repaired. Data were recorded on runs 249 and 250 . Runs 251 and 252 had yet more solder joint problems in the flow meter, which again were repaired. Data were recorded during runs 253 through 266 . During run 267 a large leak occurred in the test plate section, and testing was suspended.

Run 268 was used to assess the erratic response of the strain gages. When water was drained away from the test section, the erratic response of the gages ceased and the response became very stable.

All bolts were retightened to prevent water leaks that occurred during run 267. Gage 14-5 (XE01) was connected to the strip chart recorder, and testing was resumed. Runs 269 and 270 were used to 
Table 1. Data location identification

\begin{tabular}{|c|c|c|c|}
\hline $\begin{array}{c}\text { Pressure } \\
\text { tap }\end{array}$ & $\begin{array}{c}\text { Computer } \\
\text { I.D. }\end{array}$ & $\begin{array}{c}\text { Strain } \\
\text { gage }\end{array}$ & $\begin{array}{c}\text { Computer } \\
\text { I.D. }\end{array}$ \\
\hline $1411-5$ & PE-01 & $14-5$ & XE-01 \\
\hline $1314-5$ & $\mathrm{PE}-02$ & $13-5$ & $\mathrm{XE}-02$ \\
\hline $1213-5$ & PE-03 & $12-5$ & XE-03 \\
\hline $1012-5$ & PE-04 & $14-4$ & XE-04 \\
\hline $1411-4$ & PE-05 & $13-4$ & XE-05 \\
\hline $1314-4$ & PE-06 & $12-4$ & XE-06 \\
\hline $1213-4$ & PE-07 & $14-3$ & XE-07 \\
\hline $1012-4$ & PE-08 & $13-3$ & XE-08 \\
\hline $1411-3$ & PE-09 & $12-3$ & XE-09 \\
\hline $1314-3$ & PE-10 & $14-2$ & XE-10 \\
\hline $1213-3$ & PE-13 & $13-2$ & $\mathrm{XE}-11$ \\
\hline $1012-3$ & PE-14 & $12-2$ & XE-12 \\
\hline $1411-2$ & $\mathrm{PE}-15$ & $14-1$ & $X E-13$ \\
\hline $1314-2$ & PE-16 & $13-1$ & XE-14 \\
\hline $1213-2$ & $\mathrm{PE}-17$ & $12-1$ & $X E-15$ \\
\hline $1012-2$ & PE-18 & & \\
\hline $1411-1$ & PE-19 & & FE-01 Flow meter \\
\hline $1314-1$ & PE-20 & & PE-29 Pressure upstream \\
\hline $1213-1$ & $\mathrm{PE}-21$ & & PE-30 Pressure downstream \\
\hline $1012-1$ & PE-22 & & \\
\hline
\end{tabular}




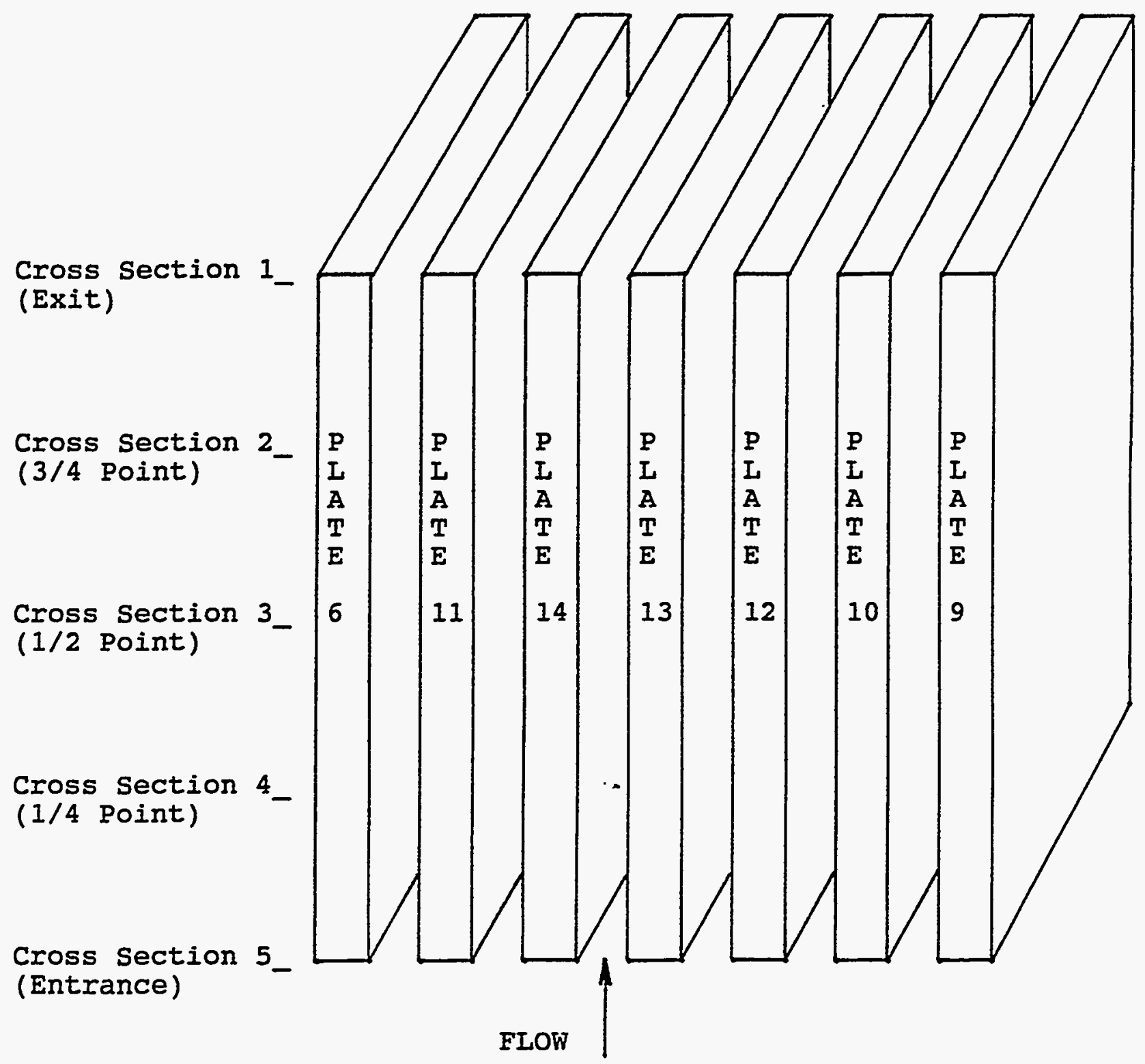

Fig. 12. Plate number and section location. 
stabilize conditions for running the tests. Flow data were collected on runs 271 through 282 . During this series of tests, the flow data started out in agreement with previous data, but then pressures needed to produce a given flow rate drastically increased. Tests were suspended and the test section removed from the flow loop. An inspection revealed that silicone gasket material had broken free from the flow straightener flanges, flowed upward through the flow straightener, and partially blocked some of the flow channels in the test plate section (Fig. 13). In the very deformed channel openings shown in Fig. 13, silicone material, which cannot be seen in the photograph, is wedged in the channels some $150 \mathrm{~mm}$ or so along the flow path. 


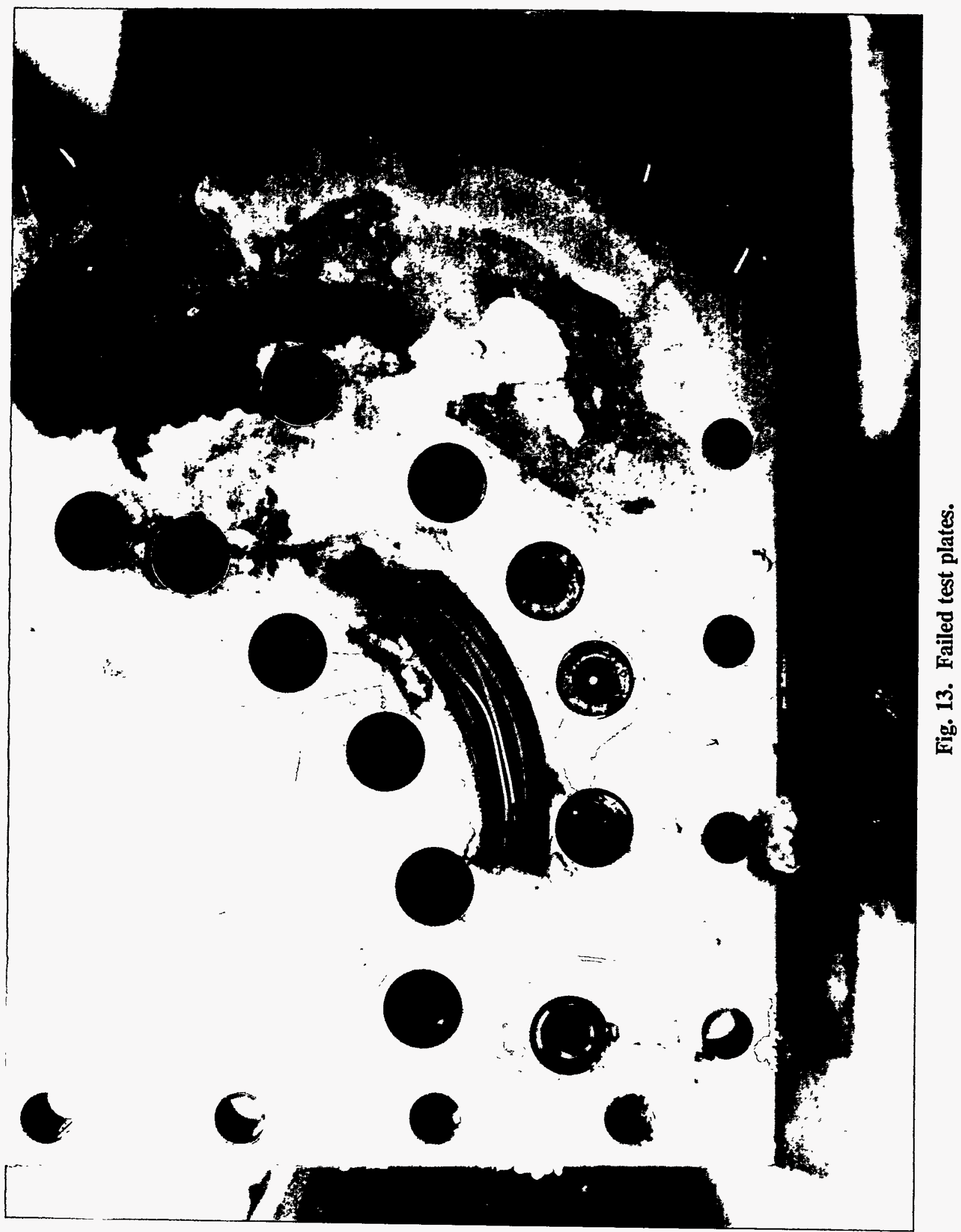





\section{DATA ANALYSIS}

The data was grouped into two series to help in assessing it. Series I contains all of the data from runs 207 through 268 and is reflective of tests that had no channel blockage. Series II contains all of the data from runs 269 through 282 and reflects the problems that could occur from flow blockage.

From a design viewpoint, the location of the maximum plate load is needed so that the most critical plate deformation can be assessed. The pressure differences or pressure loads for the central plate, plate 13, at the five instrumented cross sections for several flow velocities are illustrated in Fig. 14. This is a typical deformed shape for the circular arc test plates, and it is observed that the maximum pressure loads occur at the one-quarter and one-half cross sections, with the pressure loads at the one-quarter cross section having the largest values in all but one instance. Working with flat plates, Smissaert observed a pressure difference with respect to the plate length. ${ }^{3}$ The experimental work with involute plates reported by Swinson also noted the pressure difference variation with respect to length. ${ }^{1,2}$ In the work with involute plates, the maximum pressure loads occurred at the entrance and the three-quarter cross section.

Equation (1) was developed from data obtained using epoxy plate models that required flow velocities of about one-fifth the velocity magnitudes expected in the operating reactor. Thus, one of the objectives of these tests was to supply data at prototypic velocities that could be used to examine the validity of Eq. (1) at a higher velocity range. Equation (1) was developed to yield the maximum average pressure load on the plates, which in this case occurs at the one-quarter and one-half cross sections. The average pressure loads at these cross sections are compared with the pressure load calculated using Eq. (1), (Fig. 15). The results indicate the validity of Eq. (1) to calculate the maximum average pressure loads at prototypic velocities.

Having the average pressure load for design purposes is important information; however, knowing how much variation in the pressure load for a specific plate from the average pressure load is more important. The plate that has the largest variation that increases the pressure load is the plate that would most likely be susceptible to plate failure; this is the rationale for multiple plate tests. Because the maximum pressure loads for these tests occurred at the one-quarter and one-half cross sections, the pressure loads at these cross sections for the three central plates are illustrated in Figs. 16 and 17. It is observed that the pressure load on the central plate, plate 13, agrees with the calculated pressure load from Eq. (1); however, the pressure load on plate 14 is larger and is, therefore, the critical load. At the planned operating flow velocity of $25 \mathrm{~m} / \mathrm{s}$, the pressure load on plate 14 is approximately $45 \%$ larger than the average pressure load. This is a large pressure load variation between plates at a specific cross section, and it would be helpful to know whether or not this is typical. An examination of other works shows that this much variation should be expected. Working with flat plates, Smissaert showed pressure load variations in some instances on the order of $60 \%$ above the average pressure load. ${ }^{3}$ The experimental work using involute plates ${ }^{1,2}$ had pressure load variations on the order of $40 \%$, and some isolated points were even higher. Groninger and Kane did not report pressure load data but did report deflection data. ${ }^{4}$ The deflection data gave variations of $67 \%$ from the average and are reflective of the pressure load variation. Based on the somewhat limited data available, large variations in the pressure load from the average should be expected.

Examination of the stress induced in plate 14, the plate that had the critical pressure load, indicates that the maximum stress at the planned operating flow load was $23 \mathrm{MPa}$. Note that the yield stress for this material, aluminum 6061-0, is $55 \mathrm{MPa}$ at room temperature. If this same pressure load were applied to the equivalent involute plate for the lower element core, the induced stress would be $111 \mathrm{MPa}$, and plate failure would be predicted. 


\begin{tabular}{|ccc|}
\hline \multicolumn{3}{c|}{ FLOW VELOCITIES } \\
$-27.2 \mathrm{~m} / \mathrm{s}$ & $-24.4 \mathrm{~m} / \mathrm{s}$ & $-* 19.5 \mathrm{~m} / \mathrm{s}$ \\
$-14.7 \mathrm{~m} / \mathrm{s}$ & $*-10.8 \mathrm{~m} / \mathrm{s}$ & $-4.9 \mathrm{~m} / \mathrm{s}$ \\
\hline
\end{tabular}

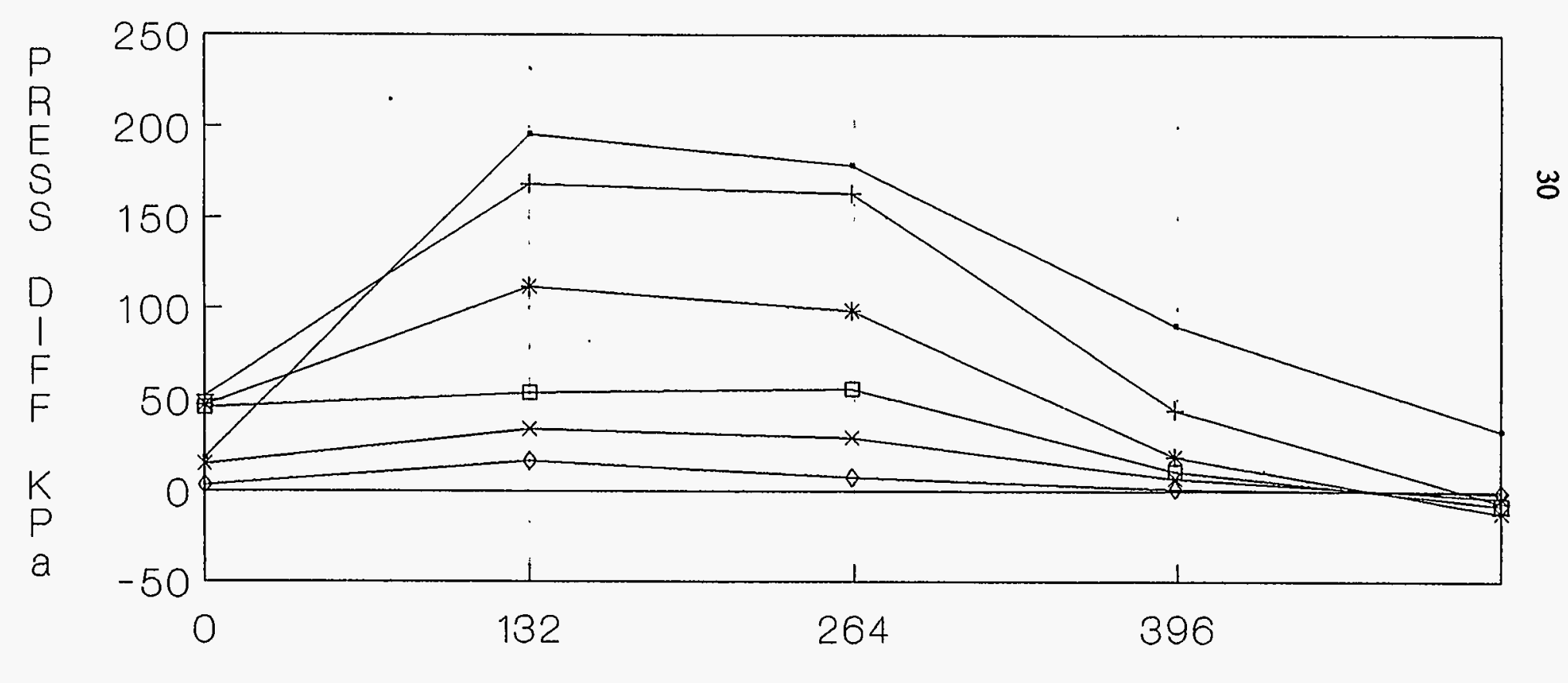

Fig. 14. Pressure difference for plate 13 with respect to distance along plate. 


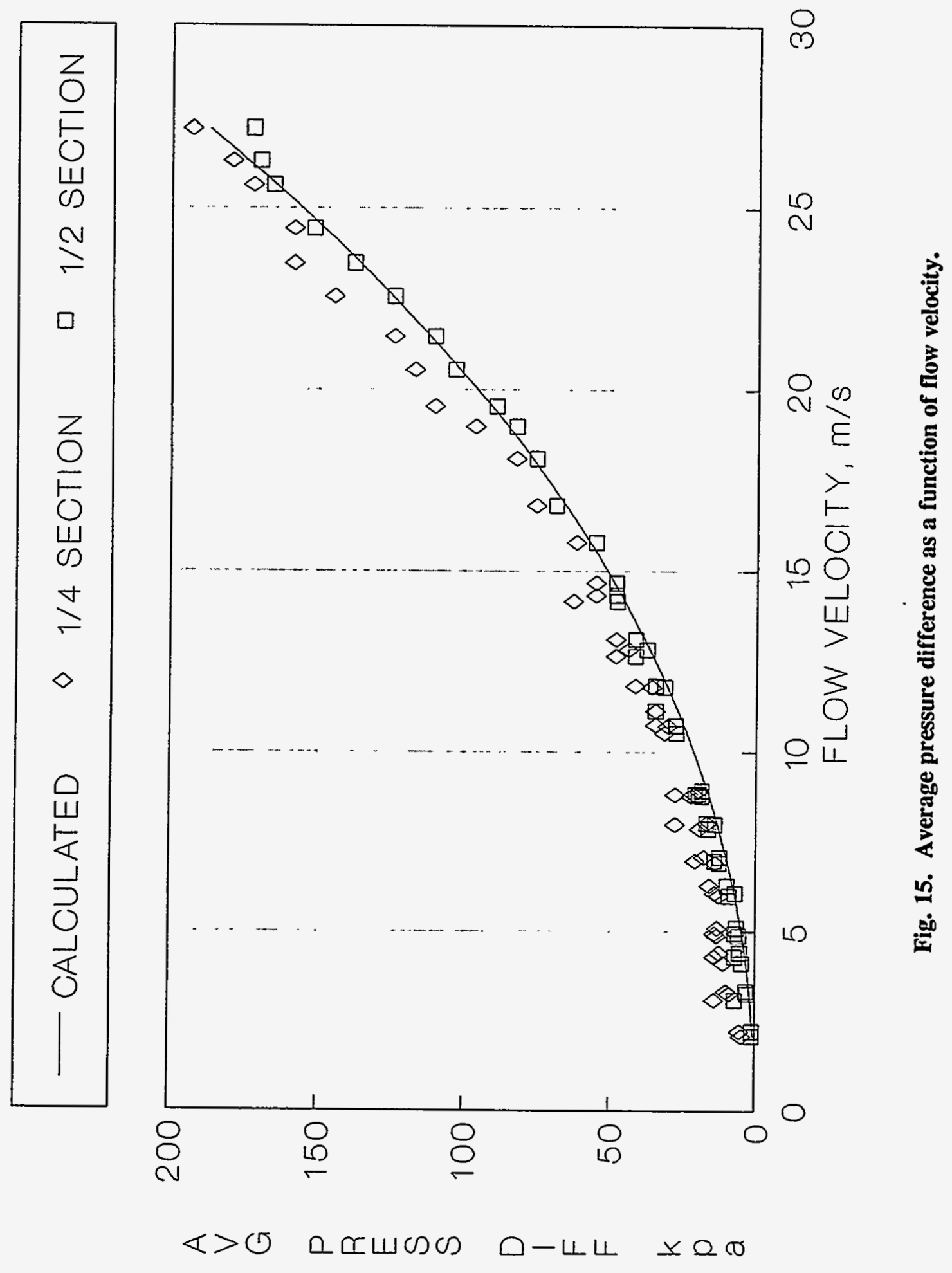



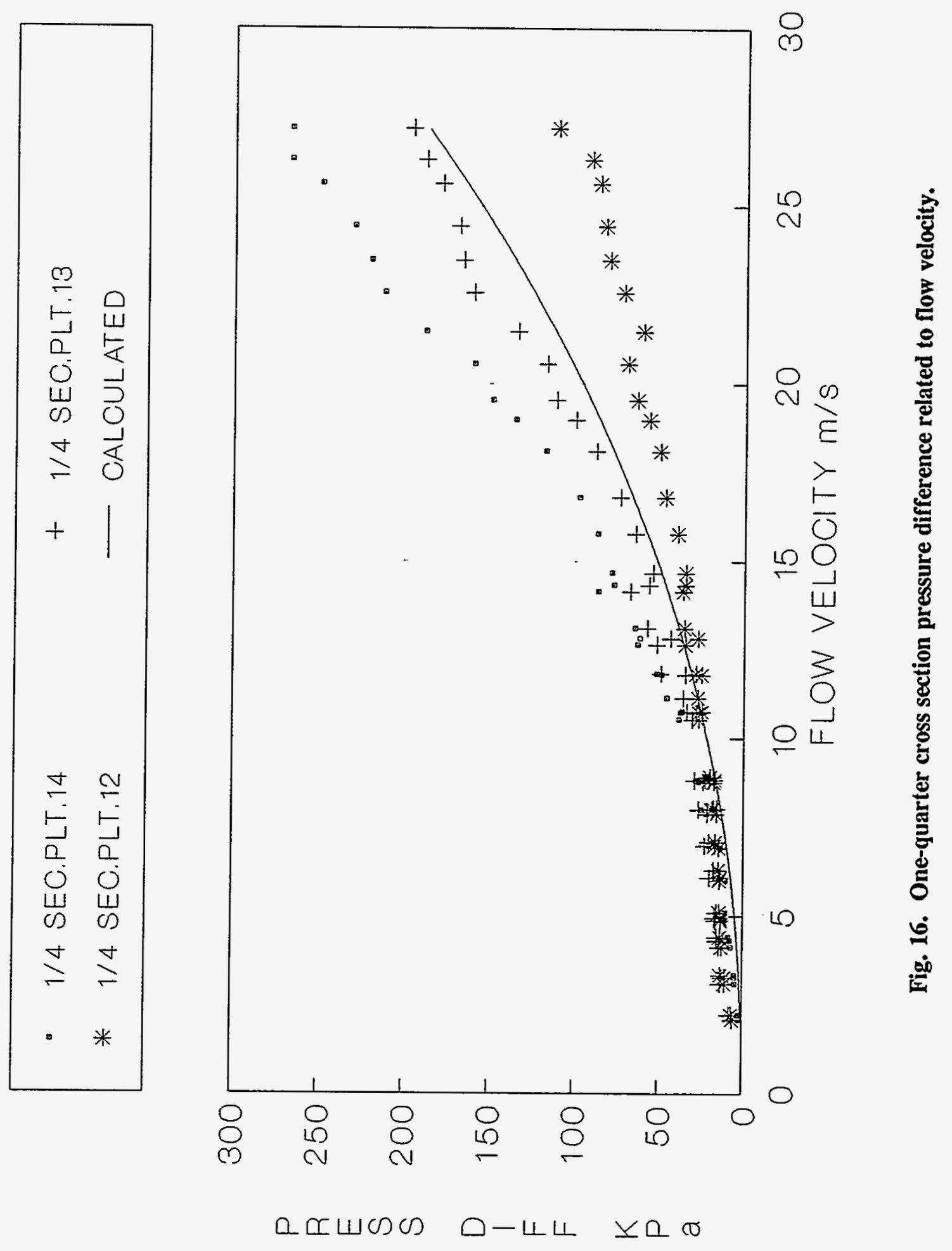

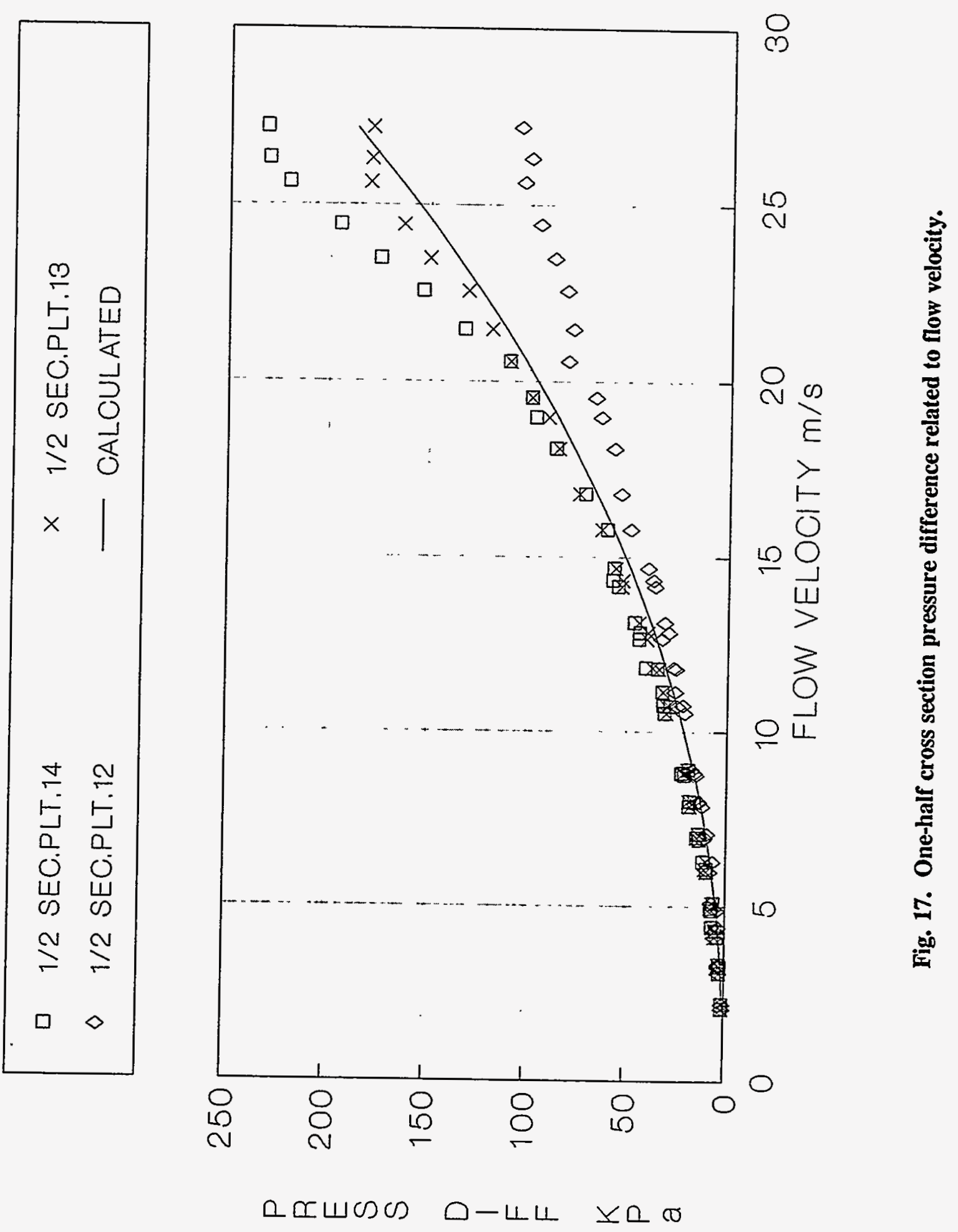
An indication of the maximum plate deflection can be found by taking the experimental pressure loads found and calculating the maximum plate deflection using the results reported in Sect. 2. For comparative purposes and to show how small the plate deflections are, the coordinate locations of the three central plates relative to the central plate at the one-quarter cross section are illustrated in Fig. 18. The deflection of each plate is found by taking its coordinate at a given flow velocity and subtracting from it the coordinate location of the plate at zero flow velocity. The $1.27-\mathrm{mm}$ distance between plates is given to illustrate the effect of plate deflection on the cooling channel. As Fig. 18 emphasizes, the deflections or changes in channel cross sections for the circular arc plates are very small. The maximum deflection occurs in plate 14 , and, at the operating flow velocity, this value is $0.04 \mathrm{~mm}$ or $3 \%$ of the channel height. As a point for comparison, the maximum deflection predicted for the equivalent involute plate is $8 \%$ of the channel height.

It was indicated in Sect. 1 that based on analysis and tests with epoxy plate models, no flutter or resonance of the prototype plates is predicted in the flow velocity range from 0 to $33 \mathrm{~m} / \mathrm{s} .^{1.2}$ Because of the approximate nature of this dynamic model analysis, some additional data on the transient response of aluminum plates at prototypic flow velocities would be helpful. Therefore, useable data from these tests on circular arc plates were analyzed for the potential of plate flutter.

Gages 12-2 and 13-5 did not experience any short circuit problems from the coolant as did all the other gages. Gage 12-2 is located on plate 12 at the three-quarter cross section, and gage 13-5 is located on the central plate at the entrance. The leading edges of the plates have a free boundary in contact with the coolant flow and are more sensitive to vibration; therefore, data from gage 13-5 will be very helpful in assessing the potential of plate vibrations.

On runs 208 through 216, gage 13-5 was connected to the strip chart recorder, which had a sensitivity of 20 microstrain. During these runs, which corresponded to flow velocities of 3.3 to $8.8 \mathrm{~m} / \mathrm{s}$, there was not only no detectable vibration but also no detectable strain ( $\$ 20$ microstrain). On all the other runs, 217 through 268, gage 13-5 was connected to the computer recorder, which during one run would scan gage 13-5 (and all of the other gages) three times in a 30-s interval in the loaded condition. The consistency (variation) of these three readings taken in a 30-s interval, while the plate was loaded, gives an indication of whether a plate is in a transient deformation pattern or in a steady deformation pattern. The possibility of a plate being transiently deformed and having its three load scans yielding essentially the same value is not very likely. Of the 40 data runs in which gage 13-5 was connected to the computer recorder, the consistency of the three recorded readings per run showed 34 runs within 4 microstrain, 5 runs within 16 microstrain, and 1 run with a 46-microstrain variation. The run before the 46-microstrain variation had a 6-microstrain variation, and the run after had a 5-microstrain variation. It appears that the 46-microstrain variation was a singular reading. Also, if the 46-microstrain variation were an actual variation caused by the plate transiently deforming, it would only add $0.006 \mathrm{~mm}$ more to the maximum deflection in the plate. Based on the data from gage 13-5, no significant vibration is evident in the central plate. Gage 12-2 was connected to the computer recorder during all of the tests, and the consistency of the loaded recordings had 1 run with a 28-microstrain variation, 1 run with a 15-microstrain variation, 5 runs with 10 -or-less microstrain variations, and 42 runs with 5 -or-less microstrain variations. Based on the data from gage 12-2, it appears that there is no significant vibration in plate 12 in the flow velocity range of these tests.

Gages 13-3 and 12-3, located on plates 13 and 12 at the mid-length cross sections, did not become erratic until run 246. The data from these gages, which included flow velocities up to $14 \mathrm{~m} / \mathrm{s}$, correlated with gages 13-5 and 12-2 in showing no evidence of plate vibration through this lower flow velocity range.

Gage 13-2, located on plate 13 at the three-quarter cross section, was connected to the strip chart recorder during runs 217 through 268. Because this gage was connected to the strip chart recorder, its response relative to time could be observed, and the intermittent erratic response could be separated from 


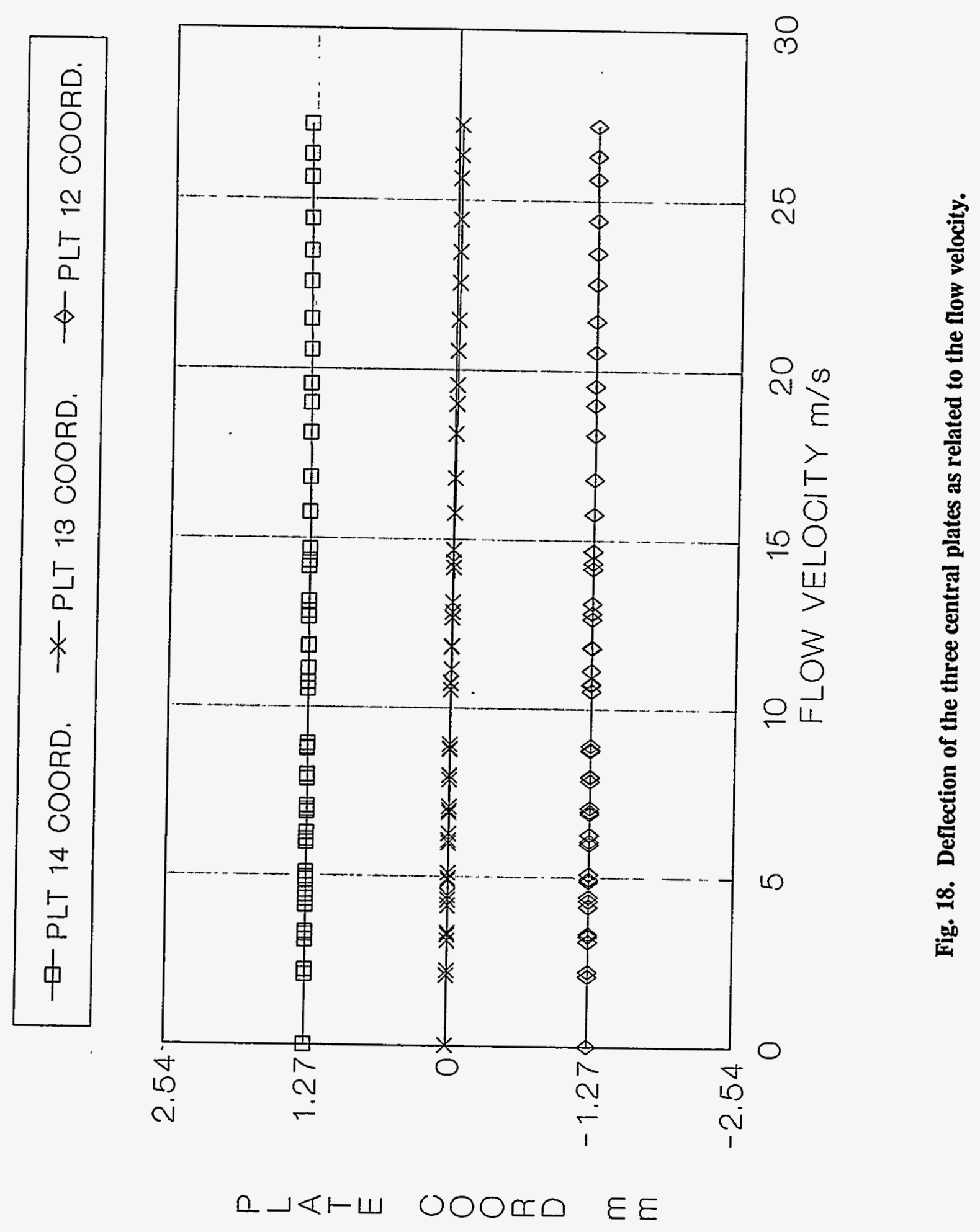


the stable response. A calibration resistor was introduced into the gage's bridge circuit during some of the gage's stable response periods to verify that readings taken during these stable periods were correct. Figure 19 is a copy of the response of gage 13-2 on run 264 and is typical of the intermittent erratic and stable response of the gage. The record began at the left and moved to the right with a chart velocity of 5 $\mathrm{cm} / \mathrm{min}$. The flow velocity started at zero and was manually adjusted to $25.6 \mathrm{~m} / \mathrm{s}$. It can be observed on the chart record that the gage response leveled off at about the half-way point of the test and corresponds to the time that the flow velocity reached $25.6 \mathrm{~m} / \mathrm{s}$. When the run started, the gage response was erratic and then became stable. The stable response of the gage indicates no vibration of the plate. When the test ended, the pump was turned off and the gage returned to its initial state as can be seen at the right of the record. Figure 20 shows the gage response on the next run, 265 , that had a flow velocity of $26.3 \mathrm{~m} / \mathrm{s}$ and essentially exhibited stable response throughout the test run.

None of the valid strain gage data indicated a vibration problem for the aluminum circular arc plates in the flow velocity range 0 to $27.2 \mathrm{~m} / \mathrm{s}$.

The rest of this section will be used to report the observations from the Series II data, which came as a result of channel flow blockage. The average pressures upstream and downstream from the test section were recorded. The pressure drops, which are the upstream pressure minus the downstream pressure, as a function of flow velocity for Series I and Series II data are compared in Fig. 21. What is evident is that Series II data started in agreement with Series I data. Before Series II data ended, the pressure drop was twice that of Series I. The blockage material had the greatest effect on the plate pressure loads at the entrance region of the plate section, which of course would be expected. The pressure load on the plates at the entrance is shown in Fig. 22. The effect of the blockage was most significant, reaching pressure loads of $1000 \mathrm{KPa}$ as compared to the $250 \mathrm{KPa}$ pressure loads realized in the unblocked data. Calculations show that yielding of the circular arc plates should start at pressure loads of $572 \mathrm{KPa}$. Examining Fig. 22, it can be seen that, in between the runs that bracketed the $572 \mathrm{KPa}$ pressure load on plate 14, the pressure loads associated with plates 12 and 13 changed abruptly. Although it cannot be conclusive, it does offer some evidence that any pressure loads on the plates that induce stresses beyond the yield should be avoided. 


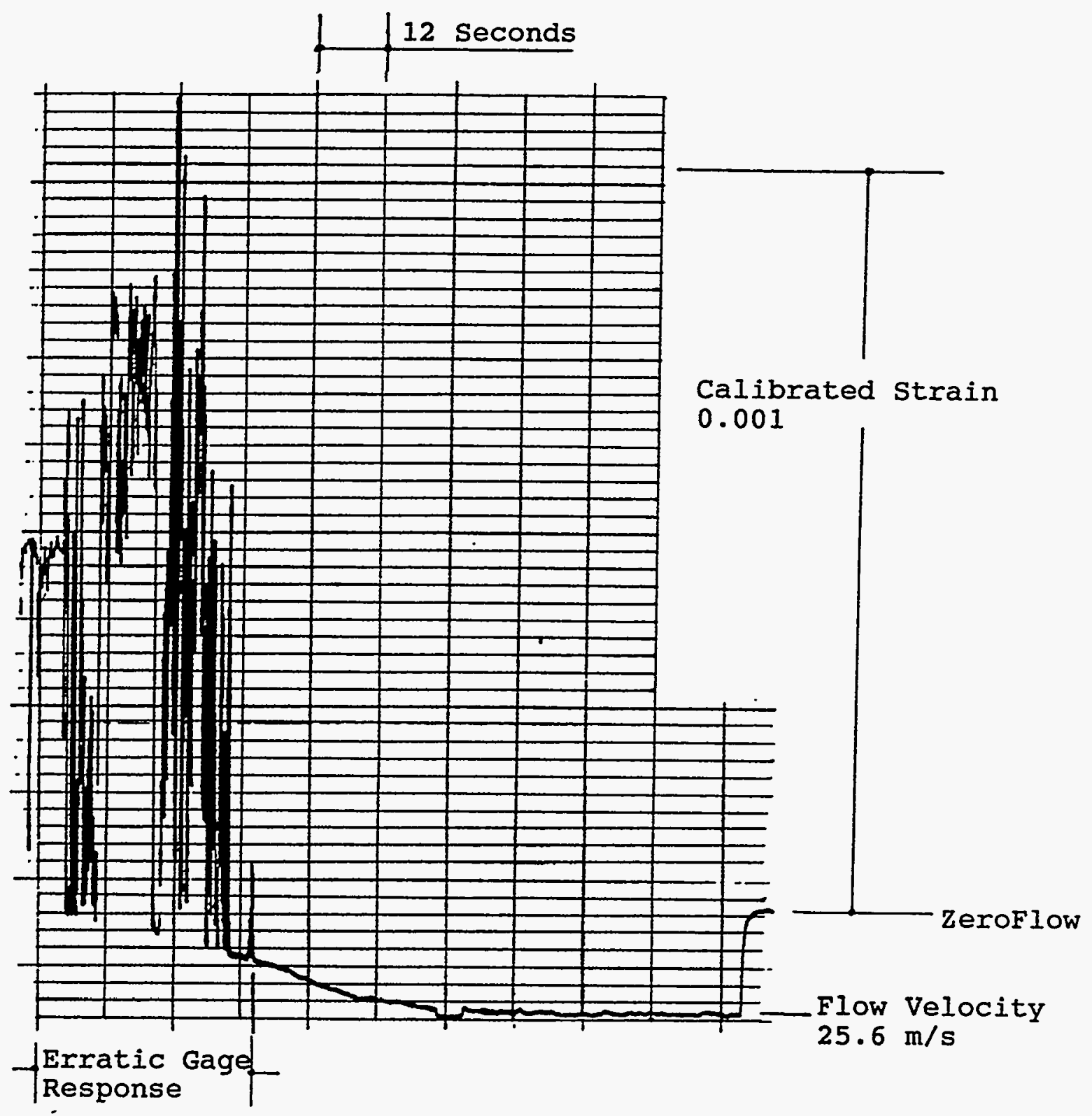

Fig. 19. Gage 13-2 transient response for run 264. 


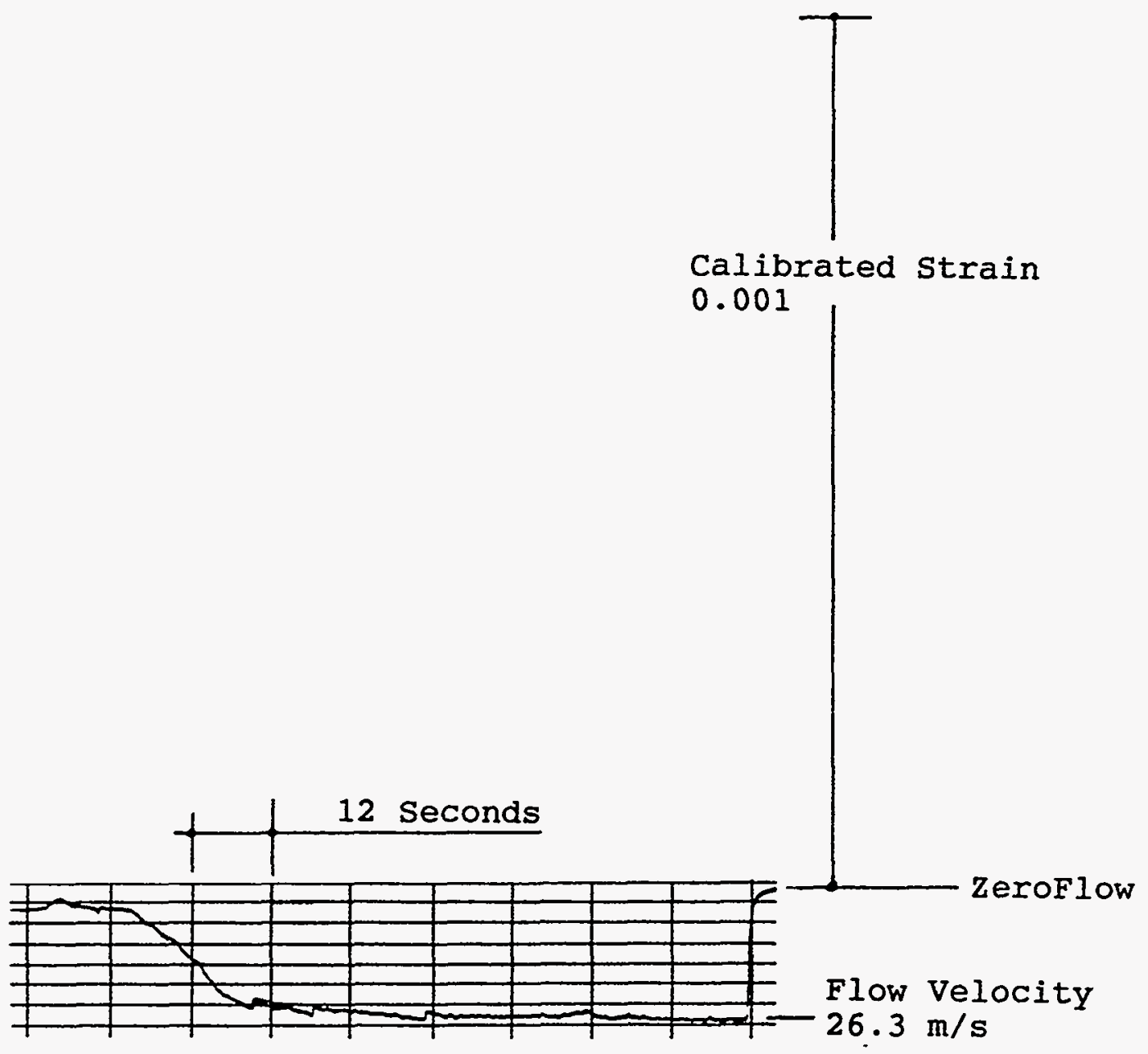

Fig. 20. Gage 13-2 transient response for run 265 . 


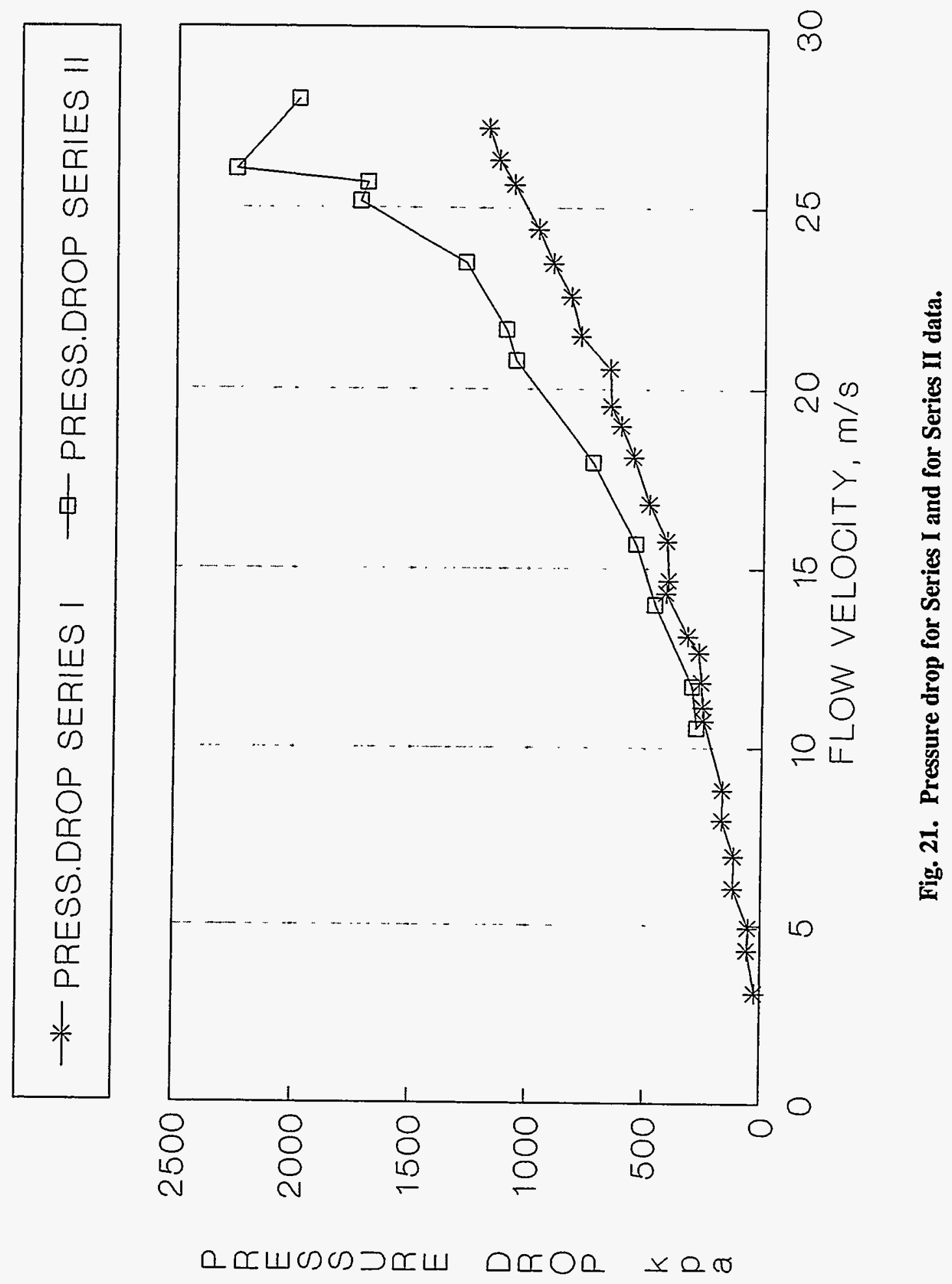



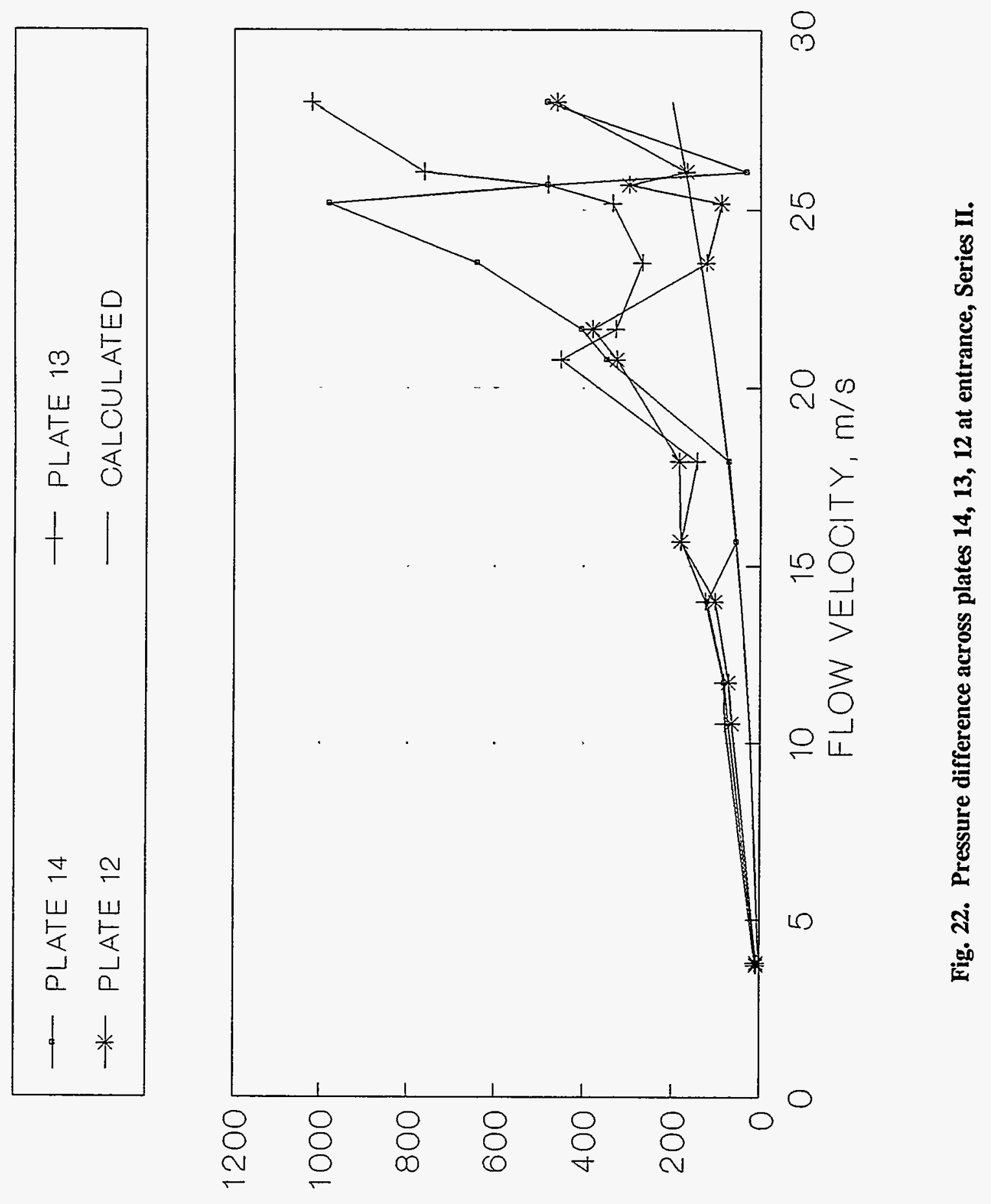

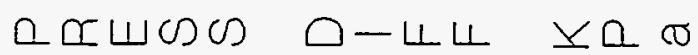




\section{CONCLUSIONS}

In Sect. 1 are six questions that need answers to help with a structural assessment of the aluminum fuel plates to coolant flow. The answers to these questions as determined from these tests on circular arc plates are given as follows.

1. The maximum plate deflection at an operating flow velocity of $25 \mathrm{~m} / \mathrm{s}$ is expected to be $0.04 \mathrm{~mm}$ or $3 \%$ of the channel height.

2. The maximum plate stress at an operating flow velocity of $25 \mathrm{~m} / \mathrm{s}$ is expected to be $23 \mathrm{MPa}$.

3. There is no evidence of an unstable plate collapse in the flow velocity range from to $27.2 \mathrm{~m} / \mathrm{s}$.

4. There is no evidence of plate vibration or flutter in a flow velocity range from 0 to $27.2 \mathrm{~m} / \mathrm{s}$.

5. Equation (1),

$$
\frac{\Delta p}{\rho V^{2}}=0.040\left(\frac{V h}{v}\right)^{0.177}
$$

gives a reasonable approximation for finding the average maximum flow load on a plate from coolant flow. The highest maximum flow load at the operating flow velocity of $25 \mathrm{~m} / \mathrm{s}$ is expected to be $43 \%$ above the average maximum flow load.

6. Analysis of an involute plate for the ANS lower fuel element subjected to the flow loads from these tests indicate that the plates could fail before the coolant reaches a velocity of $25 \mathrm{~m} / \mathrm{s}$. The substitution of circular arc plates for the involute plates would avoid this stress problem. 


\section{REFERENCES}

1. W. F. Swinson et al., Fuel Plate Stability Experiments and Analysis for the Advanced Neutron Source, ORNL/TM-12353, Martin Marietta Energy Systems, Inc., Oak Ridge Natl. Lab., May 1993.

2. W. F. Swinson et al., Follow-Up Fuel Plate Stability Experiments and Analyses for the Advanced Neutron Source, ORNL/TM-12629, Martin Marietta Energy Systems, Inc., Oak Ridge Natl. Lab., November 1993.

3. G. E. Smissaert, "Static and Dynamic Hydro-Elastic Instabilities in MTR-Type Fuel Elements. Part I. Introduction and Experimental Investigation," Nucl. Eng. Design 7, 535-546 (1968).

4. R. D. Groninger and J. J. Kane, "Flow Induced Deflections of Parallel Flat Plates," Nucl. Sci. Eng. 16, 218 (1963).

5. W. F. Swinson et al., An Examination of the Elastic Structural Response of the Advanced Neutron Source Fuel Plates, ORNL/TM-12712, Martin Marietta Energy Systems, Inc., Oak Ridge Natl. Lab., September 1994. 


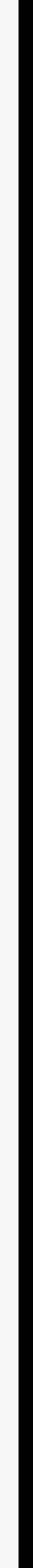


Appendix A: MODEL THEORY

A-1 



\section{A-3}

A theory for going from model data to prototype data with steady-state loading and with transient loading is given as follows.

The principal variables for the plates are taken as

$\delta$ plate deflection,

$E$ modulus of elasticity,

$\mu_{a}$ Poisson's ratio,

$L$ dimension parameter, and

$\rho_{a}$ plate density.

The plate density is a variable involving mass and is related to inertia effects. Because of the relation to inertia properties, density is often used to characterize dynamic motion but is not needed in characterizing steady-state deformation. An example of this is seen in the deflection of a simple beam. If the beam deformation is steady, density does not appear in the solution; however, if the deformation is transient, density appears in the solution.

The principal variables for the coolant are assumed to be

$V$ coolant velocity,

$\mu$ coolant viscosity, and

$\rho$ coolant density.

The necessary dimensionless terms or independent variables needed to describe the interaction of the fluid and structure with these principal variables are

$\pi_{1}=\delta / L, \quad \pi_{2}=\mu_{a}, \quad \pi_{3}=\mu V /(E L), \quad \pi_{4}=V^{2} \rho / E, \quad$ and $\pi_{s}=V^{2} \rho_{a} / E$.

To go from model data to prototype data, the dimensionless terms as related to the model must match the dimensionless terms as related to the prototype, subject to the constraint that the variables selected represent the primary variables when the plates are loaded by the coolant flow.

The first dimensionless term, $\pi_{1}$, relates the measured model deflection to the prototype deflection. In the case of the epoxy plate models, the models were full scale, and therefore the measured model deflection represented the prototype deflection. ${ }^{1,2}$

The second dimensionless term, $\pi_{2}$, depends on the material property, Poisson's ratio. The match between this property for the epoxy plates and aluminum plates could not be achieved. However, the term is considered to be a secondary term in that Poisson's ratio for aluminum is 0.33 and that for epoxy is 0.35. In plate theory Poisson's ratio takes the form of $\left(1-\mu_{2}{ }^{3}\right)$ in solutions, and the difference in this term whether using 0.33 or 0.35 is small.

The third dimensionless term, $\pi_{3}$, because it includes fluid viscosity, is related to the friction force in the direction of the flow velocity; thus, its effect on the plate deflection in a direction perpendicular to the flow velocity is considered small.

The fourth dimensionless term, $\pi_{4}$, relates the equivalent prototype velocity to the experimental model velocity for a stable deformation configuration. Dynamic terms such as $\pi_{5}$, which includes plate density, are not variables in a structure that is statically deformed. Therefore, for the epoxy plate model using the same coolant,

$$
\left(V_{\text {procotype }}\right)^{2} \cdot E_{\text {model }}=\left(V_{\text {model }}\right)^{2} \cdot E_{\text {procoxype }},
$$


and for the lower element epoxy plate tests with the modulus of elasticity that was found,

$$
V_{\text {procotype }}=5.034 \cdot V_{\text {model }} \text {. }
$$

Equation (A2) was used to relate the equivalent prototype velocity using aluminum plates to the model test velocity when the plates were in a stable deformed shape. ${ }^{1}$

The fifth dimensionless term, $\pi_{5}$, is a variable for relating dynamic deformation. If a transient event such as flutter occurs at a measured model flow velocity during a model test, the equivalent flow velocity at which this will occur in the prototype can be estimated. This estimate is made by assuming that during the dynamic event the dominant variable is $\pi_{s}$, then

$$
\left(V_{\text {procotypec }}\right)^{2} \cdot \rho_{\text {procotype }} \cdot E_{\text {model }}=\left(V_{\text {model }}\right)^{2} \cdot \rho_{\text {model }} \cdot E_{\text {prolotype }} \cdot
$$

For the epoxy model tests of ref. 1 Eq. (A3) reduces to

$$
V_{\text {procotype }}=3.27 \cdot V_{\text {model }} \text {. }
$$

A transient event occurring in the tests on epoxy plates at a given model velocity was related to the prototype velocity through Eq. (A4). Tests run on the epoxy plates found some flutter type motion at about $10 \mathrm{~m} / \mathrm{s}$ model flow velocity. ${ }^{2}$ Using Eq. (A4), it is estimated that the aluminum prototype plates would experience some flutter type motion at $33 \mathrm{~m} / \mathrm{s}$. This value is the basis for the comment in the introduction that no significant flutter is anticipated in the involute plates in the flow velocity range of 0 to $33 \mathrm{~m} / \mathrm{s}$. 
Appendix B: ESTIMATE OF FLOW BLOCKAGE EFFECTS

B-1 



\section{B-3}

Blockage of the flow channels is a concern in evaluating the design of the fuel element plates. This is a complicated problem in that the size of the blockage is unknown, the blockage location is unknown, and, therefore, the flow field associated with these unknown blockage parameters is unknown. However, some conservative assumptions can be made, and estimates for the size of blockage and location of the blockage required for failure can be made.

First, if total blockage in a channel occurs, plate failure is inevitable. The pressure in the channel that is past the blockage becomes the exit pressure, and for ANS design cases this is on the order of 900 $\mathrm{KPa}$ below the entrance pressure of the unblocked channels. None of the proposed fuel plates can support this magnitude of pressure load.

The effect of partial blockage can be estimated by using the solution given in Eqs. (10) and (11) for $M_{O F}, N_{O F}$, and $V_{O F}$. These are the boundary unknowns for a circular arc plate subjected to a concentrated force $F$ located at $\theta_{c}$ (Fig. 4). The area of the wake that follows the blockage is based on the wake area per unit length of the plate. Therefore, the wake area per unit length is the dimension of the blockage along the arc length. The concentrated force $F$ is conservatively estimated as

$$
F=\mathrm{p}_{d y n} \cdot A_{b},
$$

where

$$
\begin{aligned}
\mathrm{p}_{d y n}= & \text { dynamic pressure of the coolant } \\
A_{b}= & \text { area of the wake per unit length or the } \\
& \text { length of the flow blockage along the } \\
& \text { arc length. }
\end{aligned}
$$

By placing $F$ at different locations on the arc, the boundary unknowns can be evaluated. The magnitude of $F$ necessary to cause yielding in the plate for each different location of $F$ can be found. The size of blockage necessary to cause failure can be estimated through Eq. (B1).

Using this approach for the circular arc plate described in this report, the following information was determined.

1. The largest stresses occur when the blockage is centered on the arc.

2. The largest stresses occur at the center of the arc in contrast to occurring at the boundaries for the pressure load.

3. If $12 \%$ of the channel with respect to the channel arc length is blocked, the calculated stresses predict yielding in the plate at a coolant flow velocity of $25 \mathrm{~m} / \mathrm{s}$.

To help in evaluating this technique, a comparison was made with some similar work done on the involute plates of the lower element. The thermal-hydraulics section had an experiment that involved a single flat rectangular channel with $25 \%$ blockage at the edge. Using a laser velocimeter, the average velocity in a small area was determined at many locations and used to establish the flow velocity field. From the velocity field, a pressure distribution was calculated. There is some question in calculating the pressure distribution in that in highly turbulent flow there is an unknown amount of the flow energy in the form of rotational energy and how this is taken into account in calculating pressures is uncertain. However, this calculated pressure field was used as input to a finite element program of the involute plate to calculate the maximum stress. In comparing this finite element result with the estimated result reported in this section, both approaches predicted the same location in the plate for maximum stress, although the estimated result was $32 \%$ higher than the finite element result. 


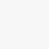


Appendix C: THERMAL STRESSES IN THE PLAN OF THE ARC WITH ONE BOUNDARY SUPPORT FLOATING

C-1 



\section{C-3}

Plate stresses in the plane of the arc that result from the plate being at one temperature and the support boundaries at a different temperature can be calculated by using energy techniques. The boundary conditions are assumed to be such that one support cylinder is free to rotate relative to the other support cylinder. The radial distance between support cylinders is assumed to be constant, which requires all of the deformation to occur in the plates, although, in reality, the cylinders do deform and reduce the stresses. The outer support cylinder is free to rotate (Fig. C.1). Equilibrium yields

$$
\begin{gathered}
V_{o}=N_{l} \sin \left(\theta_{a}\right)-V_{l} \cos \left(\theta_{a}\right), \\
N_{o}=N_{l} \cos \left(\theta_{a}\right)+V_{l} \sin \left(\theta_{a}\right), \\
M_{o}=M_{l}+N_{l} r_{c}\left[1-\cos \left(\theta_{a}\right)\right]-V_{l} r_{c} \sin \left(\theta_{a}\right),
\end{gathered}
$$

where

$\mathrm{V}_{o}, N_{o}, M_{o}$ are the shear load, the tangent load, and the moment load on the plate at the inside support cylinder;

$\mathrm{V}_{l}, N_{l}, M_{l}$ are the shear load, the tangent load, and the moment load on the plate at the outside support cylinder;

$r_{c}$ is the radius of the circular arc plate;

$\theta_{a}$ is the included angle of the circular arc plate.

The moment equation at any location $\theta$ is

$$
M=M_{l}+N_{l} r_{c}[1-\cos (\theta)]-V_{l} r_{c} \sin (\theta) .
$$

The so-called axial load equation, which is better described as the tangent load equation, at any location $\theta$ is

$$
N=N_{l} \cos (\theta)+V_{l} \sin (\theta) .
$$

It is more convenient to rewrite Eqs. (C2) and (C3) so that the force components are normal and tangent to the support cylinders because the boundary conditions are better described in terms of those directions.

These equations become

and

$$
\begin{aligned}
M= & M_{l}+f_{r d} r_{c}\left[\sin \left(\theta_{e}+\phi_{e}\right)-\sin \left(\theta_{e}+\phi j \cos (\theta)-\cos \left(\theta_{e}+\phi_{e}\right) \sin (\theta)\right]\right. \\
& +f_{a r c} r_{c}\left[\cos \left(\theta_{c}+\phi_{e}\right)-\cos \left(\theta_{e}+\phi_{e}\right) \cos (\theta)+\sin \left(\theta_{e}+\phi_{\jmath} \sin (\theta)\right]\right.
\end{aligned}
$$

$$
N=f_{r o}\left[\sin \left(\theta_{e}+\phi_{e}\right) \cos (\theta)+\cos \left(\theta_{e}+\phi_{e}\right) \sin (\theta)\right]+f_{\alpha}\left[\cos \left(\theta_{e}+\phi_{e}\right) \cos (\theta)-\sin \left(\theta_{e}+\phi_{e}\right) \sin (\theta)\right],
$$




\section{C-4}

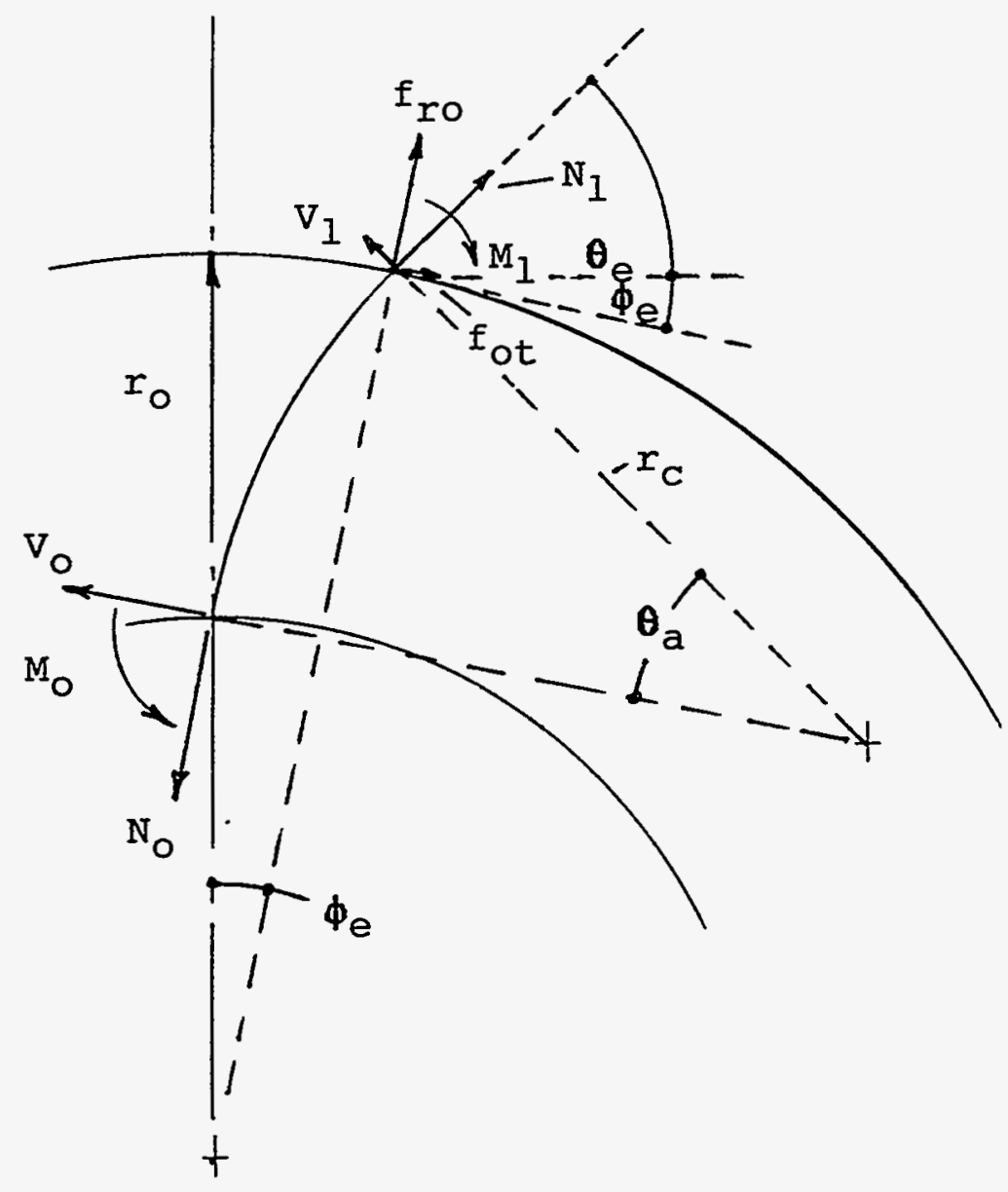

Fig. C1. Plate free body diagram with thermal load. 


\section{C-5}

where

$f_{\text {ro }}$ is the component of the plate outer boundary force along the radial direction of the outside support, cylinder,

$f_{\alpha}$ is the component of the plate outer boundary force along the tangential direction of the outside support cylinder,

$\theta_{c}$ is the circular arc plate outer tangent angle,

$\phi_{c}$ is the support cylinder angle to the outer plate boundary.

There are three thermal boundary conditions that must be satisfied to yield a solution for this case.

1. The plate, being at a higher temperature than the support boundaries, tries to expand but is constrained by the support cylinders. To satisfy this condition, the plate is allowed to expand thermally by $\alpha \cdot \Delta T \cdot l_{x}$, where $\alpha$ is the coefficient of expansion, $\Delta T$ is the temperature difference between the plates and the support boundaries, and $l_{r}$ is the radial projection of the plate. The plate displacement in the radial direction, $\delta_{r o}$ is produced by force, $f_{r o}$. By applying enough force, $f_{r o}$, the plate displacement in the radial direction, $\delta_{\text {ro }}$, can be made to offset the thermal expansion and satisfy this boundary constraint or

$$
\delta_{r o}=-\alpha\left[r_{0}-r_{i} \cos \left(\phi_{e}\right)\right] \Delta T,
$$

where $r_{o}$ and $r_{i}$ are the outer and inner radii of the support cylinders.

2. The outer support cylinder is free to rotate and results in an outer plate boundary displacement of $\delta_{\alpha}$. The outer plate boundary displacement is also equal to $r_{o} \cdot \Delta \theta$, where $\Delta \theta$ is the angle at which the outer boundary of the plate rotates and the angle at which the outer support cylinder rotates. This constraint is expressed as

$$
\Delta \theta \cdot r_{0}=\delta_{\alpha}
$$

3. When the outer support cylinder rotates, a moment is produced in the plate and equilibrium requires

$$
M_{l}+f_{\alpha} r_{o}=0
$$

Using energy techniques, the displacement terms, $\delta_{\text {so }}$ and $\delta_{\alpha}$, and the rotation term, $\Delta \theta$, can be expressed as

$$
\begin{aligned}
& \delta_{r o}=\frac{r_{c}}{E} \int_{0}^{\theta_{a}}\left(\frac{M}{I} \frac{\partial M}{\partial f_{r o}}+\frac{N}{A} \frac{\partial N}{\partial f_{r o}}\right) d \theta, \\
& \delta_{o t}=\frac{r_{c}}{E} \int_{0}^{\theta_{c}}\left(\frac{M}{I} \frac{\partial M}{\partial f_{o t}}+\frac{N}{A} \frac{\partial N}{\partial f_{o t}}\right) d \theta
\end{aligned}
$$




$$
\Delta \theta=\frac{I_{c}}{E I} \int_{0}^{\theta_{d}} M \frac{\partial M}{\partial M_{1}} d \theta
$$

Using Eqs. (C4) and (C5) to expand Eqs. (C9), (C10), and (C11), the results can be inserted into the boundary equations, Eqs. (C6), (C7), and (C8), and a solution found for the plate boundary loads.

Using this technique of solution, the boundary loads for the circular arc plate of this report are

$M_{o}=0.0240 \mathrm{~N} /{ }^{\circ} \mathrm{C}, \quad N_{o}=-0.946 \mathrm{~N} /\left(\mathrm{m}^{\circ} \mathrm{C}\right), V_{o}=0.149 \mathrm{~N} /\left(\mathrm{m}^{\circ} \mathrm{C}\right)$, and

$M_{l}=-0.0320 \mathrm{~N} /{ }^{\circ} \mathrm{C}, N_{l}=-0.315 \mathrm{~N} /\left(\mathrm{m}^{\circ} \mathrm{C}\right)$, and $V_{l}=-0.946 \mathrm{~N} /\left(\mathrm{m}^{\circ} \mathrm{C}\right)$.

The magnitude of the maximum normal stresses at these locations calculate to be

$\sigma_{0}=84.4 \mathrm{Kpa} /{ }^{\circ} \mathrm{C}$ and $\sigma_{1}=109.9 \mathrm{Kpa} /{ }^{\circ} \mathrm{C}$.

These stress values will be multiplied by the average temperature difference between the plates and the support cylinders. In the operation of the reactor, these values then will be superimposed on the stresses induced by the pressure load. Final evaluation will also have to account for any temperature variations within the plates. 
Appendix D: DATA COLLECTED

D-1 
. 


\section{D-3}

The data collected was computer controlled, and the basic information was voltage readings, which are included in this appendix.

Section 3 includes information on how these voltage readings were translated into pressures, strains, and/or flow rate. The conversion constants are

for pressure readings, 10 volts $=3.447 \mathrm{MPa}$;

for strain readings, 1 microvolt $=1$ microstain; and

for flow rate, liters/s $=($ volt rdg -1$) \cdot(5.126)$.

The data set number corresponds to the same numbered experimental flow run, which is described in Sect. 4. Each datum comes from a specific location in the experiment, and each location is assigned an identification number, which is described in Sect. 4. Table I references the identification number to a computer voltage reading. 


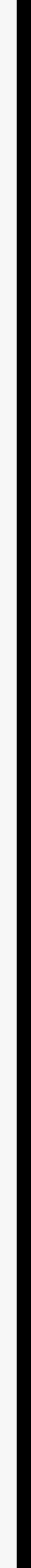


Arentive :

ANE_ME_TESTS

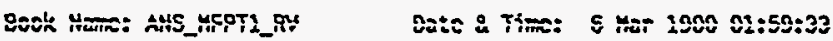

Püge Lascription:

OATA SET HQ. 207

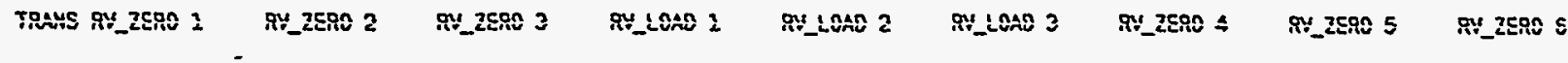

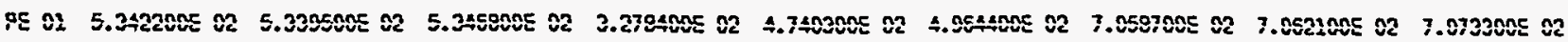

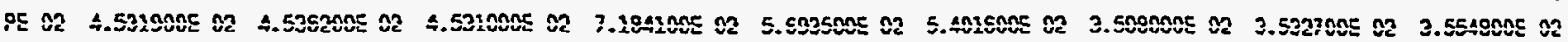

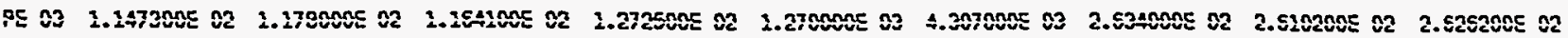

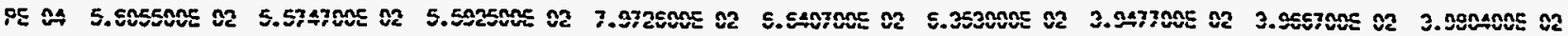

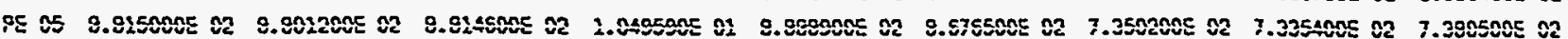

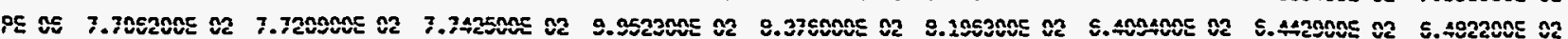

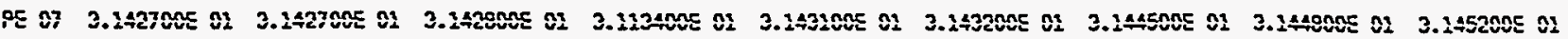

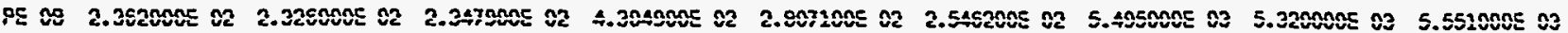

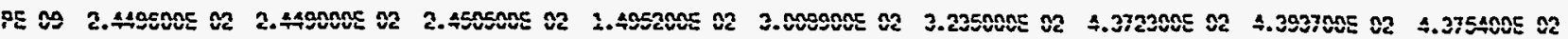

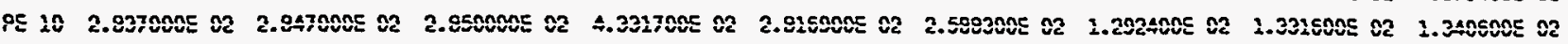

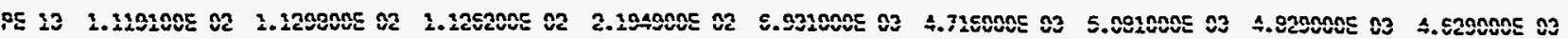

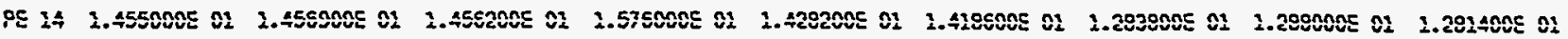

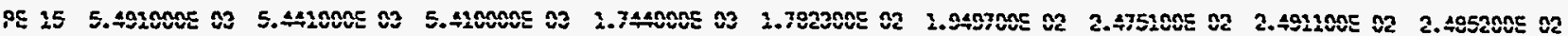

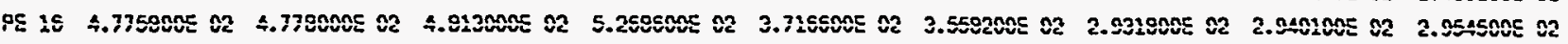

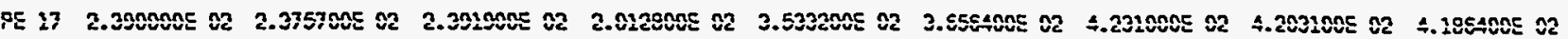

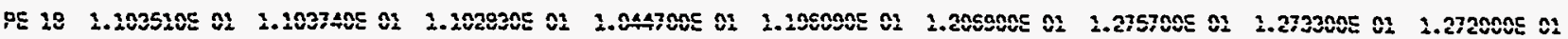

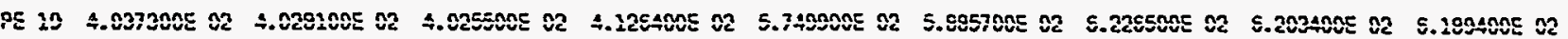

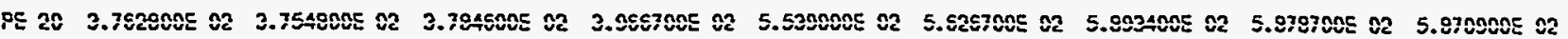

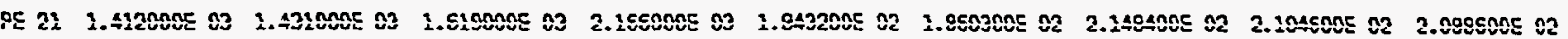

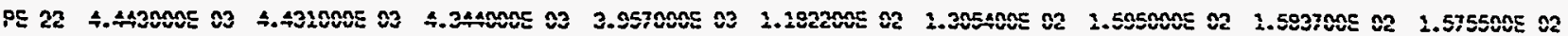

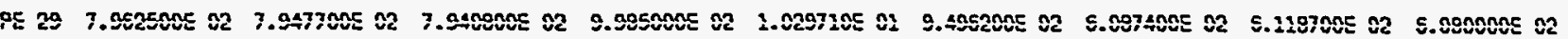

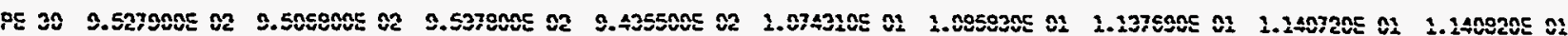

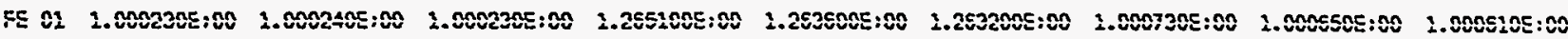

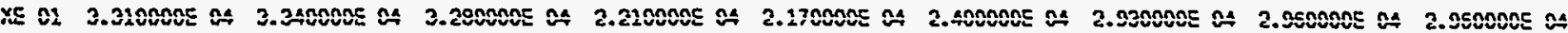

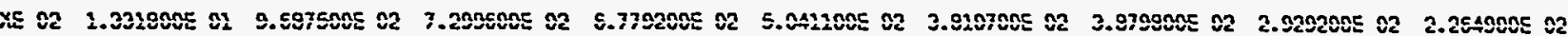

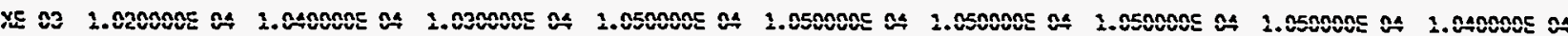

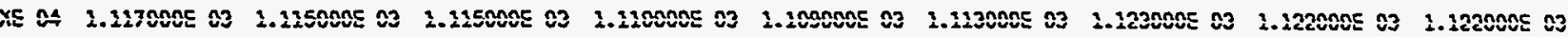

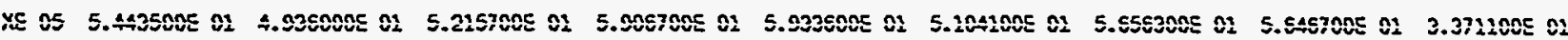

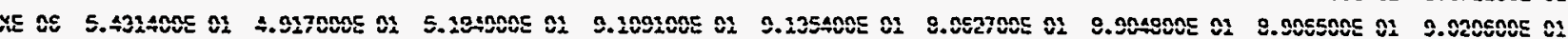

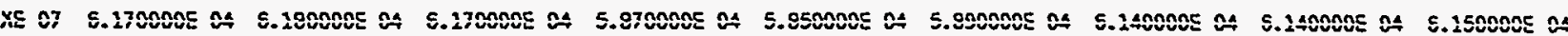

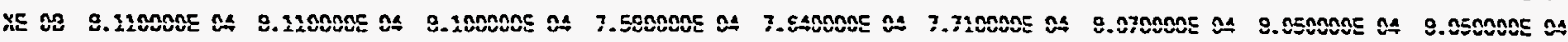

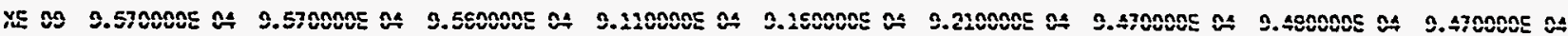

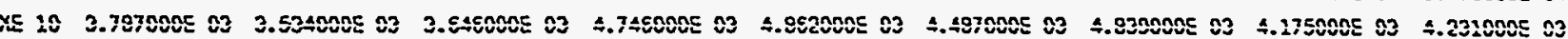

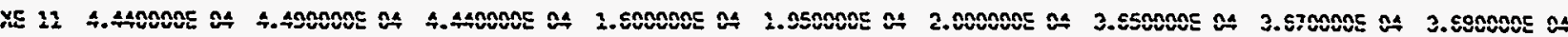

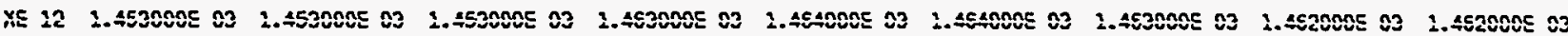

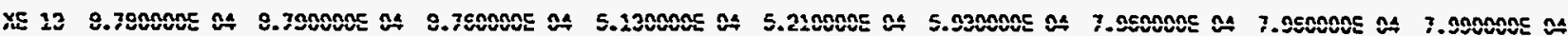

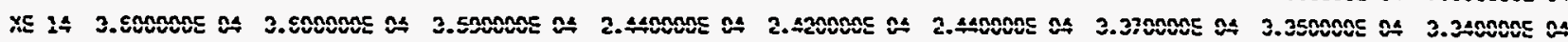

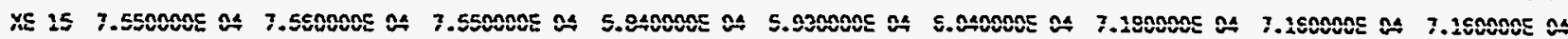

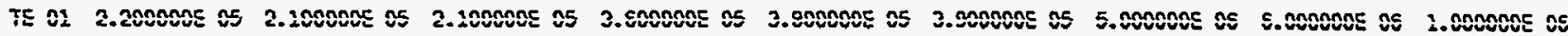

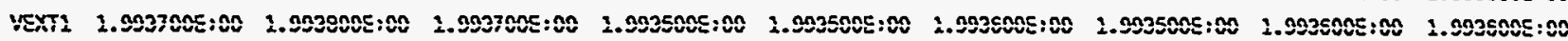

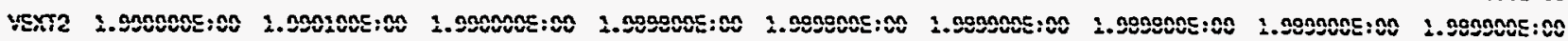

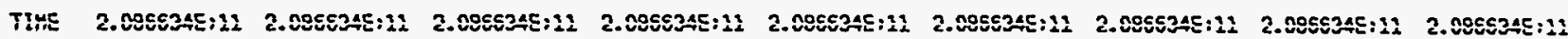




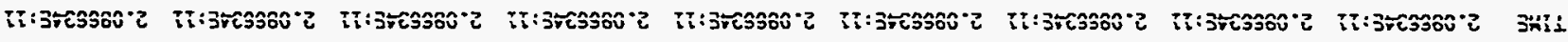

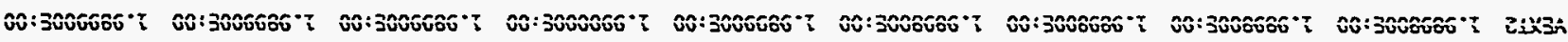

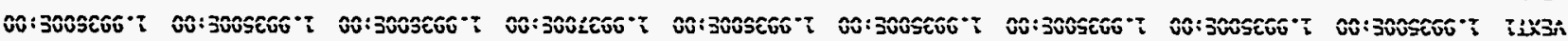

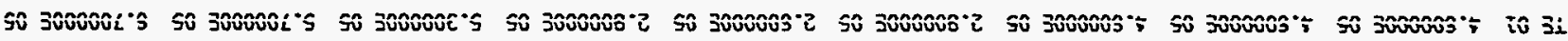
O0 3000025"

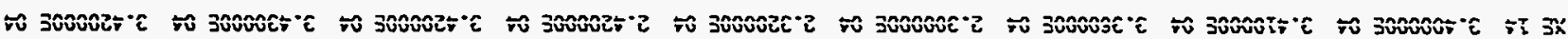

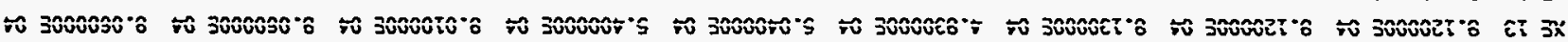

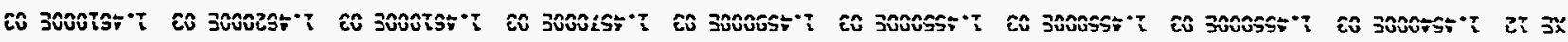

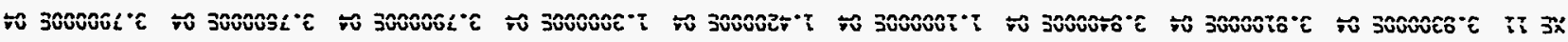

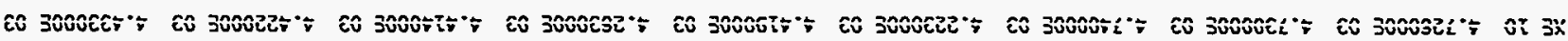

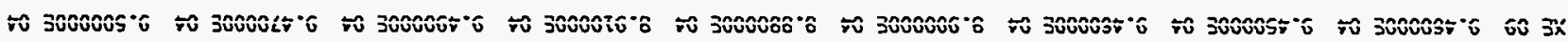

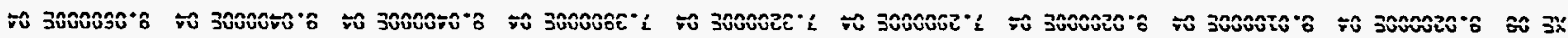

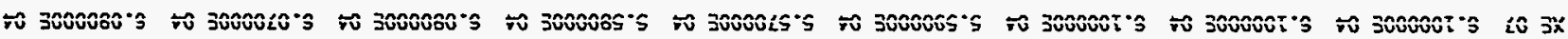

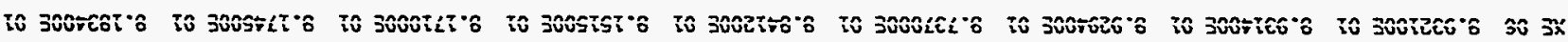

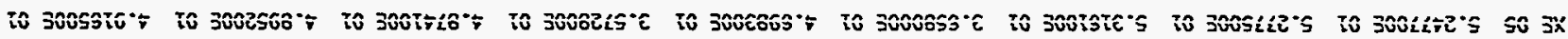

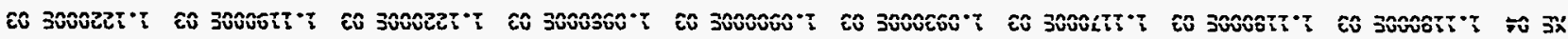

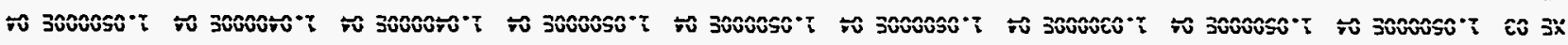
¿0 300L6

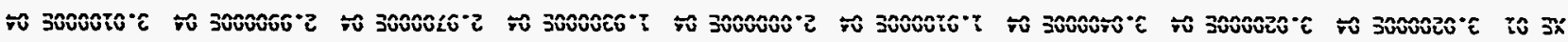

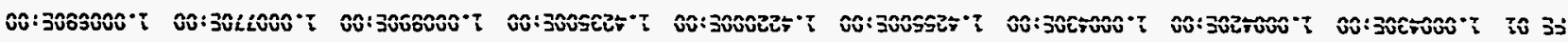

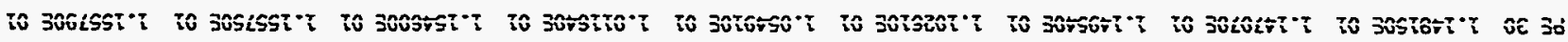

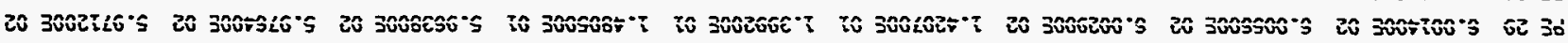

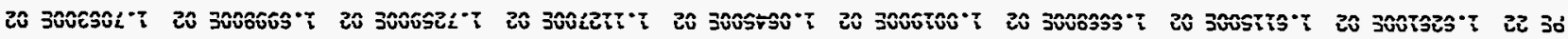

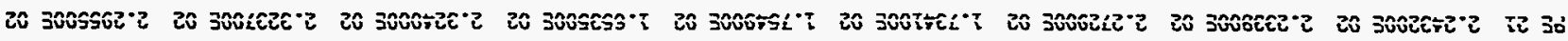

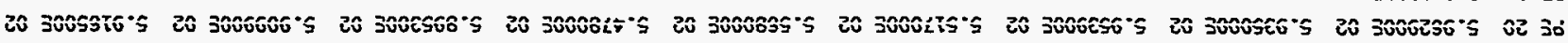

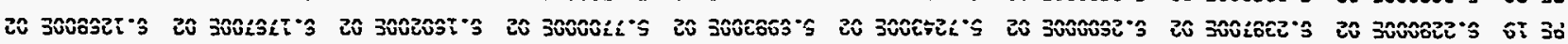

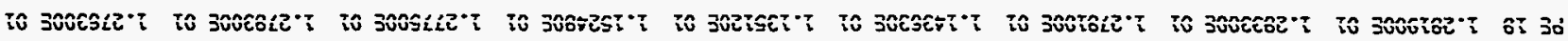
20 300ZSSE"

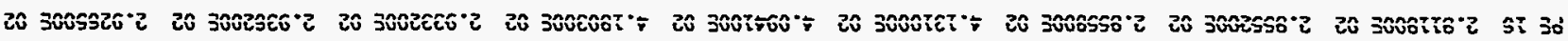

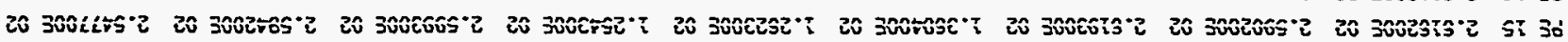

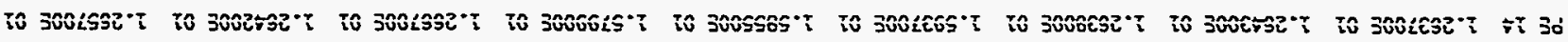

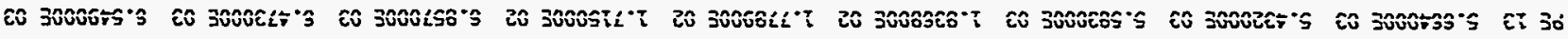

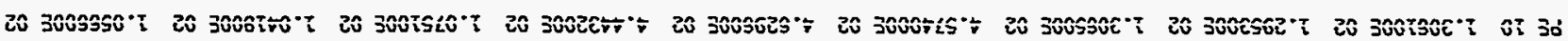

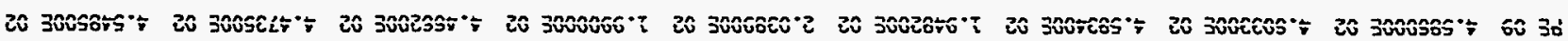

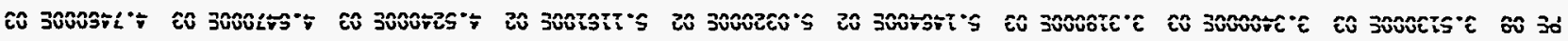

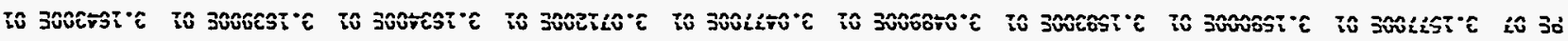

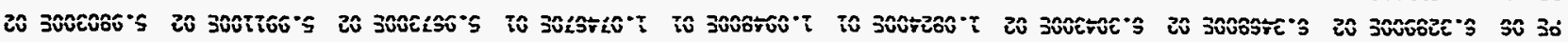

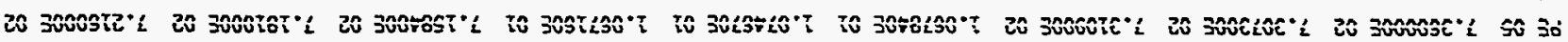

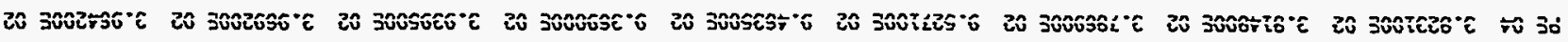

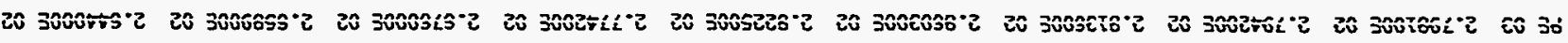

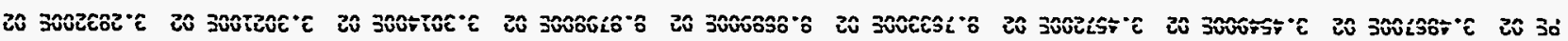

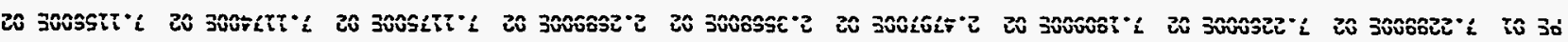

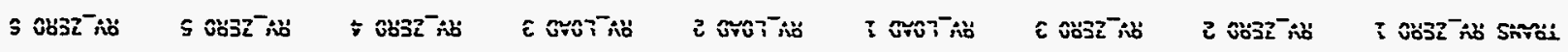

Sỗ : 
Arthition

AUE_:MOTSSTS

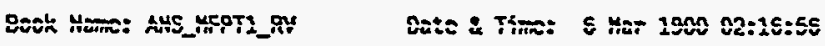

Page lescriptioni:

GATA ScT 208

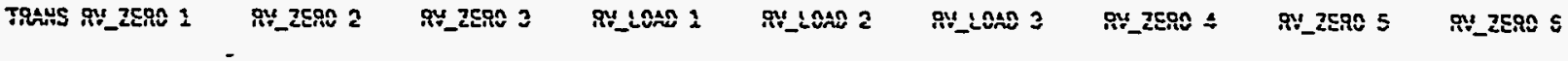

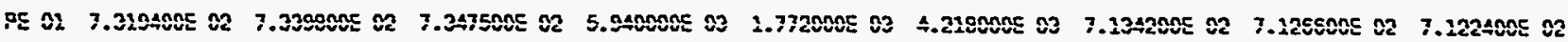

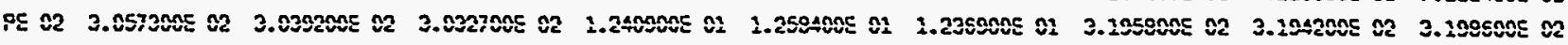

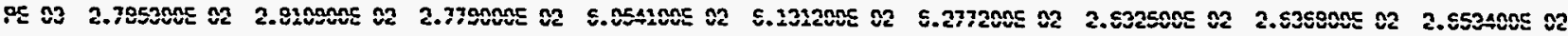

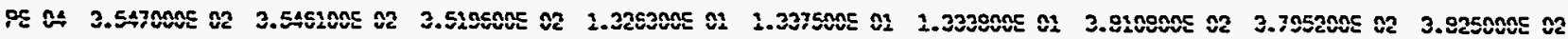

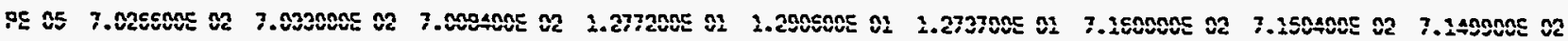

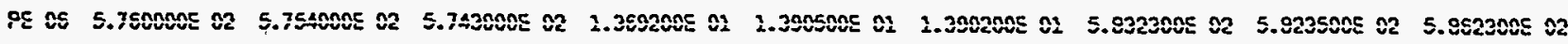

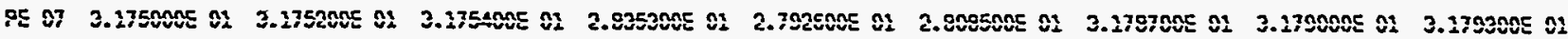

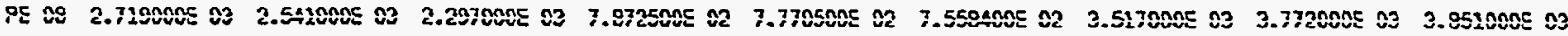

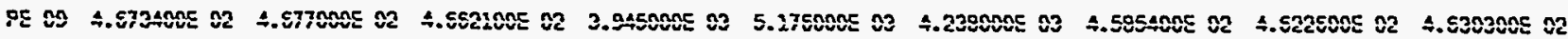

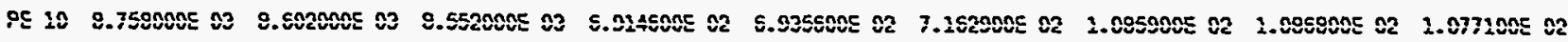

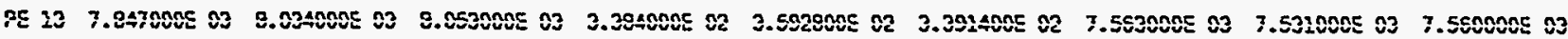

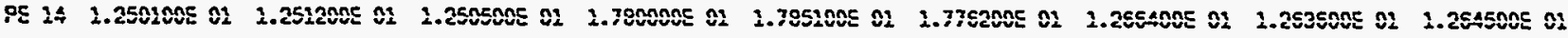

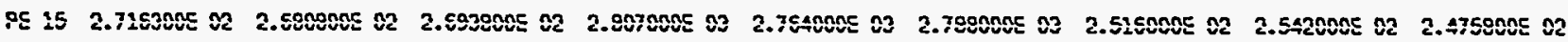

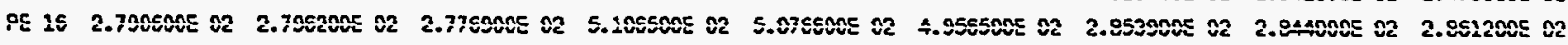

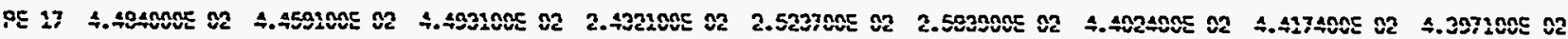

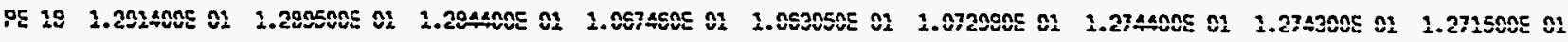

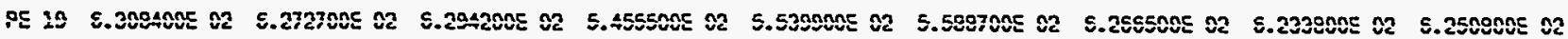

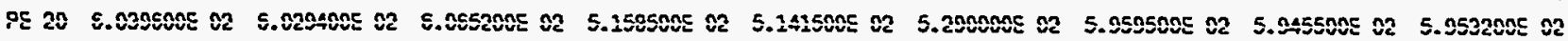

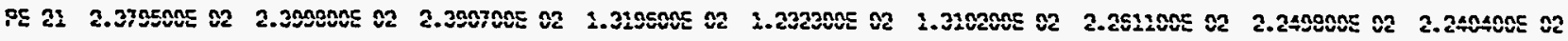

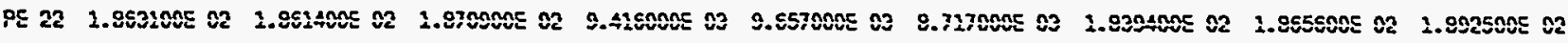

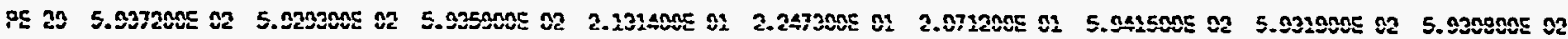

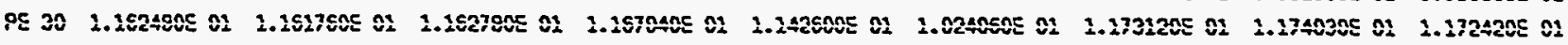

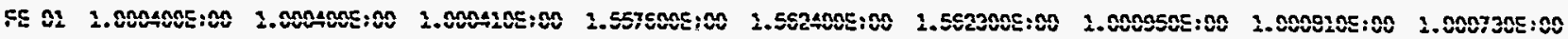

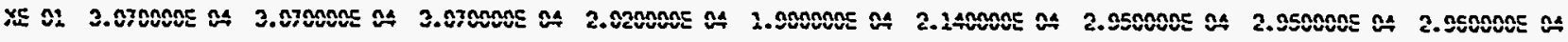

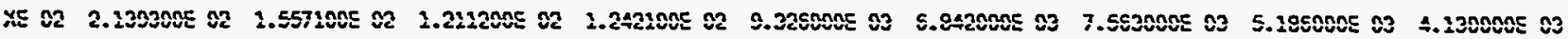

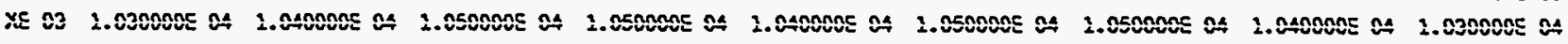

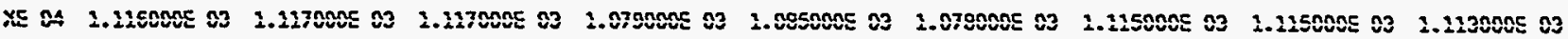

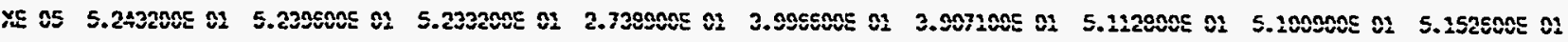

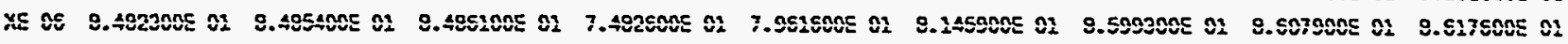

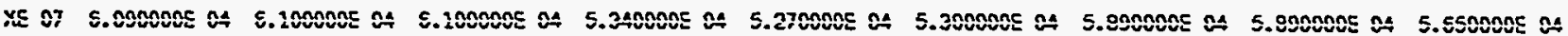

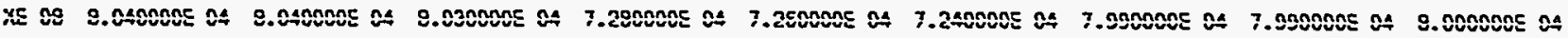

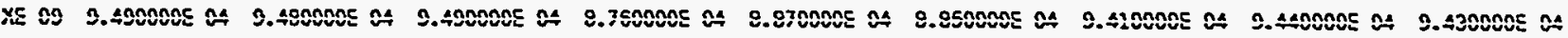

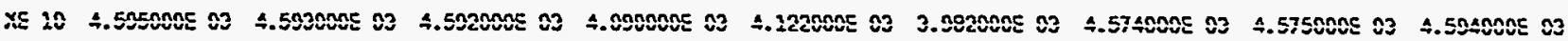

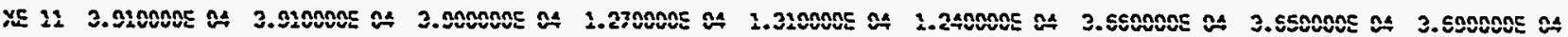

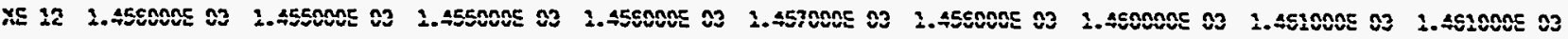

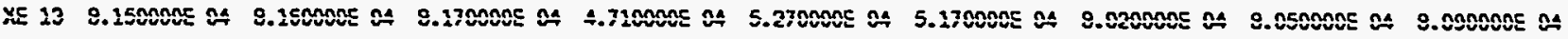

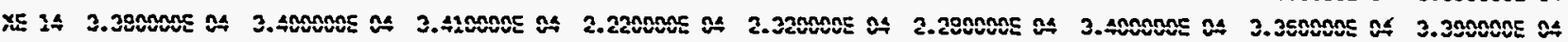

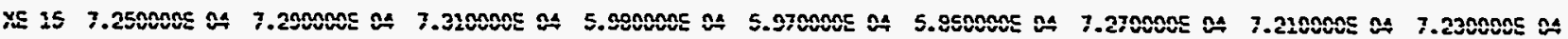

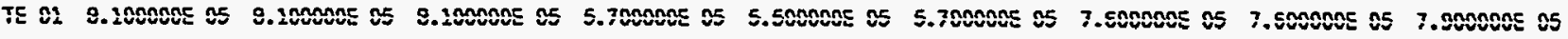

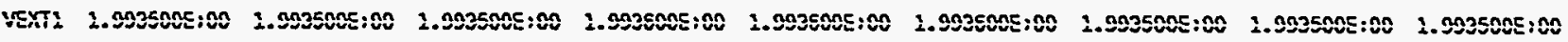

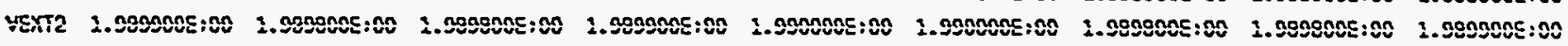

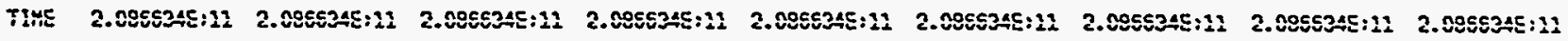


Page expeription:

DATA STS 210

TRAMS

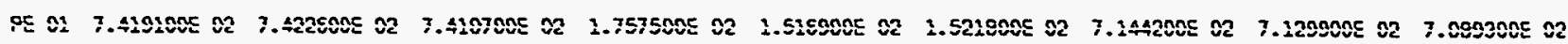

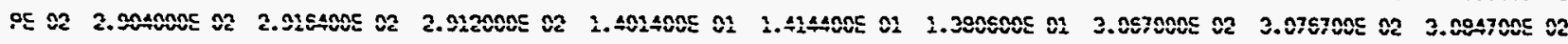

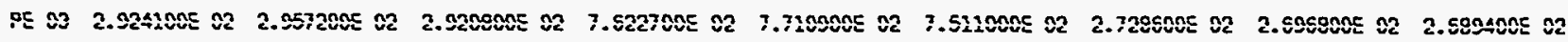

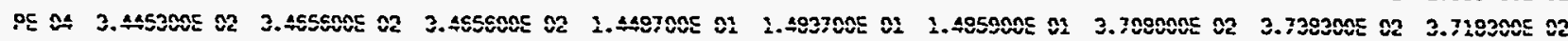

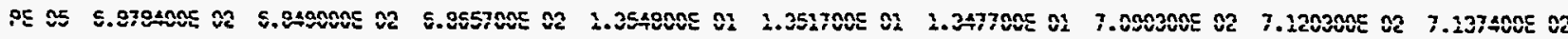

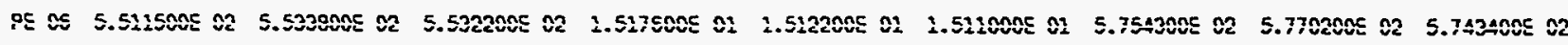

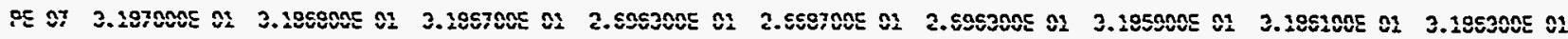

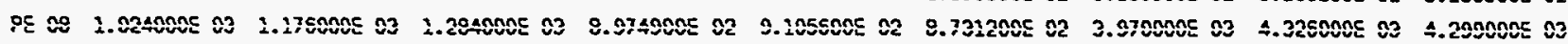

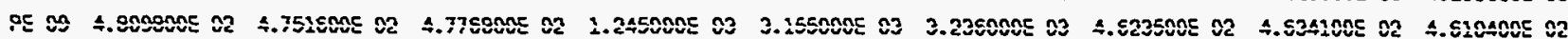

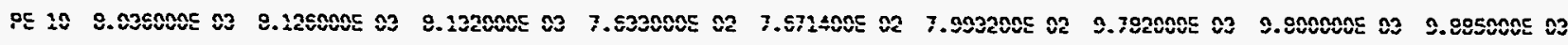

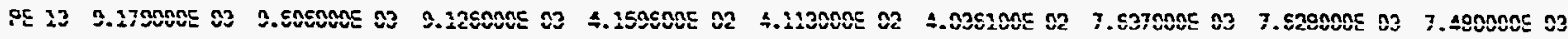

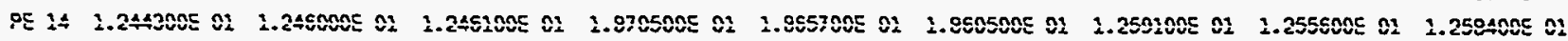

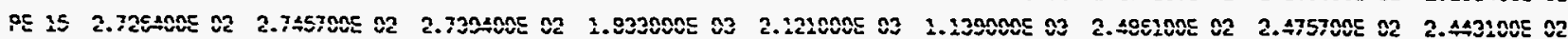
IE IE z.

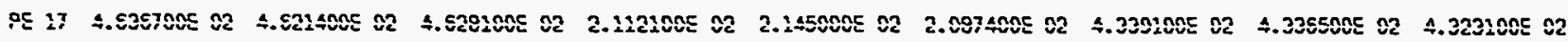

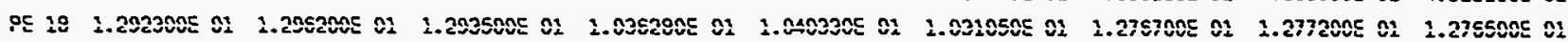

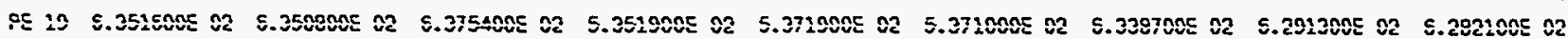

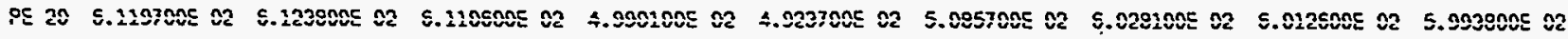

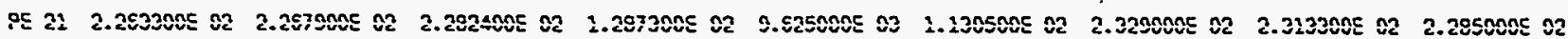

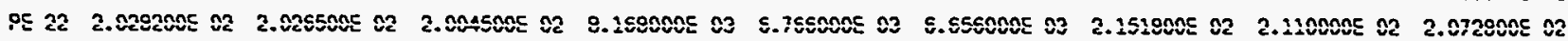

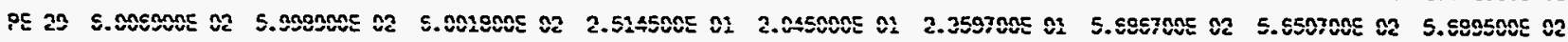

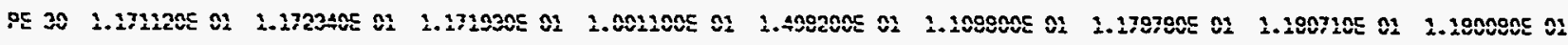

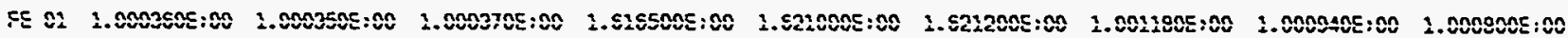

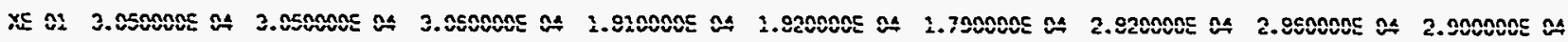

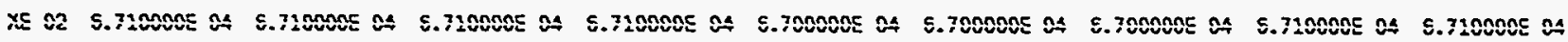

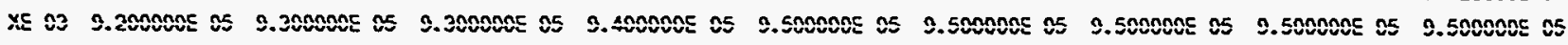

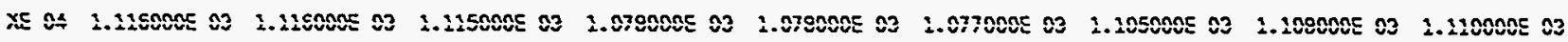

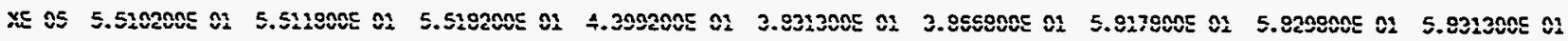

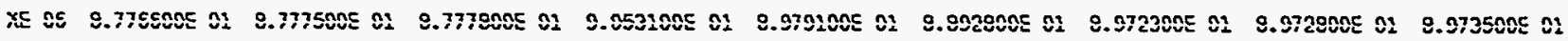

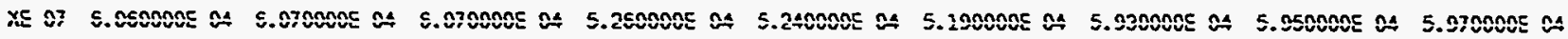

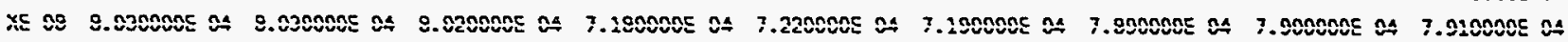

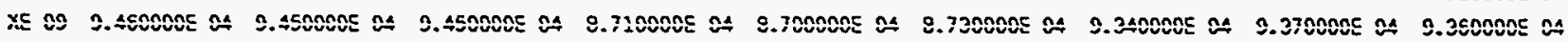

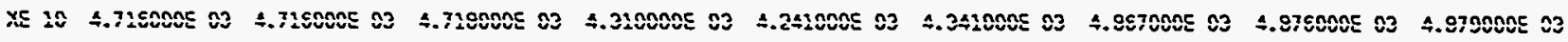

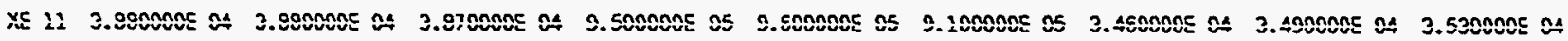

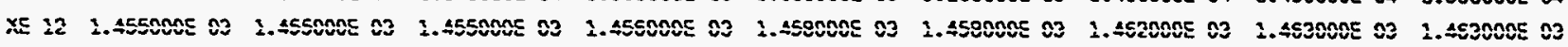

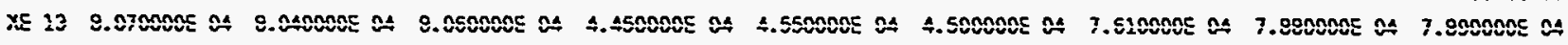

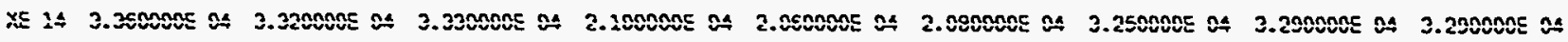

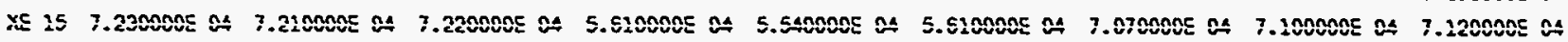
TE A2 5.

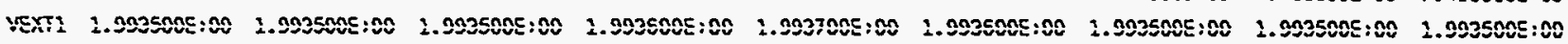

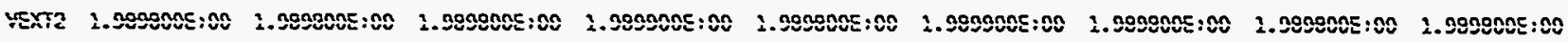

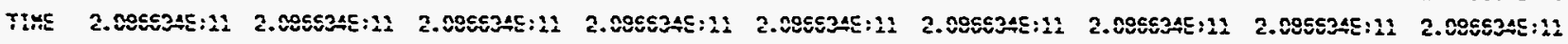




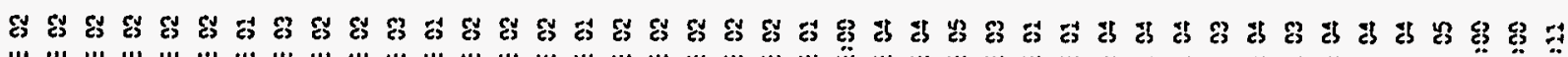

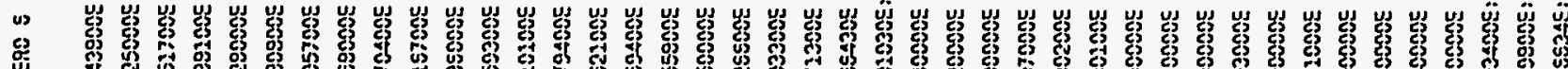

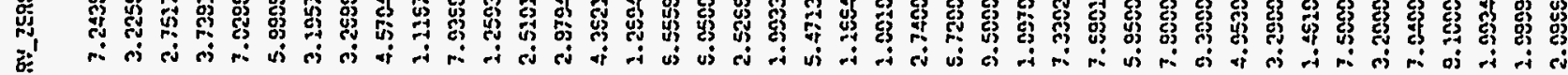

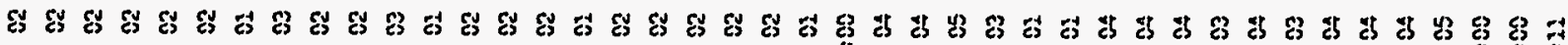

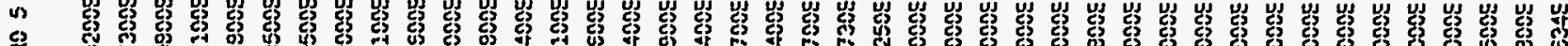

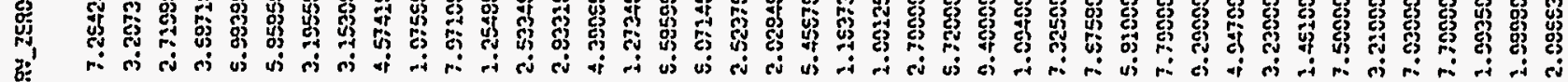

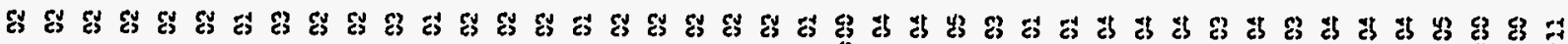

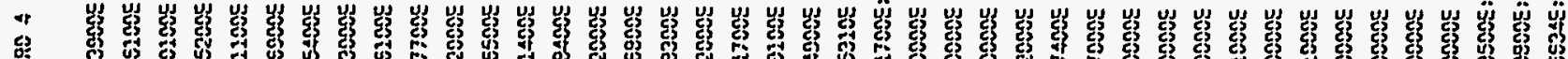

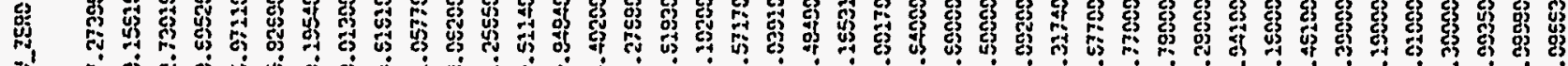

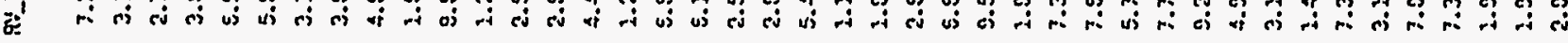

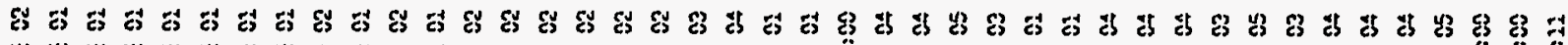

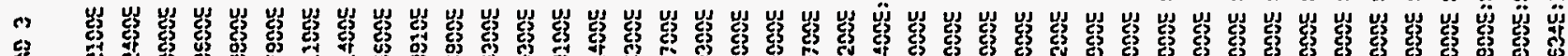

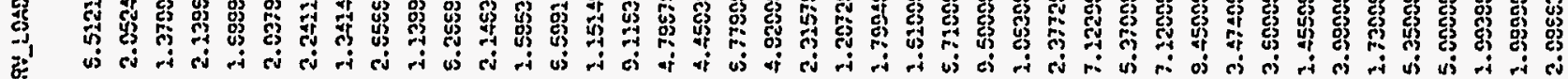

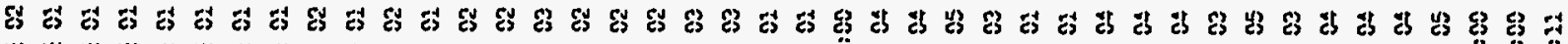

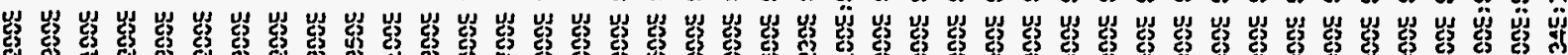

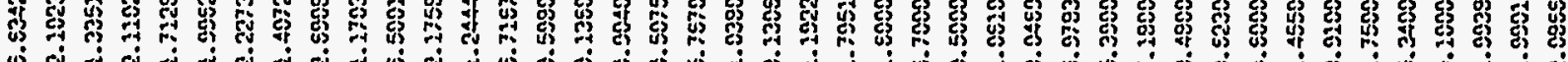

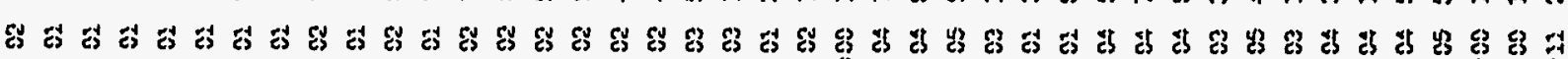

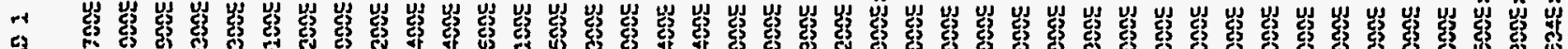

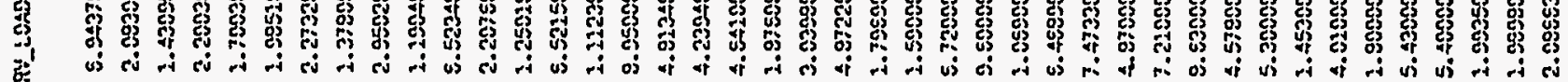

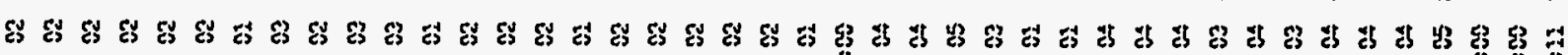

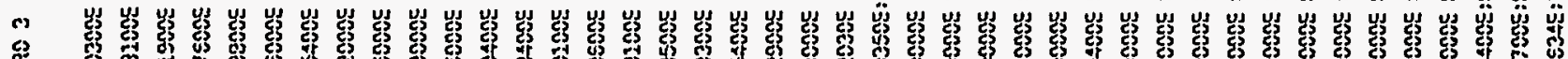

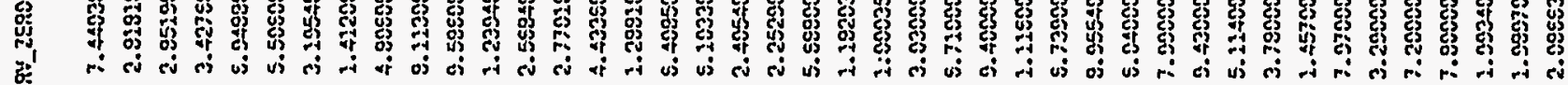

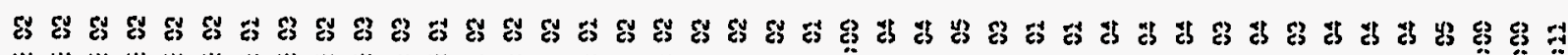

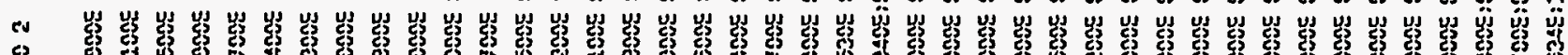

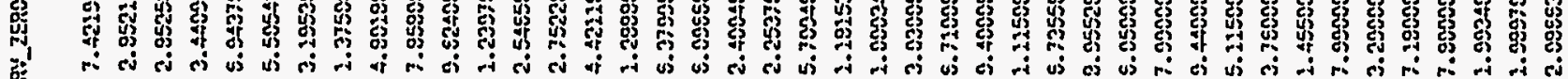

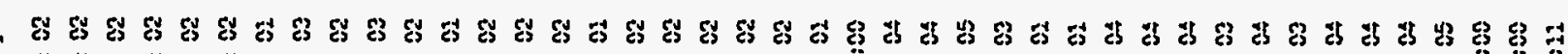

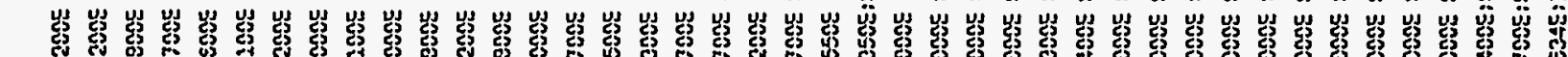

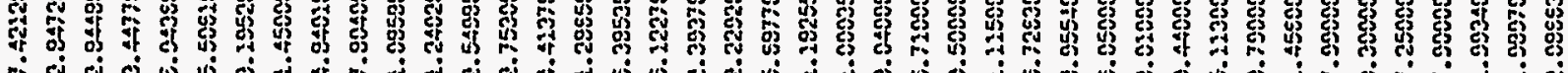

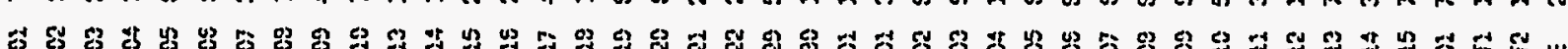

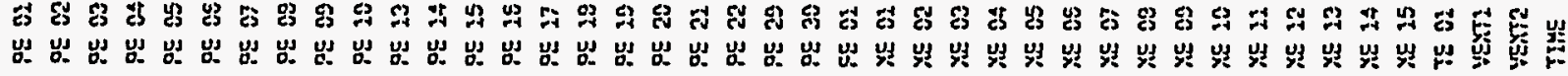


Arstive

Ams_m:_tests

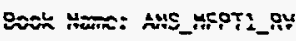

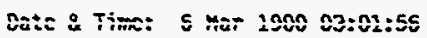

Page Deseription:

EATA SET HO. 223

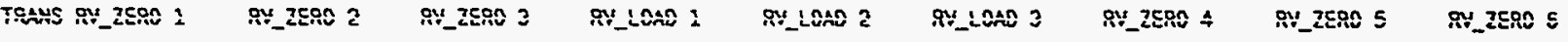

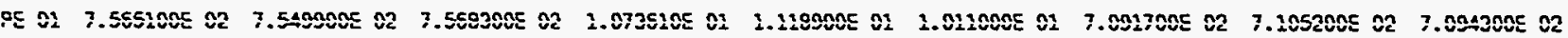
of 02 2.

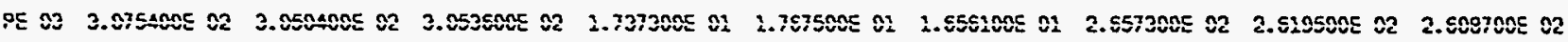

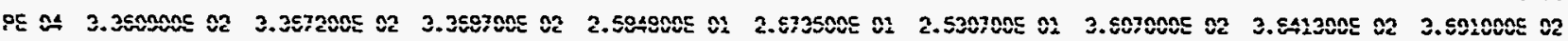

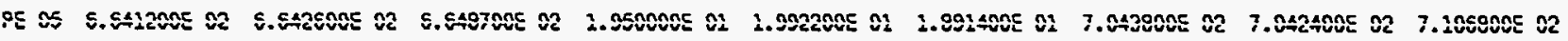

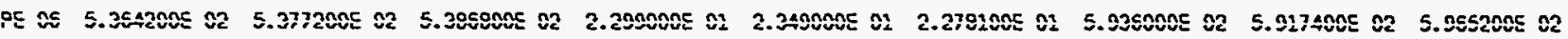

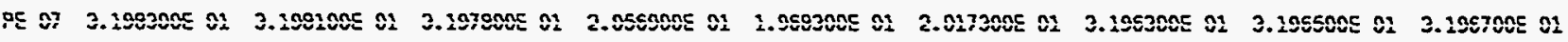

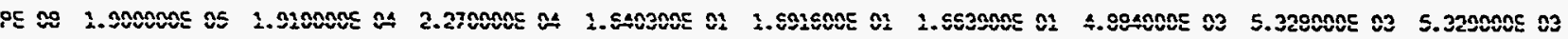

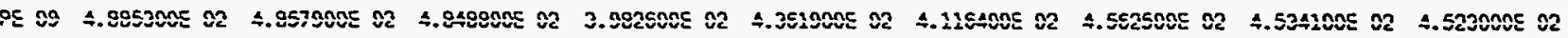
ic is 7.77 .

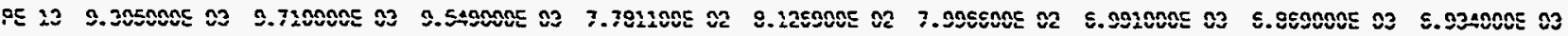

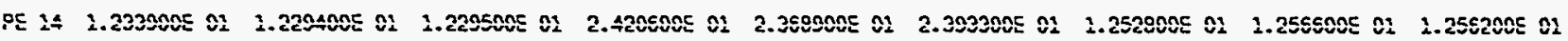

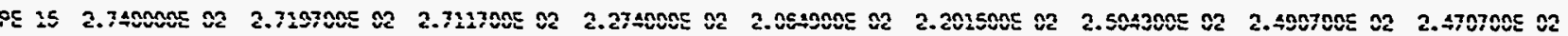

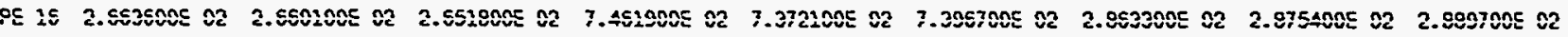
oc 27 4.5975005 02 4.

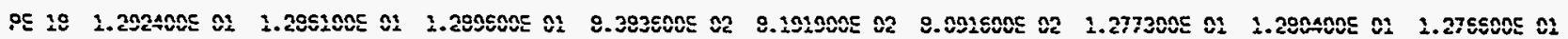

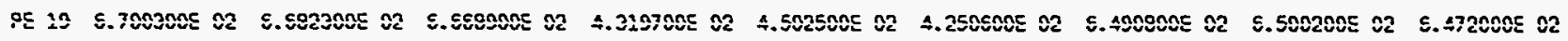

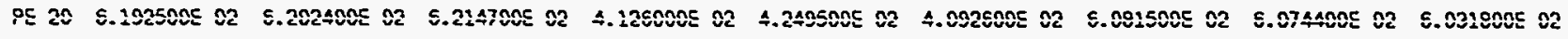

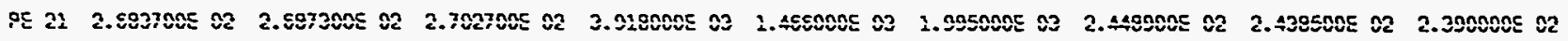

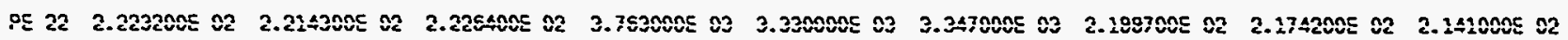

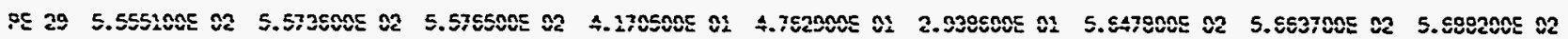

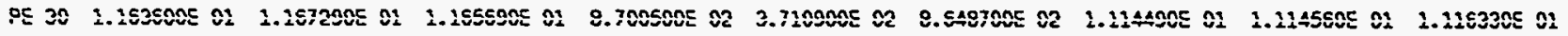

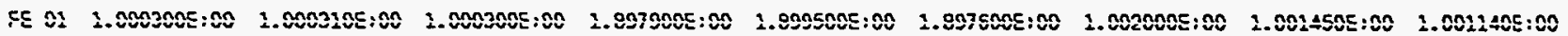

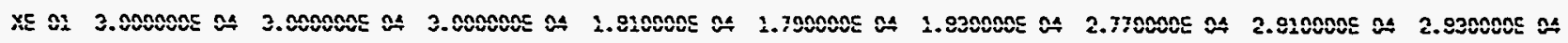

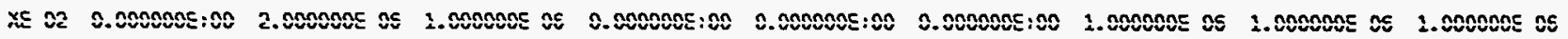

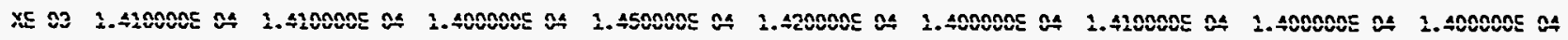

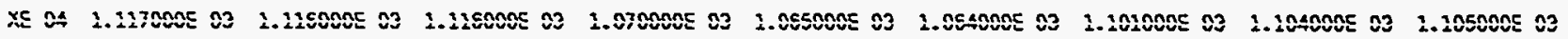

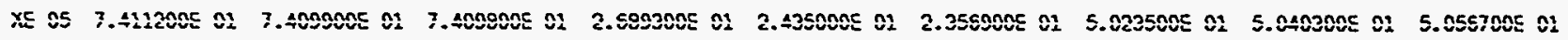

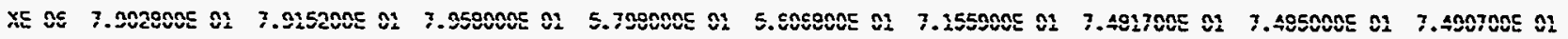

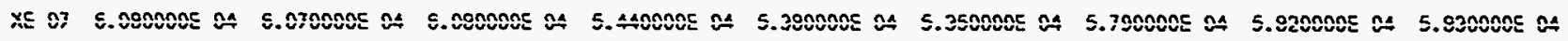

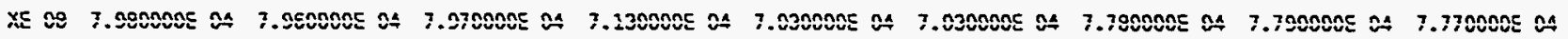

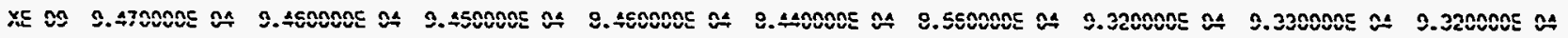

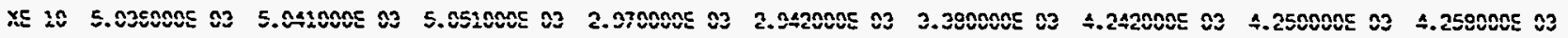

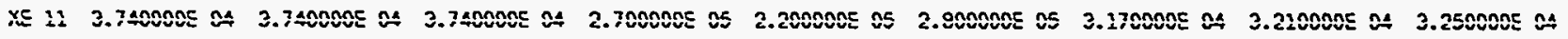

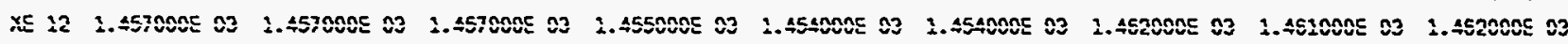

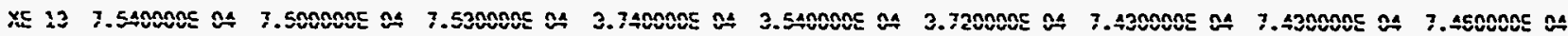

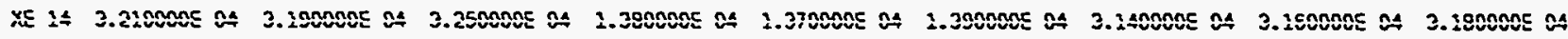

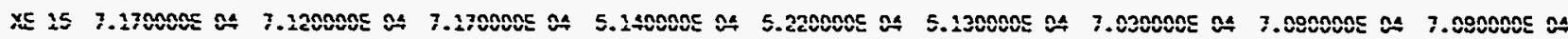

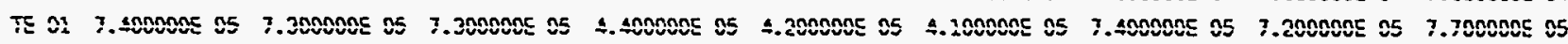

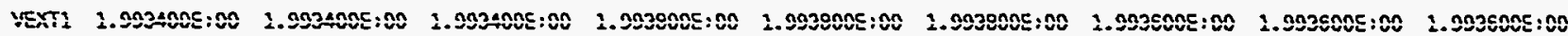

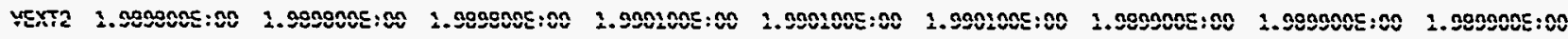

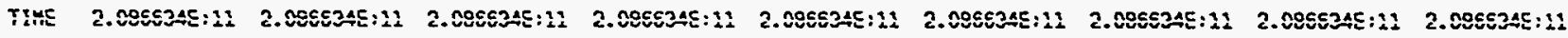


Pege sescription: DATA SET iv. 223

T⿱口八中⿰㇀丶㇀

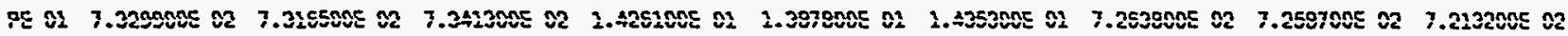

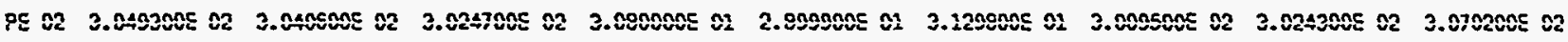

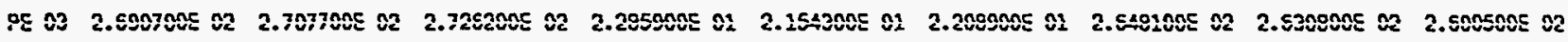

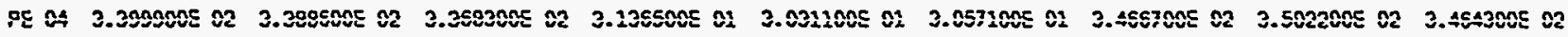

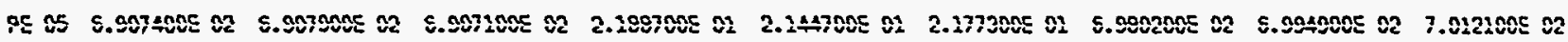

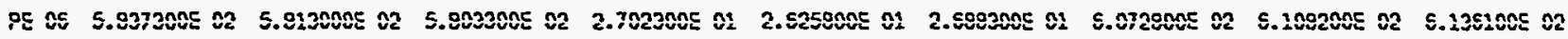

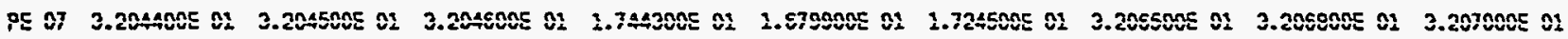

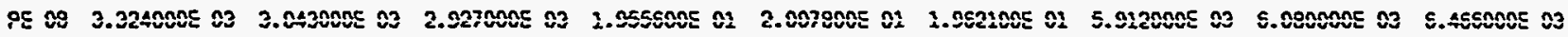

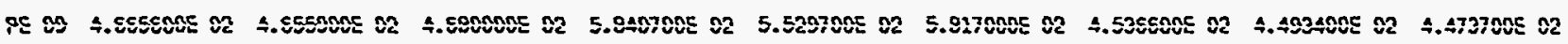

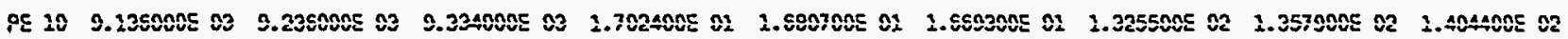

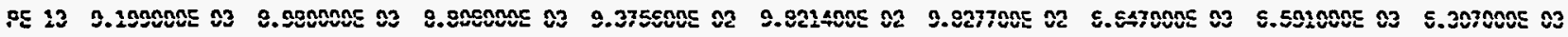

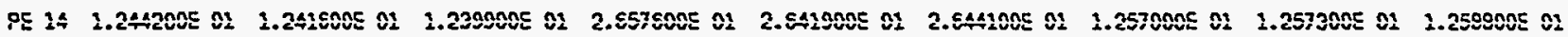

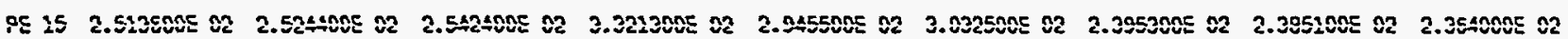

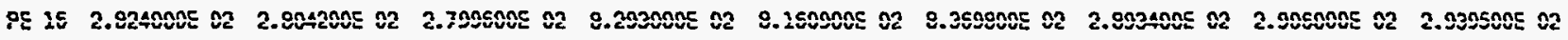

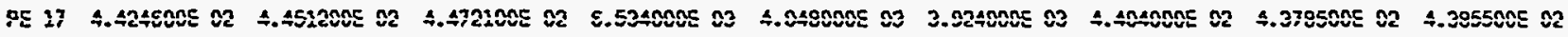

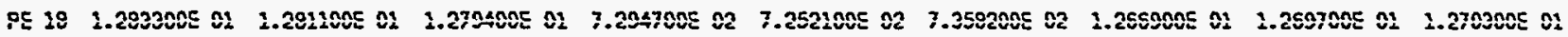

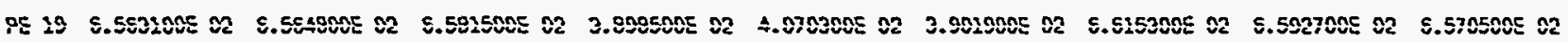

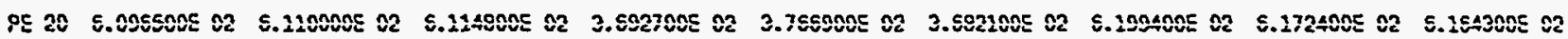

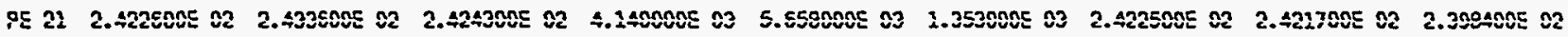

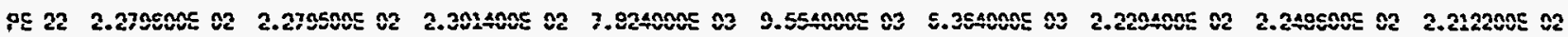

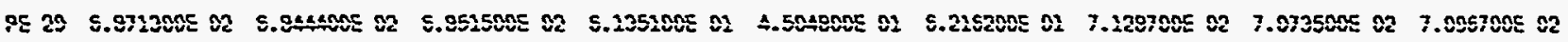

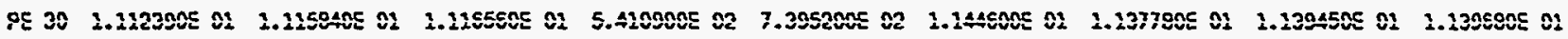

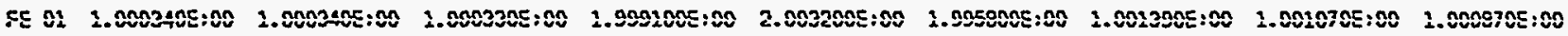

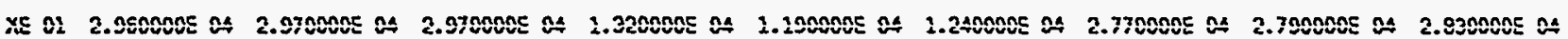

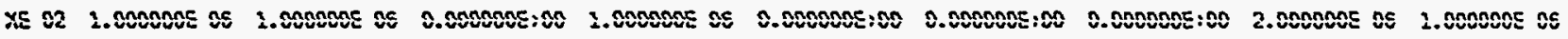

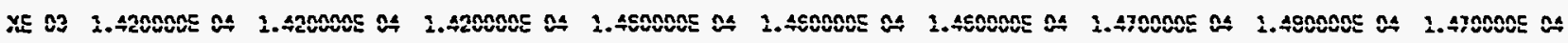

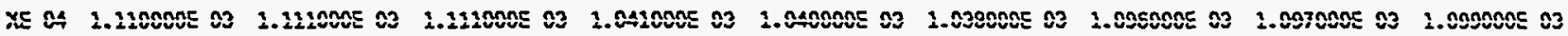

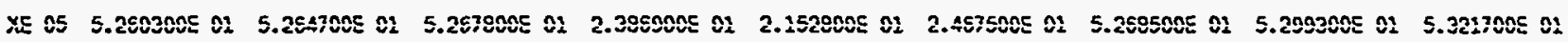

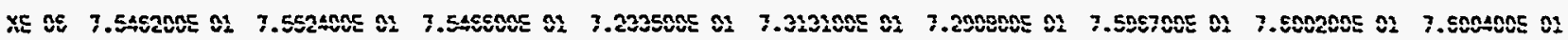

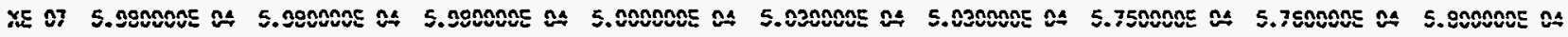

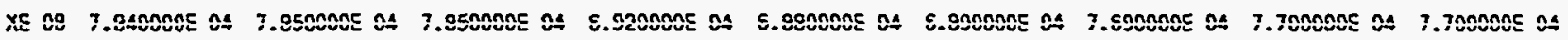

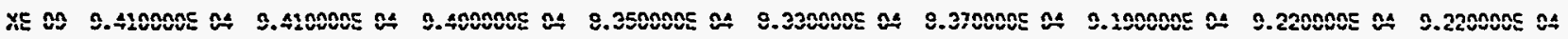
XE $10 \quad 4.330000503 \quad 4.331000503 \quad 4.327000503 \quad 3.307000503 \quad 3.345000503 \quad 3.323000503 \quad 4.345000503 \quad 4.358000503 \quad 4.355000503$

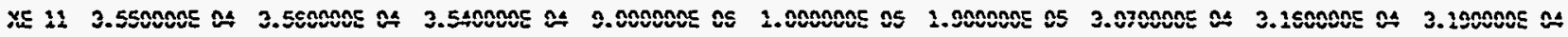

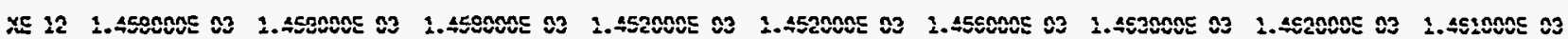

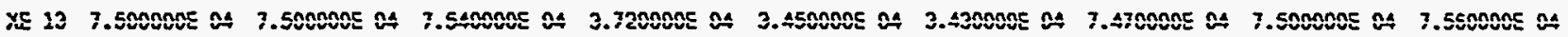

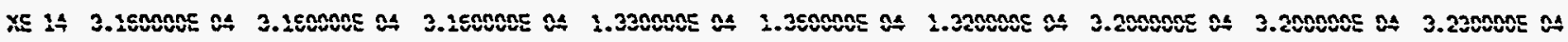

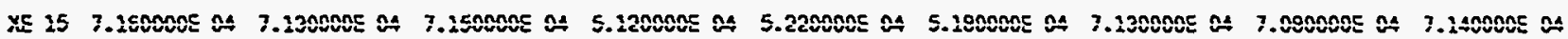

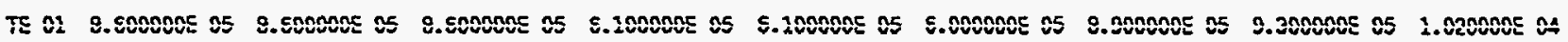

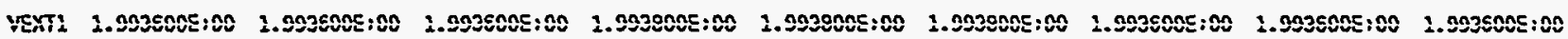

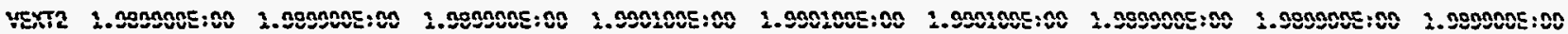

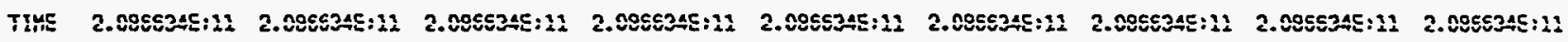


Arivive:

AYS_m?_TESTS

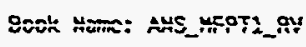

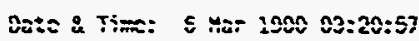

Pinge vescriptivin:

RATA SET M. 214

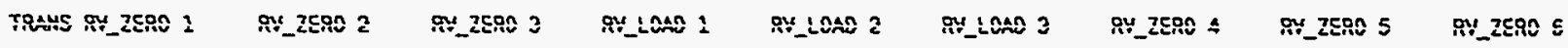

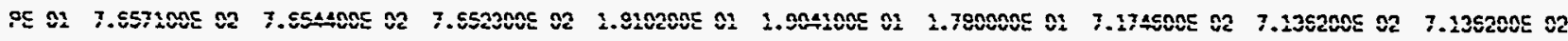

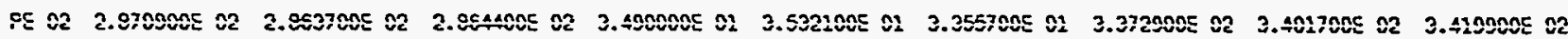

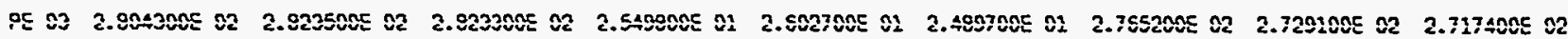

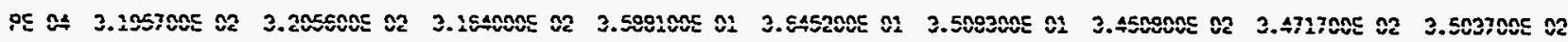

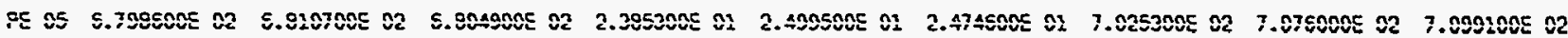

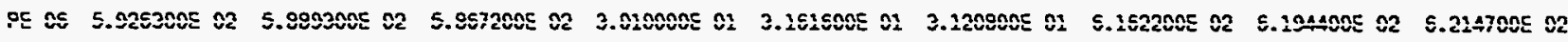

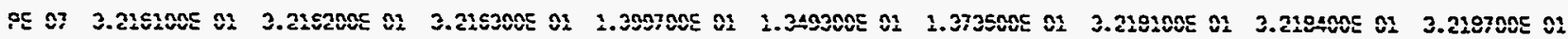

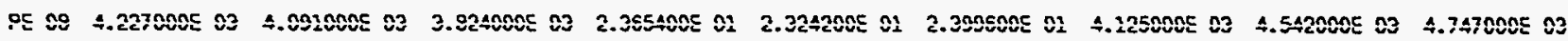

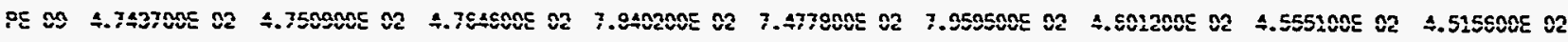

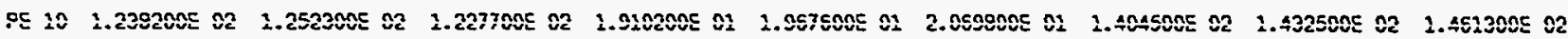

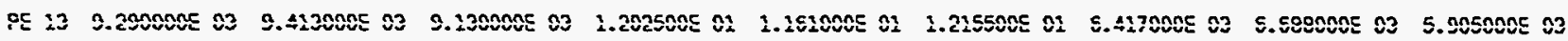

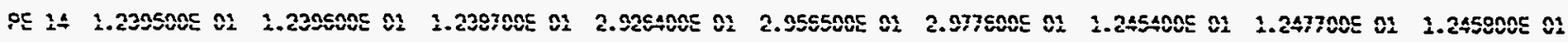

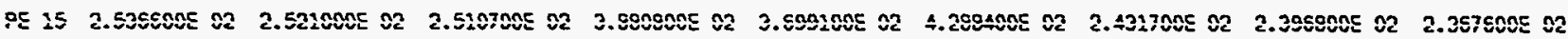

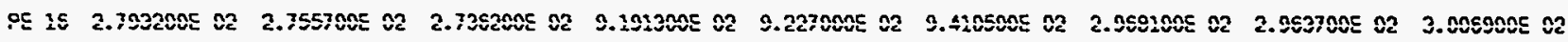

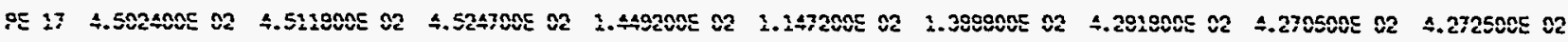

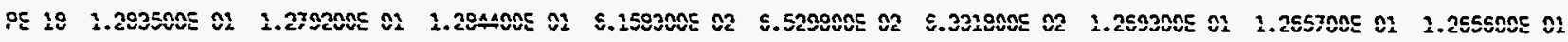

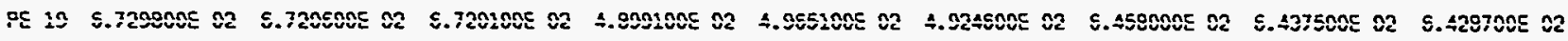

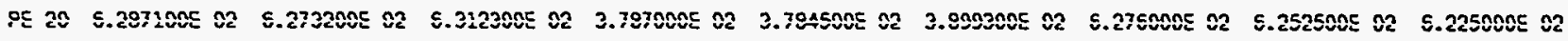

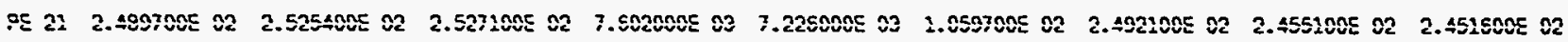

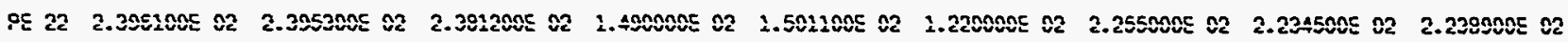

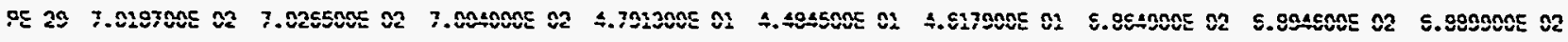

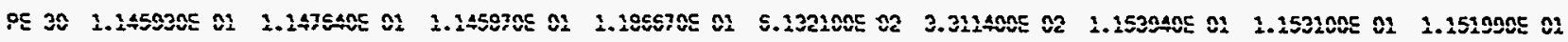

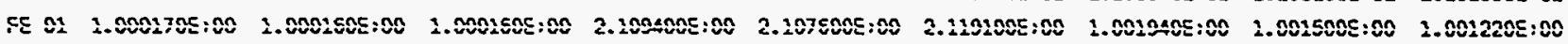

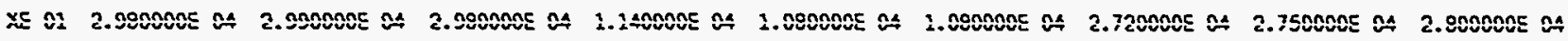

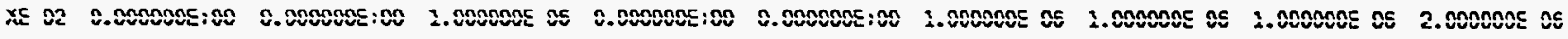

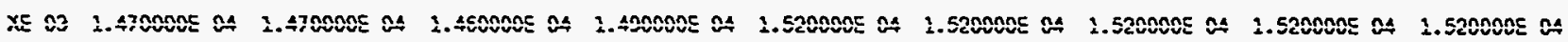

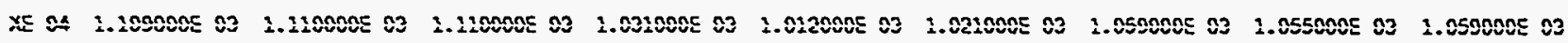

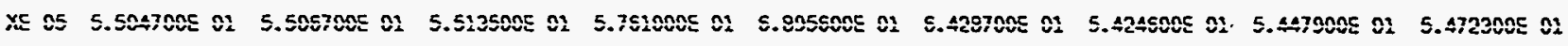

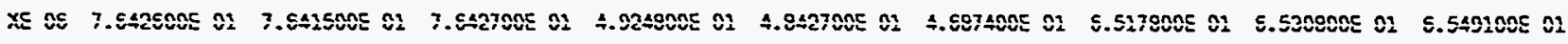

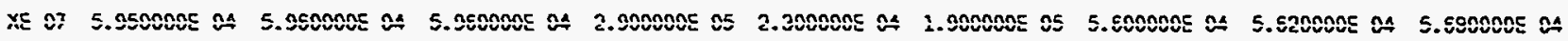
xE ca 7.

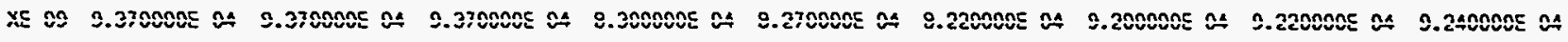

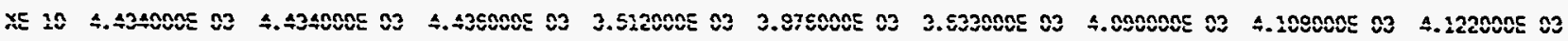

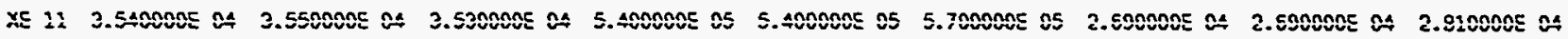

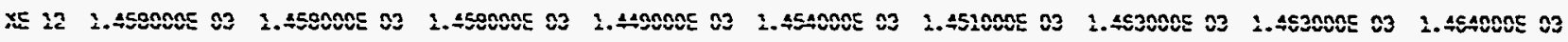

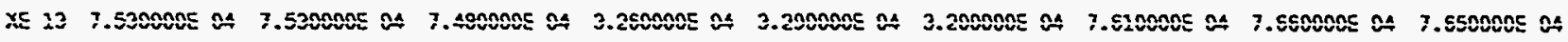

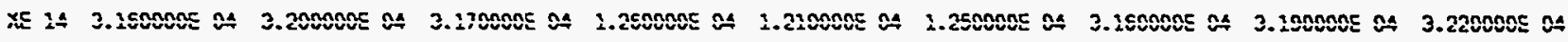

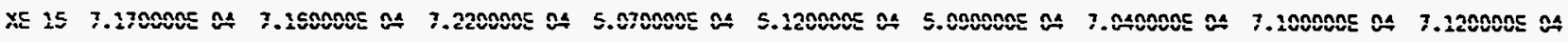

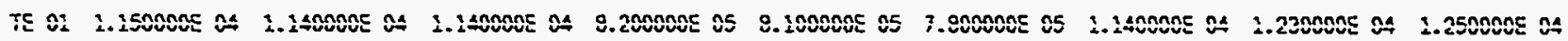

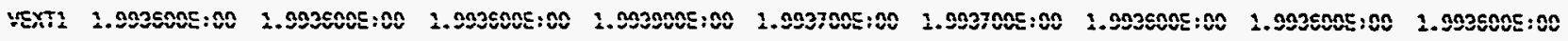

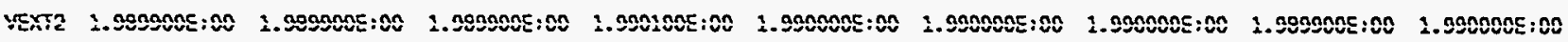

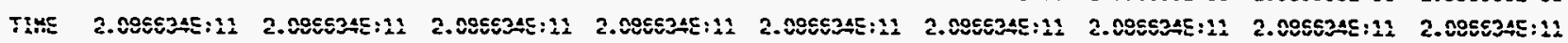


Artiston:

AHE_HEP_TESTS

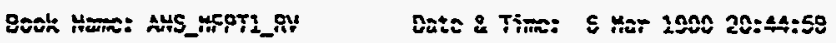

Page Lesctiptiom:

DATA SET YO. 235

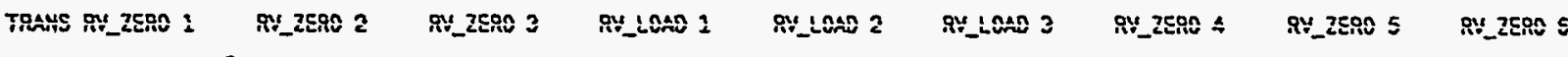

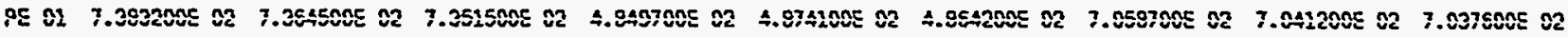

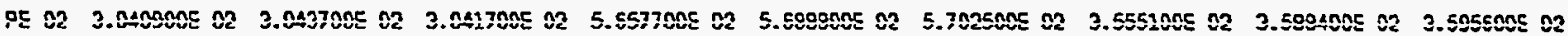

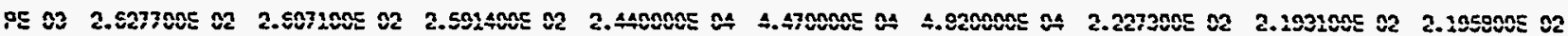

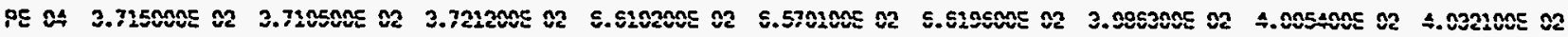

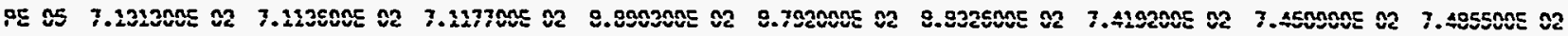

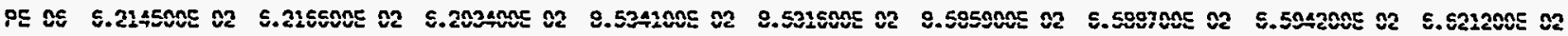

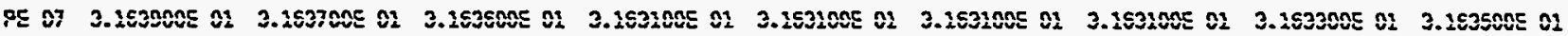

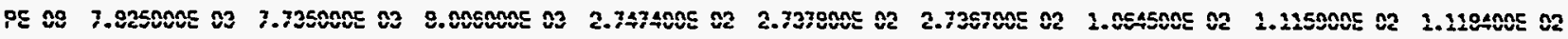

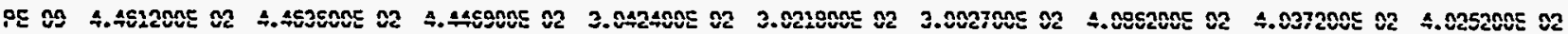

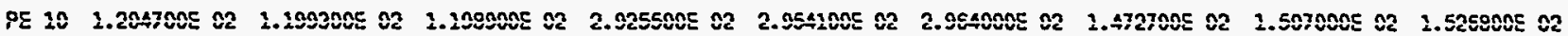

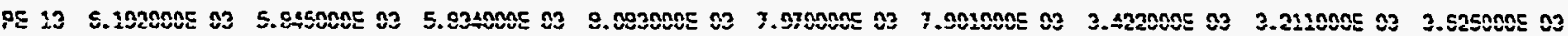

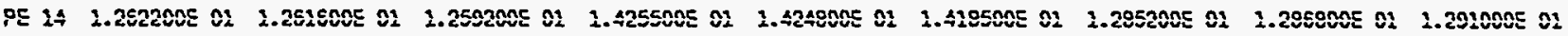

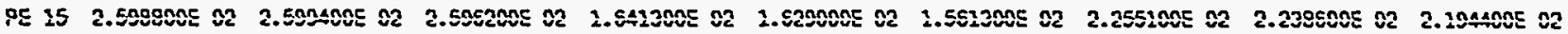

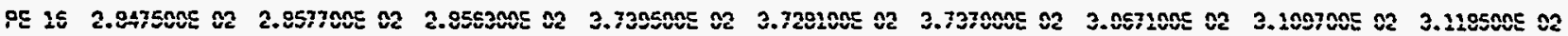

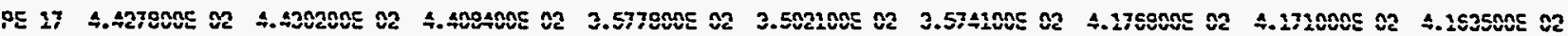

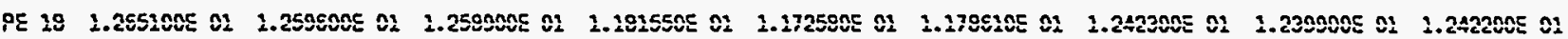

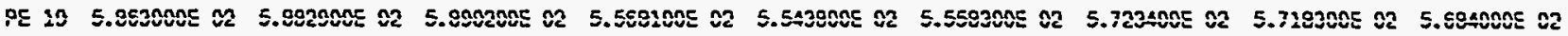

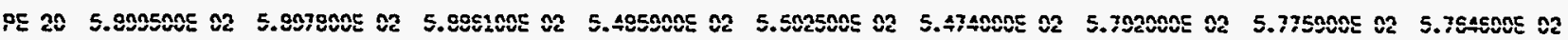

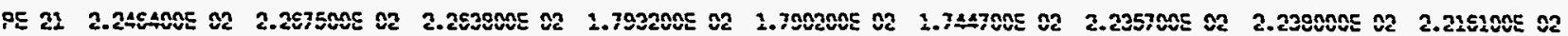

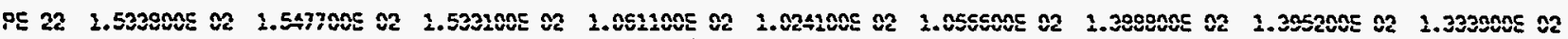

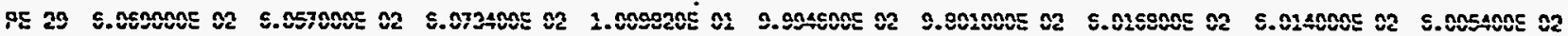

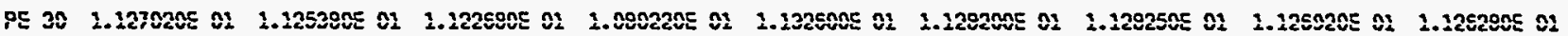

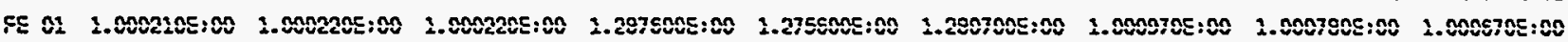

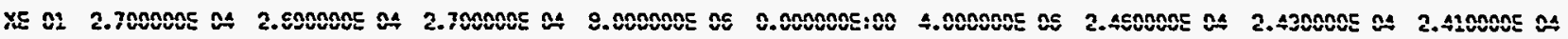

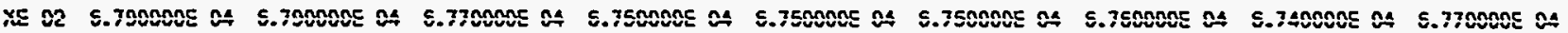

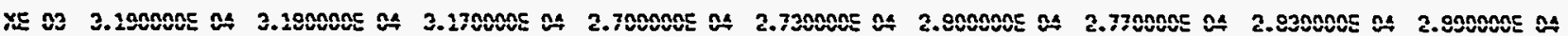

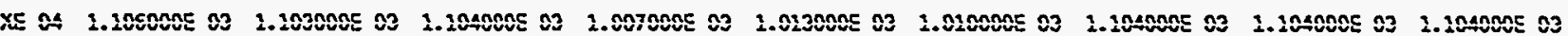

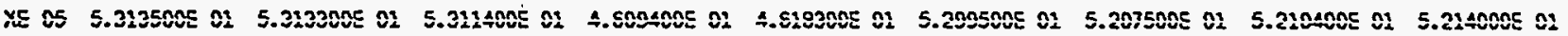

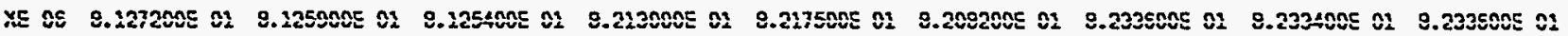

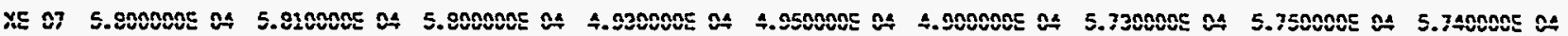

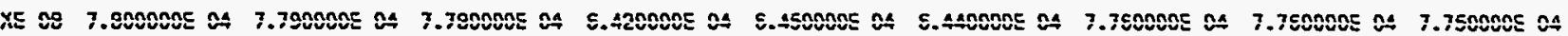

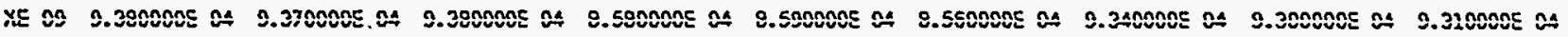

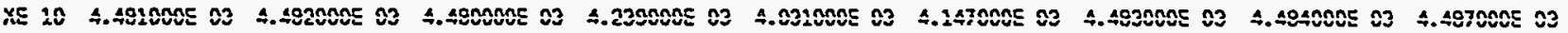

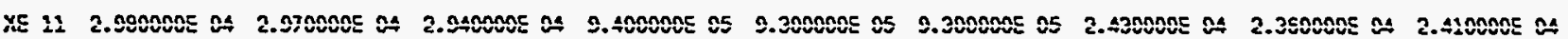

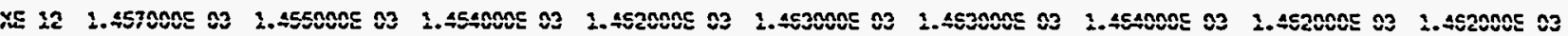

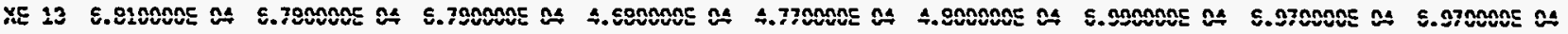

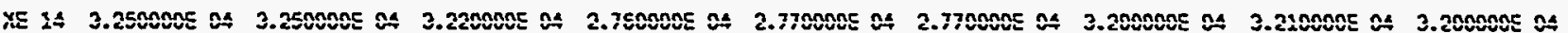

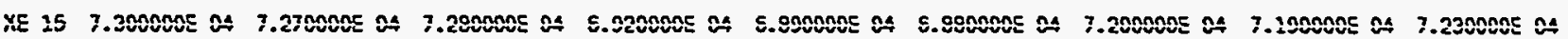

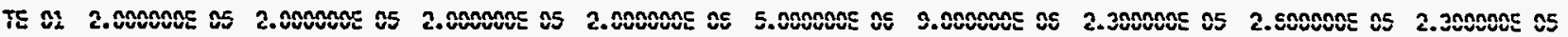

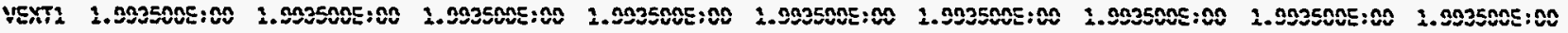

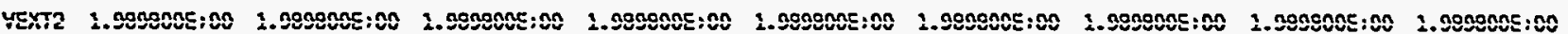

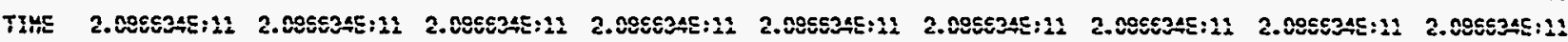


:

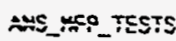

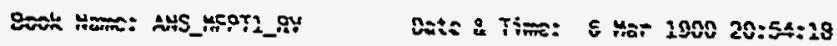

Page Description:

DATA SET KE. - 218

TRA4S RE_ZERO-2 RE_ZERE-2 RU_ZERO-3 RV_LOAO-2 RV_LOAO-2 RY_LOAD-3 RV_ZERO-4 RY_ZERO-5 RV_ZERO-6

PCE- $02-7.2169005-02-7.2285005-02-7.2256005-02-2.42900 E-02-2.488500 E-02-2.384100 E-02-7.2170005-02-7.124200 E-02-7.103900 E-02$ PE-02 3.5A1500E-02 3.532200E-02 3.513500E-02 8.605400E-02 $8.680400 E-82$ 8.565800E-02 $3.555800 E-02 \quad 3.575500 E-02 \quad 3.568600 E-02$

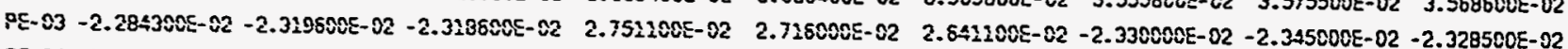

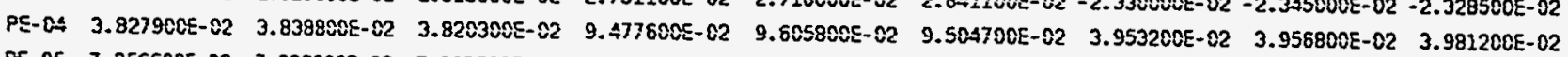

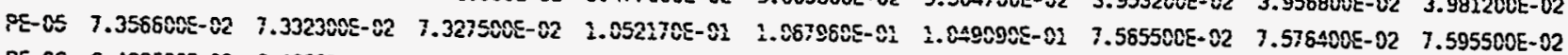

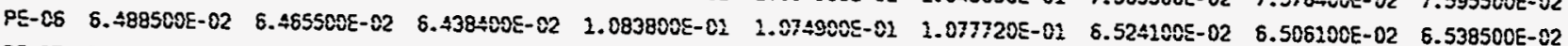
PE- $07-3.272500 E-01-3.172800 E-01-3.1727005-01-3.0762005-01-3.091400 E-01-3.074700 E-01-3.1746005-01-3.274600 E-01-3.275000 E-01$ $\begin{array}{llllllllll}\text { PE-08 } & 1.011800 E-02 & 1.021400 E-\$ 2 & 1.0395005-02 & 4.972000 E-02 & 5.183700 E-02 & 4.911300 E-02 & 1.144900 E-02 & 1.1521 C 0 E-02 & 1.171400 E-02\end{array}$ PE- $89-4.2695005-02-4.196800 E-02-4.195300 E-02-1.9472005-02-1.919100 E-02-1.925700 E-02-4.1216005-02-4.101100 E-02-4.093400 E-02$ PE-10 $2.451600 E-02 \quad 1.418500 E-02 \quad 1.424400 E-02 \quad 4.455300 E-02 \quad 4.440200 E-02 \quad 4.525300 E-02 \quad 1.819100 E-02 \quad 1.854600 E-02 \quad 1.874100 E-02$

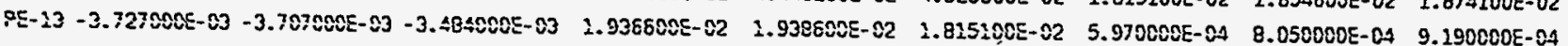

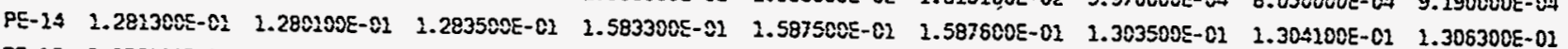

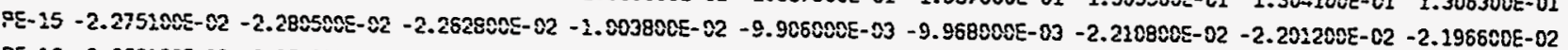
FE-15 3.053100E- 02 3.254200E- $22 \quad 3.0459005-02 \quad 4.261500 E-02 \quad 4.2578005-02 \quad 4.152800 E-02 \quad 3.024300 E-02 \quad 3.052300 E-02 \quad 3.051200 E-02$ QE- $37-4.213200 E-02-4.223400 E-02-4.2214005-02-3.101600 E-02-3.086100 E-02-3.066100 E-02-4.254800 E-02-4.249800 E-02-4.235700 E-02$ PE-18 - $2.250800 E-01-1.246800 E-02-1.248900 E-01-1.127990 E-02-3.112130 E-01-1.114540 E-01-1.249300 E-01-1.245200 E-01-1.242500 E-01$

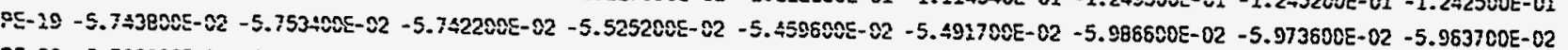

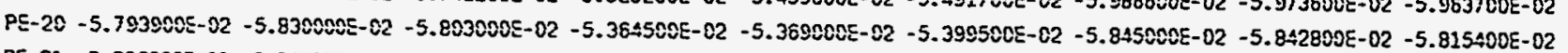

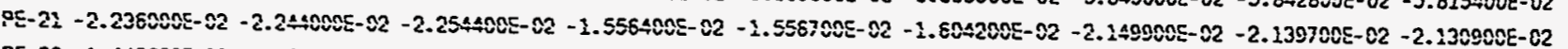
$P E-22-1.448600 E-02-1.456800 E-02-1.431900 E-02-8.5370005-03-7.397000 E-03-8.409000 E-03-2.4730005-02-1.4526005-02-1.463400 E-02$ $\begin{array}{lllllllllll}P E-29 & 6.0467005-02 & 6.034200 E-02 & 6.023800 E-02 & 1.385500 E-01 & 1.214500 E-01 & 1.285500 E-01 & 6.069600 E-02 & 6.059900 E-02 & 6.073900 E-02\end{array}$

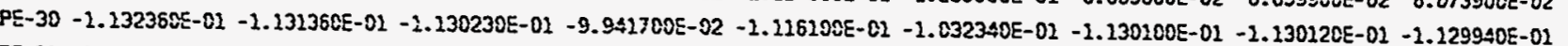
FE-02 $1.000400 E+00 \quad 1.000300 E+00 \quad 3.000420 E+00 \quad 1.417900 E+00 \quad 1.416300 E+00 \quad 1.428100 E+00 \quad 2.001100 E+00 \quad 1.000760 E+00 \quad 1.000600 E+00$ $X E-01-2.620000 E-04-2.610000 E-04-2.6100005-04 \quad 1.900000 E-05 \quad 7.000000 E-06-3.000000 E-05-2.420000 E-04-2.470000 E-04-2.490000 E-04$

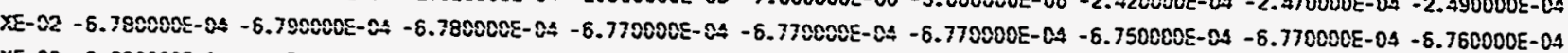
$X E-03-3.280000 E-04-3.270000 E-04-3.270000 E-04-3.290000 E-04-3.2500005-04-3.240000 E-04-3.290000 E-04-3.280000 E-04-3.280000 E-04$ XE-0. $-1.102000 E-03-1.1020005-03-1.2010005-03-9.790000 E-04-9.830000 E-04-9.920000 E-04-1.090000 E-03-1.093000 E-03-1.092000 E-03$ $\begin{array}{llllllllll}x E-05 & 5.295100 E-02 & 5.297000 E-01 & 5.299800 E-01 & 5.335400 E-01 & 6.008500 E-01 & 6.527800 E-01 & 6.751300 E-01 & 6.737100 E-01 & 6.725600 E-01\end{array}$

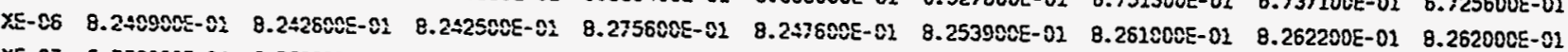
$X E-07-5.750000 E-04-5.750000 E-04-5.760000 E-04-4.570000 E-04-4.600000 E-04-4.590000 E-04-5.720000 E-04-5.730000 E-04-5.740000 E-04$ XE- $88-7.740000 E-04-7.7300005-04-7.7300005-04-6.270000 E-04-6.270000 E-04-8.3 .00000-04-7.6900008-04-7.720000 E-04-7.690000 E-04$ XEE-09 -9.320000E- $04-9.320000 E-04-9.320000 E-04-8.460000 E-04-8.430000 E-04-8.450000 E-04-9.2100005-04-9.210000 E-04-9.210000 E-04$ XE- $20-4.5110005-83-4.5100005-03-4.5120005-03-4.5010005-03-4.552000 E-03-4.771000 E-03-4.9220005-03-4.9180005-03-4.920000 E-03$ $X E-11-2.710000 E-04-2.720000 E-04-2.6800005-04 \quad 1.350000 E-04 \quad 1.320000 E-04 \quad 1.220000 E-04-2.370000 E-04 \quad-2.430000 E-04-2.460000 E-04$ XE-12 - $-1.4530005-83-1.4560005-03-1.455000 E-03-1.462000 E-03-1.481000 E-03-1.462000 E-03-1.466000 E-03-1.464000 E-03-1.464000 E-03$ $X E-13-6.830000 E-04-6.800000 E-04-6.810000 E-04-4.440000 E-04-4.530000 E-04-4.540000 E-04-6.830008 E-04-8.840000 E-04-6.840000 E-04$ XE-14 -3.210000E-04 -3.180000E-04 -3.200000E-04 -2.660000E-04 -2.680000E-04 -2.680000E-04 -3.090000E-04 -3.060000E-04 -3.150000E-04 $X E-15-7.270000 E-04-7.260000 E-04-7.270000 E-04-6.870000 E-04-6.850000 E-04-8.880000 E-04-7.230000 E-04-7.250000 E-04-7.270000 E-04$ TE- $02-3.3000005-05-3.300000 E-05-3.300000 E-05-1.3000005-05-2.500000 E-05-1.200000 E-05-3.500000 E-05-3.500000 E-05-4.500000 E-05$ VEXT1 $1.9935 C 0 E+00 \quad 1.993508 E+C 0 \quad 1.993500 E+00 \quad 1.993500 E+00 \quad 1.993400 E+00 \quad 1.993400 E+00 \quad 1.993400 E+00 \quad 1.993400 E+00 \quad 1.993400 E+00$

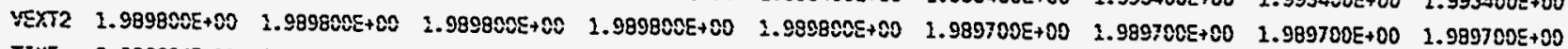

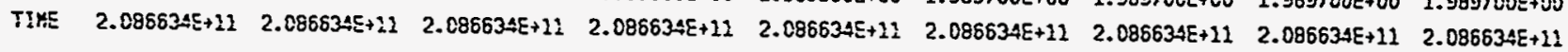


Arative Name:

AHS_YF:_TESTS

Book Hemre: AHS_MFPT1_Ry Oate \& Time: 6 Yar 1900 21:15:17

Page Description:

DATA SET $90 .-217$

TRAYS RY_ZERO-1 RY_ZERO-2 RY_ZERO-3 RY_LOAR-1 RY_LOAD-2 RY_LOAD-3 RY_ZERO-4 RY_ZERO-5 RY_ZERO-6

$P 5-01-7.475400 E-02-7.4894005-02-7.4804005-02-2.045000 E-03-2.746000 E-03-2.205000 E-03-7.163200 E-02-7.1545005-02-7.120200 E-02$ PE-02 3.069800E-02 3.0724CQE-\$2 $3.090600 E-02 \quad 3.133500 E-01 \quad 1.153100 E-01 \quad 1.141920 E-01 \quad 3.468700 E-02 \quad 3.470100 E-02 \quad 3.459100 E-02$ PE-03 -2.618500E-02 -2.627000E-02 -2. E42000E-02 5.297200E-02 $5.2531005-02 \quad 5.347900 E-02-2.306308 E-02 \quad-2.253600 E-02 \quad-2.264000 E-02$ PE-04 3.562G80E-02 3.562800E-02 $3.574400 E-02 \quad 1.235500 E-01 \quad 1.220500 E-01 \quad 1.243000 E-01 \quad 4.029700 E-02 \quad 4.020300 E-02 \quad 4.054500 E-02$ PL- $05 \quad 7.332600 E-02 \quad 7.336000 E-02 \quad 7.342500 E-02 \quad 2.217100 E-02 \quad 1.221300 E-01 \quad 3.205800 E-01 \quad 7.480800 E-02 \quad 7.532300 E-02 \quad 7.544300 E-02$ PE-06 $\quad 6.200900 E-02 \quad 6.202700 E-02 \quad 6.208200 E-02 \quad 1.287800 E-01 \quad 1.303600 E-01 \quad 1.298000 E-01 \quad 5.492400 E-02 \quad 8.505800 E-02 \quad 6.537500 E-02$ PE- 87 -3.279500E- $02-3.1792005-02-3.1791005-02-2.9238005-01-2.9065005-01-2.9028005-01-3.1776005-01-3.1778005-01-3.1780005-01$ PE-CB $\quad 8.3560 C 0 E-03 \quad 8.346000 E-03 \quad 8.4000005-03 \quad 7.079000 E-02 \quad 6.949700 E-02 \quad 7.223200 E-02 \quad 1.097200 E-02 \quad 1.106100 E-02 \quad 1.046900 E-02$

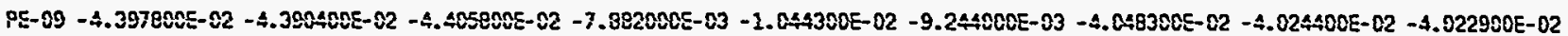
PE-10 1.630300E-02 $1.644100 E-02 \quad 1.632600 E-02 \quad 6.133900 E-02 \quad 6.207200 E-02 \quad 6.155200 E-02 \quad 1.906300 E-02 \quad 1.947900 E-02 \quad 2.001600 E-02$

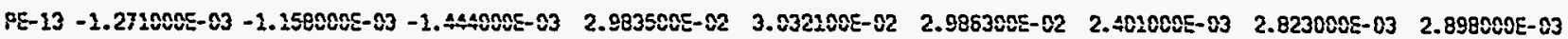
PE- $14 \quad 1.274900 E-01 \quad 1.273500 E-01 \quad 2.274000 E-01 \quad 1.736400 E-01 \quad 1.720300 E-01 \quad 1.718100 E-01 \quad 1.290500 E-01 \quad 1.290200 E-01 \quad 1.291700 E-01$ $P E-25-2.433200 E-02-2.4214005-02-2.4277005-02-5.3220005-03-3.9820005-03-2.382000 E-03-2.180200 E-02-2.241500 E-02-2.116000 E-02$ PE-16 2.835100E-02 2.854000E-02 2.859000E-02 $4.764500 E-02 \quad 4.813000 E-02 \quad 4.8421005-02 \quad 3.0525005-02 \quad 3.089500 E-82 \quad 3.118600 E-02$ $8 E-17-4.392100 E-02-4.402100 E-02-4.412000 E-02-2.642000 E-02-2.561300 E-02-2.720100 E-02-4.247800 E-02-4.2 C 8000 E-02-4.219800 E-02$

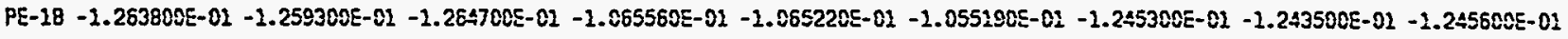
gE-19-6.166500E- $02-6.1521005-02-8.173300 E-02-5.359700 E-02-5.304900 E-02-5.343800 E-02-5.832700 E-02-5.837200 E-02-5.783200 E-02$ PE-20 -5.951000E- $02-5.9681005-02-5.9763005-02-5.211400 E-02-5.059700 E-82-5.176700 E-\$ 2 \quad-5.871500 E-02-5.880900 E-02-5.848700 E-02$ PE- $21-2.244100 E-02-2.2432005-02-2.259800 E-02-1.3259005-02-2.318800 E-02-1.273100 E-82-2.086300 E-02-2.0490000 E-02-2.055400 E-02$ PE-22 - 1.690800E-02 - - $.678508 E-02-1.653800 E-02-6.169000 E-03-6.280000 E-03-5.763000 E-03-1.559900 E-02-1.540000 E-02-1.508800 E-02$ PE-29 $\quad 6.236300 E-02 \quad 6.2499005-02 \quad 5.212700 E-02 \quad 1.736900 E-01 \quad 1.9303005-01 \quad 1.496500 E-01 \quad 6.104800 E-02 \quad 6.131900 E-02 \quad 6.092400 E-02$ PE-30 -1.132060E-01 -1.131650E-01 -2.132890E-01 -1.109080E-01 -1.205140E-01 -9.297300E-02 -1.131520E-01 -1.132890E-01 -1.132070E-01 FE-01 $1.000170 E+00 \quad 1.000170 E+00 \quad 1.000180 E+00 \quad 1.524900 E+00 \quad 1.524700 E+00 \quad 1.525800 E+00 \quad 1.001040 E+00 \quad 1.000790 E+00 \quad 1.000640 E+00$ XE- $01-2.710000 E-04-2.700000 E-04-2.710000 E-04 \quad 4.300000 E-05 \quad 4.100000 E-05 \quad 4.000000 E-05-2.370000 E-04 \quad-2.400000 E-04$-2.410000E-04

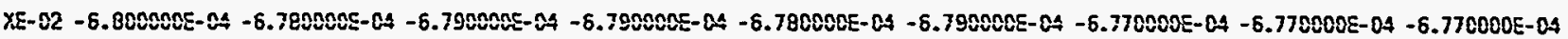
XEE-03 -3.190000E-04 -3.190000E-04 -3.190000E-04 -3.100000E-04 -3.090000E-04 -3.100000E-04 -3.080000E-04 -3.080000E-04 -3.070000E-04 XE-04 - $1.202000 E-03-1.203000 E-03-1.103000 E-83-9.770000 E-04-9.730000 E-04-9.780000 E-04-1.090000 E-03-1.093000 E-03-1.094000 E-03$

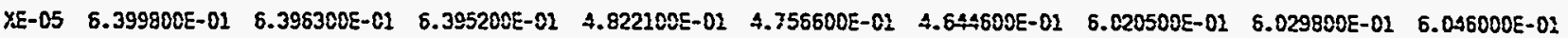

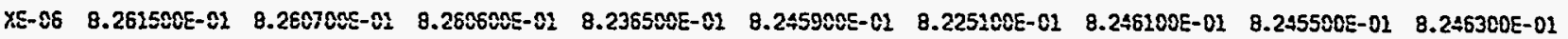
XE-07 -5.870000E-04 -5.860000E-04 -5.870000E-04 -4.610000E-04 -4.610000E-04 -4.620000E-04 -5.570000E-04 -5.680000E-04 -5.700000E-04 XE- $88-7.7700005-04-7.7600005-04-7.7600005-04-6.310000 E-04-6.3300005-04-6.2900005-04-7.6600005-04-7.660000 E-04-7.660000 E-04$ XE-09 -9.370000E-04 -9.360000E-04 -9.360000E-04 -8.380000E-04 -8.400000E-04 -8.230000E-04 -9.140000E-04 -9.140000E-04 -9.160000E-04 XE-10 - $4.8280005-03-4.8250005-03-4.828000 E-03-4.338000 E-03-4.189000 E-03-4.2520005-03-4.704000 E-03-4.710000 E-03-4.717000 E-03$ YE-11 2.280000E-05 2.200000E-05 2.300000E-05 2.200000E-05 $2.300000 E-05 \quad 2.300000 E-05 \quad 2.300000 E-05 \quad 2.300000 E-05 \quad 2.400000 E-05$ $X E-12-1.4580005-03-1.4580005-03-1.458000 E-03-1.4620005-03-1.462000 E-03-1.461000 E-03-1.454000 E-03-1.453000 E-03-1.463000 E-03$ XE-13-6.770000E-04 -6.780000E-04 -6.770000E-04 -4.270000E-04 -4.160000E-04 -4.220000E-04 -6.810000E-04 -8.780000E-04 -6.810000E-04 XE-14 -3.190000E- $04-3.3300005-04-3.140000 E-84,-2.570000 E-0.4-2.5900005-04-2.560000 E-04-3.130000 E-04-3.0000005-04-3.120000 E-04$ XE-15 -7.29C000E-04 -7.270000E-04 -7.280COOE-04 -6.790000E-04 -6.78CO00E-04 -6.760000E-04 -7.180000E-04 -7.220000E-04 -7.210000E-04 TE-01 -3.600000E- $05-3.800000 E-05-3.700000 E-05-1.2000080 E-05-9.069000 E-06-7.000000 E-06-3.400000 E-05-4.100000 E-05-4.300000 E-05$ $\begin{array}{lllllllllll}\text { VEXT1 } & 1.993400 E+00 & 1.993500 E+00 & 1.993400 E+00 & 1.993500 E+00 & 1.993500 E+00 & 1.993500 E+00 & 1.993500 E+00 & 1.993500 E+C D & 1.993500 E+00\end{array}$

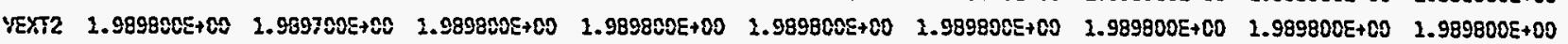

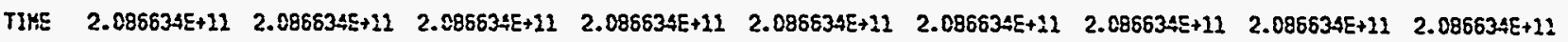


Arthive hanc:

AUS_*CO_TESTS

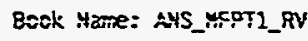

Cate \& Time: 6 Mar 1900 21:26:15

iage Sescription:

DATA SCT H0.-218

TRAYS SY Z Z $250-1$

RY_ZERR-2 RY_ZERO-3 RY_LARO-2

$R Y$ LOAD-3

RY_ZERO-A

RY_ZERO-5

RV_ZERD-6

8E- $02-7.3815005-02-7.391500 E-02-7.389400 E-02 \quad 2.282700 E-02 \quad 2.710100 E-02 \quad 3.168500 E-02-7.105600 E-02 \quad-7.118800 E-02-7.093500 E-02$

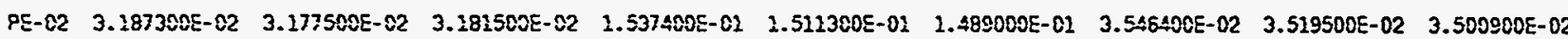

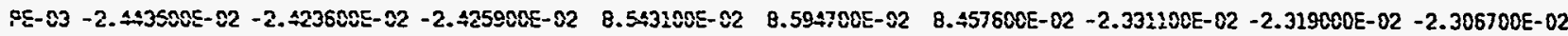
PE-04 3.654000E- $22 \quad 3.663600 E-02 \quad 3.649300 E-02 \quad 1.527700 E-02 \quad 1.603200 E-01 \quad 1.573500 E-02 \quad 3.757600 E-02 \quad 3.794500 E-02 \quad 3.783200 E-02$

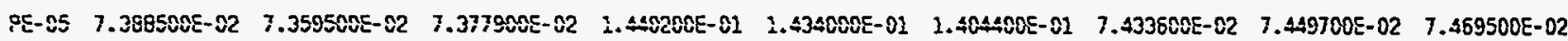
PE-26 6.422800E- $22 \quad 6.401500 E-02 \quad 5.391400 E-02 \quad 1.574200 E-02 \quad 1.625200 E-01 \quad 1.620600 E-02 \quad 6.389800 E-02 \quad 6.417800 E-02 \quad 6.433400 E-02$

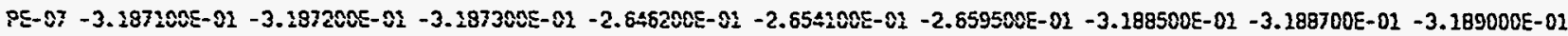
$\begin{array}{lllllllllll}P E-08 & 5.591000 E-03 & 5.440000 E-03 & 5.526000 E-03 & 1.005710 E-02 & 1.001210 E-02 & 9.722800 E-02 & 7.760000 E-03 & 7.963000 E-03 & 8.512000 E-03\end{array}$

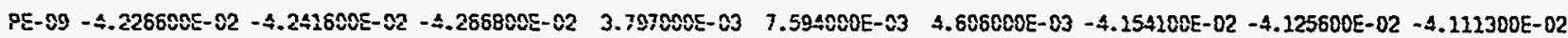
$\begin{array}{llllllllll}P E-20 & 1.859600 E-02 & 1.857000 E-02 & 1.852500 E-02 & 8.183900 E-02 & 8.175400 E-\$ 2 & 8.134500 E-02 & 1.563800 E-02 & 1.576400 E-02 & 1.599700 E-02\end{array}$ \begin{tabular}{lllllllllll}
\hline .13 & $1.53900005-93$ & $1.927000 E-03$ & $1.742000 E-03$ & $4.758800 E-02$ & $4.572700 E-02$ & $4.594700 E-02$ & $3.940000 E-04$ & $3.220000 E-04$ & $7.160000 E-04$
\end{tabular}

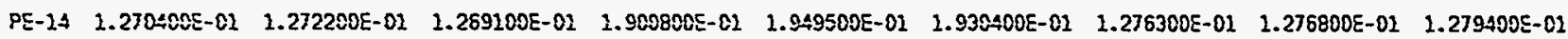

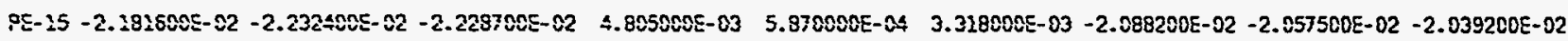
PE-15 3. $300600 E-82 \quad 2.994900 E-02 \quad 3.021000 E-\$ 2 \quad 5.534480 E-82 \quad 5.468900 E-02 \quad 5.477700 E-82 \quad 3.064500 E-02 \quad 3.061200 E-02 \quad 3.099300 E-02$ $P E-17-4.285600 E-02-4.293300 E-02-4.312500 E-02-2.037600 E-02-1.918900 E-02-1.899800 E-02-4.238300 E-02-4.204000 E-02-4.196500 E-02$ PCE-18 - $1.252800 E-01-1.252500 E-01-1.253000 E-01-2.003760 E-01-9.809500 E-82-1.007020 E-01-1.2518005-01-1.246800 E-01-1.244400 E-01$ PE- $19-5.8722005-02-5.891100 E-\$ 2-5.8984005-02-5.1220005-02-5.174500 E-02-5.447200 E-02-5.8792005-\$ 2-5.863800 E-02-5.839700 E-02$ PE-20 -5.978100E-02 -5.990900E- $22-5.989600 E-02-4.794400 E-02-5.093500 E-02-4.926500 E-02-5.035100 E-02-6.007600 E-02-5.974500 E-02$ PE- 22 -2.109500E- $02-2.204900 E-82-2.099300 E-\$ 2-1.053000 E-02-1.197900 E-02-1.042108 E-02-2.1727005-02-2.115000 E-02-2.066200 E-02$ $P E-22-1.654800 E-02-1.636700 E-02-1.647300 E-02-3.601000 E-03-3.463000 E-03-2.912000 E-03-1.692800 E-02-1.661400 E-02-1.636900 E-02$

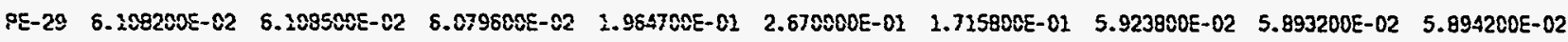
$P E-30-1.235240 E-01-1.234360 E-02-1.134900 E-02-5.456600 E-02-1.107560 E-01 \quad-1.295100 E-01-1.130310 E-01-1.134810 E-01-1.236640 E-01$ FE-02 $1.000170 E+00 \quad 2.000170 E+\infty 0 \quad 3.0001805+00 \quad 3.647900 E+00 \quad 1.645800 E+00 \quad 1.6 \$ 6300 E+00 \quad 1.001600 E+00 \quad 1.001170 E+00 \quad 1.000960 E+00$ XE-01 -2.630000E-04 -2.630000E-04 -2.630000E-04 5.200000E-C5 4.700000E-CS 5.100008E-05 -2.340000E-04 -2.390000E-04 -2.420000E-04 XE- $82-8.800000 E-04-8.780000 E-04-6.7900005-04-6.800000 E-04-6.800000 E-04-6.790000 E-04-6.750000 E-04-6.770000 E-04-6.780000 E-04$ $X E-83-3.560000 E-04-3.560000 E-04-3.550000 E-04-3.600000 E-04-3.260000 E-04-3.070000 E-04-3.020000 E-04-3.030000 E-04-3.090000 E-04$ $x E-04-2.098000 E-03-2.093000 E-03-1.097000 E-03-0.580000 E-04-9.550000 E-04-9.550000 E-04-1.0700005-03-1.080000 E-03-1.082000 E-03$

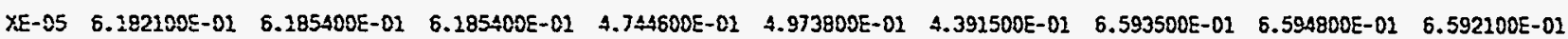
$\begin{array}{llllllllll}X E-06 & 8.240500 E-01 & 8.240800 E-02 & 8.240800 E-01 & 8.243300 E-01 & 8.026700 E-01 & 8.0058 C 0 E-01 & 7.967000 E-01 & 7.969100 E-01 & 7.972000 E-01\end{array}$ XE- $07 \quad-5.800000 E-04-5.800000 E-04-5.820000 E-04-3.450000 E-04-4.460000 E-04-4.430000 E-04-5.500000 E-04-5.540000 E-04-5.560000 E-04$ $X E-0 E-7.700000 E-0 \leqslant-7.720000 E-04-7.7000005-04-6.220000 E-04-6.190000 E-04-6.270000 E-04-7.560000 E-04-7.580000 E-04-7.580000 E-04$ XE-09 - $9.270000 E-04-9.260000 E-05-9.270000 E-04-8.260000 E-04-8.200000 E-04-8.170000 E-04-9.020000 E-04-9.020000 E-04-9.040000 E-04$ XE- $10-4.759000 E-03-4.7600005-03-4.759000 E-03-4.320000 E-03-4.128000 E-03-4.063000 E-03-4.7720005-03-4.779000 E-03-4.784000 E-03$

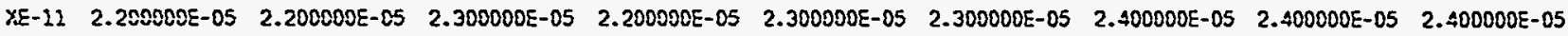
$X E-12-1.459000 E-03-1.459000 E-83-1.458008 E-03-1.460008 E-03-1.459000 E-03-1.460000 E-03-1.465000 E-03-1.466080 E-03-1.465000 E-03$ $X E-13-6.700000 E-04-6.670000 E-04-6.690000 E-04-3.940000 E-04-3.910000 E-04-3.910000 E-04-6.680000 E-04-5.670000 E-04-6.730000 E-04$ XE-14 -3.15E000E-04 -3.1 S0000E-04 -3.130000E- $24-2.5200005-04-2.480000 E-04-2.490000 E-04-3.080000 E-04-3.050000 E-04-3.090000 E-04$ $X E-15-7.300000 E-04-7.260000 E-04-7.270000 E-04-6.740000 E-04-6.720000 E-04-6.710000 E-04-7.220000 E-04-7.240000 E-04-7.260000 E-04$ TE- $01-5.500000 E-05-5.5000005-05-5.500000 E-05-2.900000 E-05-2.800000 E-05-2.700000 E-05-4.200000 E-05-6.500080 E-05-6.100000 E-05$ $\begin{array}{lllllllllll}\text { VEXT: } & 1.993400 E+00 & 2.993500 E+00 & 1.993400 E+00 & 1.993500 E+00 & 1.993500 E+00 & 1.993600 E+00 & 1.993400 E+00 & 1.993500 E+00 & 1.993500 E+00\end{array}$ $\begin{array}{lllllllllll}\text { VEXT2 } & 1.999800 E+00 & 1.989800 E+00 & 2.989800 E+00 & 1.989800 E+00 & 1.989900 E+00 & 1.989900 E+00 & 1.989800 E+00 & 1.989800 E+00 & 1.989800 E+00\end{array}$

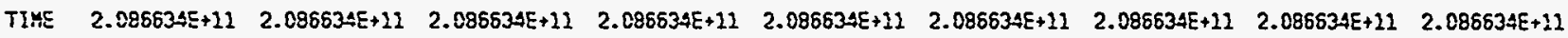


irchive Heme:

AHS_YFP_TESTS

Bosk Hame: AYS_MFPT__RY Cate \& Time: 6 Mar 2980 21:36:13

Page Description:

CATA SET MC. -219

TRAHS RY_ZERO-1 RY_ZERO-2 RY_ZERD-3 RV_LOAD-1 RY_LOAD-2 RV_LOAD-3 RY_ZERO-4 RV_ZERO-5 RV_ZERO-6

PE- 01 -7.429700E-02 -7.424500E-02 -7.432000E-02 5.940200E-02 5.901300E-02 5.868400E-02 -7.141700E-02 -7.2445C0E-02 -7.128300E-02 PE-02 3.174COCE-02 $3.167700 E-02 \quad 3.360700 E-02 \quad 1.864400 E-02 \quad 1.976400 E-01 \quad 2.911800 E-02 \quad 3.436500 E-02 \quad 3.435000 E-02 \quad 3.460800 E-02$

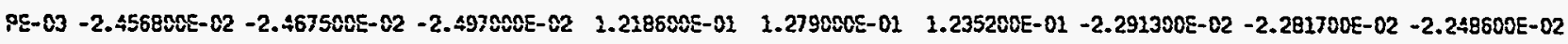
PE-04 3.543100E-02 $3.549700 E-02 \quad 3.533700 E-02 \quad 2.052100 E-01 \quad 2.083500 E-01 \quad 2.026900 E-01 \quad 3.777000 E-02 \quad 3.757400 E-02 \quad 3.779600 E-02$

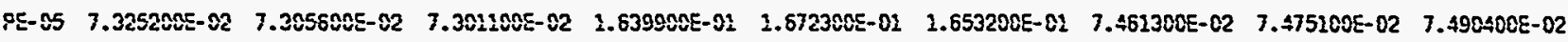
$\begin{array}{lllllllllll}P E-06 & 6.202400 E-\$ 2 & 6.205900 E-02 & 6.198100 E-02 & 1.790900 E-02 & 1.853600 E-01 & 1.813600 E-01 & 6.159400 E-02 & 6.190900 E-02 & 5.203700 E-02\end{array}$

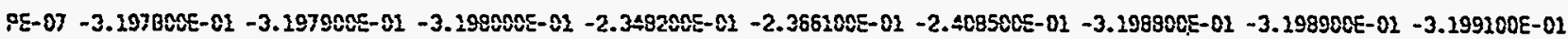
PE-C8 $6.434000 E-03 \quad 6.810000 E-03 \quad 6.497000 E-03 \quad 1.307800 E-01 \quad 1.281600 E-01 \quad 1.265900 E-01 \quad 8.180000 E-03 \quad 8.170000 E-03 \quad 8.162000 E-03$

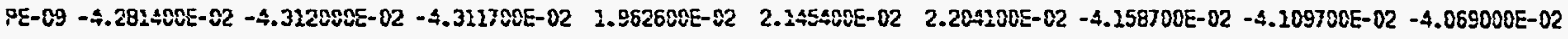
$\begin{array}{lllllllllll}P E-10 & 1.416000 E-02 & 1.401900 E-02 & 1.395900 E-02 & 2.040780 E-01 & 1.079370 E-02 & 1.032000 E-02 & 1.565100 E-02 & 1.598900 E-02 & 1.508100 E-02\end{array}$

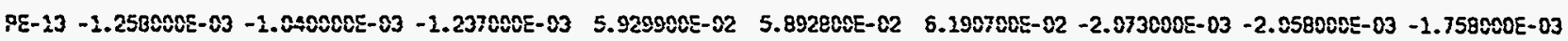

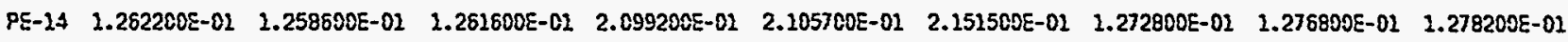
PE-15 -2.168200E-02 $-2.175300 E-02 \quad-2.156900 E-02 \quad 3.563300 E-02 \quad 3.212900 E-02 \quad 1.251500 E-02 \quad-1.994700 E-02 \quad-1.961800 E-02 \quad-1.945000 E-02$ FE-15 2.983900E-02 $2.979000 E-02 \quad 2.986700 E-02 \quad 6.277500 E-02 \quad 6.269700 E-02 \quad 8.462600 E-02 \quad 3.131000 E-02 \quad 3.1 \frac{14400 E-02}{3} \quad 3.131800 E-02$ $P E-17$ - $4.320400 E-02-4.306200 E-02-4.328400 E-02-1.5645008-82-1.121208 E-02-1.2028008 E-02-4.184800 E-82-4.179000 E-02-4.169200 E-02$ PE-19 - - $.253600 E-02-1.254500 E-02-1.254300 E-02-9.215900 E-02-9.052900 E-02-9.104000 E-02-1.238400 E-02-1.235300 E-01-1.236600 E-01$

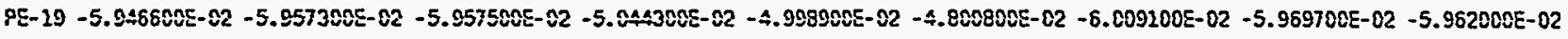
PE-20 - $5.080100 E-02-6.047900 E-02-6.061900 E-02-4.588000 E-02-4.573800 E-02-4.711100 E-02-6.007700 E-02-5.971600 E-02-5.969500 E-02$ PE-21 - 2.182180E-02 -2.171000E- $02-2.273600 E-02-5.0240005-03-5.976800 E-03-6.427000 E-03-2.195600 E-02-2.159600 E-02-2.130800 E-02$

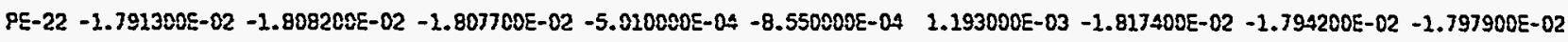
$\begin{array}{llllllllll}P E-29 & 5.960300 E-82 & 5.954800 E-02 & 5.927200 E-02 & 2.731400 E-02 & 2.955800 E-01 & 2.857100 E-01 & 5.792600 E-02 & 5.791500 E-02 & 5.759500 E-02\end{array}$

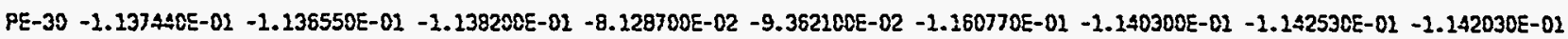
$\begin{array}{lllllllllll}F E-01 & 1.000350 E+00 & 1.000350 E+00 & 2.000340 E+00 & 1.762500 E+00 & 1.760300 E+00 & 1.762600 E+00 & 1.001490 E+00 & 1.001160 E+00 & 1.000960 E+00\end{array}$ XE-01 -2.660000E-04 -2.650000E-04 -2.670000E-04 7.200000E-05 7.600000E-05 7.900000E-05 $-2.320000 E-04 \quad-2.380000 E-04$-2.410000E-04 XE-02 -6.790000E-04 -6.780000E-04 -6.790000E-04 -6.810000E-04 -6.790000E-04 -6.780000E-04 -6.760000E-04 -6.780000E-04 -6.770000E-04 $X E-03-4.560000 E-04-4.560000 E-04-4.560000 E-04-3.820000 E-04-3.800000 E-04-3.920000 E-04-4.650000 E-04-4.660000 E-04-4.650000 E-04$ XE-04 -1.097000E- $03-1.097000 E-03-1.098000 E-03-9.430000 E-04-9.370000 E-04-9.430000 E-04-1.072000 E-03-1.075000 E-03-1.076000 E-03$ $\begin{array}{lllllllllll}X E-05 & 6.616900 E-01 & 6.627500 E-01 & 6.822200 E-01 & 5.885200 E-01 & 6.422800 E-01 & 5.694100 E-02 & 6.965200 E-01 & 7.012500 E-01 & 4.922200 E-01\end{array}$

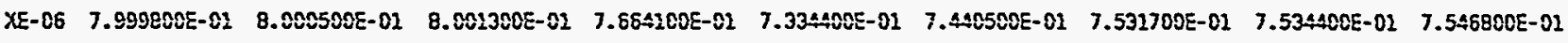

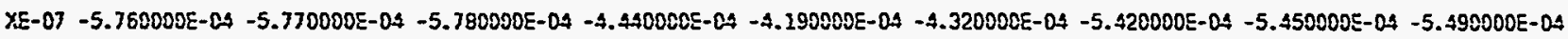
XE-00 -7.700000E-04 -7.700000E-04 -7.700C00E-04 -6.230000E-04 -6.260800E-04 -6.210000E-04 -7.520000E-04 -7.510000E-04 -7.520000E-04 XE- $09-9.2300005-04-9.230000 E-04-9.240000 E-04-8.130000 E-04-8.080000 E-04-8.090000 E-04-8.960000 E-04-8.970000 E-04-9.000000 E-04$ $X E-10-4.814000 E-03-4.8250005-03-4.827000 E-03-4.272000 E-03-4.474000 E-03-4.497000 E-03-4.750000 E-03-4.703000 E-03-4.193000 E-83$ $\begin{array}{lllllllllll}X E-11 & 2.3000005-05 & 2.300000 E-05 & 2.300000 E-05 & 2.300000 E-05 & 2.300000 E-05 & 2.300000 E-05 & 2.400000 E-05 & 2.500000 E-05 & 2.400000 E-05\end{array}$ $X E-12-2.462000 E-03-1.4600005-03-1.459000 E-03-1.460000 E-03-2.458000 E-03-1.460000 E-03-1.468000 E-03-1.466080 E-03-1.465000 E-83$ XE-13 -6.780000E-04 -6.750000E-0. $-6.770000 E-04-3.650000 E-04-3.650000 E-04-3.630000 E-04-6.660000 E-04-6.590000 E-04-6.620000 E-04$ $X E-14-3.130000 E-04-3.060000 E-04-3.120000 E-04-2.390000 E-04-2.410000 E-04-2.360000 E-04-3.070000 E-04-3.060000 E-04-3.070000 E-04$ XE-15 -7.280000E-04 -7.250000E-04 -7.280000E-04 -6.680000E-04 $-6.670000 E-04-6.660000 E-04-7.220000 E-04-7.230000 E-04-7.240000 E-04$ TE- $01-7.400000 E-05-7.500000 E-05-7.400000 E-05-5.200000 E-05-4.900000 E-05-4.700000 E-85-6.700000 E-05-8.500000 E-05-8.500000 E-05$ VEXT2 $1.993400 E+00 \quad 2.993400 E+00 \quad 1.993400 E+00 \quad 1.993500 E+00 \quad 1.993500 E+00 \quad 1.993400 E+00 \quad 1.993400 E+00 \quad 1.993500 E+00 \quad 1.993500 E+00$

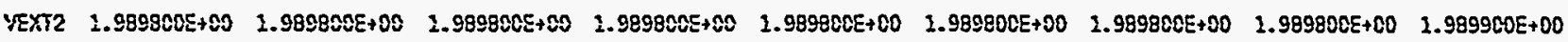

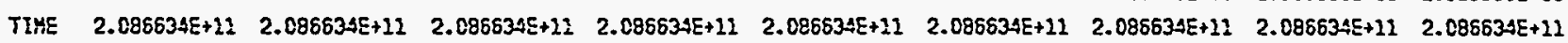




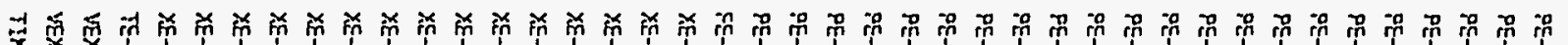

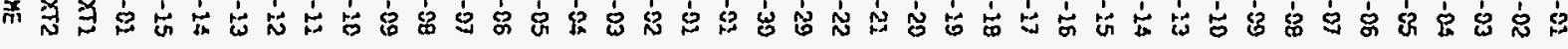
N م

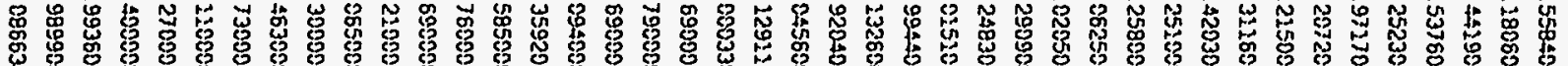

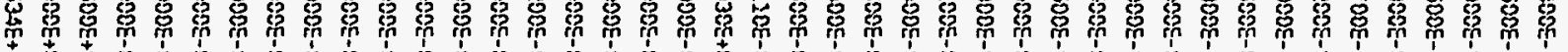

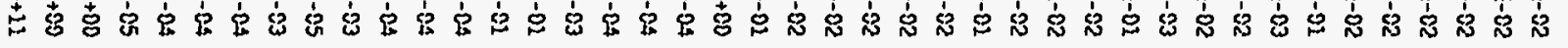

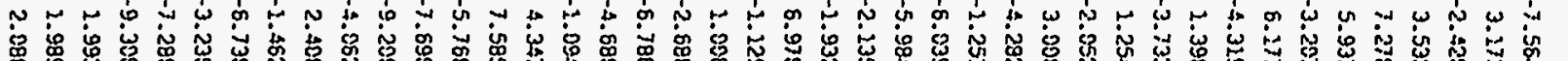

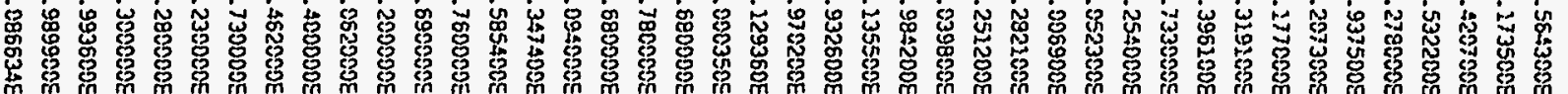

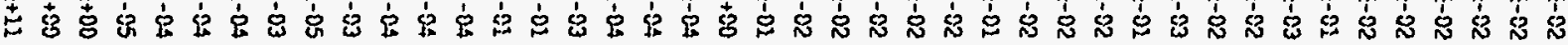
N

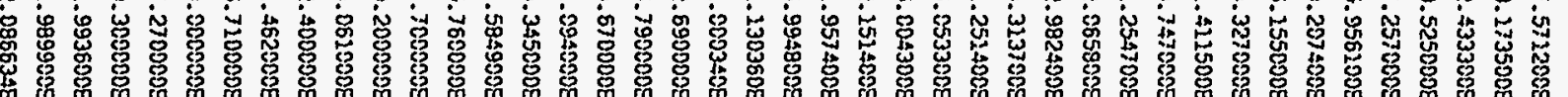

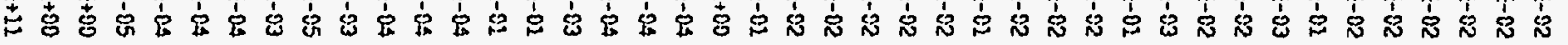

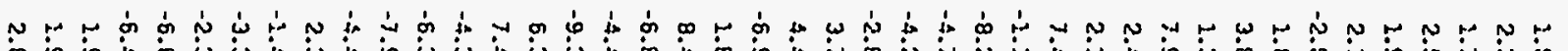

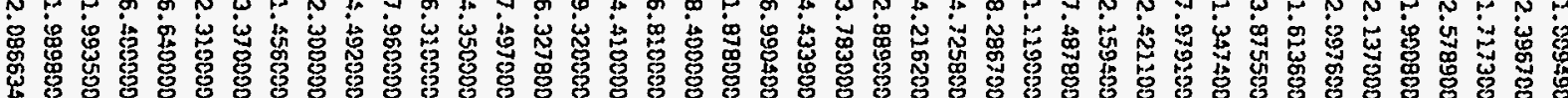

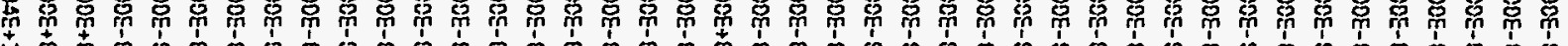

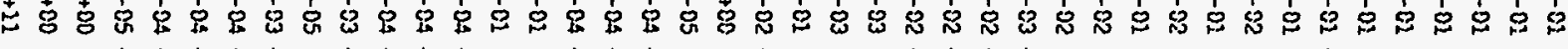
N م

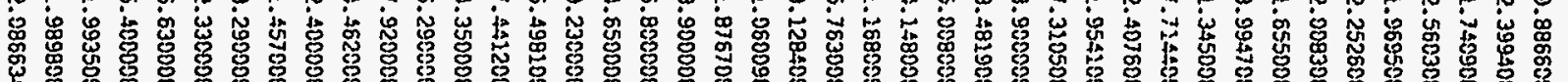

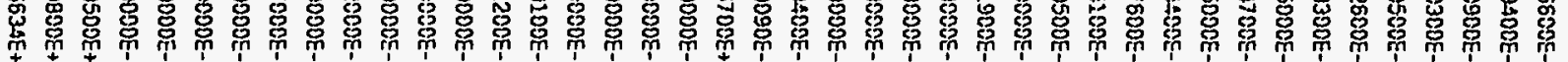

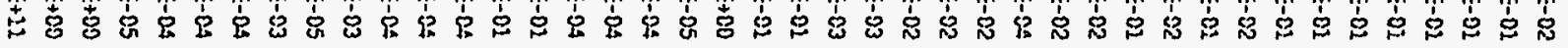

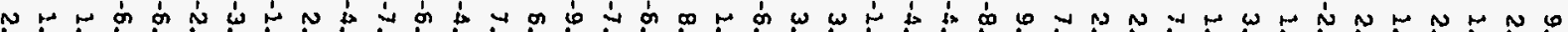

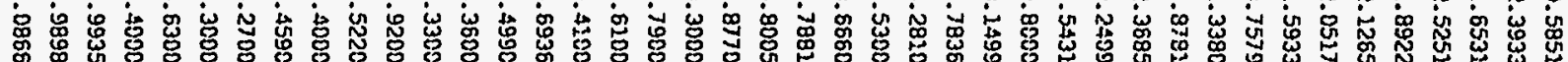

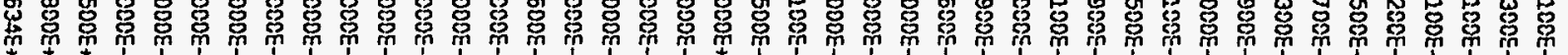

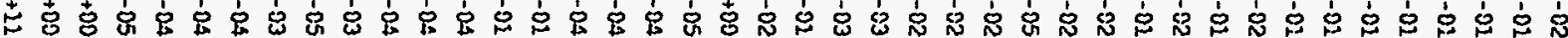

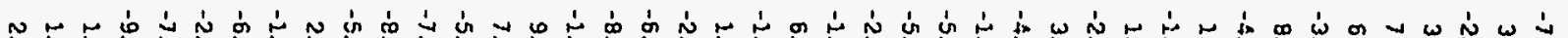

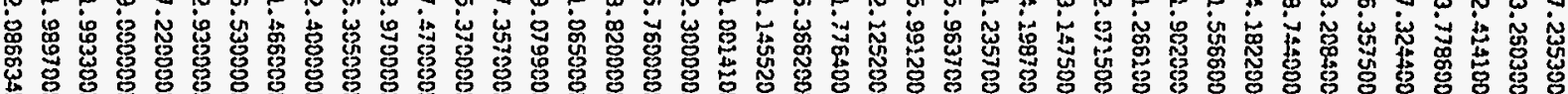

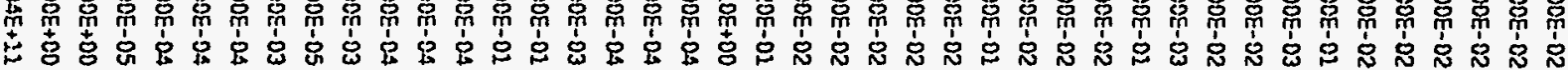

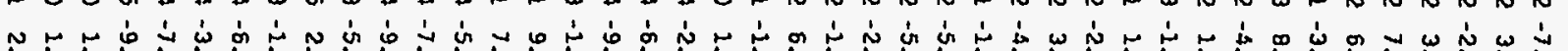

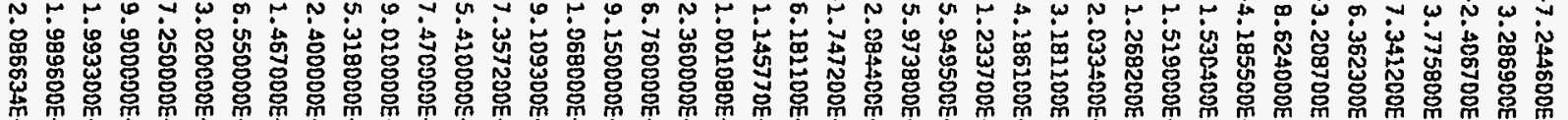

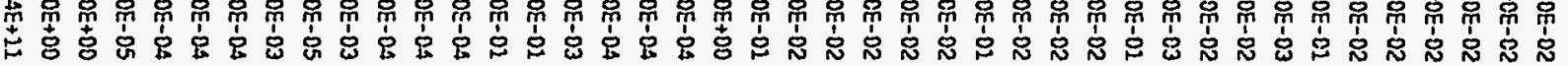

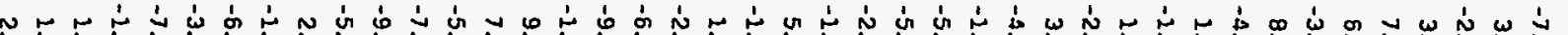

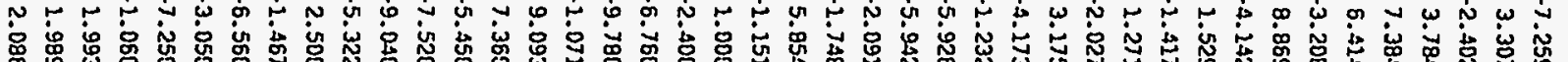

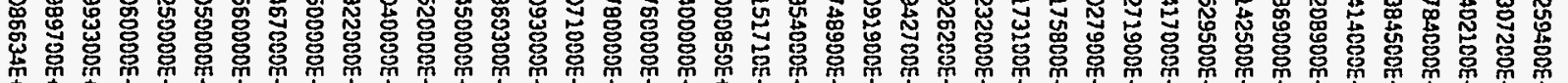

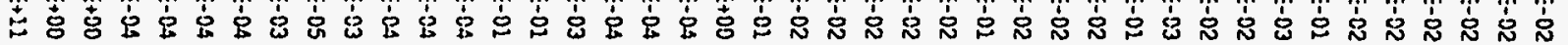

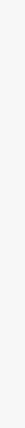

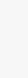
.

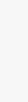


Page Description:

DATA SET HO.-222

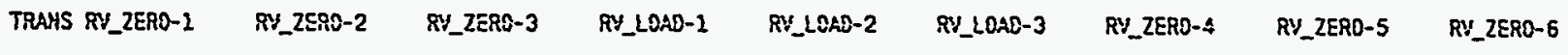

PE-02 -7.577700E-02 -7.5962005-02 -7.593500E-02 1.507800E-02 1.47.4500E-01 $3.557300 E-01$-7.299700E-02 -7.272900E-02 -7.289300E-02 PE-02 3.057800E-02 $3.021700 E-02 \quad 2.994200 E-02 \quad 3.0 E 8300 E-02 \quad 3.053300 E-01 \quad 3.240700 E-01 \quad 3.595400 E-02 \quad 3.584100 E-02 \quad 3.571500 E-02$ PE- 03 -2.591700E-02 -2.837600E- 02 -2.820300E-02 2.203600E-02 2.180100E-01 2.297600E-02 $-2.338500 E-02 \quad-2.307500 E-02$-2.293700E-02 PE-04 3.421200E-02 $3.388500 E-02 \quad 3.382800 E-02 \quad 3.124100 E-01 \quad 3.106500 E-01 \quad 3.212600 E-01 \quad 3.708500 E-02 \quad 3.719100 E-02 \quad 3.726200 E-02$ PE-05 T.171800E-02 7.140108E-02 7.244200E-02 $2.189800 E-01 \quad 2.214000 E-01 \quad 2.226400 E-01 \quad 7.452800 E-02 \quad 7.397700 E-02 \quad 7.315200 E-02$

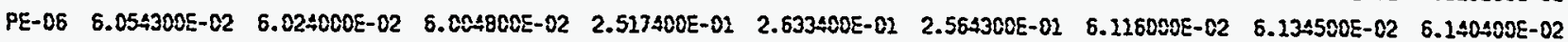

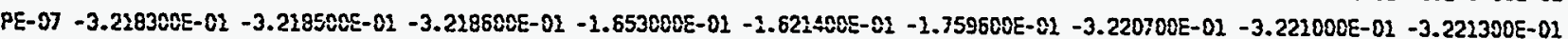
PE-08 4.910000E-03 4.934000E-03 5.100000E-03 2.202400E-02 2.097300E-01 2.007700E-01 $8.758000 E-03 \quad 8.824000 E-03 \quad 8.607000 E-03$

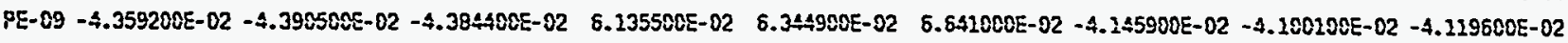

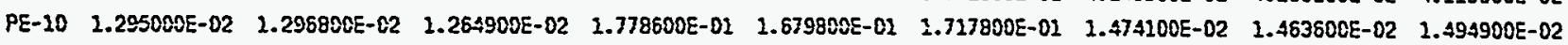

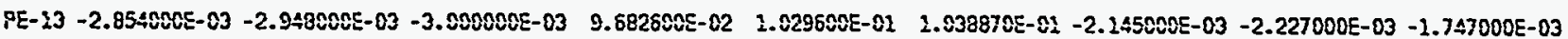
PE-24 1.243000E-01 $1.244700 E-01 \quad 1.242300 E-01 \quad 2.663400 E-01 \quad 2.633500 E-01 \quad 2.772800 E-01 \quad 1.263400 E-01 \quad 1.264800 E-01 \quad 1.262300 E-01$

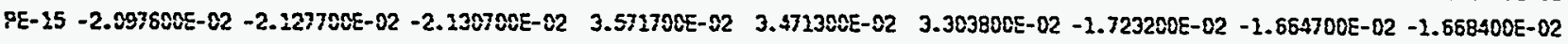
PE-16 3.084100E-02 $3.074200 E-02 \quad 3.047700 E-02 \quad 8.242500 E-02 \quad 8.725700 E-02 \quad 8.432500 E-02 \quad 3.239300 E-02 \quad 3.285500 E-02 \quad 3.314600 E-02$

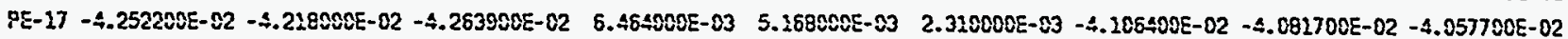

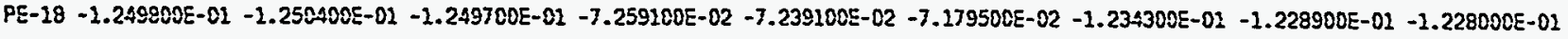
$P E-12-8.0285085-02-6.003200 E-02-6.021200 E-02-4.679900 E-02-4.490700 E-02-4.254000 E-02-5.936600 E-02-5.888900 E-02-5.892700 E-02$ PE-20 -5.975300E-02 -5.001500E-02 -6.012800E-02 -3.691208E-02 -3.678600E-02 -3.866100E-02 -6.084900E-02 -6.062300E-02 -6.054200E-02 PE-22 -2.149380E-02 $-2.254400 E-02-2.2742005-02 \quad 3.011000 E-03 \quad 5.827000 E-03 \quad 4.709000 E-03 \quad-2.128800 E-02 \quad-2.093400 E-02 \quad-2.097900 E-02$ PE-22 - - $.920100 E-02$ - $1.937000 E-02 \quad-1.912800 E-02 \quad 1.150900 E-02 \quad 9.042000 E-03 \quad 8.441000 E-03-1.802200 E-02-1.766400 E-02-1.735300 E-02$ \begin{tabular}{lllllllllll}
\hline$E-29$ & $5.485600 E-02$ & $5.517700 E-02$ & $5.489580 E-02$ & $6.314308 E-02$ & $5.961500 E-01$ & $2.781008 E-01$ & $6.495600 E-02$ & $6.535800 E-02$ & $6.584700 E-02$
\end{tabular} PE-30 -2.160739E-02 -2.259C89E-02 - 2.160200E-02 -8.0237C0E-02 -8.028-00E-02 -5.888700E-02 -1.117130E-02 - 1.217750E-01 - $1.120930 E-02$

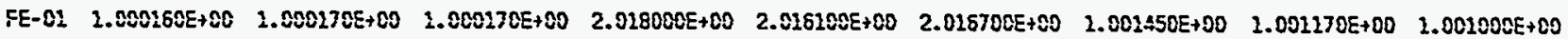
XE-02 -2.550000E-04 -2.850000E-04 -2.680000E-04 8.900000E-05 9.700000E-05 9.000000E-05 $-2.390000 E-04 \quad-2.420000 E-04 \quad-2.440000 E-04$

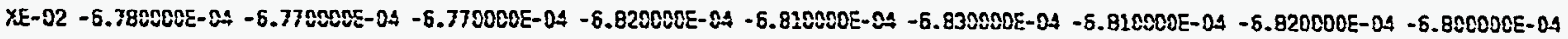
$X E-03$-9.860000E-04 -9.860000E-04 -9.850000E-04 -4.630000E-04 -4.290000E-04 -5.210000E-04 -8.100000E-04 -5.960000E-04 -5.880000E-04 XE-04 -1.092000E-03 -2.092000E-03 - $2.092000 E-03-9.230000 E-04-9.060000 E-04-9.130000 E-04-1.058000 E-03-1.061000 E-03-1.062000 E-03$ $\begin{array}{llllllllllll}X E-05 & 9.216200 E-01 & 9.228300 E-01 & 9.234600 E-01 & 2.694700 E-01 & 5.911000 E-01 & 2.511000 E-01 & 7.789200 E-01 & 8.192000 E-01 & 7.173900 E-01\end{array}$

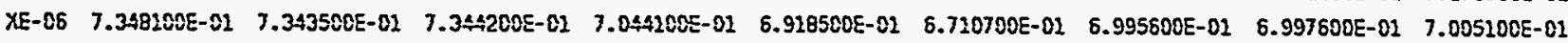
YE-07 -5.740000E-04 -5.750000E-04 -5.740000E-04 -4.310000E-04 -4.330000E-04 -4.320000E-04 -5.330000E-04 -5.37C000E-04 -5.410000E-04 XE- $88-7.680000 E-04-7.700000 E-04-7.700000 E-04-6.2800005-04-6.260000 E-04-6.260000 E-04-7.430000 E-04-7.430000 E-04-7.460000 E-04$ XE-09 $-9.230000 E-04-9.230000 E-04-9.240000 E-04-7.810000 E-04-7.820000 E-04-7.810000 E-04-8.970000 E-04-9.000000 E-04-9.020000 E-04$ $X E-20-5.378000 E-03-5.379000 E-03-5.378000 E-03-3.341000 E-03-4.09 .4000 E-03-3.052000 E-03-4.814000 E-03-4.937000 E-03-4.6890005-03$ XE-11 2.300000E-15 $2.300000 E-05 \quad 2.300000 E-05 \quad 2.300000 E-05 \quad 2.400000 E-05 \quad 2.500000 E-05 \quad 2.400000 E-05 \quad 2.400000 E-05 \quad 2.500000 E-05$ XE-12 - $2.464000 E-03-1.454000 E-03-1.463000 E-03-2.451000 E-03-1.453000 E-03-1.357000 E-03-1.465000 E-03-1.467000 E-03-1.267000 E-03$ XEE-13 -6.710000E-0. $-6.700000 E-04-6.700000 E-04-3.240000 E-04-3.160000 E-04-3.140000 E-04-6.550000 E-04-6.550000 E-04-6.570000 E-04$ YE-14 -3.130000E-04 -3.308000E- $24-3.130000 E-04-2.170000 E-04-2.130000 E-04-2.260000 E-04-3.050000 E-04-3.110000 E-04-3.120000 E-04$ XE-25 -7.280000E-04 -7.290000E-04 -7.250000E-04 -6.630000E-04 -6.640000E-04 -6.620000E-03 -7.230000E-04 -7.230000E-04 -7.230000E-04

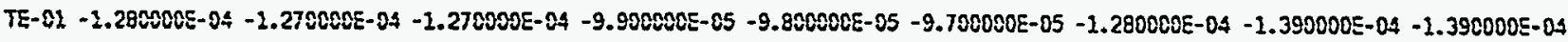
VEXT1 $2.993300 E+C D \quad 1.993300 E+00 \quad 1.993300 E+00 \quad 1.993600 E+00 \quad 1.993500 E+00 \quad 1.993700 E+C 0 \quad 1.993500 E+00 \quad 1.993400 E+00 \quad 1.993500 E+00$ VEXT2 $1.98970 C E+00 \quad 2.989780 E+00 \quad 1.989700 E+00 \quad 1.989900 E+00 \quad 1.989900 E+00 \quad 1.990100 E+00 \quad 1.989800 E+00 \quad 1.989800 E+C 0 \quad 1.989800 E+00$

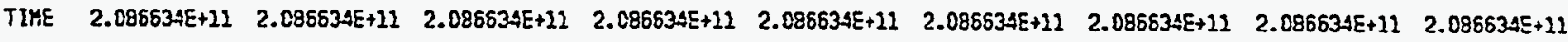


Page Eescripticn:

DATA SET $40 .-222$

TRAYS RY_ZERO-1 RY_ZERO-2 RY_ZERO-3 RY_LOLO-1 RY_LOAD-2 RV_LOAD-3 RV_ZERO-4 RY_ZERO-5 RY_ZERO-6

$8 E-02-7.706900 E-02-7.716000 E-02-7.707800 E-02 \quad 2.006400 E-01 \quad 1.918800 E-02 \quad 2.043300 E-02 \quad-7.392400 E-02-7.300700 E-02 \quad-7.402200 E-02$ 8E- $02 \quad 3.043000 E-02 \quad 3.049300 E-02 \quad 3.051500 E-02 \quad 3.735800 E-01 \quad 3.715800 E-01 \quad 3.874800 E-02 \quad 3.041900 E-02 \quad 3.013500 E-02 \quad 3.030300 E-02$

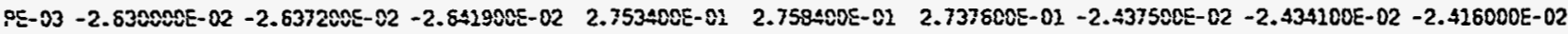
PE-04 3.439EODE-02 $3.423860 E-02 \quad 3.440700 E-02 \quad 3.740400 E-01 \quad 3.610200 E-01 \quad 3.542800 E-01 \quad 3.637700 E-02 \quad 3.617200 E-02 \quad 3.643700 E-02$

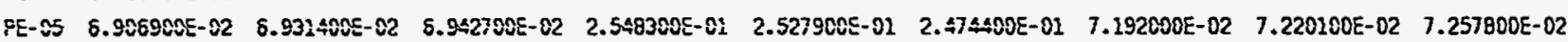
:E-06 5.752900E-02 $5.767300 E-02 \quad 5.766100 E-02 \quad 3.115400 E-01 \quad 3.260400 E-01 \quad 3.085500 E-01 \quad 6.070600 E-02 \quad 6.076900 E-02 \quad 6.075600 E-02$ PE- $07-3.229300 E-01-3.220300 E-01-3.229300 E-01-1.289900 E-01-1.305000 E-01-1.360400 E-02-3.228200 E-01-3.228400 E-01-3.228500 E-01$ 8E- $89 \quad 3.156000 E-03 \quad 3.412000 E-03 \quad 3.273000 E-83 \quad 2.582400 E-01 \quad 2.350020 E-01 \quad 2.344500 E-01 \quad 8.739000 E-03 \quad 8.440000 E-03 \quad 8.473000 E-03$

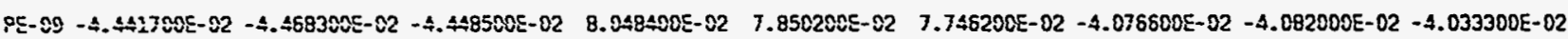
$\begin{array}{lllllllllll}P E-10 & 1.329500 E-02 & 2.324400 E-02 & 1.335200 E-\$ 2 & 1.908500 E-01 & 2.068800 E-01 & 2.022300 E-01 & 1.707700 E-02 & 1.736900 E-02 & 1.750600 E-02\end{array}$

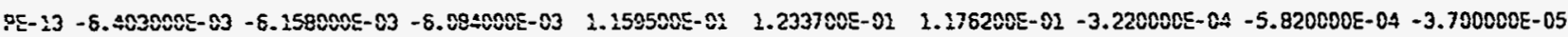

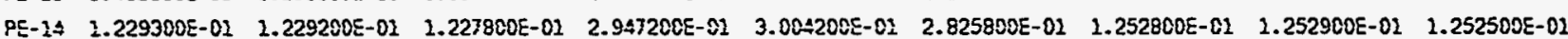

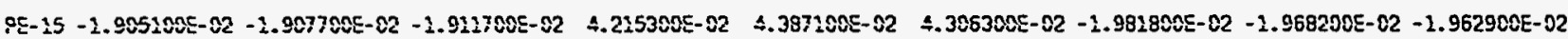
PE-16 $\quad 3.047200 E-02 \quad 3.045700 E-02 \quad 3.931900 E-02 \quad 9.903000 E-02 \quad 9.024300 E-02 \quad 9.561400 E-02 \quad 3.328600 E-02 \quad 3.321200 E-02 \quad 3.382600 E-02$ $P E-17-4.278000 E-02-4.283808 E-02-4.287180 E-82 \quad 1.350500 E-\$ 2 \quad 1.328400 E-02 \quad 3.248 \Omega 00 E-02-4.052908 E-02 \quad-4.019580 E-02$ - $3.996100 E-02$

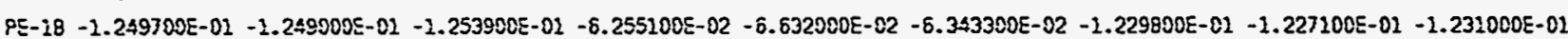

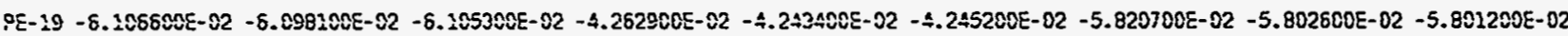

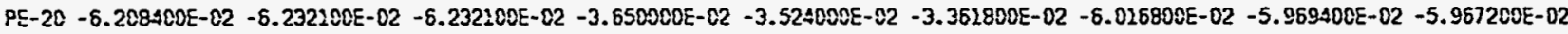

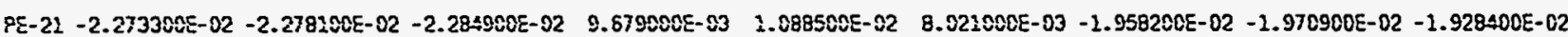
PE-22 - 2.035300E-02 -2.018700E-02 -2.031800E-02 1.526500E-02 $1.4573020 E-02 \quad 1.641600 E-02-1.809400 E-02 \quad-1.769500 E-02 \quad-1.787700 E-02$ $\begin{array}{lllllllllll}\text { PE-29 } & 6.848600 E-02 & 5.944800 E-02 & 6.903908 E-02 & 5.913800 E-01 & 5.527600 E-01 & 6.175700 E-01 & 7.117700 E-02 & 6.979900 E-02 & 7.114500 E-02\end{array}$ $P E-30-2.115800 E-02-1.115380 E-02-1.213670 E-01-1.139910 E-01 \quad-9.111100 E-02 \quad-5.252600 E-02-1.140100 E-01-1.144770 E-01-1.242600 E-01$

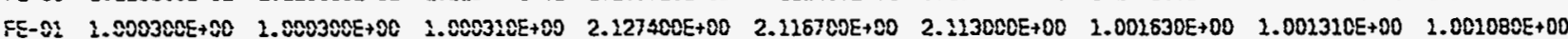
XE-02 $-2.770000 E-04 \quad-2.790000 E-04 \quad-2.3700000-04 \quad 9.300000 E-05 \quad 9.000000 E-05 \quad 8.500000 E-05-2.430000 E-04 \quad-2.460000 E-04 \quad-2.510000 E-04$ XE- $02-5.780000 E-04-6.790000 E-04-6.790000 E-04-6.820000 E-04-6.790000 E-04-6.800000 E-04-6.780000 E-0.4-6.770000 E-04-6.780000 E-04$ $X E-03-6.050000 E-04-6.050000 E-04-6.040000 E-04-5.310000 E-04-5.3700005-04-4.760000 E-04-4.360000 E-04-4.350000 E-04-4.410000 E-04$ XE-04 -1.097000E-03 -1.097000E-03 - - $1.097000 E-03-9.240000 E-04$-9.280000E-04 -9.290000E-04 -1.038000E-03 - $1.050000 E-03-1.050000 E-03$

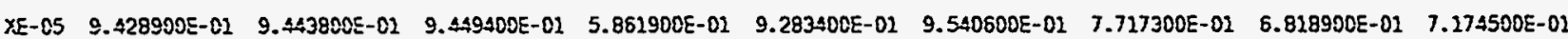

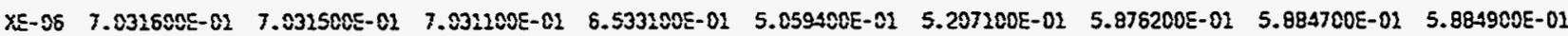
XE-07 -5.820000E-04 -5.830000E-04 -5.820000E-04 -4.530000E-04 -4.350008E-04 -2.830000E-04 -5.130000E-04 -5.200000E-04 -5.250000E-04 $x E-08-7.7700005-04-7.7600005-04-7.7700005-04-6.430000 E-04-6.340000 E-04-6.360000 E-04-7.390000 E-04-7.410000 E-04-7.4300005-04$ $x E-09-9.320000 E-04-9.310000 E-04-9.310000 E-04-8.000000 E-04-7.9100005-04-7.880000 E-04-8.950000 E-04-8.990000 E-044-9.010000 E-04$

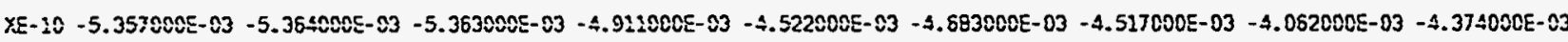

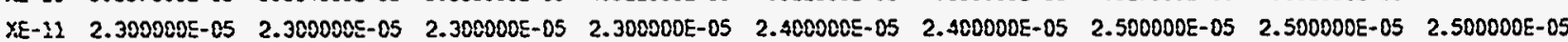
$X E-12-1.465000 E-83-1.4650005-03-1.465000 E-03-1.4550005-03-1.455000 E-03-1.455000 E-03-1.467000 E-03-1.468000 E-03-1.470000 E-03$ $X E-13-6.750000 E-04-6.740000 E-04-6.730000 E-04-3.070000 E-04-3.220000 E-04-3.120000 E-04-6.510000 E-04-6.530000 E-04-5.550000 E-04$ XE-14 -3.175050E-04 -3.150000E-04 -3.250000E-04 -2.160000E-04 -2.120000E-04 -2.110000E-04 -3.120000E-04 -3.110000E-04 -3.020000E-04 XE-15 -7.260000E-04 -7.300000E-04 -7.270000E-04 -6.620000E-04 - $5.650000 E-04-6.600000 E-04-7.240000 E-04-7.260000 E-04-7.250000 E-04$ TE- $01-1.330000 E-04-1.320000 E-04$ - $-1.300000 E-04$-9.500000E-05 - $9.500000 E-05-9.400000 E-05-1.260000 E-04-1.330000 E-04-1.330000 E-04$ $\begin{array}{lllllllllll}V E X T 2 & 2.993300 E+00 & 1.993300 E+00 & 1.993300 E+00 & 1.993500 E+00 & 2.993400 E+00 & 1.993400 E+00 & 1.993500 E+00 & 1.993500 E+00 & 1.993500 E+00\end{array}$ $\begin{array}{lllllllllll}v E X 2 & 1.989600 E+00 & 1.989600 E+00 & 2.989700 E+00 & 1.989700 E+00 & 1.989800 E+00 & 1.989700 E+00 & 1.989800 E+00 & 1.989800 E+00 & 1.999800 E+00\end{array}$ TIME $2.886634 E+12 \quad 2.086534 E+11 \quad 2.086634 E+11 \quad 2.086634 E+11 \quad 2.086534 E+12 \quad 2.886634 E+12 \quad 2.086634 E+11 \quad 2.086634 E+112 \quad 2.086634 E+11$ 
Archifive Metre:

AYS_MFP_TESTS

Eook Hane: AHS_YFPT1_RY Cate \& Time: 6 Yar 1900 22:16:47

Page Eescription:

EATA SET NO. -223

TRAYS RY_ZERO-1 RY_ZERO-2 RY_ZERO-3 RY_LOAD-1 $\quad$ RY_LOAD-2 $\quad$ RY_LOAR-3 RY_ZERE-4 $\quad$ RY_ZERC-5 RV_ZERO-6

PE- 01 -7.697100E-02 -7.879500E-02 -7.700000E-02 2.995200E-01 2.861300E-01 2.989200E-01 $-7.398900 E-02-7.386400 E-02-7.413400 E-02$ PE-02 2.723600E-02 2.728800E-02 $2.707800 E-02 \quad 4.867300 E-01 \quad 5.199000 E-01 \quad 4.947800 E-02 \quad 2.893300 E-02 \quad 2.904100 E-02 \quad 2.906200 E-02$ PE-03 -2.618000E-02 -2.606700E-02 -2.617400E-02 3.8698005-01 4.107800E-01 3.848200E-01 -2.330600E-02 -2.301900E-02 -2.37.3100E-02 PE-04 3.304800E-02 3.2865C0E-02 3.296200E-02 4.911700E-02 $5.213100 E-01 \quad 4.97$. $200 E-01 \quad 3.626300 E-02 \quad 3.640900 E-02 \quad 3.652200 E-02$

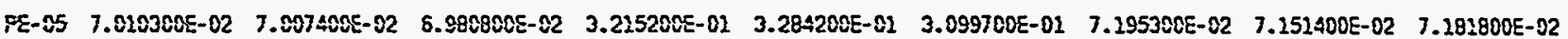
PE-06 5.797200E-02 5:793300E-02 5.787000E-02 4.151700E-01 $4.242600 E-01 \quad 3.994500 E-02 \quad 6.035200 E-02 \quad 6.003380 E-02 \quad 6.024700 E-02$ PE-07 -3.234400E-01 -3.234400E-02 -3.234500E-02 -3.704700E-02 -5.507100E-02 - 4.722000E-02 -3.235600E-01 -3.235900E-01 -3.236200E-01 PE-08 $4.939000 E-03 \quad 4.805000 E-03 \quad 4.73500 C E-03 \quad 3.710600 E-81 \quad 3.400100 E-02 \quad 3.518900 E-02 \quad 3.705000 E-03 \quad 3.654000 E-03 \quad 3.655000 E-03$ PE-09 -4.240400E-02 -4.254600E-02 -4.257000E-02 1.395900E-01 1.297200E-01 1.290300E-01 -4.132800E-02 -4.134700E-02 -4.083500E-02 PE-10 $1.522100 E-02 \quad 1.505600 E-02 \quad 1.496500 E-02 \quad 2.780600 E-01 \quad 2.798800 E-01 \quad 2.613200 E-01 \quad 1.635500 E-02 \quad 1.655200 E-02 \quad 1.573500 E-02$

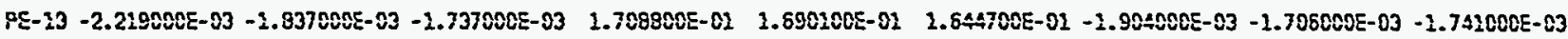
PE-14 1.232500E-01 1.2325COE-01 $1.232600 E-01 \quad 3.654900 E-01 \quad 3.728500 E-01 \quad 3.593400 E-02 \quad 1.252100 E-01 \quad 1.255800 E-01 \quad 1.253400 E-01$ EE-15 -2.101300E-02 -2.128300E-02 -2.122900E-02 $6.385800 E-02 \quad 7.5 A 2600 E-02 \quad 6.696900 E-02-2.024400 E-02 \quad-2.005300 E-02$-2.004100E-02 $P E-16 \quad 3.213500 E-02 \quad 3.179000 E-02 \quad 3.173600 E-02 \quad 1.184820 E-01 \quad 1.219800 E-81 \quad 1.244000 E-02 \quad 3.299500 E-02 \quad 3.296700 E-02 \quad 3.307000 E-02$ PE-17 - $4.157500 E-02-4.184200 E-02-4.170700 E-02 \quad 3.474900 E-02 \quad 3.938300 E-02 \quad 3.527100 E-02-4.048100 E-02-4.026100 E-02-4.025000 E-02$ PE-18 - 2.247700E-02 -1.246200E-01 -2.250800E-02 -4.125200E-02 -4.658300E-02 -4.720700E-02 -1.225100E-01 -1.224700E-02 -1.224200E-01

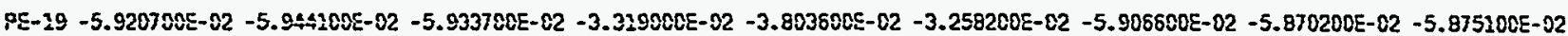
$P E-20-6.081600 E-02-6.088800 E-02-6.096600 E-02-2.334000 E-02-2.262500 E-02 \quad-2.463400 E-02-5.987800 E-02-5.943600 E-02-5.938400 E-02$ PE-21 -2.0786C0E-02 -2.078300E-02 -2.061700E-02 1.887000E-02 2.106600E-02 2.4833C0E-02 -2.012800E-02 - $1.989300 E-02$ - $2.976500 E-02$ PE-22 -1.956500E-02 -1.948400E-02 - $2.945600 E-02 \quad 2.964300 E-02 \quad 3.128100 E-02 \quad 3.119700 E-02 \quad-1.887000 E-02 \quad-1.863100 E-02 \quad-1.824800 E-02$

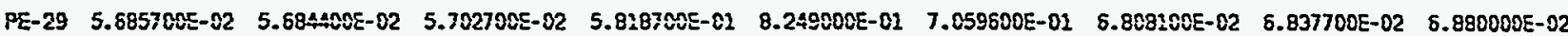
PE-30 - $1.151330 E-02-1.152470 E-02$-1.254270E-01 -8.899500E-02 - $6.310300 E-02-1.205700 E-02-1.239220 E-02-1.140410 E-02-1.143150 E-01$ FE-01 $1.000320 E+00 \quad 1.000320 E+00 \quad 1.000310 E+00 \quad 2.365200 E+00 \quad 2.360600 E+00 \quad 2.361500 E+00 \quad 1.002030 E+00 \quad 1.00159 C E+00 \quad 1.001330 E+00$ YEE-01 -2.760000E-04 -2.770000E-04 -2.770000E-04 9.500300E-05 $2.140000 E-04$ 1.140000E-04 $-2.350000 E-04-2.420000 E-04$-2.480000E-04 $X E-02-6.770000 E-04-6.770000 E-04-6.770000 E-04-6.790000 E-04-6.800000 E-04-6.830000 E-04-6.780000 E-04-6.760000 E-04-8.780000 E-04$ XEE-03 - $4.610000 E-04-4.600000 E-04-4.590000 E-04-5.070000 E-04-5.330000 E-04-5.900000 E-04-5.190000 E-04-5.190000 E-04-5.290000 E-04$

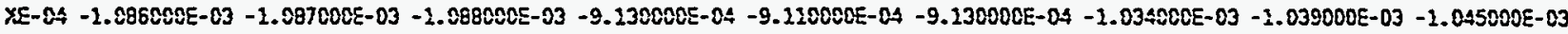
XEE-05 7.448900E-01 7.392600E-01 $7.279000 E-02 \quad 5.767300 E-01 \quad 3.638600 E-01 \quad 6.936200 E-01 \quad 8.514300 E-01 \quad 7.115800 E-01 \quad 9.004200 E-01$

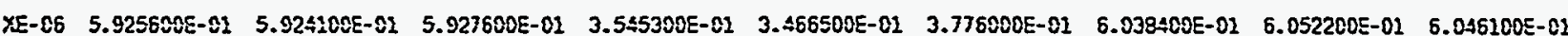
XEE-07 -5.710000E-04 -5.720000E-04 -5.740000E-04 -4,260000E-04 -4.050000E-04 -4.060000E-04 -4.930000E-04 -5.080000E-04 -5.030000E-04 XE-08 -7.710000E-04 -7.720000E-04 -7.720000E-04 -6.210000E-04 -6.220000E-04 -6.200000E-04 -7.350000E-04 -7.370000E-04 -7.140000E-04 XEE-09-9.260000E-04 -9.250000E-04 -9.270000E-04 -7.740000E-04 -7.750000E-04 -7.680000E-04 -8.920000E-04 -8.930000E-04 -8.990000E-04 $X E-10-4.482000 E-03-4.474000 E-03-4.436000 E-03-3.299000 E-03-2.825000 E-03-3.5760005-03-4.784000 E-03-4.386000 E-03-4.958000 E-03$ XE-12 2.300000E-05 2.400000E-05 $2.500000 E-05 \quad 2.400000 E-05 \quad 2.400000 E-05 \quad 2.400000 E-05 \quad 2.400000 E-05 \quad 2.500000 E-05 \quad 2.400000 E-05$ $x E-12-1.467000 E-03-1.467000 E-03-1.4650005-03-1.457000 E-03-1.452000 E-03-1.456000 E-03-1.471000 E-03-1.4710005-03-1.471000 E-03$ XE-13 -6.690000E-04 -6.690000E-04 -6.670000E-04 -2.640000E-04 -2.750000E-04 -2.640000E-04 -6.190000E-04 -6.250000E-04 -6.280000E-04 XE-14 -3.37C000E-04 -3.150000E-04 -3.140000E-04 -1.880000E-04 -1.850000E-04 -1.710000E-04 -3.060000E-0.4-3.000000E-04 -3.110000E-04 XE-15 -7.310000E-04 -7.330000E-04 -7.310000E-04 - $8.560000 E-04-6.530000 E-04$-6.530000E-04 -7.220000E-04 -7.240000E-04 -7.250000E-04 TE- $02-1.350000 E-04-1.350000 E-04-1.340000 E-04-9.900000 E-05-9.600000 E-05-9.700000 E-05-1.320000 E-04-1.380000 E-04-1.490000 E-04$ $\begin{array}{lllllllllll}V E X T 1 & 1.993500 E+00 & 2.993500 E+00 & 1.993500 E+00 & 1.993700 E+00 & 1.993700 E+00 & 1.993600 E+00 & 1.993400 E+00 & 1.993500 E+00 & 1.993400 E+00\end{array}$

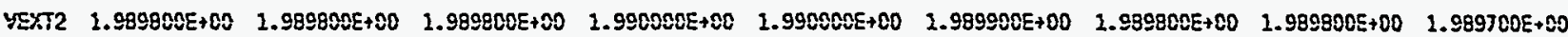
TIME 2.08563AE+12 $2.086534 E+11 \quad 2.085634 E+12 \quad 2.086634 E+11 \quad 2.086534 E+11 \quad 2.086634 E+12 \quad 2.086634 E+12 \quad 2.096634 E+11 \quad 2.086634 E+12$ 
Page Cescription:

DATA SET NO.-224

TRAYS RY_ZERO-1 RY_ZERO-2 RV_ZERO-3 RV_LOAD-1 RY_LOAQ-2 RY_LOAD-3 RV_ZERO-4 RY_ZERO-5 RV_ZERO-6

PE- 01 - 5.936400E- $\$ 2 \quad-5.9332005-02-5.934000 E-02 \quad 1.920800 E-01 \quad 1.971300 E-01 \quad 2.10310 C E-01 \quad-7.209800 E-02 \quad-7.210900 E-02-7.218100 E-02$ $\begin{array}{llllllllll}P E-02 & 4.265900 E-02 & 4.277500 E-02 & 4.287800 E-02 & 3.608800 E-02 & 4.028000 E-01 & 3.886600 E-01 & 3.137800 E-02 & 3.130200 E-02 & 3.105900 E-02\end{array}$

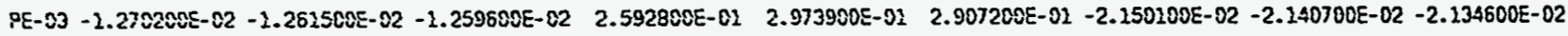
PE-0.4 5. $447600 E-02 \quad 5.458500 E-02 \quad 5.460800 E-02 \quad 3.728400 E-01 \quad 3.825700 E-01 \quad 4.005200 E-02 \quad 4.066600 E-02 \quad 4.083500 E-02 \quad 4.055000 E-02$

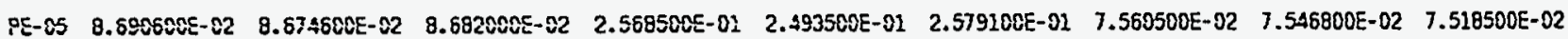
PE- DS T.843300E- $02 \quad 7.793100 E-02 \quad 7.831200 E-02 \quad 3.874600 E-02 \quad 2.984900 E-02 \quad 3.1395 C 0 E-02 \quad 6.584900 E-02 \quad 6.608900 E-02 \quad 6.603000 E-02$ PE- $07-3.264900 E-01-3.1649005-01-3.164900 E-02-1.293200 E-01-1.361700 E-01-1.250000 E-01-3.168200 E-01-3.168700 E-01-3.169200 E-01$ $\begin{array}{llllllllll}P E-08 & 2.150700 E-02 & 2.189400 E-02 & 2.161700 E-02 & 2.548200 E-01 & 2.565100 E-01 & 2.558800 E-01 & 9.075000 E-03 & 8.956000 E-03 & 8.945000 E-03\end{array}$

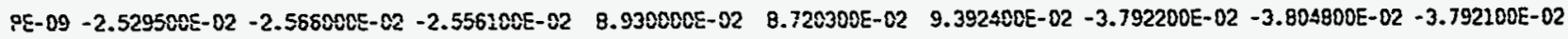
$\begin{array}{llllllllll}P E-10 & 2.960200 E-02 & 2.933500 E-02 & 2.962300 E-02 & 2.018800 E-01 & 1.960980 E-02 & 2.080200 E-01 & 2.032900 E-02 & 2.024900 E-02 & 2.023700 E-02\end{array}$ $\begin{array}{llllllllll}P E-13 & 9.270000 E-03 & 9.271000 E-03 & 9.198000 E-03 & 2.281400 E-02 & 1.259000 E-01 & 1.278700 E-01 & 1.085000 E-03 & 8.650000 E-04 & 7.140000 E-04\end{array}$ PE-14 $1.409400 E-02 \quad 1.409400 E-01 \quad 1.408000 E-02 \quad 2.975900 E-01 \quad 3.059600 E-01 \quad 3.079500 E-01 \quad 1.284500 E-01 \quad 1.284600 E-01 \quad 1.285300 E-01$ PE- 25 - $5.297000 E-03 \quad-5.263000 E-03-5.102000 E-03 \quad 4.755700 E-02 \quad 4.242000 E-02 \quad 3.935400 E-82-1.629200 E-02 \quad-1.625900 E-02$ - $1.633000 E-02$ PE-15 4.554000E-82 4.565200E-02 4.583500E-02 $9.717400 E-02$ 9.675600E-02 $9.528100 E-02 \quad 3.475300 E-02 \quad 3.490500 E-02 \quad 3.470300 E-02$ $\begin{array}{llllllllll}\text { PE- } 17 & -2.802600 E-02 & -2.789900 E-02 & -2.790600 E-02 & 2.972400 E-02 & 1.682100 E-02 & 1.717500 E-02 & -3.669800 E-02 & -3.618000 E-02 & -3.610900 E-02\end{array}$

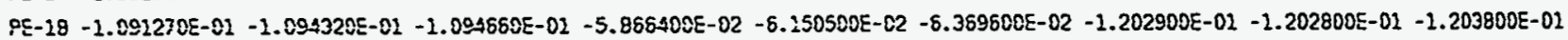
PE-19-4.016300E- $\$ 2$ - $4.0012805-02-3.990500 E-02-3.609900 E-02-3.870900 E-02-3.835100 E-02-5.442200 E-02-5.418900 E-02-5.413700 E-02$ PE-20 - $4.311400 E-02-4.323000 E-02-4.317800 E-02-3.264100 E-02-2.396100 E-02-2.862300 E-02-5.535700 E-02 \quad-5.535200 E-02 \quad-5.523300 E-02$

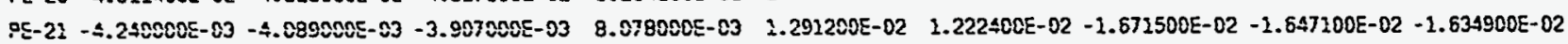
$\begin{array}{llllllllll}P E-22 & 2.356000 E-03 & 2.484000 E-03 & 2.345000 E-03 & 2.023300 E-02 & 2.378500 E-02 & 2.003900 E-02 & -1.057600 E-02 & -1.050700 E-02 & -1.041800 E-02\end{array}$ PE-29 7.581200E-02 7.526100E-02 7.548700E-02 5.219000E-02 $5.356700 E-01 \quad 4.726400 E-01 \quad 5.998200 E-02 \quad 5.997500 E-02 \quad 5.977500 E-02$

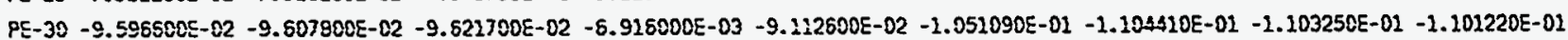

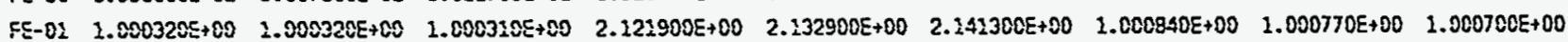
$X E-01-2.860000 E-04 \quad-2.890000 E-04 \quad-2.870000 E-04 \quad 3.900000 E-05 \quad 4.000000 E-05 \quad 3.400000 E-05 \quad-2.710000 E-04 \quad-2.710000 E-04 \quad-2.720000 E-04$ $X E-02-6.820000 E-04-6.820000 E-04-6.819000 E-04-6.870000 E-04-6.880000 E-04-6.870000 E-04-6.830000 E-04-6.830000 E-04-6.820000 E-04$ $x E-03-5.290000 E-04-5.280000 E-04-8.290000 E-04-5.940000 E-04-8.250000 E-04-8.990000 E-04-9.970000 E-04-1.012000 E-03-1.036000 E-03$ $x E-04-1.121000 E-03-2.120000 E-03-1.120000 E-03-9.770000 E-04-9.780000 E-04-9.820000 E-04-1.116000 E-03-1.116000 E-03-1.114000 E-03$ $X Y=-05 \quad 9.403200 E-02 \quad 9.403400 E-01 \quad 9.402800 E-02 \quad 8.124 .000 E-02 \quad 6.482100 E-01 \quad 6.348300 E-01 \quad 7.370500 E-01 \quad 8.115500 E-01 \quad 8.425500 E-01$ $\begin{array}{llllllllll}X E-06 & 7.798708 E-01 & 7.796800 E-02 & 7.801000 E-02 & 6.614200 E-01 & 6.613500 E-01 & 6.621200 E-01 & 7.347300 E-01 & 7.344900 E-01 & 7.343600 E-01\end{array}$ XE-07 -5.990000E-04 -6.000000E-04 -6.000000E-04 - $4.610000 E-04-4.550000 E-04-4.610000 E-54-5.840000 E-04-5.810000 E-04-5.820000 E-04$ $X E-88-7.9880005-04-7.9700005-04-7.960000 E-0 \leq-8.700000 E-04-6.650000 E-04-6.710000 E-04-7.880000 E-04-7.890000 E-04-7.8800005-04$ $x E-09-9.520000 E-04-9.510000 E-04-9.510000 E-04-8.300000 E-04-8.310000 E-04-8.260000 E-044-9.420000 E-04-9.420000 E-04-9.420000 E-04$ XE- $10-5.5570005-03-5.55 \$ 0005-03-5.555000 E-03-4.1400005-03-4.2720005-03-3.780000 E-03-4.872000 E-03-5.087000 E-03-5.169000 E-03$

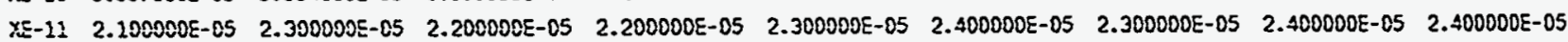
$X E-12-1.4700005-03-2.4690005-03-1.469000 E-03-2.459000 E-03-1.457000 E-03-1.462000 E-03-1.473000 E-03-1.473000 E-03-1.472000 E-03$ $X E-13-6.5200005-04-6.520000 E-04-6.490000 E-04-3.120000 E-04-3.280000 E-04-3.220000 E-04-6.570000 E-04-6.580000 E-04-6.550000 E-04$ XE- 14 -3.3900COE- $24-3.380000 E-04-3.390000 E-04-2.230000 E-04-2.300000 E-04$-2.04C000E-04 -3.160000E-04 -3.160000E-04 -3.160000E-04 XE-15 -7.280000E-04 -7.240000E-04 -7.240000E-04 -6.600000E-04 -6.570000E-04 -6.580000E-04 -7.280000E-04 -7.260000E-04 -7.250000E-04

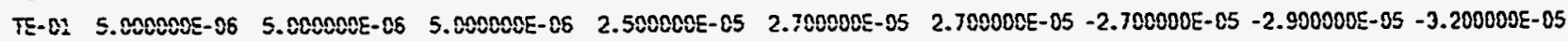
$\begin{array}{llllllllll}\text { VEXTI } & 1.993300 E+00 & 1.993300 E+00 & 1.993300 E+00 & 1.993500 E+00 & 1.993500 E+00 & 1.993500 E+00 & 1.993400 E+00 & 1.993400 E+00 & 1.993400 E+00\end{array}$ $\begin{array}{llllllllll}\text { VEXT2 } & 1.989600 E+00 & 1.989608 E+00 & 2.989600 E+00 & 2.989800 E+00 & 1.989800 E+00 & 1.989900 E+00 & 1.989900 E+00 & 1.989700 E+00 & 1.989700 E+00\end{array}$

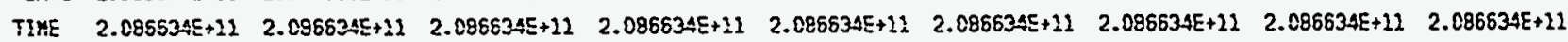


Page Description:

DATA SET Y0.-225

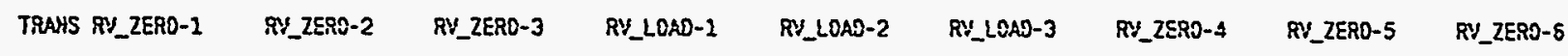

PE-01 -7.427600E-02 -7.432100E-02 -7.414400E-02 2.933900E-81 2.900500E-01 2.809700E-01 $-7.253300 E-02 \quad-7.257300 E-02 \quad-7.274400 E-02$ PE-02 2.880700E-02 2.874300E-02 2.8515C0E-02 $\quad 4.903300 E-01 \quad 4.994400 E-01 \quad 4.731100 E-02 \quad 3.027600 E-02 \quad 3.039000 E-02 \quad 3.054600 E-02$

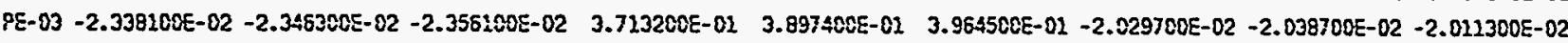
PE-04 3.733700E-02 3.707500E-02 $3.733000 E-02 \quad 4.962800 E-01 \quad 4.995908 E-01 \quad 5.051500 E-01 \quad 3.891200 E-02 \quad 3.931300 E-02 \quad 3.943200 E-02$ PE-05 7.307800E- $02 \quad 7.295700 E-02 \quad 7.299500 E-02 \quad 3.182500 E-01 \quad 3.171400 E-01 \quad 3.082700 E-01 \quad 7.332700 E-02 \quad 7.314980 E-02 \quad 7.299900 E-02$ PE-06 $5.242100 E-02 \quad 6.249800 E-02 \quad 6.261800 E-02 \quad 4.164900 E-01 \quad 4.249100 E-01 \quad 4.106400 E-01 \quad 6.680500 E-02 \quad 6.699800 E-02 \quad 6.6635 C 0 E-02$ PE-07 - 3.1807C0E- $01-3.180900 E-01$-3.181008E-01 -4.584000E-02 -5.938300E-02 -5.188800E-02 -3.285100E-01 -3.185500E-01 -3.186000E-01 PE-08 $\quad 5.891000 E-03 \quad 5.983000 E-03 \quad 6.042000 E-03 \quad 3.516200 E-02 \quad 3.421400 E-02 \quad 3.423100 E-02 \quad 7.421000 E-03 \quad 7.537000 E-03 \quad 7.445000 E-03$ PE-09 -4.049100E-02 -4.060708E-02 -4.585000E-02 $2.320700 E-02 \quad 1.353200 E-02 \quad 1.287800 E-01 \quad-3.7313005-02$-3.731000E-02 $-3.702100 E-02$ $\begin{array}{lllllllllll}P E-10 & 1.769300 E-02 & 1.753900 E-02 & 3.745000 E-02 & 2.874100 E-01 & 2.789000 E-02 & 2.696000 E-01 & 1.818000 E-02 & 1.799900 E-02 & 1.772700 E-02\end{array}$

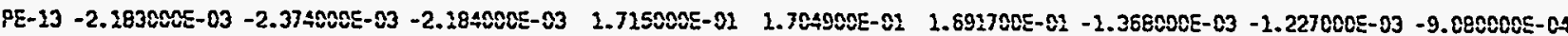
PE-14 1.26210CE-02 2.251100E-01 $2.262300 E-02 \quad 3.581100 E-01 \quad 3.570600 E-01 \quad 3.583600 E-01 \quad 1.289300 E-01 \quad 1.288000 E-01 \quad 1.285500 E-01$

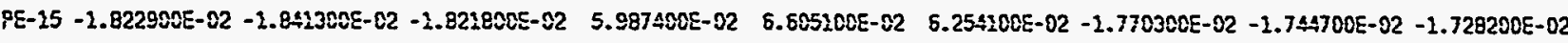
$P E-16 \quad 3.271500 E-02 \quad 3.239700 E-02 \quad 3.272600 E-02 \quad 1.272440 E-01 \quad 1.244100 E-01 \quad 3.194960 E-01 \quad 3.388500 E-02 \quad 3.377100 E-02 \quad 3.407900 E-02$

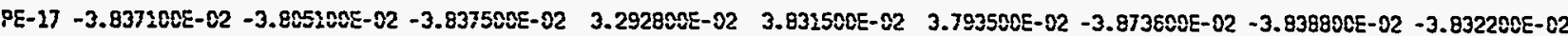

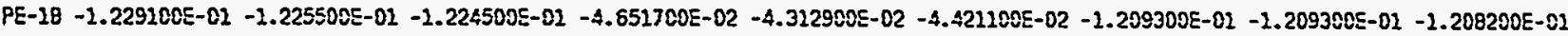
PE-19 -5.546900E-02 -5.572200E-02 -5.571800E-02 - 3.267700E-02 -3.204000E-02 - 3.2475C0E-02 -5.587200E-02 -5.585500E-02 -5.565200E-02 PE-20 -5.642400E-02 -5.683700E-02 -5.660400E-02 -2.042500E-02 -1.962600E-02 -2.044400E-02 -5.639200E-02 $-5.629800 E-02$-5.509900E-02

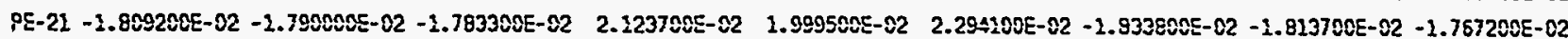
$P E-22$-1.293000E-02 $-1.274600 E-82$-1.278600E-02 $3.065000 E-02 \quad 3.036500 E-02 \quad 3.287600 E-02-1.3616005-02 \quad-1.329800 E-02 \quad-1.342400 E-02$ PE-29 5.887600E-02 5.890800E-02 $5.924208 E-02 \quad 6.899300 E-01 \quad 8.225100 E-01 \quad 8.057100 E-01 \quad 5.559500 E-02 \quad 5.533200 E-02 \quad 5.517400 E-02$

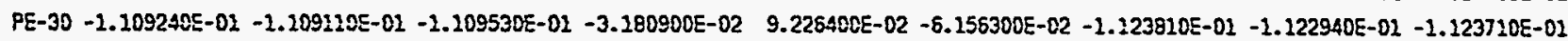

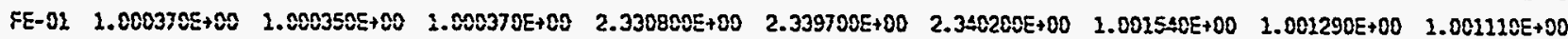
XEE-01 -2.720000E-04 -2.720000E-04 -2.720000E-04 7.300000E-05 7.100000E-05 7.400000E-C5 $-2.570000 E-04 \quad-2.510000 E-04$-2.640000E-004 XE-02 -6.830000E-04 -6.830000E- $04-6.820008 E-04-6.900000 E-04-6.870000 E-04-6.890000 E-04-6.840000 E-04-6.840000 E-84-6.850800 E-04$ $X E-03-2.034000 E-03-1.034000 E-03-1.032000 E-03-7.500000 E-04-2.035000 E-03-8.240000 E-04-8.320000 E-04-8.740000 E-04-8.750000 E-04$ YE-04 -1.217000E- $03-1.11 E 000 E-03-1.1150005-03-9.510000 E-04-9.580000 E-04-9.610000 E-84-3.096000 E-03-1.0970005-03-1.0980005-03$ YE-05 8.190300E-02 $8.193300 E-02 \quad 8.184500 E-01 \quad-2.953000 E-03 \quad-1.947000 E-03$-1.955000E-03 $-2.024000 E-03 \quad-2.021000 E-03 \quad-2.021000 E-03$ $\begin{array}{llllllllll}X E-06 & 7.353500 E-01 & 7.353200 E-01 & 7.352500 E-02 & 5.499800 E-01 & 5.528600 E-01 & 5.402300 E-01 & 7.237600 E-01 & 7.241300 E-01 & 7.245800 E-02\end{array}$ XE-07 -5.870000E-04 -5.870000E-0. $-5.870000 E-04$ 4.900000E-05 2.000000E-05 $\quad 4.800000 E-05-5.590000 E-04 \quad-5.590000 E-04 \quad-5.630000 E-04$ XE- $88-7.8600005-04-7.850000 E-04-7.8600005-04-5.4400005-04-6.510000 E-04-6.470000 E-04-7.7100005-04-7.710000 E-04-7.730000 E-04$ XE-09 -9.410000E-04 -9.410000E-04 - $9.4100005-04-7.970000 E-84-7.990000 E-04-8.0000005-04-9.220000 E-04-9.2100005-04-9.240000 E-04$ $X E-10-5.080000 E-03-5.082000 E-03-5.076000 E-03 \quad-2.305000 E-03-2.3340005-03-2.163000 E-03-2.717000 E-03-2.7210005-03-2.7220005-03$ YE-12 2.200000E-05 $\quad 2.400000 E-05 \quad 2.400000 E-05 \quad 2.3000000 E-05 \quad 2.500000 E-05 \quad 2.400000 E-05 \quad 2.400000 E-05 \quad 2.400000 E-05 \quad 2.500000 E-05$ $X E-12-1.471000 E-03-1.470000 E-03-1.4690 C 0 E-03-2.452000 E-03-1.4530005-03-1.454000 E-03-1.474000 E-03-1.475000 E-03-1.474000 E-03$ XE-13 - 5.490000E-04 -6.470000E-04 -6.480000E-04 -2.850000E-04 -2.930000E-04 -2.790000E-04 -6.4800005-04 -6.490000E-04 -6.510000E-04 XE-14 -3.350C00E-04 -3.0900005-04 -3.070000E-04 -1.730000E-04 -1.760000E-04 -3.680000E-04 -3.030000E-04 -3.050000E-04 -3.08C0C0E-04 $X E-15-7.240000 E-04-7.210000 E-04-7.210000 E-04-6.550000 E-04-6.540000 E-04-5.520000 E-04-7.210000 E-04-7.240000 E-04-7.250000 E-04$ TE-01 - 4.500000E-05 - $4.5000005-05-4.500000 E-05$-2.600000E-05 -2.300000E-05 -2.600000E-05 - 7.200000E-05 - T.100000E-05 -8.400000E-85 VEXT2 $1.993400 E+00 \quad 1.993400 E+00 \quad 1.993400 E+00 \quad 1.993900 E+00 \quad 1.993900 E+00 \quad 1.993900 E+00 \quad 1.993900 E+00 \quad 1.993900 E+00 \quad 1.993900 E+00$ VEXT2 $2.989708 E+C O \quad 1.989700 E+00 \quad 1.989700 E+C 0 \quad 1.990200 E+00 \quad 1.990200 E+00 \quad 1.990200 E+00 \quad 1.990200 E+00 \quad 1.990200 E+00 \quad 1.990200 E+00$

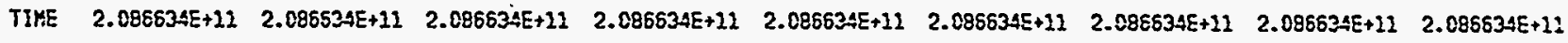


Arative izme:

ATS_MEP_TESTS

8cok Name: ANS_MFPTI_RV

Date \& Time: 7 har 1900 00:55:39

Page Description:

DATA SET HO. -225

TRAYS RY_ZERO-2 RY_ZERO-2 RY_ZERO-3 RY_LOAO-1 $\quad R Y_{-} L O A D-2 \quad R Y_{-} L O A D-3 \quad$ RV_ZERO-4 $\quad$ RV_ZERO-5 RY_ZERO-6

FE- $01-7.508100 E-02-7.515500 E-02-7.506800 E-02 \quad 3.707800 E-01 \quad 3.658100 E-01 \quad 3.424700 E-01-7.210000 E-02-7.198400 E-02-7.203200 E-02$ $\begin{array}{llllllllll}P E-02 & 2.747500 E-02 & 2.741500 E-D 2 & 2.729700 E-02 & 5.061900 E-01 & 5.848900 E-01 & 5.797300 E-01 & 3.092700 E-02 & 3.108200 E-02 & 3.115400 E-02\end{array}$

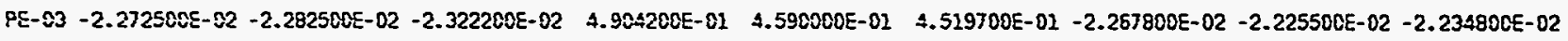

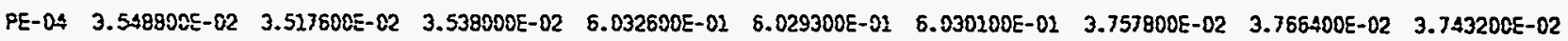

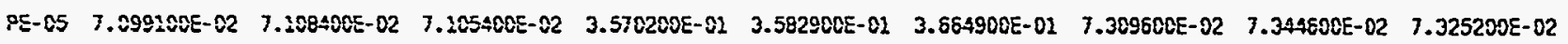
PE- $08 \quad 6.269300 E-02 \quad 6.236600 E-02 \quad 6.210500 E-02 \quad 4.829400 E-01 \quad 4.969300 E-01 \quad 4.914600 E-02 \quad 6.532900 E-02 \quad 6.569400 E-02 \quad 6.576700 E-02$

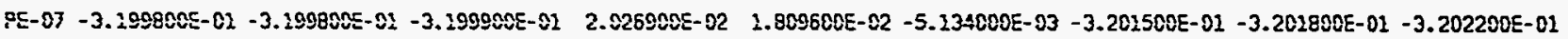
$\begin{array}{llllllllllll}P E-88 & 4.770000 E-03 & 4.856000 E-03 & 4.535000 E-03 & 4.050400 E-02 & 3.970100 E-02 & 4.102100 E-01 & 5.513000 E-03 & 5.313000 E-03 & 6.427000 E-03\end{array}$

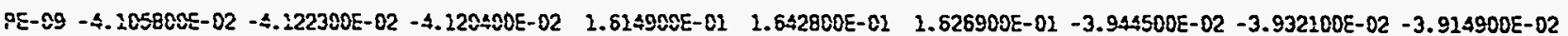
PE-10 $1.350400 E-02 \quad 1.355800 E-02 \quad 1.350400 E-02 \quad 3.171200 E-01 \quad 3.287200 E-01 \quad 3.131300 E-02 \quad 1.609200 E-02 \quad 1.600400 E-02 \quad 1.573200 E-02$

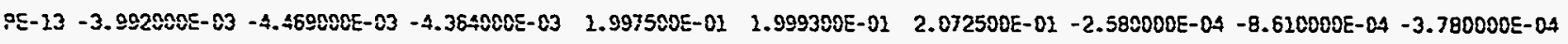
PE-14 $1.252400 E-02 \quad 3.254000 E-02 \quad 1.253600 E-02 \quad 4.037100 E-02 \quad 4.059800 E-01 \quad 4.088200 E-02 \quad 1.278000 E-01 \quad 1.279500 E-01 \quad 1.275900 E-01$

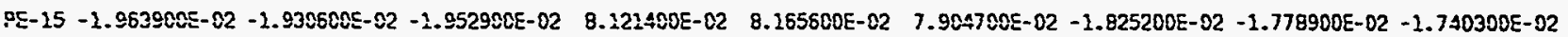
PE-15 3.235900E-02 $3.157700 E-02 \quad 3.137700 E-02 \quad 1.356900 E-01 \quad 1.3790005-02 \quad 1.382200 E-01 \quad 3.350500 E-02 \quad 3.344200 E-02 \quad 3.359300 E-02$

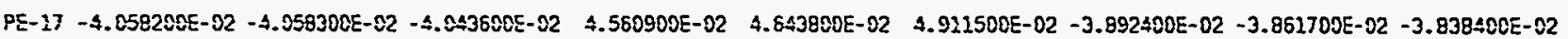
PE-19 $-1.234 \triangle 00 E-02-2.235900 E-02-1.233900 E-02 \quad-2.693200 E-02-2.501200 E-02-2.767700 E-02-1.212500 E-02-1.212600 E-01-1.212900 E-01$ PE- $19-5.725700 E-\$ 2-5.727100 E-82-5.715400 E-02-2.463500 E-02-2.725900 E-02-2.797300 E-02-5.554500 E-02-5.494500 E-02-5.485600 E-02$

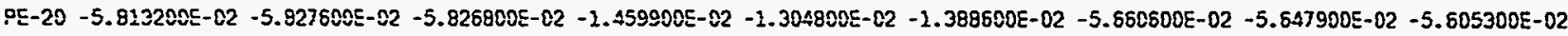
EE-22 - $1.963700 E-02-2.970500 E-82-1.041700 E-02 \quad 3.654800 E-02 \quad 3.312300 E-02 \quad 2.903400 E-02-1.746600 E-02-1.716100 E-02-1.6952005-02$ PE-22 - 1.586500E-02 -1.597800E-02 - $1.591408 E-02 \quad 4.123800 E-02 \quad 3.597700 E-02 \quad 4.027100 E-02-1.375800 E-02 \quad-1.344500 E-02 \quad-1.324300 E-02$ PE-29 5.46 $3000 E-02 \quad 5.510200 E-02 \quad 5.483900 E-02 \quad 8.639800 E-01 \quad 9.085600 E-01 \quad 8.208200 E-01 \quad 6.232000 E-02 \quad 5.993500 E-02 \quad 5.839600 E-02$

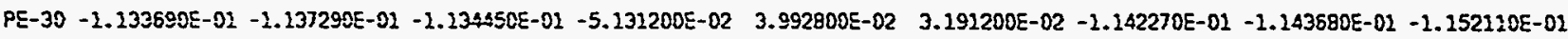
EE-02 $1.000350 E+00 \quad 1.000350 E+00 \quad 1.000350 E+00 \quad 2.494700 E+00 \quad 2.503300 E+00 \quad 2.497800 E+00 \quad 1.001870 E+00 \quad 1.001510 E+00 \quad 1.001250 E+00$ $X E-02 \quad-2.720000 E-04 \quad-2.730000 E-04 \quad-2.740000 E-04$ 3.000000E-04 $8.800000 E-05$ 9.100000E-05 $-2.430000 E-04 \quad-2.490000 E-04 \quad-2.520000 E-04$ $x E-02-6.830000 E-04-6.820000 E-04-6.820000 E-04-6.900000 E-04-6.890000 E-04-6.900000 E-04-6.850000 E-04-6.860000 E-04-6.850000 E-04$ XEE-03 - $9.310000 E-04-8.310000 E-04-8.300000 E-04-7.650000 E-04-5.780000 E-04-4.870000 E-04-4.200000 E-04-4.200000 E-04-4.180000 E-04$ XE-04 -2.212008E- $83-1.221000 E-03-1.211000 E-03-9.390000 E-04-9.380000 E-04-9.350000 E-04-1.074000 E-03-2.075000 E-03-2.077000 E-03$ XE-05 -2.038000E- $03-2.038000 E-03-2.037000 E-03-1.909000 E-03-1.900000 E-03-1.907000 E-03-2.895000 E-03-1.731000 E-03-1.703000 E-03$ XC-06 7.290008E-01 7.289400E-01 7.290300E-01 5.582300E-01 $6.647800 E-01 \quad 6.661400 E-01 \quad 7.776100 E-01 \quad 7.774000 E-01 \quad 7.772700 E-01$

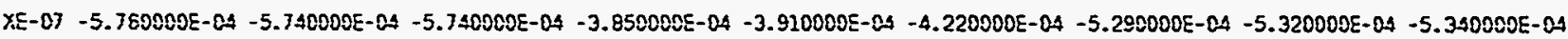
XE- $28-7.8100005-84-7.8200005-04-7.820000 E-24-5.380000 E-04-6.360000 E-04-5.370000 E-04-7.470000 E-04-7.470000 E-04-7.5000005-04$ XE-09 - $-9.3300005-04-9.330000 E-04-9.3300005-04-7.790000 E-04-7.7500005-04-7.710000 E-04-9.070000 E-04-9.060000 E-04-9.090000 E-04$ XEL-10 $-2.7550005-03-2.755000 E-03-2.754000 E-03-2.300000 E-03-2.318000 E-03-2.310000 E-03-2.837000 E-03-2.842000 E-03-2.8500005-03$ XE-11 2.200000E-05 2.400000E-05 2.400000E-05 2.300000E-05 2.400000E-05 2.500000E-05 2.400000E-05 2.400000E-05 2.500000E-05 XE- $12-1.471000 E-03-1.471000 E-03-1.4700005-03-1.450000-03-1.446000 E-03-1.446000 E-03-2.472000 E-03-2.473000 E-03-1.4730005-03$

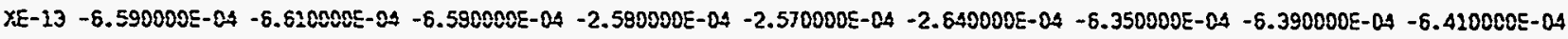
$X E-14-3.070000 E-04-3.030000 E-04-2.960000 E-04-1.330000 E-04-1.300000 E-04-1.320000 E-04-2.960000 E-04-3.040000 E-04-3.060000 E-04$ XE-15 -7.230000E-04 -7.240000E-04 -7.210000E-04 -6.470000E-04 -6.510000E-04 - 5.460000E-04 -7.240000E-04 -7.170900E-04 -7.250000E-04 TE- 01 -9.000000E- $05-9.900002 E-05-8.900000 E-05-5.300000 E-05-6.300000 E-05-5.900000 E-05-1.000000 E-04-9.600000 E-05-1.080000 E-04$ VEXTI $1.993900 E+00 \quad 2.993900 E+00 \quad 1.993900 E+00 \quad 2.993900 E+00 \quad 1.993900 E+00 \quad 1.993900 E+00 \quad 1.993800 E+00 \quad 1.993800 E+00 \quad 1.993800 E+00$

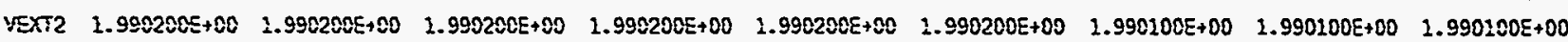

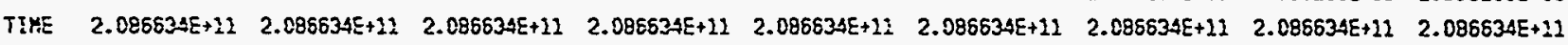


Aishive hane:

AMS_KFP_TESTS

Sook Hane: AYS_MFPTI_RY

Date \& Time: 7 Mar 1900 01:06:13

Page Description:

DATA SET N0.-227

PE-01 -7.375800E-02 -7.481900E-82 -7.389100E-02 4.388600E-01 4.427300E-01 4.316300E-01 -7.320200E-02 -7.301800E-02 -7.287300E-02 PE-02 2.906700E-02 2.889100E-02 2.892400E-02 $6.955900 E-01 \quad 6.983800 E-01 \quad 6.688200 E-02 \quad 3.227800 E-02 \quad 3.252200 E-02 \quad 3.222800 E-02$ PE-03 -2.467400E-02 -2.480500E-02 -2.481000E-02 5.541600E-01 $5.566988 E-01 \quad 5.374800 E-02$ - $1.577300 E-02$ - $1.549900 E-02$ - $1.548000 E-02$ PE-0.4 3.395700E-02 3.4193COE-02 $3.424800 E-02 \quad 6.821600 E-01 \quad 6.766200 E-01 \quad 6.891200 E-01 \quad 3.656700 E-02 \quad 3.684900 E-02 \quad 3.680400 E-02$ PE-05 7.068700E-02 7.086900E-02 7.073700E-02 4.218700E-02 $4.137700 E-01 \quad 4.143900 E-01 \quad 7.168500 E-02 \quad 7.137200 E-02 \quad 7.141800 E-02$ PE-06 $\quad 5.225100 E-02 \quad 6.223700 E-02 \quad 5.192900 E-02 \quad 5.949100 E-01 \quad 5.738900 E-01 \quad 5.706600 E-02 \quad 6.455200 E-02 \quad 6.460200 E-02 \quad 6.481200 E-02$ PE-07 -3.212500E-01 -3.212600E-02 $-3.212700 E-01 \quad 7.201000 E-02 \quad 7.085200 E-02 \quad 8.009300 E-02 \quad-3.214600 E-01 \quad-3.214900 E-01$ - $3.215300 E-01$ PE-08 3.721000E-03 3.8510C0E-C3 $3.542000 E-03 \quad 4.855900 E-01 \quad 4.670000 E-01 \quad 4.715200 E-01 \quad 7.770000 E-03 \quad 7.767000 E-03 \quad 7.958000 E-03$ PE-09 -4.228500E-02 -4.209400E-02 -4.203900E-02 1.846200E-01 1.912500E-01 1.928900E-01 -4.144000E-02 -4.138400E-02 -4.102600E-02 $P E-10 \quad 1.210800 E-02 \quad 1.224600 E-02 \quad 1.223400 E-02 \quad 3.571800 E-01 \quad 3.761400 E-02 \quad 3.821800 E-01 \quad 1.337400 E-02 \quad 2.309900 E-02 \quad 1.267800 E-02$

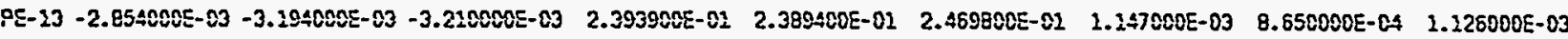
PE-14 1.251800E-01 1.248400E-01 1.249900E-01 4.578400E-01 $4.439500 E-01 \quad 4.612000 E-01 \quad 2.264600 E-01 \quad 1.261400 E-02 \quad 1.263600 E-01$

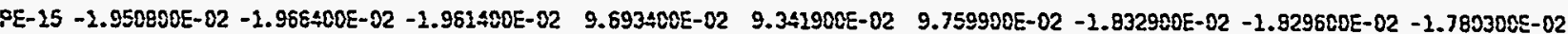
$P E-16 \quad 3.163400 E-02 \quad 3.119400 E-02 \quad 3.129800 E-02 \quad 1.546200 E-01 \quad 1.503100 E-01 \quad 1.555300 E-01 \quad 3.230900 E-02 \quad 3.257800 E-02 \quad 3.227900 E-02$ FE-17 -4.008500E-02 -4.027700E-02 -4.021000E-02 5.329000E-02 $6.1700095-02 \quad 6.156100 E-02 \quad-3.928100 E-02 \quad-3.989000 E-02 \quad-3.869900 E-02$ PE-19 -1.232400E-01 -1.239400E-01 -1.232700E-01 -1.884500E-02 - $1.576400 E-02-1.992400 E-02-1.222600 E-01$-1.225100E-01 -2.224900E-01 PE- $19-5.6565 C 0 E-02-5.6465 C 0 E-02-5.679000 E-02$-2.83780QE-02 -2.344100E-02 -2.800800E-02 -5.672400E-02 -5.597900E-02 -5.599600E-02 PE-20 -5.761900E-02 -5.763500E-02 -5.772800E-02 -7.509000E-03 $-5.123000 E-03-1.321300 E-02-5.677200 E-02-5.643900 E-02-5.628200 E-02$ PE-21 - $1.817700 E-02-1.824300 E-02-1.857600 E-02$ 4.159500E-\$2 4.302600E-02 3.632500E-02 -1.714600E-02 -1.698200E-02 - $1.654000 E-02$ PE-22 - $1.534200 E-02-1.550900 E-02$-1.544400E-02 4.767800E-02 4.963600E-02 4.083100E-02 $-1.333000 E-02$ - $1.316700 E-02$ - $1.294500 E-02$ PE-29 5.36090CE-02 5.372ACCE-02 $5.376900 E-02 \quad 9.933000 E-02 \quad 1.178000 E+00 \quad 2.036540 E+00 \quad 5.844400 E-02 \quad 6.86 \div 400 E-02 \quad 6.929500 E-02$ PE-30 - 1.150480E-02 -1.260370E-02 -1.259630E-02 -2.858000E-02 -2.355700E-02 -2.247300E-02 -1.135940E-02 -1.135440E-02 - $2.135490 E-01$ $\begin{array}{lllllllllll}\text { SE-01 } & 1.00037 E E+00 & 1.000350 E+00 & 1.030350 E+00 & 2.628200 E+00 & 2.635000 E+00 & 2.627500 E+00 & 1.002410 E+00 & 1.001880 E+00 & 2.001540 E+00\end{array}$ XE-02 -2.790000E-04 -2.790000E-04 -2.790000E-04 1.090000E-04 1.106000E-04 2.100000E-04 -2.350000E-04 -2.410000E-04 -2.470000E-04 XE- $02-6.820000 E-04-8.820000 E-04-6.810000 E-04-6.870000 E-04-6.890000 E-04-6.850000 E-04-6.850000 E-04-6.830000 E-04-6.840000 E-04$ XE-03 -4.2400COE-C4 - $4.230000 E-04-4.240000 E-04$-2.920000E-04 $-3.170000 E-04-3.340000 E-04-3.400000 E-04-3.410000 E-04-3.400000 E-04$ XE-04 - - $2.208000 E-03-1.109000 E-03-1.109000 E-03-9.150000 E-04-9.150000 E-04-9.136000 E-04-1.062000 E-03-1.056000 E-03-1.070000 E-03$ XE-05 7.275200E-01 7.615100E-01 $\quad 6.709400 E-01 \quad-1.965000 E-03 \quad-2.001000 E-03$-2.983000E-03 $2.752700 E-02 \quad 1.815000 E-02 \quad-1.107000 E-03$

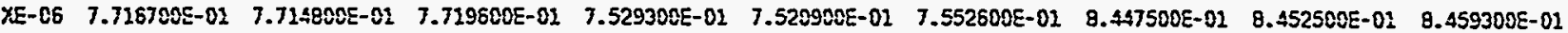
XE-07 -5.770000E-04 -5.770000E-04 -5.750000E-04 -2.070000E-04 -2.840000E-04 -1.230000E-04 -4.850000E-04 -4.890000E-04 -4.890000E-04 XE-08 -7.780000E-04 -7.770000E-04 -7.7800005-04 -6.310000E-04 -6.330000E-04 -6.320000E-04 -7.290000E-04 -7.340080E-04 -7.380000E-04 YE-09 -9.380000E-04 -9.350009E-04 -9.350000E-04 -7.490000E-04 -7.510000E-04 -7.460000E-04 -8.940000E-04 -8.960000E-04 -8.980000E-04 $X E-10-4.960000 E-03-5.030000 E-03-4.797000 E-03-2.525000 E-03-2.493000 E-03-2.535000 E-03-3.018000 E-03-3.129000 E-03-3.031000 E-03$ $\begin{array}{llllllllll}X E-11 & 2.400000 E-05 & 2.400000 E-05 & 2.400000 E-05 & 2.300000 E-05 & 2.500000 E-05 & 2.400000 E-05 & 2.400000 E-05 & 2.400000 E-05 & 2.400000 E-05\end{array}$ $X E-12-1.472000 E-03-1.471000 E-03-2.471000 E-03-1.444000 E-03-2.442000 E-03-1.445000 E-03-1.470000 E-03-1.470000 E-03-1.471000 E-03$ XE-13 -6.610000E-04 -6.620000E-04 -6.600000E-04 -2.390000E-04 -2.500000E-04 -2.360000E-04 -6.210000E-04 -6.230000E-04 - $6.260000 E-04$ XE-14 -3.060000E-04 -3.080000E-04 -3.100000E-04 -1.200000E-04 -1.260000E-04 -2.150000E-04 -2.920000E-04 -2.990000E-04 -2.980000E-04 XE-15 -7.230000E-04 -7.210000E-04 -7.220000E-04 -6.330000E-04 -6.310000E-04 -6.290000E-04 -7.130000E-04 -7.150000E-04 -7.180000E-04 TE-01 - $1.130000 E-04-2.120000 E-04-1.210000 E-04-8.100000 E-05-7.800000 E-05-7.600000 E-05-2.220000 E-04-1.100000 E-04-1.300000 E-04$ VEXT1 $1.993400 E+00 \quad 2.993400 E+00 \quad 2.993500 E+00 \quad 2.993800 E+00 \quad 2.993800 E+00 \quad 1.993900 E+00 \quad 1.993900 E+00 \quad 1.993900 E+00 \quad 1.993300 E+00$ YEXT2 $\quad 1.989700 E+00 \quad 1.989700 E+00 \quad 1.989800 E+C 0 \quad 2.990280 E+00 \quad 1.990200 E+00 \quad 1.990100 E+00 \quad 2.990100 E+00 \quad 1.990100 E+00 \quad 1.999700 E+00$

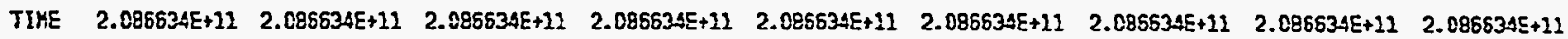


Arotire Hente:

AHS_MEP_TESTS

800k Name: A4S_YFPTI_RY

Date \& Time: 7 Mar 1900 01:16:07

Page Description:

DATA SET HO. -228

TRAYS RV_ZEFO-1 RV_ZERO-2 RY_ZERO-3 RV_LOAD-1 R R_LOAD-2 RV_LOAD-3 RY_ZERO-4 RY_ZERO-5 RY_ZERO-6

PE- 01 -7.561100E-02 - 7.529600E-02 -7.575600E-02 5.225800E-01 5.321700E-01 5.401700E-01 -5.989000E-02 -6.958600E-02 -6.955900E-02 $\begin{array}{llllllllll}P E-02 & 2.873900 E-02 & 2.855800 E-02 & 2.838100 E-02 & 7.887400 E-01 & 8.129000 E-01 & 8.273900 E-01 & 3.324200 E-02 & 3.336700 E-02 & 3.309900 E-02\end{array}$ PE- $03-1.774600 E-02-1.797500 E-02 \quad-1.777000 E-02 \quad 6.629200 E-01 \quad 6.704400 E-01 \quad 6.792300 E-01-1.676700 E-02 \quad-1.642000 E-02 \quad-1.669200 E-02$ PE-O4 3.458800E-02 $3.480200 E-02$ 3.467300E-02 $8.295400 E-01 \quad 8.417000 E-01 \quad 8.430500 E-01 \quad 3.525700 E-02 \quad 3.497800 E-02 \quad 3.505500 E-02$

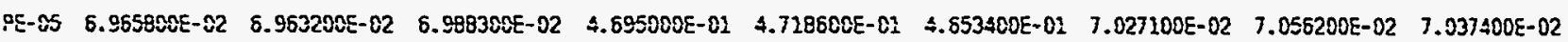
PE-08 $\quad 6.205500 E-02 \quad 6.223100 E-02 \quad 6.215400 E-02 \quad 7.031100 E-02 \quad 7.140000 E-02 \quad 7.167600 E-01 \quad 7.318200 E-02 \quad 7.312900 E-02 \quad 7.304100 E-02$

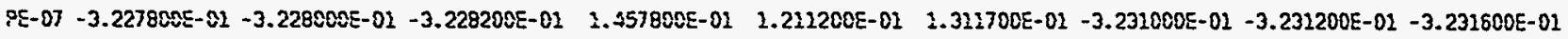
$\begin{array}{llllllllllll}\text { PE-08 } & 6.252000 E-03 & 6.108000 E-03 & 5.813000 E-03 & 5.639700 E-01 & 5.647000 E-02 & 5.618000 E-01 & 1.039700 E-02 & 1.037200 E-02 & 1.038300 E-02\end{array}$

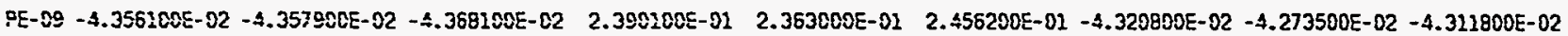
PE-10 $\quad 1.049600 E-02 \quad 3.055600 E-02 \quad 1.052500 E-02 \quad 4.457100 E-01 \quad 4.559400 E-01 \quad 4.653900 E-01 \quad 1.414900 E-02 \quad 1.408700 E-02 \quad 1.407500 E-02$

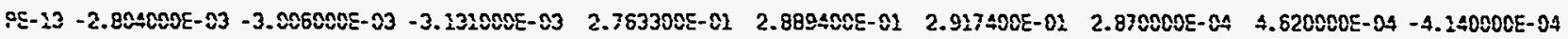
PE- $14 \quad 1.237600 E-01 \quad 1.234500 E-01 \quad 1.235100 E-02 \quad 5.070200 E-02 \quad 5.182100 E-02 \quad 5.294600 E-02 \quad 1.254600 E-02 \quad 1.264200 E-02 \quad 1.251800 E-01$

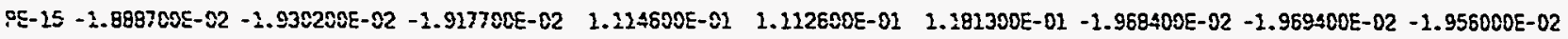
PE-16 3.037800E-02 $3.041400 E-02 \quad 3.018800 E-02 \quad 1.775500 E-01 \quad 1.828100 E-02 \quad 1.848200 E-02 \quad 3.270800 E-02 \quad 3.280900 E-02 \quad 3.220400 E-02$ PE-17 - $4.075400 E-02-4.092500 E-02-4.094800 E-02 \quad 7.901900 E-02 \quad 8.065800 E-82 \quad 8.582500 E-02-3.949200 E-02 \quad-3.929000 E-02 \quad-3.935900 E-02$ $P E-18-1.235400 E-01 \quad-1.239000 E-01 \quad-2.237200 E-01 \quad 5.233000 E-03 \quad 2.985000 E-03 \quad 8.758000 E-03 \quad-1.222600 E-01 \quad-1.220500 E-01 \quad-1.217300 E-01$

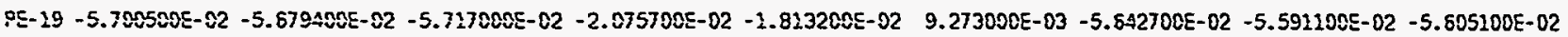
PE-20 -5.565500E-02 -5.703400E-02 -5.549500E-02 $\quad 1.740000 E-04 \quad 2.979000 E-03 \quad 2.005000 E-02 \quad-5.536500 E-02 \quad-5.507100 E-02 \quad-5.484900 E-02$

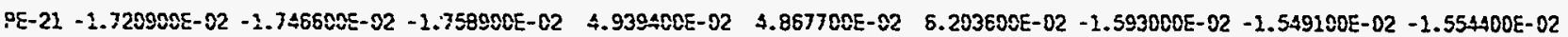
PE-22 - $1.485200 E-02-1.483900 E-02-1.481100 E-02 \quad 5.899200 E-02 \quad 5.690300 E-02 \quad 6.603000 E-02-1.480900 E-02-1.435000 E-02-1.440900 E-02$ $\begin{array}{llllllllll}P E-29 & 6.979100 E-02 & 6.986900 E-02 & 6.890900 E-02 & 1.350280 E+00 & 1.367600 E+00 & 1.200300 E+00 & 6.656200 E-02 & 5.660000 E-02 & 6.635400 E-02\end{array}$ $P E-30-1.145380 E-01-1.1454405-01 \quad-1.256820 E-02 \quad-9.940000 E-04 \quad 1.652100 E-02-3.301000 E-02-1.112400 E-01 \quad-1.114060 E-01-1.114880 E-02$ FE- $02 \quad 1.000380 E+00 \quad 2.000370 E+00 \quad 1.000370 E+00 \quad 2.390700 E+00 \quad 2.800800 E+00 \quad 2.804800 E+00-2.700000 E-05-2.780000 E-05-2.700000 E-05$ XE-01 -2.750000E-04 -2.750000E-04 -2.760000E-04 1.350000E-04 1.350000E-04 1.350000E-04 $-2.220000 E-04-2.260000 E-04-2.300000 E-04$

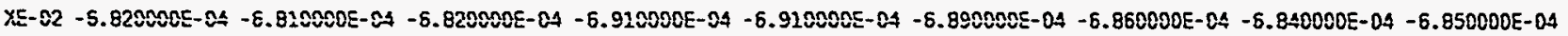
XE-03 -3.700000E-04 -3.700000E- $04-3.700000 E-04-2.440000 E-84-2.220000 E-04-3.630000 E-04-6.660000 E-04-6.660000 E-04-6.670000 E-04$ YE-DS - $1.205000 E-03-1.105000 E-03-2.206000 E-03-9.080000 E-04-9.020000 E-04-9.060000 E-04-1.085000 E-03-1.070000 E-03-1.073000 E-03$ XE-05 - $2.085000 E-03 \quad-2.085000 E-03 \quad-2.083000 E-03 \quad 4.314100 E-02 \quad 1.698900 E-02 \quad 1.458000 E-03-2.061000 E-03 \quad-2.062000 E-03-2.061000 E-03$

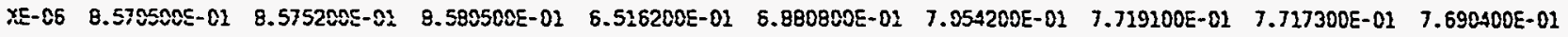
$X E-03 \quad-5.280000 E-04 \quad-5.330000 E-04 \quad-5.240000 E-04 \quad 2.40000 E-04 \quad 7.700000 E-05 \quad 9.500000 E-05-4.990000 E-04 \quad-5.060000 E-04 \quad-5.080000 E-04$ XE-08 - 7.740008E- $04-7.740000 E-04-7.740000 E-04-6.280000 E-04-6.2700005-04-6.230000 E-04-7.370000 E-04-7.390000 E-04-7.430000 E-04$ $X E-00-9.320000 E-04-9.330000 E-04-9.330000 E-04-7.120000 E-04-7.130000 E-04-7.070000 E-04-8.980000 E-04-8.940000 E-04-8.980000 E-04$

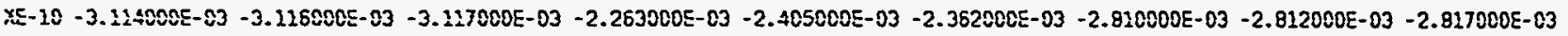
$\begin{array}{llllllllll}X E-11 & 2.400000 E-05 & 2.500000 E-05 & 2.400000 E-05 & 2.400000 E-05 & 2.500000 E-05 & 2.500000 E-05 & 2.500000 E-05 & 2.500000 E-05 & 2.600000 E-05\end{array}$ $x E-12-1.471008 E-03-1.472000 E-03-1.472000 E-03-1.434000 E-03-1.437000 E-03-1.434000 E-03-1.470000 E-03-1.468000 E-03-1.470000 E-03$ XE-13 - $6.550000 E-04-6.540000 E-04-6.570000 E-24-2.280000 E-04-2.350000 E-04-2.370000 E-04-8.320000 E-04-6.330000 E-04-6.370000 E-04$ XE-14 -3. $2800005-04-3.140000 E-04-3.090000 E-04-1.000000 E-04-1.020000 E-04-1.060000 E-04-2.940000 E-04-2.990000 E-04-2.990000 E-04$ $X E-15-7.200000 E-04-7.210000 E-04-7.230000 E-04-6.330000 E-04-6.380000 E-04-6.360000 E-04-7.200000 E-04-7.180000 E-04-7.200000 E-04$ TE- $01-1.6000005-04-1.6000005-04-1.600000 E-04-1.3200005-04-2.2600005-04-1.270000 E-04-1.570000 E-04-1.300000 E-04-1.680000 E-04$ VEXT: $1.993800 E+00 \quad 2.993800 E+00 \quad 1.993800 E+00 \quad 1.993900 E+00 \quad 1.993900 E+00 \quad 1.993900 E+00 \quad 1.993800 E+00 \quad 1.993800 E+00 \quad 1.993800 E+00$

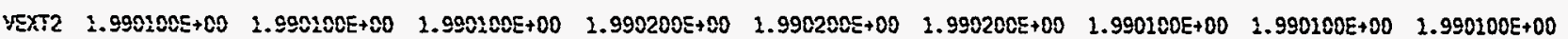

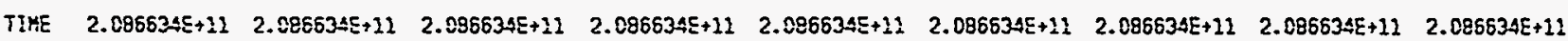


Arthive Hame:

AHS_YF:_TESTS

Book Hame: ANS_MFPTI_RV

Date \& Time: 7 yar 1900 01:29:53

Page Qescription:

OATA SET MO. -229

TRAHS RY_ZERO-2 RY_ZERO-2 RY_ZERC-3 RY_LOAD-1 $\quad$ RV_LOAD-2 $\quad$ RV_LOAD-3 $\quad$ RV_ZERO-4 $\quad$ RV_ZERO-5 RV_ZERO-6

PE- $01-7.367700 E-02-7.362900 E-02-7.383300 E-02 \quad 6.467700 E-02 \quad 6.470100 E-02 \quad 8.670200 E-01 \quad-6.588900 E-02 \quad-6.584300 E-02-6.556700 E-02$ $\begin{array}{llllllllll}P E-02 & 2.816100 E-D 2 & 2.826200 E-02 & 2.805300 E-02 & 9.740900 E-01 & 2.032320 E+00 & 1.032060 E+00 & 3.068100 E-02 & 3.078200 E-02 & 3.077400 E-02\end{array}$ PE-03 - $2.884200 E-02$ - $2.889000 E-02$ - $2.898500 E-02$ 8.327500E-01 $8.122700 E-01$ 8.223900E-02 - $1.579400 E-02$ - $1.553400 E-02$ - $1.544800 E-02$ $\begin{array}{llllllllll}P E-04 & 3.257300 E-02 & 3.245100 E-02 & 3.264700 E-02 & 9.936800 E-01 & 1.05448 C E+00 & 1.032370 E+00 & 3.338100 E-02 & 3.311800 E-02 & 3.307700 E-02\end{array}$

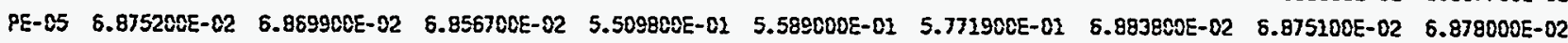
PE-06 7.022700E-02 7.017600E-02 7.039100E-02 $8.189400 E-01 \quad 8.587700 E-01 \quad 8.535600 E-01 \quad 7.366300 E-02 \quad 7.372800 E-02 \quad 7.412100 E-02$ PE-07 -3.240500E-01 $-3.240400 E-02 \quad-3.240300 E-01 \quad 2.572800 E-01 \quad 2.460300 E-01 \quad 2.5980 C 0 E-01 \quad-3.241000 E-01 \quad-3.241300 E-01 \quad-3.241600 E-01$ $\begin{array}{lllllllllll}P E-08 & 7.907000 E-03 & 7.674000 E-03 & 7.655000 E-03 & 7.033900 E-02 & 6.949300 E-01 & 7.081300 E-01 & 1.207800 E-02 & 1.241200 E-02 & 1.261000 E-02\end{array}$ PE-09 -4.65780CE-02 -4.666200E-02 -4.671900E-02 2.960000E-01 $3.040300 E-01 \quad 3.091500 E-01 \quad-4.455900 E-02 \quad-4.428600 E-02 \quad-4.411700 E-02$ PE-10 1.028400E-02 $2.011800 E-02 \quad 1.012900 E-02 \quad 5.480400 E-02 \quad 5.542900 E-01 \quad 5.527100 E-02 \quad 1.399700 E-02 \quad 1.408300 E-02 \quad 1.403500 E-02$

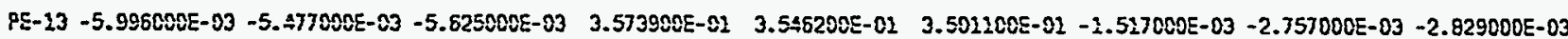

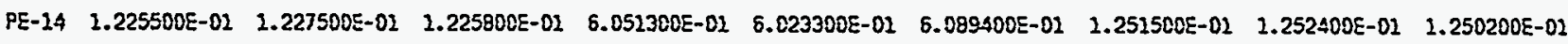
PE-15 -2.089000E-02 -2.30530CE-02 -2.100700E-02 1.404502E-01 $1.453500 E-02 \quad 1.388800 E-02-1.997900 E-02$ - $2.978100 E-02$ - $1.993100 E-02$ $\begin{array}{lllllllllll}P E-16 & 2.992500 E-02 & 3.001000 E-02 & 3.027100 E-02 & 2.190800 E-01 & 2.257000 E-01 & 2.182200 E-02 & 3.250600 E-02 & 3.273800 E-02 & 3.246000 E-02\end{array}$ PE-17 -4.207500E-02 -4.193600E-02 -4.204500E- $\$ 2 \quad 1.130900 E-02 \quad 1.243000 E-02 \quad 1.138000 E-01 \quad-3.949000 E-02 \quad-3.937000 E-02 \quad-3.926600 E-02$ PE-19 $-1.250400 E-01 \quad-1.250900 E-02 \quad-1.249800 E-01 \quad 3.345600 E-02 \quad 3.078700 E-02 \quad 3.561300 E-02 \quad-1.232300 E-01 \quad-1.234600 E-01 \quad-1.229300 E-01$

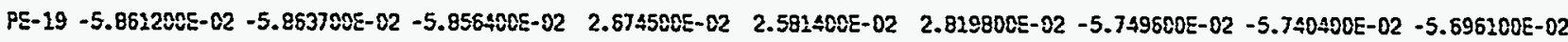
PE-20 -5.706700E-02 -5.709000E- $22 \quad-5.711700 E-02 \quad 3.537200 E-02 \quad 3.228100 E-02 \quad 3.315700 E-02 \quad-5.545400 E-02 \quad-5.515200 E-02 \quad-5.487500 E-02$

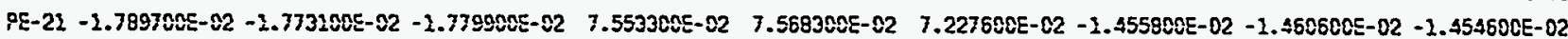
PE-22 - $2.747900 E-02 \quad-1.746800 E-02 \quad-1.749108 E-02 \quad 8.114500 E-02 \quad 8.159100 E-02 \quad 8.383600 E-02 \quad-2.508800 E-02 \quad-1.488000 E-02 \quad-1.453300 E-02$ PE-29 5.57ABOCE-02 $\quad 5.549800 E-02 \quad 5.565200 E-02 \quad 1.546500 E+00 \quad 1.547100 E+C 0 \quad 1.442400 E+00 \quad 6.733700 E-02 \quad 6.695800 E-02 \quad 6.681200 E-02$

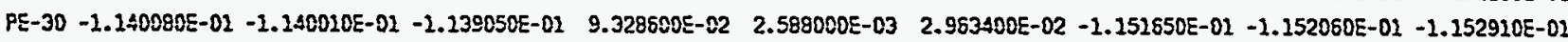
FE- 01 -3.300000E- 05 -3.100000E-05 $-3.0000005-05 \quad 3.766280 E-02 \quad 3.068200 E-02 \quad 3.842200 E-01 \quad 0.000000 E+00-2.700000 E-05-2.5 C 0000 E-05$ XEE-02 -2.600000E-03 $-2.600000 E-04 \quad-2.600000 E-04 \quad 1.510000 E-04 \quad 1.550000 E-04 \quad 1.460000 E-04-2.180000 E-04 \quad-2.220000 E-04 \quad-2.150000 E-04$ $X E-02-6.800000 E-04-6.800000 E-04-E .8000005-04-6.840000 E-04-6.840000 E-04-6.830000 E-04-6.850000 E-04-6.830000 E-04-6.830000 E-04$ XE-03 - $6.770000 E-04-6.760000 E-04-6.760000 E-04-3.890000 E-04-1.630000 E-04-2.210000 E-04-2.520000 E-04-2.520000 E-04-2.530000 E-04$ XE-04 - - $.1110005-03-1.110000 E-03-1.110000 E-83-9.060000 E-04-9.100000 E-04-9.110000 E-04-1.063000 E-03-1.058000 E-03-1.069000 E-03$ XEE-05 $-2.074000 E-03 \quad-2.072000 E-03 \quad-2.071000 E-03 \quad 9.591000 E-03 \quad 5.384000 E-03 \quad 1.1388008-01 \quad-2.092000 E-03 \quad-2.096000 E-03 \quad-2.083000 E-03$ XE-06 7.942600E-01 7.946600E-01 7.952000E-01 $5.614900 E-02 \quad 5.616700 E-01 \quad 5.471100 E-01 \quad 7.222200 E-01 \quad 7.240500 E-02 \quad 7.237500 E-01$ XEE-07 -5.750000E-04 -5.760000E-04 -5.770000E-04 2.220000E-04 2.210000E-04 2.170000E-04 -5.050000E-04 $-5.1000000 E-04$-5.150000E-04 XE- 88 -7.650000E-04 -7.840080E-04 -7.8E00005-04 -6.340000E-04 -6.4300005-04 -6.340000E- $24-7.3600005-04-7.380000 E-04-7.410000 E-04$ XE-09 -9.410000E-04 - $-9.420000 E-04-9.400000 E-04-6.650000 E-04-6.630000 E-04-6.550000 E-04-9.020000 E-04-9.030000 E-04-9.070000 E-04$ $X E-10-2.949000 E-03-2.951000 E-03-2.950000 E-03-2.916000 E-03-1.848000 E-03-1.9450005-03-2.673000 E-03-2.691000 E-03-2.689000 E-03$ XE-21 $2.300000 E-05 \quad 2.400000 E-05 \quad 2.500000 E-05 \quad 2.400000 E-05 \quad 2.500000 E-05 \quad 2.500000 E-05 \quad 2.500000 E-05 \quad 2.500000 E-05 \quad 2.400000 E-05$ XE-12 - $2.473000 E-03-1.473000 E-03-1.473000 E-03-1.431008 E-03-1.427000 E-03-1.427000 E-03-1.469000 E-03-1.468000 E-03-1.468000 E-03$ $X E-13-6.550000 E-04-6.580000 E-04-6.570000 E-04-1.600000 E-04-1.370000 E-04-1.190000 E-04-6.170000 E-04-6.170000 E-04-6.230000 E-04$

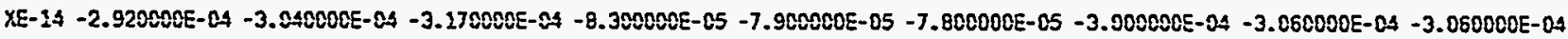
XE-15 -7.220000E-04 -7.220000E-04 -7.240000E-04 -6.160000E-04 -6.190000E-04 -6.270000E-04 -7.120000E-04 -7.140000E-04 -7.170000E-04 TE-02 - - $.600000 E-04-2.580000 E-04-2.580000 E-04-1.130000 E-04-1.120000 E-04-2.270000 E-04-1.630000 E-04-1.590000 E-04-1.630000 E-04$ VEXT2 $2.993800 E+00 \quad 1.993800 E+00 \quad 1.993800 E+00 \quad 1.993900 E+00 \quad 1.993900 E+00 \quad 1.993900 E+00 \quad 1.993900 E+00 \quad 3.993900 E+00 \quad 1.993900 E+00$ VEXT2 $2.990100 E+00 \quad 1.990200 E+00 \quad 1.990100 E+00 \quad 1.990300 E+00 \quad 1.990200 E+00 \quad 2.990200 E+00 \quad 1.990200 E+00 \quad 1.990200 E+00 \quad 1.990200 E+00$

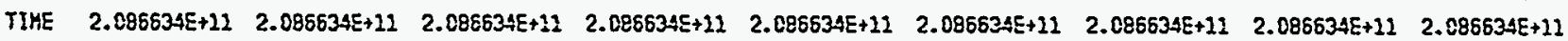


Arative name:

AYS_YP:TESTS

Book Matre: ANS_FFPTI_RY

Date \& Time: 2 Mat 1900 00:25:47

Page Description:

DATA SET HO. -230

TRAYS RV_ZERO-1 RY_ZERO-2 RY_ZERD-3 RV_LOAD-1 $\quad$ RV_LOAD-2 $\quad$ RY_LOAD-3 $\quad$ RV_ZERO-4 RV_ZERO-5 RV_ZERO-5

$P E-02-4.354500 E-02-4.360090 E-02-4.360700 E-02 \quad 3.476900 E-02 \quad 4.059900 E-02 \quad 3.779000 E-02-6.597800 E-02-8.609700 E-02-8.516700 E-02$ PE-02 5.347100E-02 $5.375900 E-02 \quad 5.359400 E-02 \quad 1.450800 E-01 \quad 1.521500 E-01 \quad 1.510300 E-01 \quad 3.212900 E-02 \quad 3.227500 E-02 \quad 3.214700 E-02$ PE-03 -2.780000E-02 -4.880000E-03 -5.740000E-04 8.576700E-02 8.644500E-02 8.651300E-02 -2.233000E-02 -2.226000E-02 -2.264400E-02 $\begin{array}{lllllllllll}P E-C 4 & 6.527900 E-02 & 6.528380 E-02 & 6.536700 E-02 & 1.623100 E-01 & 1.615100 E-02 & 1.560700 E-02 & 4.699100 E-02 & 4.666900 E-02 & 4.683300 E-02\end{array}$

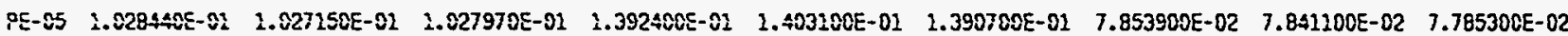
PE-06 9.578100E-02 $9.578500 E-02 \quad 9.585200 E-02 \quad 1.573100 E-01 \quad 3.574100 E-01 \quad 1.573600 E-01 \quad 7.097900 E-02 \quad 7.099700 E-02 \quad 7.123200 E-02$

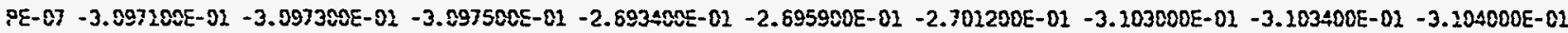
\begin{tabular}{lllllllllll}
\hline$E-08$ & $3.855600 E-02$ & $3.858200 E-02$ & $3.838800 E-02$ & $1.002700 E-01$ & $9.505000 E-02$ & $9.271100 E-02$ & $1.708000 E-02$ & $1.712100 E-02$ & $1.697400 E-02$
\end{tabular}

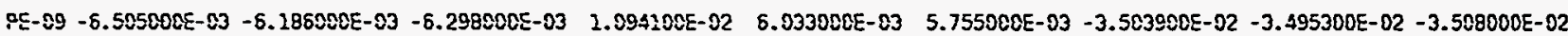
PE-10 $4.757200 E-02 \quad 4.734700 E-02 \quad 4.754800 E-02 \quad 8.363300 E-02 \quad 8.733600 E-02 \quad 8.344100 E-02 \quad 1.552900 E-02 \quad 2.6621005-02 \quad 1.654600 E-02$

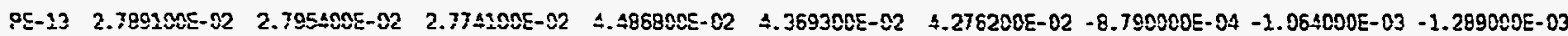

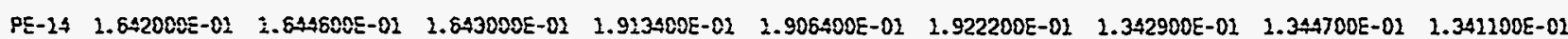

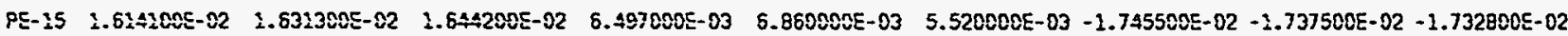
PE-16 $5.885100 E-02 \quad 6.910900 E-02 \quad 6.881900 E-02 \quad 5.097100 E-02 \quad 5.948500 E-02 \quad 5.8178008-02 \quad 3.670500 E-02 \quad 3.683600 E-02 \quad 3.653700 E-02$ $P E-17-1.949000 E-03-2.001000 E-03-2.279000 E-03 \quad-1.341900 E-02-1.579700 E-02-1.358700 E-02-3.513600 E-02-3.515400 E-02-3.506300 E-02$ PE-19 - - .292100E- $02-8.791900 E-02-8.7195005-02-9.5346005-02-9.549200 E-02-9.592600 E-02-1.202100 E-02-1.205200 E-02-1.206100 E-01$ PE-19-1.291200E- $02-1.269500 E-02-1.276700 E-02-4.690400 E-02-4.468500 E-02-4.683000 E-02-5.054200 E-02-5.024300 E-02-5.025200 E-02$ P5-20 - $1.033900 E-82-1.0529005-02-1.037800 E-02-4.102100 E-02-3.929-00 E-02-4.159900 E-02-4.743800 E-02-4.750900 E-02-4.720600 E-02$

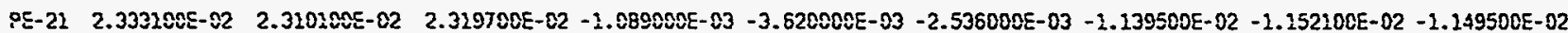
PE-22 3.411700E-02 3.411100E-02 3.407800E-02 8.707000E-03 $5.885000 E-03 \quad 9.247000 E-03 \quad-1.613000 E-03 \quad-1.459000 E-03 \quad-1.297000 E-03$ PE-29 T. $260280 E-02 \quad 7.046300 E-02 \quad 7.037100 E-02 \quad 2.2712005-01 \quad 2.376400 E-02 \quad 2.138000 E-01 \quad 5.588900 E-02 \quad 5.588400 E-02 \quad 6.561800 E-02$ PE-30 -5.552400E-02 -5.543800E-02 $-5.551000 E-02-3.231100 E-02-1.009000 E-01-9.184000 E-02-1.060360 E-01-1.059510 E-01-1.062100 E-01$

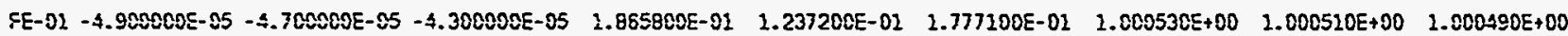
XEE-02 -3.430000E-04 -3.430000E-04 -3.420000E-04 1.400000E-05 5.000000E-05 - 7.000000E-06 $1.230000 E-04 \quad 1.320000 E-04 \quad 1.390000 E-04$ XE- $02-7.880000 E-04-7.880008 E-04$-7.888000E-04 -7.890008E-04 -7.900000E-04 -7.910000E-04 -7.920000E-04 -7.930000E-04 -7.910000E-04 YE $03-3.302000 E-03-1.303000 E-03-1.302000 E-03-1.304000 E-03-1.303000 E-03-1.302000 E-03-2.307000 E-03-1.305000 E-03-1.306000 E-03$ XE- $04-1.4360005-03-1.4380005-03-1.436000 E-03-1.346000 E-03-1.343000 E-03-1.335000 E-03-2.279000 E-03-1.279000 E-03-1.275000 E-03$ $X E-05-3.519000 E-03-3.518000 E-03-3.527000 E-03-3.448000 E-03-3.416000 E-03-3.416000 E-03-3.563000 E-03-3.564000 E-03-3.562000 E-03$

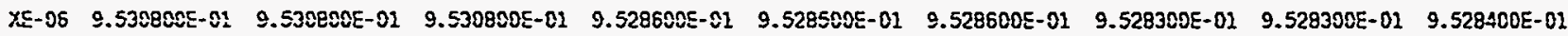
XE-07 -8.030000E-04 -8.010000E-04 -8.220000E-04 -7.280000E-04 -7.230000E-04 -7.240000E-04 -7.230000E-04 -7.230000E-04 -7.220000E-04 $X E-08-1.034000 E-03-1.033000 E-03-1.034000 E-03-1.011000 E-03-1.021000 E-03-1.010000 E-03-1.014000 E-03-1.014000 E-03-1.015000 E-03$ $x E-09-1.235000 E-03-1.235000 E-03-1.2350005-03-1.140000 E-03-1.140000 E-03-1.141000 E-03-1.107000 E-03-1.108000 E-03-1.106000 E-03$ XE- $20-3.9700005-03-3.9700005-03-3.970000 E-03-3.746000 E-03-3.715000 E-03-3.715000 E-03-3.664000 E-03-3.665000 E-03-3.662000 E-03$ XE-21 $3.400000 E-05 \quad 3.300000 E-05 \quad 3.300000 E-05 \quad 3.300000 E-05 \quad 3.400000 E-05 \quad 3.300000 E-05 \quad 3.400000 E-05 \quad 3.400000 E-05 \quad 3.300000 E-05$ $x E-12-1.704000 E-03-1.7050005-03-1.704000 E-03-1.6960005-03-1.697000 E-03-1.697000 E-03-1.704000 E-03-1.7030005-03-1.702000 E-03$ XE-13 -1.007000E-03 - $2.009000 E-03-1.009000 E-03-5.080000 E-04-5.130000 E-04-6.240000 E-04-6.700000 E-04-6.580000 E-04-6.700000 E-04$ $x E-14-4.5308005-04-4.520000 E-04-4.5200005-04-3.5200005-04-3.540000 E-04-3.540000 E-04-3.490000 E-04-3.480000 E-04-3.470000 E-04$ XE-15 -8.860000E-04 -8.870000E-04 -8.860000E-04 -6.080000E-04 - $6.110000 E-04-6.110000 E-04-5.510000 E-04-5.510000 E-04-5.500000 E-04$ TE- $01-2.500000 E-05-2.600000 E-05-2.800000 E-05-1.100000 E-05-1.100000 E-05-1.000000 E-05-2.300000 E-05-2.600000 E-05-2.700000 E-05$ $\begin{array}{lllllllllll}\text { VEXT2 } & 1.993900 E+00 & 1.993900 E+C 0 & 1.993900 E+00 & 1.993900 E+00 & 1.993900 E+00 & 1.993900 E+00 & 1.993900 E+00 & 1.993900 E+00 & 1.993900 E+00\end{array}$ VEXT2 $\quad 1.990300 E+00 \quad 1.990300 E+00 \quad 1.990300 E+00 \quad 1.990300 E+00 \quad 1.990300 E+00 \quad 1.990300 E+00 \quad 1.990300 E+00 \quad 1.990300 E+00 \quad 1.990300 E+00$

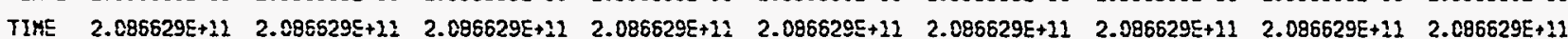


Aretrive Hame:

AHS_MF:_TESTS

Book Hame: ANS_MFPT1_RV Date \& Time: 1 Mar 1900 00:37:27

Page Description:

OATA SET NO.-231

TRAYS RY_ZERO-1 RV_ZERO-2 RY_ZERO-3 RY_LOAD-1 $\quad$ RY_LOAD-2 R R_LOAD-3 RV_ZERO-4 RV_ZERO-5 RV_ZERO-6

PE-01 -6.910300E-02 - $6.896900 E-02-8.917200 E-02 \quad 3.561800 E-01 \quad 1.675900 E-02 \quad 1.639800 E-01-6.701100 E-02-6.723500 E-02-6.764000 E-02$ PE-02 2.962600E-02 $2.973500 E-02 \quad 2.955600 E-02 \quad 2.9538 C 0 E-01 \quad 3.002300 E-02 \quad 2.991400 E-02 \quad 3.0326 C 0 E-02 \quad 3.049800 E-02 \quad 3.016100 E-02$ PE-03 -2.473300E-02 -2. $460900 E-02-2.439200 E-02 \quad 2.237200 E-02 \quad 2.170200 E-02 \quad 2.179100 E-02 \quad-2.424400 E-02 \quad-2.433500 E-02-2.424500 E-02$ PE-04 4.341600E-02 $4.373100 E-02 \quad 4.359700 E-02 \quad 3.189200 E-01 \quad 3.153900 E-02 \quad 3.177000 E-01 \quad 4.202000 E-02 \quad 4.209200 E-02 \quad 4.159700 E-02$

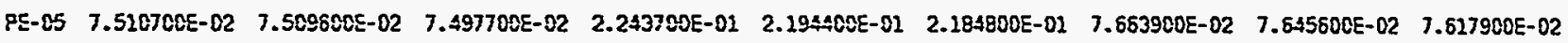
$\begin{array}{llllllllll}P E-06 & 6.851800 E-02 & 6.862800 E-02 & 6.846100 E-02 & 2.802300 E-02 & 2.665500 E-01 & 2.7200005-01 & 6.807400 E-02 & 6.821500 E-02 & 6.828100 E-02\end{array}$ PE-07 -3.127600E-02 - -3.217800E-01 -3.227900E-01 -1.691600E-02 -1.815800E-01 -1.644900E-01 -3.120300E-01 -3.120600E-01 -3.120900E-01 PE-C8 $1.415400 E-02 \quad 1.409000 E-02 \quad 1.400200 E-02 \quad 2.059400 E-02 \quad 1.929000 E-01 \quad 2.094000 E-02 \quad 1.575100 E-02 \quad 1.583900 E-02 \quad 1.594100 E-02$ PE-09 -3.658900E-02 -3.610000E-02 -3.6829COE-02 $6.059600 E-02 \quad 5.967200 E-02 \quad 6.120400 E-02 \quad-3.581800 E-82$ - $3.583800 E-02$ - $3.583500 E-02$ PE-10 1.365900E-02 $1.480500 E-02 \quad 1.470400 E-02 \quad 1.521400 E-01 \quad 1.656200 E-02 \quad 1.548700 E-01 \quad 1.608300 E-02 \quad 1.579500 E-02 \quad 1.576300 E-02$

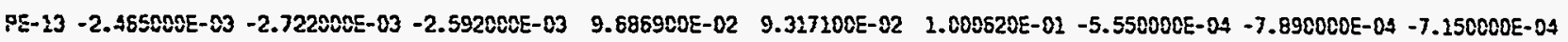
PE-14 $1.317900 E-01 \quad 1.318600 E-01 \quad 3.317500 E-02 \quad 2.680700 E-01 \quad 2.555100 E-01 \quad 2.688800 E-01 \quad 1.324900 E-01 \quad 1.325500 E-01 \quad 1.323700 E-01$ PE-15 - 2.844700E-02 - $2.821600 E-02-2.844000 E-02$ 3.120000E-02 3.109200E-02 3.089800E-02 -1.749900E-02 -1.7491005-02-1.7512005-02 PE-16 3.56240CE-02 $3.551700 E-02 \quad 3.550200 E-02 \quad 8.711500 E-02 \quad 8.991700 E-02 \quad 8.913100 E-02 \quad 3.532700 E-02 \quad 3.540300 E-02 \quad 3.623300 E-02$

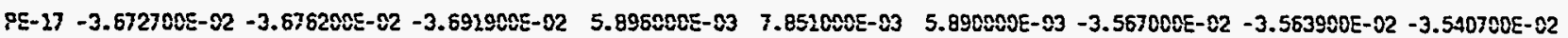
PE-28 -1.212900E-01 - $1.218500 E-01-2.218200 E-02$-6.690100E-02 $-6.851000 E-02-7.422500 E-02-1.208200 E-02-1.211800 E-02-1.204900 E-02$ PE-19 -5.149000E- $02-5.2440005-82$-5.251400E-02 -3.949200E-02 -4.157400E-02 -4.274900E-02 -5.108500E-02 -5.086800E-02 -5.108200E-02 PE-20 -4.832100E-02 -4.830700E-02 - $4.842500 E-02$-3.657600E-02 -3.549800E-02 -3.178500E-02 -4.813600E-02 -4.815900E-02 -4.811900E-02 PE-21 - $1.183600 E-02-1.185800 E-02-1.168800 E-02 \quad 9.269000 E-03 \quad 9.954000 E-03 \quad 1.071000 E-02-1.170200 E-02 \quad-1.151200 E-02-1.157200 E-02$ PE-22 -3.446000E-03 -3.669000E-03 $-3.723000 E-03 \quad 2.205200 E-02 \quad 1.850800 E-02 \quad 2.0944900 E-02 \quad-3.155000 E-03 \quad-2.992000 E-03 \quad-3.116000 E-03$ $\begin{array}{lllllllllll}P E-29 & 6.562900 E-02 & 6.552900 E-02 & 6.508900 E-02 & 4.702800 E-01 & 5.059300 E-01 & 3.973300 E-01 & 6.580800 E-02 & 6.539700 E-02 & 6.560300 E-02\end{array}$ PE-30 -1.059070E-02 -1.059830E-01 -1.057780E-02 -6.996800E-02 -7.203200E-02 -3.578000E-02 - $1.080990 E-01-1.083310 E-01-1.081250 E-01$

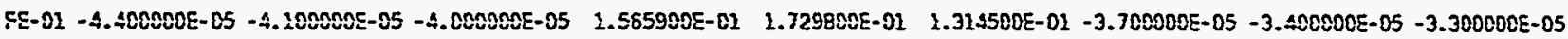
YEE-01 2.280000E-04 2.250000E-04 2.240000E-04 2.100000E-05 2.000000E-05 3.000000E-05 1.140000E-04 1.160000E-04 1.120000E-04 XE-02 -7.86000CE-04 -7.860000E-04 -7.860000E-04 -7.910000E-04 -7.900000E-04 -7.910000E-04 -7.880080E-04 -7.8900005-04 -7.890000E-04 $X E-03-2.044000 E-03-1.044000 E-03-1.044000 E-03-2.045000 E-03-1.045000 E-03-1.044000 E-03-1.054000 E-03-1.055000 E-03-1.070000 E-03$ XE-04 - - 2.252000E-03 -1.254000E-03 -1.253000E-03 - $2.305000 E-03-1.299000 E-03-1.275000 E-03-1.257000 E-83-1.251000 E-03-1.253000 E-03$ XE-05 - 3.575000E-03 -3.574000E-03 -3.574000E-03 2.885900E-02 -2.012000E-03 -4.890000E-02 -3.578000E-03 -3.579000E-03 -3.578000E-03

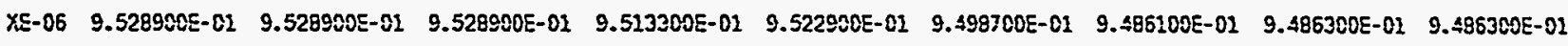
XE-07 -7.010000E-04 -7.010000E-04 -7.010000E-04 -7.050000E-04 -7.050000E-04 -7.050000E-04 -6.900000E-04 -6.900000E-04 -6.890000E-04 XE-08 - $1.013000 E-03-1.013000 E-03-1.013000 E-03-1.0020005-03-1.003000 E-03-1.008000 E-03-1.017000 E-03-1.015000 E-03-1.017000 E-83$ $X E-09-1.217000 E-03-1.118000 E-03-1.218000 E-03-1.140000 E-03-1.0920005-03-1.098000 E-03-1.0530005-03-2.052000 E-03-1.065000 E-03$ $X E-10-3.737000 E-03-3.739000 E-03-3.741000 E-03-3.922000 E-03-3.798000 E-03-3.795000 E-03-3.802000 E-03-3.8010 C C E-03-3.802000 E-03$ YEE-11 $3.200000 E-05 \quad 3.300000 E-05 \quad 3.200000 E-05 \quad 3.200000 E-05 \quad 3.200000 E-05 \quad 3.300000 E-05 \quad 3.300000 E-05 \quad 3.400000 E-05 \quad 3.300000 E-05$ $X E-12-1.599000 E-03-1.6990005-03-1.599000 E-03-1.695000 E-03-1.6970005-03-1.701000 E-03-1.707000 E-03-1.706000 E-03-1.706000 E-03$ YE-13 - $6.830000 E-04-6.880000 E-04-6.880000 E-04-6.670000 E-04-6.670000 E-04-6.310000 E-04-7.580000 E-04-7.600000 E-04-7.570000 E-04$ XE-14 -3.530000E-04 -3.630000E-04 -3.620000E-04 -3.540000E-04 -3.570000E-04 -3.510000E-04 -3.500000E-04 -3.490000E-04 -3.490000E-04 XE- $25-5.390000 E-04-5.370000 E-04-5.360000 E-04-5.3900000 E-04-6.390000 E-04-6.370000 E-04-5.520000 E-04-5.490000 E-04-5.500000 E-04$ TE-01 - $-4.300000 E-05-4.100000 E-05-4.000000 E-05-2.3080000-05-2.000000 E-05-1.800000 E-05-3.300000 E-05-3.506000 E-05-3.600000 E-05$ VEXT2 $1.993900 E+00 \quad 1.993900 E+00 \quad 1.993900 E+00 \quad 1.993900 E+00 \quad 1.993900 E+00 \quad 1.993900 E+00 \quad 1.993900 E+00 \quad 1.993900 E+00 \quad 1.993900 E+00$ $\begin{array}{lllllllllll}\text { YEXT2 } & 1.990300 E+00 & 1.990300 E+00 & 1.990300 E+00 & 1.990380 E+00 & 1.990300 E+00 & 1.990300 E+00 & 1.990300 E+00 & 1.990300 E+00 & 1.990300 E+00\end{array}$

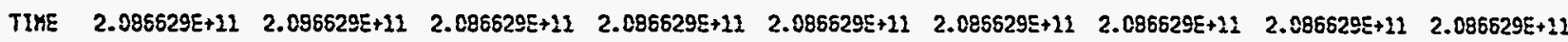


itchive Hzane:

ANS_re?_TESTS

SoOk Hame: AHS_MFOTI_RY

Date \& Time: 1 Mar 1900 02:09:47

Page Description:

EATA SET $\$ 0 .-232$

TRAYS RY ZERO-1

RE_ZERO-2 RY_ZERO-3

RY_LCAD-I

RY_LOAD-2

RI_LOAD-3

RY_ZERO-4

RV_ZERO-5

RV_ZERO- 6

PE- $02-5.461100 E-02-6.450800 E-02-5.503200 E-02 \quad 3.190900 E-01 \quad 3.215100 E-01 \quad 1.188400 E-01 \quad-6.908800 E-02-6.892200 E-02-6.895800 E-02$ $\begin{array}{llllllllll}P E-02 & 3.427700 E-02 & 3.403500 E-02 & 3.434800 E-02 & 2.548200 E-01 & 2.567000 E-02 & 2.455400 E-01 & 2.946500 E-02 & 2.952300 E-02 & 2.922000 E-02\end{array}$

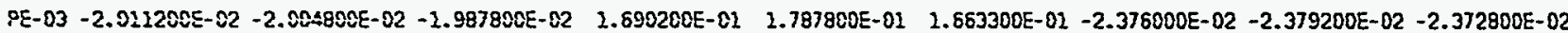
PE-04 4.581608E-02 $4.572700 E-02 \quad 4.592000 E-02 \quad 2.581200 E-01 \quad 2.560500 E-02 \quad 2.540000 E-01 \quad 4.170500 E-02 \quad 4.159600 E-02 \quad 4.141700 E-02$

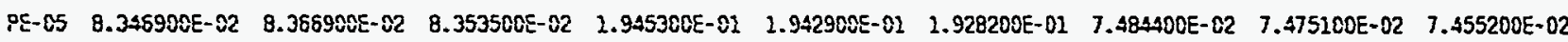
PE-05 7.532000E-02 $7.556500 E-02 \quad 7.566500 E-02 \quad 2.425400 E-02 \quad 2.424800 E-02 \quad 2.375600 E-01 \quad 6.740400 E-02 \quad 6.750300 E-02 \quad 6.746100 E-02$

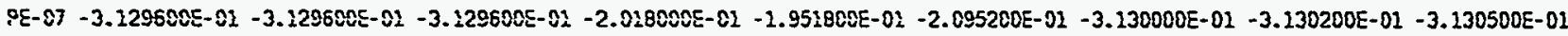
$\begin{array}{llllllllll}P E-08 & 2.030100 E-02 & 2.056700 E-02 & 2.038200 E-02 & 2.696500 E-02 & 1.758800 E-02 & 1.655200 E-02 & 2.499700 E-02 & 1.539500 E-02 & 1.536700 E-02\end{array}$ PE-09 -2.513100E- $02-2.495500 E-02-2.467800 E-02$ 4.555400E-02 4.289000E-02 4.378300E-02 -3.618100E-02 $-3.584900 E-02 \quad-3.583700 E-02$ $\begin{array}{llllllllll}P E-10 & 2.709400 E-02 & 2.710408 E-02 & 2.724200 E-02 & 2.428700 E-01 & 1.434000 E-01 & 1.431200 E-01 & 1.641400 E-02 & 1.624800 E-02 & 1.610500 E-02\end{array}$ $\begin{array}{llllllllll}9 E-13 & 9.3610000-03 & 9.127000 E-03 & 9.590000 E-03 & 3.870000 E-02 & 8.178400 E-02 & 8.200500 E-02 & -9.100000 E-05 & 5.500000 E-05 & 1.000000 E-04\end{array}$ PE-14 $1.445600 E-02 \quad 1.446600 E-01 \quad 1.443400 E-01 \quad 2.419200 E-01 \quad 2.387700 E-02 \quad 2.490800 E-01 \quad 1.315100 E-01 \quad 1.323200 E-01 \quad 1.314500 E-01$ $.8 E-15$ - $2.869000 E-03-1.682000 E-03-1.631000 E-03 \quad 2.226400 E-02 \quad 2.534900 E-02 \quad 2.602400 E-02-1.785800 E-02-1.791500 E-02-1.769300 E-02$ PE-16 5.008200E-02 $5.014400 E-02 \quad 5.032300 E-02 \quad 7.693400 E-02 \quad 7.576400 E-02 \quad 8.094700 E-02 \quad 3.513200 E-02 \quad 3.602300 E-02 \quad 3.648200 E-02$

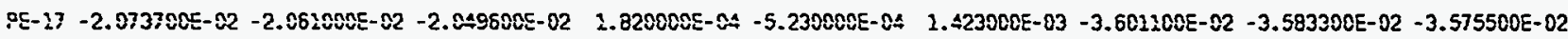
$P E-18-1.056360 E-02-1.053050 E-02-1.055290 E-01-7.690900 E-02-7.743100 E-02-3.797700 E-02-1.205300 E-01-1.209400 E-01-1.204500 E-01$ :E-19-3.192700E- $\$ 2-3.1436005-02-3.140900 E-02-4.192500 E-02-4.345400 E-02-4.107800 E-82-5.172600 E-02-5.162700 E-02-5.172800 E-02$ PE-20 -2.870000E-02 -2.892800E-02 -2.868500E-02 -3.762200E-02 -3.702300E-02 -3.789000E-02 -4.867100E-02 -4.868200E-02 -4.841100E-02

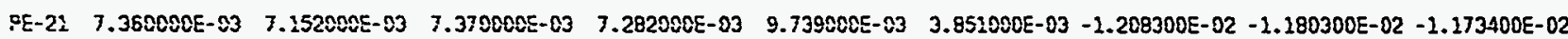
$\begin{array}{llllllllll}P E-22 & 1.541800 E-02 & 1.534300 E-02 & 1.512000 E-02 & 1.589000 E-02 & 1.582200 E-02 & 1.503600 E-02 & -3.314000 E-03 & -3.584000 E-03 & -3.535000 E-03\end{array}$ $\begin{array}{llllllllll}\text { PE-29 } & 6.955680 E-82 & 8.943200 E-02 & 6.938100 E-02 & 3.590500 E-81 & 3.719900 E-01 & 4.018400 E-01 & 6.520400 E-02 & 6.523600 E-02 & 6.498100 E-02\end{array}$ PE-30 - 7.563000E-02 -7.584200E-02 -7.5E4900E-02 - $2.071120 E-01-3.874500 E-02-6.521100 E-02-2.080410 E-01-1.077960 E-01-1.090250 E-01$

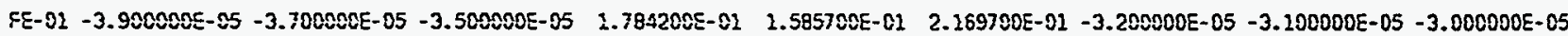
XE-01 -3.600000E-04 -3.590000E-04 -3.620000E-04 - $1.730000 E-04-1.760000 E-04-1.790000 E-04-1.340000 E-04-1.330000 E-04-1.350000 E-04$ XE-02 -7.850000E-04 -7.870009E-04 -7.870000E-04 -7.890000E-04 -7.910000E-04 -7.890000E-04 -7.890000E-04 -7.990000E-04 -7.9000000E-04 YE- $03-1.2050005-03-1.2030005-03-1.204000 E-03-1.205000 E-03-1.205000 E-03-1.205000 E-03-1.206000 E-03-1.207000 E-03-1.208000 E-03$ $x E-84-1.435000 E-03-1.435000 E-03-1.435000 E-03-2.323000 E-03-1.327000 E-03-1.331000 E-03-1.354000 E-03-1.353000 E-03-1.352000 E-03$ $X E-05-3.5800005-03-3.5810005-03-3.5800005-03-3.063000 E-03-3.358000 E-03-3.399000 E-03-3.596000 E-03-3.586000 E-03-3.586000 E-03$ XE- 08 9.488900E- $01 \quad 9.489000 E-01 \quad 9.489000 E-01$ 9.493000E-01 9.493100E-02 9.489000E-01 $9.489200 E-01 \quad 9.489200 E-01 \quad 9.4893 C 0 E-01$ $x E-07-8.000000 E-04-7.990000 E-04-8.000000 E-04-7.080000 E-04-7.130000 E-04-6.960000 E-04-6.820000 E-04-6.850000 E-04-6.870000 E-04$ XE-08 - $2.034000 E-03-1.0340005-03-1.0340005-03-1.017000 E-03-1.017080 E-03-1.017000 E-03-1.024000 E-03-1.023000 E-03-1.023000 E-03$ $X E-09-1.234000 E-03-1.234000 E-03-1.234000 E-03-2.115000 E-03-1.116000 E-03-1.116000 E-03-1.1070005-03-1.110000 E-03-1.110000 E-03$ $X E-10-3.957000 E-03-3.957000 E-03-3.957000 E-03-3.831000 E-03-3.835000 E-03-3.846080 E-03-3.867000 E-03-3.869000 E-03-3.869000 E-03$ $X E-11 \quad 3.300000 E-05 \quad 3.300000 E-05 \quad 3.100000 E-05 \quad 3.100000 E-05 \quad 3.100000 E-05 \quad 3.200000 E-05 \quad 3.200000 E-05 \quad 3.1000000 E-05 \quad 3.200000 E-05$ $X E-12-1.7100005-83-1.709000 E-03-1.7$ SQ080E- $03-1.705000 E-03-1.707000 E-03-1.710000 E-03-1.714000 E-03-1.713000 E-03-1.713000 E-03$ $X E-13-1.015000 E-03-1.014000 E-03-1.025000 E-03-6.850000 E-04-6.920000 E-04-7.020000 E-04-7.750000 E-04-7.820000 E-04-7.870000 E-04$ XE- $14-4.530000 E-04-4.520000 E-04-4.530000 E-04-3.590000 E-04-3.610000 E-03-3.630000 E-04-3.680000 E-04-3.670000 E-04-3.670000 E-04$ XE-15 -8.890000E-04 -8.890000E-04 -8.900000E-04 -7.370000E-04 -7.390000E-04 -7.430000E-04 -7.240000E-04 -7.200000E-04 -7.210000E-04 TE- $02-1.700000 E-05-1.700000 E-05-1.700000 E-05 \quad 1.000000 E-06 \quad 4.008000 E-06 \quad 6.0000005-06-9.0000005-05-9.000000 E-06-1.100000 E-05$ $\begin{array}{lllllllllll}\text { VEXT1 } & 1.993900 E+00 & 1.993900 E+C D & 1.993900 E+00 & 1.993900 E+C D & 1.993900 E+C 0 & 1.993900 E+C 0 & 1.993900 E+00 & 1.993900 E+00 & 1.993900 E+00\end{array}$

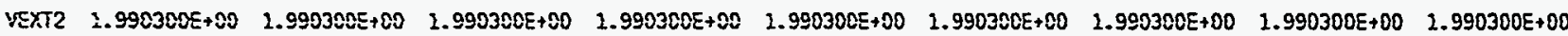

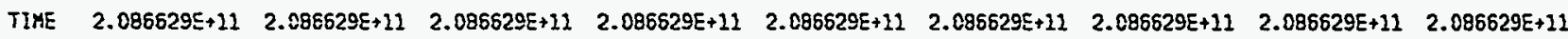


Bcok Mame: AYS_XFPTI_Ry Q Date \& Time: 1 Mar 1900 04:00:57

Page Description:

DATA SET NO.-233

TRAHS RY_ZERO-1 RY_ZERO-2 RY_ZERO-3 RY_LOAD-1 $\quad$ RY_LOAD-2 $\quad$ RY_LOAD-3 RY_ZERO-4 RV_ZERO-5 RY_ZERO-6

PE-02 -6.996400E-02 -7.003000E-02 - 7.019100E-02 4.992900E-02 5.855800E-02 5.380000E-02 -6.905000E-02 -6.903100E-02 -6.909900E-02 PE-02 $3.019100 E-02 \quad 3.005200 E-02 \quad 3.022200 E-02 \quad 1.649000 E-02 \quad 1.698700 E-01 \quad 1.709500 E-01 \quad 3.070700 E-02 \quad 3.054700 E-02 \quad 3.028500 E-02$ $P E-03-2.359000 E-\$ 2 \quad-2.345900 E-\$ 2 \quad-2.355700 E-02 \quad 1.029530 E-01 \quad 1.0 \$ 7720 E-01 \quad 9.620200 E-02 \quad-2.160100 E-02 \quad-2.168000 E-02 \quad-2.155900 E-02$ PE-O4 4.145000E-02 4.166700E-02 4.275300E-02 1.854100E-01 $1.832300 E-01$ 1.804400E-01 $4.271300 E-02 \quad 4.2593 C 0 E-02 \quad 4.294800 E-02$

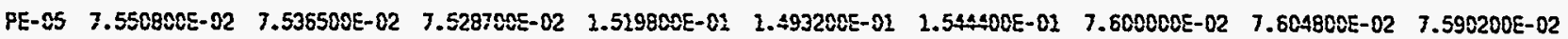
$\begin{array}{llllllllll}P E-06 & 6.832 C 00 E-02 & 6.810300 E-02 & 6.841200 E-02 & 1.743800 E-01 & 1.725300 E-01 & 1.808700 E-01 & 6.947400 E-02 & 6.955000 E-02 & 6.966600 E-02\end{array}$

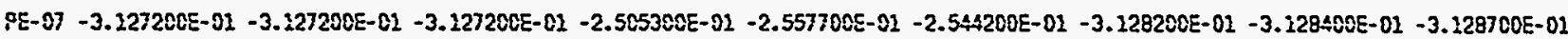
PE-C8 $1.527200 E-02 \quad 2.517600 E-02 \quad 1.509100 E-02 \quad 2.241900 E-02 \quad 1.122100 E-01 \quad 1.224800 E-01 \quad 1.729900 E-02 \quad 1.749600 E-02 \quad 1.737100 E-02$ $P E-89-3.457900 E-02 \quad-3.478400 E-02 \quad-3.465400 E-02 \quad 1.266200 E-02 \quad 1.418100 E-02 \quad 1.698600 E-02 \quad-3.378400 E-02 \quad-3.378800 E-02 \quad-3.374800 E-02$ PE-10 $1.657900 E-02 \quad 1.633800 E-02 \quad 1.843900 E-02 \quad 9.333100 E-02 \quad 9.436100 E-02 \quad 9.467500 E-02 \quad 1.713500 E-02 \quad 1.757200 E-02 \quad 1.757400 E-02$ PE-13 2.090000E-04 3.020000E-04 $1.720000 E-04 \quad 5.2778008-02 \quad 5.122900 E-02 \quad 5.099000 E-02 \quad 4.220000 E-04 \quad 3.703000 E-03 \quad 2.036000 E-03$ $\begin{array}{lllllllllll}P E-14 & 1.305700 E-01 & 1.307500 E-01 & 1.305800 E-02 & 2.029200 E-01 & 2.020100 E-01 & 2.024200 E-01 & 1.329500 E-01 & 1.330200 E-01 & 1.328700 E-01\end{array}$ PE- $15-2.681300 E-02-1.678000 E-02-1.671400 E-02 \quad 8.289000 E-03 \quad 8.503000 E-03 \quad 9.028000 E-03-1.632000 E-22$ - $1.634300 E-02$ - $2.606600 E-02$ $\begin{array}{llllllllll}P E-16 & 3.62350 \Omega E-02 & 3.630900 E-02 & 3.616600 E-02 & 6.051200 E-02 & 6.396000 E-02 & 6.187200 E-02 & 3.732300 E-02 & 3.735000 E-02 & 3.770700 E-02\end{array}$ $P E-17-3.653300 E-02-3.653500 E-02-3.675500 E-02-1.310200 E-02-1.299300 E-02-1.248700 E-02-3.584200 E-02-3.572700 E-02-3.558700 E-02$ PE-18 $-1.213600 E-01-1.214700 E-01-1.213500 E-02 \quad-9.190900 E-02-9.377000 E-02-8.976000 E-02-1.203100 E-02-1.206400 E-01-1.201800 E-01$ $P E-19-5.045800 E-02-5.047600 E-02-5.031800 E-02$ - $4.687080 E-02$ - $4.689600 E-02-4.512400 E-02$-5.002300E-02 $-4.975500 E-02$-4.970600E-02 PE-20 - 4.897700E-02 - 4.920100E-02 -4.902800E-02 - 4.494100E-02 -3.963100E-02 -4.364600E-02 -4.832900E-02 -4.836500E-02 -4.815700E-02 PE-21 - $2.128200 E-02-1.124500 E-02-1.140800 E-02-5.290000 E-04$ 9.660000E-04 -2.3470C0E-03 - $1.169000 E-02-1.170100 E-02-1.152100 E-02$ PE-22 $-3.083000 E-03 \quad-2.950000 E-03 \quad-2.975000 E-03 \quad 1.075500 E-02 \quad 8.955000 E-03 \quad 8.777000 E-03 \quad-2.260000 E-03 \quad-2.016000 E-03 \quad-1.902000 E-03$ PE-29 $5.342300 E-02 \quad 6.359300 E-02 \quad 6.347900 E-02 \quad 2.229000 E-02 \quad 2.964000 E-01 \quad 2.264100 E-02 \quad 6.422400 E-02 \quad 6.448700 E-02 \quad 6.520500 E-02$ $P E-30-1.071670 E-01-1.069260 E-02$-1.069720E-01 -7.488500E-02 -9.718400E-02 -1.092190E-02 -1.075210E-01 -1.073720E-01 - $2.074900 E-01$ FE- $02 \quad 2.000440 E+C 0 \quad 1.000440 E+C 0 \quad 1.000450 E+00 \quad 1.672000 E+C 0 \quad 1.676500 E+00 \quad 1.674800 E+C 0 \quad 1.002190 E+00 \quad 1.001080 E+00 \quad 1.000870 E+00$ XE-02 -3.290000E-04 -3.290000E-04 -3.290000E-04 -2.060000E-04 -2.000000E-04 -2.040000E-04 -3.140000E-04 -3.130000E-04 -3.130000E-04 XE-02 -7.970000E-04 -7.950000E-04 -7.950000E-04 -7.880000E-04 -7.88С000E-04 -7.890000E-04 -7.948000E-04 -7.950000E-04 -7.940000E-04 $X E-03-1.231000 E-03-1.230000 E-03-1.230000 E-03-1.232000 E-03-1.232000 E-03-2.232000 E-03-1.233000 E-03-1.233000 E-03-1.234000 E-03$ XE- $04-1.407000 E-03-1.407000 E-03-1.407000 E-83-1.360000 E-03-1.361000 E-03-1.355000 E-03-1.386000 E-03-1.385000 E-03-1.385000 E-03$ XE-05 -3.609000E-03 -3.607000E-03 - 3.608000E-03 -3.043000E-03 1.621000E-03 -3.492000E-03 -3.601000E-03 -3.600000E-03 -3.601000E-03

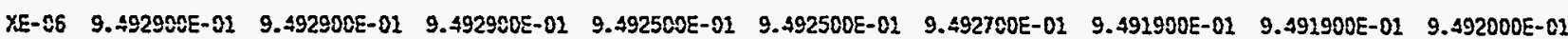
XE-07 -3.290000E-04 -3.310000E-04 -3.470000E-04 -2.140000E-04 -2.100000E-CL -2.020000E-04 -3.730000E-04 -3.840000E-04 -3.920000E-04 XE-08 - $1.037000 E-03-1.035000 E-03-2.035000 E-03-1.018000 E-03-1.020000 E-03-1.027000 E-03-1.035000 E-03-1.035000 E-03-1.034000 E-03$ XE-09 - $1.227000 E-03-1.225000 E-03-1.226000 E-03-1.209000 E-03-1.208000 E-03-1.204000 E-03-1.227000 E-03-1.225000 E-03-1.224000 E-03$ $X E-10-3.891000 E-03-3.891000 E-03-3.990000 E-03-3.859000 E-03-3.855000 E-03-3.855000 E-03-3.900000 E-03-3.898000 E-03-3.898000 E-03$ XE-11 2.400000E-05 $2.500000 E-05 \quad 2.500000 E-05 \quad 2.500000 E-05 \quad 2.600000 E-05 \quad 2.600000 E-05 \quad 2.500000 E-05 \quad 2.500000 E-05 \quad 2.500000 E-05$ XE- $12-1.722000 E-03-1.722000 E-03-1.721000 E-03-1.724080 E-03-1.725000 E-83-1.727000 E-03-1.729000 E-03-1.728000 E-03-1.729000 E-03$ XE-13 -6.090000E-04 -6.180000E-04 -6.120000E-04 -5.210000E-04 -5.220000E-02 -5.310000E-04 -6.950000E-04 -6.950000E-04 -6.940000E-04 XE-14 -3.750000E- 84 -3.740C00E-04 -3.750000E-04 -3.540000E-04 -3.680000E-04 -3.670600E-04 -3.820000E-04 -3.83C000E-04 -3.820000E-04 XEE-15 -9.250000E-C. $-8.240000 E-04-8.230000 E-04-7.120000 E-04-7.190000 E-04-7.250000 E-04-8.150000 E-04-8.160000 E-04-9.170000 E-04$

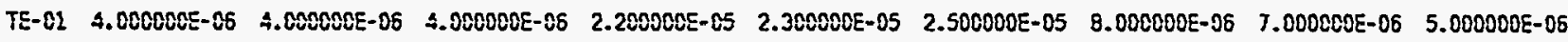
$\begin{array}{lllllllllll}\text { VEXT1 } & 1.993800 E+00 & 1.993800 E+00 & 1.993800 E+00 & 1.993800 E+00 & 1.993800 E+00 & 1.993800 E+00 & 2.993800 E+00 & 3.993800 E+00 & 1.993800 E+00\end{array}$ $\begin{array}{lllllllllll}\text { VEXT2 } & 1.990200 E+00 & 2.99010 C E+C D & 1.990100 E+00 & 1.990100 E+00 & 1.990100 E+00 & 1.990100 E+00 & 1.990100 E+C 0 & 1.990100 E+00 & 1.990100 E+00\end{array}$

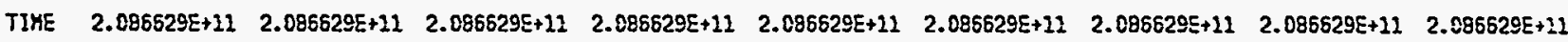


Page Description:

DATA SET NO.-234

TRAHS RY_ZERD-1 RV_ZERO-2 RY_ZERO-3 RV_LOAD-1 $\quad$ RV_LOAD-2 $\quad$ RV_LOAD-3 RV_ZERO-4 RV_ZERO-5 RY_ZERO-6

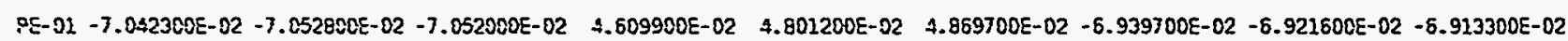
$\begin{array}{llllllllll}P E-02 & 3.035500 E-02 & 3.012300 E-02 & 3.018000 E-02 & 3.576800 E-01 & 1.640500 E-01 & 1.607900 E-02 & 3.073100 E-02 & 3.050200 E-02 & 3.031700 E-02\end{array}$ PE- 03 -2.293600E-02 - 2.291100E-02 $-2.251600 E-02 \quad 9.520700 E-02 \quad 9.718100 E-02 \quad 9.883100 E-02 \quad-2.209600 E-02 \quad-2.164300 E-02 \quad-2.185700 E-02$ $\begin{array}{lllllllllll}P E-04 & 4.145500 E-02 & 4.181200 E-02 & 4.160900 E-02 & 1.720000 E-01 & 1.700280 E-02 & 2.741300 E-01 & 4.296300 E-02 & 4.311700 E-02 & 4.282600 E-02\end{array}$ $\begin{array}{lllllllllll}P S-05 & 7.523300 E-02 & 7.500200 E-02 & 7.500500 E-02 & 2.489700 E-01 & 1.480800 E-01 & 1.487300 E-01 & 7.543700 E-02 & 7.652000 E-02 & 7.523200 E-02\end{array}$ $\begin{array}{lllllllllll}P E-06 & 6.839980 E-02 & 6.833300 E-02 & 6.825900 E-02 & 1.723400 E-01 & 1.731100 E-02 & 2.728100 E-01 & 5.957600 E-02 & 6.961600 E-02 & 6.910600 E-02\end{array}$

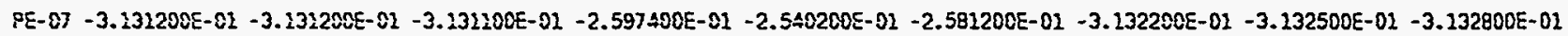
$\begin{array}{lllllllllll}P E-08 & 1.356100 E-02 & 1.374500 E-02 & 1.389900 E-02 & 1.063030 E-02 & 1.026240 E-01 & 1.089670 E-02 & 1.300500 E-02 & 1.310700 E-02 & 1.297300 E-02\end{array}$

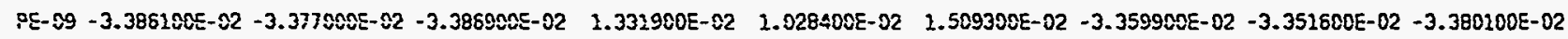
$\begin{array}{llllllllll}P E-10 & 1.659500 E-02 & 1.538400 E-02 & 1.525700 E-02 & 8.682400 E-02 & 8.919900 E-02 & 9.057100 E-02 & 1.775200 E-02 & 1.782200 E-02 & 1.778700 E-02\end{array}$

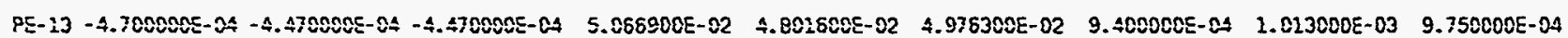
$\begin{array}{lllllllllll}P E-14 & 1.307900 E-02 & 1.305700 E-02 & 2.305200 E-02 & 2.008300 E-01 & 1.942000 E-01 & 2.985800 E-02 & 1.3216005-02 & 1.319900 E-01 & 1.322200 E-01\end{array}$

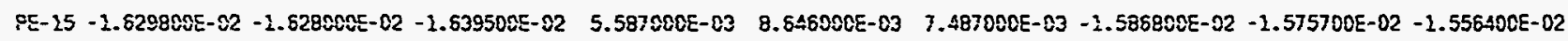
PE-16 3.641800E-02 3.654000E-02 $3.643400 E-02 \quad 5.924700 E-02 \quad 6.270700 E-02 \quad 6.056700 E-02 \quad 3.754200 E-02 \quad 3.767000 E-02 \quad 3.770200 E-02$

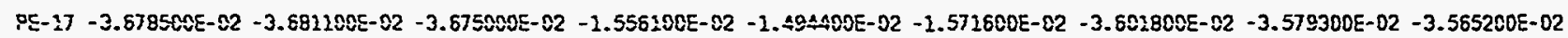

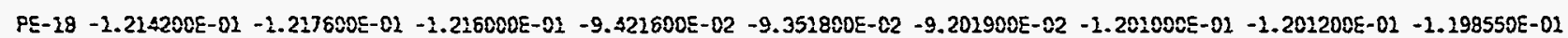
$.8 E-19-5.054700 E-02-5.071700 E-02-5.028400 E-02-4.400300 E-02-4.425500 E-02-4.371200 E-02-5.0213005-02-4.995600 E-02-4.968400 E-02$ PE-20 -4.903408E-02 -4.905900E-02 -4.983700E-02 -4.1832005-02 -4.089400E-02 -4.026900E-82 -4.E57500E- $82-4.843900 E-02-4.829600 E-02$

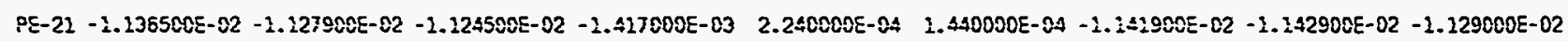
PE-22 $-2.666000 E-03 \quad-2.492000 E-03 \quad-2.592000 E-03 \quad 8.574000 E-03 \quad 9.301000 E-03 \quad 5.739000 E-03 \quad-2.700000 E-03 \quad-2.339000 E-03 \quad-2.129000 E-03$ $\begin{array}{lllllllllll}P E-29 & 6.518700 E-02 & 6.533900 E-02 & 6.523200 E-02 & 2.621900 E-02 & 2.9482005-01 & 2.750300 E-02 & 6.268300 E-02 & 6.2556 C 0 E-02 & 6.244600 E-02\end{array}$

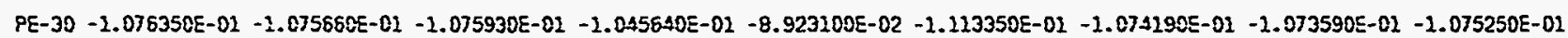
$\begin{array}{lllllllllll}\text { CE- } 01 & 1.000260 E+00 & 1.000250 E+00 & 1.000250 E+C 0 & 1.550600 E+00 & 2.652900 E+00 & 1.655800 E+C 0 & 1.0012805+00 & 2.001040 E+00 & 1.080910 E+00\end{array}$

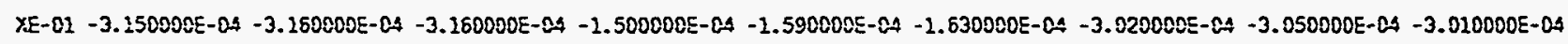
XE-02 - - 7.970000E-04 -7.950000E-04 -7.950000E-04 -7.890000E-09 -7.900000E-04 -7.890000E-04 -7.950000E-04 -7.970000E-04 -7.980000E-04 XE- $03-3.770000 E-04-3.770000 E-04-3.770000 E-04-3.800000 E-04-3.790000 E-04-3.800000 E-04-3.760000 E-04-3.770000 E-04-3.760000 E-04$ $X E-04-2.403000 E-03-1.401000 E-03-1.401000 E-03-1.367000 E-03-1.3650005-03-1.363000 E-03-1.411000 E-03-1.410000 E-03-1.410000 E-03$ XE- $05-3.615000 E-03-3.615000 E-03-3.515000 E-03-3.260000 E-03-3.4800005-03-3.502000 E-83-3.6050005-03 \quad 1.533500 E-02 \quad 1.926900 E-02$

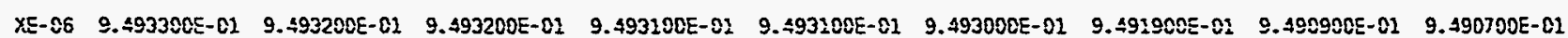
XE-07 - $4.290000 E-04-4.300000 E-04-4.300000 E-04-2.870000 E-04-2.840000 E-04-2.770000 E-04-3.950000 E-04-3.970000 E-04-3.990000 E-04$ $x E-88-1.0370005-03-1.0360005-03-1.0380005-03-1.006000 E-03-1.0040005-03-1.006000 E-03-1.0390005-03-1.038000 E-03-1.0360 C 0 E-03$ $X E-09-1.230000 E-03-1.229000 E-03-2.229000 E-03-1.298000 E-03-1.197000 E-03-1.199000 E-03-1.2220005-03-1.224000 E-03-1.223000 E-03$ $x E-10-3.891000 E-03-3.891000 E-03-3.891000 E-03-3.829000 E-03-3.825000 E-03-3.927000 E-03-3.595000 E-03-3.944000 E-03-3.957000 E-03$ $\begin{array}{llllllllll}X E-11 & 2.300000 E-05 & 2.500000 E-05 & 2.400000 E-05 & 2.400000 E-05 & 2.400000 E-05 & 2.500000 E-05 & 2.3000005-05 & 2.500000 E-05 & 2.500000 E-05\end{array}$ $X E-12-1.727000 E-03-1.725000 E-03-1.725000 E-03-1.732000 E-03-1.733000 E-03-2.733000 E-03-1.735000 E-03-1.735000 E-03-1.734000 E-03$ XE-13 - $-6.650000 E-04-6.9500005-04-6.950000 E-04-4.830000 E-04-4.810000 E-04-4.840000 E-04-7.5000005-04-7.140000 E-04-6.810000 E-04$ $X E-14-3.570000 E-04-3.570000 E-04-3.550000 E-04-3.270000 E-04-3.260000 E-04-3.250000 E-04-3.560000 E-04-3.590000 E-04-3.610000 E-04$ XE-15 -8.250000E-04 -8.130000E-04 -8.228000E-04 -6.490000E-04 -6.550000E-04 -6.500000E-04 -8.090000E-04 -8.130000E-04 -8.130000E-04

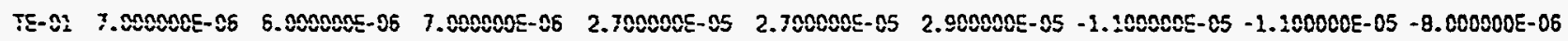
$\begin{array}{lllllllllll}\text { VEXT1 } & 1.993700 E+00 & 1.993700 E+00 & 1.993700 E+00 & 1.993700 E+00 & 1.993700 E+00 & 1.993700 E+00 & 1.99370 \varepsilon E+00 & 1.993700 E+00 & 1.993700 E+00\end{array}$ $\begin{array}{lllllllllll}\text { YEXT2 } & 1.990008 E+00 & 1.990080 E+00 & 1.990000 E+00 & 2.990000 E+00 & 1.990000 E+00 & 1.990000 E+00 & 1.990000 E+00 & 1.998000 E+00 & 1.990000 E+00\end{array}$

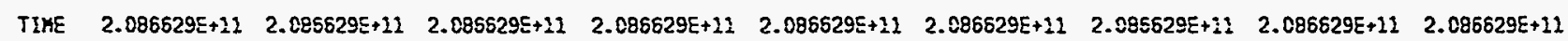


Archive Mank:

AMS_MFP_TESTS

Book Yame: ANS_yspT1_RV Late \& Time: 1 has 1900 22:51:57

Page Description:

DATA SET HO.-235

TRAYS RY_ZERO-2 RY_ZERO-2 RY_ZERO-3 RV_LOAD-1 RY_LOAD-2 RV_LOAD-3 RV_ZERO-4 RV_ZERO-5 RV_ZERO-6

PE- $02-5.248300 E-02-5.165800 E-02-5.250600 E-02-2.401300 E-02-2.546600 E-02-2.322500 E-02-6.589900 E-02-6.697200 E-02-6.675200 E-02$ PE-02 4.631800E-02 4.651300E-02 4.643900E-02 $9.156900 E-02 \quad 8.848000 E-02 \quad 9.037400 E-02 \quad 3.094300 E-02 \quad 3.112500 E-02 \quad 3.109900 E-02$ PE- 03 -9.77000CE-03 -9.762000E-03 -9.832000E-03 3.279500E-02 3.231800E-02 3.24240CE-02 $-2.059000 E-02$-2.050980E-02 -2.069700E-02 $\begin{array}{lllllllllll}P E-04 & 5.794700 E-02 & 5.793300 E-02 & 5.785200 E-02 & 9.923000 E-02 & 2.013290 E-02 & 1.003390 E-02 & 4.223700 E-02 & 4.227500 E-02 & 4.208300 E-02\end{array}$ PE-05 9.353100E-02 $\quad 9.340700 E-02 \quad 9.352200 E-02 \quad 3.109230 E-02 \quad 3.121910 E-02 \quad 1.124888 E-02 \quad 7.772700 E-02 \quad 7.785300 E-02 \quad 7.791800 E-02$ PE-06 $8.785300 E-02 \quad 8.776400 E-02 \quad 8.781700 E-02 \quad 2.201400 E-01 \quad 1.198460 E-02 \quad 1.220400 E-02 \quad 7.310900 E-02 \quad 7.353200 E-02 \quad 7.364200 E-02$ PE- $07-3.232880 E-01-3.132800 E-01-3.132800 E-01-3.039100 E-01-3.036300 E-01$-3.022100E-01 $-3.133400 E-01-3.133600 E-01-3.133900 E-01$ $\begin{array}{lllllllllll}P E-08 & 2.823500 E-02 & 2.823800 E-02 & 2.838100 E-02 & 5.682500 E-02 & 5.573600 E-02 & 5.837200 E-02 & 1.935500 E-02 & 1.941400 E-02 & 1.933600 E-02\end{array}$ $18-09-2.726800 E-02-3.741900 E-02-1.758100 E-02-2.090000 E-02-1.225800 E-02-8.975000 E-03-3.348300 E-02-3.357900 E-02-3.389000 E-02$ $\begin{array}{lllllllllll}P E-10 & 3.392100 E-02 & 3.390800 E-02 & 3.313400 E-02 & 5.036000 E-02 & 5.153300 E-02 & 5.107100 E-02 & 1.957700 E-02 & 1.977900 E-02 & 1.987400 E-02\end{array}$ PE-13 1.666800E-02 $1.642700 E-02 \quad 1.627500 E-02 \quad 2.373700 E-02 \quad 2.432400 E-02 \quad 2.3248 \Omega 0 E-02 \quad 2.522000 E-03 \quad 2.572000 E-03 \quad 3.065000 E-03$

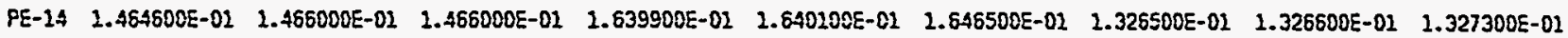
PE-15 2.317000E-03 2. 2.868000E-03 $\quad 1.979000 E-03 \quad-3.394000 E-03 \quad-2.324000 E-03 \quad-2.025080 E-03 \quad-1.338700 E-02 \quad-1.356600 E-02 \quad-1.348300 E-02$ $\begin{array}{lllllllllll}P E-16 & 5.331900 E-02 & 5.307300 E-02 & 5.339000 E-02 & 4.951500 E-02 & 4.980000 E-02 & 4.919500 E-02 & 3.933700 E-02 & 3.951400 E-02 & 3.956800 E-02\end{array}$ PE-17 -1.913600E-02 - $1.900300 E-02-1.907600 E-02-2.278700 E-02-2.398000 E-02-2.316100 E-02-3.319100 E-02-3.308000 E-02-3.294100 E-02$ PE-18 -1.029040E-01 -1.025390E-01 - $1.029470 E-01-1.046760 E-01-1.055660 E-01-1.039830 E-01-2.182260 E-01-1.180500 E-02-1.181510 E-01$ PE-19 - 3.214300E-02 -3.151300E-02 -3.22880CE-02 -4.661700E-02 -4.432900E-02 -4.468300E-02 -4.699508E-02 - $4.722000 E-02-4.689400 E-02$ $P E-20-3.012500 E-02-3.019000 E-02-3.036500 E-02-4.365500 E-02-4.275500 E-02-4.313100 E-02-4.542200 E-02-4.540400 E-02-4.543500 E-02$

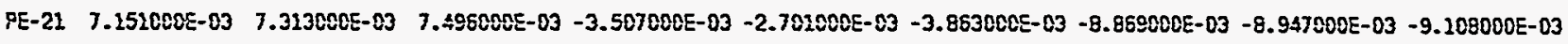
PE-22 $1.476900 E-02 \quad 1.494400 E-02 \quad 1.525400 E-82 \quad 4.489000 E-03 \quad 4.641000 E-03 \quad 4.217000 E-03 \quad 4.380000 E-04 \quad 6.070000 E-04 \quad 6.390000 E-04$

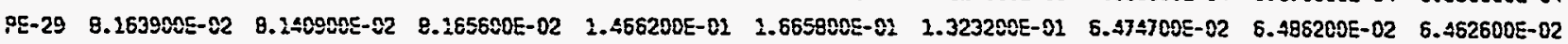
PE-30 - $9.769500 E-02-8.788000 E-02-8.781500 E-02-1.054950 E-01-1.019020 E-02-9.956900 E-02-1.051540 E-01-1.049130 E-01-1.052420 E-01$ CE- $01 \quad 1.000480 E+00 \quad 2.000460 E+00 \quad 1.000460 E+00 \quad 1.420000 E+00 \quad 2.423200 E+00 \quad 1.426400 E+00 \quad 1.000720 E+00 \quad 1.000580 E+00 \quad 1.000620 E+00$ YE-02 -2.300000E-04 -2.310000E-04 -2.300000E-04 -5.300000E-05 -6.100000E- 05 -7.200000E-05 -2.900000E-04 -2.870000E-04 -2.890000E-04 XE-02 -8.040008E-04 -8.020000E-04 -9.020000E-04 -7.900000E-04 -7.9100005-04 -7.930800E-04 -8.030300E-04 -8.020000E-04 -8.010000E-04 XE-03 -4.320000E-04 -4.310000E-04 -4.310000E-04 -4.320000E-04 -4.320000E-04 -4.320000E-04 -4.450000E-04 -4.470000E-04 -4.550000E-04 XE-04 - 1.193000E-03 - $1.193000 E-03-1.193000 E-03-1.304000 E-03-1.312000 E-03-1.315000 E-03-1.419000 E-03-1.418000 E-03-1.418000 E-03$ XE-05 $-3.578000 E-03 \quad-3.575000 E-03 \quad-3.575000 E-03 \quad 2.088900 E-02 \quad 9.658000 E-03 \quad 1.592400 E-02 \quad 6.402000 E-03 \quad 2.994900 E-02 \quad 1.853600 E-02$ XE-05 9.4966C0E-01 $9.496600 E-01$ 9.496608E-01 $9.492000 E-01$ 9.495100E-01 $9.494200 E-02 \quad 9.494500 E-01 \quad 9.493400 E-02 \quad 9.494000 E-01$ YE-07 5.200000E-04 5.300000E-04 5.430000E-04 -3.720000E-04 -3.860000E-04 -3.930000E-04 -4.070000E-04 -4.050000E-04 -3.970000E-04 YE- $09-9.760000 E-04-9.750008 E-04-9.7400805-04-1.019800 E-03-1.0180005-03-1.021000 E-03-1.039000 E-03-1.039000 E-03-1.038000 E-03$ $X E-09-1.143000 E-03-1.141000 E-03-1.240000 E-03-1.180000 E-03-1.2770005-03-2.279000 E-03-1.234000 E-03-1.233000 E-03-1.232000 E-03$ XE- $10-3.356800 E-03-3.3550005-03-3.352000 E-03-4.356000 E-03-3.452000 E-03-3.513000 E-03-3.874000 E-03-3.927000 E-03-3.910000 E-03$ YE-11 2.300000E-05 2.500000E-0S 2.400000E-05 2.400000E-0S 2.400000E-05 2.500000E-05 2.500000E-05 $2.500000 E-05 \quad 2.500000 E-05$

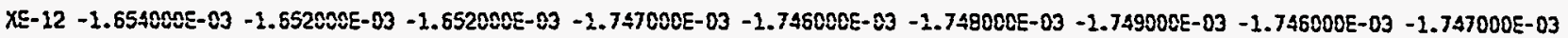
XE-13 -2.010000E-04 -1.970000E-04 -1.910000E-04 -1.720000E-04 -1.900000E-04 -1.960000E-04 $-5.700000 E-04 \quad-5.750000 E-04-5.8500005-04$ XE-24 -2.640000E-04 -2.630000E-04 -2.630000E-04 -2.3700005-04 -2.390800E-04 -2.4C0000E-04 -3.008000E-04 -3.880000E-04 -3.060000E-04 $X E-15-7.730000 E-04-7.710800 E-04-7.710000 E-04-4.720000 E-04-4.830000 E-04-4.880000 E-04-8.050000 E-04-8.060000 E-04-9.100000 E-04$ TE-01 T.00COOOE-06 6.000000E-06 8.000000E-05 2.400000E-05 2.500000E-05 2.700000E-05 $0.000000 E+00-2.000000 E-06 \quad-4.000000 E-06$ VEXTI $1.993700 E+00 \quad 1.9937 C 0 E+00 \quad 1.993700 E+00 \quad 1.993700 E+00 \quad 1.993700 E+00 \quad 1.993700 E+00 \quad 1.993700 E+00 \quad 1.993700 E+00 \quad 1.993700 E+00$

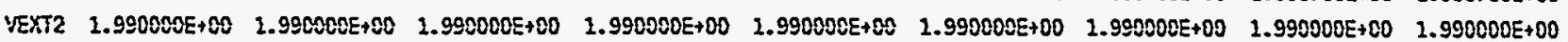

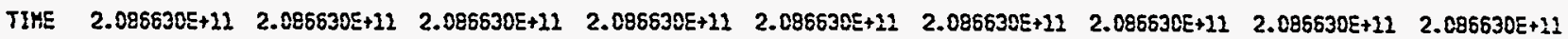


Page Description:

DATA SET H0.-236

TRANS RY_ZERO-1 RY_ZERO-2 RY_ZERO-3 RY_LOAD-1 $\quad R Y_{-}$LOAO-2 $\quad$ RV_LOAD-3 RY_ZERO-4 RV_ZERO-5 RY_ZERO-6

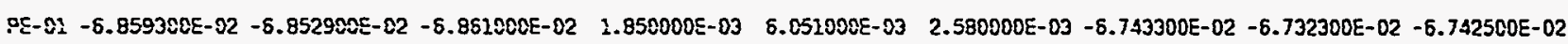
PE-02 3.046700E-02 3.042400E-02 $3.043100 E-02 \quad 1.130870 E-01 \quad 1.094420 E-01 \quad 1.083930 E-01 \quad 3.089000 E-02 \quad 3.080200 E-02 \quad 3.096400 E-02$ QE- 33 -2. 285900E- 02 -2.189300E-02 -2.194600E-02 5.243000E-02 4.898500E-02 4.965100E-02 -2.037400E-02 -2.027300E-02 -2.017700E-02 $\begin{array}{llllllllll}P E-04 & 4.203500 E-02 & 4.099800 E-02 & 4.076300 E-02 & 1.198920 E-01 & 1.215400 E-01 & 1.187450 E-01 & 4.151500 E-02 & 4.133300 E-02 & 4.105600 E-02\end{array}$

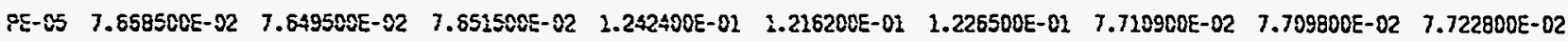
PE-06 7.243400E-02 $\quad 7.134500 E-02 \quad 7.157100 E-02 \quad 1.353900 E-01 \quad 1.331200 E-01 \quad 1.378400 E-01 \quad 7.236700 E-02 \quad 7.254400 E-02 \quad 7.240700 E-02$ $P C-07-3.143000 E-02-3.243000 E-01-3.143000 E-01-2.887900 E-01-2.873700 E-01-2.909300 E-01-3.144200 E-01 \quad-3.144400 E-01-3.144700 E-01$ PE-28 $1.656700 E-02 \quad 1.6717$ EEE-02 $\quad 1.633900 E-02 \quad 7.086800 E-02 \quad 7.092500 E-02 \quad 7.157900 E-02 \quad 1.591900 E-02 \quad 2.623500 E-02 \quad 1.597700 E-02$ $P E-09-3.465400 E-02-3.433100 E-02-3.460300 E-02-3.807000 E-03-6.084000 E-03-3.470000 E-03-3.431800 E-02-3.431800 E-02-3.408200 E-02$ PE-10 $1.802400 E-02 \quad 1.762800 E-02 \quad 1.772900 E-02 \quad 6.322800 E-02 \quad 6.403800 E-02 \quad 5.950700 E-02 \quad 1.975500 E-02 \quad 1.990300 E-02 \quad 1.959200 E-02$ $\begin{array}{llllllllll}9 E-13 & 2.776000 E-03 & 2.439000 E-03 & 1.351000 E-03 & 3.171100 E-02 & 3.275000 E-02 & 3.115600 E-02 & 3.2780005-03 & 3.507000 E-03 & 3.371000 E-03\end{array}$

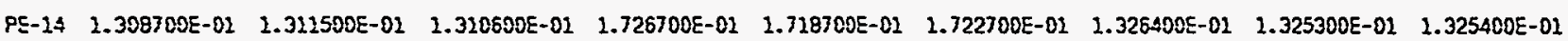
CE- 15 - $1.429200 E-02 \quad-2.449600 E-02-1.452100 E-02 \quad 6.290000 E-04 \quad 1.799000 E-03 \quad 8.900000 E-04-2.379800 E-02 \quad-1.351200 E-02 \quad-1.353400 E-02$ PE-16 3.840900E-02 $3.795300 E-02 \quad 3.828900 E-02 \quad 5.314400 E-02 \quad 5.350000 E-02 \quad 5.264900 E-02 \quad 3.880700 E-02 \quad 3.925400 E-02 \quad 3.915400 E-02$

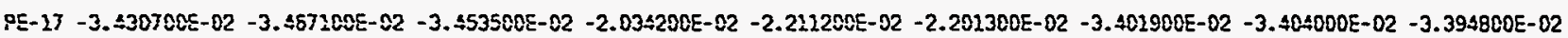
PE-18 -1.193730E-01 - - $2.190870 E-01-1.294680 E-01-1.023240 E-01-1.033530 E-01-1.022260 E-01-1.183170 E-01-1.186500 E-01-1.181770 E-01$ 8E-19-4.769900E- $82-4.776208 E-02-4.769108 E-02-4.521200 E-02-4.671200 E-02-4.403000 E-02-4.747106 E-02-4.715700 E-02-4.704100 E-02$ $P E-20-4.652800 E-02-4.661600 E-02-4.6742005-02-4.092600 E-02-4.291700 E-02-4.168700 E-02-4.6582005-02-4.631200 E-02-4.639100 E-02$ $.8 E-21-9.130000 E-03-8.9990005-03-9.835000 E-03-2.460800 E-03-1.937000 E-03-2.652000 E-03-8.230000 E-03-8.209000 E-03-8.301000 E-03$ PE-22 -9.870000E-04 - $1.005000 E-03 \quad-2.186000 E-03 \quad 4.682000 E-03 \quad 4.881000 E-03 \quad 6.199000 E-03 \quad-6.950000 E-04 \quad-3.420000 E-04 \quad-4.870000 E-04$ PE-29 6. $667100 E-02 \quad 6.495200 E-02 \quad 6.499700 E-02 \quad 1.520100 E-02 \quad 2.592400 E-01 \quad 1.711200 E-01 \quad 5.269800 E-02 \quad 6.147500 E-02 \quad 6.151100 E-02$ $P E-30-1.055420 E-01-1.057960 E-01-1.059210 E-01-1.105400 E-02-1.073470 E-01-1.074810 E-02-1.056120 E-02-1.057290 E-01-1.057660 E-01$

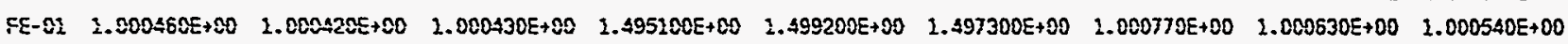
XE- $01-2.910000 E-04-2.9000005-04-2.900000 E-24-7.500000 E-05-7.2000005-05-7.400000 E-05-2.940000 E-04-2.970000 E-04-2.980000 E-04$ XE-02 -8.030000E-04 -8.020000E-04 -8.020000E-04 -7.950000E-04 -7.950000E-04 -7.960000E-04 -8.040000E-04 -8.050000E-04 -8.040000E-04 XE-03 - $4.810000 E-04-4.810000 E-04-4.810000 E-04-4.820000 E-04-4.810000 E-04-4.820000 E-04-4.820000 E-04-4.820000 E-04-4.850000 E-04$ XE-04 - - $2.423000 E-03-1.4210005-03-1.423000 E-03-1.311000 E-03-1.310000 E-03-1.310000 E-03-1.420000 E-03-1.421000 E-03-1.418000 E-03$ XE- CS $-3.517000 E-03 \quad-3.616000 E-03 \quad-3.617000 E-03 \quad 3.108700 E-02 \quad 2.533500 E-02 \quad 4.738800 E-02 \quad-1.716000 E-03 \quad 4.390000 E-04 \quad 9.054000 E-03$

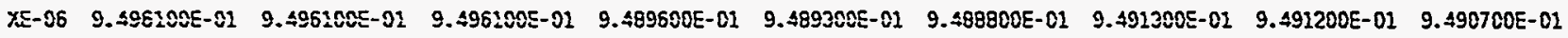
XE-07 $2.000000 E-06 \quad-1.0000005-06 \quad 2.200000 E-05-3.760000 E-04-3.700000 E-04 \quad-3.690000 E-04-2.870000 E-04 \quad-2.600000 E-04 \quad-2.440000 E-04$ $X E-08-1.033000 E-03-1.031000 E-03-2.0320005-83-1.014000 E-03-1.0220005-03-1.017000 E-03-1.038000 E-03-1.037000 E-03-1.036000 E-03$ $X E-09-1.231000 E-03-1.229000 E-03-2.229000 E-03-1.173000 E-03-1.172000 E-03-1.272000 E-03-1.228000 E-03-1.226000 E-03-1.226000 E-03$ $X E-10-3.8770005-03-3.877080 E-03-3.8770805-83-3.567000 E-03-3.56 \$ 000 E-03-3.574800 E-03-3.854000 E-03-3.863000 E-03-3.887000 E-03$ $\begin{array}{lllllllllll}X E-11 & 2.400000 E-05 & 2.500000 E-05 & 2.500000 E-05 & 2.5000000 E-05 & 2.500000 E-05 & 2.500000 E-05 & 2.500000 E-05 & 2.500000 E-05 & 2.500000 E-05\end{array}$ $x E-12-1.7400005-03-1.740000 E-03-1.739000 E-03-1.747000 E-83-1.748000 E-03-1.748000 E-03-1.748000 E-03-1.747000 E-03-1.748000 E-03$ $X E-13-6.240000 E-04-6.220000 E-04-8.240000 E-04-1.770000 E-04-1.670000 E-04-1.750000 E-04-3.820000 E-04-4.000000 E-04-3.890000 E-04$ XE- $24-3.160000 E-04-3.050000 E-04-3.280000 E-04-2.370000 E-04-2.320000 E-04-2.330000 E-04-3.020000 E-04-3.070000 E-04-2.910000 E-04$ XE-15 -8.070000E-04 -8.040000E-04 -8.050000E-04 $-4.670000 E-04-4.630000 E-04-4.740000 E-04-8.020000 E-04-8.070000 E-04-8.070000 E-04$ TE-01 - $2.500000 E-05-1.600000 E-05-1.600000 E-05$ 1.000000E-06 7.000000E-06 $1.000000 E-06-1.200000 E-05-1.200000 E-05-1.400000 E-05$ VEXT2 $2.993700 E+00 \quad 1.993708 E+00 \quad 2.993700 E+00 \quad 1.993700 E+00 \quad 1.993700 E+C 0 \quad 1.993700 E+00 \quad 1.993700 E+00 \quad 1.993700 E+00 \quad 1.993700 E+00$ VEXT2 $1.990000 E+00 \quad 1.990000 E+00 \quad 1.990000 E+00 \quad 1.990000 E+00 \quad 1.990000 E+00 \quad 1.990000 E+00 \quad 1.990000 E+00 \quad 1.990000 E+00 \quad 1.990000 E+00$

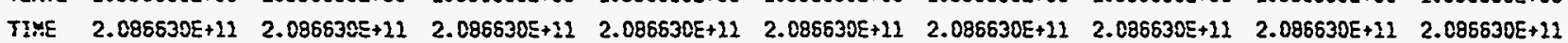


Archive Yzare:

AMS_MFP_TESTS

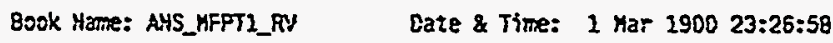

Page Description:

DATA SET MO. -237

TRAHS RY_ZERO-1 RV_ZERO-2 RV_ZERO-3 RV_LOAD-1 RV_LOAD-2 RV_LOAD-3 RV_ZERO-4 RV_ZERO-5 RY_ZERO-5

PE-02 -6.9385COE-02 -6.938200E-02 -6.91\$200E-02 -2.354200E-02 -2.201600E- $\$ 2-2.386200 E-02-6.788000 E-02-6.780900 E-02-6.787100 E-02$ PE-C2 2.993400E-02 $3.015700 E-02 \quad 2.995400 E-02 \quad 3.954900 E-02 \quad 8.246400 E-02 \quad 8.284100 E-02 \quad 3.055500 E-02 \quad 3.030400 E-02 \quad 3.047200 E-02$ PE-03 -2.2159QCE-02 -2.20910CE-02 -2.226700E-02 2.389100E-02 2.501400E-02 2.593100E-02 $-2.068800 E-02 \quad-2.059400 E-02 \quad-2.054900 E-02$ PE-D4 4.032300E-02 4.035400E-02 4.050300E-02 $9.274600 E-02 \quad 9.208100 E-02 \quad 9.201900 E-02 \quad 4.063500 E-02 \quad 4.075500 E-02 \quad 4.092400 E-02$ PE- $\$ 5$ 7.639900E-02 7.62980CE-02 7.610800E-02 $3.088470 E-01 \quad 1.073260 E-01 \quad 1.073570 E-01 \quad 7.683100 E-02 \quad 7.675200 E-02 \quad 7.687600 E-02$ $\begin{array}{llllllllll}P E-06 & 6.967300 E-02 & 6.935700 E-02 & 6.948900 E-02 & 1.133470 E-02 & 1.232350 E-02 & 1.132120 E-02 & 7.154100 E-02 & 7.167200 E-02 & 7.129400 E-02\end{array}$

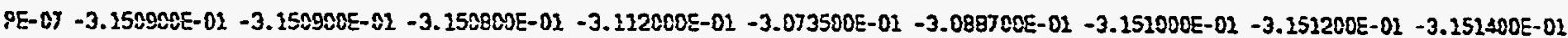
PE-CQ $\quad 1.2782 C 0 E-02 \quad 1.304200 E-02 \quad 1.289900 E-02 \quad 5.0660000 E-02 \quad 4.989300 E-02 \quad 5.094500 E-02 \quad 1.630700 E-02 \quad 1.635000 E-02 \quad 1.668700 E-02$

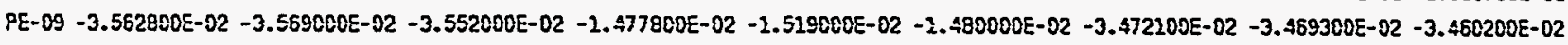
PE-10 1.782400E-02 $1.759600 E-02 \quad 1.787900 E-02 \quad 4.532100 E-02 \quad 4.662400 E-02 \quad 4.550200 E-02 \quad 3.954200 E-02 \quad 1.959000 E-02 \quad 1.974400 E-02$ PE-13 4.630000E-04 5.950000E-04 $6.520000 E-04 \quad 1.989600 E-02 \quad 2.156700 E-02 \quad 2.140500 E-02 \quad 3.425000 E-03 \quad 2.241000 E-03 \quad 2.178000 E-03$

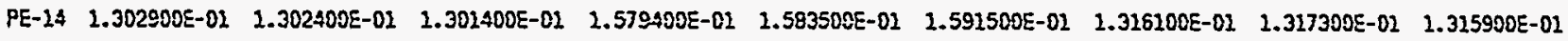
FE-15 - $1.505800 E-02-3.500300 E-02-1.5049005-02-4.307000 E-83-4.486000 E-03-4.151000 E-03-1.445200 E-02-1.420000 E-02-1.398400 E-02$ PE- $26 \quad 3.773100 E-02 \quad 3.806200 E-02 \quad 3.799900 E-02 \quad 4.855100 E-02 \quad 4.919700 E-02 \quad 4.958000 E-02 \quad 3.905400 E-02 \quad 3.923300 E-02 \quad 3.914200 E-02$ FE-27 -3.5607CQE- $\$ 2-3.538360 E-02-3.5 \div 3600 E-02-2.563300 E-02-2.393600 E-02-2.524800 E-02-3.446900 E-02-3.404000 E-02-3.394800 E-02$

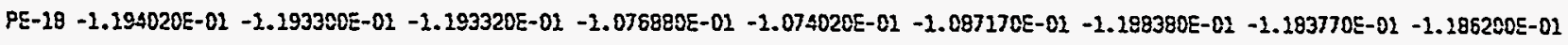
PE-19-4.847900E-02 - 4.876500E-02 -4.841100E-02 -4.852300E-02 -4.550000E-82 -4.657308E-02 -4.729400E-02 -4.733200E-02 -4.732200E-02 PE-20 - $4.777600 E-02-4.787000 E-02-4.783200 E-02-4.451300 E-02-4.505600 E-02-4.272600 E-02-4.693400 E-02-4.664500 E-02-4.841600 E-02$ PE-21 - $8.4680005-03-8.4470005-03-8.512800 E-03$ - $4.6210005-03-5.229000 E-03-4.44200005-03-8.5370005-03-8.508000 E-03-8.3570005-03$ PE-22 $-2.439000 E-03 \quad-2.554000 E-03 \quad-2.627000 E-03 \quad 2.690000 E-03 \quad 3.080000 E-03 \quad 3.979000 E-03 \quad-1.201000 E-03 \quad-9.150000 E-04 \quad-1.236000 E-03$

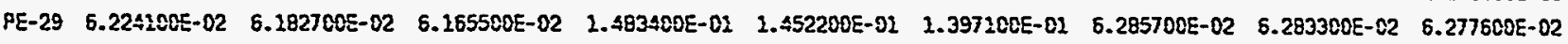
$P E-30-1.060510 E-02$-2.059510E-01 -1.059010E-02 -1.109990E-02 -9.001400E-02 -7.965400E-02 -1.062230E-01 -1.062270E-02 - $2.063240 E-01$ FE-01 $1.000210 E+00 \quad 2.000210 E+00 \quad 1.000190 E+00 \quad 1.393600 E+00 \quad 1.392800 E+00 \quad 1.396000 E+00 \quad 1.001050 E+00 \quad 1.000930 E+00 \quad 1.000710 E+00$ XE-02 -2.890000E-04 -2.900000E-04 -2.900000E-04 -9.000000E-05 -9.300000E-25 -9.200000E-05 -2.830000E-04 -2.940000E-04 -2.990000E-04 XE-02 -8.0500005-04 -8.050000E-04 -8.050000E-04 -7.990000E-04 -7.990000E-04 -7.990000E-04 -8.050000E-04 -8.070000E-04 -8.080000E-04 $X E-03-5.230000 E-04-5.230000 E-04-5.220000 E-04-5.230000 E-04-5.240000 E-04-5.240000 E-04-5.260000 E-04-5.260000 E-04-5.270000 E-04$ $X E-04-1 . \$ 190005-03-1.418000 E-03-1.417000 E-03-1.323000 E-03-1.320000 E-03-1.323000 E-03-1.419000 E-03-1.418000 E-03-1.417000 E-03$ XE-05 2.049300E-02 $5.2470005-03 \quad 3.929000 E-03 \quad 9.334000 E-03 \quad 5.741000 E-03 \quad 5.058300 E-02 \quad 5.855400 E-02 \quad 7.327900 E-02 \quad 7.7890005-01$ XE-05 9.490900E-01 9.491600E-01 $9.491700 E-01 \quad 9.491100 E-01 \quad 9.491300 E-01 \quad 9.488400 E-01 \quad 9.488200 E-01 \quad 9.487500 E-01 \quad 9.447500 E-01$ XE-07 -2.500000E-04 -2.470000E-04 -2.470000E-04 -3.930000E-04 -3.840000E-04 -3.840000E-04 -3.640000E-04 -3.430000E-04 -3.420000E-04 XE- $89-1.037000 E-03-1.036000 E-03-1.035000 E-03-1.016000 E-03-1.019000 E-03-1.018000 E-03-1.043000 E-03-1.042000 E-03-1.041000 E-03$ $X E-09-1.231000 E-03-2.232000 E-03-2.230000 E-03-2.175000 E-03-1.175000 E-03-1.173000 E-03-1.230000 E-03-1.226000 E-03-1.228000 E-03$ XE-10 -3.928000E-03 - -3.901000E-03 -3.898000E-03 -3.475000E-03 -3.483000E-03 -3.619000E-03 -4.017000E-03 -4.063000E-03 - $6.036000 E-03$ XE-11 $2.400000 E-05 \quad 2.400000 E-05 \quad 2.500000 E-05 \quad 2.400000 E-05 \quad 2.500000 E-05 \quad 2.500000 E-05 \quad 2.500000 E-05 \quad 2.500000 E-05 \quad 2.500000 E-05$ XE-12 - $2.7420005-03-1.7$ A2000E-03 - $2.7420005-03-1.751000 E-03-1.752000 E-03-1.752000 E-03-1.754000 E-03-1.752000 E-03-1.750000 E-03$ XE-13 -5.500000E-04 -5.5C0000E-04 -4.980000E-04 -1.510000E-04 -1.610000E-04 -1.630000E-04 -3.810000E-04 -3.890000E-04 -4.090000E-04 $X E-14-2.980008 E-04-3.010008 E-04-3.000000 E-04-2.450000 E-04-2.450000 E-04-2.440000 E-04-2.900000 E-04-2.930000 E-04-2.930000 E-04$ XE-15 -8.080000E-04 -8.030000E-04 -8.000000E-04 -5.030000E-04 -5.070000E-04 -5.080000E-04 -8.050000E-04 -8.070000E-04 -8.080000E-04 TE-01 - 1. .00000E-05 - 1.400000E-05 - $1.400000 E-05$ 1.300000E-05 1.400000E-05 1.400000E-05 - $1.200000 E-05-1.300000 E-05-1.700000 E-05$

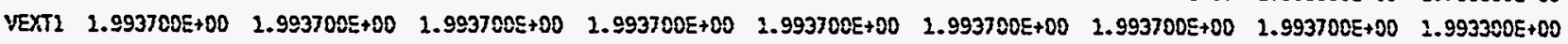

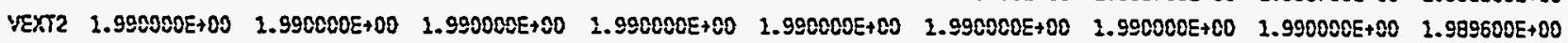

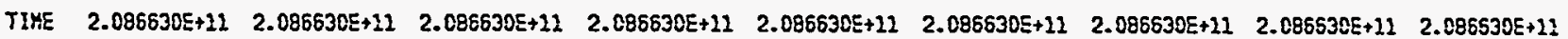


Page Description:

DATA SET HO.-238

TRAYS RY_ZERO-1 RY_ZERO-2 RY_ZERO-3 RY_LOAD-1 RV_LOAD-2 $\quad$ RY_LOAD-3 RV_ZERO-4 RV_ZERO-5 RV_ZERO-6

$P E-02-6.939300 E-\$ 2 \quad-6.933800 E-02-8.954500 E-02 \quad 1.838600 E-02 \quad 1.272600 E-02 \quad 1.577400 E-02-8.874700 E-02 \quad-6.874600 E-02-6.880200 E-02$ $\begin{array}{llllllllll}P E-02 & 3.003100 E-02 & 3.024100 E-02 & 3.039000 E-02 & 1.230700 E-02 & 1.253200 E-01 & 1.243300 E-02 & 3.032900 E-02 & 3.030400 E-02 & 3.035300 E-02\end{array}$

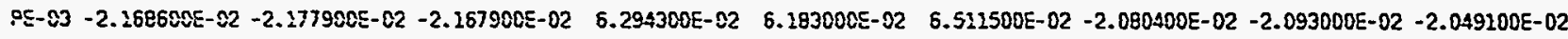
PE-04 4.036300E-02 3.996900E-02 $4.011100 E-02$ 2.327400E-01 $1.348300 E-01 \quad 1.353700 E-02 \quad 4.048600 E-02 \quad 4.024700 E-02 \quad 4.030500 E-02$ PE-05 T.622500E-02 $7.613600 E-02 \quad 7.626500 E-02 \quad 1.323500 E-01 \quad 1.323800 E-02 \quad 3.298200 E-01 \quad 7.654000 E-02 \quad 7.6 C 8100 E-02 \quad 1.640000 E-02$ PE- $06 \quad 7.004600 E-02 \quad 6.959700 E-02 \quad 5.930500 E-02 \quad 1.477900 E-02 \quad 1.450500 E-02 \quad 1.462100 E-01 \quad 7.116500 E-02 \quad 7.081700 E-02 \quad 7.059300 E-02$

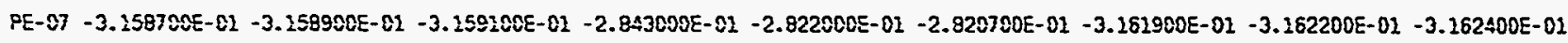
PE- 02 1.530700E-02 $2.521700 E-02 \quad 1.499800 E-02 \quad 8.134400 E-82 \quad 8.240100 E-02 \quad 7.929000 E-02 \quad 1.638900 E-02 \quad 1.626200 E-02 \quad 1.653600 E-02$

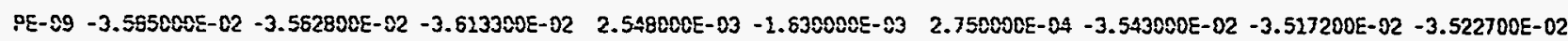
$\begin{array}{llllllllll}P E-10 & 1.919300 E-02 & 1.902300 E-02 & 1.899100 E-02 & 6.899000 E-02 & 6.697100 E-02 & 6.868300 E-02 & 1.925100 E-02 & 1.942100 E-02 & 1.981400 E-02\end{array}$ $\begin{array}{lllllllllll}95-13 & 2.422000 E-83 & 3.211000 E-83 & 7.3300005-04 & 3.526000 E-02 & 3.697800 E-02 & 3.600000 E-02 & 3.177000 E-03 & 3.896000 E-03 & 4.557000 E-83\end{array}$

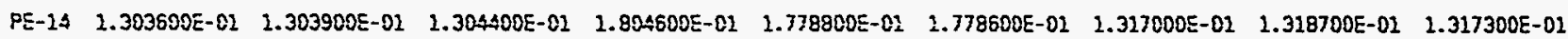
PE- 15 - $1.408 E 00 E-02-1.432500 E-02$ - $2.456000 E-02 \quad 5.240080 E-03 \quad 4.616000 E-03 \quad 5.282000 E-03 \quad-1.440600 E-02 \quad-1.418600 E-02 \quad-1.385700 E-02$ $\begin{array}{llllllllll}P E-16 & 3.847500 E-02 & 3.830900 E-02 & 3.817200 E-02 & 5.555100 E-02 & 5.621300 E-02 & 5.688200 E-02 & 3.889300 E-02 & 3.898100 E-02 & 3.903700 E-02\end{array}$ PE- $27-3.4071000-02-3.432000 E-02-3.427800 E-02-2.888500 E-02-1.8914005-02-1.824300 E-02-3.433600 E-02-3.441600 E-02-3.417400 E-02$ PE-18 -1.292340E-01 - $1.193800 E-02-1.193090 E-01$-9.997000E-02 $-9.969200 E-02-9.805700 E-02-2.187290 E-01-2.187170 E-01-1.288180 E-01$ PE-19 - $4.937100 E-02-4.935200 E-02-4.8524005-02-4.497200 E-82-4.5843005-82-4.535200 E-02-4.779300 E-02-4.822600 E-02-4.776900 E-02$ $P E-20-4.667900 E-02-4.689800 E-02-4.660800 E-02-4.355200 E-02-4.214700 E-02-4.323000 E-02-4.705700 E-02-4.678600 E-02-4.672500 E-02$ PE- 21 -8. $\$ 42000 E-83-8.561000 E-03-8.767009 E-03-2.026000 E-03 \quad-5.1700005-04-8.600000 E-05-8.877000 E-03-8.644000 E-03-8.449000 E-03$ PE-22 - $1.852000 E-03$ - $1.595000 E-03 \quad-1.783000 E-03 \quad 5.247000 E-03 \quad 3.860000 E-03 \quad 5.851000 E-03-2.131000 E-03 \quad-1.968000 E-03 \quad-1.549000 E-03$ $\begin{array}{cccccccccc}P E-29 & 6.756400 E-02 & 6.903600 E-02 & 6.832200 E-02 & 2.139000 E-01 & 2.013100 E-01 & 2.618400 E-01 & 6.487000 E-02 & 6.592400 E-02 & 6.649200 E-02\end{array}$

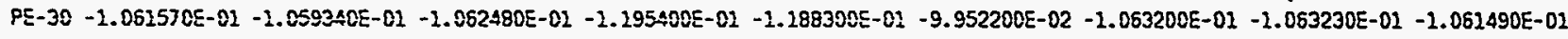

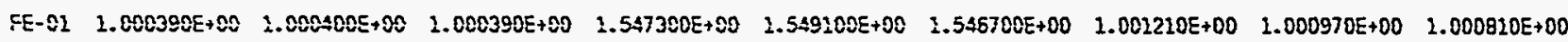
XE-01 -2.980000E-04 -2.970000E-04 -2.990000E-04 -5.800000E-05 $-8.500000 E-05-5.700000 E-05-2.950000 E-04-2.920000 E-04-3.000000 E-04$ $X E-02-8.080000 E-04-8.070000 E-04-8.070000 E-04-8.020000 E-04-8.0300005-04-8.030000 E-04-8.200000 E-04-8.100000 E-04-8.110000 E-04$

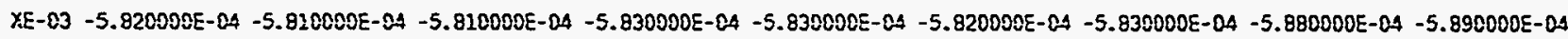
XE-04 - - $.410000 E-03-2.308000 E-03-1.409000 E-03-1.292000 E-03-1.296000 E-03-1.295000 E-03-1.411000 E-03-1.411000 E-03-1.412000 E-03$

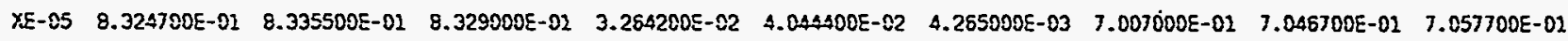
$X E-06$ 9. $X E-07-1.780000 E-04-1.750000 E-04-2.730000 E-04-3.490000 E-04-3.460000 E-04-3.400000 E-04-3.250000 E-04-3.200000 E-04-3.040000 E-04$ XE-08 - $1.0360005-03-1.0360085-03-1.036000 E-03-1.0080005-03-1.0090005-03-1.007000 E-03-1.039000 E-03-1.038000 E-03-1.039000 E-03$ $X E-09-1.226000 E-03-1.226000 E-03-1.226000 E-03-1.158000 E-03-1.153000 E-03-1.154000 E-03-1.213000 E-03-1.213000 E-03-1.213000 E-03$ $X E-10-6.1990005-03-6.201000 E-03-6.200000 E-03-4.500300 E-03-3.543000 E-03-3.459000 E-03-5.815000 E-03-5.829000 E-03-5.837000 E-03$ $\begin{array}{llllllllll}X E-11 & 2.500000 E-05 & 2.500000 E-05 & 2.600000 E-05 & 2.500000 E-05 & 2.500000 E-05 & 2.600000 E-05 & 2.500000 E-05 & 2.600000 E-05 & 2.600000 E-05\end{array}$ XE- $12-1.745000 E-03-1.7440005-03-1.743000 E-03-1.748000 E-03-1.748000 E-03-1.748000 E-03-1.753000 E-03-1.751000 E-03-1.751000 E-03$ $X E-13-4.850000 E-04-5.100000 E-04-4.930000 E-04-1.280000 E-04-1.280000 E-04-1.260000 E-04-4.1900005-04-4.020000 E-04-4.240000 E-04$ XE-14 -2.920000E-04 -2.960000E-04 -2.830000E-04 -2.350000E-04 -2.350000E-04 -2.310000E-04 -3.280000E-04 -2.710000E-04 -2.660000E-04 XE- $25-7.990000 E-04-8.000000 E-04-8.010000 E-04-4.910000 E-04-4.930000 E-04-4.890000 E-04-8.070000 E-04-8.110000 E-04-8.090000 E-04$ TE- $01-3.800000 E-05-3.9000005-05-3.900000 E-05-2.300000 E-05-2.500000 E-05-2.000000 E-05-4.800000 E-05-5.500000 E-05-5.300000 E-05$ VEXT2 $1.993300 E+00 \quad 2.993200 E+00 \quad 1.993300 E+00 \quad 1.993700 E+00 \quad 1.993700 E+00 \quad 1.993700 E+00 \quad 1.993300 E+00 \quad 1.993300 E+00 \quad 1.993300 E+00$

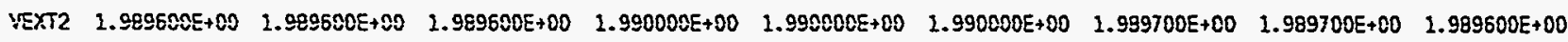
$\begin{array}{lllllllll}\text { TIME } 2.885630 E+12 & 2.886630 E+11 & 2.086630 E+12 & 2.086530 E+12 & 2.086630 E+12 & 2.086630 E+12 & 2.086630 E+12 & 2.086630 E+12 & 2.086630 E+11\end{array}$ 
Archive Meare:

AUS_MFP_TESTS

8cok Mame: AHS_MFPTI_GN

Qate \& Time: 1 Mar 1900 23:42:23

Page Eescription:

DATA SET MD.-239

TRAYS RV_ZERO-1

RN_ZERO-3

RY_LOAD-1

RY_LOAD-2

RY_LOAD-3

RY_ZERO-4

RY_ZERO-5

RV_ZERD-6

PE-01 -6.9696COE-02 -6.990500E-02 -6.986100E-02 3.984600E-02 $4.256200 E-02 \quad 3.616800 E-02-6.925600 E-02 \quad-6.939300 E-02 \quad-5.945600 E-02$ PE-02 2.974200E-02 2.979500E-02 2.980600E-02 $1.515500 E-02 \quad 1.540300 E-01 \quad 1.469600 E-01 \quad 2.987900 E-02 \quad 3.008800 E-02 \quad 3.018500 E-02$ PE-03 -2.19840EE-02 -2.200009E-02 -2. $293908 E-02 \quad 8.798200 E-02 \quad 8.711900 E-02 \quad 8.857400 E-02 \quad-2.075000 E-02 \quad-2.099500 E-02 \quad-2.111900 E-02$ PE-04 3.981600E-02 $3.980500 E-02 \quad 3.981300 E-02 \quad 1.580900 E-01 \quad 1.582000 E-01 \quad 1.639900 E-01 \quad 3.990700 E-02 \quad 3.994000 E-02 \quad 3.993000 E-02$

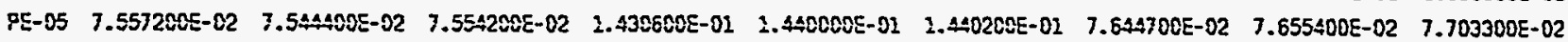
PE-C6 $8.931300 E-02 \quad 6.925100 E-02 \quad 6.920200 E-02 \quad 1.651400 E-02 \quad 1.698500 E-02 \quad 1.674300 E-01 \quad 7.026200 E-02 \quad 7.049400 E-02 \quad 3.075000 E-02$

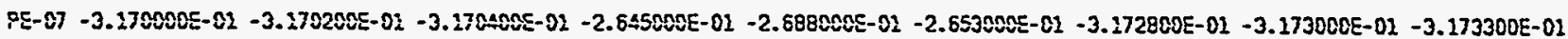
$\begin{array}{lllllllllll}P E-08 & 2.289200 E-02 & 2.28050 E E-02 & 1.28930 C E-02 & 9.970800 E-02 & 1.009610 E-02 & 1.022250 E-01 & 1.363700 E-02 & 1.366000 E-02 & 1.376300 E-02\end{array}$ PE-09 -3.63990CE-02 -3.6505CQE-02 $-3.6510000 E-02 \quad 3.042700 E-02 \quad 8.349000 E-03 \quad 8.032000 E-03 \quad-3.572900 E-02 \quad-3.585700 E-02 \quad-3.566400 E-02$ $\begin{array}{llllllllll}P E-10 & 1.837800 E-02 & 1.839600 E-02 & 1.829700 E-02 & 8.664200 E-02 & 8.227100 E-02 & 8.382500 E-02 & 1.895000 E-02 & 1.880500 E-02 & 1.903800 E-02\end{array}$ $\begin{array}{lllllllllll}P E-13 & 2.420000 E-03 & 2.208000 E-03 & 2.674000 E-03 & 4.579200 E-02 & 4.440500 E-02 & 4.706700 E-02 & 1.058008 E-03 & 1.291000 E-03 & 1.790000 E-03\end{array}$ PE- $24 \quad 1.305600 E-02 \quad 1.305100 E-02 \quad 1.303000 E-02 \quad 1.901800 E-02 \quad 1.905700 E-02 \quad 3.917700 E-01 \quad 1.310900 E-02 \quad 1.312400 E-01 \quad 1.314700 E-02$

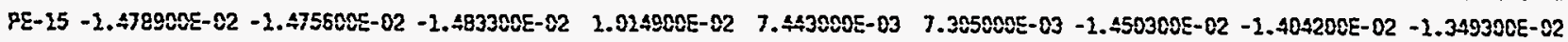
PE-16 3.832800E-ع2 $3.394208 E-02 \quad 3.811300 E-02 \quad 5.962300 E-02 \quad 5.932500 E-02 \quad 5.922400 E-02 \quad 3.919600 E-02 \quad 3.881200 E-02 \quad 3.877200 E-02$ PE- $27-3.482400 E-02$ - $3.458500 E-02-3.483300 E-02$ - $2.632500 E-02-1.665080 E-02-1.701800 E-02-3.490900 E-02-3.465300 E-02$-3.453900E-02 PE-18 -1.199120E-01 - $1.195570 E-02-1.200000 E-01 \quad-9.451600 E-02-9.575000 E-02-9.528200 E-02-1.192250 E-02-1.195420 E-01-2.189370 E-02$ PE- $29-4.8568005-82-4.8652085-82-4.8531005-82-4.327 .008 E-02-4.616600 E-02-4.239000 E-02-4.831900 E-02-4.817600 E-02-4.791100 E-02$ PE-20 - 4.780500E-02 -4.767600E-02 - $4.7651005-02-4.081300 E-02-4.2336005-02-4.1536005-02-4.762800 E-02-4.732900 E-02-4.730300 E-02$ PE-21 -8.925000E-03 -8.904000E-03 -8.627000E-03 -2.910000E-03 3.964000E-03 $6.700000 E-04 \quad-9.078000 E-03-8.751000 E-03-8.672000 E-03$ $P E-22 \quad-3.050000 E-03 \quad-3.184000 E-03 \quad-3.284000 E-83 \quad 5.324000 E-03 \quad 7.542000 E-03 \quad 6.883000 E-03 \quad-2.935000 E-03 \quad-3.044000 E-03 \quad-2.818000 E-03$ PE-29 T.513500E-02 T.461000E-02 T.439800E-02 $2.952000 E-01 \quad 2.519400 E-01 \quad 1.577600 E-81 \quad 7.238600 E-02 \quad 7.342500 E-02 \quad 7.406900 E-02$

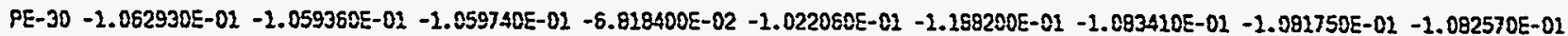
FE-01 $1.000380 E+00 \quad 2.000370 E+00 \quad 1.00037 C E+00 \quad 1.629500 E+00 \quad 1.529808 E+00 \quad 1.625700 E+00 \quad 1.001500 E+00 \quad 1.001110 E+00 \quad 1.000910 E+00$ YE-02 -3.090000E-04 -3.100000E-04 -3.220000E-04 -3.700000E-05 -3.800000E-05 -3.400000E-05 -2.870000E-04 -2.920000E-04 -2.930000E-04 $x E-02-8.100000 E-04-8.100000 E-04-8.100800 E-04-8.260800 E-04-8.080800 E-04$-8.080000E-04 -8.120000E-04 -8.140000E-04 -8.140000E-04 XE-03 -6.330000E-04 -6.330000E-04 -6.330000E-04 -6.350000E-04 -6.350000E-04 -6.360000E-04 -6.350000E-04 -6.360000E-04 -6.390000E-04 XE-04 -1.411000E-03 -1.410000E-03 -1.412800E-03 -1.283000E-03 -1.276080E-03 -1.277000E-03 - $1.404000 E-03-1.404000 E-03-1.406000 E-03$ XE-05 7.259800E-02 $7.262400 E-01 \quad 7.263200 E-01 \quad 1.433900 E-02 \quad 3.346000 E-03 \quad 1.565000 E-02 \quad 6.716800 E-02 \quad 6.737800 E-02 \quad 6.711100 E-01$ $X E-06$ 9.450300E-01 9.450300E-01 9.450200E-01 9.489900E-01 9.490400E-01 9.489800E-01 9.452400E-01 9.452300E-01 9.452490E-01 $X E-07-2.360000 E-04-2.080000 E-04-1.000000 E-04-3.400000 E-04-3.320000 E-04-3.320000 E-04-3.210000 E-04-3.0500005-04-2.950000 E-04$ $X E-08-1.036000 E-03-1.037000 E-03-1.035000 E-03-1.003000 E-03-1.001000 E-03-1.002000 E-03-1.038000 E-03-1.037000 E-03-1.038000 E-03$ $X E-09-1.217000 E-03-1.215000 E-03-1.216000 E-03-1.147000 E-03-1.145000 E-03-1.145000 E-03-1.211000 E-03-1.2090005-03-1.210000 E-03$ $X E-10-5.911000 E-03-5.913000 E-03-5.9180005-03-3.461000 E-03-3.419000 E-03-3.47 \$ 000 E-03-5.729000 E-03-5.742000 E-03-5.741000 E-03$ XE-11 2.500000E-05 2.500000E-05 2.500000E-05 2.500000E-05 2.500000E-05 2.600000E-05 $2.500000 E-05 \quad 2.600000 E-05 \quad 2.600000 E-05$ XE- $12-1.745000 E-03-1.746000 E-03-1.746000 E-03-1.744000 E-03-1.746800 E-03-1.747000 E-03-1.753000 E-03-1.752000 E-03-1.7520005-03$ XE-13 -5.560000E-04 -5.460000E-04 -5.550000E-04 -1.120000E-04 -1.130000E-04 -1.070000E-04 -4.050000E-04 -4.290000E-04 -4.550000E-04 XE-14 -2.900000E-04 -2.910000E-04 -2.930300E-04 -2.290000E-04 -2.280000E-04 -2.250000E-04 -2.840000E-04 -2.840000E-04 -2.870000E-04 XE-15 -8.070000E-04 -8.070000E-04 -8.060000E-04 -4.860000E-04 -4.800000E-04 -4.750000E-04 -8.080000E-04 -8.120000E-04 -8.160000E-04 TE-01 - T.3000005-05 - T.30C000E-05 - T.300000E- $05-5.300000 E-05-5.200000 E-05-4.900000 E-05-8.000000 E-05-7.800000 E-05-8.30 C 0005-05$ $\begin{array}{lllllllllll}\text { VEXT1 } & 1.993300 E+00 & 1.993300 E+00 & 1.993300 E+00 & 1.993700 E+00 & 1.993700 E+00 & 1.993700 E+00 & 1.993300 E+00 & 1.993300 E+00 & 1.993300 E+00\end{array}$ $\begin{array}{lllllllllll}\text { VEXT2 } & 1.989600 E+80 & 1.989600 E+00 & 1.999600 E+00 & 1.990000 E+00 & 1.990000 E+C 0 & 1.990000 E+C 0 & 1.989700 E+00 & 1.999700 E+00 & 1.989700 E+00\end{array}$

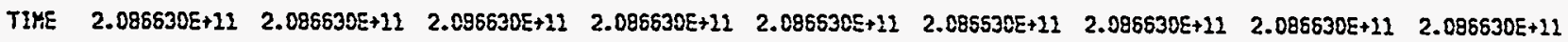


Arethite Heme:

AYS__:Pי_TESTS

8ocke Hame: ANS_MFPT1_RY

Date \& Time: 2 Mar 1900 23:58:21

Page Deseription:

DATA SET NO. -2\$0

TRAYS RY_ZERO-1 RV_ZERO-2 RY_ZERC-3 RY_LOAD-2 $\quad$ RY_LOAD-2 $\quad$ RY_LOAD-3 $\quad$ RV_ZERO-4 $\quad$ RV_ZERO-5 RV_ZERO-6

$9 E-01-7.039000 E-02-7.023608 E-02-7.030000 E-02 \quad 9.012300 E-02 \quad 8.235 \$ 00 E-02 \quad 8.410500 E-02 \quad-6.962000 E-02 \quad-5.947500 E-02 \quad-6.984900 E-02$

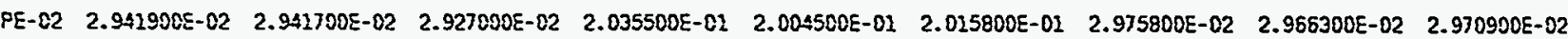
PE- 03 -2.220100E- 02 -2.246800E- $\$ 2$-2.246000E-02 2.340800E-01 1.341200E-01 $1.333000 E-01 \quad-2.142600 E-02$-2.120500E-02 $-2.143900 E-02$ PE-04 3.889900E-02 $3.925300 E-02 \quad 3.905300 E-02 \quad 2.072400 E-01 \quad 2.126000 E-01 \quad 2.098300 E-01 \quad 3.999400 E-02 \quad 3.923000 E-02 \quad 3.915500 E-02$ $\begin{array}{llllllllll}P E-05 & 7.537400 E-02 & 7.527400 E-02 & 7.525300 E-02 & 1.753000 E-02 & 1.750200 E-01 & 1.599400 E-01 & 7.618000 E-02 & 7.615900 E-02 & 7.606700 E-02\end{array}$ PS-06 $5.852300 E-02 \quad 5.844800 E-02 \quad 6.842500 E-02 \quad 2.153200 E-02 \quad 2.140200 E-01 \quad 2.032700 E-01 \quad 6.975100 E-02 \quad 6.979200 E-02 \quad 6.965800 E-02$

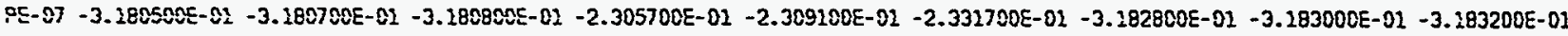
$\begin{array}{lllllllllll}P E-08 & 1.837000 E-02 & 1.025680 E-02 & 1.025200 E-02 & 1.350000 E-02 & 1.324200 E-01 & 1.357500 E-01 & 1.068800 E-02 & 1.099300 E-02 & 1.105000 E-02\end{array}$

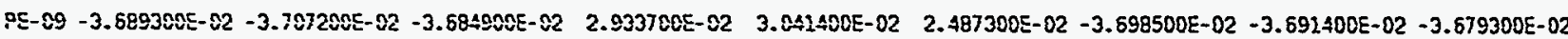
PE-10 $1.763100 E-02 \quad 1.798200 E-02 \quad 1.795500 E-02 \quad 1.093830 E-01 \quad 1.116740 E-02 \quad 1.121250 E-01 \quad 1.885000 E-02 \quad 1.893600 E-02 \quad 1.901300 E-02$

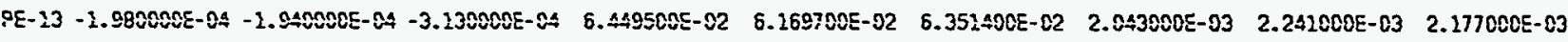

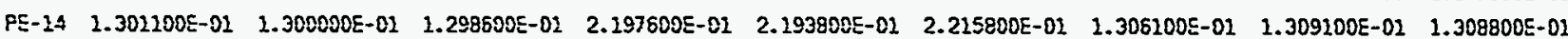
SE- 15 - $1 . .22000 E-02-1.446700 E-82-1.461800 E-02 \quad 1.692200 E-02 \quad 1.519700 E-02 \quad 1.763700 E-02 \quad-1.371400 E-02 \quad-1.359200 E-02 \quad-1.363300 E-02$ PE-16 3.834200E-02 $3.839000 E-02 \quad 3.828400 E-82 \quad 7.0952005-02 \quad 6.815000 E-02 \quad 6.802900 E-02 \quad 3.859200 E-02 \quad 3.879300 E-02 \quad 3.871500 E-02$

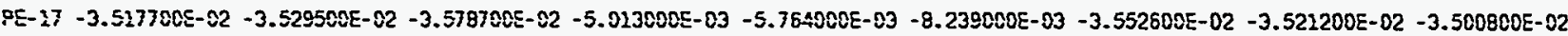
PE-18 - - 201400E-01 $-1.199690 E-02-1.196500 E-01-8.689000 E-02-8.447300 E-02-8.566200 E-02-1.192030 E-02-1.193780 E-02-1.192970 E-02$ $P E-19-4.920500 E-02-4.924400 E-02-4.992100 E-02-4.397100 E-02-4.333700 E-02-4.187100 E-02-4.871000 E-02-4.850900 E-02-4.856500 E-02$ $P E-20-4.801800 E-02-4.784000 E-82-4.793800 E-02-3.985300 E-02-3.963200 E-02-4.028000 E-02-4.828400 E-02-4.840500 E-02-4.799500 E-02$ PE-22 -8.728000E-03 -8.572000E-03 -8.684000E-03 5.624000E-83 $4.921000 E-03 \quad 1.659000 E-03-8.716000 E-03-8.718000 E-03-8.7260005-03$ PE-22 -3.775000E-03 - $4.095000 E-03-4.206000 E-03 \quad 1.065000 E-02 \quad 3.363400 E-02 \quad 7.964000 E-03 \quad-3.551000 E-03 \quad-3.570000 E-03 \quad-3.447000 E-03$ PE-29 7.582700E- $02 \quad 7.585500 E-02 \quad 7.588200 E-02 \quad 3.666200 E-01 \quad 5.873308 E-01 \quad 3.762108 E-02 \quad 7.353200 E-02 \quad 7.353200 E-02 \quad 7.372200 E-02$

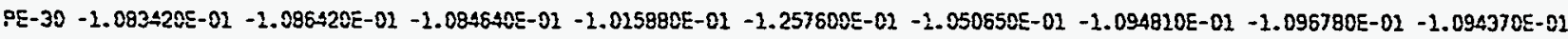

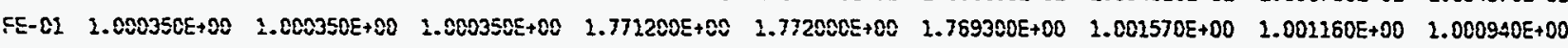
$x E-01-3.090000 E-04-3.090000 E-04-3.080000 E-04-2.500000 E-05-3.200000 E-05-2.400000 E-05-2.870000 E-04-2.860000 E-04-2.920000 E-04$

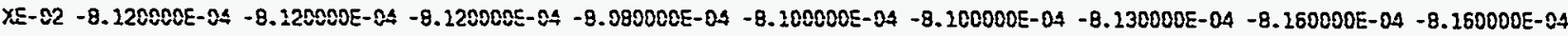
XE- $03-6.700000 E-04-6.700000 E-04-6.7200005-04-6.720000 E-04-6.720000 E-04-6.730000 E-04-6.730000 E-04-5.750000 E-04-6.740000 E-04$ XE-04 - $1.409080 E-03-1.409000 E-03-1.410000 E-03-1.250000 E-03-1.233000 E-03-1.233000 E-03-1.393000 E-03-1.397000 E-03-1.397000 E-03$ $X E-05 \quad 6.637400 E-02 \quad 6.655200 E-01 \quad 6.593400 E-01 \quad-2.340000 E-03 \quad-2.742000 E-03 \quad-3.098000 E-03 \quad 6.705900 E-01 \quad 6.731500 E-01 \quad 6.742400 E-01$ $X E-86 \quad 9.453580 E-01 \quad 9.453300 E-02 \quad 9.453100 E-01 \quad 9.489000 E-01 \quad 9.48 B 400 E-01 \quad 9.488600 E-02 \quad 9.448900 E-01 \quad 9.448800 E-01 \quad 9.4488 C 0 E-01$ $X E-07 \quad 4.400000 E-05 \quad 4.800000 E-05 \quad 4.400000 E-05-3.110000 E-04 \quad-3.070000 E-04-3.050000 E-04-3.100000 E-04 \quad-3.080000 E-04 \quad-2.880000 E-04$ $X E-98-2.036000 E-03-1.036000 E-03-1.0370005-03-9.970000 E-04-9.950000 E-04-9.950000 E-04-1.036000 E-03-1.0340005-03-1.035000 E-03$ XE-09 - $1.214000 E-03-1.215000 E-03-1.214000 E-03-1.136000 E-03-1.134000 E-03-1.231000 E-03-1.210000 E-03-1.207000 E-03-1.208000 E-03$ XE- $10-5.739000 E-03-5.745000 E-03-5.753000 E-03-3.371000 E-03-3.377000 E-03-3.378000 E-03-5.723000 E-03-5.726000 E-03-5.741000 E-03$ $\begin{array}{llllllllll}X E-11 & 2.500000 E-05 & 2.600000 E-05 & 2.600000 E-05 & 2.500000 E-05 & 2.500000 E-05 & 2.500000 E-05 & 2.700000 E-05 & 2.500000 E-05 & 2.600000 E-05\end{array}$ XE- $12-1.7470005-03-1.747000 E-03-2.7450005-83-1.743080 E-03-1.743000 E-03-1.744000 E-03-1.754000 E-03-1.752000 E-03-1.751000 E-03$ $X E-13-6.120000 E-04-6.130000 E-04-6.130000 E-04-6.400000 E-05-4.300000 E-05-3.300000 E-05-4.070000 E-04-4.590000 E-04-4.220000 E-04$ $X E-14-2.920000 E-04-2.800000 E-04-2.950000 E-04-2.138000 E-04-2.120000 E-04-2.200000 E-04-3.120000 E-04-2.810000 E-04-2.810000 E-04$ XEE-15 -8.090000E-04 -8.110000E-04 -8.080000E-04 -4.610000E-04 -4.570000E-04 - $4.530000 E-04-7.950000 E-04-7.750000 E-04-8.040000 E-04$ TE- $01-9.5000005-95-9.500000 E-05-9.4000005-05-7.100000 E-05-6.9000005-05-5.880000 E-05-9.600000 E-05-8.900000 E-05-1.050000 E-04$ YEXT1 $1.993300 E+00 \quad 1.993300 E+00 \quad 1.993300 E+00 \quad 1.993700 E+00 \quad 1.993700 E+00 \quad 1.993700 E+00 \quad 1.993300 E+00 \quad 1.993300 E+00 \quad 1.993300 E+00$ $\begin{array}{llllllllll}Y E X T 2 & 1.989700 E+00 & 1.989700 E+00 & 1.989700 E+00 & 1.990000 E+00 & 1.990000 E+00 & 1.990000 E+00 & 1.989700 E+00 & 1.989700 E+00 & 1.989700 E+00\end{array}$

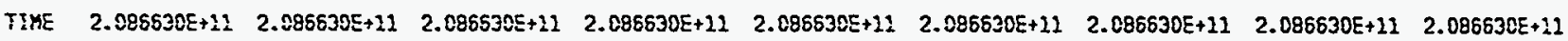


Page Description:

DATA SET N0.-241

TRAAS RY_ZERO-1 RV_ZERO-2 RV_ZERO-3 RY_LOAD-1 $\quad$ RY_LOAD-2 $\quad$ RV_LOAD-3 $\quad$ RV_ZERO-4 $\quad$ RV_ZERO-5 RV_ZERO-6

PE-01 -7.073400E-02 -7.075900E-02 -7.072800E-02 1.112400E-01 $1.149100 E-01 \quad 1.187700 E-01 \quad-6.978900 E-02 \quad-6.988700 E-02 \quad-7.006600 E-02$ $\begin{array}{llllllllll}P E-02 & 2.899300 E-02 & 2.934400 E-02 & 2.922900 E-02 & 2.433200 E-02 & 2.587400 E-02 & 2.545700 E-02 & 2.951900 E-02 & 2.974500 E-02 & 2.967200 E-02\end{array}$ PE-03 -2.216500E-02 -2.232900E-02 $-2.236100 E-02 \quad 1.8 C 8 A 00 E-01 \quad 1.780400 E-01 \quad 1.797800 E-01 \quad-2.195500 E-02 \quad-2.196100 E-02 \quad-2.175500 E-02$ $\begin{array}{llllllllll}P E-04 & 3.862500 E-02 & 3.842500 E-02 & 3.837900 E-02 & 2.501600 E-01 & 2.641800 E-01 & 2.522800 E-01 & 3.880300 E-02 & 3.897900 E-02 & 3.884200 E-02\end{array}$

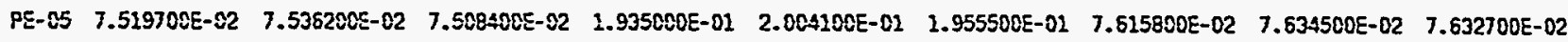
PE-06 5.772400E-02 $\quad 6.730400 E-02 \quad 6.743200 E-02 \quad 2.381400 E-02 \quad 2.394600 E-02 \quad 2.452200 E-01 \quad 6.840300 E-02 \quad 6.883000 E-02 \quad 6.880400 E-02$ PE- $07-3.189500 E-02-3.289608 E-01-3.189800 E-01-2.032900 E-02-2.036400 E-01-2.102 C 00 E-01-3.191500 E-02-3.291700 E-01-3.191900 E-01$ $\begin{array}{lllllllllll}P E-08 & 8.954000 E-03 & 8.685000 E-03 & 8.461000 E-03 & 1.695500 E-01 & 1.578300 E-01 & 1.716800 E-01 & 1.128300 E-02 & 1.139300 E-02 & 1.139600 E-02\end{array}$ PE-09 - 3.773900E-02 -3.770E00E-02 -3.772400E-02 4.602400E-02 $4.224400 E-02 \quad 4.374200 E-02-3.695700 E-02 \quad-3.683300 E-02 \quad-3.651500 E-02$ $\begin{array}{llllllllll}P E-10 & 1.785600 E-02 & 1.786800 E-02 & 1.801800 E-02 & 1.425900 E-01 & 1.454200 E-01 & 1.355600 E-02 & 1.887500 E-02 & 1.902100 E-02 & 1.900700 E-02\end{array}$ PE-13 5.990000E-04 $5.700000 E-04 \quad 6.320000 E-04 \quad 7.989000 E-82 \quad 7.819200 E-02 \quad 8.286200 E-02 \quad 7.760000 E-04 \quad 7.5800005-04 \quad 5.280000 E-04$ PE-14 $1.293500 E-02 \quad 1.293600 E-02 \quad 1.291800 E-02 \quad 2.412400 E-01 \quad 2.420700 E-01 \quad 2.369200 E-02 \quad 1.302500 E-02 \quad 1.303800 E-01 \quad 1.306300 E-02$

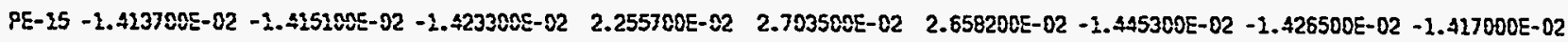
$\begin{array}{llllllllll}P E-25 & 3.798200 E-02 & 3.801300 E-02 & 3.830200 E-02 & 7.7099005-02 & 7.952100 E-02 & 7.843500 E-02 & 3.832500 E-02 & 3.876000 E-02 & 3.973000 E-02\end{array}$ PE-17 -3.550000E-02 -3.579600E-02 -3.554700E-02 -4.800000E-04 -1.570000E-04 -2.638000E-03 -3.530200E-02 -3.53今800E-02 -3.529800E-02 $P E-18-1.198290 E-02-1.205500 E-02-1.200500 E-02-7.481700 E-02-7.641900 E-02-7.703200 E-02-1.195040 E-01-1.195250 E-02-1.192850 E-01$ PE-19 - $4.907800 E-02-4.939800 E-02-4.930600 E-02-3.822100 E-02-4.2113205-02-4.064500 E-82-4.896900 E-02-4.905000 E-02-4.090600 E-02$ PE-20 - $4.832300 E-02-4.824300 E-02-4.817100 E-02-3.607400 E-02-3.630500 E-02-3.658200 E-82-4.804508 E-02-4.792500 E-02-4.785300 E-02$

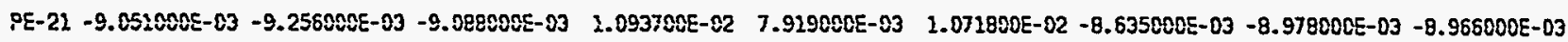
PE-22 - $4.416000 E-03 \quad-4.637000 E-03 \quad-4.374000 E-03 \quad 1.528600 E-02 \quad 3.227700 E-02 \quad 1.755200 E-02 \quad-4.3710005-03 \quad-4.272000 E-03 \quad-4.097000 E-03$

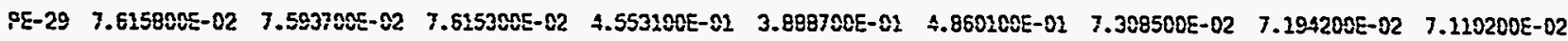

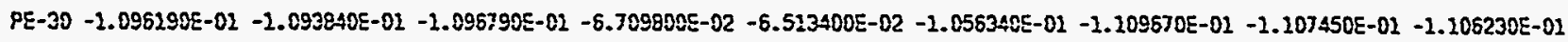
FE-02 $\quad 2.000350 E+00 \quad 2.000370 E+00 \quad 1.000360 E+00 \quad 1.887580 E+00 \quad 1.883300 E+00 \quad 1.891400 E+00 \quad 1.001550 E+00 \quad 2.001190 E+00 \quad 1.000970 E+00$ YE-01 -3.100000E- $04-3.080000 E-04-3.090000 E-04-2.000000 E-05-9.000000 E-06-2.000000 E-05-2.890000 E-04-2.940000 E-04-2.990000 E-04$ XE-02 -8.140000E-04 -E. 240000E-04 -8.240000E-04 -8.130000E-04 -8.120000E-04 -8.130080E-04 -8.180000E-04 -8.200000E-04 -8.200000E-04 XE- $03-6.880000 E-04-6.870000 E-04-6.870 C 00 E-04-6.870000 E-04-6.880000 E-04-6.880000 E-04-6.880000 E-04-6.890000 E-04-6.880000 E-04$ $X E-04-1.398000 E-03-1.398000 E-03-1.398000 E-03-1.173000 E-03-2.178000 E-03-1.273080 E-03-1.387000 E-03-1.388000 E-03-1.391000 E-03$ $X E-05 \quad 6.834300 E-01 \quad 6.833900 E-01 \quad 6.835800 E-01 \quad 8.359400 E-02 \quad-3.920000 E-04$-1.594000E-03 -3.620000E-03 -3.623000E-03 $-3.624000 E-03$ XE-06 $9.4489005-01$ 9.448900E-01 $9.448900 E-01 \quad 9.472500 E-01$ 9.469800E-01 $9.453400 E-01 \quad 9.441000 E-01 \quad 9.441000 E-01 \quad 9.441000 E-01$ $X E-07-6.900000 E-05-7.800000 E-05-7.800000 E-05-2.890000 E-04-2.770000 E-04-2.740000 E-04-2.940000 E-04-2.870000 E-04-2.780000 E-04$ $X E-08-2.0340005-03-1.035000 E-03-1.0360005-03-9.890000 E-04-9.920000 E-03-9.900000 E-04-1.030000 E-03-1.031000 E-03-1.0310005-03$ $X E-09-1.223000 E-03-1.212000 E-03-1.213000 E-03-1.124000 E-03-1.123000 E-03-1.124000 E-03-1.205000 E-03-1.206000 E-03-1.207000 E-03$ YE-10 -5.790000E-03 -5.789000E-03 $-5.790000 E-03-3.504000 E-03-3.366000 E-03-3.354000 E-03-3.822000 E-03-3.827000 E-03-3.841000 E-03$ $\begin{array}{llllllllll}X E-12 & 2.500000 E-05 & 2.500000 E-05 & 2.600000 E-05 & 2.500000 E-05 & 2.500000 E-05 & 2.600000 E-05 & 2.500000 E-05 & 2.500000 E-05 & 2.700000 E-05\end{array}$

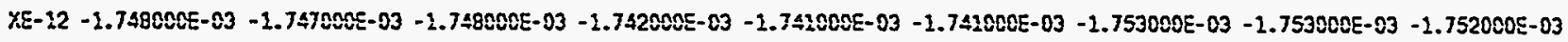
XE-13 -5.140000E-04 -5.260000E-04 -5.290000E-04 -2.500000E-05 - $9.000000 E-06$-2.000000E-06 $-3.900000 E-04-3.970000 E-04$-4.410000E-04 XE-14 -2.86C000E-04 -2.890000E-04 -2.870000E-C4 -1.900000E-04 -1.940000E-04 -1.850000E-04 -2.990000E-04 -2.810000E-04 -2.790000E-04 XE-15 -7.980000E-04 -8.020000E-04 -8.010000E-04 -4.160000E-04 -4.260000E-04 -4.290000E-04 -7.920000E-04 -7.940000E-04 -8.030000E-04 TE-02 - $-1.090000 E-04-1.0900005-04-2.090000 E-04-9.400000 E-05-8.300000 E-05-8.400000 E-05-1.070000 E-04-1.180000 E-04-1.130000 E-04$ $\begin{array}{llllllllllll}\text { VEXT2 } & 1.993300 E+00 & 1.993300 E+00 & 1.993300 E+00 & 1.993500 E+00 & 1.993700 E+00 & 1.993700 E+00 & 1.993700 E+00 & 1.993700 E+00 & 1.993700 E+00\end{array}$ $\begin{array}{lllllllllll}\text { VEXT2 } & 1.989703 E+00 & 1.989700 E+00 & 1.989700 E+C 0 & 2.990809 E+C 0 & 1.990000 E+00 & 1.990080 E+C 0 & 1.990000 E+00 & 1.990 C 00 E+00 & 1.990000 E+00\end{array}$

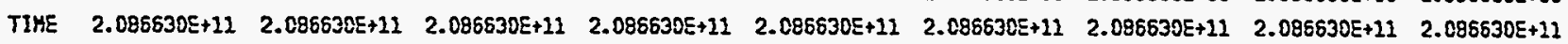




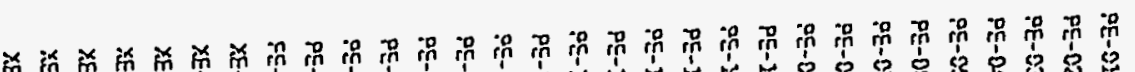

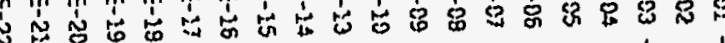

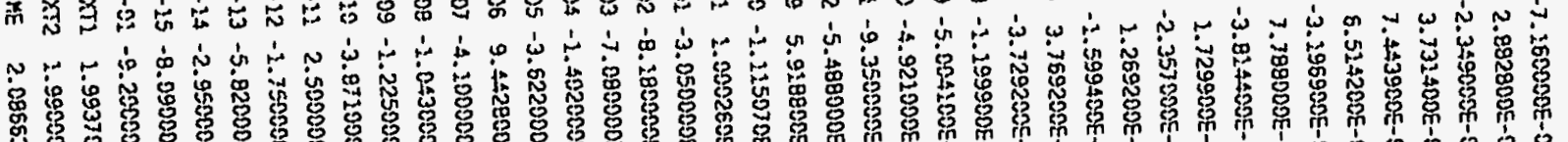

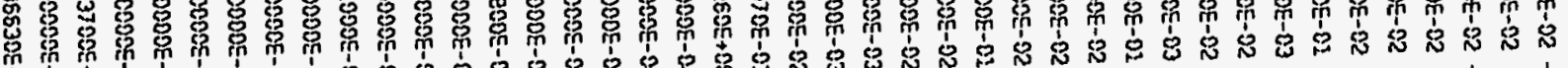

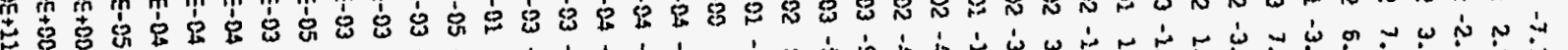

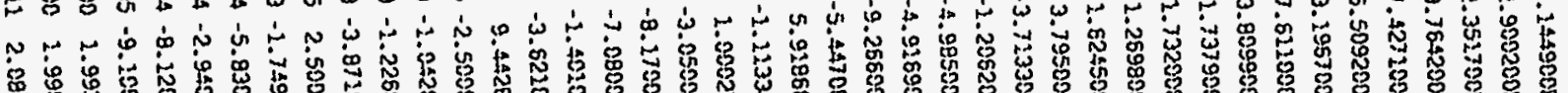

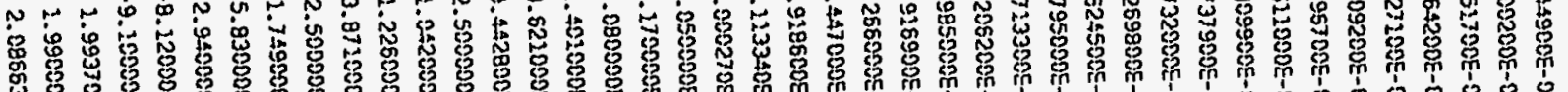

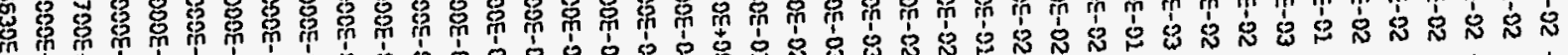

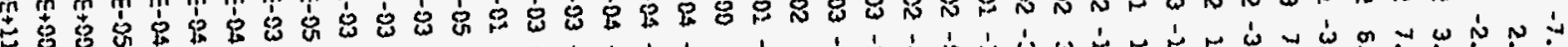
N

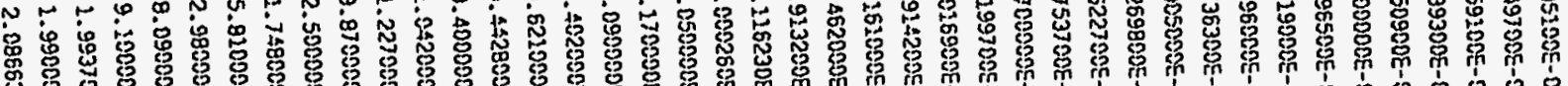

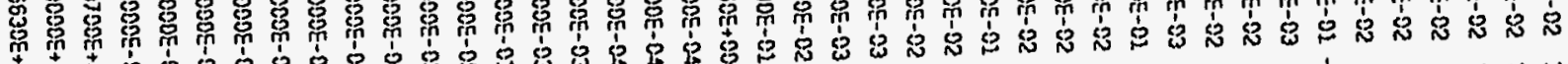

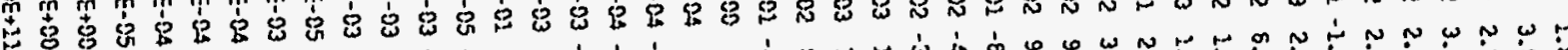

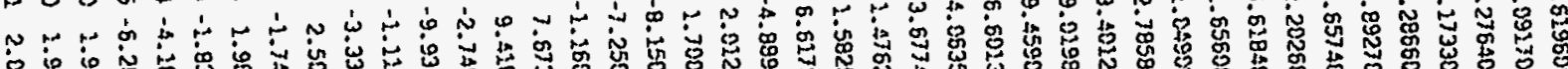

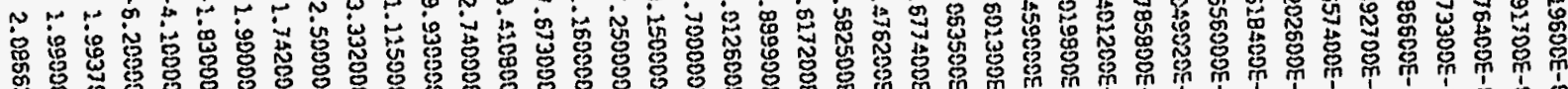

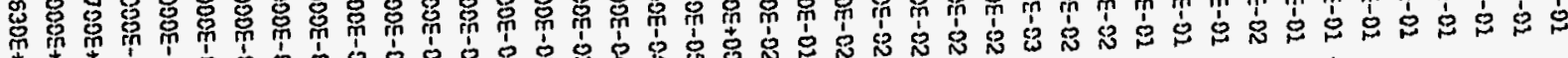

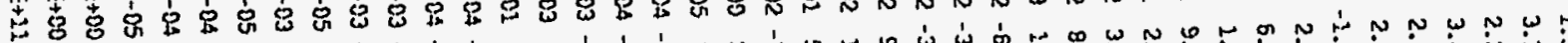

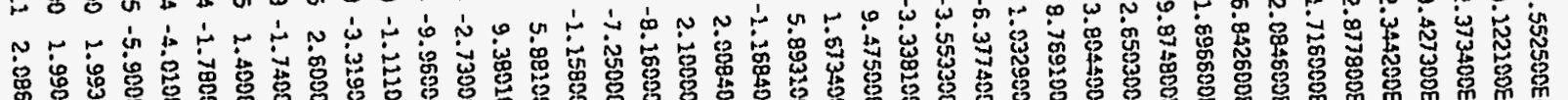

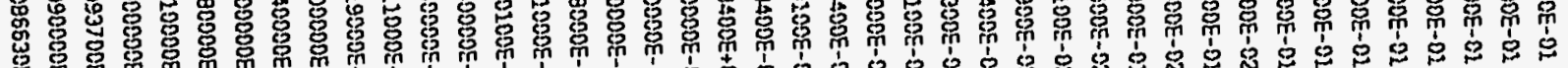

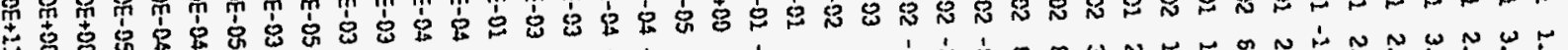

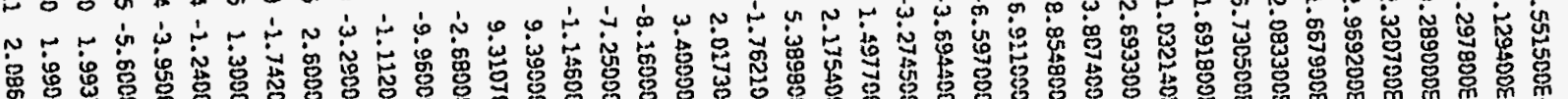

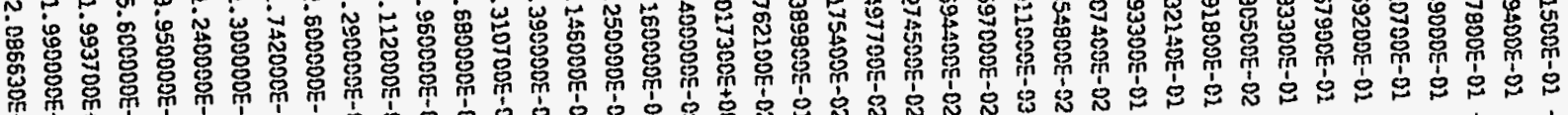

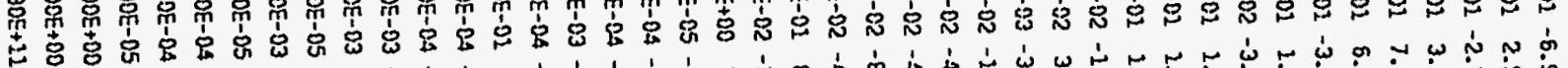

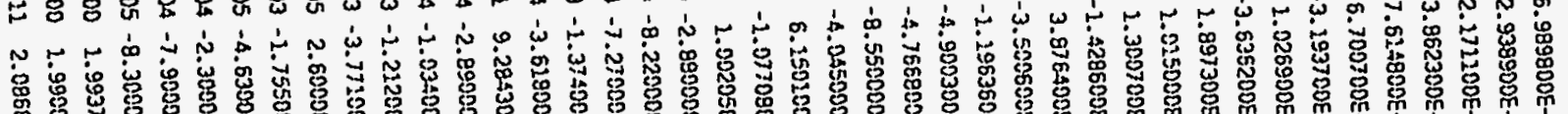

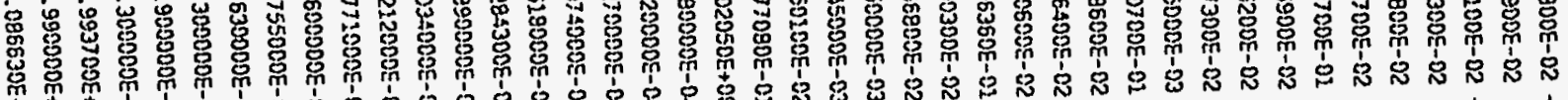

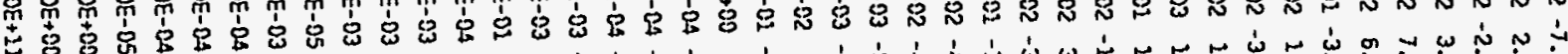
N-

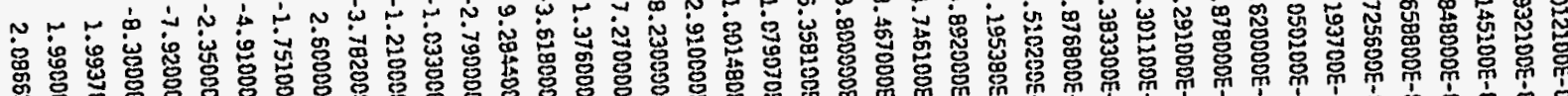

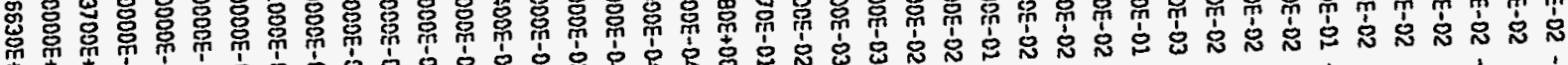

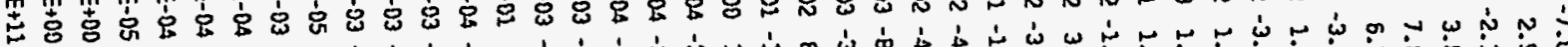

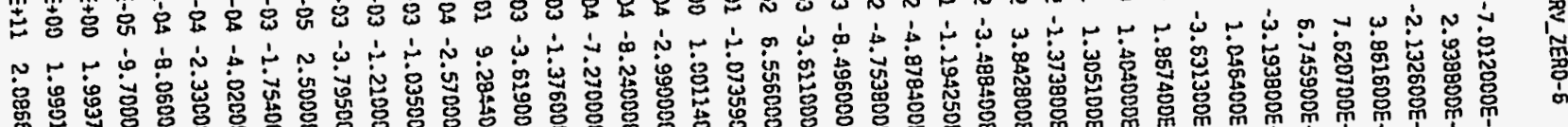
. 
Page Description:

DATA SET HO. -243

TRAYS RY_ZERO-1 RY_ZERO-2 RY_ZERO-3 RV_LOAD-2 RY_LOAD-2 $\quad$ RE_LOAD-3 RY_ZERO-4 $\quad$ RN_ZERO-5 RY_ZERO-6

PE-02 -7.146800E-02 -7.255700E-02 -7.171300E-02 1.908600E-02 2.077700E-01 $1.982900 E-02$-7.029800E-02 -7.021000E-02 -7.032400E-02 PE-02 2.993200E-02 2.859600E-02 $2.881200 E-02 \quad 3.446600 E-01 \quad 3.651400 E-01 \quad 3.537400 E-02 \quad 2.974400 E-02 \quad 2.972200 E-02 \quad 2.956200 E-02$ PE-03 -2.225300E-02 -2.226800E-02 -2.2366C0E-02 2.655ACOE-01 2.832800E-01 2.703900E-02 - $1.926300 E-02 \quad-1.915100 E-02 \quad-1.878400 E-02$ PE-04 3.810700E-02 $3.785900 E-02 \quad 3.774100 E-02 \quad 3.740000 E-02 \quad 3.704100 E-02 \quad 3.794400 E-01 \quad 3.822800 E-02 \quad 3.864800 E-02 \quad 3.847900 E-02$

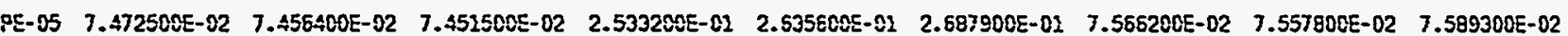
$\begin{array}{llllllllll}\text { PE- } 06 & 5.587000 E-02 & 6.639200 E-02 & 5.611500 E-02 & 3.193300 E-01 & 3.328 A 00 E-01 & 3.429200 E-02 & 6.721000 E-02 & 6.747000 E-02 & 6.694900 E-02\end{array}$

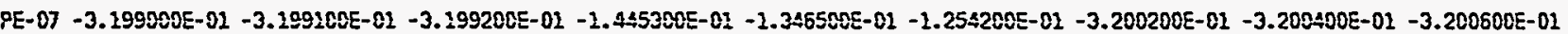
PE-00 $9.851009 E-03 \quad 9.801000 E-03 \quad 9.820000 E-03 \quad 2.442900 E-01 \quad 2.551300 E-02 \quad 2.528500 E-01 \quad 1.055700 E-02 \quad 1.049600 E-02 \quad 1.030000 E-02$ PE- $99-3.702300 E-02$-3.691700E-02 -3.684000E-02 $9.658400 E-02 \quad 7.804900 E-02 \quad 9.153400 E-02-3.616700 E-02 \quad-3.610900 E-02-3.622200 E-02$ $P E-10 \quad 1.911100 E-02 \quad 1.823100 E-02 \quad 1.82250 \Omega E-02 \quad 2.120600 E-02 \quad 2.00 \Omega 500 E-01 \quad 1.958100 E-01 \quad 1.895200 E-02 \quad 1.858900 E-02 \quad 1.862600 E-02$

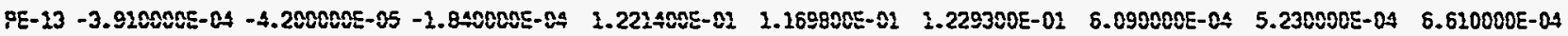
PE-14 1.288200E-02 2.288100E-01 $1.291200 E-01 \quad 2.981300 E-02 \quad 2.9336005-01 \quad 2.996900 E-02 \quad 1.3021005-01 \quad 1.304500 E-01 \quad 1.305400 E-01$

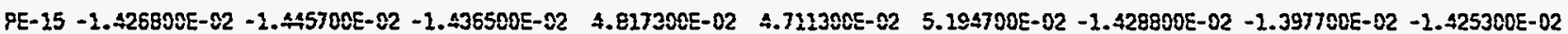
$\begin{array}{lllllllllll}P E-16 & 3.825300 E-02 & 3.815500 E-02 & 3.836800 E-02 & 9.816900 E-02 & 1.029340 E-01 & 1.011620 E-02 & 3.900800 E-02 & 3.903200 E-02 & 3.909400 E-02\end{array}$

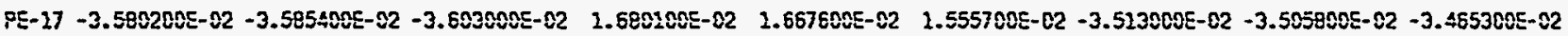

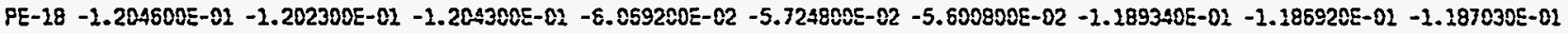
PE-19-4.950000E-02 - $4.951700 E-02-4.911900 E-02-3.459300 E-02-3.339200 E-02-3.125800 E-02-4.845300 E-02-4.876000 E-02-4.854400 E-02$ $P E-20-4.812100 E-02-4.829200 E-02-4.822400 E-02-2.658400 E-02-2.595900 E-02-3.025800 E-02-4.805900 E-02-4.789600 E-02-4.741500 E-02$ PE-21 -8.318000E-03 -8.724000E-03 -8.753000E-03 2.20E100E-02 2.189300E- $02 \quad 1.9292005-02-8.278000 E-03-8.371000 E-03-8.246000 E-03$ PE-22 -4.300000E-03 - $4.5250 C 0 E-03 \quad-4.332000 E-03 \quad 2.451980 E-02 \quad 2.2219005-02 \quad 2.431500 E-02 \quad-3.818000 E-03 \quad-3.487000 E-03 \quad-3.385000 E-03$ PE-29 7.742000E-02 7.703700E-02 $7.693900 E-02 \quad 6.722700 E-01 \quad 4.565200 E-01 \quad 5.357200 E-01 \quad 7.620600 E-02 \quad 7.633900 E-02 \quad 7.658300 E-02$ $P E-30-1.076860 E-02-1.073220 E-02-1.077240 E-02-2.2039000 E-02-6.273300 E-02-1.362500 E-02-1.109290 E-01-2.108440 E-01-1.109550 E-01$ FE-02 $2.000320 E+00 \quad 1.000320 E+00 \quad 1.000310 E+00 \quad 2.116900 E+08 \quad 2.113200 E+00 \quad 2.126400 E+00 \quad 1.001760 E+00 \quad 1.001280 E+00 \quad 1.001020 E+00$ XEE-02 -2.980000E-04 -2.980000E-04 -2.970000E-04 4.500000E-05 2.900000E-05 2.800000E-05 -3.080000E-08 $-3.210000 E-04-3.090000 E-04$ XE- 22 -8.220000E-04 -8.220000E-04 -8.210000E-04 -8.210000E-04 -8.200000E-04 -8.210000E-04 -9.250000E-04 -8.270000E-04 -8.280000E-04 XE-03 -7.400000E-04 -7.400000E-04 -7.400000E-04 -7.420000E-04 -7.420000E-04 -7.420000E-04 -7.430000E-04 -7.430000EE-04 -7.440000E-04 XE- $04-1.387000 E-03-1.386000 E-03-1.386000 E-03-1.123000 E-03-1.131000 E-03-1.127000 E-03-1.353200 E-03-1.355000 E-03-1.355000 E-03$ $X E-85-3.617000 E-03-3.617000 E-83-3.615000 E-03-3.476000 E-83-3.4830005-03-3.484000 E-83-3.633000 E-03-3.633000 E-03-3.635000 E-03$

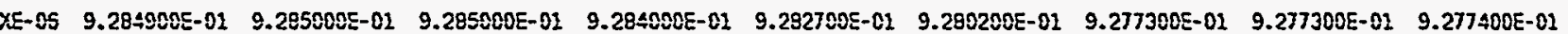
XE-07 -1.650000E-04 -1.720000E-04 -2.720000E-04 -2.580000E-04 -2.540000E-04 -2.440000E-04 -2.070000E-04 -2.050000E-04 -2.120000E-04 XE-08 - $1.039000 E-03-1.039000 E-03-1.039000 E-03-9.900000 E-04-9.910000 E-04-9.930000 E-04-1.027000 E-03-1.028000 E-03-1.029000 E-03$ $X E-09-1.218000 E-03-1.217000 E-03-1.217000 E-03-1.103000 E-03-1.0950005-03-1.103000 E-03-1.2050005-03-1.204000 E-03-1.203000 E-03$ $X E-10-3.814000 E-03-3.012000 E-03-3.809000 E-03-3.2720005-03-3.273000 E-03-3.270000 E-03-3.799000 E-03-3.799000 E-03-3.800000 E-03$ XE-11 $2.500000 E-05 \quad 2.500000 E-05 \quad 2.600000 E-05 \quad 2.5000005-05 \cdot 2.500000 E-05 \quad 2.600000 E-05 \quad 2.600000 E-05 \quad 2.700000 E-05 \quad 2.600000 E-05$ $X E-12-1.750000 E-03-1.749000 E-03-1.749000 E-03-1.738000 E-03-1.741000 E-03-1.740000 E-03-1.752000 E-03-1.753000 E-03-1.753000 E-03$ XE-13 -5.360000E-04 -5.090000E-04 -5.000000E-04 4.000000E-05 4.100000E-05 5.500000E-05 -3.750000E-04 -3.940000E-04 -4.220000E-04

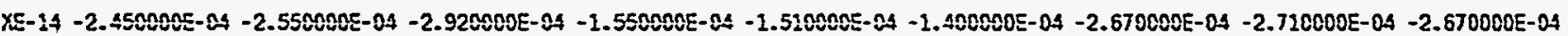
XE-25 -8.110000E-04 -9.070000E-04 -8.090000E-04 -3.930000E-04 -3.950000E-04 -3.890000E-04 -7.950000E-04 -9.0300000E-04 -7.990000E-04 TE-01 - $1.0500005-04-1.050000 E-04-1.060000 E-04-8.200000 E-05-7.9000085-05-7.900000 E-05-1.000000 E-04-1.190000 E-04-1.170000 E-04$

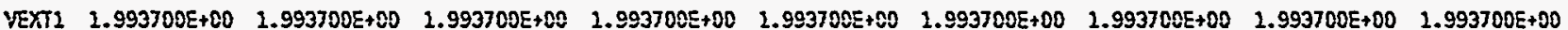

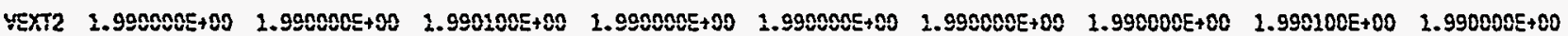
TIME $2.086630 E+112.086630 E+12 \quad 2.086530 E+12 \quad 2.086630 E+12 \quad 2.085630 E+12 \quad 2.086630 E+112.086530 E+112.085630 E+112.086630 E+11$ 
irchive Hzere:

AHS_MP_icSTS

Eook :ame: AHS_MFPT1_RY Date \& Time: 2 Mar 1900 D0:31:29

Page Description:

DATA SET HO. -24S

TRANS RY_ZERO-1 RY_ZERO-2 RY_ZERO-3 RY_LOAD-1 RY_LOAD-2 RV_LOAD-3 RV_ZERO-4 RY_ZERO-5 RV_ZERO-5

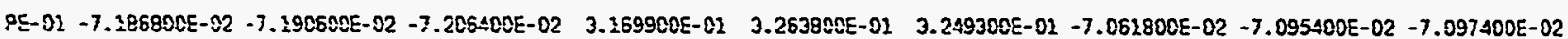
PE-02 2.851200E-02 2.841700E-02 $2.850000 E-02 \quad 5.161300 E-01 \quad 5.074100 E-01 \quad 5.236300 E-02 \quad 2.908700 E-02 \quad 2.930000 E-02 \quad 2.895500 E-02$ PE- 03 -1.966800E-02 - $2.974200 E-02$-2.006500E-02 4.383300E-01 4.013900E-01 4.074800E-01 -1.865800E-02 -2.869300E-02 - $1.830300 E-02$ PE-04 $3.755900 E-02 \quad 3.731400 E-02 \quad 3.750900 E-02 \quad 5.360600 E-01 \quad 5.013600 E-01 \quad 5.209300 E-01 \quad 3.825700 E-02 \quad 3.848700 E-02 \quad 3.821100 E-02$ PE-05 7.436300E-02 $7.425900 E-02 \quad 7.425200 E-02 \quad 3.535800 E-01 \quad 3.292400 E-01 \quad 3.292100 E-01 \quad 7.554400 E-02 \quad 7.602100 E-02 \quad 7.608800 E-02$

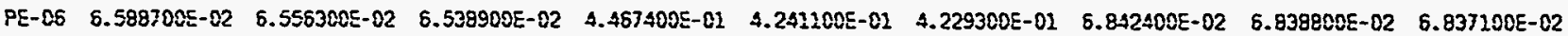

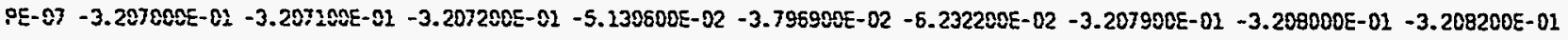
$P E-08 \quad 9.439000 E-03 \quad 9.497000 E-03 \quad 9.500000 E-03 \quad 3.511200 E-01 \quad 3.422100 E-02 \quad 3.511300 E-01 \quad 1.108600 E-02 \quad 2.095100 E-02 \quad 1.097700 E-82$ FE- $02-3.3181005-02-3.739500 E-02 \quad-3.704000 E-02 \quad 3.325700 E-01 \quad 1.275100 E-01 \quad 1.311000 E-01 \quad-3.706300 E-02 \quad-3.633300 E-02-3.657300 E-02$ $\begin{array}{llllllllll}\text { PE-10 } & 1.817400 E-02 & 2.792600 E-02 & 1.803500 E-02 & 2.746100 E-01 & 2.754300 E-02 & 2.035800 E-01 & 1.776300 E-02 & 1.775600 E-02 & 1.775200 E-02\end{array}$

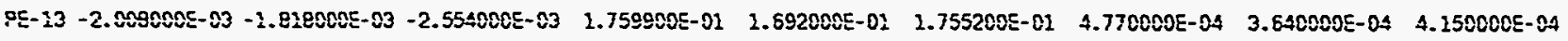

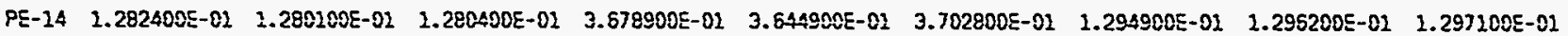

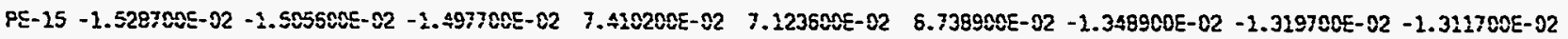
PE-16 3.837800E-02 $3.803000 E-02 \quad 3.796280 E-02 \quad 1.319500 E-01 \quad 1.2729005-01 \quad 1.215600 E-01 \quad 3.861400 E-02 \quad 3.827100 E-02 \quad 3.859100 E-02$ PE- 17 - 3.559800E-02 $-3.551400 E-02-3.580400 E-02 \quad 3.430400 E-02 \quad 3.540700 E-02 \quad 2.913800 E-02 \quad-3.510500 E-02 \quad-3.512200 E-02 \quad-3.522000 E-02$

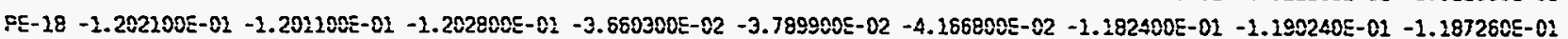

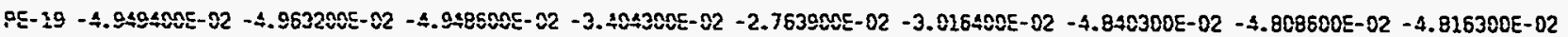
PE-20 - 4.831900E-02 -4.824400E-02 -4.839900E-02 -2.461800E-02 -2.166700E-02 - $1.590200 E-02-4.800100 E-02-4.722000 E-02-4.752400 E-02$ $9 E-22$ - $9.652000 E-03-8.4380005-03-8.553000 E-03 \quad 2.9024005-02 \quad 2.940900 E-02 \quad 3.868000 E-02-8.691000 E-03-8.681000 E-03-8.599000 E-03$ PE- $22 \quad-4.541000 E-03 \quad-4.645000 E-03 \quad-4.762000 E-03 \quad 3.787500 E-02 \quad 3.741200 E-02 \quad 4.064500 E-02 \quad-3.899000 E-03 \quad-3.619000 E-03 \quad-3.739000 E-03$ PE-29 7.681000E-02 T.709200E-02 $7.687500 E-02 \quad 8.730300 E-02 \quad 7.836800 E-02 \quad 9.462100 E-01 \quad 7.148600 E-02 \quad 7.219100 E-02 \quad 7.251960 E-02$

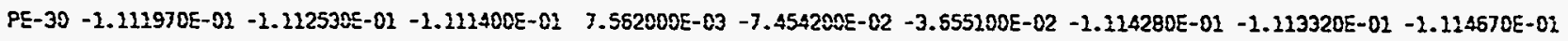
FE- $01 \quad 1.000150 E+00 \quad 1.000158 E+00 \quad 1.000140 E+00 \quad 2.368100 E+00 \quad 2.374000 E+00 \quad 2.360200 E+00 \quad 1.002110 E+00 \quad 1.001500 E+00 \quad 1.001190 E+00$ XE-01 -3.010000E-D4 -3.010000E-04 -3.030000E-04 9.400000E-05 8.700000E-05 9.600000E-05 -3.070000E-04 -3.110000E-04 -3.130000E-04 XE-02 - $-3.2400005-24-8.240000 E-04-8.240000 E-04-8.230000 E-04$-8.240000E-04 -8.230000E-04 -9.280000E-04 -8.290000E-04 -8.290000E-04 $X X E-03-8.490000 E-04-8.490000 E-04-8.4700005-04-8.440000 E-04-8.360000 E-04-8.610000 E-04-8.900000 E-04-8.820000 E-04-8.770000 E-04$ $X E-04-1.3660805-03-1.3650005-03-1.366000 E-03-1.110000 E-03-1.101000 E-83-1.103000 E-03-1.358000 E-03-1.360000 E-03-1.361000 E-03$ $X E-05-3.6350005-03-3.634000 E-03-3.636000 E-03-3.473000 E-03-3.4650005-03-3.453000 E-03-3.623000 E-03-3.626000 E-03-3.525000 E-03$

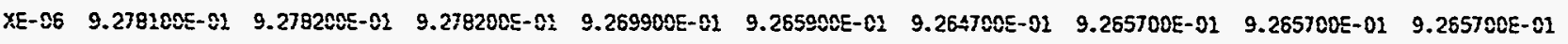
XE-07 -2.390000E-04 -2.400000E-04 -2.340000E-04 -2.340000E-04 -2.310000E-04 -2.230000E-04 -1.980000E-04 -2.000000E-04 -1.870000E-04 $x E-08-1.0390005-03-1.038000 E-03-2.039000 E-03-9.800000 E-04-9.830000 E-04-9.840000 E-04-1.027000 E-03-1.027000 E-83-1.028008 E-03$ XE- $09-2.218000 E-03-1.217008 E-03-2.219000 E-03-1.041000 E-03-1.036000 E-03-1.035000 E-03-1.1740005-03-1.2730005-03-1.173000 E-03$ XE- $20-3.7990005-03-3.7990005-03-3.8000005-03-3.2180005-03-3.2080005-03-3.2190005-83-3.780000 E-03-3.785080 E-03-3.792000 E-03$ $\begin{array}{lllllllllll}X E-11 & 2.600000 E-05 & 2.500000 E-05 & 2.600000 E-05 & 2.600000 E-05 & 2.600000 E-05 & 2.600000 E-05 & 2.600000 E-05 & 2.600000 E-05 & 2.600000 E-05\end{array}$ XE- $12-1.750000 E-03-1.751000 E-03-1.750000 E-03-1.733000 E-03-1.738000 E-03-1.731000 E-03-1.752000 E-03-1.752000 E-03-1.751000 E-03$ XE-13 -5. $460000 E-04 \quad-5.590000 E-04 \quad-5.610000 E-04$ 1.220000E-04 $1.310000 E-04 \quad 1.250000 E-04-4.670000 E-04-4.870000 E-04 \quad-5.030000 E-04$ $X E-14-2.6100005-04-2.6600005-04-2.5500005-04$-8.300000E-05 -8.000000E-05 -6.600000E-05 -2.320000E-04 -2.420000E-04 -2.500000E-04 XE-15 -8.130000E-04 -8.150000E-04 -8.140000E-04 -3.040000E-04 -3.030000E-04 -3.030000E-04 -7.980000E-04 -8.000000E-04 -8.020000E-04 TE- 01 -1.280000E- $04-1.240000 E-04-1.250000 E-04-9.600000 E-05$ - $9.700000 E-05-9.400000 E-05-1.220000 E-04-1.180000 E-04-1.240000 E-04$ $\begin{array}{lllllllllll}\text { VEXTI } & 1.993700 E+00 & 1.993700 E+00 & 1.993700 E+00 & 1.993700 E+00 & 1.993700 E+00 & 1.993700 E+00 & 1.993700 E+00 & 1.993700 E+00 & 1.993700 E+00\end{array}$ VEXT2 $2.990100 E+\infty 0 \quad 1.990000 E+00 \quad 1.990000 E+C 0 \quad 1.990000 E+C 0 \quad 1.990000 E+00 \quad 1.990000 E+00 \quad 1.990000 E+00 \quad 1.990000 E+00 \quad 1.990000 E+00$

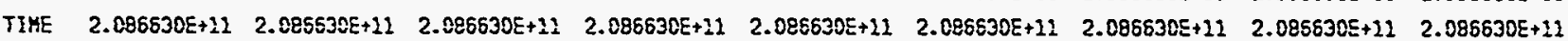


Archive Yame:

AMS_WF_TESTS

Book Manre: AMS_MFPT2_RY

Date \& Time: $2 \operatorname{Mar} 1900$ 00:39:18

Page Description:

DATA SET HO.-245

TRAYS RY_ZERO-1 RV_ZERO-2 RN_ZERO-3 RY_LOAD-1 RV_LOAD-2 $\quad$ RY_LOAD-3 RY_ZERO-4 $\quad$ RY_ZERO-5 RY_ZERO-6

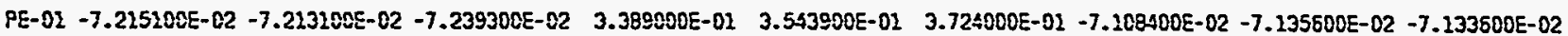
PE-02 2.828200E-02 2.7937COE-02 2.839900E-02 $5.020200 E-02 \quad 5.271800 E-02 \quad 5.580200 E-01 \quad 2.911400 E-02 \quad 2.942500 E-02 \quad 2.903500 E-02$ PE-03 - $1.930300 E-02$ - $1.938508 E-02$-1.948200E-02 4.156200E-01 4.194600E-01 4.410100E-01 $-2.108200 E-02$-2.188700E-02 -2.077300E-02 PE-04 3.701900E-02 3.694700E-02 $3.577100 E-02 \quad 5.649100 E-01 \quad 5.493300 E-01 \quad 5.725700 E-01 \quad 3.773000 E-02 \quad 3.745500 E-02 \quad 3.753600 E-02$ PE-05 7.443300E-02 7.424E00E-02 $7.433300 E-02 \quad 3.415500 E-01 \quad 3.442500 E-02 \quad 3.404100 E-02 \quad 7.539400 E-02 \quad 7.535600 E-02 \quad 7.511300 E-02$ PE-06 $6.722900 E-02$ 6.725300E-02 $6.720500 E-02$ 4.717900E-01 4.4E_500E-01 $4.690400 E-02 \quad 6.661200 E-02 \quad 6.673000 E-02 \quad 5.636300 E-02$

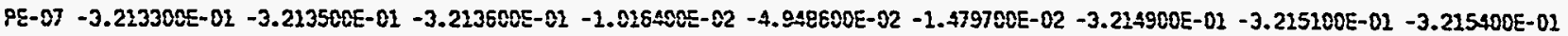

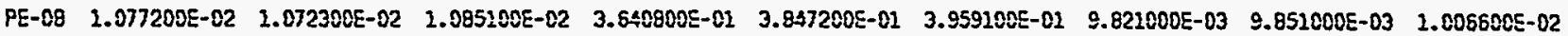

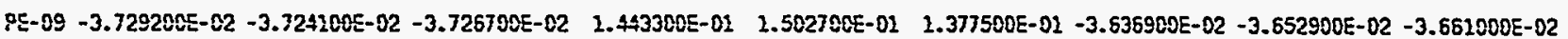
PE-10 $1.709700 E-02 \quad 1.695200 E-02 \quad 1.693300 E-02 \quad 2.9252005-02 \quad 2.932000 E-02 \quad 2.911100 E-02 \quad 1.747900 E-02 \quad 1.745900 E-02 \quad 1.735100 E-02$

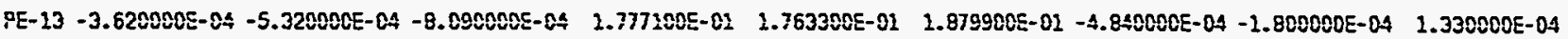

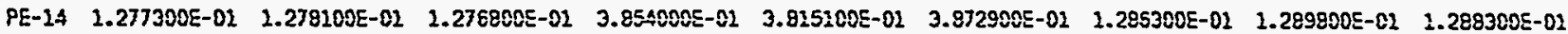

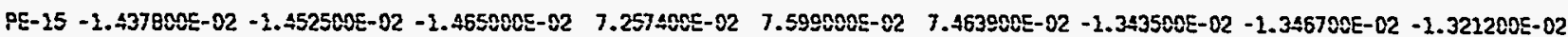
PE-15 3.786800E-02 3.807600E-02 3.807600E-02 $1.262600 E-01 \quad 1.256800 E-02 \quad 1.286100 E-02 \quad 3.864400 E-02 \quad 3.857400 E-02 \quad 3.870300 E-02$

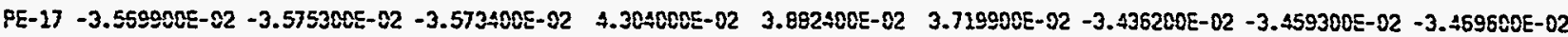
PE-19 - - 201500E-02 -1.199990E-01 -1.203900E-02 -2.542500E-02 -2.845800E-02 -3.279000E-02 -1.197170E-02 -1.190710E-01 -1.190630E-01 PE-19 - 4.909A00E-02 -4.930900E-02 -4.908500E-02 -2.5987005-02 -2.402500E-02 -2.718800E-02 -4.873C00E-02 -4.848300E-02 -4.842900E-02 PE-20 - 4.843900E-02 - $4.847400 E-02-4.861300 E-02-1.545700 E-02-1.623300 E-02-1.539200 E-02-4.785500 E-02-4.774600 E-02-4.747308 E-02$

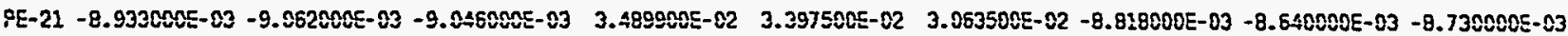
PE-22 -4.743000E-03 -4.941000E-03 -5.131000E-03 3.797300E-02 $3.592000 E-02 \quad 3.672500 E-02-4.428000 E-03 \quad-4.422000 E-03 \quad-3.9520005-03$

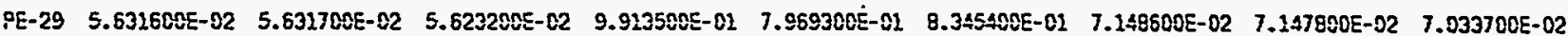

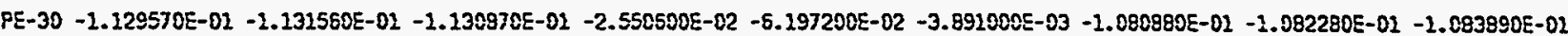
FE-02 2. $200170 E+00 \quad 1.000160 E+00 \quad 1.000170 E+00 \quad 2.410700 E+00 \quad 2.415700 E+00 \quad 2.418000 E+00 \quad 1.002200 E+00 \quad 1.001620 E+00 \quad 1.001270 E+00$ YE-02 -3.050000E-04 -3.090000E-04 -3.050000E-04 1.030000E-04 9.800000E-05 1.0800005-04 -3.080000E-04 -3.110000E-04 -3.150000E-04 XE-02 -8.250000E-04 -8.260000E-04 -8.250000E-04 -8.270000E-04 -8.250000E-04 -8.230000E-04 -8.230000E-04 -8.240000E-04 -8.2300000E-04 YE-03 -8.520000E-04 -8.530000E-04 -8.530000E-04 -9.300000E-04 -9.760000E-04 -8.990000E-04 -9.290000E-04 -9.2900000E-04 -9.330000E-04 XE-04 - $1.362000 E-03-1.363000 E-03-1.363000 E-03-1.045000 E-03-1.045000 E-03-1.050000 E-03-1.316000 E-03-1.317000 E-03-1.321000 E-03$ XE-05 -3.627000E-03 -3.627000E-03 -3.627000E-03 -3.436000E-03 -3.429000E-03 -2.341000E-03 -3.591000E-03 -3.590000E-03 -3.589000E-03 XE-06 9.266600E-01 $9.266600 E-01 \quad 9.266600 E-01 \quad 9.262980 E-01 \quad 9.262400 E-01 \quad 9.265300 E-02 \quad 9.268500 E-01 \quad 9.268600 E-01 \quad 9.268500 E-01$ XE-07 -1.610000E-04 -1.480000E-04 -1.660000E-04 -2.010000E-04 -1.930000E-04 - $2.810000 E-04-1.700000 E-04-1.5800000-04-1.360000 E-04$ XE- $08-1.036000 E-03-1.036000 E-03-1.037000 E-83-9.730000 E-04-9.760000 E-04-9.710000 E-04-1.023000 E-03-1.024000 E-03-1.025000 E-03$ XE-09 -1.182000E-03 -1.181000E-03 -1.192000E-03 -9.330000E-04 -9.420000E-04 -9.230000E-04 $-1.1690005-03-1.167000 E-03-1.2710005-03$ XE-10 -3.791000E-03 -3.7978005-83 -3.792000E- $03-3.222008 E-03-3.226000 E-03-3.214000 E-03-3.772000 E-03-3.7790005-03-3.785000 E-03$ $\begin{array}{lllllllllll}\text { XE- } 12 & 2.500000 E-05 & 2.600000 E-05 & 2.500000 E-05 & 2.600000 E-05 & 2.700000 E-05 & 2.600000 E-05 & 2.700000 E-05 & 2.700000 E-05 & 2.700000 E-05\end{array}$ XE-12 -1.749000E-03 - $1.749000 E-03-1.749000 E-03-1.723000 E-03-1.724000 E-03-1.724000 E-03-1.751000 E-03-1.752000 E-03$-1.752000E-03 XE-13 -5.260000E-04 -5.630000E-04 -5.110000E-04 1.290000E-04 1.360000E-04 1.300000E-04 -4.060000E-04 -4.350000E-04 -4.950000E-04 XE-14 -2.590000E-04 -2.590000E-04 -2.590000E-04 -4.000000E-05 -4.500000E-05 -3.600000E-05 -2.330000E-04 -2.380000E-04 -2.3600000E-09 XE-15 -8.120000E-04 -8.150000E-04 -8.110000E-04 -2.870000E-04 -2.940000E-04 -2.910000E-04 -7.920000E-04 -7.980000E-04 -8.020000E-04 TE-02 - $2.350000 E-04-1.330000 E-04-1.350000 E-04-1.0100000-04-1.030000 E-04-1.020000 E-04-1.280000 E-04-1.390000 E-04-1.480000 E-04$ $\begin{array}{lllllllllll}\text { VEXT1 } & 1.993700 E+D 0 & 1.993700 E+00 & 1.993700 E+00 & 2.993700 E+00 & 1.993700 E+00 & 1.993700 E+00 & 1.993700 E+00 & 1.993700 E+00 & 1.993700 E+00\end{array}$ YEXT2 $1.990000 E+00 \quad 1.990000 E+00 \quad 1.990000 E+00 \quad 1.990000 E+00 \quad 1.990000 E+00 \quad 1.990000 E+00 \quad 1.990000 E+00 \quad 1.990000 E+00 \quad 1.990000 E+00$

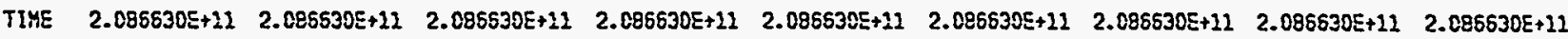


Aration y200:

ATS_HE_TESTS

Sock Hame: AUS_MFOTI_RY

Da:e \& Time: 2 Yar 1900 00:47:34

Page Eescription:

DATA SET M0.-246

TRAYS RE_ZERO-1 RE_ZERO-2 RY_ZERO-3 RY_LOAD-1 RY_LOAD-2 RY_LOAD-3 RY_ZERO-4 RV_ZERO-5 RV_ZERO-6

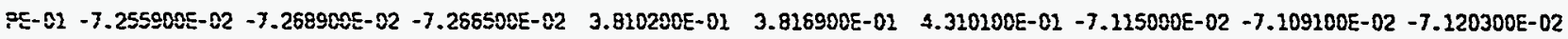
$\begin{array}{llllllllll}P E-02 & 2.811200 E-02 & 2.802600 E-02 & 2.799100 E-02 & 5.951800 E-01 & 5.994700 E-01 & 6.082800 E-01 & 2.944100 E-02 & 2.947500 E-02 & 2.954200 E-02\end{array}$

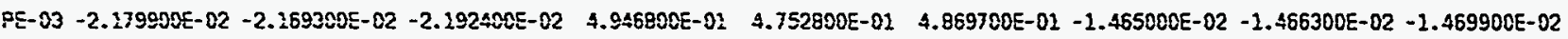
PE-04 3.641500E-02 $3.566200 E-02 \quad 3.554600 E-02 \quad 5.294800 E-01 \quad 5.794900 E-01 \quad 6.024300 E-01 \quad 3.732200 E-02 \quad 3.747600 E-02 \quad 3.711500 E-02$ PE-05 7.400900E-02 7.384500E-02 $7.402900 E-02 \quad 3.7402005-01 \quad 3.802200 E-01 \quad 3.829600 E-02 \quad 7.441600 E-02 \quad 7.440400 E-02 \quad 7.446200 E-02$

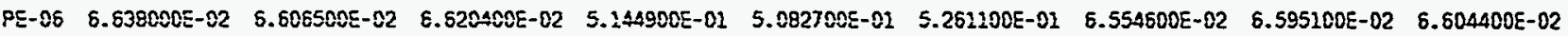

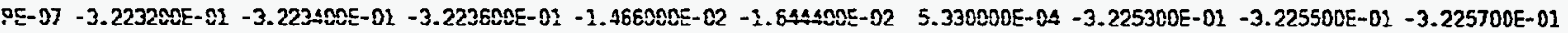
PE- 09 1.002000E-02 $\quad 9.970000 E-03 \quad 1.004000 E-02 \quad 4.035100 E-02 \quad 3.983500 E-01 \quad 3.995800 E-01 \quad 1.074900 E-02 \quad 1.107300 E-02 \quad 1.108800 E-02$

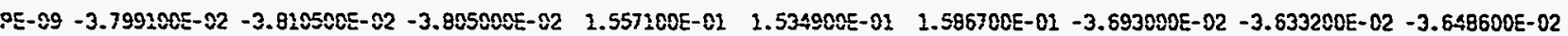
FE- $10 \quad 1.654800 E-02 \quad 1.662900 E-02 \quad 1.671100 E-02 \quad 3.386000 E-02 \quad 3.251900 E-02 \quad 3.227500 E-01 \quad 1.782700 E-02 \quad 1.778500 E-02 \quad 1.769300 E-02$

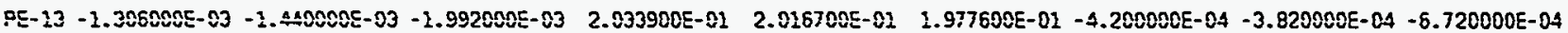

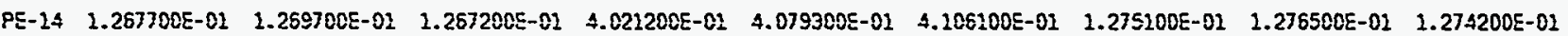

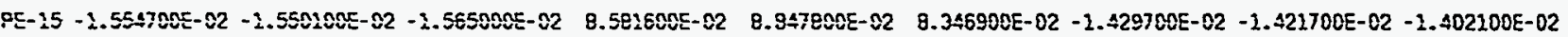
PE-15 3.985700E-82 $3.814200 E-02 \quad 3.792200 E-02 \quad 1.394700 E-02 \quad 1.431100 E-01 \quad 1.439300 E-02 \quad 3.827800 E-02 \quad 3.8511005-02 \quad 3.859600 E-02$

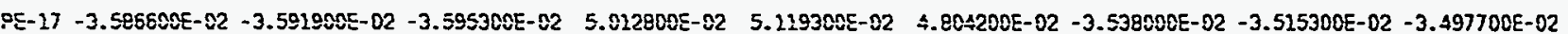

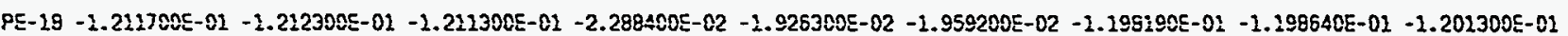
PE- 19 -4.9\$4800E- 82 - $4.993100 E-82-4.901500 E-02-7.712000 E-83-7.302000 E-03-5.000000 E-03-4.894500 E-02-4.871200 E-02-4.844300 E-02$

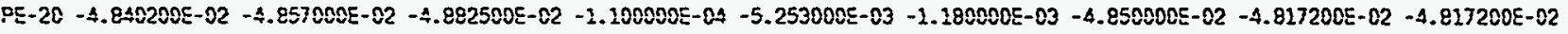
PE-21 - $9.319000 E-83-3.8590005-03-8.856000 E-03 \quad 4.780300 E-02 \quad 4.5295005-02 \quad 5.138500 E-02-8.599000 E-03-9.043000 E-03-0.873000 E-03$ PE-22 -5.463000E- 83 - 5.381000E-03 -5.542000E-03 $4.527900 E-02$ 4.633900E-02 4.875200E-02 $-5.580000 E-03$-5.208000E-03 -5.170000E-03 $\begin{array}{lllllllllll}\text { PE-29 } & 5.682200 E-92 & 5.6781005-02 & 5.679200 E-02 & 9.723700 E-02 & 8.304200 E-02 & 7.96 E 000 E-02 & 7.162500 E-02 & 7.086200 E-02 & 7.062700 E-02\end{array}$

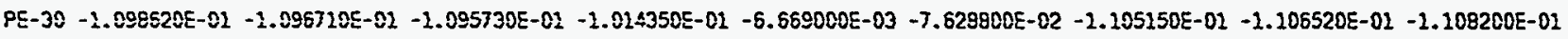

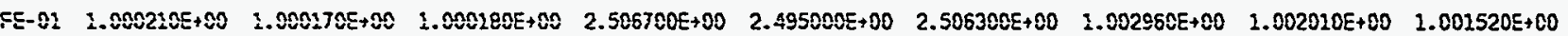

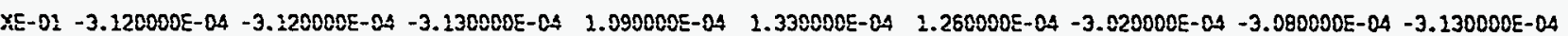
XE-02 -8.220000E-04 -8.220000E-04 -8.210000E-04 -8.290000E-04 -8.290000E-04 -8.270000E-04 -8.270000E-04 -8.268000E-04 -8.280000E-04 XE-03 -9.\$60000E-04 -9.350000E-04 -9.350000E-04 - - $1.031000 E-03-1.038000 E-03$-1.015000E-03 -9.420000E-04 -9.390000E-04 -9.400000E-04 XE- $04-1.3290005-03-1.3290085-03-2.330000 E-83-1.0410005-03-1.023000 E-03-1.0280005-83-1.389000 E-03-1.314000 E-03-1.316000 E-03$ XE-05 $-3.591000 E-03-3.584000 E-03-3.585000 E-03-3.424000 E-03-3.423000 E-03-3.412000 E-03-3.587000 E-03-3.584000 E-03-3.585000 E-03$

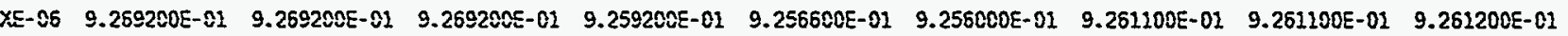
XE-07 - $1.040000 E-04-9.500000 E-05-9.000000 E-05-2.900000 E-04-2.920000 E-04-2.760000 E-04-2.590000 E-04-1.420000 E-04-1.360000 E-04$

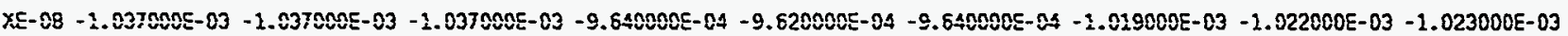
$X E-09-1.177000 E-03-1.177000 E-03-2.177000 E-03-9.150000 E-04-9.150000 E-04-9.190000 E-04-1.172000 E-03-1.173000 E-03-1.172000 E-03$ $x E-12-3.7920005-83-3.7930005-03-3.794000 E-03-3.178000 E-03-3.172080 E-03-3.186000 E-03-3.735000 E-03-3.749000 E-03-3.751000 E-03$ $\begin{array}{llllllllll}x E-12 & 2.500000 E-05 & 2.600000 E-05 & 2.700000 E-05 & 2.600000 E-05 & 2.600000 E-05 & 2.700000 E-05 & 2.8000000 E-05 & 2.700000 E-05 & 2.500000 E-05\end{array}$ XE-12 - $1.7500005-83-1.749000 E-03-1.750000 E-83-1.7160005-03-1.713000 E-03-1.716000 E-03-1.75 \$ 000 E-03-2.754000 E-03-1.752000 E-03$ $x E-13 \quad-5.080000 E-04 \quad-5.280000 E-04 \quad-5.380000 E-04 \quad 1.280000 E-04 \quad 2.280000 E-04 \quad 1.290000 E-04 \quad-3.760000 E-04 \quad-4.670000 E-04 \quad-5.030000 E-04$ XE- $14-2.5600005-04-2.820000 E-04-2.580000 E-04-2.100000 E-05 \quad 1.700000 E-05 \quad 2.200000 E-05-2.150000 E-04-2.150000 E-04-2.170000 E-04$ $x E-15-8.220000 E-04-8.2200005-04-8.210000 E-04-3.220000 E-04-3.270000 E-24-3.130000 E-04-7.940000 E-04-7.980000 E-04-8.000000 E-04$ TE- $01-1.5700005-04-1.5500005-04-2.660000 E-04-1.370000 E-04-1.360000 E-04-1.340000 E-04-2.570000 E-04-1.690000 E-04-1.720000 E-04$ $\begin{array}{lllllllllll}\text { VEXI1 } & 1.993700 E+00 & 1.993700 E+00 & 1.993700 E+00 & 1.993700 E+00 & 1.993700 E+00 & 1.993700 E+00 & 1.993700 E+00 & 1.993700 E+00 & 1.993700 E+00\end{array}$ VEXT2 $1.990000 E+00 \quad 2.990000 E+00 \quad 1.990000 E+00 \quad 1.990000 E+00 \quad 1.990000 E+00 \quad 2.990000 E+00 \quad 1.990000 E+00 \quad 1.990080 E+00 \quad 1.990000 E+00$

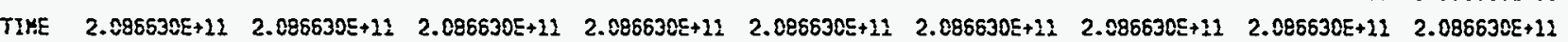


Arative Yzune:

AHS_MFP_TESTS

Bock Harre: AMS_MFPT1_BV Date \& Time: 2 Mar 1900 01:00:25

Page Description:

DATA SET HO.-247

TRANS RY_ZERO-2 RV_ZERO-2 RY_ZERO-3 RV_LOAD-1 $\quad$ RY_LOAD-2 $\quad$ RY_LOAD-3 $\quad$ RY_ZERO-4 $\quad$ RV_ZERO-5 RY_ZERO-6

PE-01 -7.379200E-02 -7.392600E-02 -7.353000E-02 5.059400E-01 4.795100E-01 5.048300E-01 $-7.203800 E-02 \quad-7.192100 E-02 \quad-7.189100 E-02$ $\begin{array}{llllllllll}P E-02 & 2.868200 E-02 & 2.835500 E-02 & 2.836500 E-02 & 7.509700 E-01 & 7.557000 E-02 & 7.447300 E-02 & 2.965600 E-02 & 2.983700 E-02 & 2.985200 E-02\end{array}$ PE-03 -1.684400E-02 -1.689200E-02 -1.693100E-82 5.567700E-01 5.877100E-02 5.888500E-01 - $1.666300 E-02$ - 2.675600E-02 -1.55C800E-02 PE-04 3.593200E-02 3.511700E-02 $3.577300 E-02 \quad 7.333300 E-02 \quad 7.256400 E-02 \quad 7.358000 E-01 \quad 3.627200 E-02 \quad 3.612100 E-02 \quad 3.521300 E-02$ PC-05 7.338600E-02 7.32.5500E-02 7.353100E-02 $4.436400 E-02 \quad 4.322800 E-01$ 4.443200E-01 $7.393100 E-02 \quad 7.406500 E-02 \quad 7.394900 E-02$

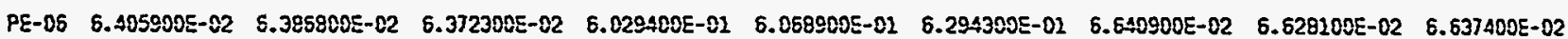

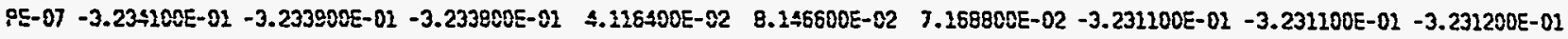
PE-08 1.039100E-02 1.030200E-02 1.039200E-02 4.929500E-01 $5.046600 E-02 \quad 4.897600 E-01 \quad 1.076700 E-02 \quad 1.090100 E-02 \quad 1.132800 E-02$

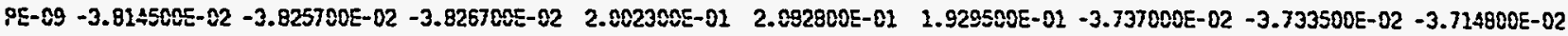

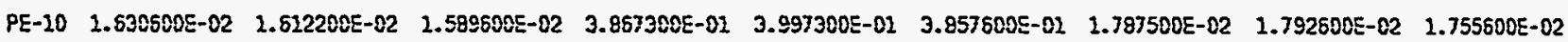
PE- 13 -3.536000E-03 -3.E25000E-03 -3.466000E-03 2.410000E-01 2.419200E-01 2.404900E-01 -8.380000E-04 -1.245000E-03 -1.233000E-03 PEE-14 1.237000E-01 $1.240000 E-01 \quad 1.236700 E-01 \quad 4.622200 E-01 \quad 4.549200 E-01 \quad 4.657200 E-01 \quad 1.259100 E-01 \quad 1.272200 E-01 \quad 1.274500 E-01$

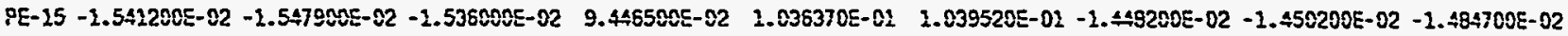
PE-16 $\quad 3.772400 E-02 \quad 3.755200 E-02 \quad 3.794600 E-02 \quad 1.605700 E-01 \quad 1.634400 E-01 \quad 1.638500 E-01 \quad 3.799200 E-02 \quad 3.787400 E-02 \quad 3.798700 E-02$

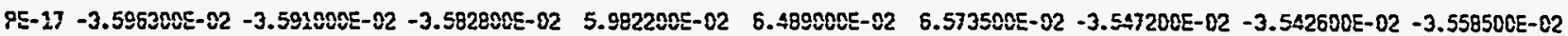

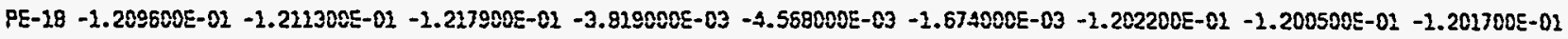
PE-19 - $4.975000 E-02-4.9978005-02-4.995100 E-02-2.0863008-02-1.948300 E-02-1.771200 E-02-4.8803000-02-4.863 E 00 E-02-4.852700 E-02$ PE-20 -4.9283005-02 -4.922600E-02 -4.938100E-02 -7.556000E-03 -1.215400E-02 -9.443000E-03 -4.889300E-02 -4.872300E-02 -4.017500E-02 CEE-21 - 8.874000E-03 -9.01200CE-03 -9.045000E-03 4.564000E-02 4.285900E-02 4.482600E-02 -8.827000E-03 -8.762000E-03 -8.582000E-03 8E-22 - $5.897000 E-03-6.620000 E-03-6.884000 E-03 \quad 5.317800 E-02 \quad 5.350900 E-02 \quad 5.444700 E-02 \quad-5.875000 E-03 \quad-5.527000 E-03 \quad-5.512008 E-03$ $\begin{array}{lllllllllll}P E-29 & 5.627100 E-02 & 5.525200 E-02 & 5.632900 E-02 & 1.209200 E+00 & 1.139500 E+00 & 1.055220 E+00 & 7.128700 E-02 & 7.115300 E-02 & 7.145800 E-02\end{array}$ $P E-30-1.121590 E-02 \quad-1.124450 E-02 \quad-1.122430 E-02 \quad-2.716400 E-02 \quad 2.327600 E-02 \quad 8.327000 E-03 \quad-1.133560 E-01 \quad-1.231970 E-01 \quad-1.132440 E-01$

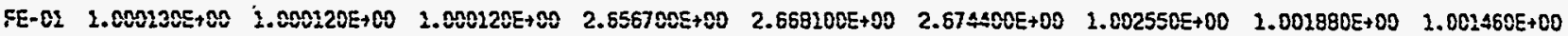
YEE-02 -3.150000E-04 -3.230000E-04 -3.140000E-04 1.410000E-04 1.410000E-04 1.4500005-04 -2.990000E-04 -3.050000E-04 -3.090000E-04 XE- $22-8.240000 E-04-8.240000 E-04-8.240000 E-04-8.280000 E-04-8.280000 E-04-8.260000 E-04-8.300000 E-04-8.320000 E-04-8.310000 E-04$ XE-03 -9.370000E-04 -9.380000E-04 -9.420000E-04 -4.413000E-03 -5.428000E-03 -1.782000E-03 -9.990000E-04 -9.300000E-04 -8.790000E-04

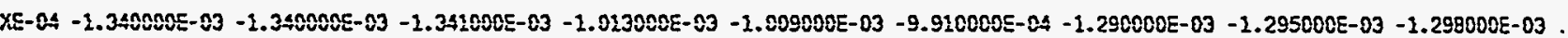
$X E E-05-3.591000 E-03-3.5820005-03-3.582000 E-03-3.495000 E-83-3.4830005-03-3.451000 E-03-3.572000 E-03-3.572000 E-03-3.570000 E-03$ XE-06 9.262200E-02 9.252300E-01 9.262300E-01 9.241900E-01 $9.2781005-01$ 9.3277005-01 $9.3445005-01 \quad 9.341600 E-01 \quad 9.338500 E-01$

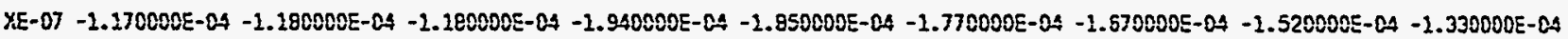
$X E-88-1.0460005-03-1.04 E 000 E-03-1.0460005-03-9.620000 E-04-9.620000 E-04-9.560000 E-04-1.0240 C 0 E-03-3.022000 E-03-1.027000 E-03$ X.E-09 - $2.292000 E-03-1.190000 E-03-2.291000 E-03-8.930000 E-04-8.810000 E-03-8.890000 E-03-1.168000 E-03-1.1670 C 0 E-03-1.173000 E-03$

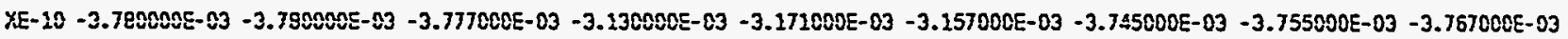
$\begin{array}{llllllllll}X E-11 & 2.500000 E-05 & 2.600000 E-05 & 2.600000 E-05 & 2.600000 E-05 & 2.600000 E-05 & 2.600000 E-05 & 2.600000 E-05 & 2.700000 E-05 & 2.700000 E-05\end{array}$ XE- 12 - $1.752000 E-03-1.751000 E-03-1.750000 E-03$ - $1.706000 E-03-1.705000 E-03-1.706000 E-03-2.752000 E-03-1.751000 E-03-1.754000 E-03$ XE-13 -5.620000E-04 -5.430000E-04 -5.340000E-04 1.450000E-04 1.540000E-04 1.640000E-04 -4.630000E-04 - $4.840000 E-04-5.060000 E-04$ $X E-14-2.7700005-04-2.350000 E-04-1.920000 E-04$ 5.200000E-05 5.900000E-05 5.300000E-05 -2.340000E-04 -2.210000E-04 -2.210000E-04 XE-15 -8.090000E-04 -8.200000E-04 -8.120000E-04 -2.390000E-04 -2.290000E-04 -2.130000E-04 -7.920000E-04 -7.910000E-04 -7.970000E-04 TE-02 - $1.750000 E-04-1.7400005-04-3.730000 E-04$ - $1.370000 E-04-2.300000 E-04-1.330000 E-04-1.530000 E-04-1.630000 E-04-1.710000 E-04$ $\begin{array}{lllllllllllll}\text { VEXT1 } & 1.993700 E+00 & 2.993700 E+00 & 1.993700 E+00 & 1.993700 E+00 & 1.993700 E+00 & 1.993700 E+00 & 1.993700 E+00 & 1.993700 E+00 & 1.993700 E+00\end{array}$ VEXT2 $2.990000 E+00 \quad 1.990000 E+00 \quad 1.990000 E+00 \quad 1.990000 E+00 \quad 1.990000 E+00 \quad 1.990000 E+00 \quad 1.990000 E+00 \quad 1.9900 C 0 E+00 \quad 1.990000 E+00$

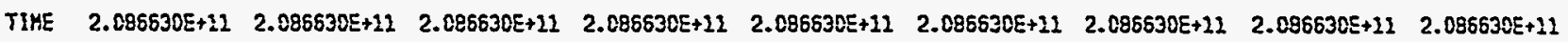


Page Description:

OATA SET MO. -2AB

TRAYS RV_ZERO-1 RV_ZERO-2 RV_ZERO-3 RI_LOAD-1 RV_LOAD-2 RY_LOAD-3 RV_ZERO-A RV_ZERO-5 RV_ZERO-6

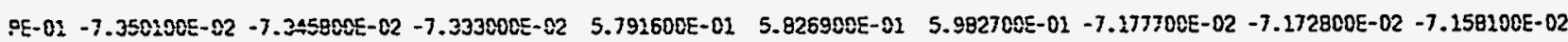
$\begin{array}{llllllllll}P E-02 & 2.852900 E-02 & 2.920100 E-02 & 2.833100 E-02 & 8.703200 E-02 & 8.703900 E-01 & 8.786800 E-01 & 2.967300 E-02 & 2.960900 E-02 & 2.972100 E-02\end{array}$

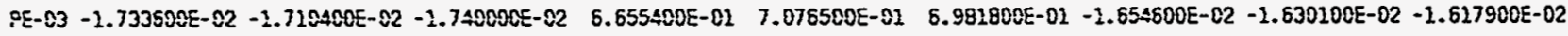
FE- 03 3.585100E-02 $3.565500 E-02 \quad 3.570400 E-02 \quad 8.505100 E-02 \quad 8.326580 E-01 \quad 8.430900 E-01 \quad 3.571500 E-02 \quad 3.576000 E-02 \quad 3.570900 E-02$

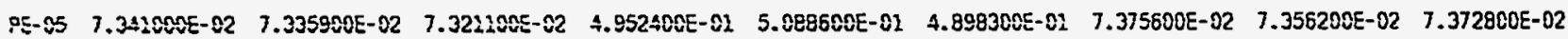
$\begin{array}{llllllllll}P E-\$ 8 & 5.605300 E-02 & \varepsilon .597300 E-02 & \varepsilon .585300 E-02 & 6.798600 E-02 & 6.925900 E-01 & 6.718600 E-01 & 6.535300 E-02 & 6.653000 E-02 & 6.699500 E-02\end{array}$

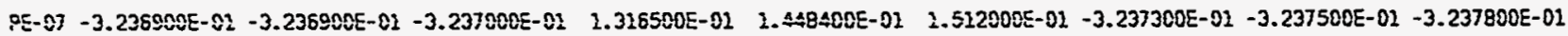

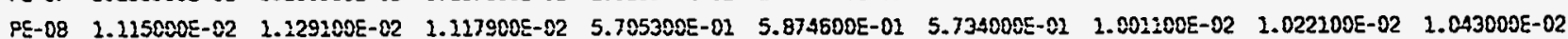
$P E-09-3.8548005-02-3.842400 E-02 \quad-3.849108 E-02 \quad 2.446300 E-02 \quad 2.464200 E-01 \quad 2.446500 E-01 \quad-3.924200 E-02 \quad-3.901600 E-02-3.927900 E-02$ PE-10 $1.588600 E-02 \quad 1.675100 E-02 \quad 1.678100 E-02 \quad 4.647000 E-01 \quad 4.715200 E-01 \quad 4.662400 E-01 \quad 1.8557005-02 \quad 1.869300 E-02 \quad 1.868200 E-02$

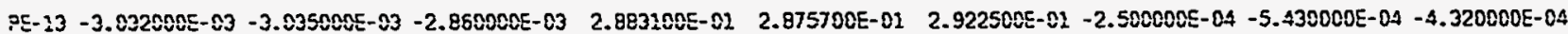
$\begin{array}{lllllllllll}P E-14 & 1.255500 E-01 & 1.254900 E-02 & 1.257000 E-01 & 5.192000 E-01 & 5.320800 E-01 & 5.317000 E-01 & 1.275900 E-01 & 1.279200 E-02 & 1.277800 E-01\end{array}$

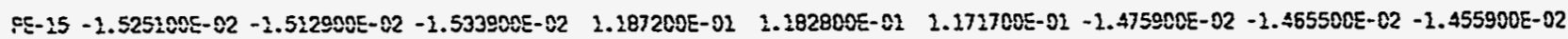
$\begin{array}{llllllllll}P E-16 & 3.758700 E-02 & 3.748700 E-02 & 3.767100 E-02 & 2.848700 E-02 & 1.828800 E-02 & 1.844900 E-01 & 3.791100 E-02 & 3.775400 E-02 & 3.769300 E-02\end{array}$

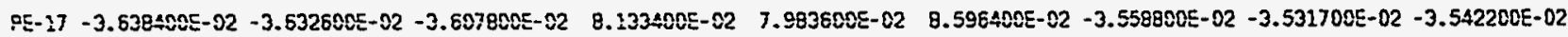

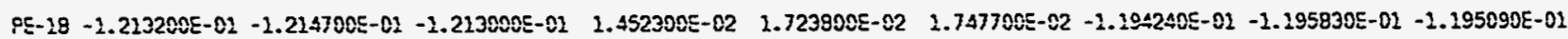
PE-19-4.902100E- $\$ 2-4.8984005-82-4.884000 E-82-2.222800 E-02-1.161800 E-02-9.882000 E-03-4.835500 E-02-4.893000 E-02-4.082900 E-02$ $P E-20-4.938200 E-02-4.926900 E-02-4.9096005-02-2.005000 E-03-3.899000 E-03-1.592000 E-03-4.811500 E-02-4.7893005-02-4.814700 E-02$

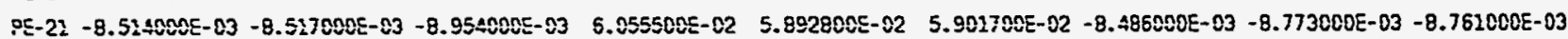
$P E-22-6.257080 E-03-6.178000 E-03 \quad-6.437000 E-83 \quad 6.490800 E-02 \quad 5.660300 E-02 \quad 6.785600 E-02 \quad-5.574000 E-03 \quad-5.583000 E-03-5.711000 E-03$

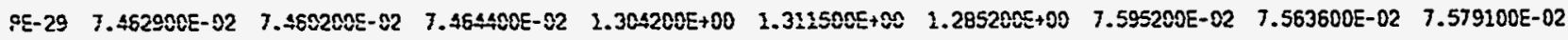

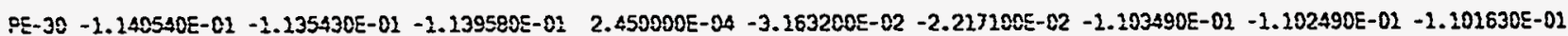

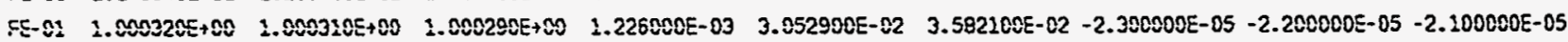
XE-01 $-3.240000 E-04 \quad-3.240000 E-04-3.250000 E-04 \quad 1.900000 E-04 \quad 1.920000 E-04 \quad 1.960000 E-04-2.790000 E-04 \quad-2.850000 E-04 \quad-2.890000 E-04$ XE-02 - $9.260000 E-04-8.260000 E-04-8.260000 E-04-8.290000 E-04-8.2900005-04-8.260008 E-04-8.330000 E-04-8.330000 E-04-8.310000 E-04$ $X E-03-6.510000 E-04-6.500000 E-04-6.490000 E-04-2.976000 E-03-4.252000 E-03-6.485000 E-03-4.892000 E-03-4.840000 E-03-4.356000 E-03$ XE- $04-1.320000 E-03-2.320000 E-03-1.3210005-03-9.710000 E-04-9.670000 E-04-9.5300005-06-1.281000 E-03-1.285000 E-03-1.289000 E-03$ $X E-05-3.5850005-03-3.584000 E-03-3.583000 E-03-2.741000 E-03 \quad 1.8940005-03-2.026000 E-03-3.564000 E-03-3.567000 E-03-3.572000 E-03$

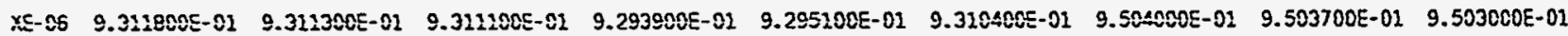
XE-07 2.020000E-04 2.170000E-04 2.050000E-04 -1.290000E-04 -1.150000E-04 -1.0600005-04 -1.280000E-04 -1.1000005-04 -9.100000E-05 XE- $09-2.043000 E-03-1.043000 E-03-1.0330005-03-9.5400005-04-9.530000 E-04-9.500000 E-04-1.020000 E-03-1.021000 E-03-1.0250005-03$ $X E-09-1.1910005-03-1.190000 E-03-1.190000 E-03-8.210000 E-04-7.840000 E-04-7.830000 E-04-1.245000 E-03-1.149000 E-03-1.149000 E-03$ XE- $10-3.799000 E-83-3.900000 E-03-3.900000 E-03-3.121000 E-03-3.132000 E-03-3.143000 E-03-3.748000 E-03-3.797000 E-03-3.795000 E-83$

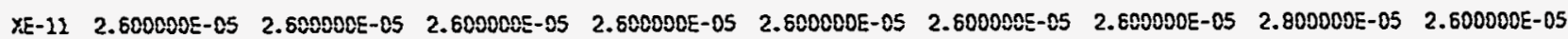
$X E-12-2.754000 E-23-2.753003 E-03-1.753000 E-03-1.695000 E-03-1.692000 E-03-1.694000 E-03-1.747000 E-03-1.752000 E-03-1.749000 E-03$ XE-13 -5.920000E-04 -5.880000E-04 -5.930000E-04 1.670000E-04 1.8000005-04 1.840000E-04 -4.600000E-04 -4.850000E-04 -5.000000E-04

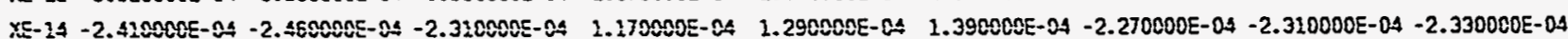
$X E-15-8.230000 E-04-8.090000 E-04-8.130000 E-04-2.080000 E-04-1.970000 E-04-1.9200005-04-7.730000 E-04-7.820000 E-04-7.820000 E-04$ T5- $02-3.9200005-04-1.9200005-04-1.9100005-04-1.540000 E-04-1.5200005-04-1.5100005-04-1.830000 E-04-1.890000 E-04-1.910000 E-04$ VEXT1 $1.993700 E+00 \quad 1.993700 E+00 \quad 1.993700 E+00 \quad 1.993700 E+00 \quad 1.993700 E+00 \quad 1.993700 E+00 \quad 1.993700 E+00 \quad 1.993700 E+00 \quad 1.993700 E+00$

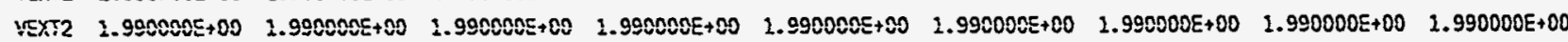

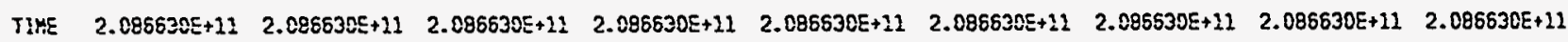


Arativite Hame:

AYYS_MP_TSSTS

Book Mame: AHS_MFPTI_RV Cate \& Time: 2 Mar 1900 03:28:24

Page Rescription:

DATA SET MO.-249

TRANS RV_ZERO-1 RY_ZERO-2 $\quad$ RV_ZERO-3 RV_LOAD-1 $\quad$ RY_LOAD-2 $\quad$ RY_LOAD-3 $\quad$ RY_ZERO-4 RV_ZERO-5 RY_ZERO-6

PE-01 -7.051000E-02 -7.043000E-02 -7.029400E-02 4. 497000E-01 4.196200E-01 4.791300E-01 -6.901300E-02 -6.894000E-02 -5.911000E-02 PE-02 3.033900E-02 $3.041200 E-02 \quad 3.046000 E-02 \quad 7.024500 E-01 \quad 7.161500 E-02 \quad 6.897100 E-02 \quad 3.2672005-02 \quad 3.253300 E-02 \quad 3.285500 E-02$ PE- 03 -2.159900E-02 -2.150800E-02 -2.261700E-02 5.5933005-01 5.553500E-01 5.229900E-01 -1.440000E-02 -1.434100E-02 -1.414400E-02 PE-Q4 4.227400E-02 4.205900E-02 4.226700E-02 $6.738100 E-02 \quad 6.731300 E-01 \quad 6.972900 E-01 \quad 4.192100 E-02 \quad 4.266100 E-02 \quad 4.231300 E-02$ PE- 05 7.621300E-02 $7.515200 E-02 \quad 7.649100 E-02 \quad 3.996608 E-02$ 4.267700E-01 4.028800E-02 7.8157C0E-02 $7.798800 E-02 \quad 7.803600 E-02$ PE- $05 \quad 6.656500 E-02 \quad 5.643 .00 E-02 \quad 6.647700 E-02 \quad 5.741400 E-01 \quad 5.832600 E-01 \quad 5.698200 E-02 \quad 7.163000 E-02 \quad 7.252000 E-02 \quad 7.173700 E-02$

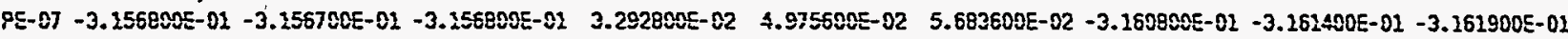
PE-C9 $\quad 9.081000 E-03 \quad 8.9740005-03 \quad 9.0720005-03 \quad 4.623500 E-02 \quad 4 . E 36500 E-01 \quad 4.869000 E-01 \quad 1.6354005-02 \quad 1.612800 E-02 \quad 1.526700 E-02$

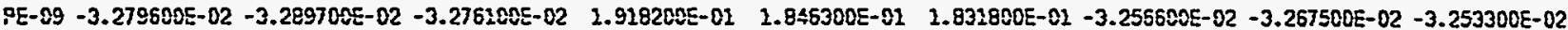
PE-10 $\quad 1.935300 E-02 \quad 1.922800 E-02 \quad 1.923200 E-02 \quad 3.491400 E-01 \quad 3.800000 E-02 \quad 3.753300 E-02 \quad 2.102500 E-02 \quad 2.120500 E-02 \quad 2.133400 E-02$ PE-13 - - $2.510000 E-04 \quad-2.720000 E-04 \quad 2.500000 E-05 \quad 2.297700 E-02 \quad 2.310400 E-01 \quad 2.324900 E-02 \quad 1.8330005-03 \quad 2.467000 E-03 \quad 2.628000 E-03$

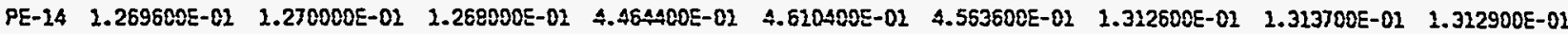

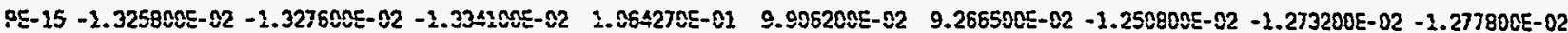
$\begin{array}{lllllllllll}P E-16 & 3.932800 E-02 & 3.910400 E-02 & 3.918300 E-02 & 3.528200 E-01 & 1.608900 E-01 & 1.577100 E-01 & 3.941900 E-02 & 3.954900 E-02 & 3.951300 E-02\end{array}$

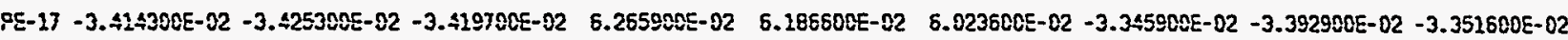

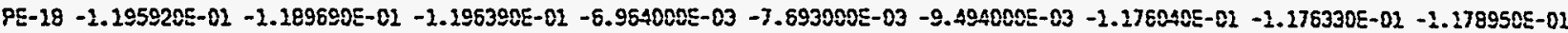

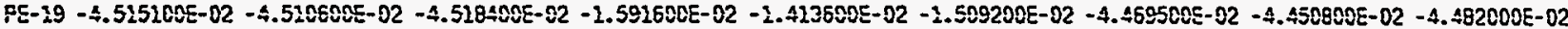
PE-20 - $4.542000 E-02-4.526000 E-02-4.564800 E-02-4.620000 E-02-6.427000 E-03-1.989000 E-03-4.445400 E-02-4.4402002-02-4.450300 E-02$

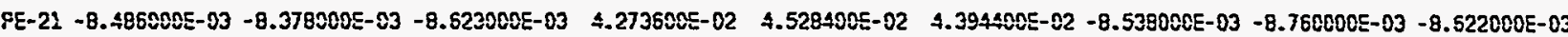
PE-22 7.570000E-04 5.250000E-04 5.000000E-04 5.460200E-02 5.298900E-02 5.505500E-22 7.780000E-04 7.970000E-04 5.210000E-04 PE-29 7.964200E-02 7.978000E-02 7.9E7200E-02 9.7564C0E-02 8.793500E-01 $1.040150 E+007.7626005-02 \quad 7.728980 E-02 \quad 7.685200 E-02$ PE-30 - $2.069520 E-02$-2.0711905-02 -1.069240E-02 -2.155000E-02 2. E42300E-02 -1.273800E-02 -2.054080E-01 -1.065940E-01 -1.053140E-01

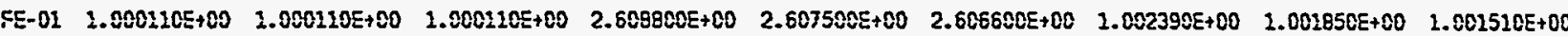
XE-01 -3.250000E-04 -3.250000E-04 -3.250000E-04 1.290000E-04 2.330000E-04 1.340000E-04 -3.160000E-04 -3.230000E-04 -3.250000E-04 XEE-02 -8.520000E-04 -8.512000E-04 -8.500000E-04 -8.550000E-04 -8.540000E-04 -8.530000E-04 -8.550000E-04 -8.570000E-04 -8.568000E-04 XE- $03-2.486000 E-03-2.485000 E-03-2.485000 E-03-2.579000 E-03-4.787000 E-03-8.3170005-03-4.615000 E-03-4.567000 E-03-4.549000 E-03$ XE- $04-1.410000 E-03-1.409000 E-03-1.409000 E-03-9.860000 E-04-9.820000 E-04-9.730000 E-04-1.309000 E-03-1.317000 E-03-2.324000 E-03$ XE-05 - -3.639000E-03 - -3.639000E-03 - -3.637000E-03 -3.448000E-03 -3.436000E-03 -3.4390005-03 -3.603000E-03 -3.601080E-03 -3.606000E-03

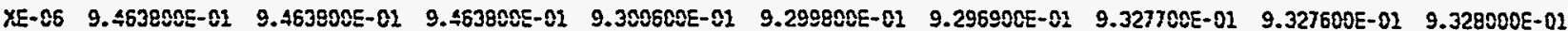
XE-07 1.870000E-04 1.980000E-04 1.960000E-04 -2.610000E-04 -2.540000E-04 -2.490000E-04 -2.620000E-04 -2.450000E-04 -2.250000E-04 XE-08 -1.203000E-03 -1.100000E-03 -1.1000005-03 - 2.004000E-03 - $1.006000 E-03$ - 1.009000E-03 - $1.079000 E-03-1.082000 E-03-1.092000 E-03$

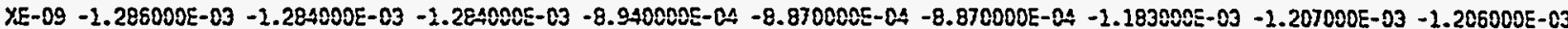
$X E-10-3.953000 E-03-3.952000 E-03-3.953000 E-03-3.242000 E-03-3.232000 E-03-3.225000 E-03-3.651000 E-03-3.710000 E-03-3.732000 E-03$ $\begin{array}{llllllllll}Y E-11 & 2.300000 E-05 & 2.400000 E-05 & 2.500000 E-05 & 2.500000 E-05 & 2.600000 E-05 & 2.600000 E-05 & 2.600008 E-05 & 2.600000 E-05 & 2.600000 E-05\end{array}$ $X E-12-1.7640005-03-1.763000 E-03-1.7630005-03-2.703000 E-03-1.7020005-03-1.704000 E-03-1.763000 E-03-1.76 \div 000 E-03-1.763000 E-03$ XE-13 -6.520000E-04 -6.650000E-04 -6.600000E-04 8.700000E-05 8.600000E-05 7.000000E-05 -4.930000E-04 $-5.340000 E-04-5.690000 E-04$ XE- 14 -2.580000E-04 -2.460020E-04 -2.460000E-04 9.400000E-05 8.700000E-05 9.500000E-05 -2.580C00E-04 -2.690800E-04 -2.570000E-04 XE- $25-7.880000 E-04-7.850000 E-04$-7.870000E-04 - $1.080000 E-04$-1.000000E-04 -7.900000E-05 -7.250000E-04 -7.250000E-04 -7.270000E-04 TE-01 -2.000000E-05 -2.000000E-05 -2.8000ع0E-05 7.000000E- 86 3.000000E-06 7.000000E-06 -4.200000E-05 -4.300000E-05 -4.900000E-05 VEXT2 $1.993700 E+00 \quad 1.993700 E+00 \quad 1.993500 E+00 \quad 2.993700 E+00 \quad 1.993700 E+00 \quad 1.99370 C E+00 \quad 1.993708 E+00 \quad 1.993700 E+00 \quad 1.993700 E+00$ WEXT2 $1.990800 E+00 \quad 1.990000 E+00 \quad 1.990000 E+00 \quad 1.990080 E+00 \quad 1.990000 E+00 \quad 1.990000 E+00 \quad 1.990000 E+00 \quad 1.990000 E+00 \quad 1.990000 E+00$ TIHE $2.086630 E+12 \quad 2.085530 E+12 \quad 2.086530 E+12 \quad 2.086630 E+11 \quad 2.086630 E+11 \quad 2.086630 E+112.085630 E+112.086630 E+12 \quad 2.0866530 E+11$ 
Arenive Yante:

AYS_YMP_TESTS

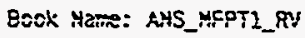

Date \& Time: 2 Kat 1900 $03: 37: 21$

Page Destription:

DATA SET YO. -250

TRAHS RE_ZERO-1 RY_ZERO-2 RY_ZERO-3 RY_LOAD-1 RY_LOAD-2 $\quad$ RY_LOAD-3 RV_ZERO-4 $\quad$ RY_ZERO-5 RY_ZERO-6

PE- 01 -7.040200E- 22 -7.035900E-02 -7.072200E-02 5.724200E-01 5.762300E-01 6.055400E-01 -6.918100E-02 -6.916600E-02 -6.906300E-02

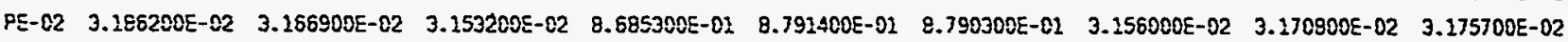
PE- 03 - $2.489500 E-82$ - $2.491100 E-02-1.478600 E-02 \quad 6.839000 E-01 \quad 6.828300 E-01 \quad 7.075000 E-02$ - $1.265300 E-02$ - $1.227300 E-02$ - $1.235400 E-02$ PE-04 A.200500E-02 4.051600E-02 4.094900E-02 8.564900E-02 $8.287700 E-01$ 8.490100E-01 $4.118100 E-02 \quad 4.117800 E-02 \quad 4.140700 E-02$

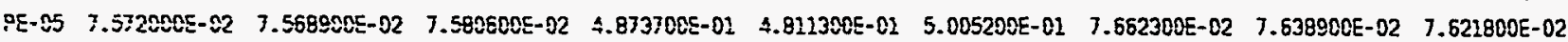
PE-05 7. $7855800 E-02 \quad 7.075500 E-02 \quad 7.081400 E-02 \quad 6.971700 E-01 \quad 7.039000 E-01 \quad 7.056400 E-01 \quad 7.1155005-02 \quad 7.126000 E-02 \quad 7.106600 E-02$

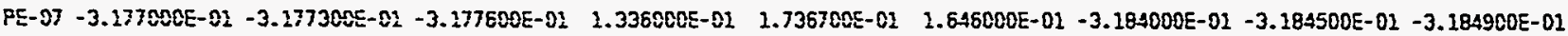
PE-08 $1.514700 E-02 \quad 2.604900 E-02 \quad 1.605900 E-02 \quad 5.900900 E-01 \quad 5.828200 E-02 \quad 5.822900 E-01 \quad 1.427000 E-02 \quad 1.436200 E-02 \quad 1.464400 E-02$

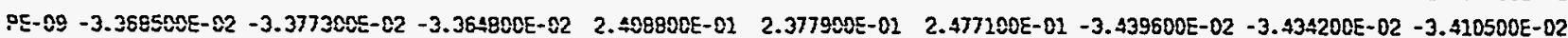
PE-10 2.004700E-02 $\quad 2.992300 E-02 \quad 2.007500 E-02 \quad 4.631700 E-01 \quad 4.570200 E-02 \quad 4.730900 E-01 \quad 2.205900 E-02 \quad 2.098600 E-02 \quad 2.110000 E-02$

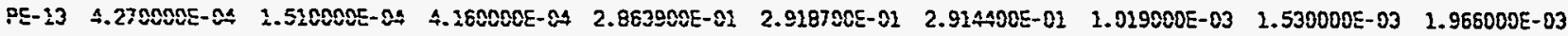
PE- $14 \quad 2.294200 E-02 \quad 1.295500 E-02 \quad 1.291500 E-02 \quad 5.287400 E-01 \quad 5.342500 E-01 \quad 5.235200 E-01 \quad 1.309100 E-01 \quad 1.309500 E-01 \quad 1.310100 E-01$

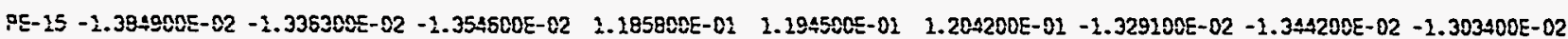
PE-15 3.897100E- $22 \quad 3.856500 E-02 \quad 3.880500 E-02 \quad 1.882200 E-02 \quad 1.879700 E-01 \quad 1.865700 E-01 \quad 3.896900 E-02 \quad 3.883600 E-02 \quad 3.872100 E-02$

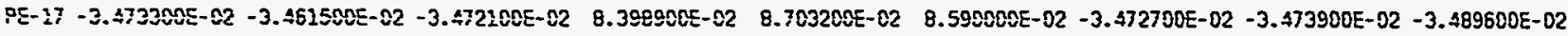

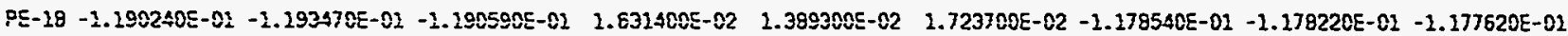

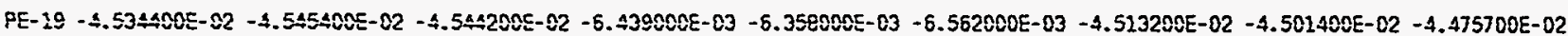
$P E-20-4.5994005-82-4.512100 E-82-4.624500 E-02-3.890000 E-04 \quad 2.209000 E-03 \quad 4.659000 E-03-4.603600 E-02 \quad-4.609500 E-02-4.608900 E-02$

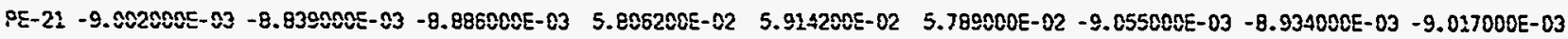
PE-22 -8.970000E-04 -7. $440000 E-04 \quad-6.838000 E-04 \quad 6.994300 E-02 \quad 7.242100 E-02 \quad 7.500800 E-02 \quad-9.790000 E-04 \quad-1.103000 E-03 \quad-1.195000 E-03$

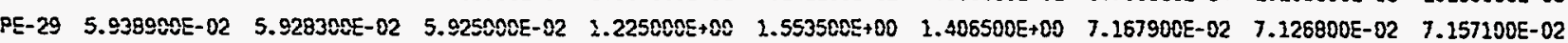

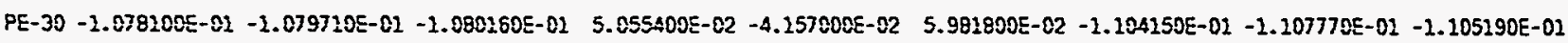
FE- $02 \quad 1.000370 E+00 \quad 1.2003085+00 \quad 3.000390 E+00 \quad 2.810100 E+00 \quad 2.825200 E+00 \quad 2.835700 E+00 \quad 1.002710 E+00 \quad 1.002050 E+00 \quad 1.001650 E+00$ XEE-01 -3.280000E-04 -3.270000E-04 -3.270000E-04 1.590000E-04 2.650000E-04 1.730000E-04 -3.080000E-04 -3.1300000E-04 -3.180000E-04 XE- 02 - $8.510080 E-04$-8.500000E- $04-8.490000 E-04-8.560000 E-04-8.540000 E-04$-8.580000E-04 -8.580000E-04 -9.570000E-04 -8.580000E-04 $X E-03-7.199000 E-03-7.170000 E-03-7.251000 E-03-2.721500 E-02-3.509700 E-02-3.051300 E-02-2.635400 E-02-2.643400 E-02-2.672100 E-02$ XE- $04-1.347000 E-03-1.346000 E-03-2.3450005-03-9.150000 E-04-9.090000 E-04-9.0408005-04-1.289000 E-03-1.297000 E-03-1.303000 E-03$ XE- $05-3.599000 E-03-3.6000005-03-3.600000 E-03-3.521000 E-03-3.526000 E-03-3.528000 E-03-3.605000 E-03-3.605000 E-03-3.599000 E-03$

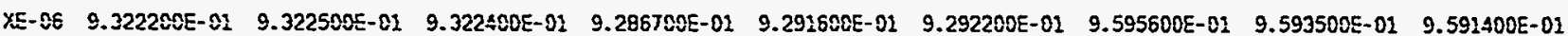

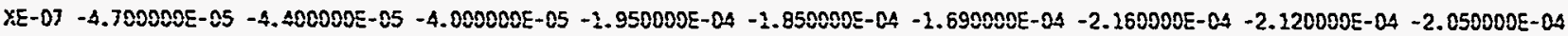
$X E-08-1.093000 E-03-1.082000 E-03-1.082000 E-03-9.890000 E-04-9.920000 E-04-9.870000 E-04-1.055000 E-03-1.065000 E-03-1.068000 E-03$ $X E-00-2.251000 E-03-1.249000 E-03-1.250000 E-03-8.180000 E-03-8.130000 E-04-8.070000 E-04-1.199000 E-03-1.199000 E-03-1.209000 E-03$ XE- $10-3.823000 E-03-3.822000 E-03-3.8250005-03-3.137000 E-03-3.1350005-83-3.210000 E-03-3.733000 E-03-3.758000 E-03-3.782000 E-03$ XE-11 2.500000E-05 2.500000E-05 2.500000E-05 2.600000E-05 2.600000E-05 2.600000E-05 2.600000E-05 $2.600000 E-05 \quad 2.600000 E-05$ XE- $12-1.7520005-03-1.7600005-03-1.7610005-03-1.689800 E-03-1.6900005-03-1.693000 E-03-1.758000 E-03-1.758000 E-03-1.758000 E-03$ $X E-13-5.620000 E-0 S-6.630000 E-04-6.620000 E-04 \quad 2.000000 E-04$ 1.220000E-04 1.280000E-04 $-4.900000 E-04 \quad-5.270000 E-04 \quad-5.530000 E-04$ XE-14 -2.470000E- 04 -2.340000E-04 -2.360008E- 04 1.580000E-04 $1.5600005-04$ 1.660000E-04 -1.990000E-04 -1.910000E-04 -2.120000E-04 $X E-15-7.630000 E-04-7.570000 E-04-7.560000 E-04 \quad 1.320000 E-04 \quad 1.370000 E-04 \quad 1.390000 E-04 \quad-5.190000 E-04 \quad-5.510000 E-04 \quad-5.720000 E-04$ TE-02 -9.300000E- $05-9.300000 E-05-9.4000005-05-6.9000005-05-6.700000 E-05-7.300000 E-05-1.070000 E-04-1.050000 E-04-1.120000 E-04$ $\begin{array}{llllllllllll}\text { VEXT1 } & 1.993700 E+00 & 1.993700 E+00 & 1.993700 E+00 & 1.993700 E+00 & 1.993700 E+00 & 1.993700 E+00 & 1.993700 E+00 & 1.993700 E+C 0 & 1.993600 E+00\end{array}$ YEXT2 $1.990000 E+00 \quad 1.990000 E+00 \quad 1.990000 E+00 \quad 1.990000 E+00 \quad 1.990000 E+00 \quad 1.990000 E+00 \quad 1.990000 E+00 \quad 1.990000 E+00 \quad 1.990000 E+00$

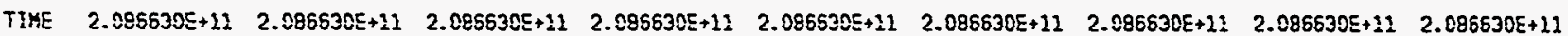


Arensive yzente:

AYS_YFP_TESTS

Book Hame: AHS_MePTI_RY Date \& Time: $2 \operatorname{Mar} 1900$ 03:46:26

Page Description:

OATA SET NO.-25I

TRAHS RY_ZERO-2 RE_ZERO-2 RY_ZERO-3 RV_LOAD-1 RY_LOAD-2 RV_LOAD-3 RY_ZERO-4 RY_ZERO-5 RV_ZERO-6

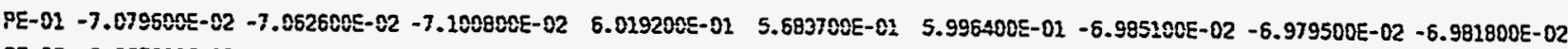
PE-02 3.057200E-02 3.056700E-02 $3.032900 E-02 \quad 8.942700 E-01 \quad 8.836900 E-01 \quad 8.5548005-02 \quad 3.1076005-02 \quad 3.150900 E-02 \quad 3.107400 E-02$

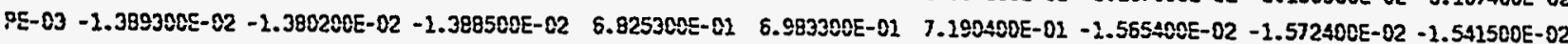
PE-04 3.928400E-02 $3.930000 E-02 \quad 3.931200 E-02 \quad 8.365700 E-02 \quad 8.531800 E-02 \quad 8.569200 E-02 \quad 3.932000 E-02 \quad 3.940300 E-02 \quad 3.9478005-02$ PE-05 7.501400E-02 7.498ACOE-02 $7.589200 E-02$ 4.871500E-01 4.529700E-02 5.012300E-02 7.523700E-02 7.510000E-02 $7.505 A 00 E-02$ PE-08 7.108509E-02 7.077700E-02 7.090600E-02 7.069100E-01 $5.921200 E-02 \quad 7.232200 E-02 \quad 5.978200 E-02 \quad 6.979300 E-02 \quad 5.9710005-02$

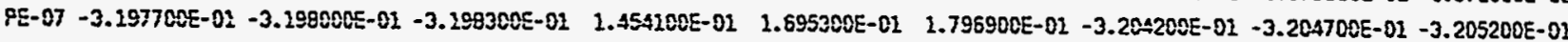
PE-08 2.410400E-02 2.421900E-02 $1.433200 E-02 \quad 5.965800 E-02 \quad 5.035200 E-02 \quad 5.941900 E-01 \quad 1.001500 E-02 \quad 2.000300 E-02 \quad 9.7340005-03$

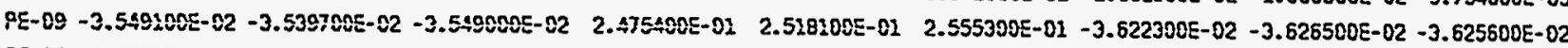
PE-10 2.962100E-02 $2.973700 E-02 \quad 1.974100 E-02 \quad 4.765600 E-02 \quad 4.723980 E-02 \quad 4.729400 E-02 \quad 2.009700 E-02 \quad 2.010000 E-02 \quad 1.999800 E-02$

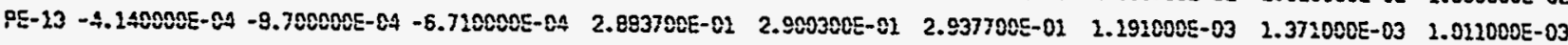

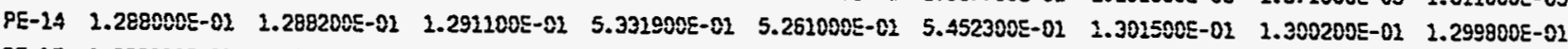

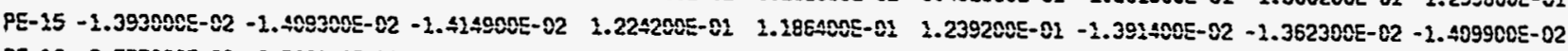
PE-16 3.777000E-\$2 $3.792100 E-02 \quad 3.7797005-02 \quad 2.883900 E-02 \quad 1.871400 E-01 \quad 1.9277005-02 \quad 3.823200 E-02 \quad 3.805800 E-02 \quad 3.813000 E-02$

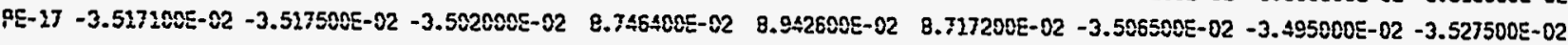
PE-18 -1.189810E-01 $-1.195240 E-02$-1.197130E-02 $1.257600 E-02 \quad 1.610600 E-02 \quad 1.737300 E-82$-1.181040E-01 $-1.181420 E-01$ - $1.286100 E-01$ $P E-29-4.5471005-02-4.5773005-82-4.5911005-02-7.5270005-03-5 . \$ 26000 E-03-9.236000 E-03-4.604500 E-02-4.599000 E-02-4.607100 E-02$ $P E-20$ - 4.743200E-02 -4.727900E-02 - $4.742900 E-02 \quad 2.233000 E-03 \quad 4.257000 E-03 \quad 3.547000 E-03-4.702200 E-02 \quad-4.676500 E-02 \quad-4.670400 E-02$ PE-21 - $-9.1600005-03$ - $9.2920005-03-9.321000 E-03 \quad 6.143000 E-02 \quad 6.129600 E-02 \quad 5.876200 E-02-9.474000 E-03 \quad-9.4210000 E-03-9.490000 E-03$ PE-22 -2.156000E-03 $-2.3230005-03 \quad-2.280000 E-03 \quad 7.118500 E-02 \quad 6.684600 E-02 \quad 7.0752005-02 \quad-2.8280005-03 \quad-2.973000 E-03 \quad-2.865000 E-03$ PE-29 5.640100E-02 $5.539500 E-02 \quad 5.629500 E-02 \quad 1.356900 E+00 \quad 1.2250005+00 \quad 1.294000 E+00 \quad 7.036400 E-02 \quad 6.948700 E-02 \quad 6.901900 E-02$

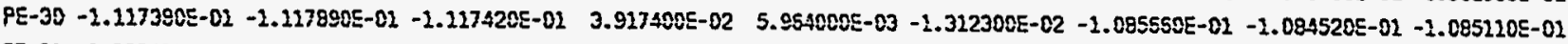

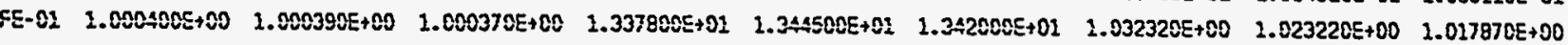

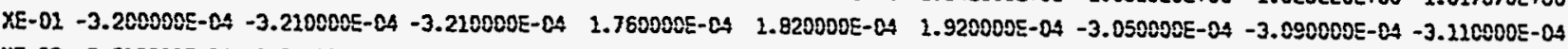
XE-02 -8.510000E- $04-8.500000 E-04-8.510000 E-04-8.560000 E-04-8.540000 E-04-8.5200005-04-8.610000 E-04-8.600000 E-04-8.580000 E-04$ $X E-03-2.0307005-02-2.023100 E-02-1.993700 E-02-3.204400 E-02-3.654900 E-02-3.605000 E-02-3.531100 E-02-3.505700 E-02-3.644900 E-02$ XE-04 -1.3340005-03 - $2.333000 E-03-1.333000 E-83-8.840000 E-04-8.830000 E-04-8.740000 E-04-1.276000 E-03-1.282000 E-03-1.289000 E-03$ XEE-05 -3.586000E-03 -3.582000E-03 -3.582000E-03 $9.172000 E-03-2.955000 E-03-3.051000 E-03-3.5030005-03 \quad-3.505000 E-03-3.605000 E-03$ XE-06 9.591700E-01 9.591800E-01 9.591900E-01 9.33530CE-01 9.309600E-01 9.304900E-01 9.648200E-01 9.849400E-01 9.6501C0E-01 $X E-07-2.650000 E-04-2.630000 E-04-1.610000 E-04-1.150000 E-04-2.100000 E-04-1.010000 E-04-1.620000 E-04-2.000000 E-04-1.790000 E-04$ XE-08 - $2.074000 E-03-1.0730005-03-1.073000 E-03-2.740000 E-04-9.690000 E-04-9.662000 E-04-1.050000 E-03-1.051000 E-03-1.053000 E-03$ $X E-09-1.2400005-03-1.2390005-03-1.240000 E-03-7.820000 E-04-7.770000 E-04-7.700000 E-04-2.180000 E-03-1.2000005-03-1.205000 E-03$ $X E-10-3.8790005-03-3.880000 E-03-3.8790000-03-3.123000 E-03-3.213000 E-03-3.103000 E-03-3.735000 E-03-3.765000 E-03-3.787000 E-03$ $X E-112.600000 E-85 \quad 2.500000 E-05 \quad 2.800000 E-05 \quad 2.500000 E-05 \quad 2.600000 E-05 \quad 2.700000 E-05 \quad 2.700000 E-05 \quad 2.700000 E-05 \quad 2.700000 E-05$ $X E-12-2.759000 E-03-2.758000 E-03-2.75 E 000 E-03-1.696000 E-23-1.6830005-03-1.683008 E-03-1.756000 E-03-1.756000 E-03-1.756000 E-03$ $X E-13-6.420000 E-04-6.430000 E-04-6.420000 E-04 \quad 2.130000 E-04$ 1.2500005-04 1.2900005-04 $-4.960000 E-04 \quad-5.2400000 E-04-5.490000 E-04$ $X E-14-2.5500005-04 \quad-1.770000 E-04 \quad-1.790000 E-04 \quad 2.170000 E-04 \quad 2.180200 E-04 \quad 2.320000 E-04-2.970000 E-04 \quad-2.030000 E-04-2.050000 E-04$ $X E-15-6.440000 E-04 \quad-6.450000 E-04 \quad-6.470000 E-04 \quad 1.250000 E-04 \quad 1.310000 E-04 \quad 1.200000 E-04-5.110000 E-04 \quad-5.370000 E-04 \quad-5.580000 E-04$ TE-01 -1. 490000E- $04-1.5000005-04-2.500000 E-04-1.200000 E-04-1.200000 E-04-1.190000 E-04-1.580000 E-04-1.590000 E-04-1.5500005-04$ $\begin{array}{lllllllllll}\text { VEXT2 } & 2.993700 E+00 & 2.993600 E+00 & 1.993700 E+00 & 1.993700 E+00 & 1.993700 E+00 & 1.993700 E+00 & 1.993700 E+00 & 1.993700 E+00 & 1.993700 E+00\end{array}$

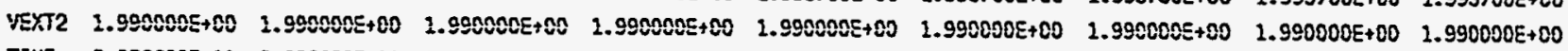

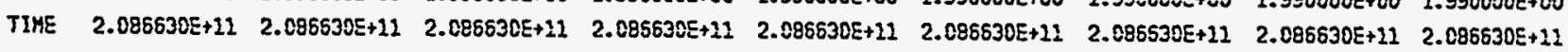


Archive

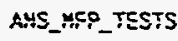

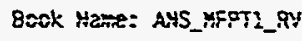

Date \& Time: 2 Yar 1900 03:57:21

Page Eescription:

DATA SET Y0.-252

TRA4S an z580-2

RY_LOAD-1

RV_LOAD-2

RV_LOAD-3

RY_ZERO-4

RY_ZERO-5

RV_ZERO-6

PE- $02-7.2671005-02-7.264500 E-02-7.269500 E-02-7.305900 E-02-7.310100 E-02-7.292900 E-02-7.292200 E-02-7.281000 E-02-7.283100 E-02$ $\begin{array}{llllllllll}P E-02 & 2.896609 E-02 & 2.904000 E-02 & 2.9093005-02 & 2.915100 E-02 & 2.904800 E-02 & 2.886300 E-02 & 2.915500 E-02 & 2.887600 E-02 & 2.904000 E-02\end{array}$ PE- 03 -1.881500E-02 -1.89-300E-02 - $2.865600 E-02-1.878700 E-02$ - $1.862700 E-02-1.871000 E-02-1.960200 E-02-1.979400 E-02-1.864200 E-02$ PE- 04 3.747300E-\$2 $3.368000 E-02 \quad 3.740100 E-02 \quad 3.785200 E-02 \quad 3.368200 E-02 \quad 3.371000 E-02 \quad 3.757500 E-02 \quad 3.747200 E-02 \quad 3.756100 E-02$

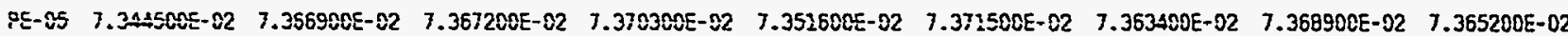
$\begin{array}{llllllllll}\text { PE-06 } & 6.756900 E-02 & 6.742000 E-02 & 5.750200 E-02 & 5.757500 E-02 & 6.759100 E-02 & 6.749400 E-02 & 5.765200 E-02 & 6.751700 E-02 & 6.772900 E-02\end{array}$

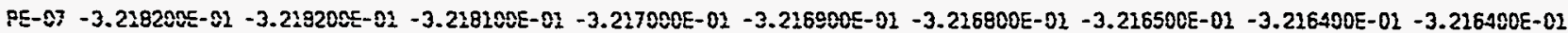
PE-08 $7.244000 E-03 \quad 7.059000 E-03 \quad 7.2 \varepsilon 1000 E-03 \quad 7.300000 E-03 \quad 7.602000 E-03 \quad 7.6900005-03 \quad 7.915000 E-03 \quad 7.835000 E-03 \quad 7.681000 E-03$ PE-09 -3. 800000E- $82-3.82 \$ 800 E-02-3.801300 E-02-3.778900 E-02-3.811600 E-02-3.774000 E-02-3.757600 E-02-3.7503005-02-3.769600 E-02$ PE-10 $1.796400 E-82 \quad 1.795300 E-02 \quad 1.798900 E-82 \quad 1.850600 E-02 \quad 1.855500 E-02 \quad 1.864500 E-02 \quad 1.858000 E-02 \quad 1.860300 E-82 \quad 1.874900 E-02$ PE- $13-2.5500005-03-2.3190005-03-2.547000 E-03-1.5570005-03-1.437000 E-03-1.4520000-03-2.7750005-03-1.3540005-03-1.4280005-03$

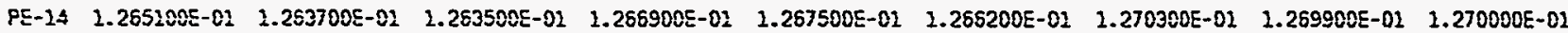
$P E-15-1.5206005-02-1.502300 E-02-1.551400 E-02-1.446600 E-02-1.428100 E-02-1.427600 E-\$ 2-1.428800 E-02-1.434700 E-02-1.437600 E-02$ PE- $16 \quad 3.709 A 00 E-02 \quad 3.741300 E-02 \quad 3.712800 E-02 \quad 3.765400 E-02 \quad 3.777500 E-02 \quad 3.7606000-02 \quad 3.808000 E-02 \quad 3.765100 E-02 \quad 3.763200 E-02$

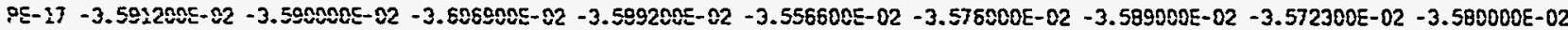
PE-19 -1.205900E-01 - $1.206400 E-02-1.199160 E-02-1.193220 E-01-1.296450 E-01-1.190910 E-01-1.199640 E-01-1.190890 E-02-1.195450 E-02$

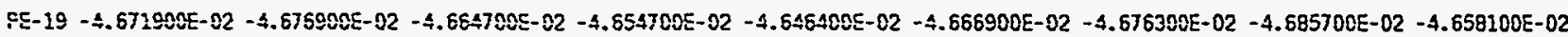
PE-20 - 4.830000E-02 -4.828900E-\$2 - 4.7823005-02 -4.7770005-02 -4.781300E-02 - 4.786000E-02 -4.7488800E-02 -4.7754005-02 -4.747000E-02 PE-21 - $9.8750005-03-1.8223005-82-1.023800 E-02-1.0056005-02-9.903000 E-03-9.788000 E-03-1.020600 E-02-1.020000 E-02-1.003600 E-02$ $P E-22-4.511000 E-03-4.229000 E-83-4.331000 E-03-4.3260005-03-4.371000 E-03-4.085000 E-03-3.977000 E-03-4.248000 E-03-4.024000 E-03$ PE-29 5.556120E- $02 \quad 5.528700 E-02 \quad 5.566100 E-02 \quad 5.579000 E-02 \quad 5.555300 E-02 \quad 5.555206 E-02 \quad 5.589500 E-02 \quad 5.553700 E-02 \quad 5.563800 E-02$ PE-30 -2.099810E-02 -2.098950E-02 -1.0970905-01 -1.094820E-01 -1.097360E-01 - $1.0952905-01-1.100020 E-01-1.099270 E-02-1.098790 E-02$

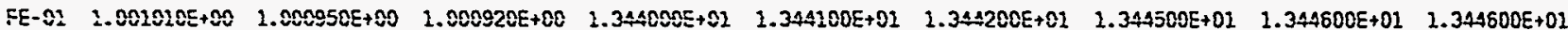
YE- $02-3.300000 E-04-3.290000 E-04-3.290000 E-04-3.3600005-04-3.370000 E-04-3.370000 E-04-3.390000 E-04-3.390000 E-04-3.390000 E-04$ $X E-02-8.5200005-04-8.520000 E-04-8.500000 E-04-8.550000 E-04-8.550000 E-04-8.560000 E-04-8.550000 E-04-8.540000 E-04-8.550000 E-04$ $x E-03-3.792300 E-02-3.797300 E-02-3.780200 E-02-3.333300 E-02-3.336300 E-02-3.338600 E-02-3.330200 E-02-3.333200 E-02-3.331000 E-02$ $X E-04-1.3390005-03-1.339000 E-03-1.3390005-03-1.345000 E-03-1.345000 E-03-1.347000 E-03-1.348000 E-03-1.349000 E-03-1.349000 E-03$ $X E-05-3.565000 E-03-3.563000 E-03-3.5550000 E-03-3.597000 E-03-3.593000 E-03-3.592000 E-03-3.594000 E-03-3.586000 E-03-3.584000 E-03$

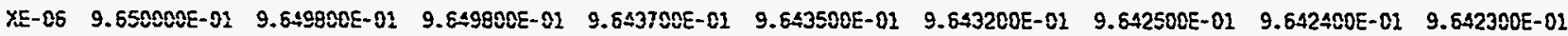
$x E-07 \quad 5.000000 E-05 \quad 7.000000 E-05 \quad 4.200000 E-05-1.490000 E-04-1.300000 E-04-1.310000 E-04-1.0500005-04-9.900000 E-05-1.020000 E-04$ $x E-88-1.0730005-03-1.073800 E-03-1.0740005-03-1.8800005-03-1.080000 E-03-1.079000 E-03-1.082000 E-03-1.082000 E-03-1.081000 E-03$ XE-09 -1.246000E-03 -1.246000E-03 - 1.245000E-03 -1.251000E-03 -1.251000E-03 - $1.251000 E-03-2.254000 E-03-2.253000 E-03-1.253000 E-03$

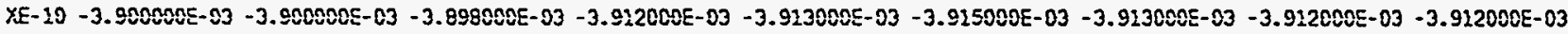
$\begin{array}{llllllllll}X E-12 & 2.500000 E-05 & 2.600000 E-05 & 2.6000000 E-05 & 2.600000 E-05 & 2.500000 E-05 & 2.6000000 E-05 & 2.600000 E-05 & 2.600000 E-05 & 2.600000 E-05\end{array}$ $X E-12-2.758000 E-03-1.756000 E-\$ 3-2.757090 E-03-2.759008 E-03-1.759000 E-03-1.759000 E-03-1.750000 E-03-1.758000 E-03-1.759000 E-03$ XE-13 - $5.530000 E-04-6.5400005-04-6.540000 E-04-6.770000 E-04-6.750000 E-04-6.750000 E-04-6.740000 E-04-6.770000 E-04-6.690000 E-04$ XE- $14-2.040000 E-04-2.000000 E-03-1.980000 E-04-2.750000 E-03-1.790000 E-04-2.980000 E-04-1.920000 E-04-1.920000 E-04-1.940000 E-04$ $x E-15-6.920000 E-04-6.930000 E-04-6.930000 E-04-7.020000 E-04-6.990000 E-04-6.980000 E-04-6.990000 E-04-6.970000 E-04-6.960000 E-04$ TE- $21-1.730000 E-04-1.7200005-04-1.710000 E-04$ - $1.540000 E-04-1.540000 E-04-1.530000 E-04-1.500000 E-04-1.520000 E-04-1.500000 E-04$ $\begin{array}{lllllllllll}\text { VEXT1 } & 1.993700 E+00 & 1.993700 E+00 & 1.993600 E+00 & 1.993700 E+00 & 1.993700 E+00 & 1.993700 E+00 & 1.993600 E+00 & 2.993500 E+00 & 1.993700 E+00\end{array}$ VEXT2 $1.990000 E+00 \quad 1.990000 E+00 \quad 1.990000 E+C 0 \quad 1.990000 E+00 \quad 1.990000 E+C 0 \quad 1.990000 E+00 \quad 1.990000 E+00 \quad 1.9900 C 0 E+00 \quad 1.990080 E+00$

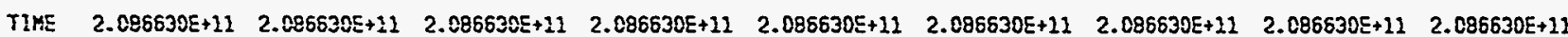


Arative

A4S_YFP_TESTS

Book Mañ: A4S_YEPTI_RY

Q2:e \& Time: 2 har 1980 04:\$2:34

Page Description:

DATA SET NO.-253

TRAYS RY_ZERO-1 RY_ZERO-2 RY_ZERO-3 RY_LOAD-1 RE_LOAD-2 RE_LOAD-3 RN_ZERO-4 FV_ZERO-5 RV_ZERO-6

PE- 01 -7.285600E- 02 -7.297300E-02 $-7.296700 E-02 \quad 5.966300 E-01 \quad 5.849500 E-02 \quad 6.007900 E-01 \quad-7.092400 E-02 \quad-7.101300 E-02 \quad-7.100400 E-02$ $\begin{array}{lllllllllll}P E-02 & 2.865500 E-02 & 2.871200 E-02 & 2.885600 E-02 & 8.990400 E-02 & 9.1591005-01 & 9.155800 E-01 & 3.182200 E-02 & 3.179100 E-02 & 3.152300 E-02\end{array}$ PE-03 - 1.9E610EE-02 -1.972200E-02 - $1.9625005-02 \quad 7.138400 E-02 \quad 7.029200 E-02 \quad 7.249300 E-02$ - $1.505300 E-02$ - $2.518000 E-02$ - $1.499700 E-02$ PE-04 4.COT600E-02 4.019500E-02 4.018900E-02 $8.624200 E-01 \quad 8.947000 E-01 \quad 8.795300 E-01 \quad 4.102600 E-02 \quad 4.1100005-02 \quad 4.090900 E-02$ PE-05 7.549000E-02 7.524700E-02 7.530E00E-02 5.029COCE-01 $5.054200 E-01 \quad 5.147000 E-02 \quad 7.622300 E-02 \quad 7.613500 E-02 \quad 7.636600 E-02$ PE-06 $\quad 8.739200 E-02 \quad 6.728300 E-02 \quad 6.725200 E-02 \quad 6.899600 E-02 \quad 7.4595005-02 \quad 7.299 E 00 E-01 \quad 7.022100 E-02 \quad 6.9967005-02 \quad 7.009100 E-02$

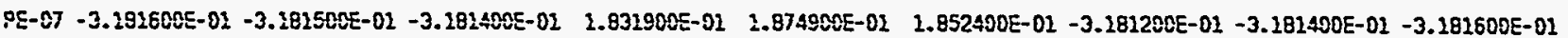
PE-C9 $\quad 8.497000 E-03 \quad 8.433000 E-03 \quad 8.630000 E-03 \quad 6.136100 E-01 \quad 6.044400 E-02 \quad 6.129000 E-01 \quad 1.590800 E-02 \quad 1.591700 E-02 \quad 1.816300 E-02$ PE- 09 -3.4905005-02 $-3.500900 E-02 \quad-3.499700 E-02 \quad 2.6142005-01 \quad 2.574700 E-02 \quad 2.631400 E-01 \quad-3.403000 E-02 \quad-3.392000 E-02 \quad-3.408300 E-02$ PE-10 1.927000E-02 $1.911100 E-02 \quad 1.927500 E-02 \quad 4.800900 E-01 \quad 4.723000 E-01 \quad 4.940200 E-02 \quad 2.1268005-02 \quad 2.095900 E-02 \quad 2.100500 E-02$ PE-23 -2.0E6000E-03 $-2.992000 E-03 \quad-1.849000 E-03 \quad 2.935700 E-01 \quad 2.973500 E-01 \quad 2.986900 E-01 \quad 1.824000 E-03 \quad 1.421000 E-03 \quad 1.037000 E-03$ $\begin{array}{llllllllllll}P E-14 & 1.261600 E-02 & 1.261300 E-01 & 1.263500 E-01 & 5.360000 E-01 & 5.439800 E-01 & 5.450000 E-02 & 1.304600 E-01 & 1.304400 E-02 & 1.305800 E-01\end{array}$

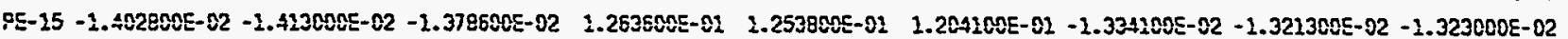
PE-16 $\quad 3.798000 E-02 \quad 3.783600 E-02 \quad 3.820200 E-02 \quad 1.901900 E-02 \quad 1.933900 E-02 \quad 1.916900 E-01 \quad 3.934800 E-02 \quad 3.8999005-02 \quad 3.901000 E-02$

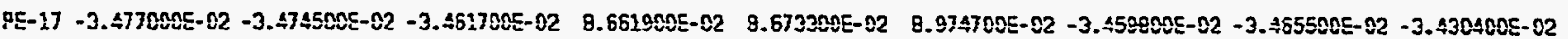

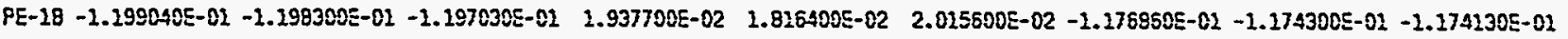
PE- $19-4.532600 E-02-4.557500 E-02-4.536800 E-02-5.367000 E-03-5.369000 E-03-4.904000 E-83-4.480400 E-02-4.467900 E-02-4.475000 E-02$ PE-20 -4.644500E-02 -4.642400E-02 -4.654000E-02 $4.804000 E-03 \quad 3.898000 E-03 \quad 3.487000 E-03$-4.539700E-02 $-4.617800 E-02 \quad-4.662200 E-02$

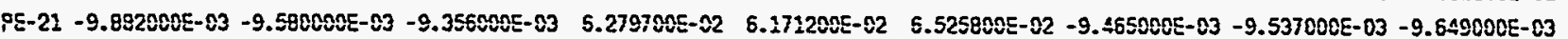
PE-22 - $-9.900000 E-04 \quad-9.0300005-04 \quad-1.007000 E-03 \quad 7.451000 E-02 \quad 7.374300 E-02 \quad 7.672700 E-02 \quad-1.1380005-03 \quad-9.490000 E-04 \quad-2.032000 E-03$ $\begin{array}{llllllllll}P E-29 & 5.952900 E-02 & 5.924300 E-02 & 5.975200 E-02 & 1.283300 E+00 & 2.353880 E+00 & 1.430300 E+00 & 6.173300 E-02 & 6.243400 E-02 & 6.152800 E-02\end{array}$

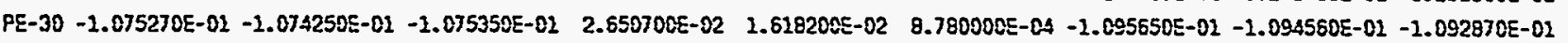

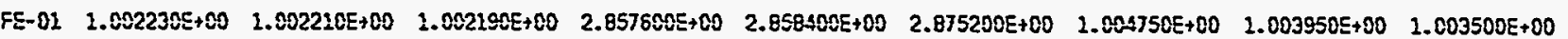
YE-02 -3.580000E-04 -3.680000E-04 -3.690000E-04 1.820000E-04 1.810000E-04 1.830000E-04 -2.850000E-04 -2.990000E-04 -3.090000E-04 XE-02 -8.5900C0E-04 -8.58C0CCE-04 -8.570600E-04 -8.620008E-04 -8.640000E-04 -8.640000E-04 -8.610000E-04 -8.590000E-04 -8.620000E-04 YE- $03-3.841900 E-02-3.842400 E-02-3.842100 E-02-5.244400 E-02-5.703000 E-02-6.596700 E-02-4.240400 E-02-4.225500 E-02-4.173400 E-02$ XE-04 - 1.402000E- 03 -1.402000E-03 - $1.401000 E-03-9.020000 E-04-8.970000 E-04-8.950000 E-04-1.296000 E-03-2.303000 E-83-1.314000 E-03$ XE-05 -3.6010005- $23-3.602000 E-03-3.600000 E-83-3.516000 E-03-3.502000 E-03-2.004000 E-03-3.570000 E-03-3.571000 E-03-3.570000 E-03$

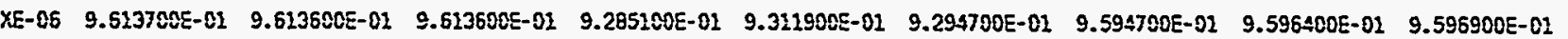
XE-07 4.530000E-04 4.840000E-04 5.060000E-04 -1.610000E-04 -2.500000E-04 -1.380000E-04 -2.030000E-04 -2.0400005-04 - $1.790000 E-04$ $X E-88-1.306000 E-03-1.206000 E-03-1.106000 E-03-9.900000 E-04-9.910000 E-04-9.850000 E-04-1.071000 E-03-1.072000 E-03-1.075000 E-03$ $X E-09-1.285000 E-03-1.284000 E-03-1.283000 E-03-7.880000 E-04-7.880000 E-04-7.820000 E-04-1.1750005-03-1.2880005-03-2.1990005-03$ $X E-10-3.949000 E-03-3.951000 E-03-3.955000 E-03 \quad-3.146000 E-03-3.135000 E-03-3.127000 E-03-3.655000 E-03-3.692000 E-03-3.725000 E-03$ XE-12 2.400000E-05 2.400000E-05 $2.400000 E-05 \quad 2.400000 E-05 \quad 2.600000 E-05 \quad 2.6000000 E-05 \quad 2.500000 E-05 \quad 2.500000 E-05 \quad 2.500000 E-05$ XE- $12-1.767000 E-03-2.767000 E-03-1.766000 E-03-1.687000 E-03-1.686000 E-03-1.686000 E-03-1.759000 E-03-1.760000 E-03-2.760000 E-03$ $X E-13 \quad-7.190000 E-04 \quad-6.750000 E-0.5-6.850000 E-04 \quad 7.700000 E-05 \quad 9.000000 E-05 \quad 9.600000 E-05-4.950000 E-04 \quad-5.310000 E-04 \quad-5.590000 E-04$ XE-14 -2.060000E-04 -2.010080E-84 -3.820000E-04 2.390000E-04 2.480000E-04 2.570000E-04 -1.330000E-04 - $1.710000 E-04$ - $1.390000 E-04$ XE-15 -7.170000E-04 -7.170000E-04 -7.250000E-04 2.120000E-04 1.230000E-04 1.360000E-04 -5.360000E-04 -5.730000E-04 -5.940000E-04 TE-01 - $-4.500000 E-05-4.6000005-05$ - $4.6000005-05-3.5000005-05-1.200000 E-05-1.000000 E-05-4.000000 E-05-4.500000 E-85-5.000000 E-05$ VEXTI $1.993700 E+00 \quad 1.993600 E+00 \quad 1.993700 E+00 \quad 1.993700 E+00 \quad 1.993700 E+00 \quad 1.993700 E+00 \quad 1.993700 E+00 \quad 1.993700 E+00 \quad 1.993600 E+00$

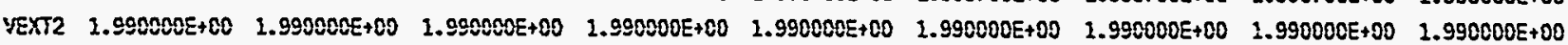

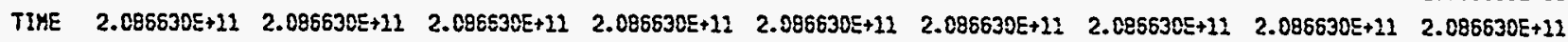




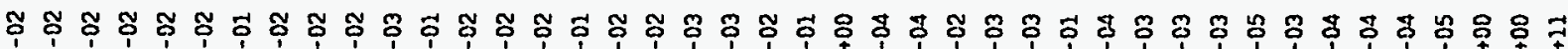

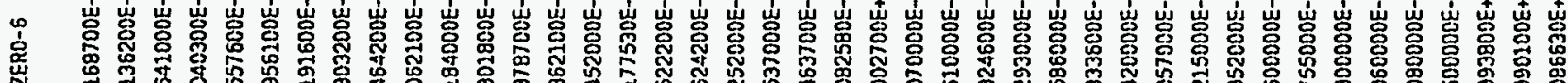

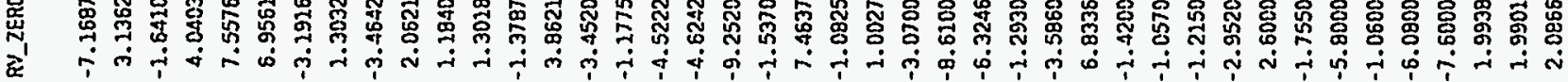

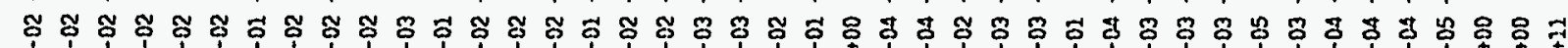

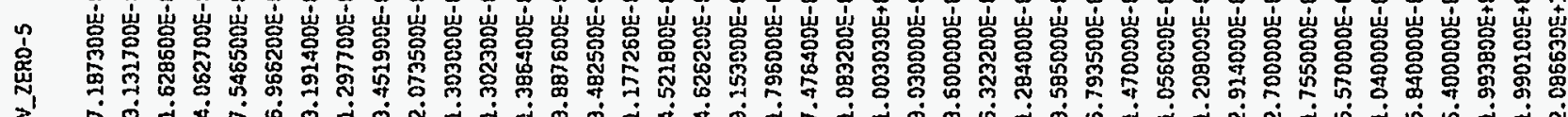

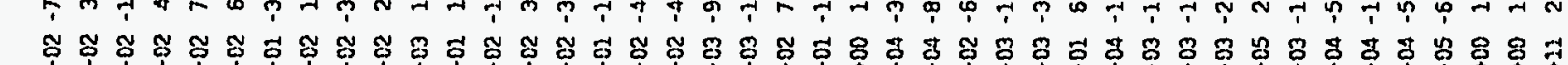

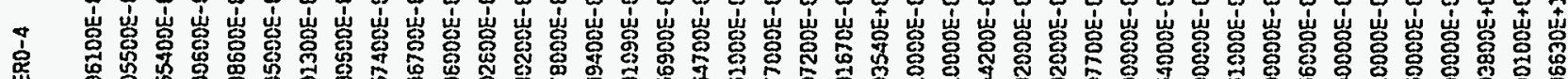

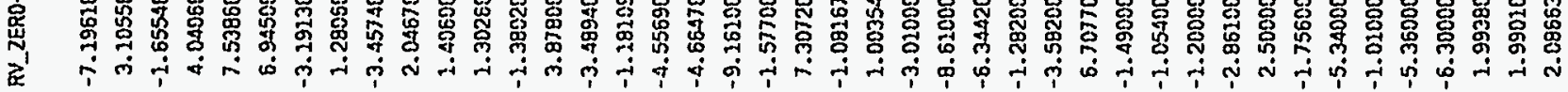

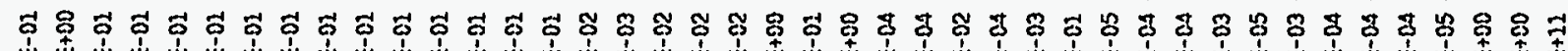

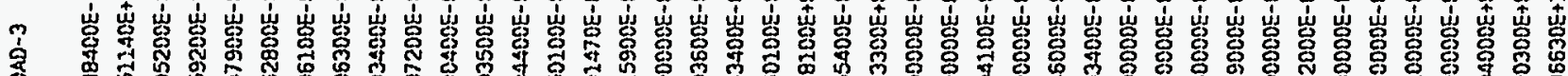

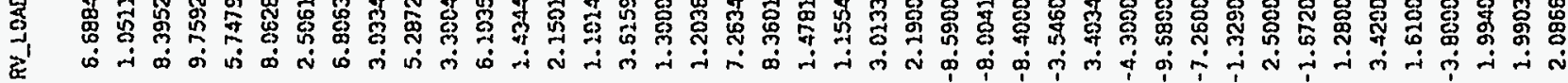

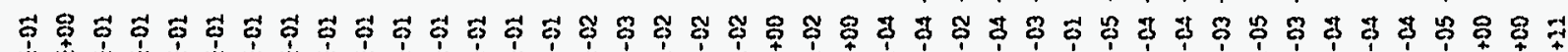

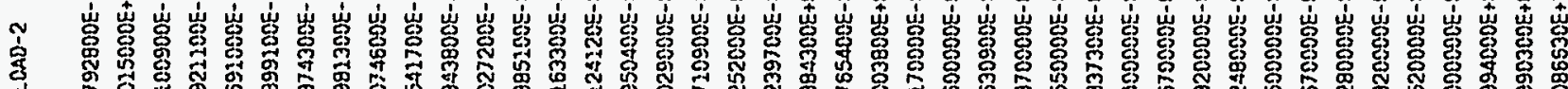

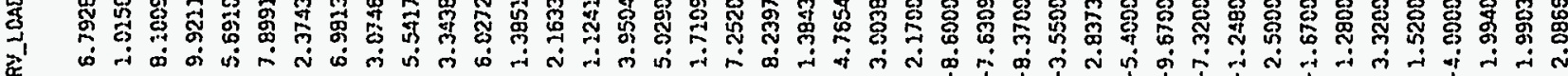

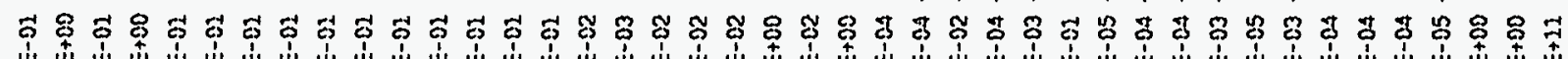

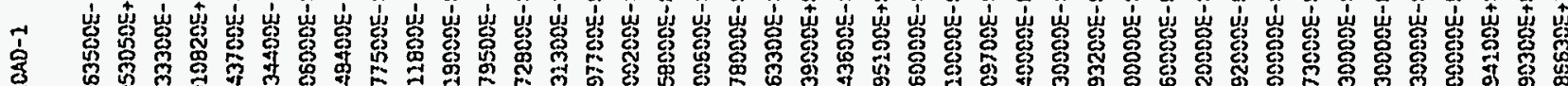

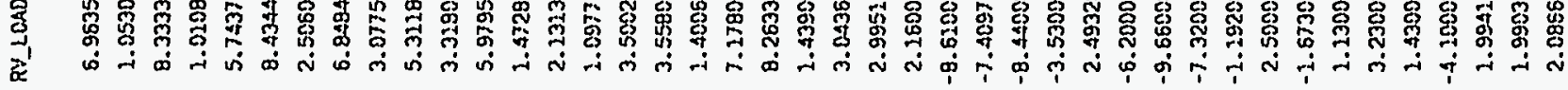

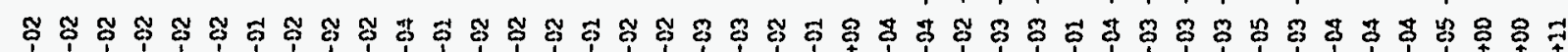

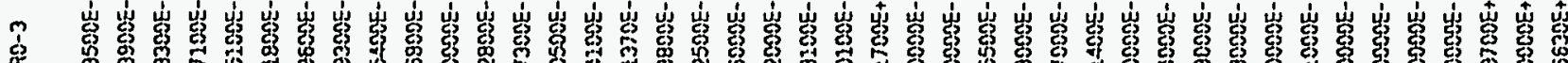

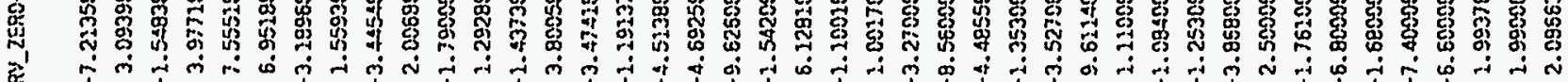

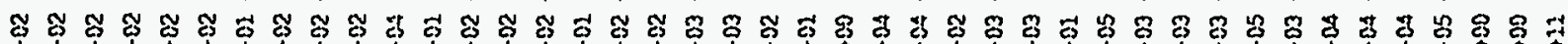

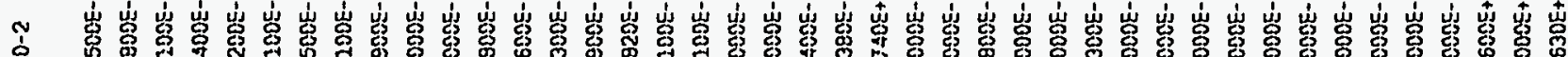

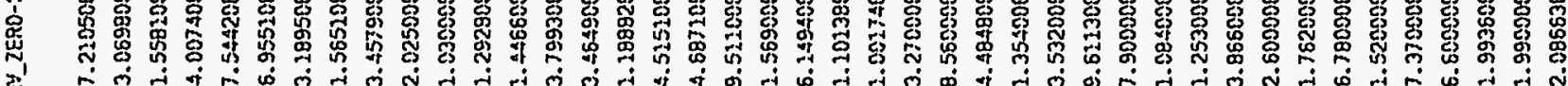

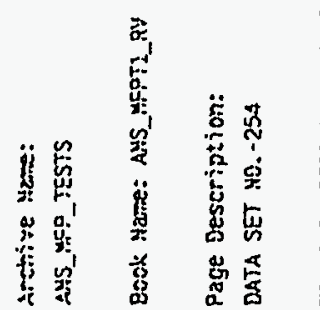

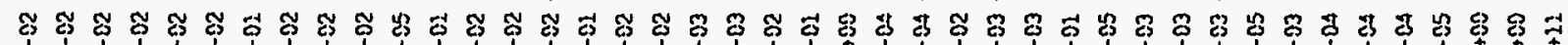

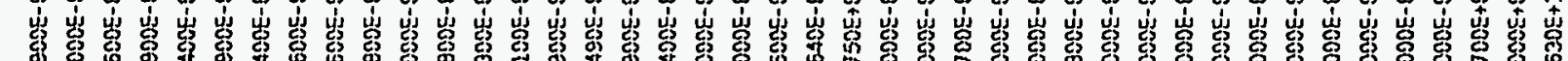

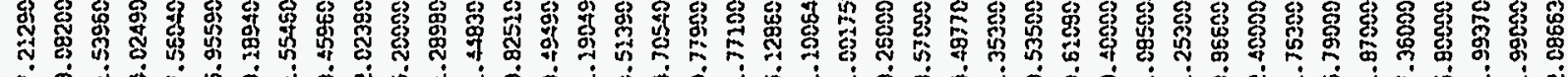

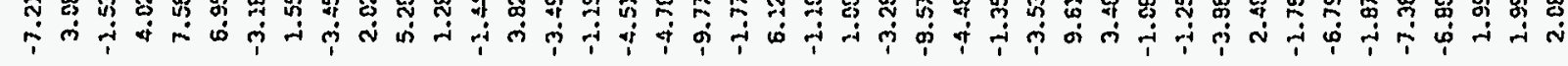

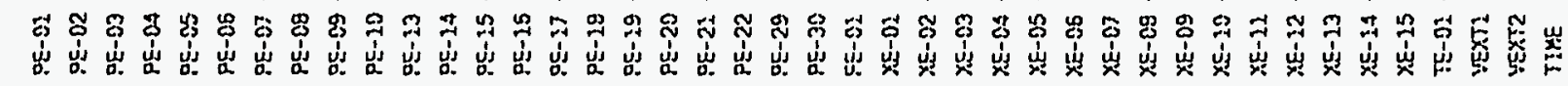


Arahive inger:

AYS_MFP_TESTS

Book Hame: A:TS_MFPTI_RY

Cate \& Time: 2 Mar 1980 05:00:35

Page Description:

OATA SET MO.-255

TRAAS RY_ZERO-2 RY_ZERO-2 RY_ZERO-3 RY_LOAD-1 RV_LOAD-2 RY_LOAD-3 RV_ZERO-4 RY_ZERO-5 RV_ZERO-6

PE-02 -7.240800E-02 -7.233800E-02 -7.248200E-02 7.351400E-02 7.58Q400E-02 7.4879C0E-01 -7.162000E-02 -7.175500E-02 -7.192500E-02 $\begin{array}{llllllllll}P E-02 & 3.0753 C 0 E-02 & 3.057200 E-02 & 3.055200 E-02 & 2.130230 E+00 & 1.235050 E+00 & 1.161050 E+00 & 3.1036005-02 & 3.074500 E-02 & 3.073200 E-02\end{array}$ PE-03 - - $.630100 E-02-2.657800 E-02-1.651200 E-02 \quad 9.306900 E-02 \quad 9.011400 E-01 \quad 9.174700 E-02 \quad-1.695900 E-02 \quad-1.701000 E-02 \quad-1.701400 E-02$ $\begin{array}{lllllllllll}P E-04 & 4.002200 E-02 & 3.981100 E-02 & 3.973700 E-02 & 1.139120 E+00 & 1.111150 E+00 & 1.155460 E+00 & 4.013600 E-02 & 4.035000 E-02 & 4.044300 E-02\end{array}$

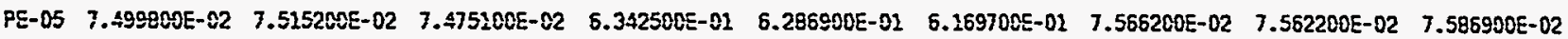
$\begin{array}{llllllllll}P E-05 & 6.946100 E-02 & 6: 924300 E-02 & 6.933800 E-02 & 8.890500 E-02 & 8.940500 E-02 & 9.093100 E-02 & 5.929100 E-02 & 6.910900 E-02 & 6.919100 E-02\end{array}$ PE-07 - -3.1969CCE-01 $-3.197100 E-01$-3.197300E-01 3.186ECOE-02 $2.936508 E-01 \quad 3.824900 E-01 \quad-3.200100 E-01 \quad-3.200300 E-01$-3.200400E-01

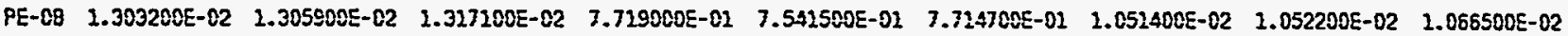

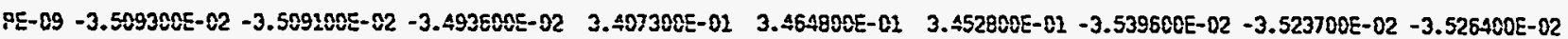
PE-10 2.043500E-02 2.048400E-02 2.039500E-02 $\quad 6.285100 E-02 \quad 5.929800 E-01 \quad 6.171900 E-01 \quad 2.098100 E-02 \quad 2.095400 E-02 \quad 2.073400 E-02$

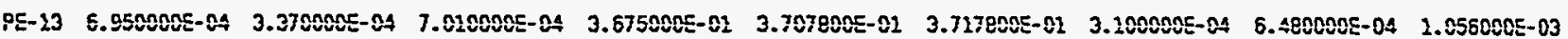

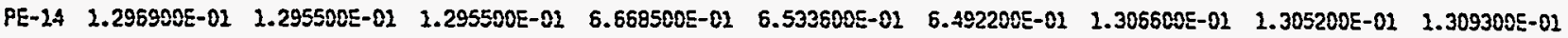

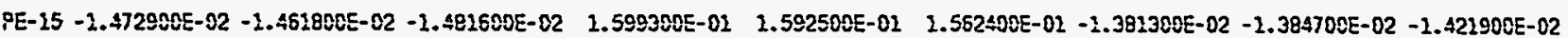
PE-16 3.835400E-02 $3.7802005-02 \quad 3.799000 E-02 \quad 2.380800 E-02 \quad 2.356200 E-02 \quad 2.362800 E-01 \quad 3.842700 E-02 \quad 3.838700 E-02 \quad 3.844000 E-02$

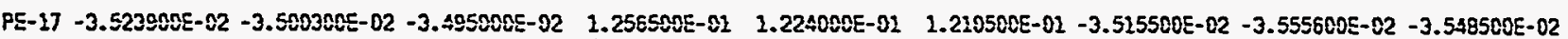
PE-28 - $1.191120 E-02$-1.192520E-01 $-1.294320 E-02 \quad 5.584700 E-02 \quad 5.194500 E-02 \quad 5.801800 E-02 \quad-1.182040 E-01 \quad-1.187330 E-01 \quad-2.185670 E-01$

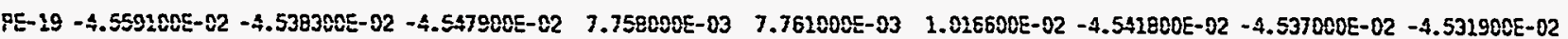
PE-20 - $4.702400 E-02 \quad-4.895900 E-02 \quad-4.898900 E-02 \quad 2.022700 E-02 \quad 2.141300 E-02 \quad 2.094400 E-02-4.735400 E-02 \quad-4.719400 E-02 \quad-4.706800 E-02$

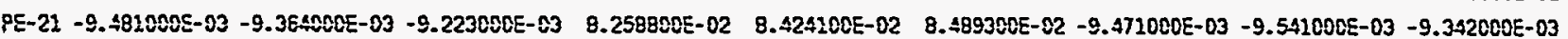
PE-22 - $1.827000 E-03 \quad-1.927000 E-03 \quad-2.033000 E-03 \quad 9.555700 E-02 \quad 9.533500 E-02 \quad 9.584500 E-02 \quad-1.078000 E-03 \quad-2.018009 E-03 \quad-2.161000 E-03$

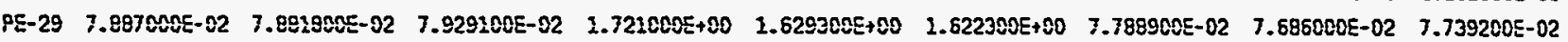
PE-30 -1.085050E-02 -1.086290E-01 $-1.085850 E-02$ E.110000E-03 $7.952700 E-02 \quad 7.080700 E-02-1.083470 E-01 \quad-1.095340 E-01 \quad-1.084940 E-01$

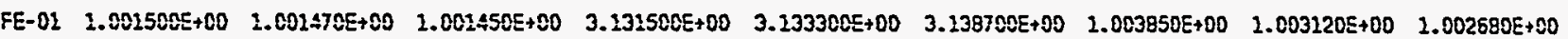
XE-01 -3.280000E-04 -3.290000E-04 -3.320000E-04 2.380000E-04 2.340000E-04 2.380000E-04 -2.920000E-04 -2.990000E-04 -2.990000E-04 XE-02 -8.580000E-04 -8.580080E-04 -8.590008E-04 -8.590080E-04 -8.5e0000E-04 -8.570000E-04 -8.510000E-04 -8.630000E-04 -8.620000E-04 XE-03 -6.191300E-02 -6.196100E-02 -6.295200E-02 - $2.378300 E-01-1.596900 E-02-1.414300 E-02-1.324700 E-01-1.309000 E-01-1.299600 E-02$ XE- $04-1.345000 E-03-1.347000 E-03-1.349000 E-03-8.190080 E-04-8.170000 E-04-8.140000 E-04-1.256000 E-03-1.2710005-03-1.279000 E-03$ XE-05 -3.595000E-03 -3.585000E-03 -3.586000E-03 -3.523000E-03 -3.50E000E-03 -3.487000E-03 -3.553000E-03 -3.560000E-03 -3.558000E-03 XE-C6 7.0\$2300E-02 $7.043800 E-01 \quad 7.049500 E-01 \quad 3.487000 E-01 \quad 3.418300 E-01 \quad 3.152000 E-01 \quad 7.230300 E-01 \quad 7.417700 E-01 \quad 7.490600 E-01$ XE-07 -6.000000E-06 -2.000000E-06 $\quad 1.600000 E-05-1.000000 E-05 \quad-5.000000 E-06 \quad 0.000000 E+00-1.2600005-04-1.250000 E-04-1.210000 E-04$ XE-08 - $1.077000 E-03-1.076000 E-03-1.077000 E-03-9.6100005-04-9.580000 E-04-9.570000 E-04-1.049000 E-03-1.051000 E-03-1.055000 E-03$ XE-09 - $1.244000 E-03-1.2450005-03-1.245000 E-03-7.010000 E-04-7.010000 E-04-7.000000 E-04-1.186000 E-03-1.199000 E-03-1.2060005-03$ XE-10 - -3.124000E- $03-3.230000 E-03-3.131000 E-03-1.476000 E-03-1.421000 E-03-1.312000 E-03-2.961000 E-03-3.054000 E-03-3.108000 E-03$ XE-12 2.500000E-05 2.600000E-05 2.600000E-05 $2.500000 E-05 \quad 2.800000 E-05 \quad 2.600000 E-05 \quad 2.500000 E-05 \quad 2.6000000 E-05 \quad 2.600000 E-05$ XE-12 -1.758000E-03 - - $.7580005-03-1.75 E 000 E-03-1.560000 E-03-1.661000 E-03-1.661000 E-03-1.747000 E-03-1.749000 E-03-1.749000 E-03$ XE-13 $-6.560000 E-04 \quad-6.570000 E-04 \quad-6.610000 E-04 \quad 3.2300005-04 \quad 1.280000 E-04 \quad 1.400000 E-04 \quad-5.020000 E-04 \quad-5.450000 E-04 \quad-5.620200 E-04$ YE-14 -1.840000E-04 -2.850008E- 24 -1.870080E-04 4.060000E-04 4.130000E-04 4.240000E-04 -5.000000E-05 -6.000000E-05 -7.600C80E-05 XE- 15 -7.660000E-04 -7.680000E-04 -7.660000E-04 2.680000E-04 1.770000E-04 1.810000E-04 $-5.160000 E-04$-5.640000E-04 $-5.920000 E-04$ TE- $01-9.500000 E-05-9.4000005-85-9.500008 E-05-5.800000 E-05-6.700000 E-05-6.600000 E-05-8.500000 E-05-9.800000 E-05-1.000000 E-04$ VEXT1 $2.993800 E+00 \quad 1.993900 E+00 \quad 2.993900 E+00 \quad 1.994100 E+00 \quad 1.994000 E+00 \quad 1.994000 E+00 \quad 1.993800 E+00 \quad 1.993900 E+00 \quad 1.993900 E+00$

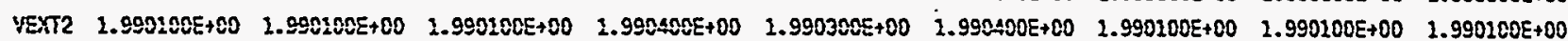

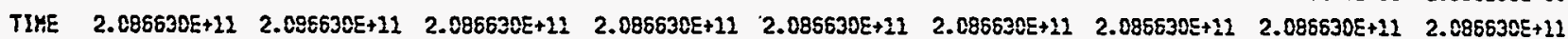




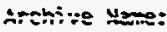

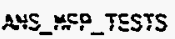

Book Yame: AHS_MfPT2_RY

Date \& Time: 2 Yar 1900 :5:09:20

Page Description:

EATA SET HO.-256

TRAHS RV_ZERO-1 RY_ZERO-2 RY_ZERO-3 RV_LOAD-1 RV_LOAD-2 RV_LOAD-3 RV_ZERE-4 RY_ZERO-5 RV_ZERO- 6

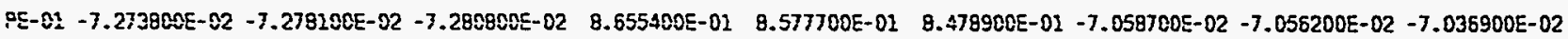

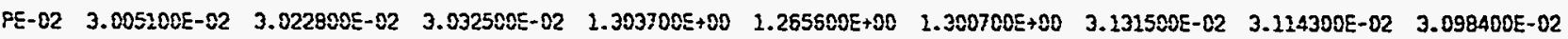

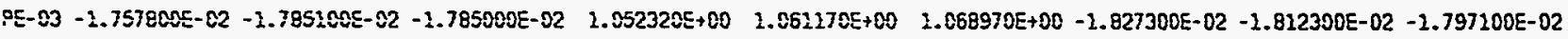
$\begin{array}{llllllllll}P E-02 & 3.953300 E-02 & 3.956800 E-02 & 3.969500 E-02 & 1.264900 E+00 & 1.261000 E+00 & 1.245600 E+00 & 3.992600 E-02 & 3.966800 E-02 & 3.957300 E-02\end{array}$ PE-05 7.422500E-02 7.427300E- 22 7.431700E-02 7.112400E-02 7.0556C0E- 01 7.072300E-01 $7.443100 E-02 \quad 7.454600 E-02 \quad 7.444400 E-02$ PE-05 $\quad 6.870300 E-02 \quad 5.857800 E-02 \quad 5.849400 E-02 \quad 3.031770 E+00 \quad 3.032560 E+00 \quad 1.0459905+00 \quad 5.917300 E-82 \quad 6.903800 E-02 \quad 6.931300 E-02$

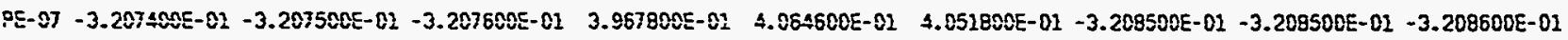
$\begin{array}{lllllllllll}\text { PE- } 08 & 9.810000 E-03 & 9.492000 E-03 & 9.621000 E-03 & 8.719300 E-01 & 8.878600 E-02 & 8.607600 E-01 & 1.448700 E-02 & 1.458000 E-02 & 1.460400 E-02\end{array}$ PE- $20-3.544000 E-02-3.544200 E-82-3.564500 E-02 \quad 3.973000 E-02 \quad 4.043500 E-01 \quad 3.903200 E-01$ - $3.544200 E-02-3.543000 E-02-3.497200 E-02$ PE-10 2.007700E-02 2.009300E-02 2.011700E-02 $7.1531005-02 \quad 7.020000 E-02 \quad 7.013900 E-02 \quad 2.0436005-02 \quad 2.041300 E-02 \quad 2.053200 E-02$

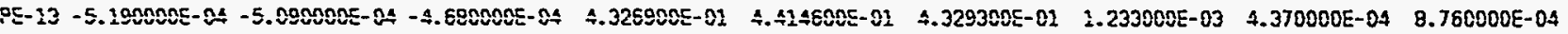

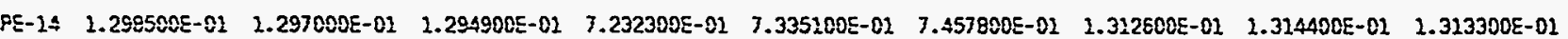

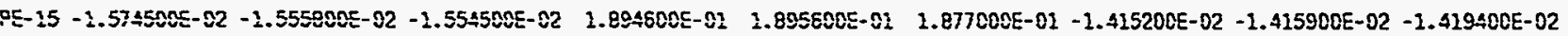
PE- $26 \quad 3.718400 E-02 \quad 3.728900 E-82 \quad 3.745500 E-02 \quad 2.6545005-01 \quad 2.576000 E-01 \quad 2.652400 E-01 \quad 3.797000 E-02 \quad 3.807500 E-02 \quad 3.807600 E-02$

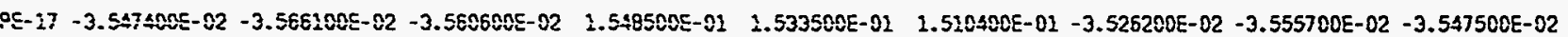
$P E-19-1.198610 E-01 \quad-1.199780 E-01 \quad-2.198750 E-01 \quad 7.892000 E-02 \quad 8.347800 E-02 \quad 8.189800 E-02 \quad-1.183570 E-01 \quad-1.181310 E-01 \quad-1.279790 E-01$ $9 E-19-4.5818005-02-4.6036005-02-4.5693005-02 \quad 5.7292005-02 \quad 6.249500 E-02 \quad 5.8693008-02-4.563108 E-02-4.583000 E-02-4.604500 E-02$ $P E-20-4.793400 E-02$ - $4.795000 E-02-4.776500 E-02 \quad 5.929700 E-82 \quad 5.946000 E-02 \quad 5.535900 E-02-4.795000 E-02$-4.803300E-02 -4.784200E-02

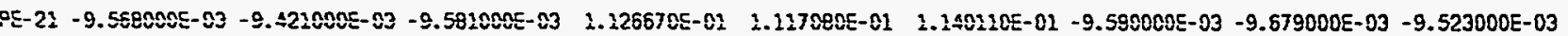
PE-22 -3.049000E-03 $-2.897000 E-03 \quad-3.279000 E-03 \quad 1.276920 E-02 \quad 1.187120 E-01 \quad 2.195630 E-01 \quad-3.059000 E-03 \quad-3.178000 E-03 \quad-2.901000 E-03$ $\begin{array}{llllllllll}9 E-29 & 7.736500 E-02 & 7.693700 E-\$ 2 & 7.750300 E-02 & 1.915900 E+00 & 1.828130 E+00 & 2.019400 E+00 & 7.689700 E-02 & 7.531900 E-02 & 7.442100 E-02\end{array}$

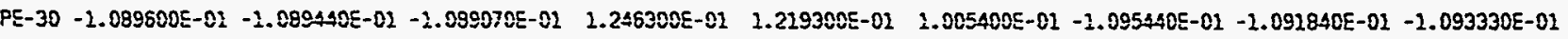
FE- $01 \quad 2.001170 E+00 \quad 1.001160 E+00 \quad 1.001150 E+00 \quad 3.295900 E+00 \quad 3.302200 E+00 \quad 3.302800 E+00 \quad 1.004110 E+00 \quad 1.003170 E+00 \quad 1.002630 E+00$ XE-02 -3.300000E-04 -3.310000E-04 -3.310000E-04 2. $290000 E-04 \quad 2.710000 E-04$ 2.700000E-04 $-2.730000 E-04 \quad-2.870000 E-04 \quad-2.740000 E-04$ $x E-82-8.6000005-04-8.610000 E-04-8.6000005-04-8.460000 E-04-8.480000 E-04-8.540000 E-04-8.620000 E-04-8.640000 E-04-8.640000 E-04$

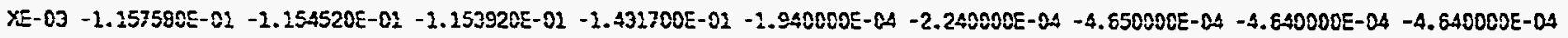
XE- $05-1.345080 E-03-1.347000 E-03-1.348000 E-03-8.060000 E-04-7.970000 E-04-7.920000 E-04-1.250000 E-03-1.265000 E-03-1.278000 E-03$ YE-05 -3.525002E-03 -3.527000E-03 -3.525008E- $83-3.557000 E-03-3.2810005-03-3.558000 E-03-3.585000 E-03-3.580000 E-03-3.583000 E-03$

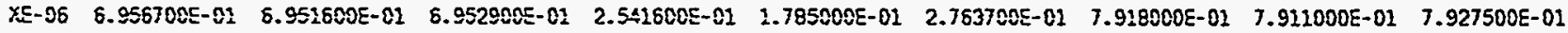

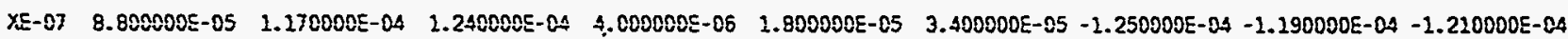
xE- $\$ 2-1.091000 E-03-2.091000 E-03-1.021000 E-03-9.430000 E-04-9.280000 E-04-9.200000 E-04-1.043000 E-03-1.047000 E-03-1.052000 E-03$ XE- $80-1.247000 E-83-1.249000 E-83-2.2490005-03-6.680000 E-04-6.630000 E-04-6.430000 E-04-1.172000 E-03-1.196000 E-03-1.192000 E-03$ $X E-10-3.1200005-03-3.1100005-03-3.1130005-03-1.0010005-03-1.1100005-03-1.094000 E-03-3.120000 E-03-3.161000 E-03-3.206000 E-03$ $\begin{array}{lllllllllll}X E-12 & 2.500000 E-05 & 2.500000 E-05 & 2.600800 E-05 & 2.500000 E-05 & 2.500000 E-05 & 2.500000 E-05 & 2.500000 E-05 & 2.500000 E-05 & 2.600000 E-05\end{array}$ $X E-12-1.7550005-03-1.755000 E-03-1.755000 E-03-1.639000 E-03-1.634000 E-03-1.6310005-03-1.737500 E-03-1.738000 E-03-1.739000 E-03$ XE-13 $-5.610000 E-04-6.610000 E-04-6.580000 E-04$ 1.020000E-04 $1.170000 E-04$ 1.250000E-04 $-5.030000 E-04-5.380000 E-04-5.550000 E-04$ XE- 24 - $1.5400005-04$ - $2.560000 E-04-2.530000 E-04$ S.590000E-04 5.930000E-04 6.198000E-04 3.700000E-05 7.000000E-06 - $1.300000 E-05$ $X E-15-7.570000 E-04$-7.500000E-04 -7.440000E-04 1.520000E-04 1.590000E-04 1.750000E-04 -5.160000E-04 -5.610000E-04 -6.000000E-04

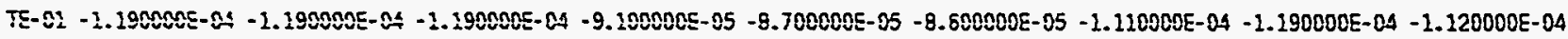
VEXT: $1.993800 E+00 \quad 1.993800 E+00 \quad 1.993800 E+00 \quad 1.994100 E+00 \quad 1.994000 E+00 \quad 1.994000 E+00 \quad 1.993700 E+00 \quad 1.993700 E+00 \quad 1.993700 E+00$

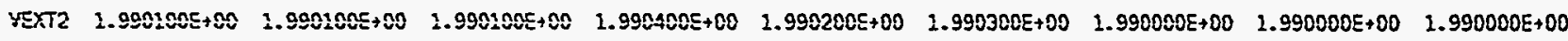
TIME 2. $285630 E+12 \quad 2.086630 E+11 \quad 2.085630 E+12 \quad 2.086530 E+12 \quad 2.096630 E+12 \quad 2.086530 E+112.086630 E+11 \quad 2.0866530 E+112.086630 E+11$ 


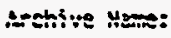

ANE_ME_TESTS

Back Hane: A4S_ufPTI_Ry Date 2 Time: 2 Mar 1900 05:18:36

iage Description:

DATA SET HO.-257

TRAHS RY_ZERO-1 RY_ZERO-2 RV_ZERO-3 RV_LOAD-2 RY_LOAD-2 RV_LOAD-3 RY_ZERO-4 RY_ZERO-5 RY_ZERO-6

PE-01 -7.171100E-02 -7.209200E-02 -7.201000E-02 9.261603E-01 9.249200E-01 9.237800E-01 -7.012300E-02 -7.038100E-02 -7.029800E-02 $\begin{array}{llllllllll}P E-02 & 3.030400 E-02 & 3.025400 E-02 & 3.041800 E-02 & 1.441700 E+00 & 1.367300 E+00 & 1.334600 E+00 & 3.127800 E-02 & 3.124500 E-02 & 3.105800 E-02\end{array}$ PE- 03 - $1.895700 E-02-1.912800 E-02-1.909100 E-02 \quad 1.150900 E+00 \quad 1.1326005+00 \quad 1.136200 E+00-1.734800 E-02-1.737600 E-02-1.695500 E-02$ PE-04 3.833800E-02 $3.895200 E-82 \quad 3.8923005-02 \quad 1.337600 E+00 \quad 1.356200 E+00 \quad 1.357200 E+00 \quad 3.916400 E-02 \quad 3.882400 E-02 \quad 3.897000 E-02$ PE-05 T.330400E-02 7.360000E-02 $7.319000 E-02 \quad 7.5914005-01 \quad 7.493200 E-01 \quad 7.577700 E-01 \quad 7.434800 E-02 \quad 7.444500 E-02 \quad 7.431100 E-02$ $\begin{array}{llllllllll}P E-06 & 6.834200 E-02 & 6.876100 E-02 & 6.836300 E-02 & 1.147390 E+00 & 1.135120 E+00 & 1.224240 E+00 & 6.870800 E-02 & 6.863300 E-02 & 5.883900 E-02\end{array}$

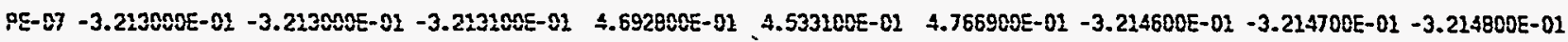
PE-08 $1.446 E 00 E-02 \quad 1.4170005-02 \quad 1.438500 E-02 \quad 9.544500 E-02 \quad 9.615800 E-01 \quad 9.549800 E-01 \quad 1.454500 E-02 \quad 1.439900 E-02 \quad 1.474000 E-02$

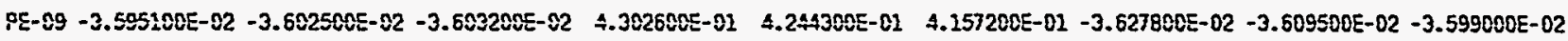
PE-10 1.975500E-02 $\quad 2.9633005-02 \quad 1.957700 E-02 \quad 7.717500 E-01 \quad 7.5753005-01 \quad 7.579800 E-01 \quad 2.075800 E-02 \quad 2.064900 E-02 \quad 2.082500 E-02$

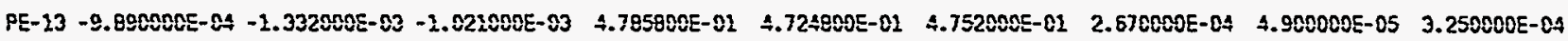
$\begin{array}{lllllllllll}\text { PE- } 14 & 1.297400 E-01 & 1.297200 E-01 & 1.297700 E-02 & 8.07 E 900 E-01 & 7.894500 E-01 & 7.848700 E-01 & 1.319200 E-01 & 1.316800 E-01 & 1.320100 E-02\end{array}$

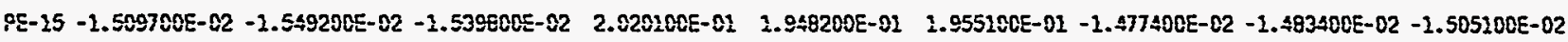
$P E-16 \quad 3.549700 E-02 \quad 3.679800 E-02 \quad 3.674500 E-02 \quad 2.852000 E-02 \quad 2.830000 E-01 \quad 2.781500 E-01 \quad 3.777800 E-02 \quad 3.763300 E-02 \quad 3.784600 E-02$

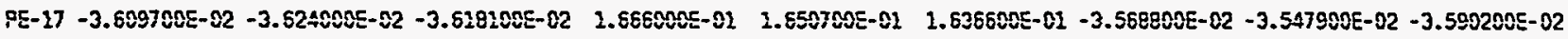

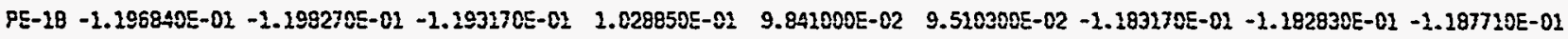
$P E-19-4.5429005-02$ - $4.622500 E-02$ - $4.505100 E-02 \quad 5.124600 E-02 \quad 2.547400 E-02 \quad 2.347400 E-02$ - $4.600500 E-02-4.5758 C 0 E-02-4.577700 E-02$ $P E-20-4.814700 E-02-4.834800 E-02-4.837200 E-02 \quad 6.344900 E-02 \quad 3.995100 E-02 \quad 3.995200 E-02 \quad-4.816600 E-02 \quad-4.8285005-02-4.845500 E-02$

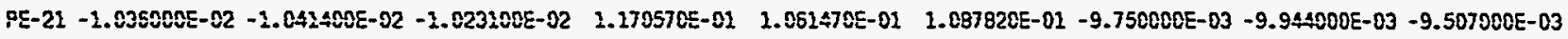
PE-22 $-3.557000 E-03 \quad-3.632000 E-03 \quad-3.344000 E-03 \quad 2.288800 E-02 \quad 3.210500 E-01 \quad 3.257700 E-02 \quad-3.539000 E-03 \quad-3.6320005-03 \quad-3.442000 E-03$ $\begin{array}{lllllllllll}85-29 & 5.5520005-02 & 5.8492005-02 & 5.855380 E-02 & 2.399408 E+00 & 2.1790805+00 & 1.884100 E+00 & 7.165000 E-02 & 7.139000 E-02 & 7.169600 E-02\end{array}$ $P E-30-1.104990 E-02 \quad-1.206720 E-02 \quad-1.105310 E-02 \quad 3.550600 E-02 \quad 1.219400 E-01 \quad 1.329700 E-01 \quad-1.130770 E-02 \quad-1.130180 E-01 \quad-1.131510 E-01$ SE-01 $1.00103 E E+00 \quad 2.001030 E+00 \quad 1.002070 E+00 \quad 3.40930 E E+00 \quad 3.309800 E+00 \quad 3.215700 E+00 \quad 1.002470 E+00 \quad 1.002140 E+00 \quad 1.001930 E+00$ YXE-01 -3.250000E-04 -3.250000E-04 -3.250000E-04 2.900000E-04 2.930000E-04 2.950000E-04 -2.880000E-04 -2.950000E-04 -2.970000E-04 XE- $02-8.590000 E-04-8.600000 E-04-8.5803005-04-8.620000 E-04-8.600000 E-04-8.640000 E-04-8.640000 E-04-8.650000 E-04-8.630000 E-04$ $X E-03-4.630000 E-04-4.620000 E-04-4.610000 E-04-2.530800 E-04-2.4900005-04-2.670000 E-04-5.880000 E-04-5.880000 E-04-5.880000 E-04$ XE-04 - - $2.341000 E-03-1.3430005-03-1.340000 E-03-7.7800005-04-7.7200005-04-7.7400005-04-1.272000 E-03-1.280000 E-03-1.284000 E-03$ XE-05 -3.565000E-03 -3.553000E-03 -3.570000E-03 $4.186000 E-03-3.646000 E-03 \quad 5.900000 E-04-3.561000 E-03 \quad-3.566000 E-03-3.564000 E-03$ $\begin{array}{lllllllllll}X E-06 & 8.355200 E-01 & 8.3773 C C E-01 & 8.396900 E-01 & 3.471500 E-01 & 6.377700 E-02 & 3.402900 E-01 & 8.918600 E-01 & 8.939800 E-01 & 8.965400 E-01\end{array}$ XE-07 $\quad 6.300000 E-05 \quad 8.800000 E-05 \quad 9.700000 E-05 \quad 4.900000 E-05 \quad 6.100000 E-05 \quad 7.600000 E-05 \quad-9.800000 E-05 \quad-8.8000000-05-7.800000 E-05$ XE- $88-1.073000 E-03-1.073000 E-03-1.073000 E-03-9.065000 E-04-9.0500005-04-8.950000 E-04-1.041000 E-03-1.043000 E-03-1.044000 E-03$ XE-09 - - 2.237000E-03 -1.236000E-03 - $1.235000 E-03-6.150000 E-04-6.100000 E-04-5.100000 E-04-1.166000 E-03-1.172000 E-03-1.175000 E-03$ XE-10 -3.513000E-03 -3.512000E-03 - -3.527000E-03 -1.289000E- $03-2.176000 E-03-1.306000 E-03-3.473000 E-03-3.508000 E-03-3.514000 E-03$ XE-11 2.500000E-05 2.600000E- $25 \quad 2.600000 E-05 \quad 2.400000 E-05 \quad 2.600000 E-05 \quad 2.600000 E-05 \quad 2.600000 E-05 \quad 2.600000 E-05 \quad 2.600000 E-05$ $X E-12-1.745000 E-03-1.734000 E-03-1.744000 E-03-1.515000 E-03-1.615000 E-03-1.612000 E-03-1.730000 E-03-1.730000 E-03-1.729000 E-03$ XE-13 - $5.550000 E-04-6.530000 E-04-8.550000 E-04$ 1.300000E-04 1.430000E-04 1.500000E-04 $-5.610000 E-04 \quad-5.770000 E-04$-5.850000E-04 XE- 24 -9. $4000008-05$-9.200000E-05 $-9.300000 E-05$ 6.720000E-04 7.060000E-04 7.150000E-04 3.000000E-05 $4.200000 E-05 \quad 2.400000 E-05$ XE-15 -7.490000E-04 -7.390000E-04 -7.510000E-04 1.510000E-04 1.610000E-04 1.710000E-04 -6.140000E-04 -6.360000E-04 -6.610000E-04 TE- $02-1.290000 E-04-2.2700005-04-1.270000 E-04-9.500000 E-05-9.300000 E-05-9.100000 E-05-1.150000 E-04-1.150000 E-04-1.160000 E-04$ $\begin{array}{llllllllllll}\text { VEXT2 } & 1.993700 E+00 & 1.993700 E+00 & 1.993700 E+00 & 1.993900 E+00 & 1.993900 E+00 & 1.993900 E+00 & 1.993700 E+00 & 1.993700 E+00 & 1.993600 E+00\end{array}$ $\begin{array}{lllllllllll}\text { VEXT2 } & 1.990000 E+00 & 1.990000 E+00 & 1.990000 E+00 & 1.990200 E+00 & 1.990100 E+00 & 1.9903 E 0 E+00 & 1.990000 E+C 0 & 1.990000 E+00 & 1.990000 E+00\end{array}$ TIME $2.086630 E+112.086530 E+12 \quad 2.086630 E+12 \quad 2.086630 E+11 \quad 2.086630 E+12 \quad 2.0866530 E+112.096630 E+112.086530 E+112.085630 E+11$ 
itchive بnes:

ALS_L_:_TESTS

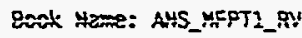

Date \& Time: 2 na: 2900 os:25:32

Page Eescription:

DATA SET MO. -258

TRAYS RY_ZERO-1 RY_ZERO-2 RY_ZERO-3 RY_LOAD-1 RY_LOAD-2 RY_LOAD-3 RY_ZERO-4 RV_ZERO-5 RY_ZERO-6

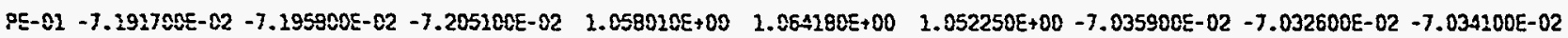
$\begin{array}{llllllllll}P E-02 & 3.050700 E-02 & 3.036000 E-02 & 3.042 E 00 E-02 & 2.550900 E+00 & 2.523400 E+00 & 2.552100 E+00 & 3.256300 E-02 & 3.171300 E-02 & 3.169200 E-02\end{array}$ $P E-03-1.844300 E-02-1.8532005-02-1.859700 E-02 \quad 1.308500 E+00 \quad 2.305800 E+00 \quad 1.309500 E+00-1.750900 E-02-1.748900 E-02-1.784600 E-02$ $P E-04 \quad 3.835500 E-02 \quad 3.848300 E-02 \quad 3.809400 E-02 \quad 3.575800 E+00 \quad 1.549300 E+00 \quad 1.567500 E+00 \quad 3.953800 E-02 \quad 3.942800 E-02 \quad 3.968400 E-02$ PE-05 7.312000E- $22 \quad 7.2939005-02 \quad 7.345300 E-02 \quad 8.3989005-02 \quad 8.476700 E-02 \quad 8.506400 E-01 \quad 7.445700 E-02 \quad 7.447200 E-02 \quad 7.460400 E-02$ $\begin{array}{lllllllllll}P E-06 & 6.7 .7000 E-02 & 6.361980 E-82 & 6.758800 E-02 & 1.283500 E+00 & 1.275900 E+00 & 1.327900 E+00 & 5.807000 E-02 & 6.810900 E-02 & 6.820300 E-02\end{array}$

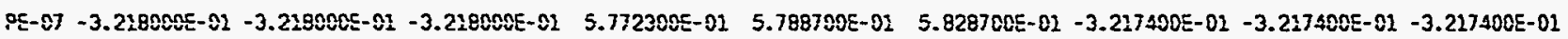
$\begin{array}{llllllllllll}P E-88 & 2.405300 E-02 & 1.393100 E-02 & 1.363900 E-\$ 2 & 1.105270 E+00 & 1.099720 E+00 & 1.106110 E+00 & 9.913000 E-03 & 9.933000 E-03 & 1.002800 E-02\end{array}$

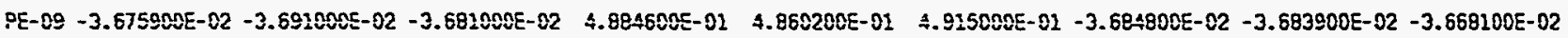
$P E-10 \quad 1.985900 E-02 \quad 2.998100 E-02 \quad 1.957800 E-02 \quad 8.812700 E-02 \quad 9.677400 E-01 \quad 8.561100 E-02 \quad 2.106100 E-02 \quad 2.107100 E-02 \quad 2.114400 E-02$

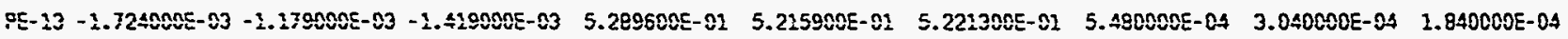
$\begin{array}{lllllllllll}P E-14 & 1.306800 E-02 & 3.3047005-01 & 1.305900 E-01 & 9.931200 E-01 & 9.978200 E-01 & 8.8457005-01 & 1.317000 E-01 & 1.319300 E-01 & 1.321500 E-01\end{array}$

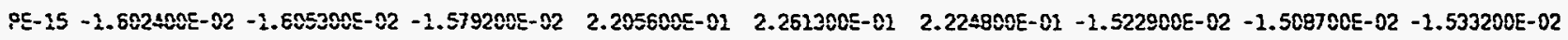
$\begin{array}{lllllllllll}P L-16 & 3.637500 E-02 & 3.629500 E-02 & 3.613500 E-02 & 3.217 \varepsilon 00 E-02 & 3.223500 E-01 & 3.238400 E-01 & 3.753800 E-02 & 3.730700 E-82 & 3.733300 E-02\end{array}$ $95-17-3.602200 E-02-3.5246005-02-3.6287005-02 \quad 1.9013005-01 \quad 1.903200 E-02 \quad 2.851000 E-01-3.567700 E-02-3.551700 E-02-3.547500 E-02$

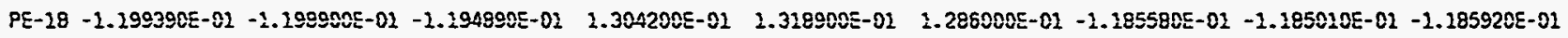

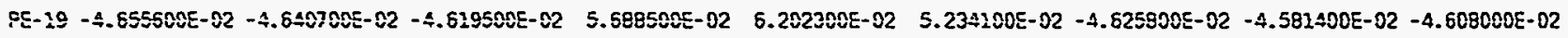

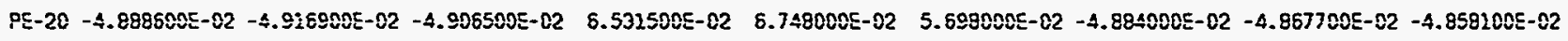

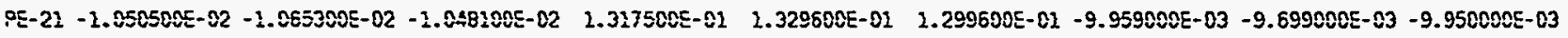
PE-22 - $4.065000 E-03 \quad-4.183000 E-03 \quad-4.053000 E-03 \quad 1.505300 E-01 \quad 1.497600 E-02 \quad 1.479000 E-01 \quad-3.786000 E-03 \quad-4.100000 E-03 \quad-3.682000 E-03$ PE-29 5.715700E-02 $5.728700 E-02 \quad 5.707100 E-02 \quad 2.2874005+09 \quad 2.322000 E+00 \quad 2.2459005+00 \quad 7.1849005-02 \quad 7.140500 E-82 \quad 7.004900 E-02$

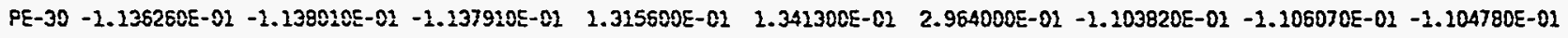

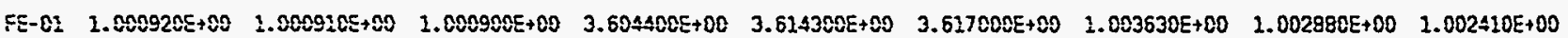
XE-02 $-3.200000 E-04 \quad-3.230000 E-04 \quad-3.230000 E-04 \quad 3.080000 E-04 \quad 3.220000 E-04 \quad 3.190000 E-04 \quad-2.750000 E-04 \quad-2.820000 E-04 \quad-2.900000 E-04$ XEL-02 -8.510000E- $04-8.510000 E-04-8.500000 E-04-8.550000 E-04-8.650000 E-04$-8.540000E-04 -9.690000E-04 -8.690000E-04 -8.670000E-04 $X E-03-5.870000 E-04-5.9700005-04-5.870000 E-04-2.520000 E-04-2.340000 E-04-3.870000 E-04-3.9300005-04-3.930000 E-04-3.930000 E-04$ XE- $04-1.3190005-03-1.321000 E-03-1.3200005-03-7.550000 E-04-7.410000 E-04-7.300000 E-04-1.241000 E-03-1.256000 E-03-2.265000 E-03$ $X E-05-3.559000 E-03-3.557000 E-03-3.559000 E-03-3.646000 E-03-3.693000 E-03-3.719000 E-03-3.560000 E-03-3.561000 E-03-3.569000 E-03$

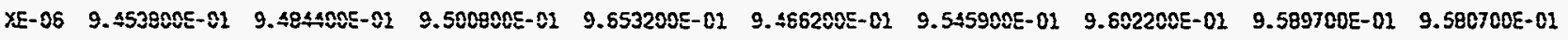

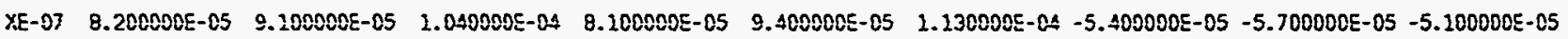
XE- $09-1.058000 E-03-1.059000 E-03-1.059000 E-03-8.580000 E-04-9.550000 E-04-8.430000 E-04-1.019000 E-03-1.023000 E-03-1.026000 E-03$ XE-09 - $1.209000 E-03-1.2080005-03-2.2110005-03-5.410000 E-04-5.4200005-04-5.370000 E-04-1.137000 E-03-1.1500005-03-2.169000 E-03$ $x E-10-3.825000 E-03-3.8280025-03-3.8320005-03-2.9550085-03-2.948000 E-03-2.943000 E-03-3.507000 E-03-3.557000 E-03-3.600000 E-03$ $\begin{array}{llllllllll}X E-12 & 2.500000 E-05 & 2.500000 E-05 & 2.500000 E-05 & 2.600000 E-05 & 2.700000 E-05 & 2.600000 E-05 & 2.600000 E-05 & 2.600000 E-05 & 2.700000 E-05\end{array}$ XE- $12-1.7350085-83-1.735008 E-03-1.7350005-03-1.580000 E-03-1.592000 E-03-1.581000 E-03-1.718000 E-03-1.717000 E-03-1.718 C 00 E-03$ XE-13 -5. $430000 E-04-6.470000 E-04-6.450000 E-04 \quad 1.300000 E-04 \quad 1.420000 E-04 \quad 1.430000 E-04-5.210000 E-04 \quad-5.430000 E-04 \quad-5.560000 E-04$

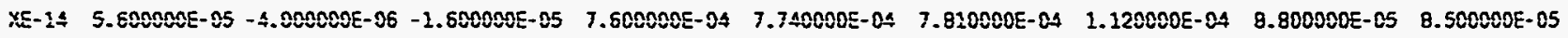
XE-15 -7.190000E-04 -7.210000E-04 -7.190000E-04 1.540000E-04 1.650000E-04 $1.710000 E-04-5.500000 E-04 \quad-5.940000 E-04-6.160000 E-04$ TE-01 -1.1E0000E-04 -1.150000E- $04-1.250000 E-04-9.1000005-05$ - $8.800000 E-05$-8.500000E- $05-1.140000 E-04-1.120000 E-04$ - $1.120000 E-04$ $\begin{array}{lllllllllll}\text { VEXT2 } & 1.993600 E+00 & 1.993600 E+00 & 1.993600 E+00 & 1.993600 E+00 & 1.993600 E+00 & 1.993600 E+00 & 1.993600 E+00 & 1.993600 E+00 & 1.993600 E+00\end{array}$

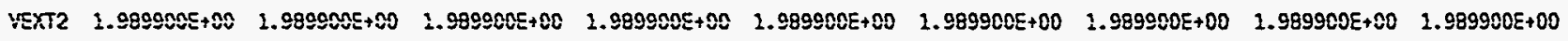

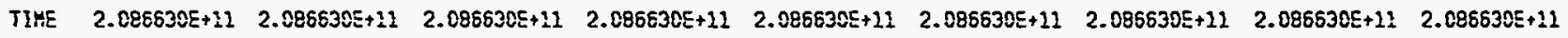


intike

Aus_usp_tests

Sookl Hzme: AMS_MFPTI_RY

Date \& Time: 2 Mar 1900 05:35:02

Page Description:

DATA SET H0.-259

TRAHS RV_ZERO-1 RV_ZERO-2 RV_ZERO-3 RV_LOAD-1 RV_LOAD-2 $\quad$ RV_LOAD-3 RV_ZERO-4 RY_ZERO-5 RV_ZERO-6

FE-01 - 7.154800E-02 -7.149000E-02 -7.259200E-02 9.502500E-01 9.512100E-01 9.571700E-01 -7.889400E-02 -7.075000E-02 -7.075300E-02 $\begin{array}{llllllllll}P E-02 & 3.087300 E-02 & 3.117100 E-02 & 3.098400 E-02 & 1.376800 E+00 & 2.391900 E+00 & 2.366100 E+00 & 3.113600 E-02 & 3.113600 E-02 & 3.107400 E-02\end{array}$ PE-03 - $2.847900 E-02-2.869000 E-02 \quad-1.869500 E-02 \quad 1.157100 E+00 \quad 1.259200 E+00 \quad 1.179000 E+00-1.977600 E-02 \quad-2.006900 E-02 \quad-1.994200 E-02$ PE-04 3.902300E-02 $3.919900 E-02 \quad 3.895700 E-02 \quad 1.397900 E+00 \quad 1.415200 E+00 \quad 1.405600 E+00 \quad 3.970400 E-02 \quad 3.953900 E-02 \quad 3.969500 E-02$

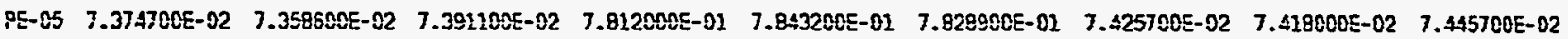
$\begin{array}{lllllllllll}P E-06 & 8.739000 E-02 & 6.735400 E-02 & 8.7242005-02 & 1.211200 E+00 & 1.285500 E+00 & 2.207200 E+00 & 6.801300 E-02 & 5.790500 E-02 & 5.779700 E-02\end{array}$

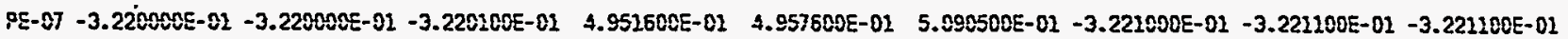
PE- $08 \quad 1.010700 E-02 \quad 1.002800 E-02 \quad 9.790000 E-03 \quad 3.002000 E+00 \quad 1.004270 E+00 \quad 1.020460 E+00 \quad 1.354600 E-02 \quad 1.347900 E-02 \quad 1.349700 E-02$

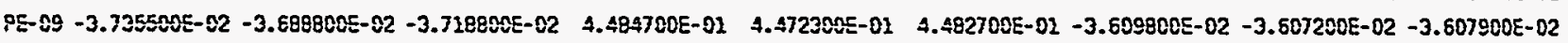
PE-10 2.023600E-02 2.025400E-02 $2.030200 E-02 \quad 7.875200 E-02 \quad 8.042000 E-01 \quad 7.930000 E-21 \quad 2.102600 E-02 \quad 2.103200 E-02 \quad 2.104600 E-02$ PS-13 -5.000000E-05 -3.8900005-04 -1.470000E-04 4.804500E-02 4.825800E-02 4.870300E-02 8.400000E-05 -0.500000E-04 -3.780000E-04 PE-14 1.305300E-01 $1.309200 E-02 \quad 1.309200 E-01 \quad 8.210700 E-02 \quad 9.101200 E-01 \quad 8.077700 E-01 \quad 1.3020005-02 \quad 1.302000 E-02 \quad 1.302000 E-01$ PE-15 - $1.622500 E-02-1.622500 E-02-1.650700 E-02 \quad 2.093800 E-02 \quad 2.050300 E-02 \quad 2.075700 E-02-1.584800 E-02 \quad-1.573800 E-02$-1.552400E-02 PE-15 3.635100E-02 $3.610400 E-02 \quad 3.659300 E-82 \quad 3.001800 E-02 \quad 3.025900 E-02 \quad 2.986900 E-02 \quad 3.708700 E-02 \quad 3.699400 E-02 \quad 3.670700 E-02$

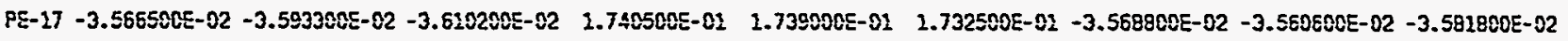

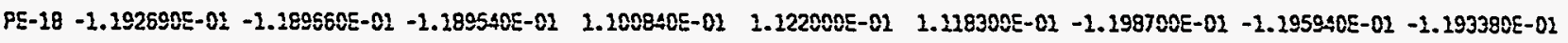

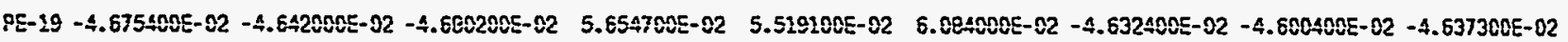
PE-20 -4.916600E-02 -4.905900E-02 -4.909400E-02 $5.2148005-02 \quad 5.391100 E-02 \quad 5.184800 E-02$ - $4.9474005-82$ - $4.917700 E-02$-4.929000E-02

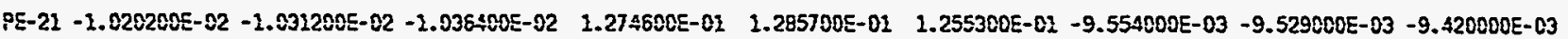
PE-22 - $4.401000 E-03-4.125000 E-03 \quad-4.076000 E-03 \quad 1.414500 E-01 \quad 1.404400 E-01 \quad 1.395700 E-01 \quad-4.850000 E-03 \quad-4.549000 E-03 \quad-4.698000 E-03$ $\begin{array}{llllllllll}P E-29 & 5.8805005-02 & 5.8799005-02 & 5.989600 E-02 & 2.296900 E+00 & 2.348100 E+00 & 2.164100 E+00 & 7.475800 E-02 & 7.517800 E-02 & 7.564100 E-02\end{array}$

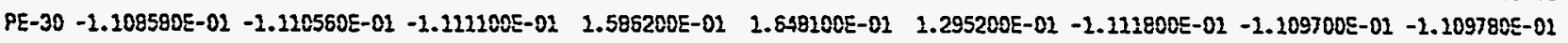
EE-01 $1.000870 E+00 \quad 1.000870 E+00 \quad 3.000840 E+00 \quad 3.483100 E+00 \quad 3.4826005+00 \quad 3.489800 E+00 \quad 1.004120 E+00 \quad 1.0032005+00 \quad 1.002670 E+00$ XE-01 -3.080000E-04 -3.050000E-04 -3.090000E-04 2.940000E-04 2.950000E-04 2.990000E-04 -2.670000E-04 -2.830000E-04 -2.990000E-04

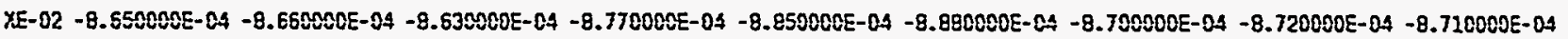
YE-03 -3.920000E-04 -3.920000E-04 -3.920000E-04 - $1.548000 E-03-7.100000 E-04-5.150000 E-04-3.8500005-04-3.840000 E-04-3.840000 E-04$ XE-04 -1.320000E- $03-1.320008 E-03-1.3190005-83-7.3600005-24-7.3900005-04-7.280000 E-04-1.224000 E-03-1.239000 E-03-1.251000 E-03$ $X E-05-3.564000 E-03-3.562000 E-03-3.564000 E-03-2.345000 E-03-3.698000 E-03-3.700000 E-03-3.573000 E-03-3.5750005-03-3.5870005-03$ XEE-06 9.727600E-01 $9.720500 E-01 \quad 9.722300 E-01 \quad 9.715100 E-01$ 9.748500E-01 $9.761300 E-01 \quad 9.735300 E-01 \quad 9.738900 E-01 \quad 9.744400 E-01$ XE-07 -7.000000E-06 3.000000E-05 $\quad 3.100000 E-05 \quad 1.630000 E-04 \quad 3.720000 E-04$ 1.820000E-04 $-2.600000 E-05$-3.600000E-05 $-3.100000 E-05$ XE-08 - - $2.0490005-03-1.049000 E-03-1.050000 E-03-6.7100005-04-6.650000 E-04-6.650000 E-04-9.770000 E-04-9.810000 E-04-9.8400005-04$ XE-09 - 2.209000E-03 - $1.209000 E-03-1.2100005-03-5.190000 E-04-5.140000 E-04-5.000000 E-004-1.109000 E-03-1.125000 E-03-1.138000 E-03$ $x E-10-3.8610005-03-3.860500 E-03-3.8580005-03 \quad-2.988000 E-03-2.971000 E-03-2.965000 E-03-3.588000 E-03-3.5720005-03-3.517080 E-03$ XE-11 2.500000E-05 2.600000E-05 2.600000E- $25 \quad 2.600000 E-05 \quad 2.500000 E-05 \quad 2.500000 E-05 \quad 2.600000 E-05 \quad 2.600000 E-05 \quad 2.600000 E-05$ XE-12 - - 2.727000E-03 -1.725000E-03 - $1.725000 E-03-1.595000 E-03-2.594000 E-03-1.593000 E-03-1.712000 E-03-1.712000 E-03-1.714000 E-03$ YE-13 - $-6.390000 E-04-6.430000 E-04-E .390000 E-04$ 2.070000E-04 2.150000E-04 2.220000E-04 $-4.300000 E-04 \quad-4.670000 E-04$-4.880000E-04 $x E-14$ 9.000000E-06 $1.000000 E-05 \quad 5.000000 E-06 \quad 8.610000 E-04 \quad 8.720000 E-04 \quad 8.890000 E-04 \quad 1.450000 E-04 \quad 1.120000 E-04 \quad 9.200000 E-05$ YE-15 -7.430000E-C4 -7.380000E-04 -7.310000E-04 1.940000E-D4 2.020000E-04 2.070000E-04 $-5.120000 E-04$-5.570000E-04 -5.980000E-04 TE-01 - $2.160000 E-04$ - $1.160000 E-04-1.1600005-04-8.000000 E-85-7.600800 E-05-7.500000 E-05-9.800000 E-05-1.860000 E-04-1.290000 E-04$ $\begin{array}{lllllllllll}\text { VEXTL } & 2.993600 E+00 & 1.993600 E+00 & 1.993600 E+00 & 1.993600 E+00 & 1.993600 E+00 & 1.993600 E+00 & 1.993600 E+00 & 1.993600 E+00 & 1.993600 E+00\end{array}$ VEXT2 $1.989900 E+00 \quad 1.989900 E+00 \quad 1.989900 E+00 \quad 1.989900 E+00 \quad 1.989900 E+00 \quad 1.989980 E+00 \quad 1.989900 E+00 \quad 1.989900 E+00 \quad 1.989900 E+00$ $\begin{array}{llllllllll}\text { TIME } & 2.086630 E+12 & 2.086530 E+11 & 2.086630 E+12 & 2.086630 E+12 & 2.086530 E+12 & 2.086630 E+11 & 2.0865530 E+11 & 2.086530 E+11 & 2.085630 E+11\end{array}$ 


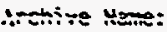

ANS_MCP_TESTS

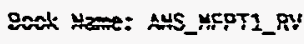

Date \& Tine: 2 Mar 1900 05:42:57

Page Description:

DATA SET NO. -260

TRAMS RY_ZERO-1 RV_ZERO-2 RV_ZERO-3 RY_LOAD-2 RV_LOAD-2 RN_LOAO-3 RV_ZERO-4 RV_ZERO-5 RY_ZERO-6

PE- $01-7.2632005-02-7.194500 E-02-7.192500 E-02 \quad 1.146600 E+00 \quad 1.174900 E+00 \quad 1.156400 E+00-7.099500 E-02-7.108800 E-02-7.099800 E-02$

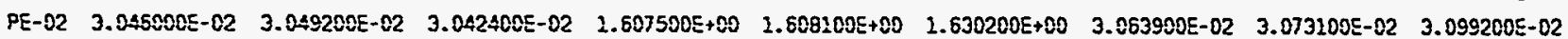
PE- $03-2.0845005-02-2.070900 E-02-2.118 E 00 E-02 \quad 1.379200 E+00 \quad 1.395000 E+00 \quad 1.381060 E+00-2.735400 E-02-1.718600 E-02-1.721000 E-02$ $\begin{array}{llllllllll}P E-04 & 3.932000 E-02 & 3.906900 E-02 & 3.917000 E-02 & 1.664300 E+00 & 1.681100 E+00 & 3.689100 E+00 & 3.947000 E-02 & 3.898200 E-02 & 3.900500 E-02\end{array}$

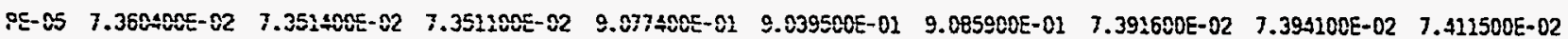
$\begin{array}{llllllllll}P E-05 & 6.772400 E-02 & 5.781500 E-02 & 6.730500 E-02 & 1.438100 E+00 & 1.45 E 400 E+00 & 1.419200 E+00 & 6.777000 E-02 & 6.797400 E-02 & 5.789400 E-02\end{array}$

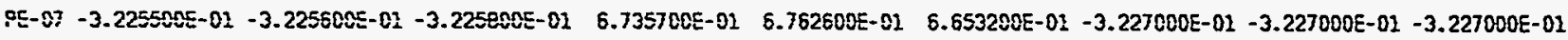
$\begin{array}{lllllllllll}P E-08 & 1.377900 E-02 & 1.347 E 00 E-02 & 1.345300 E-02 & 1.173570 E+00 & 2.183860 E+00 & 1.155590 E+00 & 1.290600 E-02 & 1.280300 E-02 & 1.28 A 500 E-02\end{array}$

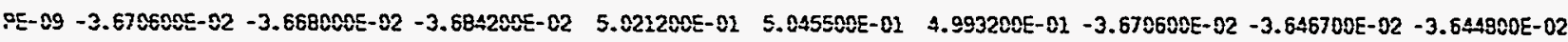
$\begin{array}{llllllllll}P E-10 & 2.072900 E-82 & 2.076700 E-02 & 2.046400 E-02 & 9.361700 E-02 & 9.5433005-02 & 9.518500 E-01 & 2.137600 E-02 & 2.126500 E-02 & 2.124100 E-02\end{array}$

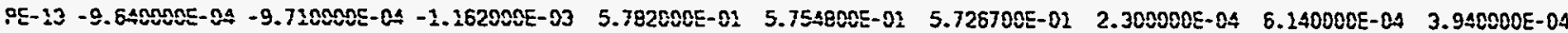

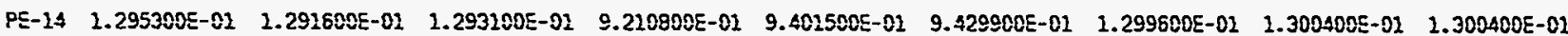

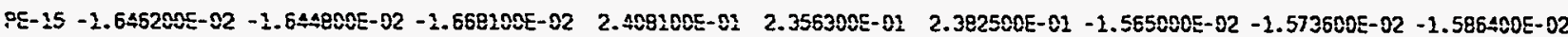

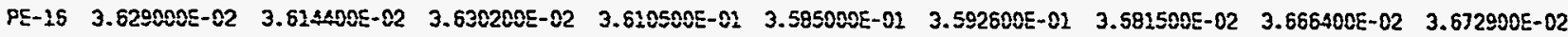

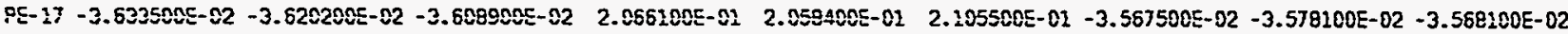

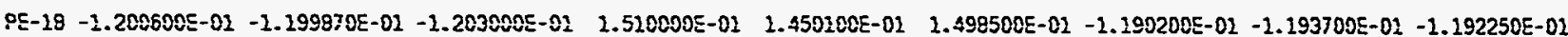
$.05-19-4.6361005-02-4.6288005-02$ - $4.646700 E-02$ 8.327580E-82 8.204900E-02 8.145600E-02 -4.660500E-02 -4.628200E-02 -4.630100E-02 PE-20 -4.930900E-02 - $4.960900 E-02$ - $4.953000 E-02 \quad 8.535400 E-02 \quad 8.456800 E-02 \quad 8.133500 E-02 \quad-4.934300 E-02 \quad-4.947000 E-02$ - $4.947900 E-02$

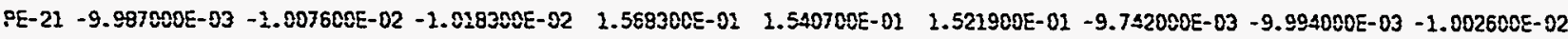
PE-22 - 5.223000E-03 $-5.228000 E-03 \quad-5.275000 E-03 \quad 2.661780 E-02 \quad 2.6346005-01 \quad 1.654700 E-01 \quad-5.204000 E-03 \quad-5.147000 E-03 \quad-5.270000 E-03$

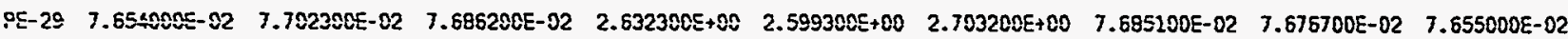

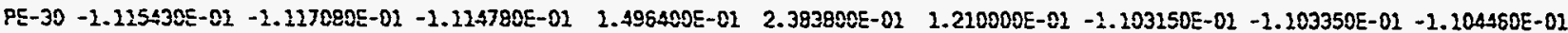

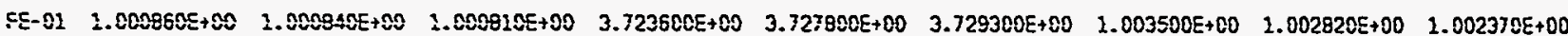
XE-02 -3.200000E-04 -3.270000E-04 -3.280000E-04 3.870000E-04 2.910000E-04 2.940000E-04 -2.690000E-04 -2.740000E-04 -2.800000E-04 XE- $02-8.670000 E-04$-8.690000E-04 -8.680002E-04 -8.690000E-04 -8.690000E-04 -8.850000E-04 -8.760000E-04 -8.750000E-04 -8.730000E-04 XE- $03-3.870000 E-04-3.850000 E-04-3.870000 E-04-1.800000 E-04-1.990000 E-04-2.670000 E-04-1.730000 E-04-1.7200005-04-1.730000 E-04$ $x E-04-1.305000 E-03-1.3100005-03-1.313000 E-03-6.020000 E-04-5.090000 E-04-6.210000 E-04-1.175000 E-03-1.191000 E-03-1.204000 E-03$ $X E-05-3.604000 E-03 \quad-3.605000 E-03-3.605000 E-03 \quad-2.909000 E-03-1.420000 E-03 \quad 1.753000 E-03-3.524000 E-03-3.524000 E-03-3.520000 E-03$ XE-06 9.778400E-01 $9.779000 E-01 \quad 9.7795005-01 \quad 9.775000 E-01 \quad 9.778500 E-01 \quad 9.788300 E-01 \quad 9.823300 E-01 \quad 9.824800 E-01 \quad 9.926400 E-01$ XE-07 5.300000E-05 $\quad 5.300000 E-05$ 7.500000E-05 $\quad 1.900000 E-04 \quad 2.000000 E-04$ 2.140000E-04 $-3.000000 E-05$-3.900000E-05 $-4.400000 E-05$ XE- $08-2.006008 E-03-1.007000 E-03-2.007000 E-03-6.450000 E-04-6.470000 E-04-6.380000 E-03-9.200000 E-04-9.240000 E-04$-9.240000E-04 $X E-09-2.193000 E-03-2.194000 E-03-1.194000 E-03-4.350000 E-04-4.210000 E-04-4.130000 E-04-1.073000 E-03-2.091000 E-03-1.205000 E-03$ $X E-10-3.8760005-03-3.881000 E-03-3.8820005-03-2.936008 E-03-2.942000 E-03-2.914000 E-03-3.600000 E-03-3.664000 E-03-3.700000 E-03$ $\begin{array}{llllllllll}X E-12 & 2.600000 E-05 & 2.700000 E-05 & 2.600000 E-05 & 2.500000 E-05 & 2.600000 E-05 & 2.500000 E-05 & 2.600000 E-05 & 2.700000 E-05 & 2.600000 E-05\end{array}$ $x E-12-1.7220005-03-2.722000 E-03-1.724000 E-03-1.559000 E-03-1.558000 E-03-1.531000 E-03-2.679000 E-03-1.682000 E-03-1.684000 E-03$ $X E-13-6.200000 E-04 \quad-6.120000 E-04-6.120000 E-04 \quad 2.120000 E-04 \quad 2.240000 E-04 \quad 2.230000 E-04-4.260000 E-04 \quad-4.520000 E-04$-4.680000E-04

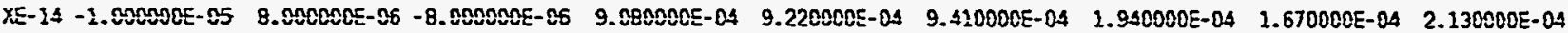
$x E-15-7.400000 E-04 \quad-7.340000 E-04-7.3790005-04 \quad 1.8000005-04 \quad 1.8000005-04 \quad 1.930000 E-04 \quad-5.660000 E-04 \quad-6.090000 E-04 \quad-6.360000 E-04$ TE-01 -1.280000E-04 - $1.2800005-04-1.270000 E-04$-1.030000E-04 - $1.0100005-24-9.900000 E-05-1.230000 E-04-1.348000 E-04-1.370000 E-04$ $\begin{array}{llllllllll}V E X T 1 & 1.993600 E+00 & 1.993600 E+00 & 1.993600 E+00 & 1.993600 E+00 & 1.993600 E+00 & 1.993600 E+00 & 2.993500 E+00 & 1.993600 E+00 & 1.993600 E+00\end{array}$

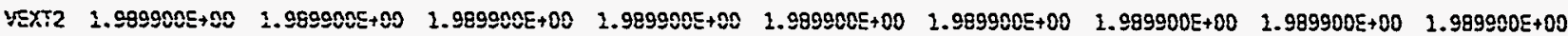
$\begin{array}{llllllllll}\text { TIYE } & 2.086530 E+12 & 2.086530 E+11 & 2.086530 E+12 & 2.086630 E+12 & 2.086530 E+12 & 2.086530 E+12 & 2.086630 E+11 & 2.085630 E+11 & 2.086630 E+11\end{array}$ 

$\begin{array}{llllllllll}P E-02 & 3.024000 E-02 & 3.029900 E-02 & 3.025900 E-02 & 2.747000 E+00 & 1.735600 E+00 & 1.769700 E+00 & 3.172800 E-02 & 3.149400 E-02 & 3.263300 E-02\end{array}$

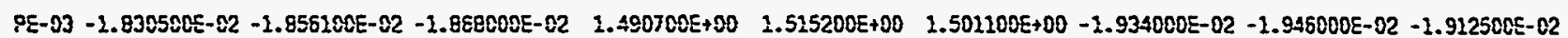
PE-04 3.807400E-02 $3.835800 E-02 \quad 3.839600 E-02 \quad 1.839600 E+00 \quad 1.850900 E+00 \quad 1.811400 E+00 \quad 3.866900 E-02 \quad 3.890300 E-02 \quad 3.913900 E-02$

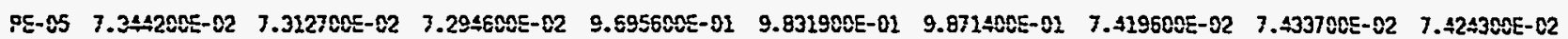
$\begin{array}{lllllllllll}P E-06 & 6.718800 E-02 & 6.717900 E-02 & 6.717200 E-02 & 1.5931005+00 & 1.579500 E+00 & 1.5773005+00 & 6.762500 E-82 & 6.7895005-02 & 6.784700 E-02\end{array}$

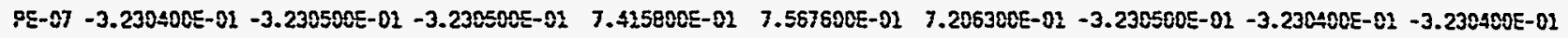
PE-CQ 2.2489005-02 $1.2708005-82 \quad 1.232800 E-02 \quad 1.247300 E+08 \quad 1.277600 E+00 \quad 1.2834005+00 \quad 8.282000 E-03 \quad 8.183000 E-03 \quad 8.331000 E-03$

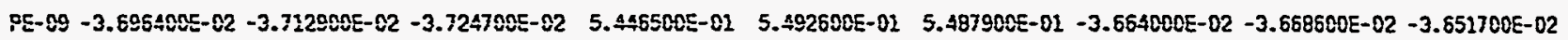
$\begin{array}{lllllllllll}P E-10 & 2.054300 E-02 & 2.055600 E-02 & 2.055700 E-02 & 1.052410 E+00 & 1.053190 E+00 & 1.057050 E+00 & 2.124700 E-02 & 2.128100 E-02 & 2.125600 E-02\end{array}$

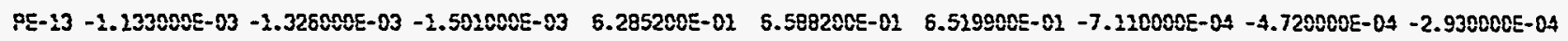

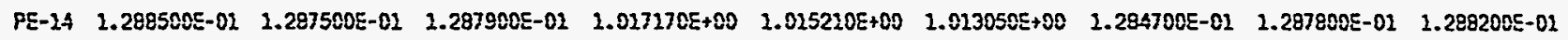

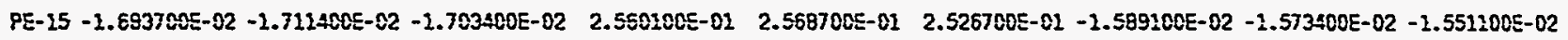
$\begin{array}{llllllllll}P E-16 & 3.605900 E-02 & 3.603700 E-02 & 3.615300 E-02 & 3.918700 E-01 & 3.9914005-01 & 3.9729005-02 & 3.666800 E-02 & 3.637800 E-02 & 3.647000 E-02\end{array}$

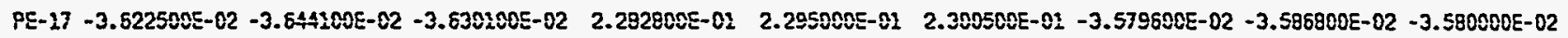
PE-18 - $1.202200 E-01 \quad-1.207700 E-01-2.203600 E-02 \quad 1.717100 E-01 \quad 1.704300 E-01 \quad 1.714100 E-01 \quad-1.201800 E-01-1.205700 E-01-1.201900 E-01$

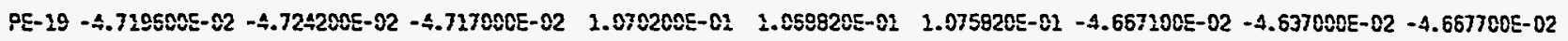

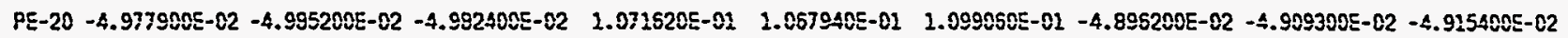
PE-22 - 2.004100E-02 -9.959000E-03 - $9.749000 E-03 \quad 1.7092005-02 \quad 1.686000 E-01 \quad 1.728400 E-01 \quad-9.948000 E-03-9.675000 E-03-9.595000 E-03$ PE-22 -5.509000E- $03 \quad-5.573000 E-03 \quad-5.734000 E-03 \quad 1.785900 E-02 \quad 1.753700 E-01 \quad 1.808600 E-01 \quad-5.833000 E-03 \quad-5.649000 E-03 \quad-6.074000 E-03$ PE-29 $7.748300 E-02 \quad 7.712200 E-02 \quad 7.711300 E-02 \quad 2.799000 E+00 \quad 2.857000 E+00 \quad 2.793100 E+00 \quad 7.400300 E-02 \quad 7.394000 E-02 \quad 7.375500 E-02$ PE-30 - 2.108570E-01 $-1.109730 E-01 \quad-1.109460 E-82 \quad 1.393000 E-01 \quad 1.861300 E-01 \quad 3.160600 E-01 \quad-1.109280 E-01 \quad-1.108410 E-02 \quad-1.109420 E-01$ FE-01 $1.000750 E+00 \quad 1.000750 E+00 \quad 1.000740 E+00 \quad 3.8531005+00 \quad 3.874200 E+00 \quad 3.0710005+00 \quad 1.003890 E+00 \quad 1.003000 E+00 \quad 1.002420 E+00$ XE-01 -3.250000E-04 -3.150000E-04 -3.150000E-04 3.090000E-04 3.240000E-04 3.250000E-C4 -2.750000E-04 -2.840000E-04 -2.890000E-04 XE- $02-8.7100005-04-8.7000005-04-8.7000305-04-9.000008 E-04-9.000080 E-04-9.020 C 00 E-04-8.790200 E-04-8.780980 E-04-8.770000 E-04$ YE-03 -1.730000E-04 -1.710000E-04 - $2.720000 E-04-2.130000 E-04-2.130000 E-04-1.990000 E-04-1.890000 E-04-1.890000 E-04-1.880000 E-04$

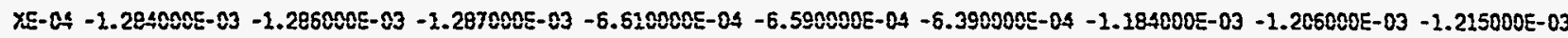
YE- $05-3.552000 E-03 \quad-3.549000 E-03 \quad-3.553000 E-03 \quad 1.741600 E-02 \quad 3.1925005-01 \quad 1.452600 E-01 \quad-3.505000 E-03 \quad-3.505000 E-03 \quad-3.494000 E-03$

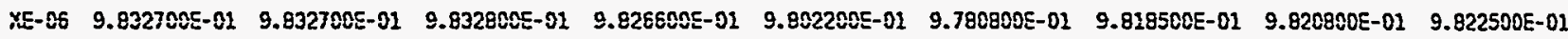
YE-07 3.000000E-05 4.200000E-05 5.700000E-C5 2.230000E-04 2.370000E-04 2.490000E-04 -1.800000E-05 -2.300000E-05 -2.400000E-05 XE- $09-2.003000 E-03-1.033000 E-03-1.004000 E-03-6.310000 E-04-6.210000 E-04-6.190000 E-04-9.410000 E-04-9.490000 E-04-9.548080 E-04$ XE-09 - $1.2730005-03-1.1740005-03-1.2760005-03-3.5800005-04-3.470000 E-04-3.5500005-04-1.0490005-03-1.052000 E-03-1.075000 E-03$ XE- $10-3.888000 E-03-3.892000 E-03-3.894000 E-03-3.204000 E-03-3.250000 E-03-3.025000 E-03-3.5280000 E-03-3.608000 E-03-3.658000 E-03$ $\begin{array}{lllllllllll}\text { YEE-11 } & 2.500000 E-05 & 2.500000 E-05 & 2.500000 E-05 & 2.600000 E-05 & 2.600000 E-05 & 2.600000 E-05 & 2.600000 E-05 & 2.700000 E-05 & 2.600000 E-05\end{array}$ YE- $12-1.702000 E-03-1.703000 E-03-1.702000 E-03-1.515000 E-03-1.514000 E-03-1.507000 E-03-1.673000 E-03-1.675000 E-03-2.675000 E-03$

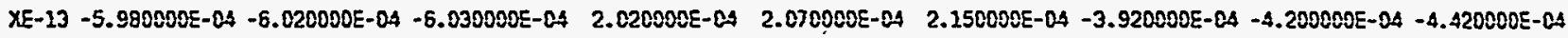
XE-14 4.580000E-05 3.100008E-05 $3.4008005-05 \quad 1.152000 E-03 \quad 1.27 E 000 E-03 \quad 1.210000 E-03 \quad 3.280000 E-04 \quad 3.110000 E-04 \quad 2.700000 E-04$

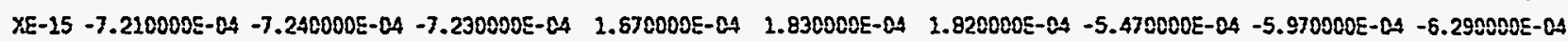
TE-01 - $1.4100005-04-1.400000 E-04-1.4000005-04-1.0400005-04-1.0200005-04-1.0100005-04-1.2100005-04-1.330000 E-04-1.280080 E-04$ VEXT2 $1.993500 E+00 \quad 1.993600 E+00 \quad 1.99360 C E+C 0 \quad 1.993500 E+00 \quad 1.993500 E+00 \quad 1.993500 E+00 \quad 1.993600 E+00 \quad 1.993600 E+C 0 \quad 1.993600 E+00$ $\begin{array}{lllllllllll}\text { YEXT2 } & 1.989900 E+00 & 1.989900 E+\infty 0 & 1.989900 E+\infty 0 & 1.989900 E+00 & 1.989800 E+00 & 1.989900 E+00 & 1.989900 E+00 & 1.989900 E+00 & 1.989900 E+00\end{array}$

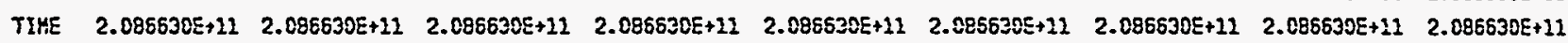


A:-7ive

ANS_يP_TESTS

Dook Hame: AYS_MFPT2_Ry Date \& Time: 2 Mar 1900 2E:00:39

Page Rescription:

DATA SET HO. -262

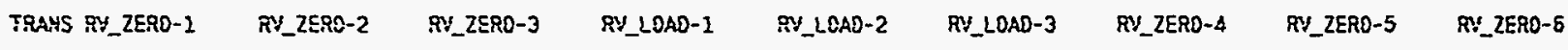

:EE- $01-7.312000 E-02 \quad-7.3130005-02-7.319000 E-02 \quad 1.418800 E+00 \quad 1.418900 E+00 \quad 1.401900 E+00-7.244700 E-02 \quad-7.240600 E-02 \quad-7.235400 E-02$ PE-02 $3.084100 E-02 \quad 3.064500 E-02 \quad 3.065400 E-02 \quad 3.847600 E+00 \quad 1.864200 E+00 \quad 3.857600 E+00 \quad 3.178600 E-02 \quad 3.18100 D E-02 \quad 3.262100 E-02$ PE- $03-2.006400 E-02 \quad-2.0395005-02-2.034400 E-02 \quad 1.6386005+00 \quad 2.613900 E+00 \quad 1.6375005+00-2.146500 E-02-2.162100 E-02-2.180500 E-02$ $\begin{array}{llllllllll}P E-04 & 3.851100 E-02 & 3.810500 E-02 & 3.847900 E-02 & 1.942300 E+00 & 3.960900 E+00 & 3.931800 E+00 & 3.895600 E-02 & 3.902600 E-02 & 3.915400 E-02\end{array}$

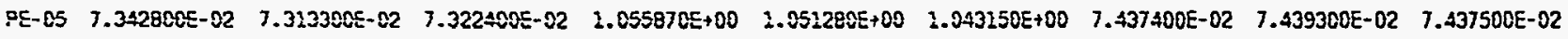
PE-05 $6.694600 E-02 \quad 6.884400 E-02 \quad 6.689800 E-02 \quad 1.670900 E+00 \quad 2.6504005+00 \quad 1.687300 E+00 \quad 6.727000 E-02 \quad 6.740908 E-02 \quad 6.732700 E-02$ CE-07 -3.2336005-01 $-3.233700 E-01 \quad-3.233700 E-02$ 8.291600E-81 $8.2584005-01$ 8.014400E-01 $-3.232200 E-01 \quad-3.232100 E-01 \quad-3.232200 E-01$ $\begin{array}{llllllllllll}P E-08 & 7.677800 E-03 & 7.649000 E-C 3 & 7.907000 E-03 & 1.351000 E+00 & 1.383000 E+00 & 1.370900 E+00 & 1.009400 E-02 & 1.011000 E-02 & 1.015000 E-02\end{array}$

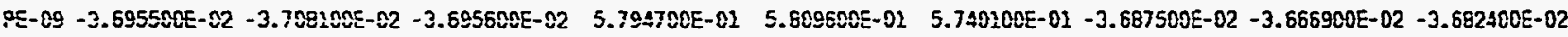

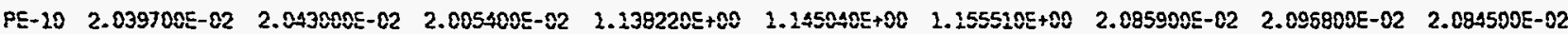

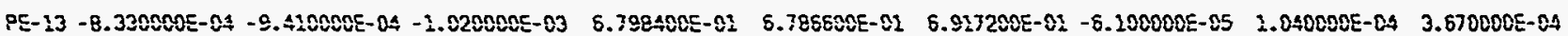

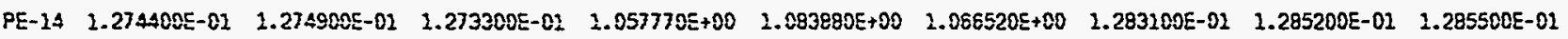

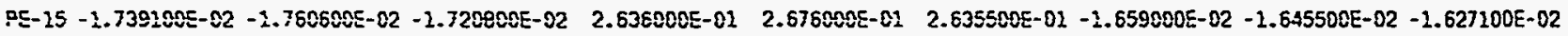
PE-15 3.529100E-02 3.553400E- $62 \quad 3.556900 E-02 \quad 4.187100 E-02$ 4. $375700 E-01 \quad 4.149400 E-01 \quad 3.597200 E-02 \quad 3.504200 E-02 \quad 3.600000 E-02$

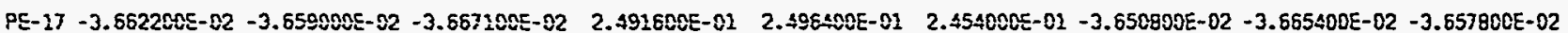

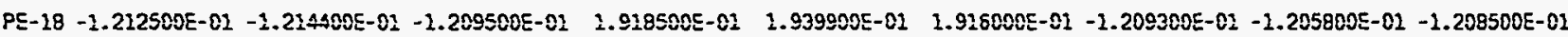

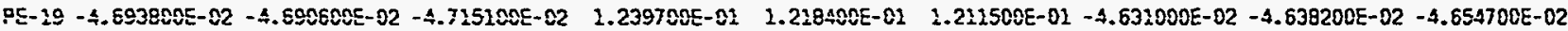
PE-20 - $4.972900 E-02-4.953200 E-02-4.993800 E-02 \quad 1.204400 E-01 \quad 1.221200 E-01 \quad 1.182100 E-01-4.999000 E-02-4.948800 E-02$ - $4.958100 E-02$

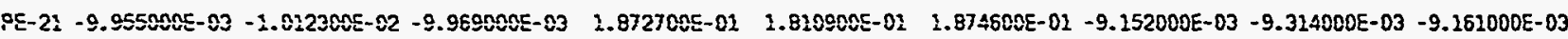
PE-22 -6.592000E-03 -6.511000E-03 -5.416000E-03 1.926000E-02 1.875700E-01 $1.911300 E-01 \quad-3.159000 E-03 \quad-5.844000 E-03 \quad-5.965000 E-03$

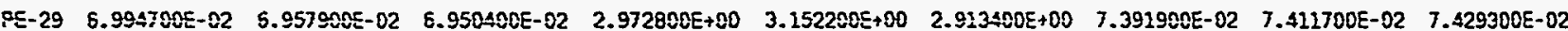

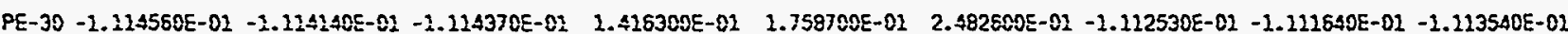

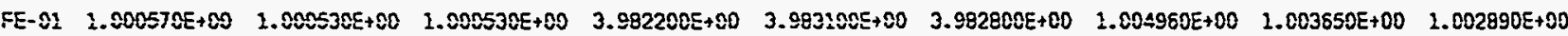
XE-01 -3.180000E-04 -3.2000005-04 -3.200000E-04 3.170000E-04 3.130000E-04 3.170000E-04 -2.770000E-04 -2.860000E-04 -2.920000E-04 $x E-02-8.730000 E-04-8.7000005-04-8.730000 E-04-9.120000 E-04-9.050000 E-04-9.100000 E-04-8.810000 E-04-8.830000 E-04-8.820000 E-04$ $X E-03-1.850000 E-84-1.950000 E-04-1.850000 E-04-3.200000 E-04-3.330000 E-04-3.100000 E-04-2.880000 E-04-2.870000 E-04-2.870000 E-04$ $x E-02-1.291000 E-03-2.293000 E-03-1.293000 E-03-5.800000 E-03-5.800000 E-04-5.590000 E-04-1.144000 E-03-1.172000 E-03-1.190000 E-03$

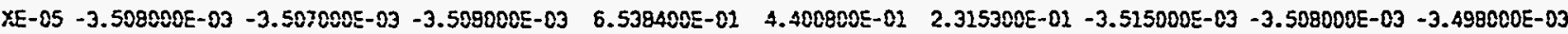

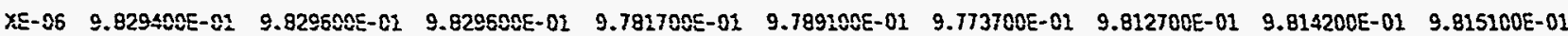
$\begin{array}{llllllllll}X E-07 & 6.300000 E-05 & 6.800000 E-05 & 7.900000 E-05 & 2.720000 E-04 & 2.880000 E-04 & 2.990000 E-04 & 1.100000 E-05 & 2.000000 E-05 & 3.000000 E-06\end{array}$ $x E-08-9.920000 E-04-9.920000 E-04-9.920000 E-04-6.150000 E-04-6.1500005-04-6.070000 E-04-9.230000 E-04-9.300000 E-04-9.350000 E-04$ $X E-09-2.154000 E-83-2.154000 E-03-1.256000 E-03-3.390000 E-04-3.3000005-04-3.320000 E-04-1.048000 E-03-1.060000 E-03-1.071000 E-03$ $X E-10-3.8600005-03-3.8590005-03-3.9610005-03-4.394000 E-03-4.241000 E-03-4.724000 E-03-3.434000 E-03-3.528000 E-03-3.599000 E-03$ $\begin{array}{lllllllllll}X E-11 & 2.500000 E-05 & 2.500000 E-05 & 2.600000 E-05 & 2.600000 E-05 & 2.600000 E-05 & 2.500000 E-05 & 2.600000 E-05 & 2.700000 E-05 & 2.700000 E-05\end{array}$ $x E-12-1.6860005-03-1.686000 E-03-1.687000 E-03-1.453000 E-03-1.455000 E-03-1.450000 E-03-1.663000 E-03-1.664000 E-03-1.666000 E-03$ $X E-13 \quad-5.920000 E-04 \quad-5.920000 E-04 \quad-5.950000 E-04 \quad 2.230000 E-04 \quad 2.190000 E-04 \quad 2.200000 E-04 \quad-3.620000 E-04$ - $3.940000 E-04 \quad-4.220000 E-04$ $X E-14 \quad 5.300000 E-05 \quad 7.700000 E-05 \quad 1.520000 E-04 \quad 1.282000 E-03 \quad 1.307008 E-03 \quad 1.320000 E-03 \quad 4.700000 E-04 \quad 4.03 C 000 E-04 \quad 3.430000 E-04$ $X E-25-7.520000 E-04-7.530000 E-04-7.500000 E-04 \quad 2.080000 E-04 \quad 2.070000 E-04 \quad 2.150000 E-04-4.930000 E-04 \quad-5.420000 E-04 \quad-5.820000 E-04$ TE- $81-1.350000 E-04-1.3400005-04-1.340000 E-03$ - $1.010000 E-04-1.010000 E-04-9.800000 E-05-1.210000 E-04-1.350000 E-04-1.260000 E-04$ $\begin{array}{lllllllllll}\text { VEXT1 } & 1.993600 E+00 & 1.993600 E+00 & 1.993600 E+00 & 1.993500 E+00 & 1.993300 E+00 & 1.993400 E+00 & 1.993600 E+00 & 1.993600 E+00 & 1.993600 E+00\end{array}$

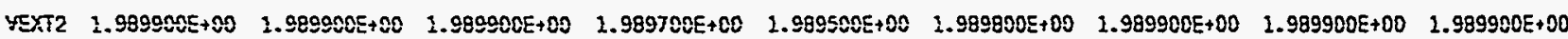

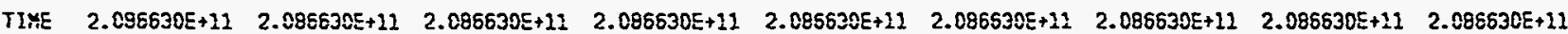


Arthise Heme:

AHS_YFP_TESTS

EOOK Name: AHS_MFPTI_RY

Oate \& Time: 2 Mar 1900 05:08:53

Page Eescription:

DATA SET MO. -263

TRANS RV_ZERO-1 RV_ZERO-2 RV_ZERO-3 RV_LOAD-1 RV_LOAD-2 RV_LOAD-3 RV_ZERO-4 RV_ZERO-5 RV_ZERO-6

PE-01 -7.3218CQE-02 -7.35040QE- $02-7.347308 E-82 \quad 1.565908 E+00 \quad 1.565700 E+00 \quad 1.537400 E+00-7.237900 E-02-7.245800 E-02-7.255700 E-02$ $\begin{array}{llllllllll}P E-02 & 3.099500 E-02 & 3.059400 E-02 & 3.103200 E-02 & 1.984800 E+00 & 1.991600 E+00 & 2.022300 E+00 & 2.981600 E-02 & 2.988900 E-02 & 2.981700 E-02\end{array}$ PE-03 -2.182000E-02 -2.209800E-02 -2.208900E-02 $2.733100 E+00 \quad 1.750500 E+00 \quad 1.790300 E+00-2.693700 E-02-1.674000 E-02 \quad-1.668900 E-02$ PE-0.4 3.849700E-02 $3.869300 E-02 \quad 3.836980 E-02 \quad 2.120700 E+00 \quad 2.076700 E+00 \quad 2.064800 E+00 \quad 3.899100 E-02 \quad 3.851900 E-02 \quad 3.893600 E-02$ $\begin{array}{llllllllll}\text { PC-CS } & 7.397700 E-02 & 7.392100 E-02 & 7.400500 E-02 & 3.127900 E+00 & -1.128000 E+00 & 1.1345 C C E+00 & 7.510100 E-02 & 7.497000 E-02 & 7.490100 E-02\end{array}$ $\begin{array}{lllllllllll}P E-06 & 6.707000 E-02 & 6.705900 E-02 & 6.704600 E-02 & 1.773800 E+00 & 1.792800 E+00 & 1.7740005+00 & 6.727700 E-82 & 6.739300 E-02 & 6.727000 E-02\end{array}$ PE- 07 -3.235008E-02 $-3.2351005-01$-3.235200E-02 9.083100E-01 $9.235700 E-01$ 9.192500E-01 -3.236400E-01 -3.236400E-01 -3.236400E-01 $P E-08 \quad 2.019900 E-02 \quad 9.985000 E-03 \quad 2.023000 E-82 \quad 1.459200 E+00 \quad 1.470880 E+00 \quad 1.4892005+00 \quad 9.1190005-03 \quad 9.332000 E-03 \quad 9.382000 E-03$

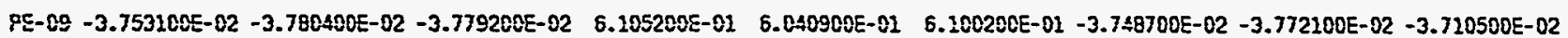
$\begin{array}{lllllllllll}P E-10 & 2.055500 E-02 & 2.065500 E-02 & 2.065300 E-02 & 1.246700 E+00 & 1.237800 E+00 & 1.223500 E+00 & 2.124900 E-02 & 2.110200 E-02 & 2.205100 E-02\end{array}$

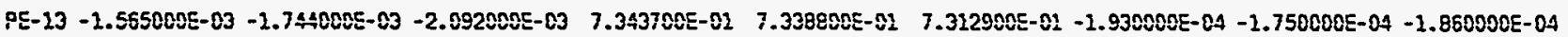

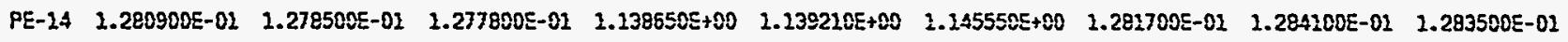

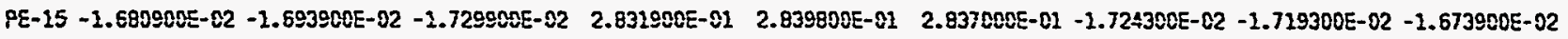
PE-16 3.562500E-02 $3.555100 E-02 \quad 3.541800 E-02 \quad 4 . E 47600 E-02 \quad 4.655400 E-01 \quad 4.635000 E-01 \quad 3.528200 E-02 \quad 3.5428005-02 \quad 3.547900 E-02$ PE- 17 -3.726500E-02 -3.731800E-02 -3.7A0200E-02 2.62 $5500 E-02$ 2.634100E-01 2.653800E-01 -3.541800E-02 -3.627700E-02 -3.607700E-02 $P E-18-2.209200 E-02 \quad-1.207700 E-02 \quad-1.212700 E-02 \quad 2.090100 E-02 \quad 2.058800 E-01 \quad 2.125500 E-01 \quad-1.211700 E-01 \quad-1.208800 E-01 \quad-1.208900 E-01$ $P E-19-4.612500 E-02-4.654500 E-02-4.6598005-02 \quad 1.512000 E-01 \quad 1.5082005-01 \quad 1.514500 E-01-4.6955005-02-4.726100 E-02-4.705700 E-02$ PE-20 -4.955400E-02 -4.952600E-02 -4.983500E-02 $1.414300 E-01 \quad 1.429100 E-01 \quad 1.455500 E-02 \quad-5.033200 E-02 \quad-5.0290005-02 \quad-4.989000 E-02$

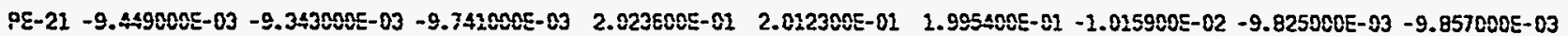
PE-22 -5.996000E-03 -7.027000E-03 -7.082000E-03 2.016700E-01 $1.998500 E-01 \quad 1.9991005-01 \quad-7.231000 E-03 \quad-7.231000 E-03 \quad-6.985000 E-03$

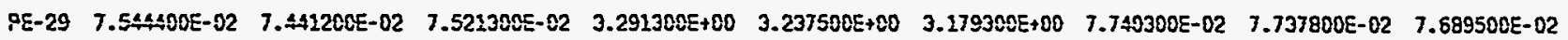

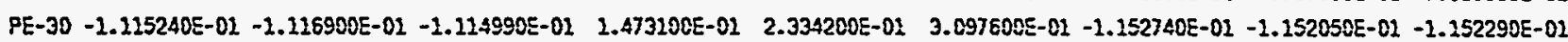
EE-02 $1.000760 E+00 \quad 1.000750 E+00 \quad 1.000730 E+00 \quad 4.105000 E+00 \quad 4.103900 E+00 \quad 4.099300 E+00 \quad 1.004910 E+00 \quad 1.003700 E+00 \quad 1.002960 E+00$ XE-02 $-3.280000 E-04 \quad-3.190000 E-04 \quad-3.210000 E-04 \quad 3.350000 E-04 \quad 3.340000 E-04 \quad 3.420000 E-04 \quad-2.530000 E-04 \quad-2.610000 E-04 \quad-2.580000 E-04$ XE- $02-8.790000 E-04-8.800000 E-04-8.790000 E-04-9.130800 E-04-9.1500005-04-9.0900005-04-8.810000 E-04-8.800000 E-04-8.790000 E-04$ XE-03 - $2.800000 E-04-2.810000 E-04-2.800000 E-04-2.380000 E-03-2.830000 E-03-2.861000 E-03-2.119000 E-03-2.129000 E-03-2.117000 E-03$ XE- $04-1.276000 E-03-1.27$ E000E-03 -1.279000E-03 -5.220000E-04 -5.160000E-04 -5.090000E-84 -1.120000E-03 -1.151000E-03 -1.168000E-03 XE-05 -3.496000E-03 $-3.496000 E-03 \quad-3.497000 E-03 \quad 1.379700 E-02 \quad 2.674500 E-02 \quad 2.440900 E-01 \quad-3.500000 E-03 \quad-3.498000 E-03 \quad-3.490000 E-03$

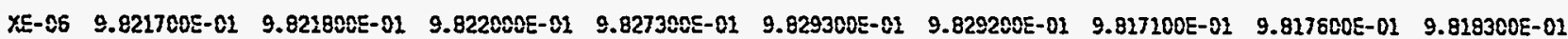
XE-07 -3.900000E-05 $-3.000000 E-05 \quad-4.700000 E-05 \quad 3.060000 E-04 \quad 3.190000 E-04 \quad 3.360000 E-04 \quad 2.400000 E-05 \quad 3.5000000 E-05 \quad-1.900000 E-05$ XE- $98-9.710000 E-04-9.710000 E-04-9.720000 E-04-5.820000 E-04-5.390000 E-04-5.400000 E-04-8.960000 E-04-9.090000 E-04-9.160000 E-04$ XE-09 -1.186000E-03 - - 1.167000E-03 - $1.1680005-03-2.940000 E-04-2.8300005-04-2.7300005-04-1.054000 E-03-1.077000 E-03-1.0860005-03$ $x \mathrm{x}-10-3.845000 E-03-3.846000 E-03-3.846000 E-03-3.026000 E-03-2.962000 E-03-3.015000 E-03-3.391000 E-03-3.499000 E-03-3.558000 E-03$ XEE-12 2.600000E-05 $2.600000 E-05 \quad 2.500000 E-05 \quad 2.600000 E-05 \quad 2.600000 E-05 \quad 2.500000 E-05 \quad 2.700000 E-05 \quad 2.6000000 E-05 \quad 2.700000 E-05$ $X E-12-1.677000 E-03-1.677030 E-03-1.676000 E-03-1.393000 E-03-1.373000 E-03-1.378000 E-03-1.651000 E-03-1.653000 E-03-1.653000 E-03$ XEE-13 $\quad-5.740000 E-04 \quad-5.750000 E-04 \quad-5.770000 E-04 \quad 2.230000 E-04 \quad 2.220000 E-04 \quad 2.390000 E-04 \quad-3.680000 E-04 \quad-4.000000 E-04 \quad-4.230000 E-04$ XE-14 2.300000E-04 2.230000E-04 2.210000E-04 $1.504000 E-03 \quad 1.5480005-03 \quad 2.645000 E-03 \quad 7.400000 E-04 \quad 7.060000 E-04 \quad 6.290000 E-04$ X.E-25 $-7.520000 E-04 \quad-7.560000 E-04 \quad-7.470000 E-04 \quad 2.020000 E-04 \quad 2.140000 E-04 \quad 2.160000 E-04 \quad-5.350000 E-04 \quad-5.890000 E-04 \quad-6.140000 E-04$ TE-02 -1.420000E-04 -1.420000E-04 -1.420000E-04 -1.880000E-04 -1.060000E-04 -1.060000E-04 -1.260000E-04 -1.340000E-04-1.380000E-04 $\begin{array}{lllllllllll}V E X T 1 & 1.993600 E+00 & 1.993600 E+00 & 2.993600 E+00 & 1.993600 E+00 & 1.993500 E+00 & 1.993500 E+00 & 1.993600 E+00 & 1.993600 E+00 & 1.993600 E+00\end{array}$ $\begin{array}{llllllllll}\text { VEXT2 } & 1.989900 E+00 & 1.989 g 00 E+00 & 1.989900 E+00 & 1.989900 E+00 & 1.989900 E+00 & 1.98998 \Omega E+00 & 1.989900 E+00 & 1.989900 E+00 & 1.989900 E+00\end{array}$

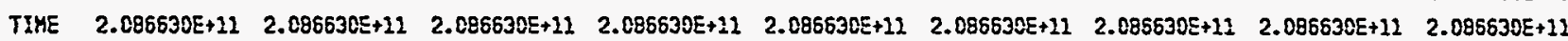


Arthive

AHS_mE_TESTS

Eook :\#2me: AHS_MFPT__RY Qate \& Time: 2 Mat 1900 05:10:51

Page Description:

DATA SET YO. -264

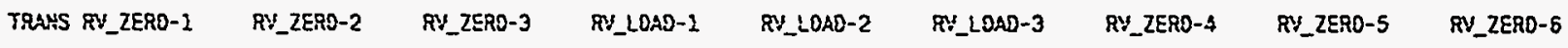

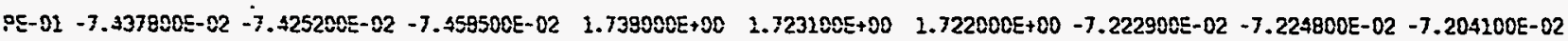
$\begin{array}{llllllllll}P E-02 & 2.920300 E-02 & 2.925500 E-02 & 2.921700 E-02 & 2.136100 E+00 & 2.133400 E+00 & 2.202400 E+00 & 3.150000 E-02 & 3.173000 E-02 & 3.177000 E-02\end{array}$ PE-03 - $1.816400 E-02-1.816700 E-02-1.600300 E-02 \quad 1.910200 E+00 \quad 1.967100 E+00 \quad 2.983500 E+00-1.902200 E-02-1.913300 E-02-1.937100 E-02$ $\begin{array}{llllllllll}P E-04 & 3.805400 E-02 & 3.786980 E-02 & 3.772700 E-82 & 2.213600 E+00 & 2.155000 E+00 & 2.315300 E+00 & 3.913800 E-02 & 3.939300 E-02 & 3.945100 E-02\end{array}$

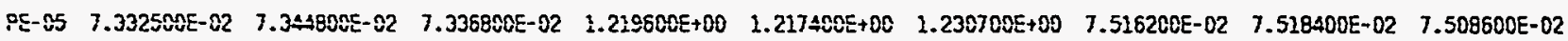
$\begin{array}{lllllllllll}9 E-86 & 8.587200 E-02 & 6.553400 E-02 & 6.586700 E-02 & 1.920500 E+00 & 1.911200 E+00 & 1.945200 E+00 & 6.713000 E-02 & 6.726600 E-02 & 6.711800 E-02\end{array}$

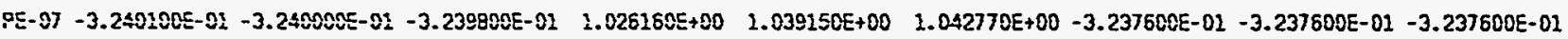
$\begin{array}{llllllllllll}\text { PE- } 88 & 8.436000 E-03 & 8.491000 E-03 & 9.528000 E-03 & 2.600300 E+00 & 1.596900 E+00 & 1.613900 E+00 & 9.430000 E-03 & 9.283000 E-03 & 9.709000 E-03\end{array}$

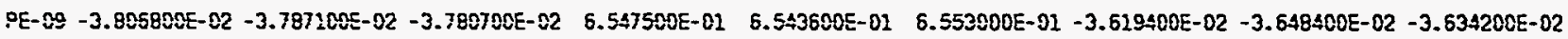
$\begin{array}{llllllllll}\text { PE-10 } & 2.017700 E-02 & 2.002800 E-02 & 1.997200 E-02 & 1.356300 E+00 & 1.364700 E+00 & 3.346300 E+00 & 2.106300 E-02 & 2.096500 E-02 & 2.097300 E-02\end{array}$ Pᄃ- 13 -2.234000E-03 -2.305000E-03 -2.192000E-03 7.960000E-01 8.043300E-01 8.118200E-01 -3.300000E-05 $-5.410000 E-04$ - $1.110000 E-04$

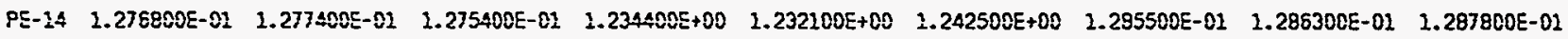
CE- $15-1.7850005-02-1.793800 E-02-1.825300 E-02 \quad 2.989200 E-02 \quad 2.995800 E-012.950100 E-01-1.637500 E-02-1.629700 E-02-1.688300 E-02$ PE-15 3.488300E- $02 \quad 3.499300 E-02 \quad 3.479500 E-02 \quad 5.313200 E-02 \quad 5.285500 E-02 \quad 5.256500 E-81 \quad 3.564500 E-02 \quad 3.528300 E-02 \quad 3.522000 E-02$

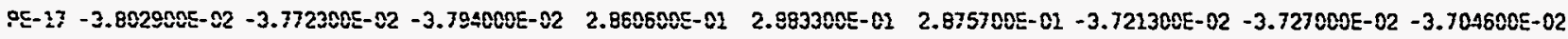

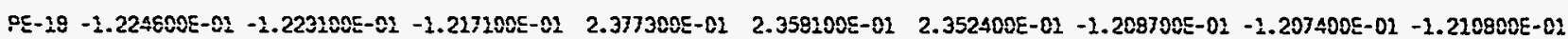

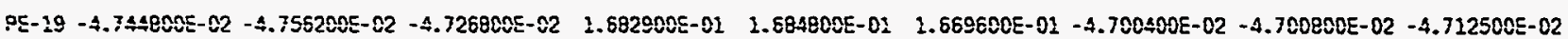

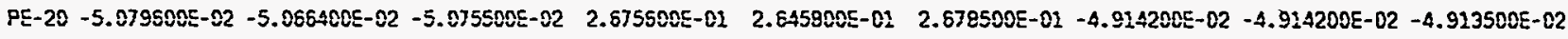
PE-21 - $-2.055500 E-02-1.0558005-02-1.0344005-02 \quad 2.1910005-01 \quad 2.270500 E-02 \quad 2.296400 E-01-1.029700 E-02-1.036500 E-02-1.039600 E-02$ PE-22 -7.722000E- 23 -7.747000E- $23 \quad-7.814000 E-03 \quad 2.274600 E-02 \quad 2.177100 E-82 \quad 2.157600 E-02-7.337000 E-03 \quad-7.368000 E-03-7.042000 E-03$ $\begin{array}{llllllllll}P E-20 & 7.147800 E-02 & 7.031500 E-02 & 7.086000 E-82 & 3.442400 E+00 & 3.554800 E+00 & 3.541000 E+00 & 7.991308 E-02 & 7.970300 E-02 & 7.978300 E-02\end{array}$

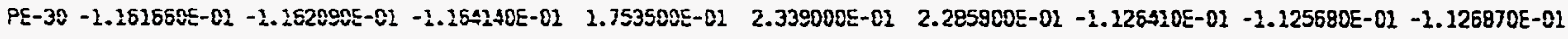

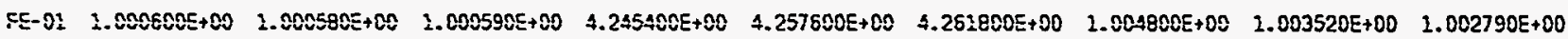
XE-02 -3.090000E-04 $-3.080000 E-04 \quad-3.090000 E-04 \quad 3.870000 E-04 \quad 3.860000 E-04 \quad 4.120000 E-04-2.340000 E-04 \quad-2.430000 E-04 \quad-2.510000 E-04$

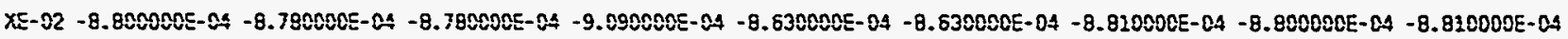
XE-03 - $1.849000 E-03-1.849000 E-03-1.849000 E-03-1.669000 E-02-1.112500 E-02-1.430700 E-02-1.933500 E-02-1.937400 E-02-1.928200 E-02$ XE- $04-1.263000 E-83-1.265000 E-03-1.2640005-03-1.250000 E-04-1.090000 E-04 \quad 2.200000 E-05-8.610000 E-04-9.230000 E-04-9.600000 E-04$ XE- $05 \quad-3.4800005-03 \quad-3.487000 E-03 \quad-3.488000 E-03 \quad 3.130900 E-02 \quad 2.297100 E-02 \quad 4.022000 E-03 \quad 5.181900 E-01 \quad 5.185700 E-01 \quad 5.188400 E-01$

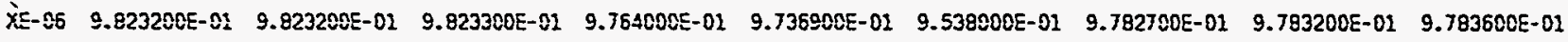
XE-07 2.700000E-04 $\quad 1.730000 E-04 \quad 1.750000 E-04 \quad 3.520000 E-04 \quad 3.590000 E-04 \quad 3.770000 E-04 \quad 2.700000 E-05 \quad 2.100000 E-05 \quad 1.900000 E-05$ XE- 28 -9.840000E-04 -9.840000E-04 -9.850000E-04 -5.030000E-04 - $4.790000 E-04-4.590000 E-04-8.800000 E-04-8.900000 E-04$-8.980000E-04 XE-09 - - $.179000 E-03-1.290000 E-03-2.279000 E-03-2.350000 E-04-2.4200005-04-2.320000 E-04-1.055000 E-03-1.072000 E-03-1.0810005-03$ XE- $10-3.843000 E-03-3.8420005-03-3.843000 E-03-5.659000 E-03-5.571000 E-03-5.546000 E-03-4.865000 E-03-4.971000 E-03-5.053000 E-03$ $\begin{array}{llllllllll}X E-11 & 2.500000 E-05 & 2.600000 E-05 & 2.500000 E-05 & 2.500000 E-05 & 2.700000 E-05 & 2.600000 E-05 & 2.600000 E-05 & 2.600000 E-05 & 2.700000 E-05\end{array}$ $x E-12-1.6680005-03-1.667000 E-03-1.667000 E-03-1.346800 E-03-1.348000 E-03-1.346000 E-03-1.665000 E-03-1.666000 E-03-1.667000 E-03$ XE-13 - $6.050000 E-04-6.070000 E-04$-E.100000E-84 2.120000E-04 2.440000E-04 $1.909000 E-03 \quad 4.500000 E-05-5.100000 E-05$ - $2.160000 E-04$ XE- 14 3.200000E-04 3.720000E-04 3.220000E-04 9.749100E-01 9.750600E-01 9.542000E-01 -4.180000E-04 -4.730000E-04 -5.050000E-04 XE- 15 -7.520080E-04 -7.520000E-04 -7.540000E-04 1.810000E-04 1.840000E-04 1.590000E-0.4 -5.080000E-04 -5.620000E-04 -6.000000E-04 TE- $02-1.480000 E-04-1.4700005-04-1.4700005-04-1.0908005-04-1.0700085-04-1.030000 E-04-1.300000 E-04-1.370000 E-04-1.320000 E-04$ $\begin{array}{llllllllllll}\text { VEXII } & 2.993600 E+00 & 1.993600 E+00 & 1.993600 E+00 & 1.993000 E+00 & 1.993000 E+00 & 1.993000 E+00 & 1.993300 E+00 & 1.993300 E+00 & 1.993300 E+00\end{array}$

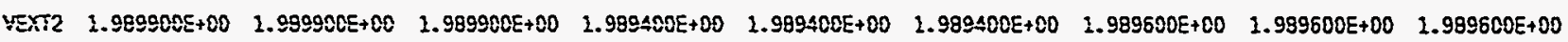
$\begin{array}{llllllllll}\text { TIME } & 2.086630 E+11 & 2.085530 E+12 & 2.086630 E+11 & 2.086530 E+11 & 2.086630 E+11 & 2.085630 E+11 & 2.086630 E+11 & 2.086630 E+11 & 2.086630 E+12\end{array}$ 
Arehive Hzare:

AHS_MFP_TESTS

Book Henre: A4S_MfPTi_RY Date \& Time: 2 Mat 1900 06:27:18

Page Description:

DATA SET YO.-2E5

TRAHS RY_ZERO-1 RY_ZERO-2 RV_ZERD-3 RV_LCAD-1 $\quad$ RY_LCAD-2 $\quad$ RY_LCAD-3 $\quad$ RV_ZERO-4 $\quad$ RV_ZERO-5 RV_ZERO-6

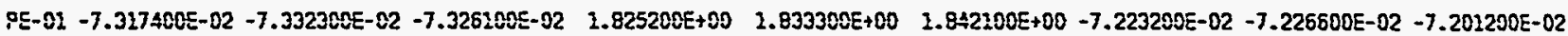
PE-02 3. 204400E-02 3.086308E-02 $3.096300 E-02 \quad 2.255400 E+00 \quad 2.206600 E+00 \quad 2.2459085+00 \quad 3.0441005-02 \quad 3.070000 E-02 \quad 3.089700 E-02$ PE-03 - $1.983800 E-02-1.992300 E-02-2.001700 E-02 \quad 2.098200 E+00 \quad 2.059200 E+00 \quad 2.077100 E+00-1.879982 E-02-1.871100 E-02-1.882900 E-02$ PE-04 3.980300E-02 $3.895900 E-02 \quad 3.893100 E-82 \quad 2.370800 E+00 \quad 2.403100 E+00 \quad 2.398600 E+00 \quad 3.900100 E-02 \quad 3.897700 E-02 \quad 3.978700 E-02$

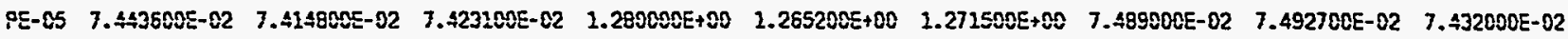
PE-06 $5.645200 E-02 \quad 6.627000 E-02 \quad 5.636700 E-02 \quad 2.0432005+00 \quad 2.017500 E+00 \quad 2.022800 E+00 \quad 5.7600005-02 \quad 6.746280 E-82 \quad 6.746600 E-02$

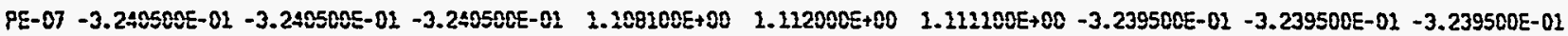

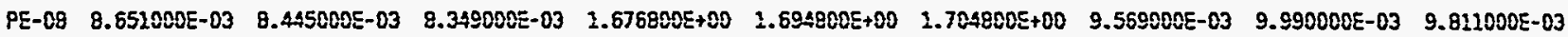
PE- 09 -3.5742005-02 -3.702300E-02 -3.664300E-02 $6.880700 E-01 \quad 6.807900 E-02 \quad 6.834300 E-02 \quad-3.634500 E-02 \quad-3.610200 E-02 \quad-3.624000 E-02$ $\begin{array}{llllllllll}P E-10 & 2.047900 E-02 & 2.049800 E-02 & 2.047500 E-02 & 1.438600 E+00 & 1.423300 E+00 & 2.383400 E+00 & 2.136500 E-02 & 2.122800 E-02 & 2.133700 E-02\end{array}$

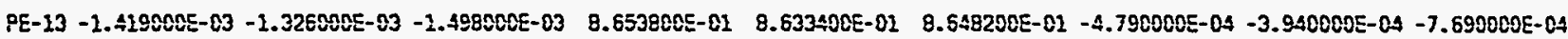
PE-14 $1.279800 E-02 \quad 1.278300 E-02 \quad 1.280400 E-01 \quad 1.292400 E+00 \quad 1.292400 E+00 \quad 1.277300 E+00 \quad 1.200200 E-02 \quad 1.293400 E-02 \quad 1.290700 E-01$

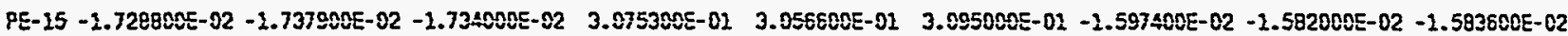
PE-15 3.454800E-02 $3.473100 E-02 \quad 3.475400 E-02 \quad 5.560700 E-02 \quad 5.558480 E-01 \quad 5.623300 E-02 \quad 3.4408005-02 \quad 3.472400 E-02 \quad 3.438400 E-02$ $\therefore E-17$-3.772400E-02 -3.7EA100E-02 $-3.816100 E-02 \quad 2.885500 E-01 \quad 2.952 E 00 E-02 \quad 2.828600 E-01 \quad-3.737608 E-02 \quad-3.703600 E-02 \quad-3.698800 E-02$

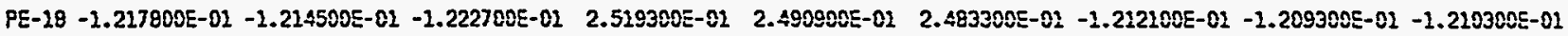
$P E-19-4.7338005-02-4.757900 E-02-4.7939005-02 \quad 2.804300 E-01 \quad 1.7726005-01 \quad 1.835500 E-01 \quad-4.831000 E-02-4.779200 E-02-4.755100 E-02$

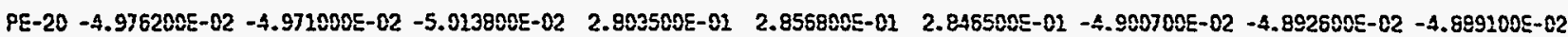

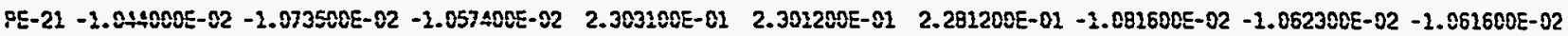
PE-22 -8.000000E-03 -8.082000E-03 $-7.918000 E-03 \quad 2.2532005-01 \quad 2.2795005-02 \quad 2.282700 E-02 \quad-8.5090005-03 \quad-8.4250005-03 \quad-8.3160005-03$ PE-29 7.952600E-02 $7.933300 E-02 \quad 3.966900 E-02 \quad 3.773400 E+00 \quad 3.928000 E+00 \quad 3.714700 E+00 \quad 1.473200 E-01 \quad 1.468500 E-01 \quad 1.468800 E-01$ PE-30 -2.129990E-02 -2.131190E-02 -1.128530E-01 2.697400E-01 2.608400E-01 2.129700E-02 $-1.109600 E-01 \quad-1.1105005-01 \quad-1.210200 E-02$ FE- $01 \quad 2.000480 E+00 \quad 1.000470 E+00 \quad 1.000460 E+00 \quad 4.337600 E+00 \quad 4.342200 E+00 \quad 4.347 E 00 E+00 \quad 1.003730 E+00 \quad 1.002940 E+00 \quad 1.002450 E+00$ YE-01 -2.970000E-04 -2.970000E-04 -2.980000E-04 4.160000E-04 4.050000E-04 4.290000E-04 -2.570000E-04 -2.620000E-04 -2.640000E-04 XE-02 -8. 800000E-04 -8.800000E-04 -8.790000E-04 -8.850000E-04 -8.600000E-04 -8.640000E-04 -8.810000E-04 -8.820000E-04 -8.810000E-04 YE $-03-1.378200 E-02-1.376900 E-02-1.376900 E-02-6.014300 E-02-8.739800 E-02-6.557300 E-02-3.580800 E-02-3.573200 E-02-3.575500 E-02$

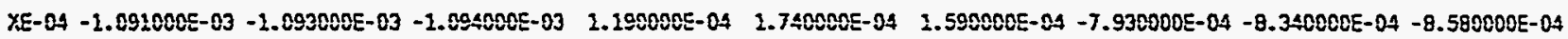
XE-05 5.189700E-01 $5.188900 E-02 \quad 5.288600 E-01 \quad-2.739000 E-03 \quad-2.608000 E-03 \quad-2.582000 E-03 \quad 2.591400 E-01 \quad 2.600300 E-012.5143005-01$ XE-06 9.7874005-01 9.787500E-01 9.787600E-01 9.6587005-01 9.678300E-01 9.625500E-01 9.824000E-01 $9.824100 E-01 \quad 9.824400 E-01$ YE-07 2.810000E-04 2.800000E-04 3.070000E-04 3.910000E-04 3.7300000 -04 $\quad 4.010000 E-04 \quad 7.600000 E-05 \quad 6.5000005-05 \quad 8.800000 E-05$ XE-08 -9.320000E-04 -9.320080E-04 -9.3300005-04 -4.360008E-04 -4.280000E-04 -4.080000E-04 -8.940000E-04 -9.040000E-04 -9.090000E-04

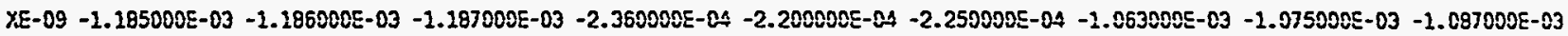
XE- $10-5.335000 E-03-5.335000 E-03-5.336000 E-03-5.443000 E-03-5.4110005-03-5.394000 E-03-4.2550005-03-4.3500005-03-4.408000 E-03$ $\begin{array}{llllllllll}x E-21 & 2.500000 E-05 & 2.600000 E-05 & 2.600000 E-05 & 2.600000 E-05 & 2.600000 E-05 & 2.700000 E-85 & 2.700000 E-05 & 2.600000 E-05 & 2.700000 E-05\end{array}$ XE- $12-1.6710005-03-1.672000 E-03-1.5730005-03-1.320000 E-03-1.326000 E-03-1.311000 E-03-1.659000 E-03-1.6610805-03-1.562000 E-03$ XE-13 -4.050000E-04 -4.200000E-04 -4.190000E-04 $1.721000 E-03 \quad 1.695000 E-03 \quad 1.774000 E-03 \quad 5.800000 E-05-9.000000 E-06$-5.500000E-05 YEE-14 -9.360000E-04 -9.330000E-04 -9.360000E-04 9.660900E-01 9.472000E-01 9.273400E-01 -2.760000E-04 -3.240008E-04 -4.020000E-04

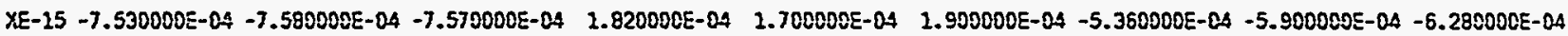
TE-01 -1.480000E-04 -1.480000E-04 -1.480000E-04 -1.250000E-04 -1.140000E-04 -1.140000E-04 -1.350000E-04 - $1.390000 E-04-1.470000 E-04$ $\begin{array}{lllllllllll}\text { VEXT2 } & 2.993300 E+00 & 2.993300 E+00 & 1.993300 E+00 & 1.993100 E+00 & 1.993100 E+00 & 1.993100 E+00 & 2.993400 E+00 & 2.993400 E+C 0 & 1.993400 E+00\end{array}$

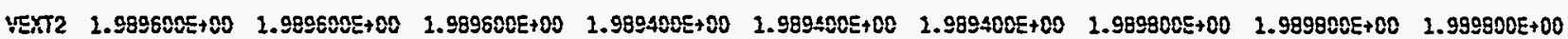

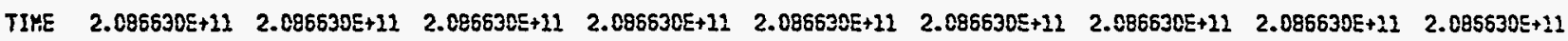


Arcinice Hene:

AYS_*F__TSSTS

Sack Hame: AYS_MFPTI_RY

Date \& Time: 2 Mar $1900 \quad 06: 36: 43$

Page Description:

DATA SET พ0.-266

TRA4S RY_ZERO-1 RV_ZERO-2 RY_ZERO-3 RV_LOAD-2 RV_LOAD-2 RY_LOAD-3 RY_ZERO-4 RV_ZERO-5 RV_ZERD-6

PE- $02 \quad-7.3923005-02 \quad-7 . \$ 03900 E-02 \quad-7.402600 E-02 \quad 2.962000 E+00 \quad 1.998900 E+00 \quad 2.967000 E+00-7.156700 E-02 \quad-7.168800 E-02 \quad-7.140300 E-02$ FE-02 $2.950700 E-82 \quad 2.993000 E-02 \quad 2.971900 E-02 \quad 2.356300 E+00 \quad 2.394400 E+00 \quad 2.372400 E+00 \quad 3.047400 E-02 \quad 3.049500 E-02 \quad 3.074500 E-02$

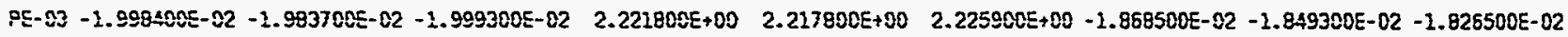

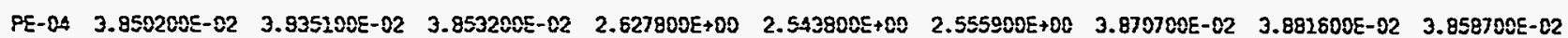

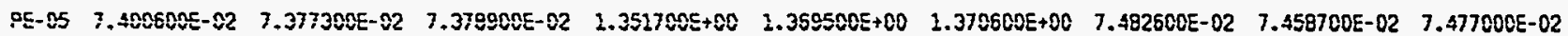
PE-DS 6.520100E-02 $\quad 6.5137005-02 \quad 6.625000 E-02 \quad 2.0999005+00 \quad 2.129300 E+00 \quad 2.1272005+00 \quad 6.699600 E-02 \quad 8.6772005-02 \quad 6.710400 E-02$

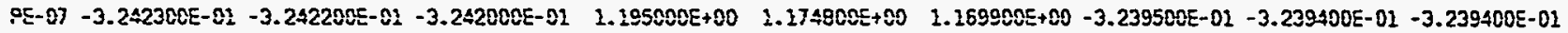
$\begin{array}{lllllllllll}P E-88 & 8.800000 E-03 & 8.6480005-83 & 8.326000 E-03 & 1.805300 E+00 & 1.809300 E+00 & 1.840500 E+00 & 8.878000 E-03 & 9.207000 E-03 & 9.012000 E-03\end{array}$

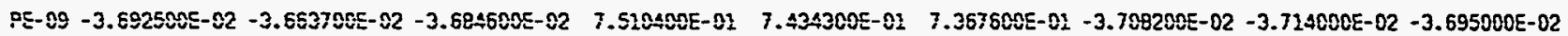
PE-10 $2.9971005-82 \quad 2.992400 E-02 \quad 2.013800 E-02 \quad 1.489600 E+00 \quad 1.482100 E+00 \quad 1.464200 E+00 \quad 2.099600 E-02 \quad 2.102600 E-02 \quad 2.121300 E-02$

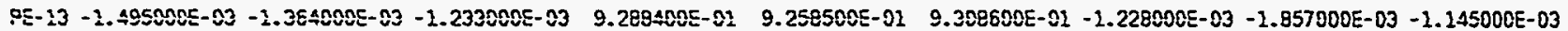

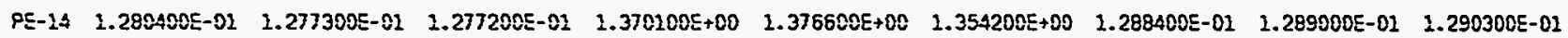

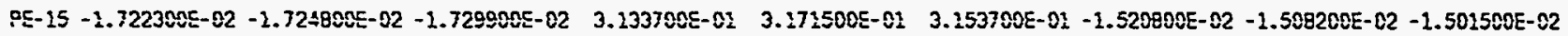
PE- $2 \varepsilon \quad 3.435300 E-02 \quad 3.429900 E-02 \quad 3.3928005-02 \quad 6.313300 E-01 \quad 6.390700 E-02 \quad 6.347100 E-01 \quad 3.407200 E-02 \quad 3.393700 E-02 \quad 3.415100 E-02$

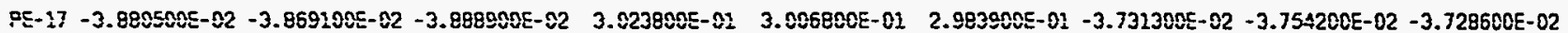

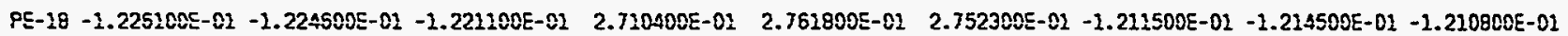

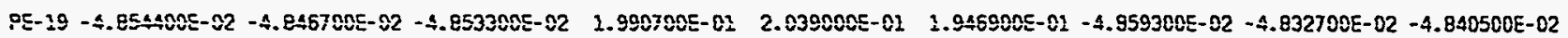

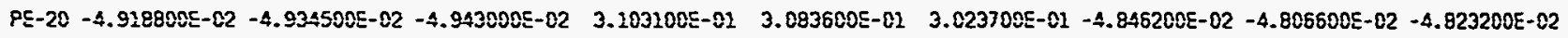

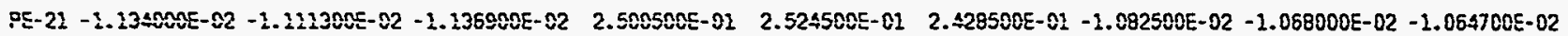
PE-22 -9.240000E- $03 \quad-9.434000 E-03 \quad-9.489000 E-03 \quad 2.455200 E-02 \quad 2.490000 E-02 \quad 2.416000 E-01 \quad-9.126000 E-03 \quad-8.994000 E-03 \quad-8.905000 E-03$ $\begin{array}{lllllllllll}P E-29 & 1.420900 E-02 & 1.400500 E-01 & 1.403400 E-02 & 3.999000 E+00 & 3.992700 E+00 & 3.970200 E+00 & 1.457300 E-02 & 1.470500 E-01 & 1.466000 E-02\end{array}$

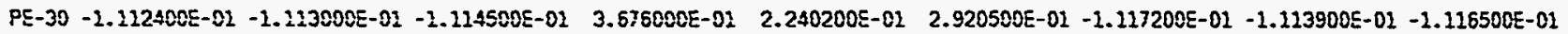
EE-02 $\quad 2.000570 E+00 \quad 1.000560 E+00 \quad 1.000560 E+00 \quad 4.452300 E+00 \quad 4.455500 E+00 \quad 4.451800 E+00 \quad 1.004740 E+00 \quad 1.003600 E+00 \quad 1.002890 E+00$ YEE- 81 -3.830000E-04 -3.030000E-04 -3.040000E-04 4. 440000E-04 4.410000E-04 4.460000E-04 -2.580000E-04 -2.570000E-04 -2.730000E-04 XE-02 -9.7900005-04 -8.700000E-04 -8.830000E- 04 -8.470008E-04 -8.480000E-04 -8.520000E-04 -8.900000E-04 -9.830000E-04 -8.820000E-04 $X E-03-2.856400 E-02-2.841300 E-02-2.842900 E-02-9.412700 E-02-1.055600 E-02-1.084320 E-02-8.498200 E-02-8.499100 E-02-8.485900 E-02$ XE-04 - $2.003000 E-03-1.002000 E-03-1.006000 E-03 \quad 1.810000 E-04 \quad 2.0100005-04 \quad 2.170000 E-04-6.860000 E-04-7.530000 E-04-7.920000 E-04$ $X E-05 \quad 2.771700 E-01 \quad 2.775200 E-01 \quad 2.777600 E-02 \quad-2.280000 E-03 \quad-2.295000 E-03 \quad-2.199000 E-03 \quad 9.257400 E-02 \quad 9.259200 E-01 \quad 9.259200 E-01$

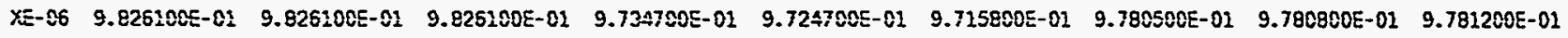
$X E-07$ 4.890000E-04 4.910000E-04 4.980000E-04 3.830000E-04 4.110000E-04 4.270000E-04 4.200000E-05 5.1000000E-05 4.700000E-05 XE- $88-9.5600005-84-9.560000 E-04-9.588000 E-04$ - $4.010000 E-04-3.8400005-84-3.750000 E-04-8.880000 E-04-8.980000 E-04-9.040000 E-04$ XE-09 - $1.290000 E-03-1.290000 E-03-1.1920005-03-1.970000 E-04-1.8000005-04-1.7300005-04-1.042000 E-03-1.055000 E-03-1.069000 E-03$ $X_{K}-10-4.626000 E-03-4.627000 E-03-4.629000 E-03-5.366000 E-03-4.884000 E-03-5.453000 E-03-6.050000 E-03-6.150000 E-03-6.2260005-03$ XE-11 2.508008E-05 2.500000E- $25 \quad 2.600000 E-05 \quad 2.600000 E-05 \quad 2.600000 E-05 \quad 2.600000 E-05 \quad 2.500000 E-05 \quad 2.700000 E-05 \quad 2.700000 E-05$ $x E-12-1.6770005-03-1.6770005-03-1.677000 E-03-1.285000 E-03-1.295000 E-03-1.291000 E-03-1.673000 E-03-1.676000 E-03-1.675000 E-03$ XE-12 $-3.5500005-04 \quad-3.690000 E-04 \quad-3.790000 E-04 \quad 1.654000 E-03 \quad 1.6940005-03 \quad 1.705000 E-03 \quad 1.0620005-04 \quad 2.400000 E-05 \quad-2.800000 E-05$ XE-14 -9.260000E-04 -8.2400005-04 - 8.180000E-04 9.613000E-01 7.919100E-01 5.174000E-02 T.060000E-04 T.200000E-04 6.700020E-04 XE- 15 -7.410000E-04 -7.460000E-04 -7.520000E-04 2.000000E-04 2.220000E-04 2.280000E-04 -4.090000E-04 -4.740000E-04 $-5.110000 E-04$ TE- $02-1.5800005-04-1.5500005-04-1.560000 E-04-1.1800005-04-1.150000 E-04-1.140000 E-04-1.380000 E-04-1.470000 E-04-1.500000 E-04$ $\begin{array}{lllllllllll}\text { VEXT1 } & 1.993400 E+00 & 1.993400 E+00 & 1.993400 E+00 & 1.993100 E+00 & 1.993200 E+00 & 1.993600 E+00 & 1.993100 E+00 & 1.993100 E+00 & 1.993100 E+00\end{array}$ VEXT2 $1.989700 E+00 \quad 1.989808 E+00 \quad 1.989780 E+C 0 \quad 1.989500 E+00 \quad 1.9896005+00 \quad 1.989900 E+\infty 0 \quad 1.989400 E+00 \quad 1.989400 E+00 \quad 1.989400 E+00$

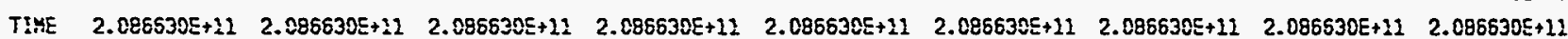


Arshive Hane:

AMS_MEP_TESTS

Book Hame: ANS_MfPT2_RV Date \& Time: 2 Mar 1900 C6:52:10

Page Destription:

DATA SET HO.-267

TRAHS FYZZERO-1 RY_ZERO-2 RV_ZERO-3 RY_LCAD-1 RV_LOAD-2 RY_LOAD-3 RV_ZERO-4 RV_ZERO-5 RV_ZERO-6 $-$

PE-02 -7.206500E-02 -7.210E00E-02 -7.226700E-02 -7.145000E-02 -7.252200E-02 -7.146500E-02 -4.742100E-02 -4.740500E-02 -4.745900E-02 PE-02 2.995100E-02 2.953600E-02 $2.990200 E-02 \quad 3.117200 E-02 \quad 3.230200 E-02 \quad 3.136700 E-82 \quad 5.6633005-02 \quad 5.655200 E-02 \quad 5.649900 E-02$

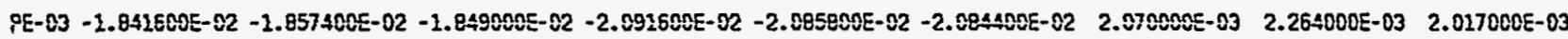

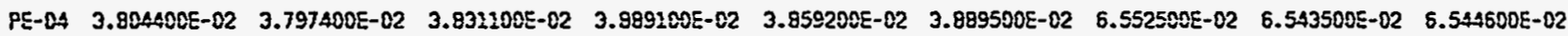
PE-05 7.419000E-02 7.404500E-02 $7.4316005-02 \quad 7.510000 E-02 \quad 7.507900 E-02 \quad 7.491700 E-02 \quad 1.009220 E-01 \quad 1.010130 E-01 \quad 1.009530 E-01$ $\begin{array}{llllllllll}P E-86 & 6.590000 E-02 & 6.668300 E-02 & 6.653900 E-02 & 6.757300 E-02 & 6.752300 E-02 & 6.729800 E-02 & 9.2782005-02 & 9.284300 E-02 & 9.276300 E-02\end{array}$

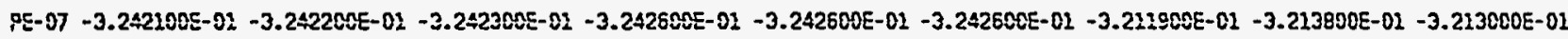
PE-08 $8.67 \varepsilon 000 E-03 \quad 8.757000 E-03 \quad 8.507000 E-03 \quad 8.979000 E-03 \quad 9.032000 E-03 \quad 9.069000 E-03 \quad 3.207120 E-02 \quad 3.196100 E-02 \quad 3.183600 E-02$ $9 E-09-3.722400 E-02-3.737020 E-02-3.7157005-02-3.674409 E-02-3.673300 E-02-3.6709005-02-8.561000 E-03-8.423000 E-03-8.405000 E-03$ $\begin{array}{llllllllll}\text { PE-10 } & 2.082300 E-02 & 2.099400 E-02 & 2.077700 E-02 & 2.064400 E-02 & 2.054100 E-02 & 2.082700 E-02 & 4.779100 E-02 & 4.754980 E-02 & 4.789900 E-02\end{array}$

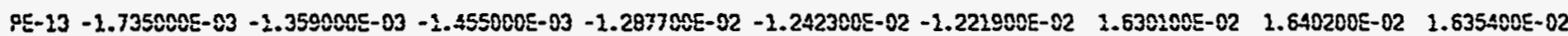

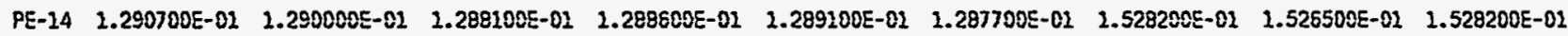

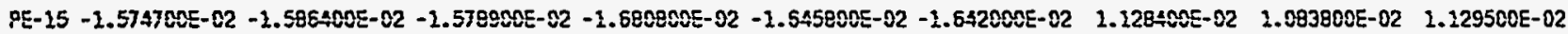
PE-16 $3.434100 E-02 \quad 3.448500 E-02 \quad 3.400000 E-82 \quad 3.409100 E-02 \quad 3.395500 E-02 \quad 3.391000 E-02 \quad 6.192400 E-02 \quad 6.1934005-02 \quad 6.184400 E-02$ $8 E-17-3.7742005-02-3.784500 E-02-3.802700 E-02-4.57 E 8020 E-82-4.582100 E-02-4.589700 E-02-1.71 \triangle 800 E-02-1.707500 E-02-1.697 B 00 E-02$

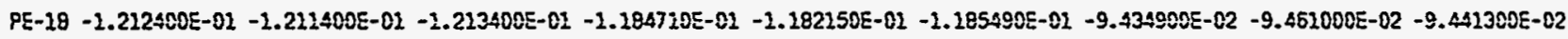
PE-19 -4.849700E- 02 - $4.8239005-02-4.830800 E-02$-5.279800E- 02 -5.28E800E-02 -5.252600E-02 -2.365700E-02 -2.382100E-02 - 2.375400E-02 PE-20 - $4.791500 E-02-4.8067005-02$ - $4.819500 E-02-4.801600 E-02-4.7897005-02-4.815800 E-02$-2.126200E-02 $-2.126000 E-02-2.103100 E-02$

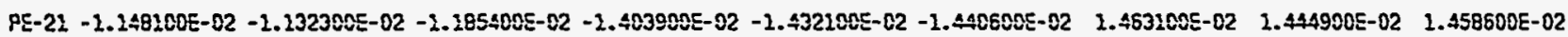
$P E-22 \quad-8.739000 E-03 \quad-8.855000 E-03 \quad-8.897000 E-03 \quad-8.802000 E-03 \quad-8.958000 E-03 \quad-8.941000 E-03 \quad 1.949000 E-02 \quad 1.939700 E-02 \quad 1.957300 E-02$ PE-29 1.411500E-01 $2.427300 E-01 \quad 1.413300 E-02 \quad 1.624980 E-01 \quad 1.6227005-01 \quad 1.6247$ COE-01 $1.897800 E-01 \quad 1.895100 E-01 \quad 1.902800 E-01$

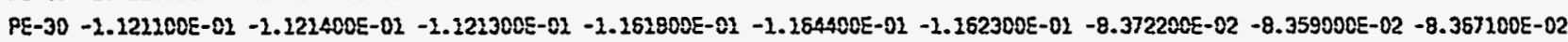
FE-01 $\quad 1.000690 E+00 \quad 1.0006 E 0 E+00 \quad 1.0006 \Omega 0 E+00 \quad 1.202110 E+00 \quad 1.001790 E+00 \quad 1.001560 E+00 \quad 1.000480 E+00 \quad 1.000490 E+00 \quad 1.000470 E+00$ XE-01 -3.100000E-04 -3.110000E-04 -3.130000E-04 -2.530000E-04 -2.740000E-04 -1.580000E-84 -3.610000E-04 -3.710000E-04 -3.690000E-04 XE-02 -9.880000E-04 -9.800000E-04 -8.820080E-04 -7.375000E-03 - 7.373000E-03 - 7.373000E-03 -7.364000E-03 -7.361000E-03 -7.357000E-03 XE-03 -5.851600E-02 -5.846800E-02 -5.839800E-02 -1.586800E-02 -1.586502E-02 - $1.586480 E-\$ 2-1.503300 E-02-1.505200 E-02-1.506000 E-02$ $X E-04 \quad-9.2400005-84 \quad-9.310000 E-04 \quad-9.3900005-04 \quad 3.608000 E-03 \quad 3.575000 E-03 \quad 3.558000 E-03 \quad 3.288000 E-03 \quad 3.284000 E-03 \quad 3.281000 E-03$

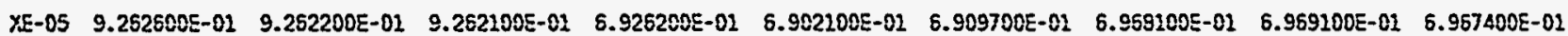

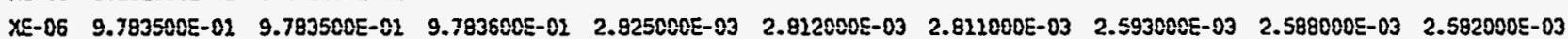
$\begin{array}{llllllllll}X E-07 & 3.180000 E-04 & 3.150000 E-0.4 & 3.360000 E-04 & 1.356000 E-03 & 1.351000 E-03 & 1.356000 E-03 & 1.579000 E-03 & 1.604000 E-03 & 1.615000 E-03\end{array}$ XE-08 -9.540000E-04 -9.550000E-04 -9.560000E- 04 -4.542000E-03 -4.554000E-03 -4.561003E-03 -4.653000E-03 - $4.655000 E-03$ - $4.656000 E-03$ XE- $09-1.172000 E-03 \quad-1.175000 E-03 \quad-1.275000 E-03 \quad 3.311000 E-03 \quad 3.292000 E-03 \quad 3.278000 E-03 \quad 3.171000 E-03 \quad 3.169000 E-03 \quad 3.168000 E-03$ XE-10 -6. \$29000E- $03-6.432000 E-03-6.4290005-03-1.395000 E-03-3.615000 E-03-3.7580005-03-3.828000 E-03-3.839000 E-03-3.829000 E-03$ $\begin{array}{llllllllll}Y E-12 & 2.600000 E-05 & 2.700000 E-05 & 2.700000 E-05 & 2.600000 E-05 & 2.700000 E-05 & 2.300000 E-05 & 2.700000 E-05 & 2.700000 E-05 & 2.700000 E-05\end{array}$ $X E-12 \quad-1.680000 E-03 \quad-1.679000 E-03 \quad-1.680000 E-03 \quad 2.740000 E-03 \quad 2.736000 E-03 \quad 2.736000 E-03 \quad 2.719000 E-03 \quad 2.719000 E-03 \quad 2.720000 E-03$ XE-13 -3.230000E-04 -3.290000E-C4 -3.490000E-04 -4.600000E-05 -5.000000E-05 -7.400000E-05 -2.920000E-04 -2.940000E-04 -2.900000E-04 YL-14 -3.810008E-04 -3.850000E- 04 -4.130000E-03 -9.643400E-01 5.990700E-01 6.170400E-01 $6.179200 E-01 \quad 6.207900 E-01 \quad 6.280500 E-01$ XE-15 -6.980000E-04 -6.920000E-04 -7.090000E-04 6.130000E-04 5.850000E-04 5.3500005-04 2.3000005-05 2.800000E-05 2.900000E-05

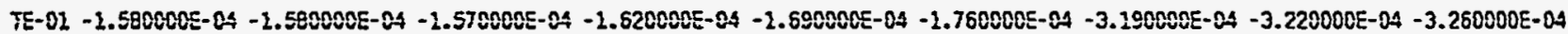

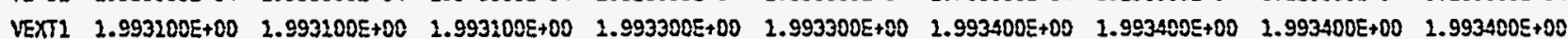
YEXT2 $1.989400 E+C D \quad 1.989 \leqslant 00 E+00 \quad 1.989400 E+00 \quad 1.989700 E+00 \quad 1.989700 E+00 \quad 1.989800 E+00 \quad 1.989800 E+00 \quad 1.989800 E+00 \quad 1.98980 R E+00$ TIYE $2.086630 E+1122.096530 E+12 \quad 2.086630 E+12 \quad 2.086630 E+12 \quad 2.085530 E+11 \quad 2.086530 E+112.086630 E+12 \quad 2.086530 E+112.086630 E+11$ 
Page Description:

BATA SET HO. -268

TRAYS RE_ZERO-1 RV_ZERO-2 RV_ZERO-3 RV_LOAD-1 RV_LOAR-2 RV_LCAD-3 SV_ZERO-4 RV_ZERO-5 RV_ZERO-6

$P E-01-6.2502005-02-6.226500 E-02-5.238500 E-02-6.236700 E-02-6.2498005-02-6.248800 E-02-6.266390 E-02-6.270500 E-02-5.244100 E-02$

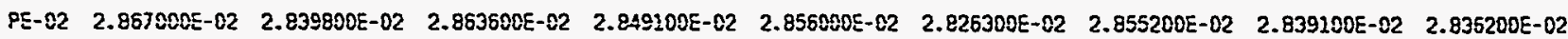

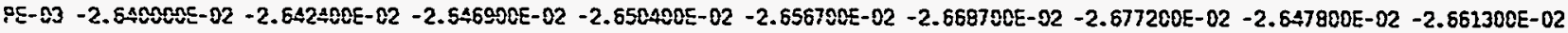
$\begin{array}{llllllllll}P S-04 & 3.759400 E-02 & 3.759600 E-02 & 3.793900 E-02 & 3.755800 E-02 & 3.748600 E-82 & 3.754809 E-82 & 3.749000 E-02 & 3.712500 E-02 & 3.739300 E-02\end{array}$

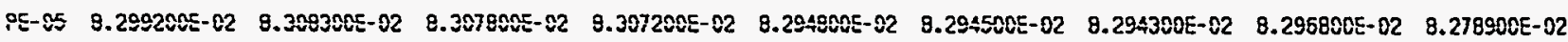
PE- 88 7.844500E- $82 \quad 7.869100 E-02 \quad 7.875400 E-02 \quad 7.878700 E-02 \quad 7.853000 E-02 \quad 7.827500 E-02 \quad 7.870800 E-02 \quad 7.844900 E-02 \quad 7.856800 E-02$

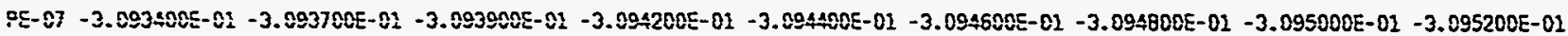
$\begin{array}{lllllllllll}P E-88 & 1.998300 E-02 & 1.878800 E-02 & 1.859200 E-02 & 1.891300 E-02 & 1.864900 E-02 & 1.871400 E-02 & 1.875400 E-02 & 1.869300 E-02 & 1.879200 E-02\end{array}$ PE-09-2.728400E-02 -2.727800E-02 - 2.733000E-02 -2.749900E-02 -2.733500E-02 -2.732700E-02 -2.760000E-02 -2.728 $-000 E-02-2.772900 E-02$ PE-10 3.117000E-02 3.118500E-82 $3.107400 E-82 \quad 3.204000 E-02 \quad 3.090200 E-02 \quad 3.100700 E-02 \quad 3.090300 E-02 \quad 3.1086005-02 \quad 3.209400 E-02$

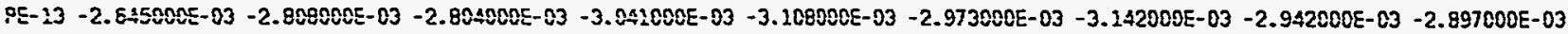
PE- $14 \quad 1.379500 E-01 \quad 1.380200 E-01 \quad 1.379700 E-01 \quad 2.378200 E-01 \quad 1.378100 E-01 \quad 1.377500 E-01 \quad 2.378400 E-02 \quad 1.379400 E-01 \quad 1.377200 E-01$ PC- $15-2.2180005-03-2.320000 E-03-2.269000 E-03-2.150000 E-93-1.994000 E-03-2.301000 E-03-1.896000 E-03-2.373000 E-03-2.186000 E-03$ PE-16 5.224600E-02 5.199100E-02 5.157500E-02 5.204900E-02 5.187900E-02 5.206900E-02 $5.191200 E-02 \quad 5.212400 E-02 \quad 5.210400 E-02$ PC-17 -2.545602E-02 -2.615600E-02 -2.641400E-02 -2.613800E-02 -2.671400E-02 -2.632600E-02 - 2.629500E-02 -2.640800E-02 -2.639400E-02 $P E-18-1.066040 E-01-1.065980 E-01-1.0693505-01-2.070150 E-01-1.068170 E-01-1.068440 E-01-1.0689805-01-1.069490 E-01-1.071950 E-01$ $P E-19-3.705308 E-82-3.7577005-02-3.746400 E-02-3.730000 E-02-3.691900 E-02-3.693400 E-02-3.726600 E-02-3.722700 E-02-3.716800 E-02$ $P E-20-2.4283005-02-2.423100 E-82-2.4549008-02-2.415500 E-02-2.419700 E-02-2.432400 E-02-2.417100 E-02 \quad-2.427800 E-02 \quad-2.421700 E-02$ PE-2i $5.6930005-03 \quad 5.715000 E-03 \quad 5.948000 E-03 \quad 5.686000 E-03 \quad 5.7330005-03 \quad 5.639000 E-03 \quad 5.584000 E-03 \quad 5.4490000 E-03 \quad 5.629000 E-03$ $\begin{array}{llllllllll}P E-22 & 1.733200 E-02 & 1.749900 E-02 & 1.735200 E-02 & 1.734800 E-02 & 1.734300 E-02 & 1.718100 E-02 & 1.714400 E-02 & 1.723100 E-02 & 1.719000 E-02\end{array}$

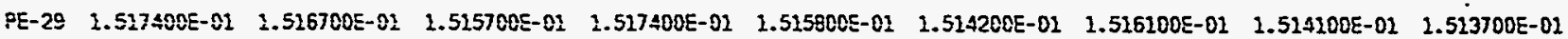
PE-30 -7.787300E-02 -7.813000E-02 -7.790300E-02 -7.788200E-02 -7.790400E-02 -7.780100E-02 -7.796800E-02 -7.799900E-02 -7.8112C0E-02 FE-01 $1.000160 E+00 \quad 1.000180 E+00 \quad 1.000280 E+00 \quad 1.000180 E+00 \quad 1.000180 E+00 \quad 1.000170 E+00 \quad 1.000190 E+00 \quad 1.000180 E+00 \quad 2.000170 E+00$

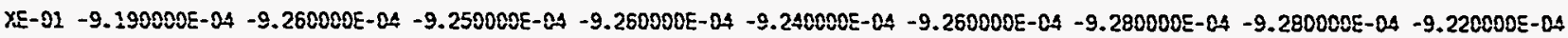
YE- $02-7.775000 E-83-7.775000 E-03-7.774000 E-83-7.774000 E-03-7.7740005-03-7.773000 E-03-7.775000 E-03-7.774000 E-03-7.775000 E-03$ $X E-03-1.328700 E-01-1.333600 E-01-1.329300 E-01-1.328300 E-01-1.328600 E-01-1.326200 E-01-1.330700 E-01-1.328600 E-01-1.330100 E-02$ $\begin{array}{lllllllllll}x E-04 & 2.074000 E-03 & 2.075000 E-03 & 2.074000 E-83 & 2.075000 E-03 & 2.075000 E-03 & 2.075000 E-03 & 2.075000 E-03 & 2.075000 E-03 & 2.075000 E-03\end{array}$

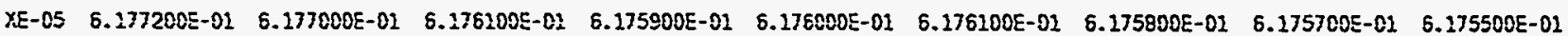
$\begin{array}{llllllllll}X E-05 & 2.273000 E-03 & 2.174000 E-03 & 2.174000 E-03 & 2.174000 E-03 & 2.174000 E-03 & 2.174000 E-03 & 2.174000 E-03 & 2.174000 E-03 & 2.174000 E-03\end{array}$ XE-07 4.120000E-04 4.120000E-04 4.130000E-04 4.240000E-04 4.120000E-04 4.130000E-04 4.130000E-0.4 4.130000E-04 4.130000E-04

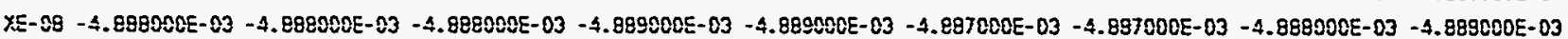
$\begin{array}{lllllllllll}X E-09 & 2.954000 E-03 & 2.955000 E-03 & 2.955000 E-03 & 2.954000 E-03 & 2.955000 E-03 & 2.955000 E-03 & 2.955000 E-03 & 2.955000 E-03 & 2.954000 E-03\end{array}$ $x E-10-4.297000 E-03-4.286000 E-03-4.286000 E-03-4.286000 E-03-4.285000 E-03-4.284000 E-03-4.284000 E-03-4.285000 E-03-4.285000 E-03$ $\begin{array}{llllllllll}X E-12 & 2.400000 E-05 & 2.500000 E-05 & 2.600000 E-05 & 2.500000 E-05 & 2.500000 E-05 & 2.600000 E-05 & 2.5000000 E-05 & 2.6000000 E-05 & 2.500000 E-05\end{array}$ $\begin{array}{lllllllllll}x E-12 & 2.606000 E-03 & 2.607000 E-03 & 2.607000 E-03 & 2.608020 E-03 & 2.606000 E-03 & 2.607000 E-03 & 2.608000 E-03 & 2.607000 E-03 & 2.608000 E-03\end{array}$ XE-13 - $2.056000 E-03-1.055000 E-03-1.054000 E-03-1.055000 E-03-1.055000 E-03-1.055000 E-03-1.055000 E-03-1.054000 E-03-1.055000 E-03$

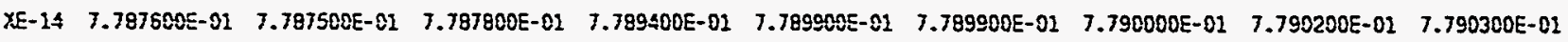
XE-15 -9.420000E-04 -9.410000E-04 -9.410000E-04 -9.410000E-04 -9.410000E-04 -9.410000E-04 -9.410000E-04 -9.420000E-04 -9.410000E-04 TE- $02-6.5000005-05-6.600000 E-05-6.6000005-05-6.700000 E-05-6.700000 E-05-6.600000 E-05-6.700000 E-05-5.700000 E-05-6.700000 E-05$ $\begin{array}{llllllllllll}\text { VEXT2 } & 1.993700 E+00 & 1.993700 E+00 & 1.993700 E+00 & 1.993700 E+00 & 1.993700 E+00 & 1.993700 E+00 & 1.993700 E+00 & 1.993700 E+00 & 1.993700 E+00\end{array}$

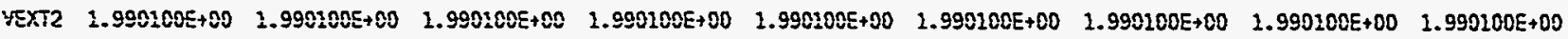

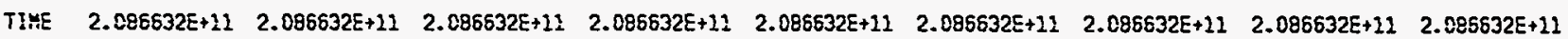


Arthive Heme:

AHS_MFP_TESTS

Sook Mame: ANS_MFPTI_RY

Date \& Time: 6 Mar 2900 23:24:25

Page Description:

DATA SET HO. -269

TRAHS RY_ZERO-1 RE_ZERO-2 RY_ZERO-3 RY_LOAD-1 RY_LOAD-2 $\quad$ RY_LOAD-3 $\quad$ RY_ZERO-4 $\quad$ RN_ZERO-5 RY_ZERO-6

$9 E-02-5.652900 E-02-5.681200 E-02-5.569800 E-02-1.052200 E-02-1.299400 E-02-1.255700 E-02-7.127900 E-02-7.124900 E-02-7.137000 E-02$ $\begin{array}{lllllllllll}P E-02 & 3.597400 E-02 & 3.605300 E-02 & 3.615500 E-02 & 1.094040 E-02 & 1.060260 E-01 & 1.077900 E-01 & 2.236500 E-02 & 2.246300 E-02 & 2.247200 E-02\end{array}$ PE-03 -2.2515ESE-02 $-2.236808 E-02 \quad-2.154900 E-02 \quad 1.864900 E-02 \quad 2.130700 E-02 \quad 1.8174005-02 \quad-3.423300 E-02 \quad-3.449800 E-02 \quad-3.419200 E-02$ $\begin{array}{llllllllll}P E-04 & 4.253400 E-02 & 4.243100 E-02 & 4.255100 E-02 & 1.057150 E-01 & 1.120760 E-01 & 1.0542 E 0 E-01 & 3.023500 E-02 & 2.826900 E-02 & 2.857400 E-02\end{array}$ $\begin{array}{llllllllll}P E-05 & 8.647300 E-02 & 8.641000 E-02 & 8.533800 E-02 & 1.252100 E-01 & 1.234800 E-01 & 1.191880 E-01 & 5.997400 E-02 & 7.003100 E-02 & 6.998800 E-02\end{array}$ PE-05 7.833700E-02 7.834800E-02 $7.804900 E-02 \quad 1.306200 E-02 \quad 1.2643005-01 \quad 3.2670005-01 \quad 6.125708 E-02 \quad 6.099300 E-02 \quad 6.094000 E-02$

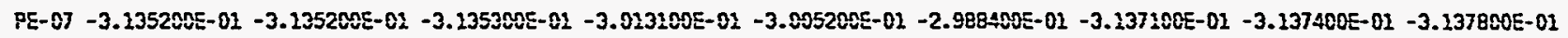
PE-C8 2.157700E-02 2.179600E-02 2.275700E-02 4.401300E-02 4.478800E-02 4.558100E-02 $5.725000 E-03 \quad 5.833000 E-03 \quad 6.394000 E-03$

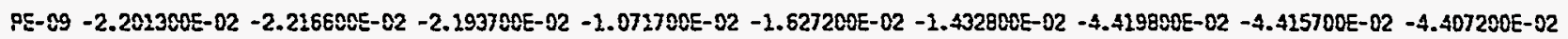
$\begin{array}{lllllllllll}P E-10 & 3.655500 E-02 & 3.645400 E-02 & 3.622200 E-02 & 7.110200 E-02 & 6.900000 E-02 & 7.026500 E-02 & 1.450500 E-02 & 1.479100 E-02 & 1.472700 E-02\end{array}$

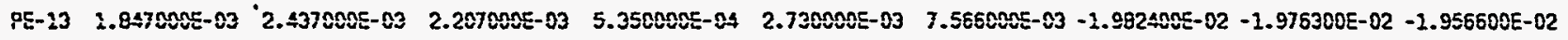
PE-14 1.434600E-02 $1.435000 E-02 \quad 1.433500 E-01 \quad 1.652700 E-01 \quad 1.661400 E-01 \quad 1.685100 E-01 \quad 1.233000 E-01 \quad 1.232200 E-01 \quad 1.234300 E-01$ PE-15 5.135000E-03 4.804000E-03 5.042000E-03 8.400000E-05 -5.130000E-04 -2.992000E-03 - $1.863700 E-02$-2.812000E-02 -1.870900E-02 PE-16 $5.990000 E-02 \quad 5.955300 E-02 \quad 5.972700 E-02 \quad 5.852900 E-02 \quad 5.900500 E-02 \quad 5.735900 E-02 \quad 3.624200 E-02 \quad 3.629600 E-02 \quad 3.636500 E-02$ $P E-17$ - $1.7 E 3500 E-02-1.751200 E-02-1.757700 E-02-4.457300 E-02-4.3424005-02-4.266400 E-02-4.437900 E-02-4.446700 E-02-4.421300 E-02$ PE-19 - 1.002750E-02 -9.966500E-02 -1.002550E-02 - $9.225800 E-02-9.400400 E-02-9.399100 E-02-1.2550005-01-1.255700 E-01-1.255500 E-01$ PE-19 -3.9781005-02 -3.07 $0000 E-02-3.114400 E-82-5.803400 E-82-5.824600 E-02-6.280100 E-02-6.109100 E-02-6.165600 E-02-6.121900 E-02$ PE-20 - - $.924000 E-02-1.932100 E-02-1.924300 E-82-3.975200 E-02-3.809500 E-02-3.859900 E-02-4.815400 E-02-4.793200 E-02-4.781800 E-02$ PE-21 2.02EE00E-02 2.018700E-02 2.003E00E-02 -3.985000E-03 -2.589000E-03 $6.995000 E-03-1.005300 E-02 \quad-1.008000 E-02-9.820000 E-03$ PE-22 2.070100E-02 2.071980E-02 2.062500E-02 -8.030000E-04 $-6.692000 E-83$-5.6550005-03 $-9.1970005-03 \quad-9.159000 E-03-9.3880005-03$ $\begin{array}{lllllllllll}P E-29 & 1.7753005-01 & 1.7722005-02 & 1.773400 E-01 & 2.867300 E-01 & 2.870200 E-01 & 2.923300 E-01 & 1.739500 E-01 & 1.737200 E-01 & 1.739200 E-01\end{array}$ PE-30 -7.235700E-02 -7.232900E-02 -7.227700E- 82 -1.045620E-02 -9.174300E-02 -8.767700E-02 -1.142500E-01 - $1.142600 E-02-1.142200 E-01$ FE-01 $\quad 1.000210 E+00 \quad 2.000110 E+00 \quad 1.000120 E+00 \quad 2.475800 E+00 \quad 1.476500 E+00 \quad 1.473100 E+00 \quad 2.000510 E+00 \quad 1.000480 E+00 \quad 1.000400 E+00$ XE-01 -1.700000E-05 - 1.700000E-05 - -2.700080E-05 -2.100000E-05 -2.200000E-05 -2.100000E-05 -1.700000E-05 -1.800000E-05 -1.700000E-05

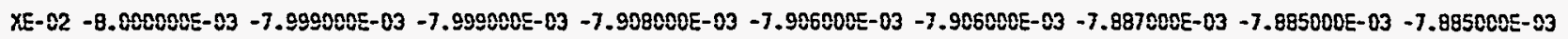
XE-03 -4.760000E-04 -4.760000E-04 -4.760000E-04 -4.830000E-04 -4.820000E-04 -4.820000E-04 -4.820000E-04 -4.820000E-04 -4.820000E-04 X5-04 - $4.6800005-84$-4.570080E-04 -4.670000E-04 6.470000E-04 6.6708005-04 6.670000E-04 4.240080E-04 4.000000E-04 3.770000E-04

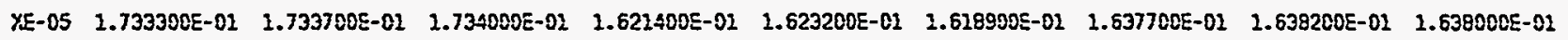
$\begin{array}{lllllllllll}X E-05 & 2.157000 E-03 & 2.157000 E-03 & 2.157000 E-03 & 2.545000 E-03 & 2.547000 E-03 & 2.5480005-03 & 2.4190005-03 & 2.417000 E-03 & 2.412000 E-03\end{array}$ XE-07 4.800000E-05 4.800000E-05 4.900000E-05 $1.022000 E-03 \quad 1.0340005-03 \quad 1.0350005-03 \quad 8.4200005-04 \quad 8.470000 E-04 \quad 8.380000 E-04$ XE-09 - $4.976000 E-03-4.975000 E-03-4.975000 E-03$ - $4.820000 E-03$-4.818000E-03 - $4.818000 E-03-4.8180005-03-4.820000 E-03-4.923000 E-03$ $\begin{array}{llllllllllll}X E-89 & 4.4550005-03 & 4.4560005-03 & 4.455000 E-03 & 4.929000 E-03 & 4.935000 E-83 & 4.9360005-03 & 4.795000 E-03 & 4.800000 E-03 & 4.801000 E-03\end{array}$ XE-10 -8.780000E-04 -8.780000E-04 -8.780000E-04 -5.300000E-05 -5.900000E-05 -5.700000E-05 - $1.740000 E-04-1.8300005-04-1.890000 E-04$ XE-11 - $-2.765000 E-03-1.7540005-03-1.764000 E-03-5.920000 E-04-6.2100005-04-5.4000005-04-9.540000 E-04-9.780000 E-04-9.800000 E-04$ $\begin{array}{llllllllll}X E-12 & 2.591000 E-03 & 2.590000 E-03 & 2.591000 E-03 & 2.665000 E-03 & 2.663000 E-03 & 2.663000 E-03 & 2.642000 E-03 & 2.640000 E-03 & 2.540000 E-03\end{array}$ XE-13 $-1.030000 E-03 \quad-2.030000 E-03 \quad-1.030000 E-03 \quad 6.540000 E-04 \quad 6.590000 E-04 \quad 6.610000 E-04 \quad 1.900000 E-04 \quad 1.540000 E-04 \quad 1.140000 E-04$ XE-1.4 -9.9586005-01 -9.958680E-01 -9.959600E-01 -9.941100E-01 -9.942600E-01 -9.941708E-01 -9.930500E-01 -9.930700E-01 -9.931200E-01 XE-15 -7.980000E-04 -7.980000E-04 -7.980000E-04 $6.950000 E-04 \quad 6.830000 E-04$ 6.800000E-04 4.990000E-04 4.780000E-04 $4.640000 E-04$

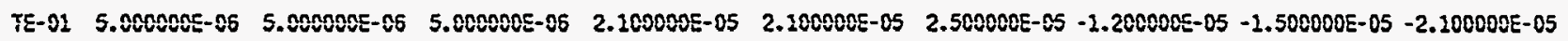

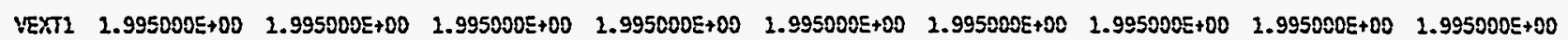
$\begin{array}{llllllllll}\text { VEXT2 } & 1.991800 E+00 & 1.991800 E+00 & 1.991800 E+C 0 & 1.991800 E+00 & 1.991800 E+00 & 1.991800 E+00 & 1.991800 E+00 & 1.991800 E+00 & 1.991800 E+00\end{array}$

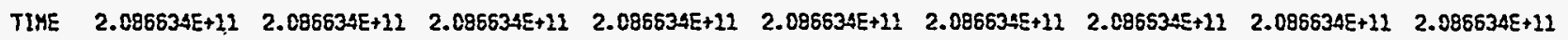


Arshive time:

AHS_KF_TESTS

Book Hzme: AMS_MFPTI_PN

Date \& Time: 6 yar 1900 23:36:54

Page Description:

QATA SET YO. -270

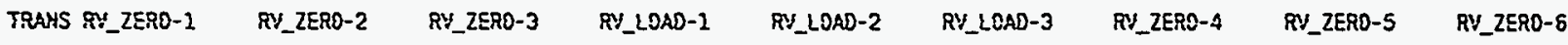

$8 E-02-7.235100 E-02-7.2400005-02-7.258800 E-02-1.181700 E-02-1.327900 E-02-1.088600 E-02-7.240800 E-02-7.237600 E-02-7.249100 E-02$ $\begin{array}{lllllllllll}P E-02 & 2.177100 E-02 & 2.197800 E-02 & 2.202100 E-02 & 1.089430 E-01 & 1.100170 E-02 & 1.080980 E-01 & 2.227800 E-02 & 2.214300 E-02 & 2.295800 E-02\end{array}$ PE- 03 -3.577500E- $02-3.584300 E-02 \quad-3.597800 E-02 \quad 1.756100 E-02 \quad 1.966900 \Omega E-02 \quad 1.750900 E-02 \quad-3.510100 E-02 \quad-3.480600 E-02 \quad-3.474000 E-02$ $\begin{array}{lllllllllll}\text { PE- } 04 & 2.748000 E-02 & 2.759800 E-02 & 2.755200 E-02 & 1.070240 E-01 & 1.067780 E-01 & 1.056470 E-01 & 2.780000 E-02 & 2.780700 E-02 & 2.763200 E-02\end{array}$

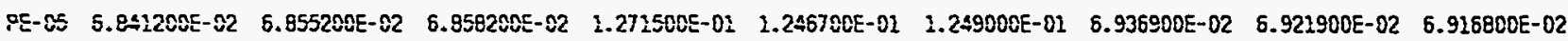
PE-06 $5.999200 E-02 \quad 8.028900 E-02 \quad 6.001900 E-02 \quad 1.295900 E-01 \quad 1.292100 E-02 \quad 1.287000 E-02 \quad 6.010000 E-02 \quad 6.029000 E-02 \quad 6.034500 E-02$

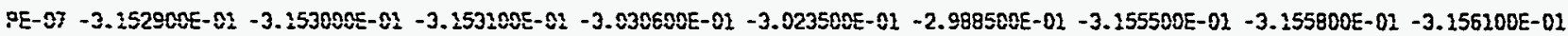
$\begin{array}{lllllllllllll}P E-08 & 4.415000 E-03 & 4.358000 E-03 & 4.565000 E-03 & 4.150700 E-02 & 4.323800 E-02 & 4.597700 E-02 & 5.992000 E-03 & 6.032000 E-03 & 6.093000 E-03\end{array}$ SE- $09-4.453100 E-02$ - $4.465200 E-02-3.451900 E-02-1.461400 E-02-1.368000 E-02-1.498900 E-02-4.506200 E-02-4.482000 E-02-4.511900 E-02$ PE-10 $1.428200 E-02 \quad 1.431800 E-02 \quad 1.410500 E-02 \quad 7.461200 E-02 \quad 7.175300 E-02 \quad 7.077500 E-02 \quad 1.452900 E-02 \quad 1.431700 E-02 \quad 1.425900 E-02$ $\therefore E-13-2.099300 E-82-2.028300 E-02-2.0562005-02-2.255000 E-03-3.0770005-03 \quad 1.054000 E-03-2.053000 E-02-2.048000 E-02-2.050000 E-02$ PE- $14 \quad 1.222000 E-01 \quad 1.228300 E-01 \quad 1.224200 E-02 \quad 1.626400 E-01 \quad 1.676300 E-01 \quad 1.652300 E-01 \quad 1.231400 E-01 \quad 1.232400 E-01 \quad 1.232300 E-01$

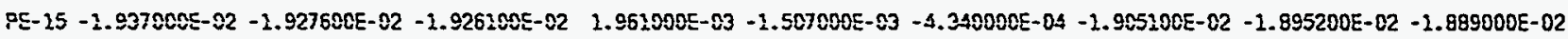
PE-15 3.561600E-02 $3.506500 E-02 \quad 3.5670000-02 \quad 5.048100 E-02 \quad 5.761600 E-02 \quad 5.906200 E-02 \quad 3.627300 E-02 \quad 3.617400 E-02 \quad 3.600700 E-02$

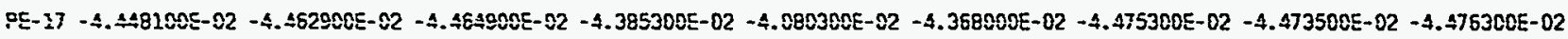

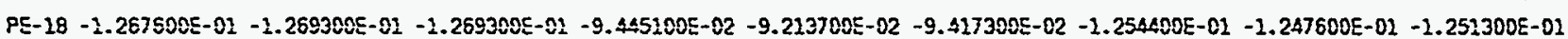

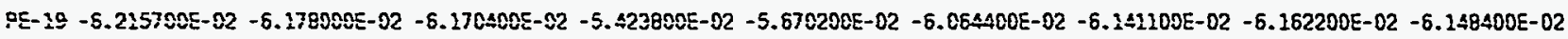
$P E-20-4.942800 E-02-4.936500 E-02-4.976500 E-82-3.894300 E-02-3.8895005-02-3.950100 E-02-4.893500 E-02-4.882800 E-02-4.886500 E-02$

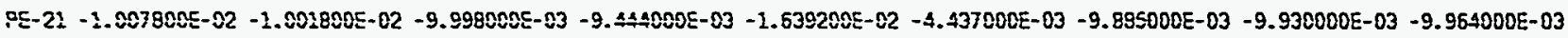
PE-22 -1.049900E- $22-1.015700 E-82-1.0279005-02-4.912000 E-03-9.103000 E-03-3.217000 E-03-1.023000 E-82-1.045900 E-02-1.040400 E-02$

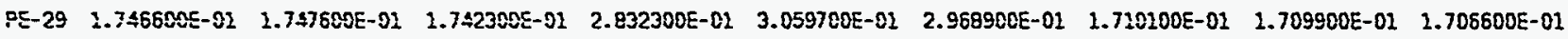

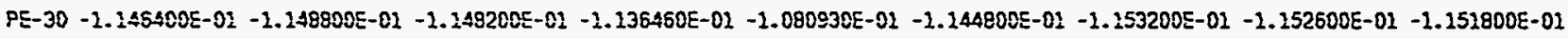

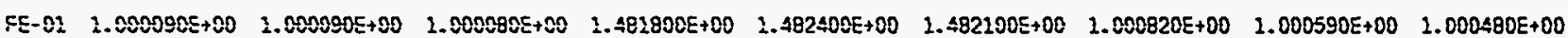
XE- $01-2.700000 E-05-1.7000005-05-1.700000 E-85-2.200000 E-05-2.000000 E-05-2.200000 E-05-1.000000 E-05-1.800000 E-05-1.800000 E-05$ $X E-02-7.8290005-03-7.8170005-03-7.816000 E-83-7.888000 E-03-7.888000 E-03-7.891000 E-03-7.874008 E-03-7.872000 E-03-7.870000 E-03$ XEL-03 - $4.900000 E-04-4.900000 E-04-4.900000 E-84$-4.920000E-04 -4.930000E-04 -4.940000E-04 -4.930000E-04 -4.920000E-04 -4.930000E-04

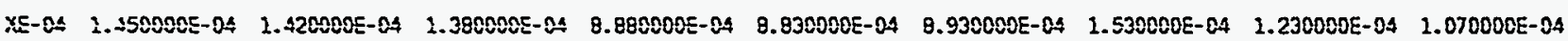
$\begin{array}{lllllllllll}X E-05 & 1.640400 E-01 & 3.640500 E-02 & 1.640300 E-02 & 1.634300 E-02 & 1.635200 E-02 & 1.633900 E-01 & 1.645300 E-01 & 1.644900 E-01 & 1.645000 E-01\end{array}$ $\begin{array}{llllllllll}X E-86 & 2.332000 E-03 & 2.332000 E-03 & 2.331000 E-03 & 2.623000 E-03 & 2.613000 E-03 & 2.611000 E-03 & 2.349000 E-03 & 2.346000 E-03 & 2.344000 E-03\end{array}$ $\begin{array}{lllllllllll}X E-07 & 1.013000 E-03 & 9.970000 E-04 & 2.021000 E-03 & 2.382000 E-03 & 1.374000 E-03 & 1.375000 E-03 & 9.310000 E-04 & 9.320000 E-04 & 9.400000 E-04\end{array}$ $X E-88-4.814000 E-03-4.814000 E-83-4.815000 E-03-4.755000 E-03-4.757000 E-03-4.756000 E-03-4.829000 E-03-4.823000 E-03-4.819000 E-03$ XEE-09 $\quad 4.747000 E-03 \quad 4.747000 E-03 \quad 4.745000 E-03 \quad 5.005000 E-03 \quad 4.998000 E-03 \quad 5.000000 E-03 \quad 4.755000 E-03 \quad 4.754000 E-03 \quad 4.752000 E-03$ $X E-10-3.170000 E-04 \quad-3.200000 E-04-3.270000 E-04 \quad 5.800000 E-85 \quad 5.808000 E-05$ 5.100000E-05 $-3.270000 E-04-3.400000 E-04-3.460000 E-04$

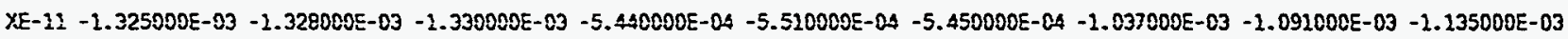
$\begin{array}{lllllllllll}X E-12 & 2.529000 E-03 & 2.528000 E-03 & 2.629000 E-03 & 2.659000 E-03 & 2.659000 E-03 & 2.657000 E-03 & 2.627000 E-03 & 2.626000 E-03 & 2.626000 E-03\end{array}$ $X E-13-3.240000 E-04 \quad-3.270000 E-04 \quad-3.500000 E-04 \quad 1.273000 E-03 \quad 1.174000 E-03 \quad 1.172000 E-03-4.000000 E-05-4.800000 E-05-9.600000 E-05$

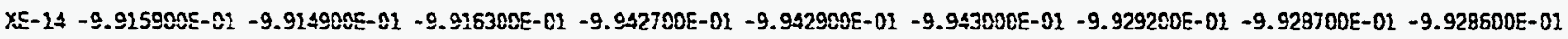
XE-15 $1.630000 E-04 \quad 1.610000 E-04 \quad 1.520000 E-04 \quad 1.066000 E-03 \quad 2.062000 E-03 \quad 1.060000 E-03 \quad 5.000000 E-04 \quad 4.830000 E-04 \quad 3.890000 E-04$

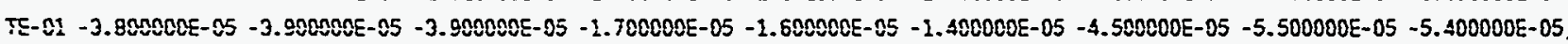
VEXT: $1.995000 E+00 \quad 1.995000 E+00 \quad 1.995000 E+00 \quad 2.995000 E+00 \quad 1.995000 E+00 \quad 1.995000 E+00 \quad 1.995000 E+00 \quad 1.995000 E+00 \quad 1.995000 E+00$ VENT $\quad 2.9918 E 0 E+00 \quad 1.991800 E+00 \quad 1.991700 E+00 \quad 1.991700 E+00 \quad 1.991800 E+00 \quad 1.991700 E+00 \quad 1.991700 E+00 \quad 1.991800 E+00 \quad 1.991700 E+00$

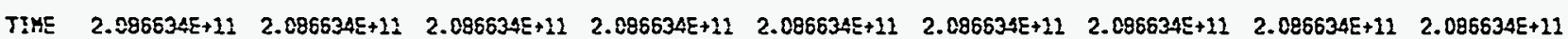


Archive Hame:

AHS_MFP_TESTS

Book Heme: AHS_MFPT2_GS

Qate \& Time: 7 hat 1900 00:05:00

Page Description:

DATA SET YO.-272

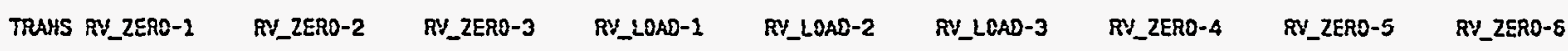

$9 E-02 \quad-7.3581005-02 \quad-7.3355005-02 \quad-7.357600 E-02 \quad 2.795500 E-02 \quad 2.830008 E-02 \quad 2.757500 E-01 \quad-7.195700 E-02 \quad-7.207700 E-02 \quad-7.212400 E-02$ PE-02 2.202200E-02 2.215900E-02 $2.212200 E-02 \quad 5.832200 E-02 \quad 6.079100 E-01 \quad 5.677000 E-01 \quad 2.320100 E-02 \quad 2.285200 E-02 \quad 2.312000 E-02$ PE-03 -3.540300E-02 -3.559600E-02 -3.579500E-02 2.857300E-01 2.994400E-02 2.808300E-01 -3.424600E-02 -3.458800E-02 -3.461000E-02 $\begin{array}{llllllllll}P E-04 & 2.700200 E-02 & 2.708000 E-02 & 2.621200 E-02 & 5.7434000 E-02 & 5.429600 E-01 & 5.232400 E-01 & 2.786900 E-02 & 2.764500 E-02 & 2.771200 E-02\end{array}$ PE-05 6.795400E-02 6.794400E-02 $6.795900 E-02 \quad 4.3314005-01 \quad 4.2095005-02 \quad 4.048100 E-01 \quad 6.870800 E-02 \quad 6.860100 E-02 \quad 6.85090 C E-02$ PE-06 5.978200E-02 5.804000E-02 5.890800E-02 4.676000E-01 4.9472005-02 5.032000E-02 5.9985005-02 $5.995600 E-02 \quad 5.990200 E-02$ PE- 07 -3.168000E-01 -3.157980E-01 -3.167900E-01 -8.394000E-03 - $1.971900 E-82-2.718500 E-02-3.167400 E-01-3.167600 E-01-3.167800 E-01$ PE-08 $\quad 4.040000 E-03 \quad 4.121000 E-03 \quad 4.4230005-03 \quad 2.326000 E-02 \quad 2.307800 E-01 \quad 2.224400 E-01 \quad 5.077000 E-03 \quad 5.225000 E-03 \quad 5.352000 E-03$ PE-09 -4.527200E-02 - 4.553100E-02 -4.586400E-02 $1.109840 E-01 \quad 1.182600 E-02 \quad 1.235500 E-01$-4.502800E-02 $-4.472200 E-02-4.457800 E-02$ PE-10 $2.400500 E-02 \quad 3.386600 E-02 \quad 2.394800 E-02 \quad 3.848300 E-01 \quad 3.622000 E-01 \quad 4.1052005-01 \quad 1.395000 E-02 \quad 1.413100 E-02 \quad 1.422800 E-02$

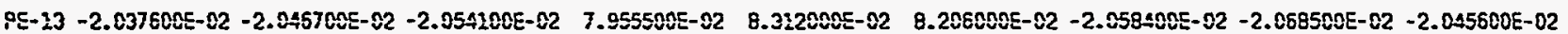
PE-14 3.217400E-01 $\quad 3.215600 E-01 \quad 3.216700 E-01 \quad 4.047700 E-01 \quad 4.042400 E-01 \quad 3.779000 E-01 \quad 1.234100 E-01 \quad 1.233200 E-01 \quad 1.231300 E-01$

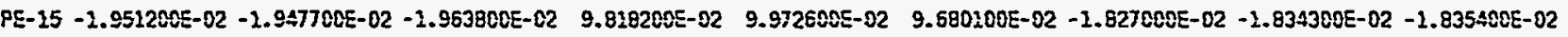
PE-16 3.592300E-02 $3.581700 E-02 \quad 3.574100 E-02 \quad 1.9780005-02 \quad 3.995500 E-02 \quad 1.936900 E-02 \quad 3.607200 E-02 \quad 3.620400 E-02 \quad 3.598100 E-02$

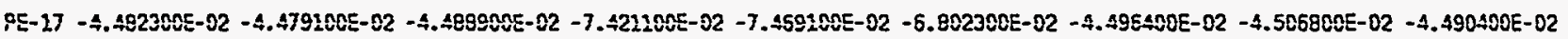

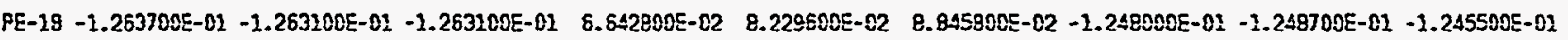
$P E-19-6.196300 E-02-6.2837005-02-6.1800005-02-2.998+005-02-4.3589005-02-5.7907005-02-6.176800 E-02-6.1923005-02-6.177100 E-02$ PE-20 -5.020400E-02 -5.030500E-02 -5.042700E-02 -2.405000E-03 - $9.142000 E-03-1.326300 E-02-4.935400 E-02-4.939300 E-02-4.934200 E-02$

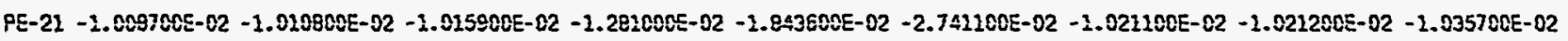
PE-22 - $1.145300 E-02-1.145300 E-02-1.245900 E-02-1.031100 E-02-1.918 E 00 E-02-9.053000 E-03-1.132600 E-02-1.124600 E-02-1.127800 E-02$

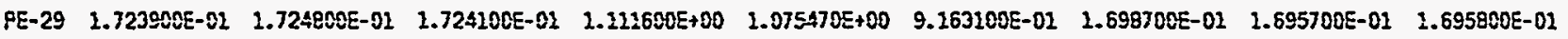

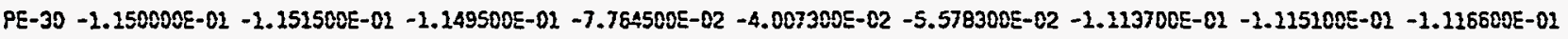

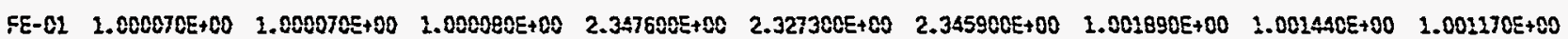
YE- $01-1.900000 E-05-1.800000 E-05-1.7000005-05-2.100000 E-05-2.100000 E-05-2.1000005-05-2.8000005-05-1.9080005-05-1.800000 E-05$ XE-02 -7.762000E-03 -7.758000E-03 -7.758000E-03 - 7.852000E-03 -7.956000E-03 -7.855000E-03 -7.835000E-03 -7.828000E-03 -7.822000E-03 XE- $03-5.060000 E-04-5.030000 E-04-5.040000 E-04-4.920000 E-04-4.8700005-04-4.830000 E-04-4.890000 E-04-4.900000 E-04-4.900000 E-04$ XE-04 $6.400000 E-85 \quad 6.300000 E-05 \quad 6.300000 E-05 \quad 2.037000 E-03 \quad 1.032000 E-03 \quad 1.035000 E-03 \quad 1.360000 E-04 \quad 1.310000 E-04 \quad 1.000000 E-04$ YEE-05 $1.557600 E-02 \quad 1.658290 E-02 \quad 1.658000 E-01 \quad 2.335190 E-02 \quad 2.215900 E-02 \quad 1.923200 E-02 \quad 2.371100 E-02 \quad 2.352900 E-02 \quad 2.291600 E-02$ XE- $06 \quad 2.323000 E-03 \quad 2.3230005-03 \quad 2.322000 E-03 \quad 2.848008 E-03 \quad 2.840008 E-03 \quad 2.835000 E-03 \quad 2.370000 E-03 \quad 2.363000 E-03 \quad 2.359000 E-03$ $\begin{array}{lllllllllll}X E-07 & 1.523000 E-03 & 2.548000 E-03 & 1.536000 E-03 & 1.439000 E-03 & 1.460000 E-03 & 1.476000 E-03 & 1.188000 E-03 & 1.204000 E-03 & 1.195000 E-03\end{array}$ YE- 08 -4.826000E-03 -4.827000E- $03-4.8260005-03-4.7880000 E-03-4.7950005-03-4.7870005-03-4.8040005-03-4.807000 E-03-4.810000 E-03$ XE-09 $\quad 4.659000 E-03 \quad 4.659000 E-03 \quad 4.659000 E-03 \quad 5.395000 E-03 \quad 5.365000 E-03 \quad 5.375000 E-03 \quad 4.763000 E-03 \quad 4.752000 E-03 \quad 4.740000 E-03$

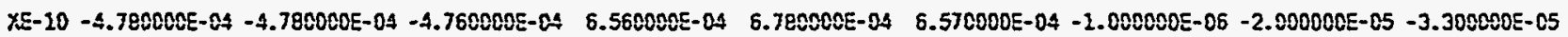
YE-12 - $1.382000 E-03-1.392000 E-03-1.381000 E-03-4.850000 E-04-4.7700005-04-4.770000 E-04-1.035000 E-03-1.202000 E-03-2.169000 E-03$ $X E-12 \quad 2.622000 E-03 \quad 2.6220005-03 \quad 2.622000 E-03 \quad 2.7540005-03 \quad 2.7530005-03 \quad 2.749000 E-03 \quad 2.519000 E-03 \quad 2.620000 E-03 \quad 2.619000 E-03$ YE-13 -4.530000E-04 -4.430000E-04 -4.770000E-04 1.232000E-03 1.244000E-03 1.236000E-03 - $1.460000 E-04 \quad-2.230000 E-04 \quad-2.700000 E-04$ $X E-14-9.907400 E-01-9.905700 E-01-9.907600 E-01 \quad-9.941400 E-01 \quad-9.941300 E-01-9.941400 E-01-9.914100 E-01-9.913300 E-01-9.912100 E-01$ YEE-25 -6.980000E- 05 -6.300000E-05 $-8.108000 E-05 \quad 1.323000 E-03 \quad 1.3200005-03 \quad 1.331000 E-03 \quad 5.950000 E-04 \quad 5.060000 E-04 \quad 3.950009 E-04$ TE- 01 - $4.000000 E-05$ - $4.000000 E-05-4.000000 E-05$ - $1.500000 E-05$-1.100000E-05 - $1.200000 E-05-4.700000 E-05-4.800000 E-05-5.100000 E-05$ VEXT1 $1.995000 E+00 \quad 1.995000 E+00 \quad 1.995000 E+C 0 \quad 1.995000 E+00 \quad 1.995000 E+00 \quad 1.995000 E+00 \quad 1.995000 E+00 \quad 1.995000 E+00 \quad 1.995000 E+00$ VEXT2 $1.991700 E+00 \quad 1.991700 E+00 \quad 1.991700 E+00 \quad 2.991800 E+00 \quad 1.991800 E+00 \quad 1.991800 E+00 \quad 1.991800 E+00 \quad 2.991800 E+00 \quad 1.991800 E+00$

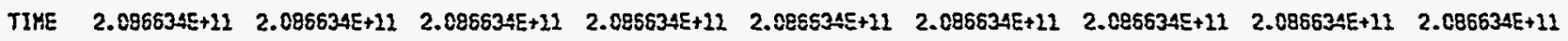


A-shive Hete:

AYS_ME:_TESTS

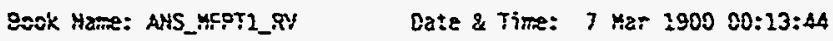

Page Description:

DATA SET Y0. -272

TRAYS RY_ZERO-2 RV_ZERO-2 RV_ZERO-3 RV_LOAD-1 FV_LOAD-2 RV_LOAD-3 RV_ZERO-4 RV_ZERO-5 RV_ZERC-6

PE- 81 -7.286800E-02 -7.314500E-02 $-7.302200 E-02 \quad 3.554700 E-01 \quad 3.464200 E-01 \quad 3.405300 E-01 \quad-7.288100 E-02 \quad-7.271800 E-02 \quad-7.272700 E-02$ $\begin{array}{llllllllll}P C-02 & 2.270300 E-02 & 2.243600 E-02 & 2.238700 E-02 & 5.971000 E-02 & 6.909100 E-01 & 6.491400 E-01 & 2.287400 E-02 & 2.397400 E-02 & 2.321400 E-02\end{array}$

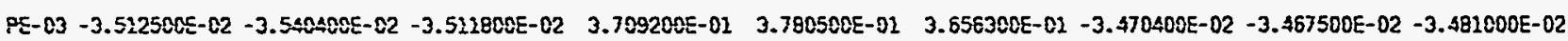
$\begin{array}{llllllllll}P E-0.4 & 2.720000 E-02 & 2.677800 E-02 & 2.702500 E-02 & 5.544900 E-01 & 6.668400 E-01 & 5.255100 E-01 & 2.768600 E-02 & 2.766600 E-02 & 2.780100 E-02\end{array}$

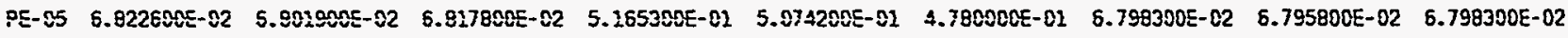
PE-05 5.922400E-02 $5.938700 E-02 \quad 5.932400 E-02 \quad 6.030600 E-02 \quad 5.979900 E-01 \quad 5.656400 E-01 \quad 5.938900 E-02 \quad 5.934200 E-02 \quad 5.932200 E-02$ $P E-07-3.176400 E-01 \quad-3.176600 E-01 \quad-3.276800 E-02 \quad 4.201300 E-02 \quad 3.794400 E-02 \quad 2.915500 E-02-3.180600 E-01 \quad-3.180900 E-01 \quad-3.191300 E-01$ $\begin{array}{llllllllll}P E-08 & 3.196000 E-03 & 3.083000 E-03 & 3.051000 E-03 & 2.693500 E-01 & 2.734100 E-01 & 2.722300 E-01 & 3.038000 E-03 & 3.086000 E-03 & 3.22 C 000 E-03\end{array}$ $P 5-89-4.539000 E-02$ - $4.4873005-02-4.548900 E-02 \quad 1.4831005-02$ 1.529700E-01 1.473000E-01 -4.522100E-02 -4.520700E-02 -4.508100E-02 PE-10 $1.361400 E-02 \quad 1.341900 E-02 \quad 1.348400 E-02 \quad 5.039600 E-01 \quad 4.354100 E-01 \quad 5.122500 E-01 \quad 1.401200 E-02 \quad 1.404900 E-02 \quad 1.394600 E-02$

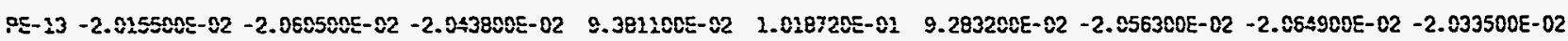
PE-14 1.217900E-01 $1.217300 E-02$ 1.217700E-02 $4.484700 E-01 \quad 4.485300 E-01 \quad 4.699900 E-01 \quad 1.225800 E-01 \quad 1.224700 E-02 \quad 1.223800 E-01$

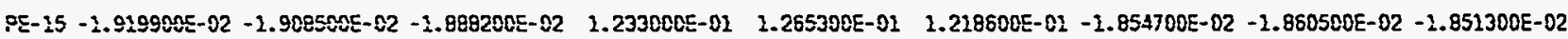
$\begin{array}{lllllllllll}P E-15 & 3.599700 E-02 & 3.604700 E-02 & 3.586400 E-02 & 2.425900 E-01 & 2.297500 E-02 & 2.293900 E-02 & 3.519400 E-02 & 3.543500 E-02 & 3.590700 E-02\end{array}$

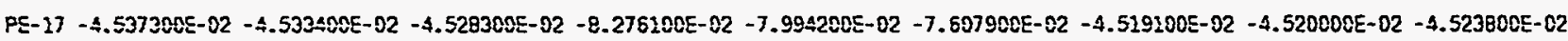

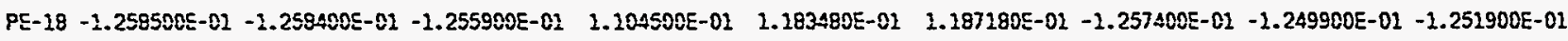
PE-19-6.173500E-02 - $-6.211500 E-02-8.169800 E-02-2.467700 E-02-4.726300 E-02-4.085000 E-02-6.205000 E-02-6.232800 E-02-6.213900 E-02$

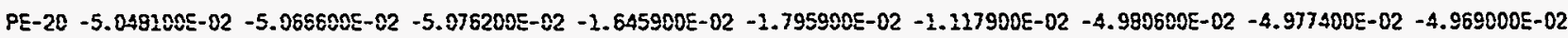
PE-21 - 9.977000E-03 - $9.8 .80000 E-03 \quad-9.9700005-03 \quad 3.644300 E-02 \quad 3.551000 E-03-1.327200 E-02-9.983000 E-03 \quad-1.017200 E-02 \quad-1.012500 E-02$ $P E-22-1.192200 E-02-1.170300 E-02-1.186200 E-02 \quad-9.845000 E-03-6.7680005-03-1.525300 E-02-1.229800 E-02-1.233700 E-02-1.211700 E-02$

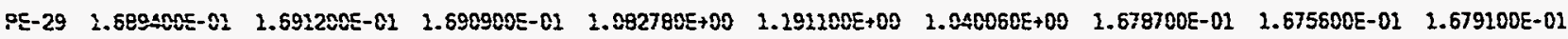
$P E-30-1.120500 E-02-1.119800 E-02-1.118900 E-02-2.953500 E-02-2.335100 E-02 \quad-5.423800 E-02 \quad-1.121200 E-01-1.119900 E-02-1.121200 E-01$ FE- $012.000290 E+00 \quad 1.000280 E+00 \quad 1.000280 E+00 \quad 2.487200 E+00 \quad 2.488300 E+00 \quad 2.486900 E+00 \quad 1.002030 E+00 \quad 1.001600 E+00 \quad 1.001310 E+00$ XE-01 -1.800000E-05 -1.800000E-05 -1.800000E-05 -2.100000E-05 -2.200000E-05 -2.000000E-05 - $1.700000 E-05-1.800000 E-05-1.800000 E-05$ XE- $82-7.7250005-83-7.7250005-83-7.724000 E-03-7.8290005-03-7.837000 E-03-7.8370005-03-7.830000 E-03-7.820000 E-03-7.812000 E-03$ $X E-03-4.950000 E-04-4.960000 E-04-4.940000 E-04-5.920000 E-04-5.800000 E-04-5.950000 E-04-6.100000 E-04-6.090000 E-04-6.090000 E-04$

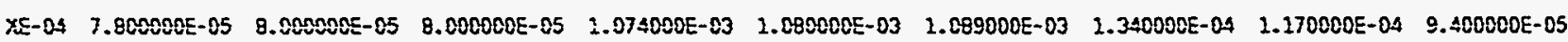
XE-05 $2.458700 E-02 \quad 2.404700 E-02 \quad 2.444700 E-02 \quad 9.580000 E-04 \quad 1.974000 E-03 \quad 1.2930005-03 \quad 5.061100 E-02 \quad 5.064300 E-02 \quad 4.977600 E-02$ $\begin{array}{llllllllll}X E-06 & 2.346000 E-03 & 2.347000 E-03 & 2.348000 E-03 & 2.889000 E-\$ 3 & 2.888000 E-03 & 2.892000 E-03 & 2.399000 E-03 & 2.389000 E-03 & 2.393000 E-03\end{array}$ $X E-07 \quad 1.513000 E-03 \quad 1.522000 E-03 \quad 1.516000 E-03 \quad 1.599000 E-03 \quad 1.613000 E-03 \quad 1.622000 E-03 \quad 1.260000 E-03 \quad 1.222000 E-03 \quad 1.217000 E-03$ $X E-08-4.815000 E-03-4.814000 E-03-4.815000 E-03-4.787000 E-03-4.781000 E-03-4.785000 E-03-4.790000 E-03-4.798000 E-03-4.796000 E-03$ XEE-09 $4.660000 E-03$ 4.661000E-03 $4.664000 E-03 \quad 5.522000 E-03 \quad 5.515000 E-03 \quad 5.532000 E-03 \quad 4.768000 E-03 \quad 4.354000 E-03 \quad 4.746000 E-03$ $X E-10-6.9000005-05-7.300000 E-05 \quad-7.200000 E-05 \quad 9.320000 E-04 \quad 8.230000 E-04 \quad 9.330000 E-04-5.800000 E-05-8.500000 E-05-1.000000 E-04$ $X E-11-1.294000 E-83-1.290000 E-03-1.291000 E-03-3.610000 E-04-3.530000 E-04-3.530000 E-04-1.0200005-03-1.102000 E-03-1.164000 E-03$ $\begin{array}{lllllllllll}X E-12 & 2.625080 E-03 & 2.52 E 000 E-03 & 2.625000 E-03 & 2.7730805-03 & 2.783000 E-03 & 2.774000 E-03 & 2.628000 E-03 & 2.628000 E-03 & 2.629000 E-03\end{array}$ $X E-13-4.460000 E-04 \quad-3.700000 E-04-3.920000 E-04 \quad 1.307000 E-03 \quad 1.306000 E-03 \quad 1.313000 E-03-1.070000 E-04 \quad-1.770000 E-04 \quad-2.300000 E-04$

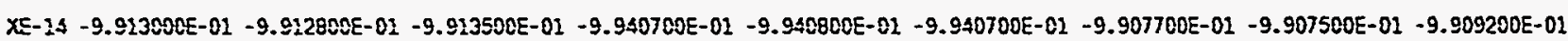
$X E-15 \quad 4.800000 E-05 \quad 4.700000 E-05 \quad 2.900000 E-05 \quad 1.346000 E-03 \quad 1.360000 E-03 \quad 1.356000 E-03 \quad 6.210000 E-04 \quad 5.100000 E-04 \quad 4.030000 E-04$ TE- $-01-5.7000005-05-5.700000 E-05-6.800000 E-05-4.500000 E-05-4.300000 E-05-4.200000 E-05-7.4 C 00005-05-8.700000 E-05-9.200000 E-05$ VEXT1 $1.995008 E+00 \quad 1.995000 E+00 \quad 1.995000 E+00 \quad 1.995000 E+00 \quad 1.995000 E+00 \quad 1.995000 E+00 \quad 1.995000 E+00 \quad 1.995000 E+00 \quad 1.995000 E+00$

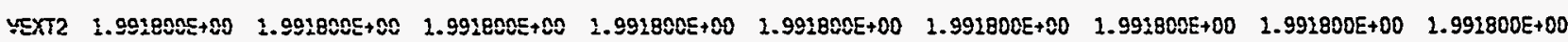

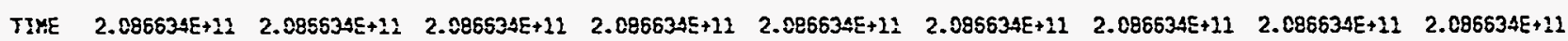


Aichitye

AHS_MFP_TESTS

Book Hame: A4S_MFPT1_RY

Date \& Time: 7 Mar 2900 00:22:29

Page Description:

DATA SET H0.-273

TRAHS RY_ZERO-1 RE_ZERO-2 $\quad$ RV_ZERO-3 RV_LOAD-1 $\quad$ RY_LOAD-2 $\quad$ RY_LOAD-3 $\quad$ RV_ZERO-4 $\quad$ RV_ZERO-5 RV_ZERO-6

PE- 02 -7.392200E-02 $-7.389100 E-02 \quad-7.377400 E-02 \quad 5.826900 E-01 \quad 5.192900 E-01 \quad 5.220400 E-01 \quad-7.348000 E-02 \quad-7.343900 E-02 \quad-7.340000 E-02$ $\begin{array}{lllllllllll}P E-02 & 2.215500 E-02 & 2.247400 E-02 & 2.213300 E-02 & 9.562900 E-01 & 9.603000 E-01 & 9.692700 E-01 & 2.278900 E-02 & 2.252700 E-02 & 2.276000 E-02\end{array}$ FE-03 -3.524E00E-02 -3.501900E-02 -3.528300E-02 5.201200E-01 $5.357200 E-02 \quad 5.383800 E-01 \quad-3.334000 E-02$-3.356000E-02 -3.367700E-02 PE-C4 2.696000E-C2 2.692400E-02 2.680400E-02 $9.1035005-01 \quad 9.360200 E-02 \quad 8.778400 E-02 \quad 2.721300 E-02 \quad 2.6982 C 0 E-02 \quad 2.711300 E-02$ $\begin{array}{llllllllll}\text { PE-0S } & 6.720600 E-02 & 6.693500 E-02 & 5.723800 E-02 & 6.620200 E-01 & 6.778700 E-01 & 6.676900 E-01 & 6.658600 E-02 & 6.655200 E-02 & 6.643000 E-02\end{array}$ PE- $06 \quad 5.798700 E-02 \quad 5.902500 E-02 \quad 5.926500 E-02 \quad 7.850900 E-02 \quad 7.596900 E-01 \quad 7.802500 E-01 \quad 5.885200 E-02 \quad 5.881000 E-02 \quad 5.875800 E-02$

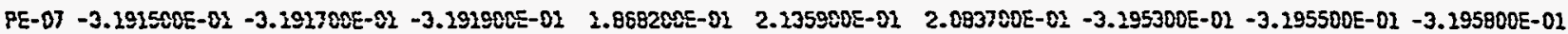
PE-C8 2.227000E-03 $2.984000 E-03 \quad 1.781000 E-03 \quad 3.685800 E-01 \quad 3.643800 E-01 \quad 3.575500 E-01 \quad 2.939000 E-03 \quad 3.133000 E-03 \quad 3.102080 E-03$ PE-09 -4.550500E- 22 -4.55390QE- 02 -4.566600E-02 2.155500E-01 2.214100E-01 2.229800E-01 -4.614100E-02 -4.603200E-02 -4.593100E-02 PE-10 1.317800E-02 $1.320100 E-02 \quad 1.285300 E-02 \quad 6.822100 E-01 \quad 6.452800 E-01 \quad 6.799900 E-01 \quad 2.407600 E-02 \quad 1.408900 E-02 \quad 1.413800 E-02$ PE- 13 -2.041800E-02 -2.047400E-02 -2.044400E-02 1.454100E-01 1.439800E-01 $1.571600 E-02-2.203100 E-02-2.090200 E-02-2.169700 E-02$

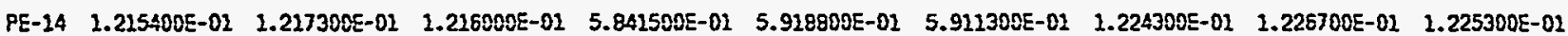

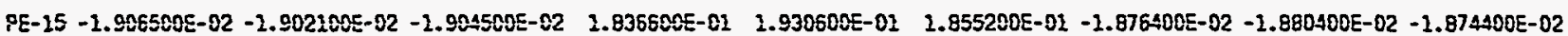
PE-16 $3.576800 E-02 \quad 3.588400 E-02 \quad 3.5673005-02 \quad 3.151 E 00 E-01 \quad 3.205300 E-01 \quad 3.167200 E-01 \quad 3.599100 E-02 \quad 3.596680 E-02 \quad 3.564600 E-02$

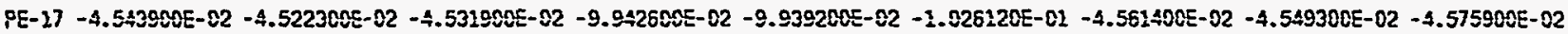

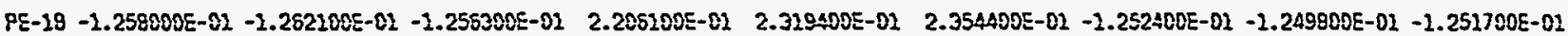
$P E-19-6.233800 E-02-6.219608 E-02-6.237900 E-02-1.445100 E-02-2.447108 E-82-2.188900 E-82-6.219500 E-02-6.233900 E-02-6.222400 E-02$

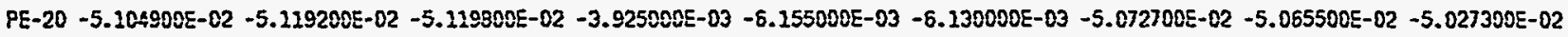
PE-21 - - $.015000 E-02-9.93 \leq 000 E-03-9.5620005-03-2.802600 E-02-1.855300 E-02-2.119500 E-02-9.936000 E-03-1.019100 E-02-1.000100 E-02$ $P E-22 \quad-1.279500 E-02-1.274000 E-02 \quad-1.269000 E-02 \quad-2.234500 E-02 \quad-2.731000 E-02-2.621500 E-02 \quad-1.317400 E-02 \quad-1.307800 E-02 \quad-1.303200 E-02$ $P E-29 \quad 1.740300 E-02 \quad 1.740280 E-02 \quad 1.745500 E-02 \quad 1.6212005+00 \quad 1.674000 E+00 \quad 1.604900 E+00 \quad 1.785900 E-01 \quad 2.791800 E-01 \quad 1.809300 E-01$

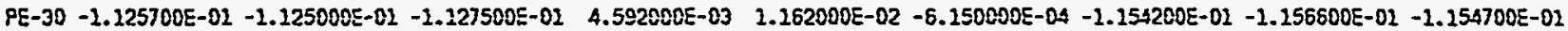
FE-01 $\quad 1.000340 E+00 \quad 2.000310 E+00 \quad 1.000320 E+00 \quad 2.772000 E+00 \quad 2.783200 E+00 \quad 2.781900 E+00 \quad 1.0028205+00 \quad 1.002080 E+00 \quad 1.001660 E+00$ XE-01 - $-2.808000 E-05-1.700000 E-05-1.800000 E-05-2.200000 E-05-2.200000 E-05-2.000000 E-05-1.900000 E-05-1.800000 E-05-1.800000 E-05$ $X E-02-7.740000 E-03-7.740030 E-03-7.738000 E-03-7.801000 E-03-7.812000 E-03-7.812000 E-03-7.837000 E-03-7.828000 E-03-7.819090 E-03$ XEE-03 -6.170000E-04 - $5.160000 E-04-6.170000 E-04-6.450000 E-04-6.380000 E-04-6.500000 E-04-5.520000 E-04-6.510000 E-04-6.510000 E-04$ YE- $04 \quad 7.280000 E-05 \quad 7.200000 E-05 \quad 7.600000 E-05 \quad 1.127000 E-03 \quad 1.208000 E-03 \quad 1.120000 E-03 \quad 1.780000 E-04 \quad 1.250000 E-04 \quad 1.020000 E-04$ $\begin{array}{llllllllll}X E-05 & 5.130900 E-02 & 5.145800 E-02 & 5.186200 E-02 & 7.812000 E-03 & 9.463000 E-03 & 1.364200 E-02 & 7.470800 E-02 & 7.625900 E-02 & 7.751800 E-02\end{array}$ $\begin{array}{llllllllll}X E-05 & 2.362000 E-03 & 2.362000 E-03 & 2.361000 E-03 & 2.946000 E-03 & 2.951000 E-03 & 2.950000 E-03 & 2.428000 E-03 & 2.405000 E-03 & 2.419000 E-03\end{array}$ $\begin{array}{llllllllll}X E-07 & 2.492000 E-03 & 1.504000 E-03 & 1.511000 E-03 & 1.555000 E-03 & 1.656000 E-03 & 1.582000 E-03 & 1.241000 E-03 & 1.285000 E-03 & 1.265000 E-03\end{array}$

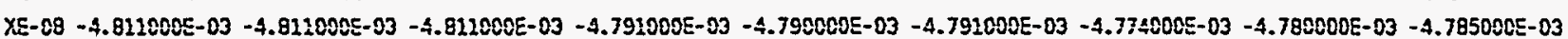
XE-09 $\quad 4.667000 E-03 \quad 4.666000 E-03 \quad 4.665000 E-03 \quad 5.7 E 4000 E-03 \quad 5.775000 E-03 \quad 5.778000 E-03 \quad 4.788000 E-03 \quad 4.774000 E-03 \quad 4.761000 E-03$ XE-10 -1.430000E-04 -1.310000E-04 -1.410000E-04 9.900000E-04 1.006000E-03 9.980000E-04 -6.100000E-05 -1.070000E-04 -1.420000E-04 $X E-11-1.250000 E-03-1.242000 E-03-1.245000 E-03-3.170800 E-04-2.990900 E-04-3.010000 E-04-9.200000 E-04-2.032000 E-03-2.085000 E-03$ $\begin{array}{lllllllllll}X E-12 & 2.631000 E-03 & 2.630000 E-03 & 2.630000 E-03 & 2.815000 E-03 & 2.833000 E-03 & 2.826000 E-03 & 2.624000 E-03 & 2.626000 E-03 & 2.624000 E-03\end{array}$ XE-13 - 4.100000E-04 -4.280000E-04 -4.270000E-04 1.319000E-03 1.319000E-03 1.316000E-03 -1.100000E-05 -8.900000E-0S -1.380000E-04 $X E-14-9.908500 E-01-9.906809 E-01 \quad-9.907100 E-01-9.939500 E-01-9.939400 E-01-9.939500 E-01-9.905100 E-01-9.902800 E-01-9.901700 E-01$ XE-15 7.080000E-05 $\quad 5.600000 E-05 \quad 5.300000 E-05 \quad 1.462000 E-03 \quad 1.474000 E-03 \quad 1.456000 E-03 \quad 6.290000 E-04 \quad 5.6500008 E-04 \quad 4.580000 E-04$ TE-01 -1.020000E-04 -1.020000E-04 -1.020000E-04 -7.700000E-05 -7.700000E-05 -7.700000E-05 -1.140000E-04 -1.110000E-04 -1.150000E-04 VEXT2 $1.995000 E+00 \quad 1.995000 E+00 \quad 1.995000 E+00 \quad 1.995000 E+00 \quad 1.995000 E+00 \quad 1.995000 E+00 \quad 1.995000 E+00 \quad 1.995000 E+00 \quad 1.995000 E+00$

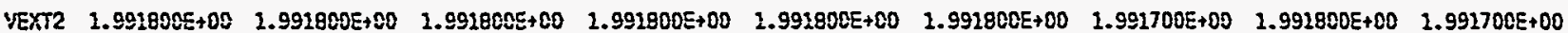
TIME 2.086634E+12 $2.086634 E+12 \quad 2.086534 E+12 \quad 2.086534 E+11 \quad 2.085634 E+11 \quad 2.085634 E+12 \quad 2.085634 E+112.086634 E+12 \quad 2.086634 E+12$ 


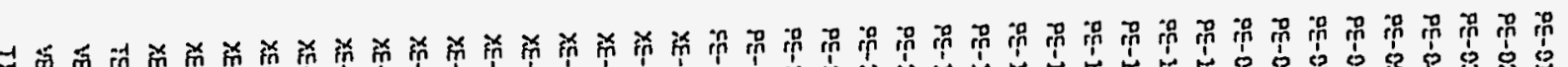

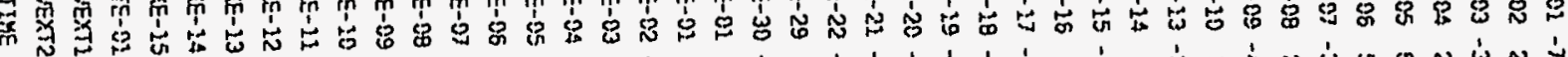

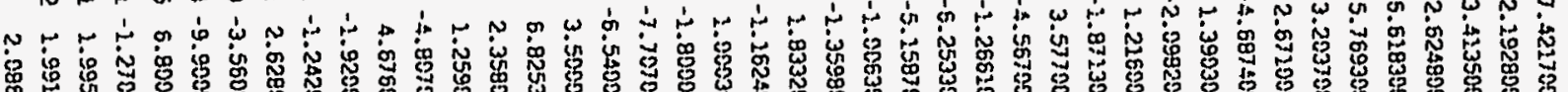

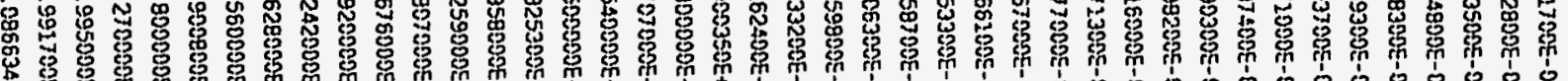

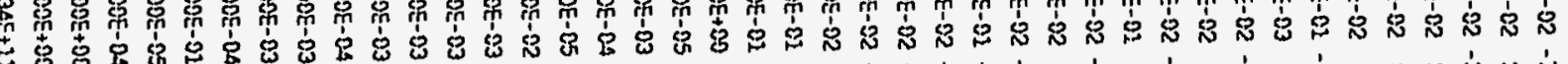
等

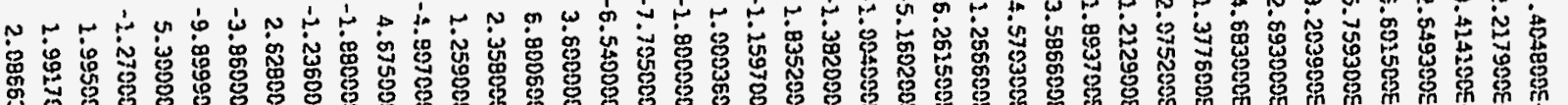

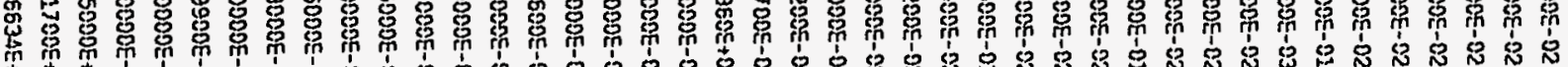

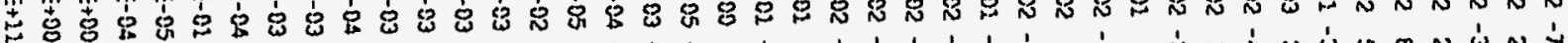

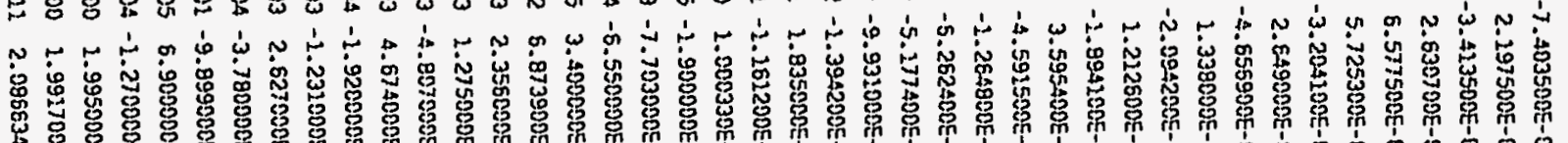

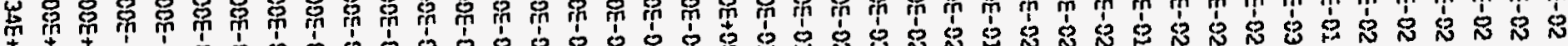

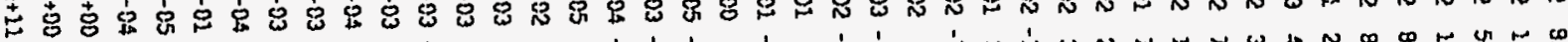

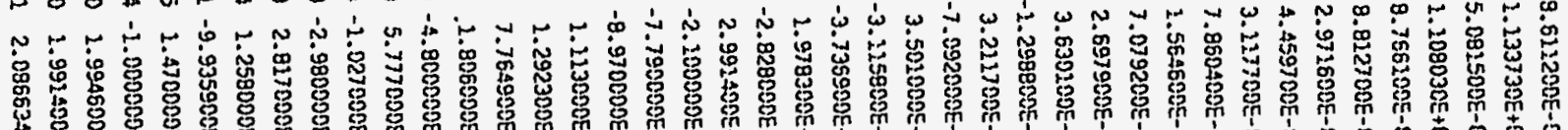

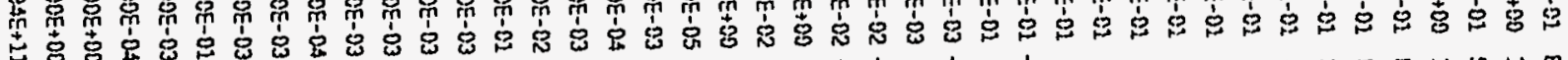

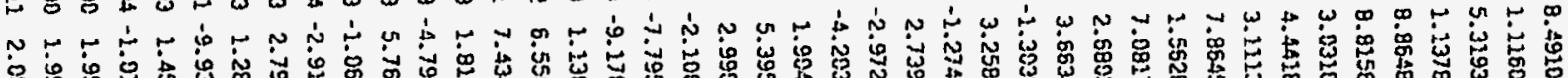

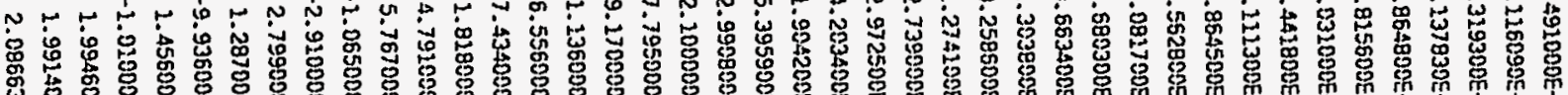

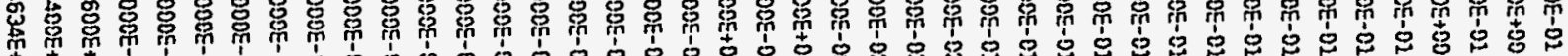

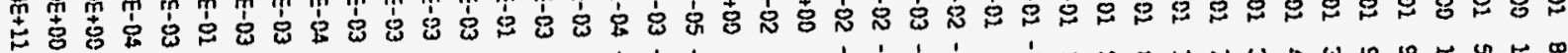

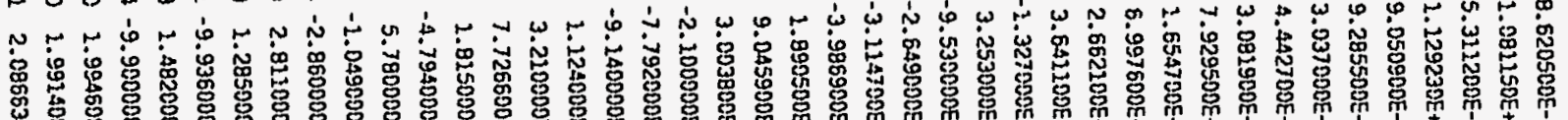

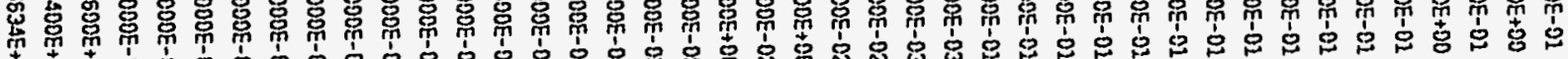

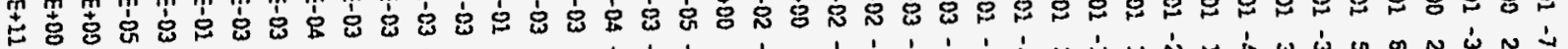

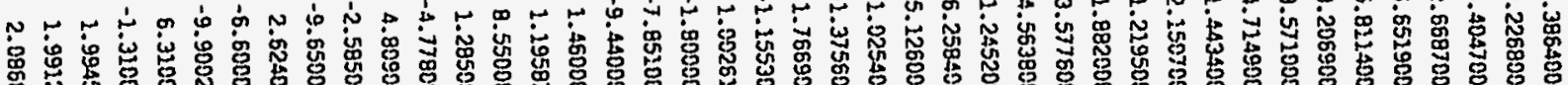

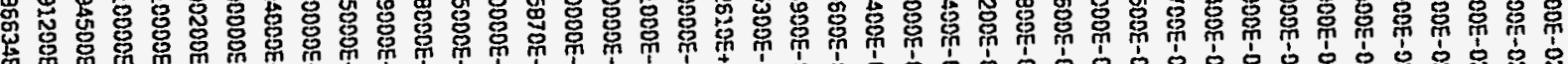

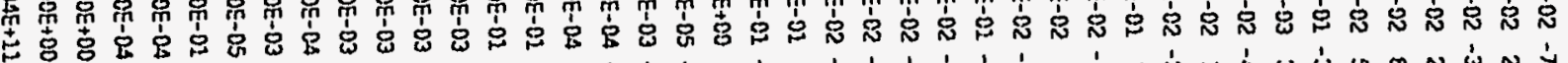
N

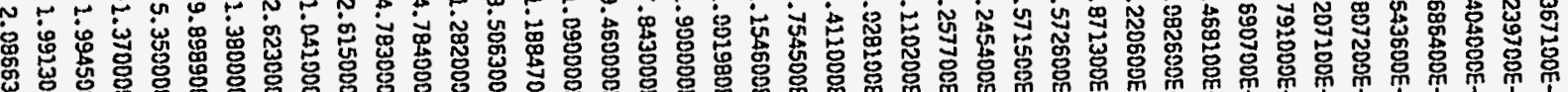

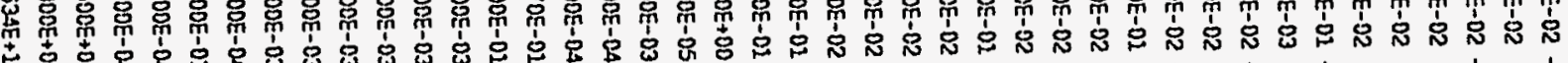
租

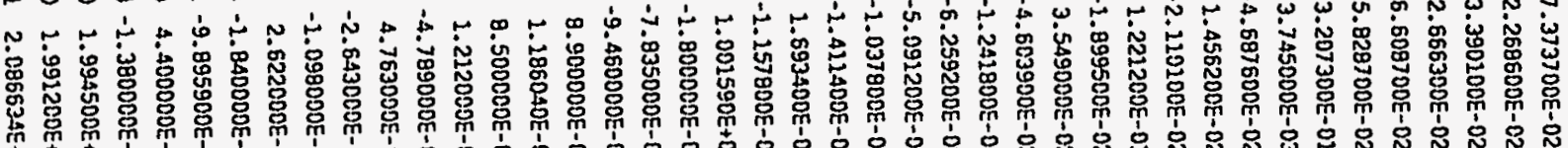


Archive Hzane:

ANS_MFP_IESTS

Beok Hame: AHS_MFPTI_RV

Cate \& Time: 7 Mar 1900 00:39:21

Page Description:

DATA SET $40 .-275$

TRAYS RV_ZERO-2 RV_ZERO-2 RY_ZERO-3 RY_LOAD-1 $\quad$ RY_LOAD-2 $\quad$ RY_LOAD-3 $\quad$ RY_ZERO-4 $\quad$ RN_ZERO-5 RY_ZERO-6

PE- $01 \quad-7.470800 E-02 \quad-7.471100 E-02 \quad-7.467900 E-02 \quad 1.095230 E+00 \quad 1.046210 E+00 \quad 1.022390 E+00-7.423500 E-02 \quad-7.429800 E-02 \quad-7.417900 E-02$ $\begin{array}{lllllllllll}P E-02 & 2.202500 E-02 & 2.191900 E-02 & 2.186500 E-02 & 1.352200 E+00 & 1.346700 E+00 & 1.364300 E+00 & 2.275900 E-02 & 2.273300 E-02 & 2.295900 E-02\end{array}$ PE-03 -3.4E668CE-02 -3. A756COE-02 -3.467000E-02 8.320000E-01 8.706200E-01 8.882400E-02 -3.339400E-02 -3.349800E-02 -3.340700E-02

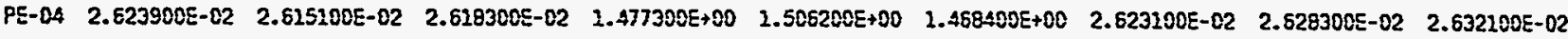
PE-C5 $\quad 6.512600 E-02 \quad 6.559500 E-02 \quad 6.498500 E-02 \quad 1.156300 E+00 \quad 1.164500 E+00 \quad 1.148300 E+00 \quad 6.541200 E-02 \quad 5.550300 E-02 \quad 6.516000 E-02$ $\begin{array}{lllllllllll}P E-05 & 5.737600 E-82 & 5.725900 E-02 & 5.726700 E-02 & 1.077730 E+00 & 1.043990 E+00 & 1.076500 E+00 & 5.772900 E-02 & 5.788500 E-02 & 5.786700 E-02\end{array}$ PE- 07 -3.214300E-01 $-3.224500 E-01 \quad-3.214700 E-01 \quad 5.275100 E-02 \quad 5.762300 E-01 \quad 5.443300 E-01 \quad-3.217200 E-01 \quad-3.217200 E-01 \quad-3.217400 E-01$ $\begin{array}{lllllllllll}P E-D O & 3.262000 E-03 & 3.246000 E-03 & 3.330000 E-03 & 5.352500 E-01 & 5.395400 E-02 & 5.390908 E-01 & 2.585000 E-03 & 2.428000 E-03 & 2.515080 E-03\end{array}$

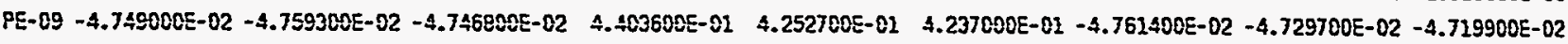
$\begin{array}{llllllllll}P E-10 & 1.352200 E-02 & 1.365700 E-02 & 1.339400 E-02 & 9.483400 E-02 & 9.524900 E-01 & 9.890000 E-01 & 1.4007005-02 & 1.426300 E-02 & 1.416800 E-02\end{array}$ PE- 13 -2.209100E-02 -2.136400E-02 -2.203500E-02 2.495508E-01 2.556280E-01 2.537900E-01 -2.122400E-02 $-2.127400 E-02$-2.233800E-02

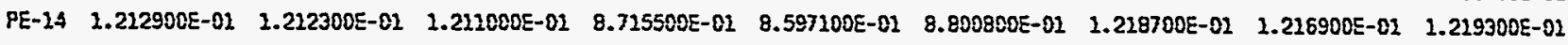
PE-15 - $1.9020005-02$ - $1.917300 E-02$ - $1.944200 E-02 \quad 3.7434005-01 \quad 3.710500 E-02$ 3.734500E-02 $-1.918500 E-02$-1.9288C0E-02 -1.912300E-02 PE-16 3.498400E-02 3.528000E-02 3.541500E-02 $4.2904095-02 \quad 4.315900 E-01 \quad 4.303500 E-02 \quad 3.500700 E-02 \quad 3.504500 E-02 \quad 3.524000 E-02$

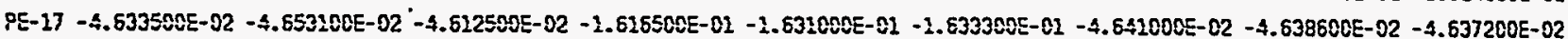

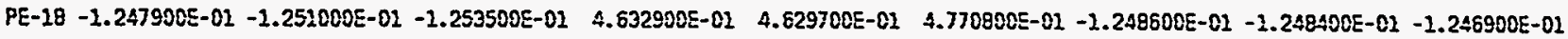
PE-19 -6.289400E-02 -6.262900E-02 -6.284600E-02 -5.3790005-03 -5.368000E-03 -4.499800E-03 -6.292200E-02 -6.297200E-02 -6.259900E-02 $P E-20 \quad-5.209200 E-02 \quad-5.167000 E-02 \quad-5.211700 E-02 \quad 2.262800 E-02 \quad 2.567300 E-02 \quad 1.251000 E-02 \quad-5.172900 E-02 \quad-5.245800 E-02 \quad-5.140900 E-02$ PE-21 - $1.033600 E-02-1.023700 E-02-1.039200 E-02-3.7403005-02-3.369900 E-02-3.726300 E-02-1.023600 E-02-1.007200 E-02-1.022600 E-02$ PE-22 - - $.461000 E-02-1.474300 E-02-1.447700 E-02-5.230200 E-02-5.517000 E-02-5.619000 E-02-1.474400 E-02-2.465400 E-02-1.479600 E-02$

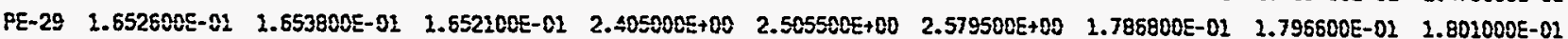
PE-30 -1.164000E-01 $-2.163900 E-02$-1.167200E-01 $9.315100 E-02 \quad 5.585500 E-02 \quad 8.110300 E-02-1.159100 E-02$ - $1.158400 E-01$ - $1.158200 E-01$

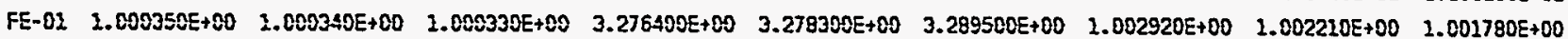
XE-02 -1.800000E-05 - 2.800000E-05 -1.800000E-05 -2.200000E-05 -2.100000E-05 -2.100000E-05 -1.800000E-05 -1.900000E-05 -1.800000E-05 XE-02 -7.728008E-03 -7.723000E- 23 -7.722000E-03 -7.735000E-03 -7.7\$2000E-03 -7.771000E-03 -7.856000E-03 -7.850000E-03 -7.843000E-03 XE-03 - $9.550000 E-04-9.550000 E-04-9.550000 E-04-9.870000 E-04-9.880000 E-04-9.900000 E-04-1.000000 E-03-9.990000 E-04-1.000000 E-03$

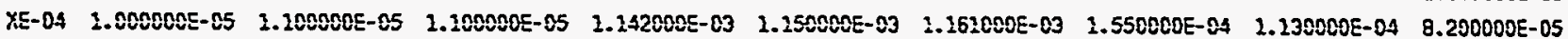

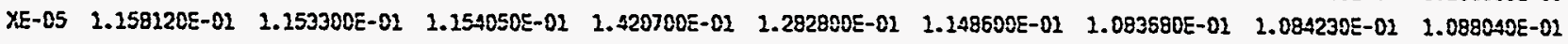

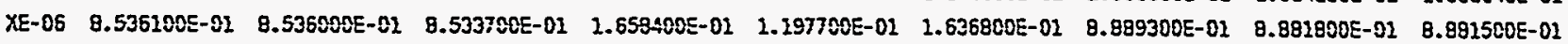
XE-07 $1.149000 E-03 \quad 1.144000 E-03 \quad 1.152000 E-03 \quad 1.870000 E-03 \quad 1.889000 E-03 \quad 1.899000 E-03 \quad 1.528000 E-03 \quad 1.445000 E-03 \quad 1.394000 E-03$ $X E-08-4.812000 E-03$ - $4.813000 E-03-4.813000 E-03-4.7840005-03-4.792000 E-03-4.798000 E-03-4.778000 E-03-4.780000 E-03-4.783000 E-03$ YE- $09 \quad 4.667000 E-03 \quad 4.667000 E-03 \quad 4.665000 E-03 \quad 5.758000 E-03 \quad 5.812000 E-03 \quad 5.802000 E-03 \quad 4.793000 E-03 \quad 4.776000 E-03 \quad 4.764000 E-03$ XE-10 -2.7240005-03 -2.722000E-03 -2.720000E-03 5.650000E-04 6.850000E-04 7.590000E-04 -2.577000E-03 $-2.627000 E-03 \quad-2.656000 E-03$ XEE-11 - -2.263000E-03 -1.264000E-03 - -2.259000E-03 -2.590000E-04 -2.560000E-04 -2.460000E-04 -9.500000E-04 $-1.035000 E-03-1.072000 E-03$ $\begin{array}{lllllllllll}X E-12 & 2.622000 E-03 & 2.622000 E-03 & 2.623000 E-03 & 2.793000 E-03 & 2.807000 E-03 & 2.797000 E-03 & 2.625000 E-03 & 2.623000 E-03 & 2.623000 E-03\end{array}$ XE-13 -3.520000E-04 $-4.000000 E-04 \quad-4.080000 E-04 \quad 1.254000 E-03 \quad 3.279000 E-03 \quad 1.272000 E-03 \quad-4.200000 E-05$ - $1.020000 E-04$ - $1.260000 E-04$ YE- $14-9.895300 E-01 \quad-9.893900 E-01-9.897300 E-01 \quad-9.936900 E-01 \quad-9.937700 E-01-9.937900 E-01-9.904000 E-02-9.901300 E-01-9.900700 E-01$ $\begin{array}{llllllllll}X E-15 & 2.900000 E-05 & 2.500000 E-05 & 1.800000 E-05 & 1.561000 E-03 & 1.578000 E-03 & 1.577000 E-03 & 6.530000 E-04 & 5.610000 E-04 & 4.470000 E-04\end{array}$ TE-01 - $1.4600005-04-1.460000 E-04-1.450000 E-04-2.270000 E-04-1.1400005-04-1.130000 E-04-1.390000 E-04-1.470000 E-04$ - $1.530000 E-04$ $\begin{array}{lllllllllll}\text { VEXT1 } & 1.994500 E+00 & 1.994500 E+00 & 1.994500 E+00 & 1.994900 E+00 & 2.994900 E+00 & 1.994800 E+00 & 1.994400 E+00 & 1.994900 E+00 & 1.994400 E+C 0\end{array}$ $\begin{array}{llllllllll}\text { YEXT2 } & 1.991300 E+00 & 1.991200 E+00 & 1.991200 E+\infty 0 & 1.991600 E+00 & 1.991608 E+00 & 1.991600 E+00 & 1.991200 E+00 & 1.991200 E+00 & 1.991200 E+00\end{array}$

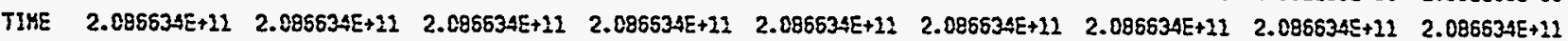


Qook Hawe: AHS_MFPT1_R! Sate \& Time: 7 Hat 1900 00:47:22

Page Rescription:

DATA SET NO. -276

TRAHS RY_ZERO-1 RY_ZERO-2 RV_ZERO-3 RV_LOAD-1 RY_LOAD-2 RV_LOAD-3 RV_ZERO-4 RV_2ERD-5 RV_ZERD-6

:CE- $02-7.5001005-02 \quad-7.495500 E-02 \quad-7.517800 E-02 \quad 1.305280 E+00 \quad 1.303500 E+00 \quad 1.296900 E+00 \quad-7.455200 E-02 \quad-7.449000 E-02 \quad-7.448900 E-02$ $\begin{array}{llllllllll}P E-02 & 2.211400 E-02 & 2.229100 E-02 & 2.195700 E-02 & 2.346200 E+00 & 2.423300 E+00 & 2.419300 E+00 & 2.258500 E-02 & 2.251200 E-02 & 2.233400 E-02\end{array}$

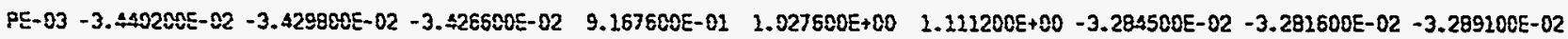
$\begin{array}{llllllllll}P E-04 & 2.544000 E-02 & 2.579800 E-02 & 2.552600 E-02 & 2.052300 E+00 & 2.024500 E+00 & 2.058400 E+00 & 2.552900 E-02 & 2.547500 E-02 & 2.524900 E-02\end{array}$ $\begin{array}{llllllllll}95-05 & 6.396203 E-02 & 6.5033005-02 & 6.513300 E-02 & 1.554500 E+00 & 1.565900 E+00 & 2.591500 E+00 & 6.538800 E-02 & 6.506300 E-02 & 6.509200 E-02\end{array}$ PE-06 $5.550300 E-02 \quad 5.556700 E-02 \quad 5.653700 E-02 \quad 1.085430 E+00 \quad 1.078040 E+00 \quad 1.060540 E+00 \quad 5.709000 E-02 \quad 5.713500 E-02 \quad 5.757100 E-02$

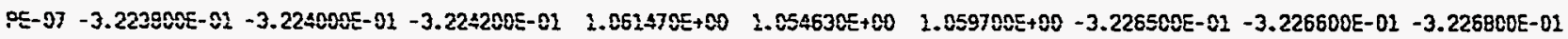
$\begin{array}{lllllllllll}P E-28 & 2.110000 E-03 & 1.9110005-03 & 2.053000 E-03 & 7.145400 E-02 & 7.056600 E-02 & 6.988000 E-01 & 2.363000 E-03 & 2.492000 E-03 & 2.572000 E-03\end{array}$ $85-09-4.786 B 005-02-4.8017005-02$ - $4.801300 E-02$ 8.261200E-01 8.229500E-01 7.954900E-01 -4.734200E-02 -4.738100E-02 - $4.711900 E-02$ PE-10 $1.366600 E-02 \quad 1.370200 E-02 \quad 3.343700 E-02 \quad 9.877100 E-01 \quad 9.590300 E-01 \quad 9.425400 E-01 \quad 1.395200 E-02 \quad 1.413500 E-02 \quad 1.404400 E-02$

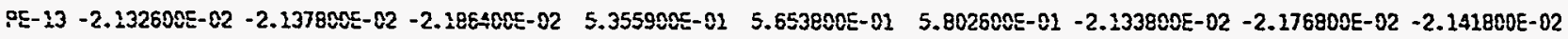
$\begin{array}{llllllllll}P E-14 & 1.212600 E-02 & 1.212200 E-02 & 1.210200 E-01 & 1.275320 E+00 & 1.171580 E+00 & 1.258400 E+00 & 1.215800 E-02 & 1.218100 E-01 & 1.220000 E-01\end{array}$

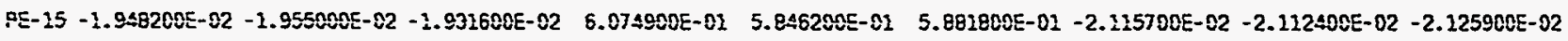
PE-15 3.519308E-02 3.507100E-02 3.503800E-02 3.463700E-01 3.356700E-02 3.287900E-01 3.5320005-02 $3.516700 E-02 \quad 3.539500 E-02$

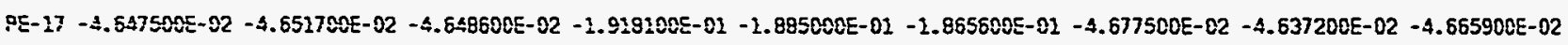

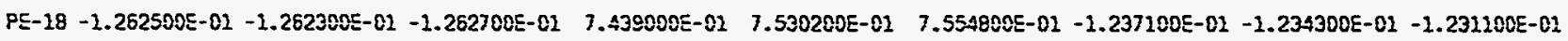

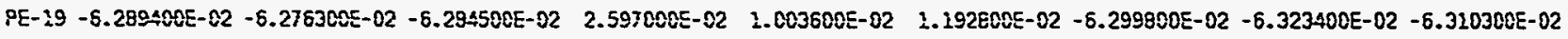

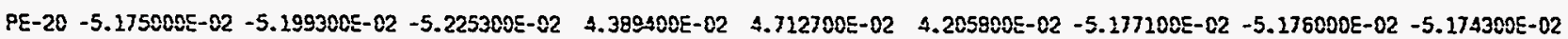

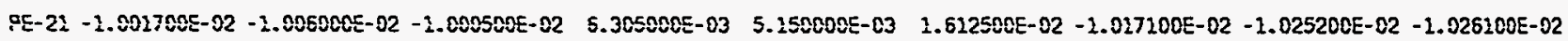

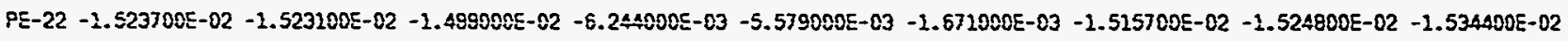

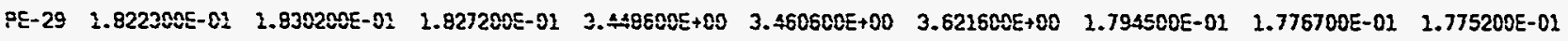

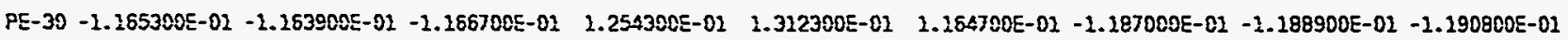

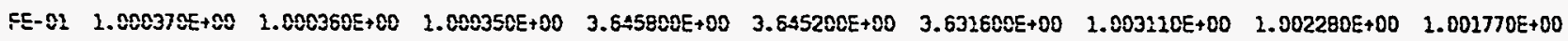
XE-02 -1.800000E- $05-1.800000 E-85-1.800000 E-05-2.100000 E-05-2.100000 E-05-2.000000 E-05-2.900000 E-05-1.900000 E-05-1.800000 E-05$ $X E-02-7.7780005-03-7.773000 E-83-7.770000 E-03-7.702000 E-83-7.5968005-03-7.704000 E-03-7.923008 E-03-7.905000 E-03-7.897000 E-03$ XE-03 - $1.007000 E-83-1.007000 E-03-1.008000 E-03-1.007000 E-83-1.005000 E-03-1.006000 E-03-1.009000 E-03-1.008000 E-03-1.009000 E-03$

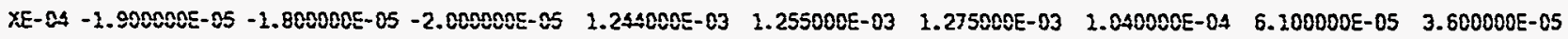
$X E-05 \quad 2.099600 E-01 \quad 1.098960 E-01 \quad 1.203620 E-01 \quad 5.493600 E-01 \quad 5.557900 E-02 \quad 5.457400 E-01 \quad 8.654500 E-02 \quad 8.654200 E-02 \quad 8.453600 E-02$ $\begin{array}{lllllllllll}X E-06 & 8.8640005-01 & 8.863400 E-01 & 9.8631005-01 & 1.510000 E-03 & 1.485008 E-03 & 1.457000 E-03 & 1.845000 E-03 & 1.827000 E-03 & 1.811000 E-03\end{array}$ $\begin{array}{lllllllllll}X E-07 & 1.121000 E-03 & 1.118000 E-03 & 1.123000 E-03 & 1.932000 E-03 & 1.939000 E-03 & 1.956000 E-03 & 1.585000 E-03 & 1.571000 E-03 & 1.539000 E-03\end{array}$ XE- $09-4.821000 E-83-4.012000 E-83-4.8120005-03$ - $4.560000 E-83-4.543000 E-03$ - $4.526000 E-03-4.777000 E-03-4.783000 E-03-4.786000 E-03$ XE-09 $\quad 4.675000 E-03 \quad 4.675000 E-03 \quad 4.672000 E-03 \quad 4.252000 E-03 \quad 4.220000 E-03 \quad 4.191000 E-03 \quad 4.122000 E-03 \quad 4.091000 E-03 \quad 4.078000 E-03$ $X E-10 \quad-2.760080 E-03 \quad-2.759090 E-03 \quad-2.766000 E-03 \quad 3.630000 E-04 \quad 3.540000 E-04 \quad 3.750000 E-04 \quad 5.500000 E-05 \quad 2.000000 E-06 \quad-3.100000 E-05$ $X E-11-1.218000 E-03-1.219000 E-03-1.223000 E-03-5.300000 E-05-5.200000 E-05-2.2000005-05-9.360000 E-04-1.032000 E-03-1.078000 E-03$ $\begin{array}{lllllllllll}X E-12 & 2.6250005-03 & 2.625000 E-03 & 2.625000 E-03 & 2.297000 E-03 & 2.273000 E-03 & 2.256000 E-03 & 2.611000 E-03 & 2.620000 E-03 & 2.620000 E-03\end{array}$ $X E-13-4.070000 E-04-3.920000 E-84 \quad-3.710000 E-04 \quad 1.250000 E-03 \quad 1.242000 E-03 \quad 1.278000 E-03-7.600000 E-05 \quad-1.360000 E-04 \quad-1.930000 E-04$ XE-14 -9.889600E-01 -9.990400E-01 $-9.890500 E-01-9.935200 E-01-9.935300 E-01 \quad-9.935400 E-01-9.899800 E-01-9.896300 E-01-9.997600 E-01$ XE-15 2.800000E-05 2.800000E-05 $3.700000 E-05 \quad 1.6770005-03 \quad 1.6390005-03 \quad 1.658000 E-03 \quad 7.970000 E-04 \quad 5.930000 E-04 \quad 4.420000 E-04$ TE- $01-1.620000 E-04-1.6200005-04-1.6100005-04-1.330000 E-04-1.2900005-04-1.3200805-04-1.620000 E-04-1.620000 E-04-1.660000 E-04$ $\begin{array}{lllllllllll}\text { VEXT2 } & 1.994500 E+00 & 1.994400 E+00 & 1.994400 E+00 & 1.994700 E+00 & 1.994700 E+00 & 1.994700 E+00 & 1.994900 E+00 & 1.995000 E+00 & 1.995000 E+00\end{array}$ $\begin{array}{llllllllll}\text { VEXTZ } & 2.991200 E+00 & 1.991200 E+00 & 1.991200 E+00 & 1.991500 E+00 & 1.991500 E+00 & 1.991500 E+00 & 1.991700 E+00 & 1.991700 E+00 & 1.991700 E+00\end{array}$

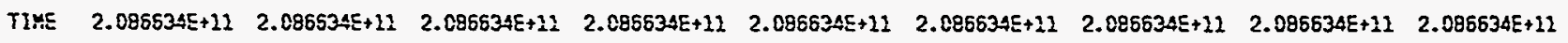


$: \cdots$

- - Page Deseription:

QATA SET MO.-237

TRANS RY_ZERO-2 RV_ZERO-2 RY_ZERO-3 RY_LOAD-1 $\quad$ RY_LOAD-2 $\quad$ RV_LEAD-3 $\quad$ RV_ZERO-4 $\quad$ RV_ZERO-5 RY_ZERO-6

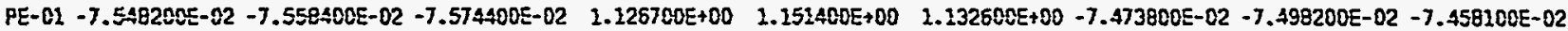
$\begin{array}{llllllllll}P E-02 & 2.159400 E-02 & 2.165200 E-02 & 2.158000 E-02 & 2.388500 E+00 & 2.390000 E+00 & 2.407300 E+00 & 2.195300 E-02 & 2.204000 E-02 & 2.205700 E-02\end{array}$ PE- 03 -3.407700E-02 -3.420900E-02 $-3.415500 E-02 \quad 1.356600 E+00 \quad 1.374900 E+00 \quad 1.365000 E+00-3.262400 E-02 \quad-3.256700 E-02 \quad-3.235400 E-02$ PE-C4 2.526500E-C2 2.500800E-02 2.503300E-C2 2.050300E+00 2.086800E+00 2. $263500 E+00 \quad 2.543600 E-02 \quad 2.524400 E-02 \quad 2.577000 E-02$ PE-C5 6.458400E-02 $6.443600 E-02 \quad 6.457100 E-02 \quad 1.658680 E+00 \quad 1.647003 E+00 \quad 1.648400 E+00 \quad 6.537100 E-02 \quad 6.534000 E-02 \quad 6.527000 E-02$ PE-06 $5.605700 E-02 \quad 5.546600 E-02 \quad 5.541700 E-02 \quad 1.335900 E+00 \quad 1.358500 E+00 \quad 1.360400 E+00 \quad 5.675700 E-02 \quad 5.665000 E-02 \quad 5.865200 E-02$

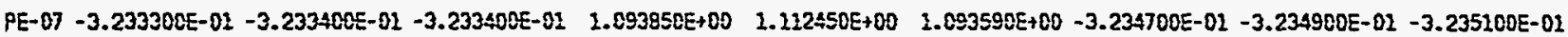
PE-09 1.941000E-03 2.641000E-03 $1.919000 E-03 \quad 7.148700 E-01 \quad 6.990900 E-02 \quad 7.012500 E-01$-9.770000E-04 $-1.022000 E-03$ - $1.019000 E-03$ PE- 09 - $4.799000 E-02$ - $4.793200 E-02$ - $4.911500 E-02$ 9.394200E-01 $8.059000 E-01$ 8.129600E-01 -4.760500E-02 -4.769300E-02 -4.755600E-02 $P E-10 \quad 2.274400 E-02 \quad 1.267100 E-02 \quad 1.274800 E-02 \quad 1.225400 E+00 \quad 1.247700 E+00 \quad 1.251100 E+00 \quad 1.369600 E-02 \quad 1.374600 E-02 \quad 1.389100 E-02$ PE-13 -2.210100E-02 -2.122000E-02 -2.115600E-02 5.389100E-01 $5.381100 E-02 \quad 5.373300 E-01 \quad-2.182300 E-02-2.299800 E-02$-2.191500E-02 PE-14 1.204800E-01 $2.204000 E-01 \quad 2.203700 E-01 \quad 1.191830 E+00 \quad 1.197180 E+00 \quad 2.2712805+00 \quad 1.214400 E-01 \quad 1.214100 E-01 \quad 1.214600 E-01$

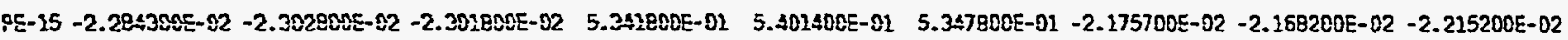
PE-15 3.457500E-02 3.456700E-02 $3.493100 E-02 \quad 4.845700 E-02 \quad 5.005100 E-02 \quad 5.082600 E-02 \quad 3.436600 E-02 \quad 3.406200 E-02 \quad 3.401900 E-02$

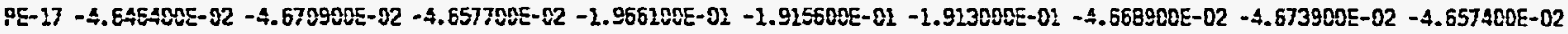

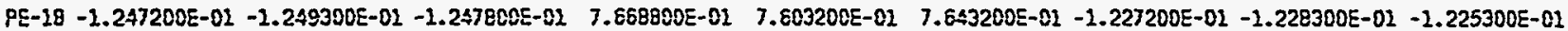
PE-19 - $5.295000 E-02-E .347900 E-02-6.344900 E-02 \quad 2.588200 E-02 \quad 1.768 C 00 E-02 \quad 2.454200 E-02-6.309200 E-02-5.331000 E-02-6.325500 E-02$ PE-20 -5.292700E-02 -5.286300E-02 -5.313500E-02 4. E27300E-02 5.023500E-02 4.913600E-02 -5.242500E-02 $-5.254600 E-02 \quad-5.180000 E-02$ PE-21 - 1.0487CEE-02 - 2.019100E-02 - $2.015500 E-02-4.398100 E-02-4.395900 E-02-6.000800 E-02-1.0178005-02-1.026400 E-02-1.029100 E-02$ PE-22 - 2.5760005-02 -1.560500E-02 -1.565300E-02 -7.562700E-02 -7.256700E-02 -8.096100E-02 -1.5750005-02 -1.587100E-02 -1.591700E-02

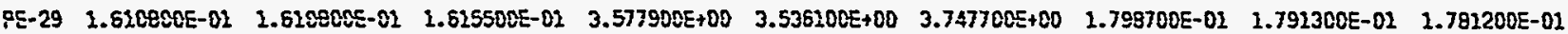

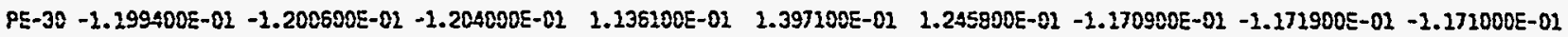
FE-01 $1.000240 E+C 0 \quad 1.000140 E+00 \quad 1.000120 E+00 \quad 3.743900 E+C 0 \quad 3.745900 E+C 0 \quad 3.759200 E+00 \quad 1.003450 E+00 \quad 1.002640 E+00 \quad 1.002130 E+00$ XE-02 - $2.800000 E-05-2.900000 E-05-1.900000 E-05-2.100000 E-05-2.200000 E-05-2.100000 E-05-1.900000 E-05-1.900000 E-05-1.800000 E-05$ $X E-82-7.797900 E-03-7.783000 E-03-7.378000 E-03-7.781000 E-03-7.7910005-03-7.793000 E-03-7.904000 E-03-7.901000 E-03-7.895000 E-03$ XE-03 - $1.024000 E-03-1.014000 E-03-1.023000 E-03-9.950000 E-04-9.950000 E-04-9.960000 E-04-1.000000 E-03-1.000000 E-03-1.001000 E-03$ XE-04 4. $400000 E-06$ 7.8000COE-06 $\quad 6.000000 E-06$ ' $1.321000 E-03 \quad 1.358 C 00 E-03 \quad 1.395000 E-03 \quad 1.180000 E-04 \quad 8.000000 E-05 \quad 6.000000 E-05$ XE-05 $\quad 6.356500 E-02 \quad 6.320300 E-02 \quad 6.335500 E-02 \quad 3.077700 E-02 \quad 3.837200 E-01 \quad 3.385700 E-02 \quad 4.837300 E-02 \quad 5.035500 E-02 \quad 5.124200 E-02$ $\begin{array}{lllllllllll}Y E-06 & 1.743000 E-03 & 1.742000 E-03 & 1.741000 E-03 & 1.3750005-83 & 2.835000 E-03 & 1.868000 E-03 & 4.795700 E-01 & 4.157600 E-01 & 4.159800 E-01\end{array}$ XE-07 $\quad 1.263000 E-03 \quad 1.272000 E-03 \quad 1.257000 E-03 \quad 1.995000 E-03 \quad 2.033000 E-03 \quad 2.055000 E-03 \quad 2.4560005-03 \quad 1.401000 E-03 \quad 1.332000 E-03$ XE-08 -4.815000E-03 -4.8150C0E-03 -4.815000E-03 -4.734000E-03 - $4.740800 E-03-4.756000 E-03-4.758000 E-03-4.776000 E-03-4.781000 E-03$ XE-09 3.991000E-03 $3.990000 E-03 \quad 3.991000 E-03 \quad 4.810000 E-03 \quad 4.852000 E-03 \quad 4.883000 E-83 \quad 4.132000 E-03 \quad 4.117000 E-03 \quad 4.102000 E-03$ XE-10 -5.600000E-05 -5.600000E-05 -5.300000E-05 1.050800E-03 9.010020E-04 1.092000E-03 -1.154000E-03 - $1.051000 E-03-1.084000 E-03$ $X E-12-1.211000 E-03-1.210000 E-03-1.205000 E-03-5.800000 E-05-5.000000 E-05-3.900000 E-05-9.220000 E-04-9.950000 E-04-1.050000 E-03$ $\begin{array}{lllllllllll}X E-12 & 2.612000 E-03 & 2.612000 E-03 & 2.512000 E-03 & 2.663000 E-03 & 2.663000 E-03 & 2.669000 E-03 & 2.623000 E-03 & 2.620000 E-03 & 2.620000 E-03\end{array}$ XE-13 $-4.390000 E-04 \quad-4.080000 E-04 \quad-4.040000 E-04 \quad 1.271000 E-03 \quad 1.283000 E-03 \quad 1.325000 E-03 \quad-5.200000 E-05 \quad-1.220000 E-04 \quad-1.870000 E-04$

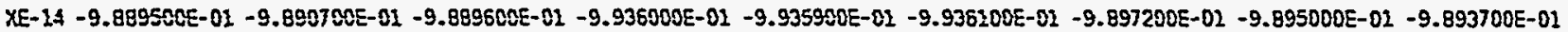
XE-15 $\quad 9.300000 E-05 \quad 9.400000 E-05 \quad 8.600000 E-05 \quad 1.839000 E-03 \quad 1.839000 E-03 \quad 1.816000 E-03 \quad 8.220000 E-04 \quad 6.570000 E-04 \quad 5.090000 E-04$ TE- 02 - $1.730000 E-04-1.730000 E-04-1.720000 E-04-1.310000 E-04-1.330000 E-04-1.290000 E-04-1.670000 E-04-1.720000 E-04-1.840000 E-04$ VEXI $2.99500 \Omega E+00 \quad 1.995000 E+00 \quad 1.995000 E+00 \quad 1.994800 E+00 \quad 1.994800 E+00 \quad 1.994800 E+00 \quad 1.994700 E+00 \quad 1.994700 E+00 \quad 1.994700 E+00$ VEXT2 $1.991700 E+00 \quad 1.991800 E+00 \quad 1.991700 E+C 0 \quad 1.991608 E+00 \quad 1.991600 E+00 \quad 1.991600 E+00 \quad 1.991500 E+00 \quad 1.991500 E+00 \quad 1.991500 E+00$ TIME 2.086634E+11 $2.086634 E+12 \quad 2.085634 E+12 \quad 2.086634 E+11 \quad 2.086634 E+11 \quad 2.085634 E+11 \quad 2.085534 E+12 \quad 2.086534 E+11 \quad 2.086534 E+11$ 
Archive

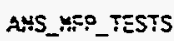

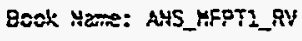

Oate \& Time: 7 Mar 1900 01:04:59

Page Description:

DATA SET NO. -278

TRAHS RV ZERO-1

\author{
RV ZE:0-2 RY ZE:Q0-3
}

RY_LOAD-1

RV_2ERO-5

RV_ZERO-6

PE- $82-7.598: 00 E-\$ 2 \quad-7.592800 E-02 \quad-7.578400 E-02 \quad 9.047000 E-01 \quad 8.242700 E-01 \quad 7.754300 E-01 \quad-7.567900 E-02 \quad-7.564600 E-02 \quad-7.566600 E-02$ $\begin{array}{llllllllll}P E-02 & 2.199700 E-02 & 2.185600 E-02 & 2.185500 E-02 & 2.778900 E+00 & 2.758700 E+00 & 2.798800 E+00 & 2.206300 E-02 & 2.228900 E-02 & 2.183600 E-02\end{array}$ CE- $83-3.316380 E-82 \quad-3.347002 E-02-3.330700 E-02 \quad 1.917900 E+00 \quad 1.910900 E+00 \quad 1.894200 E+00-3.250700 E-02-3.242800 E-02-3.250000 E-02$

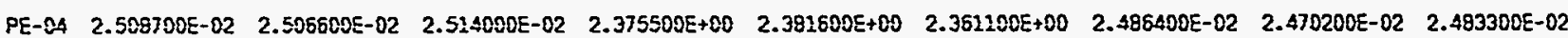

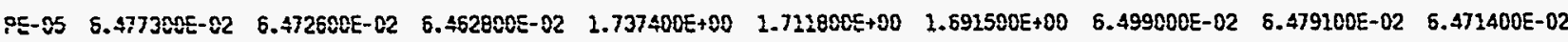
PE-06 5.542200E-02 $5.553200 E-02 \quad 5.546400 E-02 \quad 1.670900 E+00 \quad 1.698000 E+00 \quad 1.689300 E+00 \quad 5.560800 E-02 \quad 5.593700 E-02 \quad 5.575300 E-02$

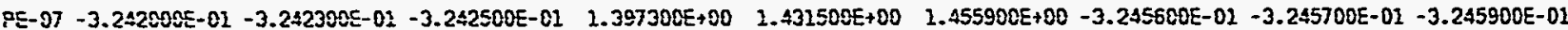
PE- $08-2.643000 E-03 \quad-1.787000 E-03 \quad-1.672000 E-03 \quad 7.655000 E-01 \quad 7.647900 E-01 \quad 7.692100 E-01 \quad-1.233000 E-03 \quad-9.150000 E-04 \quad-9.780000 E-04$

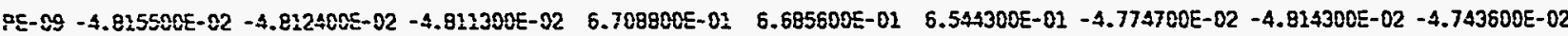
$\begin{array}{llllllllll}P E-10 & 1.303 \triangle 00 E-02 & 1.386500 E-02 & 1.308700 E-02 & 1.617600 E+00 & 1.626800 E+00 & 1.642200 E+00 & 1.362800 E-02 & 1.369700 E-02 & 1.395900 E-02\end{array}$

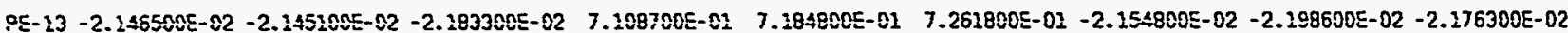
$\begin{array}{llllllllll}P E-14 & 1.205000 E-01 & 1.207900 E-01 & 1.205200 E-01 & 1.332800 E+00 & 1.345500 E+00 & 1.3386000+00 & 1.201509 E-02 & 1.203590 E-01 & 1.203900 E-01\end{array}$

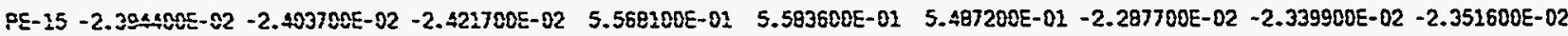
PE-15 3.437500E-\$2 $3.417200 E-02 \quad 3.423100 E-02 \quad 6.241900 E-02 \quad 8.268800 E-01 \quad 8.312000 E-01 \quad 3.490700 E-02 \quad 3.468700 E-02 \quad 3.472000 E-02$ PE-17 - $4.703500 E-02-4.6797005-02-4.703200 E-02-2.268000 E-02-2.201700 E-02-2.089900 E-02-4.676700 E-02-4.727200 E-02-4.720700 E-02$

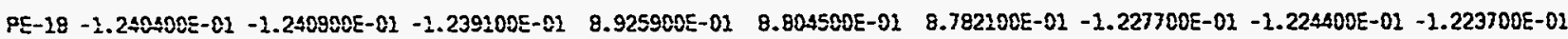

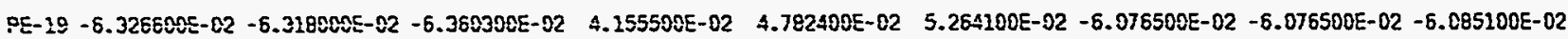

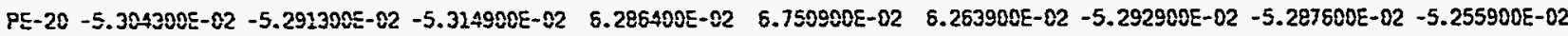
$8 E-21-2.035500 E-02-1.013200 E-02-1.020300 E-02-9.0932005-82-8.823100 E-02-9.145800 E-02-1.026880 E-02-9.895800 E-03-9.991000 E-03$ PE-22 -1.634208E-02 -1.659700E-02 -1.639900E- $02-9.8169005-82-8.935600 E-02-9.604000 E-02-1.565300 E-02-1.673709 E-02-1.669100 E-02$ PE-29 $1.810200 E-02 \quad 1.807900 E-02 \quad 1.805100 E-01 \quad 4.279700 E+00 \quad 4.224200 E+00 \quad 4.267500 E+00 \quad 1.782700 E-01 \quad 1.778100 E-01 \quad 1.775600 E-01$

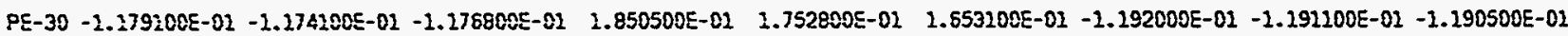
CE- $81 \quad 3.000440 E+00 \quad 1.000420 E+00 \quad 1.000420 E+00 \quad 3.999900 E+00 \quad 3.982700 E+00 \quad 3.986800 E+00 \quad 1.003710 E+00 \quad 1.082790 E+00 \quad 1.002200 E+00$ XE-02 - $1.8000005-25-1.800000 E-05-1.800000 E-05-2.100000 E-05-2.100000 E-05-2.100000 E-05-1.800000 E-05-1.900000 E-05-1.800000 E-05$ XE- $82-7.8130005-03-7.9110005-03-7.807008 E-03-7.804000 E-03-7.810000 E-03-7.802000 E-03-7.918000 E-03-7.910000 E-03-7.903000 E-03$ XE-03 - 2.0050005-03 - $1.005000 E-03-1.006000 E-03-1.063000 E-03-1.064000 E-03-1.065000 E-03-1.067000 E-03-1.065000 E-03-1.067000 E-03$

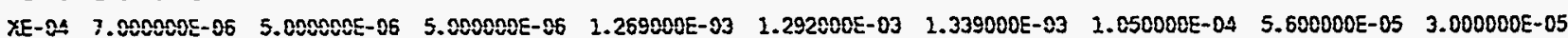
XE-05 $\quad 4.002800 E-02 \quad 4.034300 E-02 \quad 4.171400 E-02 \quad 4.712800 E-02 \quad 4.689200 E-02 \quad 7.193000 E-02 \quad-2.468000 E-03 \quad-2.512000 E-03 \quad-2.529000 E-03$ $X E-86 \quad 5.425200 E-01 \quad 5.435200 E-01 \quad 6.435300 E-01 \quad 3.164600 E-02 \quad 5.474000 E-03 \quad 2.736200 E-02 \quad 7.423100 E-01 \quad 7.445100 E-01 \quad 7.453800 E-01$ $\begin{array}{lllllllllll}X E-07 & 2.122000 E-03 & 2.129000 E-03 & 1.120000 E-03 & 1.962000 E-03 & 1.994000 E-03 & 1.986000 E-03 & 1.479000 E-03 & 1.408000 E-03 & 1.305000 E-03\end{array}$ XE-08 - $4.81 \leq 000 E-03-4.815000 E-03-4.818000 E-03$-4.799000E-03 $-4.800000 E-03-4.805000 E-03-4.779000 E-03-4.786000 E-03-4.791000 E-03$ XE-00 4.022000E-03 $4.020000 E-03 \quad 4.029000 E-03 \quad 6.220000 E-03 \quad 6.323000 E-03 \quad 6.307000 E-03 \quad 4.567000 E-03 \quad 4.536000 E-03 \quad 4.512000 E-03$ XE- $10-1.797000 E-03-1.8010005-03-2.802000 E-03 \quad 1.8\{8000 E-03 \quad 1.9050005-03-7.000000 E-06-1.721000 E-03-1.787000 E-03-1.821000 E-03$ XE-11 - - $2.209000 E-03-1.1980005-03-1.201000 E-03-3.500000 E-05-2.600000 E-05 \quad 4.000000 E-05-9.370000 E-04-1.025000 E-03-1.066000 E-03$ $\begin{array}{llllllllll}X E-12 & 2.523000 E-03 & 2.623000 E-03 & 2.623000 E-03 & 2.804000 E-03 & 2.795000 E-03 & 2.893000 E-03 & 2.632000 E-03 & 2.631000 E-03 & 2.633000 E-03\end{array}$ XE- $13-4.120000 E-04-4.140000 E-04-4.200000 E-04 \quad 1.303000 E-03 \quad 1.340000 E-03 \quad 1.359000 E-03-4.600000 E-05-1.060000 E-04 \quad-1.950000 E-04$ XE- $24-9.992600 E-02 \quad-9.891400 E-02 \quad-9.892500 E-01 \quad-9.935900 E-01 \quad-9.935800 E-01 \quad-9.934700 E-01-9.892400 E-01-9.892500 E-01-9.893700 E-01$ $\begin{array}{lllllllllll}X E-15 & 2.570000 E-04 & 1.600000 E-04 & 1.590000 E-04 & 1.887000 E-03 & 1.915000 E-03 & 1.896000 E-03 & 7.900000 E-04 & 6.030000 E-04 & 4.440000 E-04\end{array}$ TE- $01-1.970000 E-04-1.9600005-04-1.9500005-04-1.6300005-04-1.630000 E-04-2.620080 E-04-1.900080 E-04-2.970000 E-04-2.050000 E-04$ VEXT2 $1.994600 E+00 \quad 1.994600 E+00 \quad 1.994680 E+00 \quad 1.995000 E+00 \quad 2.995000 E+00 \quad 1.994900 E+00 \quad 1.994600 E+00 \quad 1.994600 E+00 \quad 1.994600 E+00$ YEXT2 $\quad 1.991300 E+00 \quad 2.991400 E+00 \quad 1.991400 E+00 \quad 1.991700 E+00 \quad 1.9918005+00 \quad 1.991700 E+00 \quad 1.991400 E+00 \quad 1.991400 E+00 \quad 1.991400 E+00$

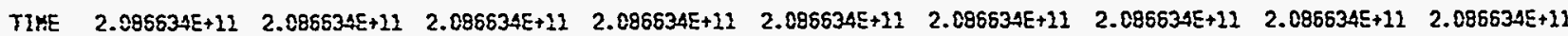


Page Description:

DATA SET $10 .-279$

TRAMS RY_ZERO-1 RE_ZERO-2 RY_ZERO-3 RY_LOAR-1 RY_LOAD-2 RY_LOAD-3 RN_ZERO-4 RY_ZERD-5 RY_ZERQ-6

PE-01 -7.637600E-02 -7.664900E-02 -7.666900E-02 $1.520200 E+00 \quad 1.564700 E+00 \quad 1.618000 E+00-7.597900 E-02-7.623800 E-02 \quad-7.613400 E-02$ $\begin{array}{llllllllll}P E-C 2 & 2.240900 E-02 & 2.151900 E-02 & 2.12060 \Omega E-Q 2 & 4.525800 E+00 & 4.491300 E+00 & 4.459400 E+00 & 2.584500 E-02 & 2.577500 E-02 & 2.598 .400 E-02\end{array}$ $P E-03-3.303500 E-02 \quad-3.296100 E-02 \quad-3.295300 E-02 \quad 3.655200 E+00 \quad 3.316200 E+00 \quad 3.201900 E+00-3.302400 E-02 \quad-3.286600 E-02 \quad-3.274600 E-02$ PE- 04 2.416300E-02 $2.366000 E-02 \quad 2.409100 E-02 \quad 3.310000 E+00 \quad 3.257600 E+00 \quad 3.277200 E+00 \quad 2.470900 E-02 \quad 2.448600 E-02 \quad 2.467400 E-02$ $\begin{array}{llllllllll}\text { PE- } 05 & 6.403800 E-02 & 6.428000 E-02 & 6.414800 E-02 & 1.544200 E+00 & 1.433900 E+00 & 1.3948 C 0 E+00 & 5.550000 E-02 & 6.539400 E-02 & 6.547900 E-02\end{array}$ $\begin{array}{llllllllll}P E-06 & 5.497300 E-02 & 5.490500 E-02 & 5.485000 E-02 & 3.344000 E+00 & 3.455900 E+00 & 3.579000 E+00 & 5.599800 E-02 & 5.605000 E-02 & 5.580000 E-02\end{array}$ $P E-07 \quad-3.2525 C 0 E-01 \quad-3.252720 E-01 \quad-3.252900 E-02 \quad 3.4077005+00 \quad 3.430400 E+80 \quad 3.380700 E+00-3.254800 E-01 \quad-3.254800 E-01 \quad-3.254900 E-01$ $P E-08-1.736 C 00 E-03 \quad-1.987000 E-03 \quad-2.014000 E-03 \quad 3.246200 E+00 \quad 3.152900 E+00 \quad 3.143300 E+00 \quad 1.207000 E-03 \quad 2.593000 E-03 \quad 1.564000 E-03$ $P E-09-4.015100 E-02-4.836800 E-02-4.859500 E-02 \quad 1.292 E 00 E+00 \quad 3.241900 E+00 \quad 1.268900 E+00-4.856500 E-02-4.802200 E-02-4.836100 E-02$ $\begin{array}{llllllllll}P E-10 & 1.309300 E-02 & 1.311300 E-02 & 1.289000 E-02 & 2.131800 E+00 & 2.133400 E+00 & 2.107900 E+00 & 1.347400 E-02 & 1.341900 E-02 & 1.353500 E-02\end{array}$

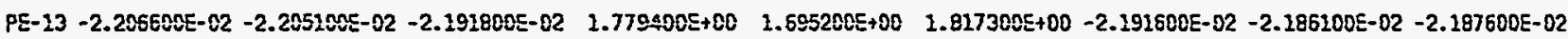

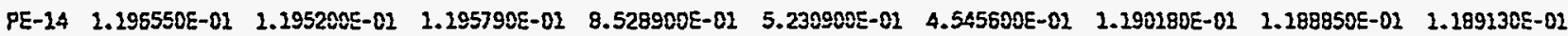

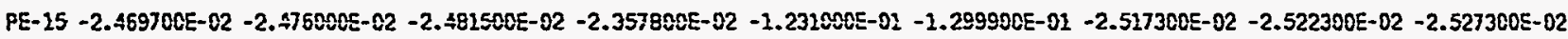
$\begin{array}{llllllllll}P E-16 & 3.432208 E-02 & 3.444800 E-02 & 3.435500 E-02 & 1.552800 E+00 & 1.550600 E+00 & 1.568500 E+00 & 3.465700 E-02 & 3.475400 E-02 & 3.4634005-02\end{array}$ PE-17 -4.726200E-02 -4.702000E-02 -4.714300E-02 1.188A00E+00 $1.184300 E+00$ 1.186400E+00 -4.270500E-02 - $4.257500 E-02-4.268600 E-02$

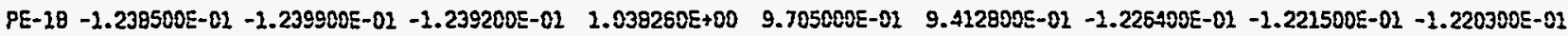
PE-19 -6.149200E-02 -6.12210CE-02 -6.238700E-02 5.437400E-02 3.284700E-02 -5.540000E-02 -5.879200E-02 -5.901000E-02 -5.9C5500E-02

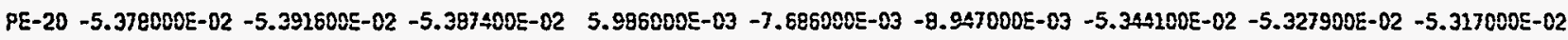

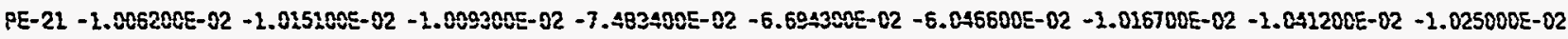
PE-22 -1.704700E-02 - $-1.713500 E-02-1.737700 E-02 \quad 2.620600 E-02 \quad 5.783500 E-02 \quad 6.800500 E-02 \quad-2.720500 E-02 \quad-1.730800 E-02-1.726400 E-02$

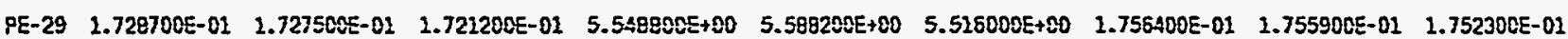

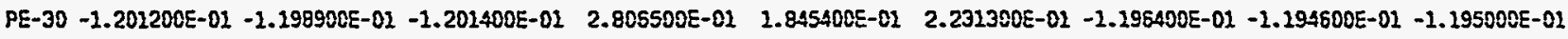

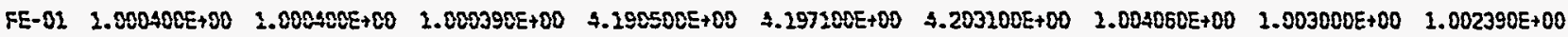
XE-01 -1.800000E-05 - -2.800000E- $05-1.800000 E-05-2.200000 E-05-2.000000 E-05-2.200000 E-05-1.9000005-05-1.800000 E-05-1.800000 E-85$ $X E-02-7.822000 E-03-7.818000 E-03-7.816000 E-93-9.023000 E-03-8.990000 E-03-8.982000 E-03-8.860000 E-03-8.855000 E-03-8.852000 E-03$ $X E-03-1.072000 E-03-2.073000 E-03-1.072000 E-03-2.0850005-03-1.0850005-03-1.085000 E-03-1.086000 E-03-1.086000 E-03-1.085000 E-03$ XE-04 $0.000000 E+00 \quad 0.000000 E+00 \quad 2.000000 E-05 \quad 6.600000 E-04 \quad 1.590002 E-03 \quad 1.731000 E-03 \quad 1.078000 E-03 \quad 1.090000 E-03 \quad 9.330000 E-04$ XE-05 $-2.515000 E-03 \quad-2.5350005-03 \quad-2.529000 E-03 \quad 2.076000 E-03 \quad 1.071400 E-02 \quad 1.777600 E-02 \quad 2.020000 E-04 \quad 1.810000 E-04 \quad 1.350000 E-04$ XEE-05 7.5227COE-01 $7.5237005-01 \quad 7.524800 E-01 \quad 9.864600 E-01 \quad 9.868900 E-01 \quad 9.852100 E-01 \quad 9.765400 E-01 \quad 9.761500 E-01 \quad 9.765700 E-01$ $X E-07 \quad 1.066000 E-03 \quad 2.060000 E-03 \quad 1.058000 E-03 \quad 4.990000 E-04 \quad 1.920000 E-04 \quad-3.800600 E-01 \quad 8.539800 E-01 \quad 9.424400 E-01 \quad 8.019200 E-01$ $X E-8 B-4.8230 C 0 E-03-4.822000 E-03$-4.825CO0E-03 -3.921C00E-03 -3.725000E-03 -3.409000E-03 -4.034000E-03 - 4.044000E-03 -4.050000E-03 XE-09 $\quad 4.422000 E-03 \quad 4.421000 E-03 \quad 4.419000 E-03 \quad 5.293000 E-03 \quad 5.010000 E-03 \quad 5.1010000 E-03 \quad 4.167000 E-03 \quad 4.132000 E-03 \quad 4.1090005-03$ XE-10 -1.910000E-83 - $1.914000 E-03-1.913000 E-03-5.930000 E-04-1.925000 E-03-1.531000 E-03-6.685000 E-03-6.863000 E-03-6.771000 E-03$ XE- 12 - $1.208000 E-03 \quad-1.204000 E-03 \quad-1.202000 E-03 \quad 2.294000 E-03 \quad 2.242000 E-03 \quad 2.214000 E-03 \quad 1.229000 E-03 \quad 1.128000 E-03 \quad 1.0540005-03$ $\begin{array}{lllllllllll}X E-12 & 2.634000 E-03 & 2.6350 \mathrm{CNE}-03 & 2.635000 E-03 & 5.855000 E-03 & 5.81 \leq 000 E-03 & 5.848000 E-03 & 4.358000 E-03 & 4.352000 E-03 & 4.338000 E-03\end{array}$ $X E-13 \quad-3.970000 E-C 4 \quad-3.810000 E-04 \quad-3.860000 E-04 \quad 2.659000 E-03 \quad 2.696000 E-03 \quad 2.8200000 E-03 \quad 7.070000 E-04 \quad 5.050000 E-04 \quad 5.7000005-04$ XE- 14 -9.891000E-01 -9.890000E-01 -9.890700E-01 -3.163800E-01 -7.456000E-01 -7.772700E-01 7.177000E-01 7.180400E-01 7.221400E-01 XEE-15 $1.930000 E-04 \quad 1.950000 E-0.4 \quad 1.790000 E-04 \quad 1.823000 E-03 \quad 1.945000 E-03 \quad 1.975000 E-03 \quad 1.017000 E-03 \quad 8.450000 E-04 \quad 6.850 C 00 E-04$ TE- $01-2.240000 E-04-2.240000 E-04-2.240000 E-04-1.940000 E-04-2.920000 E-04-1.880000 E-04-2.230000 E-04-2.200000 E-044-2.250000 E-04$ VEXT2 $1.994600 E+00 \quad 2.994500 E+00 \quad 2.994500 E+00 \quad 1.994100 E+00 \quad 1.994300 E+00 \quad 1.994300 E+00 \quad 1.993000 E+00 \quad 1.992900 E+00 \quad 1.993009 E+00$ VEXT2 $1.991400 E+00 \quad 1.991400 E+00 \quad 1.991400 E+00 \quad 1.990900 E+00 \quad 1.991000 E+00 \quad 1.990800 E+00 \quad 1.999400 E+00 \quad 1.989300 E+00 \quad 1.989400 E+00$

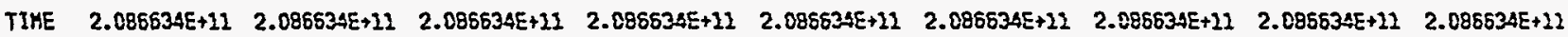


Page Description:

DATA SET MO. -280

TRAMS RY_ZERO-1

RV_ZERO-3

RV_LOAD-1

RY_LOAD-2

RY_LOAD-3

RV_Z2ERO-4

RY_ZERO-5

RV_ZERD-6

PE- $01-7.737800 E-02-7.699500 E-02-7.724300 E-02 \quad 2.637700 E+00 \quad 2.700700 E+00 \quad 2.658700 E+00-7.412900 E-02 \quad-7.399100 E-02 \quad-7.412700 E-02$ $\begin{array}{llllllllll}P E-02 & 2.566500 E-02 & 2.566700 E-02 & 2.559600 E-02 & 4.264100 E+00 & 4.268800 E+00 & 4.132100 E+00 & 2.790200 E-02 & 2.791400 E-02 & 2.802800 E-02\end{array}$ $P E-03 \quad-3.549680 E-02 \quad-3.550502 E-02 \quad-3.574100 E-02 \quad 2.388200 E+00 \quad 2.494200 E+00 \quad 2.475200 E+00 \quad-3.2101005-02 \quad-3.226900 E-02 \quad-3.209900 E-02$ $\begin{array}{llllllllll}P E-04 & 2.526000 E-02 & 2.513700 E-02 & 2.557300 E-02 & 3.430500 E+00 & 3.434800 E+00 & 3.452200 E+00 & 2.730500 E-02 & 2.733400 E-02 & 2.692100 E-02\end{array}$ $\begin{array}{llllllllll}\text { PE-\$5 } & 5.5660305-02 & 6.578808 E-02 & 6.580200 E-02 & 1.317700 E+00 & 1.291100 E+00 & 2.312800 E+80 & 6.658000 E-02 & 6.633900 E-02 & 6.668700 E-02\end{array}$ PE-06 $5.610700 E-02 \quad 5.603700 E-02 \quad 5.511300 E-02 \quad 2.886200 E+00 \quad 2.879400 E+00 \quad 2.860400 E+00 \quad 5.778900 E-02 \quad 5.743300 E-02 \quad 5.781800 E-02$ $P E-07 \quad-3.240000 E-02-3.239700 E-01 \quad-3.239500 E-01 \quad 2.589800 E+00 \quad 2.520000 E+00 \quad 2.598600 E+00-3.236500 E-01 \quad-3.236500 E-01 \quad-3.236600 E-01$ PE- 28 5.95000DE- 04 5.580000E-04 $\quad 7.6700005-04 \quad 3.234400 E+00 \quad 3.150900 E+00 \quad 3.274000 E+00 \quad 2.845000 E-03 \quad 3.050000 E-03 \quad 3.191000 E-03$ PE-09 - $4.806400 E-02-4.838100 E-02-4.808608 E-02 \quad 2.3008088 E+00 \quad 2.366800 E+00 \quad 3.324100 E+00-4.712000 E-02-4.706000 E-02-4.712800 E-02$ $\begin{array}{lllllllllll}P E-10 & 1.280300 E-02 & 1.273600 E-02 & 1.279700 E-02 & 1.728500 E+00 & 1.835500 E+00 & 1.853400 E+00 & 1.517200 E-02 & 1.514300 E-02 & 1.525700 E-02\end{array}$ $8 E-13-2.121100 E-02-2.144400 E-02-2.133200 E-02 \quad 1.155900 E+00 \quad 1.209400 E+00 \quad 1.2505 C 0 E+00-2.101000 E-02-2.101000 E-02-2.135100 E-02$ $\begin{array}{lllllllllll}P E-14 & 1.191790 E-01 & 1.193130 E-02 & 1.193760 E-01 & 1.982700 E+00 & 2.031300 E+00 & 2.061200 E+00 & 1.222500 E-01 & 1.223100 E-01 & 1.220700 E-01\end{array}$ PE- $15-2.474500 E-02-2.509200 E-02-2.515300 E-02 \quad-2.793400 E-02-2.789100 E-01-2.787000 E-02-2.357500 E-02-2.389400 E-02-2.370100 E-02$ $\begin{array}{lllllllllll}P E-16 & 3.145900 E-02 & 3.472900 E-02 & 3.479200 E-02 & 2.459500 E+00 & 1.458380 E+00 & 1.471800 E+00 & 3.492800 E-02 & 3.465900 E-02 & 3.483300 E-02\end{array}$ PE-17 - $-1.231 \leqslant 00 E-02-4.230600 E-02-4.207800 E-02 \quad 1.099130 E+00 \quad 3.051170 E+00 \quad 1.064470 E+00-4.233700 E-02-4.251500 E-02-4.264900 E-02$

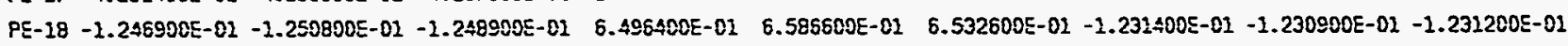
PE-19 -5.827200E-02 -5.820400E-02 -5.826000E-02 -8.517800E-02 -8.772600E-02 -9.918700E-02 $-5.7632005-02-5.788100 E-02-5.805500 E-02$ $P E-20-5.359800 E-02-5.359900 E-02-5.392900 E-02 \quad-2.452500 E-02-1.9155005-02-1.2825005-02-5.308800 E-02 \quad-5.306700 E-02-5.265700 E-02$ $P E-21-1.046 \leq 005-02-1.0210085-02-1.036300 E-02-8.4999005-02-8.658200 E-02-9.0245005-02-1.027700 E-02-1.014000 E-02-9.885000 E-03$ PE-22 - $1.652700 E-02$ - $1.655400 E-02 \quad-1.526390 E-02 \quad 9.273900 E-82 \quad 8.764700 E-02 \quad 5.566900 E-02 \quad-1.597700 E-02 \quad-1.564600 E-02 \quad-1.591500 E-02$

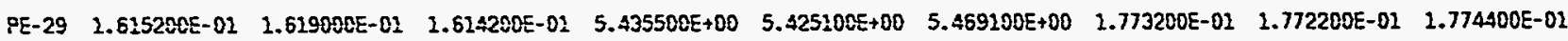

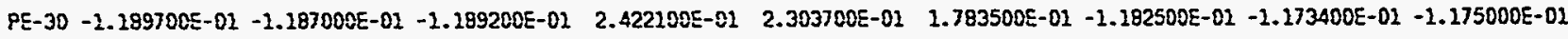
FE-O1 $\quad 2.000170 E+00 \quad 1.080288 E+00 \quad 2.000280 E+00 \quad 4.255800 E+00 \quad 4.254500 E+00 \quad 4.269200 E+00 \quad 1.004370 E+00 \quad 1.003190 E+00 \quad 1.002480 E+00$ $X E-01-2.900000 E-05-2.000000 E-05-2.000000 E-05-2.300000 E-05-2.200000 E-05-2.100000 E-05-2.000000 E-85-2.000000 E-05-1.900000 E-05$ XE- $02-8.7820005-83-8.7820005-03-8.780000 E-83-8.452000 E-03-8.443000 E-03-8.444000 E-03-7.654000 E-03-7.645000 E-03-7.653000 E-03$ XE- $03-5.880000 E-84-5.870000 E-04-5.950000 E-04-8.350000 E-04-8.270000 E-04-8.220000 E-84-8.150000 E-04-8.140000 E-04-8.240000 E-04$ $X E-04 \quad 9.620000 E-04 \quad 9.830000 E-04 \quad 9.740000 E-04 \quad 1.656000 E-03 \quad 1.647000 E-03 \quad 1.302000 E-03 \quad-3.6000005-04 \quad-4.740000 E-04 \quad-5.000000 E-04$ $X E-85 \quad 7.361000 E-03 \quad 7.286000 E-03 \quad 7.615080 E-03 \quad 7.3400005-04 \quad 1.042000 E-03 \quad 6.404000 E-03 \quad-1.461000 E-03 \quad-1.624000 E-03 \quad-1.615000 E-03$ XE-06 9.770800E-01 $9.770700 E-01 \quad 9.770700 E-01 \quad 9.919300 E-01 \quad 9.919100 E-01 \quad 1.009000 E-03 \quad-6.900000 E-05$ - $2.050000 E-04 \quad-1.260000 E-04$ $X E-07 \quad 7.913500 E-01 \quad 7.912300 E-02 \quad 7.912800 E-01 \quad-6.394700 E-02-8.845300 E-02 \quad-3.860800 E-01 \quad 9.679100 E-02 \quad 9.690600 E-01 \quad 9.703400 E-02$ XE- $08-4.1500005-83-4.156000 E-03-4.157000 E-03-3.036000 E-03-3.054000 E-03-2.849000 E-03-3.168000 E-03-3.231000 E-03-3.241000 E-03$ XE-09 $3.975000 E-03 \quad 3.976000 E-03 \quad 3.977000 E-03 \quad 4.0290005-03 \quad 3.820000 E-03 \quad 6.090000 E-04-7.600000 E-04 \quad-8.200000 E-04 \quad-8.700000 E-04$ XE- $10-6.547008 E-83-6.544000 E-03-6.549000 E-03-5.750000 E-04-6.190000 E-04-1.414000 E-03-3.328008 E-03-3.437000 E-03-3.470000 E-03$

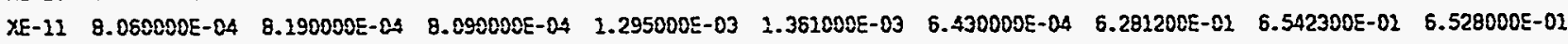
XE-12 4.15EO0尺E-03 $\quad 4.157000 E-03 \quad 4.256000 E-03 \quad 5.537000 E-03 \quad 5.517000 E-03 \quad 1.275000 E-03 \quad 6.480000 E-04 \quad 6.290000 E-04 \quad 6.150000 E-04$ $\begin{array}{lllllllllll}X E-13 & 2.590000 E-04 & 1.510000 E-04 & 1.510000 E-04 & 2.683000 E-03 & 2.761000 E-03 & 2.642000 E-03 & 9.580000 E-04 & 8.410000 E-04 & 7.520000 E-04\end{array}$

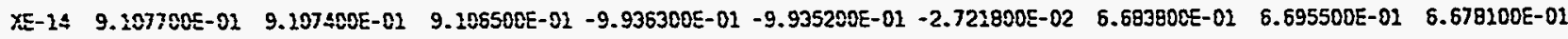
XE-15 $\quad 3.730000 E-04 \quad 3.640000 E-04 \quad 3.720000 E-04 \quad 1.465000 E-03 \quad 1.505000 E-03 \quad 1.800000 E-03 \quad 6.610000 E-04 \quad 5.310000 E-04 \quad 4.220000 E-04$

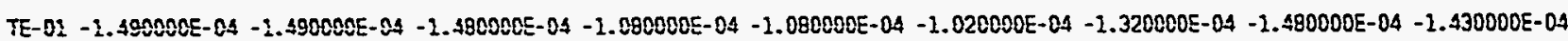
$\begin{array}{lllllllllll}V E X T 1 & 1.992900 E+00 & 1.992900 E+00 & 1.992900 E+00 & 1.995000 E+00 & 1.994800 E+00 & 1.994900 E+00 & 1.993200 E+00 & 1.993200 E+00 & 1.993200 E+00\end{array}$

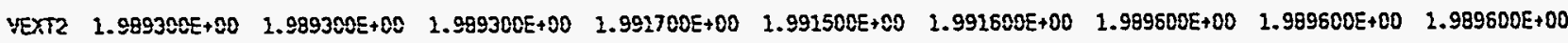

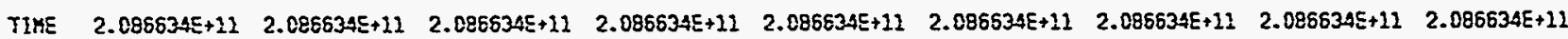


Page description:

DATA SET HO.-282

TRAHS RY_ZERO-2 RV_ZERO-2 RV_ZERO-3 RY_LOAD-1 $\quad$ RY_LOAD-2 $\quad$ RY_LOAD-3 RV_ZERO-4 $\quad$ RV_ZERO-5 RY_ZERO-6

PE-01 -7.492200E-02 -7.499800E-02 -7.503000E-02 3.680400E+00 3.694700E+00 2.881700E+00 -7.316880E-02 -7.313300E-02 -7.291900E-02 PE-02 2.782600E-02 2.754100E-02 $2.757800 E-02 \quad 4.846200 E+00 \quad 4.912700 E+00 \quad 4.869400 E+00 \quad 2.854700 E-02 \quad 2.8497 C 0 E-02 \quad 2.842800 E-02$ $P E-03 \quad-3.275400 E-02-3.305700 E-02-3.285800 E-02 \quad 1.793400 E+00 \quad 1.853000 E+00 \quad 1.836300 E+00-3.381100 E-02-3.365800 E-02-3.363900 E-02$ $\begin{array}{llllllllll}P E-04 & 2.666600 E-02 & 2.684700 E-02 & 2.664100 E-02 & 3.245100 E+00 & 3.250900 E+00 & 3.302600 E+00 & 2.308400 E-02 & 2.708500 E-02 & 2.679700 E-02\end{array}$ $\begin{array}{llllllllll}P E-05 & E .639900 E-02 & 6.614580 E-02 & 5.607800 E-02 & 3.256400 E+C O & 3.222700 E+00 & 2.579100 E+00 & 6.647300 E-02 & 6.636900 E-02 & 5.654200 E-02\end{array}$ $\begin{array}{llllllllll}P E-06 & 5.657100 E-02 & 5.705400 E-02 & 5.683100 E-02 & 3.686500 E+00 & 3.693200 E+00 & 3.588200 E+00 & 5.753600 E-02 & 5.775700 E-02 & 5.749800 E-02\end{array}$ $P E-07-3.242000 E-01 \quad-3.242100 E-01 \quad-3.242100 E-01 \quad 3.037000 E+00 \quad 3.00210 C E+00 \quad 2.962500 E+00-3.243400 E-01 \quad-3.243500 E-01 \quad-3.243700 E-01$ $\begin{array}{lllllllllll}P E-08 & 2.398000 E-03 & 2.086000 E-03 & 2.192000 E-03 & 2.511000 E+00 & 2.521100 E+00 & 2.492400 E+00 & 5.770000 E-04 & 4.670000 E-04 & 3.230000 E-04\end{array}$ PE- $09-4.760700 E-02-4.768900 E-02-4.789900 E-02 \quad 3.275409 E+00 \quad 1.363800 E+00 \quad 1.317100 E+00-4.711360 E-02-4.717700 E-02-4.683100 E-02$ $\begin{array}{llllllllll}P E-10 & 1.399200 E-02 & 2.397300 E-02 & 1.417500 E-02 & 2.284700 E+00 & 2.277900 E+00 & 2.438600 E+00 & 2.458100 E-02 & 1.504800 E-02 & 1.473100 E-02\end{array}$ PE-13 -2.25AEODE-02 -2.191200E-02 -2.135100E-02 2.156700E+00 2.198400E+00 $1.990900 E+00-1.963000 E-02-1.975400 E-02 \quad-2.009680 E-02$

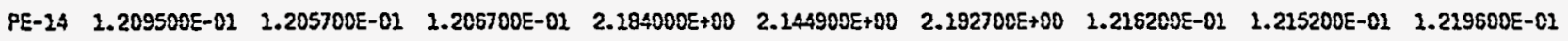
PE-15 -2.359200E-02 -2.338900E-02 - 2.364900E-02 $2.540709 E+00 \quad 1.511400 E+00 \quad 1.142900 E+00-2.361200 E-82 \quad-2.338000 E-02 \quad-2.393000 E-02$ $P E-16 \quad 3.482000 E-02 \quad 3.490200 E-02 \quad 3.461900 E-02 \quad 1.510200 E+00 \quad 1.556400 E+00 \quad 1.536500 E+00 \quad 3.491200 E-02 \quad 3.478500 E-02 \quad 3.496200 E-02$ $P E-17$-4.241600E-02 -4.271600E-02 -4.250900E-02 6.900600E-01 $6.497900 E-01 \quad 5.514600 E-01-4.253300 E-02-4.245300 E-02-4.258500 E-02$ $P E-18-1.237200 E-01-1.235100 E-01-1.232500 E-02-6.016600 E-02-2.517300 E-02-2.629300 E-02-1.230600 E-01-1.234000 E-01-1.235000 E-01$ FE- $19-5.779808 E-02-5.793700 E-02-5.790508 E-02-2.135900 E-02-3.377700 E-02-8.820800 E-02-5.752200 E-02-5.761300 E-02-5.761400 E-02$ $P E-20 \quad-5.393600 E-02 \quad-5.403900 E-02 \quad-5.425900 E-02 \quad 7.032700 E-02 \quad 7.000500 E-02 \quad 2.797500 E-02 \quad-5.400700 E-02 \quad-5.395400 E-02 \quad-5.341900 E-02$ PE-22 - $1.027900 E-02-2.008600 E-02-1.089900 E-02 \quad 8.432800 E-02 \quad 8.046400 E-02-1.119700 E-02-9.5930005-03-9.822000 E-03-1.001800 E-02$ $P E-22-1.557400 E-02-2.590600 E-02-1.530300 E-02 \quad 9.674100 E-02 \quad 1.083930 E-02 \quad 1.204460 E-01-1.529300 E-02 \quad-1.629800 E-02 \quad-1.643500 E-02$ $\begin{array}{lllllllllll}\text { PE-29 } & 2.791900 E-02 & 1.7990005-21 & 1.788280 E-01 & 5.389100 E+00 & 6.399700 E+00 & 6.377900 E+00 & 1.808500 E-01 & 2.801200 E-01 & 1.796100 E-01\end{array}$

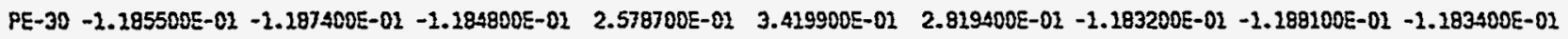

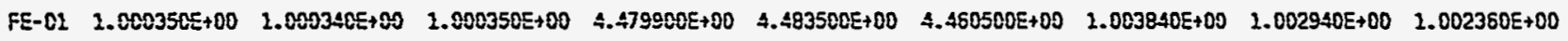
$X E-02-2.000000 E-05-2.900000 E-05-2.000000 E-05-2.300000 E-05-2.200000 E-05-2.300000 E-05-2.000000 E-05-1.900000 E-85-2.000000 E-05$ $X E-02-7.598000 E-03-7.598000 E-03-7.592000 E-03-7.676000 E-03-7.670000 E-03-7.679000 E-03-7.730000 E-03-7.723000 E-03-7.721000 E-03$ XE- $03-8.220000 E-04-8.220000 E-04-8.210000 E-04-9.430000 E-04-9.360200 E-04-9.280000 E-04-9.400000 E-04-9.390000 E-04-9.390000 E-04$ XE-04 $-5.740000 E-04 \quad-5.600000 E-04 \quad-5.880000 E-04 \quad 1.753000 E-03 \quad 1.922000 E-03 \quad 2.843000 E-03 \quad 4.920000 E-04 \quad 4.280000 E-04 \quad 5.060000 E-04$ $X X E-05 \quad-1.764000 E-03 \quad-2.818000 E-03 \quad-1.770000 E-03 \quad 2.190800 E-02 \quad 5.411000 E-03 \quad 7.371000 E-03 \quad-3.012700 E-02 \quad-2.925100 E-02 \quad-3.187200 E-01$ XE-06 -2.820000E-04 $-2.920000 E-04 \quad-2.840000 E-04 \quad 3.1940005-03 \quad 3.385000 E-03 \quad 5.248000 E-03 \quad 3.158000 E-03 \quad 3.236000 E-03 \quad 3.1210000-03$ XE-07 9.725500E-01 $9.725500 E-01 \quad 9.724500 E-01 \quad-6.992400 E-02$ - $4.935500 E-01$-4.593700E-01 $9.594800 E-01 \quad 9.492400 E-01 \quad 7.776800 E-02$ $x E-88-3.297000 E-03 \quad-3.299000 E-03 \quad-3.282000 E-03 \quad 9.847900 E-01 \quad 9.854900 E-01 \quad 9.986600 E-01 \quad 9.892500 E-01 \quad 9.899300 E-01 \quad 9.901900 E-01$ XE-09 $-2.022000 E-03 \quad-2.023000 E-03 \quad-1.024000 E-03 \quad 1.359000 E-03 \quad 1.2490005-03 \quad 1.646000 E-03 \quad 1.100000 E-05 \quad-6.300000 E-05 \quad-1.200000 E-04$

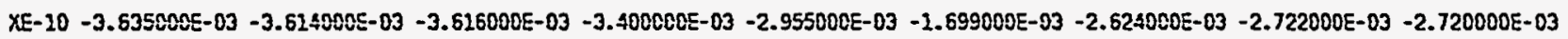
XE-12 5.984900E-01 5.911500E-01 5.806900E-01 $-9.240002 E-04 \quad-1.104000 E-03 \quad-1.629000 E-03 \quad-2.329000 E-03-2.418000 E-03-2.468000 E-03$ YE-12 $5.32008 \Omega E-04 \quad 5.3200005-04 \quad 5.298000 E-04 \quad 1.280000 E-03 \quad 1.377000 E-03 \quad 2.136000 E-03 \quad 1.495000 E-03 \quad 1.482000 E-03 \quad 1.473000 E-03$ XE-13 3.230000E-04 $3.650000 E-04 \quad 4.040000 E-04 \quad 2.956000 E-03 \quad 2.946000 E-03 \quad 3.012000 E-03 \quad 1.175000 E-03 \quad 1.043000 E-03 \quad 9.080000 E-04$

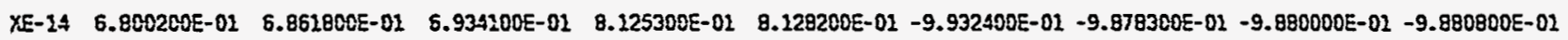
XE-15 $\quad 3.100000 E-05 \quad 2.800000 E-05 \quad-3.000000 E-05 \quad 2.236000 E-03 \quad 2.289000 E-03 \quad 2.417000 E-03 \quad 1.292000 E-03 \quad 2.047000 E-03 \quad 9.380000 E-04$ TE-02 -1.530000E-04 - - $3.530000 E-04-1.530000 E-04$-1.270000E-04 -1.280000E-04 -1.260000E-04 - $1.590000 E-04-1.700000 E-04-1.710000 E-04$ VEXT1 $1.993200 E+00 \quad 1.993200 E+00 \quad 1.993200 E+00 \quad 1.993600 E+0 D \quad 1.993700 E+00 \quad 1.994800 E+00 \quad 1.994100 E+00 \quad 1.994100 E+00 \quad 1.994200 E+00$ VEXT2 $1.989600 E+00 \quad 1.989600 E+00 \quad 1.989600 E+00 \quad 1.990100 E+00 \quad 1.990000 E+00 \quad 1.991600 E+00 \quad 1.990900 E+00 \quad 1.990900 E+00 \quad 1.991000 E+00$

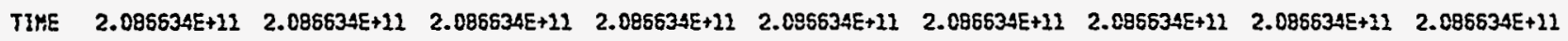


Archive Hente:

AHS_MEP_TESTS

BoOK Heme: AHS_MEPTI_RS

Eate \& Time: 7 yar 1900 81:56:22

Page Description:

CATA SET MQ. -282

TRAYS RY_ZERO-2 RY_ZERO-2 RY_ZERO-3 RY_LOAD-2 RY_LOAD-2 RY_LOAO-3 RY_ZERO-4 RV_ZERO-5 RY_ZERO-6

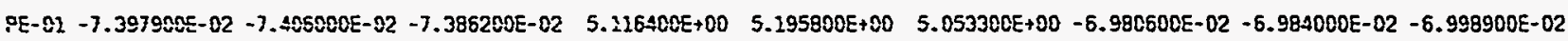
$P E-02 \quad 2.842800 E-02 \quad 2.840700 E-02 \quad 2.849800 E-02 \quad 5.379700 E+00 \quad 5.301900 E+00 \quad 5.246400 E+00 \quad 2.921100 E-02 \quad 2.945700 E-02 \quad 2.928900 E-02$ RE-83 -3.4228C0E- $02 \quad-3.416500 E-02 \quad-3.415580 E-02 \quad 2.954200 E+00 \quad 3.002100 E+00 \quad 2.979680 E+00-3.243500 E-02 \quad-3.257000 E-02 \quad-3.257300 E-02$ $\begin{array}{llllllllll}P E-04 & 2.688200 E-02 & 2.647400 E-02 & 2.638000 E-02 & 2.528 E 00 E+00 & 2.659800 E+00 & 2.716900 E+00 & 2.650000 E-02 & 2.633100 E-02 & 2.635700 E-02\end{array}$ PE- 05 6.564000E-02 $6.579600 E-02 \quad 6.596700 E-02 \quad 4.177600 E+00 \quad 4.208900 E+00 \quad 3.964500 E+00 \quad 6.581000 E-02 \quad 6.665000 E-02 \quad 6.658300 E-02$ PE-8S 5.653200E-82 5.65_EOQE- $02 \quad 5.662600 E-02 \quad 5.143100 E+00 \quad 5.236909 E+00 \quad 5.173400 E+00 \quad 5.754100 E-02 \quad 5.767300 E-02 \quad 5.743400 E-02$ $: P E-07-3.2490085-21 \quad-3.249100 E-02-3.249200 E-02 \quad 3.798700 E+00 \quad 3.873300 E+00 \quad 3.886300 E+00-3.250800 E-01 \quad-3.250900 E-01-3.251100 E-01$ PE-09 9.800000E-05 $-9.100000 E-05 \quad 1.000000 E-06 \quad 1.739300 E+00 \quad 1.809200 E+00 \quad 1.899100 E+00 \quad 7.620000 E-04 \quad 9.350000 E-04 \quad 6.240000 E-04$ PE- $99-4.7219005-92-4.765500 E-02-4.760300 E-02 \quad 3.579300 E+00 \quad 4.105800 E+00 \quad 3.971200 E+80-4.624000 E-02-4.642900 E-02-4.649200 E-02$ PE-10 $1.387600 E-02 \quad 1.384000 E-02 \quad 1.389700 E-02 \quad 3.809400 E+00 \quad 3.954400 E+00 \quad 3.740200 E+00 \quad 1.430600 E-02 \quad 1.422300 E-02 \quad 1.403500 E-02$

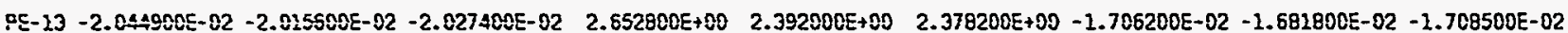

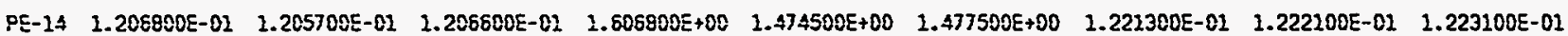
PE- $15-2.347700 E-02 \quad-2.3463005-02-2.351100 E-02 \quad 1.185600 E+00 \quad 2.069100 E+00 \quad 1.693700 E+00-2.345800 E-02 \quad-2.333100 E-02 \quad-2.340900 E-02$ PE-15 3.44J700E-02 $3.456300 E-02 \quad 3.444800 E-02 \quad 1.935900 E+00 \quad 1.290400 E+00 \quad 1.079530 E+00 \quad 3.402300 E-02 \quad 3.426900 E-02 \quad 3.432700 E-02$ $9 E-17-4.294200 E-02+4.277500 E-02-4.294500 E-02 \quad 1.559600 E+00 \quad 1.148100 E+00$ 8.257000E-01 - $1.254000 E-02-4.267600 E-02-4.282100 E-02$

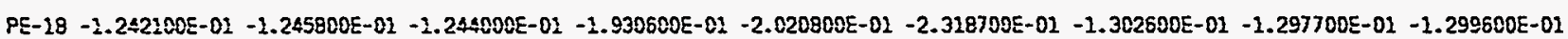
$P E-19-5.795300 E-02-5.801800 E-02-5.809500 E-02-5.986800 E-02-1.949200 E-02-2.812800 E-02-5.839400 E-02-5.835000 E-02-5.854700 E-02$ PE-20 -5. $130800 E-02 \quad-5.436400 E-02 \quad-5.445400 E-02 \quad 7.150500 E-02 \quad-2.531400 E-02 \quad-2.496500 E-02 \quad-5.436900 E-02 \quad-5.427700 E-82 \quad-5.427400 E-02$ PE-22 $-9.897000 E-03-9.718000 E-03-1.0265025-02 \quad 5.603100 E-02 \quad 3.974200 E-02 \quad 1.910500 E-02-1.016800 E-02 \quad-9.911000 E-03-1.021300 E-02$ $P E-22-1.661500 E-02-1.667500 E-02 \quad-1.669300 E-02 \quad 1.054160 E-01 \quad 9.413100 E-02 \quad 9.777600 E-02 \quad-1.705300 E-02 \quad-2.685600 E-82 \quad-1.695700 E-02$ $\begin{array}{lllllllllll}P E-22 & 1.750000 E-01 & 1.754900 E-01 & 1.755000 E-02 & 7.128700 E+00 & 7.131600 E+00 & 7.083400 E+00 & 1.783100 E-01 & 1.783400 E-01 & 1.780100 E-01\end{array}$

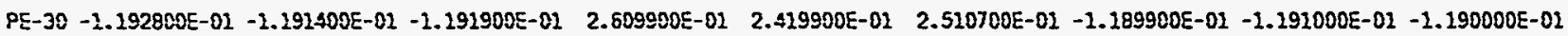
FE-01 $\quad 1.000400 E+00 \quad 1.000390 E+00 \quad 1.000370 E+00 \quad 4.319500 E+00 \quad 4.298800 E+00 \quad 4.314700 E+00 \quad 1.004840 E+00 \quad 1.003490 E+00 \quad 1.002710 E+00$ XE-01 $-2.000000 E-05 \quad-2.000000 E-05 \quad-2.000000 E-05 \quad 1.370000 E-04 \quad 2.600000 E-05 \quad 9.200000 E-05 \quad 1.270000 E-04 \quad 1.190000 E-04 \quad 1.180000 E-04$ $X E-02-7.629000 E-03-7.625000 E-03$-7.622000E-03 -9.002000E-03 -8.029000E-03 -7.679090E-03 -8.058000E-03 -8.063000E-03 -8.054000E-03 XE-03 -9.420000E-04 -9.410000E-04 -9.400000E-04 - $1.457000 E-03-1.058000 E-03-7.440000 E-04-7.950000 E-04-7.510000 E-04-7.400000 E-04$ XE- DS 5.420000E- OS $5.480000 E-24 \quad 5.510000 E-04 \quad 3.051000 E-03 \quad 3.311000 E-03 \quad 2.912000 E-03 \quad 2.810000 E-04 \quad 4.990000 E-04 \quad-2.000000 E-06$

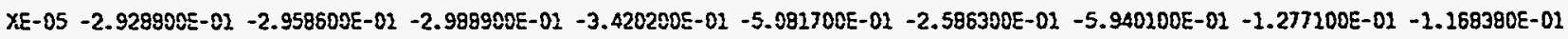
XE-05 $3.0230005-03 \quad 3.026000 E-03 \quad 3.026000 E-03 \quad 5.437000 E-03 \quad 6.433000 E-03 \quad 6.143000 E-03 \quad 4.641000 E-03 \quad 4.590000 E-03 \quad 4.560000 E-03$ $X E-07 \quad 9.852700 E-01 \quad 9.823700 E-01 \quad 9.821000 E-01 \quad-4.977200 E-01 \quad 8.650200 E-02$-2.251400E-01 $5.002700 E-01 \quad 9.283000 E-01 \quad 5.962000 E-02$

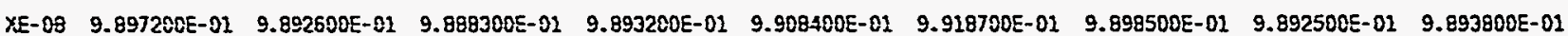
XE-09 -2.770000E-04 -2.800000E-04 -2.880000E-04 8.170000E-04 -1.380000E-04 -4.450000E-04 -8.370000E-04 -9.440000E-04 -1.025000E-03 $x E-10-2.744000 E-03-2.728000 E-03-2.730000 E-03-5.365000 E-03-4.966000 E-03-3.743000 E-03-5.599000 E-03-5.995000 E-03-6.050000 E-03$ XE-11 $-2.702000 E-03 \quad-2.709000 E-03 \quad-2.712000 E-03$ 9.380000E-01 $-7.708200 E-01$-4.203300E-02 $9.891600 E-01$ 9.876100E-01 9.874400E-01 $X E-12 \quad 1.453000 E-03 \quad 3.453000 E-03 \quad 1.453000 E-03 \quad 9.230000 E-04 \quad 2.690000 E-03 \quad 1.360000 E-04 \quad 9.390000 E-04 \quad 9.050000 E-04 \quad 9.720000 E-04$ $\begin{array}{lllllllllll}\text { XE-13 } & 5.270000 E-04 & 6.230000 E-04 & 5.890000 E-04 & 2.362000 E-03 & 2.252000 E-03 & 2.348000 E-03 & 1.168000 E-03 & 1.020000 E-03 & 9.090900 E-04\end{array}$ $X E-14$-9.871300E-01 -9.870700E-01 $-9.367600 E-01 \quad-2.088300 E-01$-9.708900E-01 $-7.499300 E-01 \quad 5.603200 E-01 \quad 5.638600 E-01 \quad 6.750500 E-01$ XE-15 $\quad 6.040000 E-04 \quad 5.160000 E-04 \quad 5.820000 E-04 \quad 3.803000 E-03 \quad 3.560000 E-03 \quad 3.617000 E-03 \quad 2.519000 E-03 \quad 2.295000 E-03 \quad 2.120000 E-03$

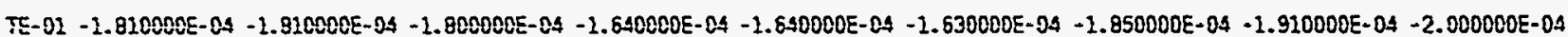
VEXT1 $1.994100 E+00 \quad 1.994200 E+00 \quad 1.994100 E+00 \quad 1.993800 E+00 \quad 1.994700 E+00 \quad 1.995000 E+00 \quad 1.993100 E+00 \quad 1.992600 E+00 \quad 1.992600 E+00$ YEXT2 $1.990900 E+00 \quad 1.990900 E+00 \quad 1.990900 E+00 \quad 1.990100 E+00 \quad 1.992000 E+00 \quad 1.992000 E+00 \quad 1.969300 E+00 \quad 1.988700 E+00 \quad 1.988700 E+00$ TIKE $2.086534 E+12 \quad 2.086534 E+11 \quad 2.086634 E+11 \quad 2.086534 E+11 \quad 2.0865634 E+11 \quad 2.086634 E+112.086634 E+112.086534 E+11 \quad 2.085634 E+11$ 


\section{Internal Distribution}

1. B. R. Appleton

2. R. L. Battiste

3. D. K. Felde

4. R. G. Gilliland

5. R. C. Gwaltney

6. W. R. Hendrich

7-10. R. L. Johnson

11. C. R. Luttrell

12. D. L. Selby
13-17. W. F. Swinson

18. C. D. West

19. G. T. Yahr.

20. ORNL Patent Office

21-22. Central Reserach Library

23. Y-12 Technical Library Document Reference Section

24-25. Laboratory Records Dept.

26. Laboratory Records, RC

\section{External Distribution}

27. U.S. Department of Energy, ANS Project Office, Oak Ridge Operations Office, FEDC, MS-8218, P.O. Box 2009, Oak Ridge, TN 37831-8218

28-29. Office of Scientific and Technical Information, P.O. Box 62, Oak Ridge, Tennessee 37831 
